\title{
32nd Annual Meeting and Pre-Conference Programs of the Society for Immunotherapy of Cancer (SITC 2017): Part One
}

National Harbor, MD, USA. 8-12 November 2017

Published: 7 November 2017

\section{About this supplement}

These abstracts have been published as part of Journal for ImmunoTherapy of Cancer Volume 5 Supplement 2, 2017. The full contents of the supplement are available online at https://jitc.biomedcentral.com/articles/supplements/volume-5-supplement-2. Please note that this is part 1 of 2.

\section{Oral presentations}

\section{1}

Identification of unique neoantigen qualities in long-term pancreatic cancer survivors

Vinod Balachandran', Marta Luksza², Julia N. Zhao', Vladimir Makarov' John Alec Moral ${ }^{1}$, Romain Remark ${ }^{3}$, Brian Herbst', Gokce Askan', Umeshkumar Bhanot ${ }^{1}$, Yasin Senbabaoglu', Danny Wells ${ }^{4}$, Charles lan Ormsby Cary ${ }^{4}$, Olivera Grbovic-Huezo', Marc Attiyeh', Benjamin Medina' Jennifer Zhang', Jennifer Loo', Joseph Saglimbeni', Mohsen Abu-Akeel', Roberta Zappasodi', Nadeem Riaz', Martin Smoragiewicz', Olca Basturk', Mithat Gönen ', Arnold J. Levine'2, Peter J. Allen', Douglas T. Fearon' ${ }^{6}$, Miriam Merad ${ }^{3}$, Sacha Gnjatic ${ }^{3}$, Christine lacobuzio-Donahue', Jedd Wolchok', Ronald DeMatteo ${ }^{7}$, Benjamin Greenbaum ${ }^{8}$, Taha Merghoub', Steven Leach

${ }^{1}$ Memorial Sloan Kettering Cancer Center, New York, NY, USA; ${ }^{2}$ Institute for Advanced Study, Princeton, NJ, USA; ${ }^{3}$ Icahn School of Medicine at Mount Sinai, New York, NY, USA; ${ }^{4}$ Parker Institute for Cancer Immunotherapy, San Francisco, CA, USA; ${ }^{5}$ University of Cambridge, Cambridge, UK; ${ }^{6}$ Cold Spring Harbor, Cold Spring Harbor, NY, USA; ${ }^{7}$ University of Pennsylvania, Philadelphia, PA, USA; ${ }^{8}$ Tisch Cancer Institute, New York, NY, USA

Correspondence: Vinod Balachandran (balachav@mskcc.org) Journal for ImmunoTherapy of Cancer 2017, 5(Suppl 2):01

\section{Background}

Pancreatic adenocarcinoma (PDAC) is a lethal cancer with $<7 \%$ of patients surviving past 5 years. T cell immunity has been linked to the exceptional outcome of the rare long-term survivors but the antigens are unknown.

Methods

To identify T cell antigens in long-term PDAC survivors, we assembled the largest cohorts of stage-matched short $[n=68$, median overall survival (OS) $0.8 \mathrm{y}$ ] and long-term PDAC survivors $(\mathrm{n}=82$, median OS 6y). To enable antigen discovery, we performed a combined analysis of whole exome sequencing, neoantigen prediction, $\mathrm{T}$ cell receptor (TCR) V $\beta$-chain sequencing, 9-color multiplexed immunohis- tochemistry, and tumor transcriptomic profiling. To assess differential neoantigen immunogenicity, we performed neoantigen fitness modeling integrating clonal genealogy, epitope homology, and $\mathrm{T}$ cell receptor affinity. To examine in vivo $T$ cell-neoantigen reactivity, we used functional assays in a subset of very long-term PDAC survivors ( $n=7$, median OS 10.5 years).

Results

We found that tumors of long-term survivors displayed 12-fold greater cytolytic $\mathrm{CD}^{+}{ }^{+} \mathrm{CD}^{+}$Granzyme- $\mathrm{B}^{+}$cells, with $>94 \%$ of intratumoral $\mathrm{T}$ cell clones unique to tumors and not shared with adjacent normal pancreatic tissue, suggesting intratumoral antigen recognition. Tumors of long-term survivors exhibited greater TCR repertoire diversity compared to tumors of short-term survivors, implying differential antigenic targets. In examining neoantigens as potential T cell targets, we found that patients with both the highest predicted neoantigen number and the greatest $\mathrm{CD}^{+} \mathrm{CD}^{+}$infiltrates together but not either parameter alone, exhibited the longest survival (median OS not reached vs. $0.8 y, P=0.004$ ). In investigating possible unique neoantigen qualities in long-term survivors, a neoantigen quality model conferring greater immunogenicity to dominant neoantigens with homology to microbial epitopes identified longterm survivors independent of confounding variables and chemotherapy (Neoantigen Quality ${ }^{\mathrm{Hi}}$ vs. Low - median OS 8.6y vs. 0.8y; $\mathrm{P}=0.002$ ) whereas a model ascribing greater immunogenicity to increasing neoantigen number did not (Neoantigen Quantity ${ }^{\text {Hi vs. Low }}$ median OS $0.8 y$ vs. 1y; $P=0.3$ ) (Fig. 1). Similarly, neoantigen quality, but not quantity, was independently prognostic of survival in a larger, independent cohort unselected by survival $(n=166$; Neoantigen

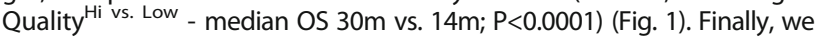
detected lasting circulating $T$ cell reactivity to both high quality neoantigens and cross-reactive antigens in PDAC survivors, including identical intratumoral clones with specificity to both ( $n=5$ of 7 patients tested). Conclusions

Our results identify neoantigens with unique qualities as $T$ cell targets in PDAC. More broadly, we identify neoantigen quality as a biomarker for immunogenic tumors that may facilitate rational application of immunotherapies. 


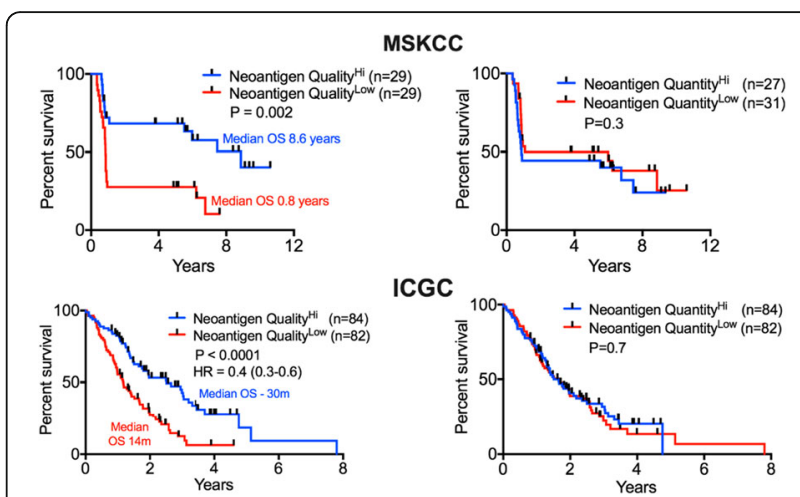

Fig. 1 (abstract 01). Neoantigen quality is prognostic of pancreatic cancer survival

02

Predictive biomarkers for response to anti-CTLA-4 and anti-PD-1 immunotherapy in melanoma patients

Priyanka Subrahmanyam ', Zhiwan Dong ${ }^{2}$, Daniel Gusenleitner ${ }^{2}$, Anita Giobbie-Hurder', Mariano Severgnini'2, Jun Zhou'², Michael Manos², Holden Maecker ${ }^{1}$, F. Stephen Hodi ${ }^{2}$

${ }^{1}$ Stanford University, Stanford, CA, USA; ${ }^{2}$ Dana-Farber Cancer Institute and Harvard Medical School, Boston, MA, USA

Correspondence: Priyanka Subrahmanyam (pri3@stanford.edu) Journal for ImmunoTherapy of Cancer 2017, 5(Suppl 2):O2

\section{Background}

Immune checkpoint blockade has greatly improved clinical outcomes in melanoma patients. However, there is an urgent need for predictive biomarkers to determine who will likely benefit most from which therapy. To date, most biomarkers of response have been identified in the tumors themselves. Biomarkers that could be assessed from peripheral blood would be even more desirable, because of ease of access and reproducibility of sampling.

Methods

We used mass cytometry (CyTOF), which is a technique similar to flow cytometry, but uses metal-ion tagged antibodies for highdimensional immune profiling. We performed CyTOF on pretreatment peripheral blood mononuclear cells from melanoma patients to identify potential biomarkers of response to anti-CTLA-4 or anti-PD-1 immunotherapy.

Results

Our studies revealed that anti-CTLA-4 and anti-PD- 1 therapies have distinct sets of candidate biomarkers. Responders to anti-CTLA-4 therapy had higher memory $\mathrm{CD} 4^{+}$and $\mathrm{CD}^{+} \mathrm{T}$ cells compared to non-responders. Furthermore, responders showed higher frequencies of $\mathrm{CD}^{+}$effector memory T cells. In anti-PD-1 (but not anti-CTLA-4) treated patients, responders had higher frequencies of CD69 and MIP-1 $\beta$ expressing NK cells. Finally, using multivariate analysis, distinct models for the prediction of response to anti-CTLA-4 and antiPD-1 were developed.

\section{Conclusions}

Our findings point to an important role for memory $\mathrm{T}$ cell subsets, as well as NK cells in response to immunotherapy, making them potential predictive biomarker candidates for response to checkpoint blockade.
03

Combining immunophenomics with a gene expression panel for improved prostate cancer recurrence prediction Nathalie Harder', Harald Hessel', Maria Athelogou', Alexander Buchner ${ }^{2}$, Christian Stief ${ }^{2}$, Thomas Kirchner ${ }^{2}$, Günter Schmidt ${ }^{1}$, Ralf Huss ${ }^{1}$

${ }^{1}$ Definiens AG, Munich, Germany; ${ }^{2}$ Ludwig-Maximilians-University

Munich, Munich, Germany

Correspondence: Nathalie Harder (nharder@definiens.com)

Journal for ImmunoTherapy of Cancer 2017, 5(Suppl 2):O3

\section{Background}

Multi-omics data provide rich information on different levels such as genome, transcriptome, function or phenotype. Integrating such comprehensive measures offers great potential for identifying improved prognostic and predictive signatures in the context of precision medicine. Here we combine gene expression data with phenomic readouts extracted from immunohistochemically stained sections of resected prostate cancer ( $\mathrm{PCa}$ ) tissue. By integrating both data sets we identify multi-variate signatures with high prognostic value regarding prostate cancer recurrence after radical prostatectomy.

Methods

Data was collected from a cohort of 23 PCa patients (Gleason-score 6-9, pT2, age $<=75 y$ ). Consecutive FFPE tissue sections were dual-stained for immune-related (CD3/CD8, CD68/CD163) and structural (CD34, CK18/ p63) components. Using Tissue Phenomics, we extracted image-based features, e.g., densities, ratios, and distances of positive cells across differently stained sections after co-registration in specific regions-ofinterest (tumor, TME, stroma) [1]. Gene data (NanoString Technologies, Seattle, nCounter PanCancer Immune Profiling Panel) was collected from sections containing tumor only and normalized w.r.t. housekeeping genes. We separately selected subsets of image-based and gene-based features by optimizing a cutoff regarding the uni-variate stratification performance (log-rank test, accuracy) on random data subsets using bootstrapping. Features from both sets were combined by Classification and Regression Trees (depth=2) and the best performing combinations were identified using cross validation. Clinical parameters (age, Gleason-score, PSA blood value) provided no prognostic value as shown by Cox regression analysis.

Results

Several tree models showed improved cross-validated stratification performance compared to all uni-variate stratifications using either feature set (Fig. 1). We found that (I) a low expression of C4B gene in combination with a low ratio of $\mathrm{CD} 8(+)$ in stroma to $\mathrm{CD} 3(+) \mathrm{CD} 8(-)$ in tumor $\left(p_{\text {crossval }}<0.05\right)$, and (II) a high expression of CD46 gene in combination with a low average distance of CD34(+) microvessels to neighboring $\mathrm{CD} 168(+) \mathrm{M} 2$ macrophages $\left(\mathrm{p}_{\text {cross Val }}<0.05\right.$ ) are strong prognostic factors for prostate cancer recurrence.

Conclusions

Our results suggest that an inhibited activity of the innate as well as the adaptive immune system provides prognostic value for prostate cancer recurrence. Both genes $\mathrm{C} 4 \mathrm{~B}$ and CD46 are related to the complement system where $\mathrm{C} 4 \mathrm{~B}$ positively correlates with complement activity while CD46 is an inhibiting complement regulator. Limited activation of CD8(+) cytotoxic T cells as well as increased activity of M2 macrophages fostering angiogenesis additionally contribute to the cancer promoting environment. Combining information from genome and phenome therefore helps gaining a broadened picture of factors promoting PCa progression.

\section{Reference}

1. Athelogou M, et al. Tumor-associated macrophages as prognostic marker for prostate cancer progression. SITC. 2016. 
04

Clinical activity of adenosine $2 \mathrm{~A}$ receptor (A2AR) inhibitor CPI-444 is associated with tumor expression of adenosine pathway genes and tumor immune modulation

Andrew Hotson ${ }^{1}$, John Powderly², Leisha Emens ${ }^{3}$, Patrick Forde ${ }^{3}$, Matthew Hellman', Lawrence Fong ${ }^{5}$, Ben Markman ${ }^{6}$, Brett Hughes' Jonathan Goldman ${ }^{8}$, Mario Sznol ${ }^{9}$, Daruka Mahadevan ${ }^{10}$,

Shivaani Kummar ${ }^{11}$, Joshua Brody ${ }^{12}$, Philip Bonomi ${ }^{13}$, Jason Luke ${ }^{14}$, Matthew Riese ${ }^{15}$, Taofeek Owonikoko ${ }^{16}$, Sherene Loi ${ }^{17}$, Amy Wiese $^{18}$, Robert Doebele ${ }^{19}$, James Lee ${ }^{20}$, Chunyan Gu ${ }^{21}$, Stephen Willingham ${ }^{21}$ Ginna Laport ${ }^{21}$, Richard Miller ${ }^{21}$, lan McCaffery ${ }^{21}$

${ }^{1}$ Corvus Pharmaceuticals, Redwood City, CA, USA; ${ }^{2}$ Carolina BioOncology Institute, Huntersville, NC, USA; ${ }^{3}$ Johns Hopkins University, Baltimore, MD, USA; ${ }^{4}$ Memorial Sloan Kettering Cancer Center, New York City, NY, USA; ${ }^{5}$ University of California, San Francisco, San Francisco, CA, USA; ${ }^{6}$ Monash Medical Centre, Clayton, Australia; ${ }^{7}$ Royal Brisbane and Women's Hospital, Herston, Australia; ${ }^{8}$ University of California, Los Angeles, Los Angeles, CA, USA; ${ }^{9}$ Yale University School of Medicine, New Haven, CT, USA; ${ }^{10}$ University of Arizona, Tucson, AZ, USA; ${ }^{11}$ Stanford University School of Medicine, Stanford, CA, USA; ${ }^{12}$ Icahn School of Medicine at Mount Sinai, New York City, NY, USA; ${ }^{13}$ Rush University Medical Center, Chicago, IL, USA; ${ }^{14}$ University of Chicago Medical Center, Chicago, IL, USA; ${ }^{15}$ Medical College of Wisconsin, Milwaukee, WI, USA; ${ }^{16}$ Emory University Hospital, Atlanta, GA, USA; ${ }^{17}$ Peter MacCallum Cancer Centre, Melbourne, Australia; ${ }^{18}$ Wayne State University, Detroit, MI, USA; ${ }^{19}$ University of Colorado Anschutz Medical Campus, Aurora, CO, USA;

${ }^{20}$ University of Pittsburgh, Pittsburgh, PA, USA; ${ }^{21}$ Corvus Pharmaceuticals, Burlingame, CA, USA

Correspondence: lan McCaffery (imccaffery@corvuspharma.com) Journal for ImmunoTherapy of Cancer 2017, 5(Suppl 2):04

\section{Background}

CPI-444, an A2AR antagonist, inhibits suppression of T cell function by adenosine and is active in multiple preclinical models. CPI- 444 is being investigated as a single agent and in combination with atezolizumab in an ongoing Phase $1 / 1 \mathrm{~b}$ clinical trial in patients with advanced cancers (NCT02655822). Biomarker investigations were conducted to explore immune modulation in serial tumor biopsies and peripheral blood as well associations between adenosine pathway genes and clinical activity.

\section{Methods}

Tumor biopsies and blood samples were analyzed in 80 patients with renal cell carcinoma (RCC: $n=35$ ), non-small cell lung cancer (NSCLC: $n=26$ ), triple negative breast cancer (TNBC: $n=17$ ), microsatellite instable colorectal cancer (MSI-CRC $n=2$ ) treated with CPI-444 100mg BID as single agent (' $\mathrm{SA}$ ' $\mathrm{n}=18$ ) or in combination with atezolizumab ('combo' $n=62$ ). Paired tumor biopsies were analyzed: gene expression profiles (Nanostring), CD8, PD-L1 and CD73 (IHC). T cell repertoires were examined by sequencing of the $\mathrm{T}$ cell receptor beta chain gene in PBMCs and tumors.

Results

Of 80 evaluable patients, 54 had progressed on prior anti-PD-(L) 1 therapy ('IO-refractory'), 26 were IO-naïve. Most patients were PD-L1 negative in archival tumor ( $80 \%$ TNBC, $91 \%$ RCC, $69 \%$ NSCLC).

Six objective responses were observed: 2 partial responses (PR) in RCC (1 SA and 1 combo); 2 PRs in NSCLC (both combo); 1 PR MSICRC (combo) and 1 PR in TNBC (combo). Notably, 3 PRs were observed in IO-refractory patients (1 RCC, 2 NSCLC) and 2 PRs were PD-L1 negative (1 RCC, 1 NSCLC).

SA and combo treatment significantly increased CD8+ cell infiltration (mean 2-fold; $95 \% \mathrm{Cl} 1.18-2.91$ ), T cell activation and interferongamma pathway gene expression in biopsies as well as $\mathrm{T}$ cell clone expansion in both tumor biopsies and periphery. Shared clonotypes expanded in matched post-dose PBMCs and tumor suggesting trafficking of T cells following CPI-444 treatment. This included IOrefractory and PD-L1-negative patients.
Patients with elevated CD73 and expression of adenosine genes in baseline tumor samples experienced significant tumor regression (best \% change: high-CD73 $-5.6 \%$ regression; low-CD73 $17.9 \%$ growth; $\mathrm{p}$-value $=0.03, \mathrm{n}=40$ ). In IO-refractory patients, CD73 expression and adenosine pathway genes were higher than IO-naïve patients at baseline suggesting adenosine pathway is a mechanism of immune escape in IO-refractory patients.

Conclusions

$\mathrm{CPI}-444$ anti-tumor activity was associated with immune-modulation of T cells in tumor and periphery, including IO-refractory and PD-L1 negative patients. Adenosine pathway is upregulated in IO-refractory patients and may be associated with clinical response to CPI-444 supporting a role for $\mathrm{CPI}-444$ in treatment of IO-refractory tumors.

Trial Registration

ClinicalTrials.gov Identifier NCT02655822

05

A dendritic cell targeting NY-ESO-1 vaccine significantly augments early and durable immune responses in melanoma patients pretreated with human Flt-3 Ligand

Nina Bhardwaj ${ }^{1}$, Philip Friedlander ${ }^{2}$, Anna Pavlick ${ }^{3}$, Marc Ernstoff ${ }^{4}$, Brian Gastman ${ }^{5}$, Brent Hanks ${ }^{6}$, Mark Albertini ${ }^{7}$, Jason Luke ${ }^{8}$,

Michael Yellin ${ }^{9}$, Tibor Keler ${ }^{9}$, Thomas Davis ${ }^{9}$, Laura Vitale ${ }^{9}$, Ana Blazquez $^{10}$, Chihiro Morishima ${ }^{11}$, Patrick Danaher ${ }^{12}$, Sarah Warren ${ }^{12}$, Bob Salim ${ }^{13}$, Bruce Hess ${ }^{11}$, Leonard D'Amico ${ }^{11}$, Lisa Lundgren ${ }^{14}$, Nora Disis ${ }^{11}$, Martin Cheever ${ }^{14}$, Steven Fling ${ }^{14}$

${ }^{1}$ Icahn School of Medicine at Mount Sinai, New York, NY, USA; ${ }^{2}$ Mount Sinai Beth Israel, New York, NY, USA; ${ }^{3}$ NYU Lagone Medical Center, New York, NY, USA; ${ }^{4}$ Roswell Park Cancer Institute, Buffalo, NY, USA;

${ }^{5}$ Cleveland Clinic, Cleveland, OH, USA; ${ }^{6}$ Duke, Durham, NC, USA;

${ }^{7}$ University of Wisconsin, Madison, WI, USA; ${ }^{8}$ University of Chicago

Medicine, Chicago, IL, USA; ${ }^{9}$ Celldex Therapeutics, Needham, MA, USA;

${ }^{10}$ Mount Sinai, New York, NY, USA; ${ }^{11}$ University of Washington, Seattle, WA, USA; ${ }^{12}$ Nanostring Technologies, Seattle, WA, USA; ${ }^{13}$ Axio Research, Seattle, WA, USA; ${ }^{14}$ Fred Hutchinson Cancer Research Center, Seattle,

WA, USA

Correspondence: Steven Fling (sfling@fhcrc.org)

Journal for ImmunoTherapy of Cancer 2017, 5(Suppl 2):O5

\section{Background}

Patients with high-risk melanoma, (AJCC TNM stage II and stage III disease), have a $20-60 \%$ recurrence rate with 5 -year overall survival (OS) between $45 \%$ and $70 \%$. The adjuvant setting is an opportunity to test prevention vaccines that may have efficacy against disease recurrence. Vaccine therapy with CDX-1401 (a fusion protein consisting of human monoclonal lgG1 antibody targeting the dendritic cell (DC) receptor DEC-205 linked to the NY-ESO-1 tumor antigen) can safely lead to humoral and cellular immunity in cancer patients with advanced malignancies that express NY-ESO-1, including melanoma. CDX-301, a recombinant human Flt3 ligand (Flt3L), safely produces increases in DC in humans and may enhance vaccine responses through increased DC number and activity. We evaluated CDX-301 and CDX-1401 combination treatment in a phase II, open-label, multicenter, randomized study of subjects with resected melanoma, to determine whether immune responses to NY-ESO-1 elicited by vaccination with CDX-1401 + poly-ICLC are substantially increased by prior expansion of circulating DC with Flt3L therapy.

Methods

60 patients with resected melanoma were randomized to two cohorts: Cohort 1 received CDX-301 (Flt3L) pretreatment (25 ug/kg SC x 10 days) in two of four monthly cycles of vaccination with CDX-1401 $(1 \mathrm{mg} \mathrm{IC})+$ poly-ICLC (2mg SC, days 1 and 2). Cohort 2 received 4 monthly cycles of vaccine with CDX-1401 and poly-ICLC without prior Flt3L. We also assessed immunogenicity to other melanoma- 
associated antigens and memory viral responses, character of PBMC subsets, and safety, tolerability and clinical efficacy of the regimens. Results

Both treatments were well tolerated with grade 1-2 AEs of chills, injection site erythema and pain, fever and myalgias most common. A substantial increase of between $\sim 15-$ to $\sim 200$-fold of innate immune cells (DC, monocytes and NK cells) was observed in subjects treated with CDX-301. Further, there was development of higher anti-NY-ESO-1 antibody titers and NY-ESO-1 specific T cells in cohort 1 vs. cohort 2, and evidence of induction of antigen-specific CD8+ T cells in cohort 1. Comparative immune cell gene expression profiling of PBMC are consistent with these differences and reveal a gene signature associated with Flt3L induction of an early and durable immune response.

\section{Conclusions}

DC mobilization with vaccines targeting DC is safe and significantly enhances vaccine responses to widely expressed tumor associated antigens.

Trial Registration

ClinicalTrials.gov Identifier NCT02129075

06

Re-educating macrophage through glutamine metabolism-mediated metabolic and epigenetic reprogramming

Ping-Chih Ho (ping-chih.ho@unil.ch)

University of Lausanne, Epalinges, Switzerland

Journal for ImmunoTherapy of Cancer 2017, 5(Suppl 2):06

\section{Background}

The different regulatory mechanisms orchestrating macrophage activation, including signaling cascades and epigenetic programming, are increasingly appreciated. Among these regulatory mechanisms, differences in the bioenergetic demands of $\mathrm{M} 1$ and $\mathrm{M} 2$ macrophages are emerging as new regulatory circuits that adjust a macrophage's behavior in response to the nutrient state in its habitation and the infected tissues. Glutamine metabolism provides synergistic support for macrophage activation and elicitation of desirable immune responses; however, the underlying mechanisms regulated by glutamine metabolism to orchestrate macrophage activation remain unclear.

Methods

We examine metabolic and epigenetic reprogramming in bone marrow-derived macrophages under M1 and M2 skewing condition. We also examine the impact of glutamine metabolism on supporting endotoxin tolerance format in bone marrow-derive macrophages in vitro and in the in vivo septic shock model. The anti-tumor responses of glutaminase 1 targeting approach was further evaluated in MC-38 and Braf/Pten melanoma-bearing mice in conjunction with or without adoptive tumor-specific T cell transfer.

Results

Here we show that the production of aa-ketoglutarate via glutaminolysis is important for alternative (M2) activation of macrophages, including engagement of fatty acid oxidation (FAO) and histone H3K27 demethylase Jmjd3-dependent epigenetic reprogramming of $\mathrm{M} 2$ genes. This M2-promoting mechanism is further modulated by a high aa-ketoglutarate/succinate ratio, while a low ratio strengthens the proinflammatory phenotype in classically (M1) activated macrophages. As such, a-ketoglutarate contributes to endotoxin tolerance following M1 activation. This study reveals new mechanistic regulations by which glutamine metabolism tailors the immune responses of macrophages through metabolic and epigenetic reprogramming. We further provide proof-of-concept evidence that targeting glutaminolysis can tailor the immune response of tumor-associated macrophages in vivo, and co- treating glutaminase inhibitor with adoptive tumor-specific $T$ cell transfer can effectively suppress tumor growth.

Conclusions

This study highlights the unrecognized mechanisms controlled by glutaminolysis to fine-tune macrophage activities and advances modulation of glutamine metabolism as an attractive strategy for harnessing macrophage-mediated immune responses. Most importantly, targeting glutaminase is able to re-educate tumor-associated macrophages and provides synergistic anti-tumor responses with adoptive cell transfer therapy. Altogether, our work uncovers new regulatory circuits orchestrated by glutamine metabolism in macrophages and reveals the potential of these mechanisms on harnessing macrophage immune responses.

07

Early FDG-PET response correlates with dose and efficacy in patients with microsatellite stable $\mathrm{MCRC}$ treated with carcinoembryonic antigen $\mathrm{T}$ cell bispecific (CEA-TCB) antibody plus atezolizumab Said Bouseida ${ }^{1}$, Federico Sandoval', Daniel Sabanes Bove ${ }^{2}$,

Vaios Karanikas', Abiraj Keelara', Tapan Nayak', Jose Saro ${ }^{3}$

${ }^{1}$ Roche Innovation Center Basel, Basel, Switzerland; ${ }^{2}$ F. Hoffmann-La Roche Ltd., Basel, Switzerland; ${ }^{3}$ Roche Innovation Center Zurich, Zurich, Switzerland

Correspondence: Said Bouseida (said.bouseida@roche.com)

Journal for ImmunoTherapy of Cancer 2017, 5(Suppl 2):07

\section{Background}

CEA-TCB (RG7802, RO6958688) is a novel T cell bispecific antibody targeting CEA on tumor cells and CD3 on T cells. An ongoing Phase Ib study (NCT02650713) is exploring the safety, tolerability and efficacy of CEA-TCB in combination with atezolizumab (anti-PD-L1). Here, we report preliminary results of ${ }^{18} \mathrm{~F}$-fluorodeoxyglucose-positron emission tomography (FDG-PET) imaging as an early pharmacodynamic marker for this novel cancer immunotherapy combination in patients with microsatellite stable (MSS) metastatic colorectal cancer (mCRC).

\section{Methods}

In this study, CEA-TCB was given weekly in combination with atezolizumab $1200 \mathrm{mg}$ every 3 weeks in patients with CEA-expressing solid tumors. On-treatment FDG-PET scans were performed at week 4 and compared with baseline. On-treatment changes in maximum standardized uptake value $\left(S U V_{\text {max }}\right)$, metabolic tumor volume (MTV) and total lesion glycolysis (TLG) were analyzed in up to 10 measurable lesions, identified at baseline by an independent reviewer, in each patient. Exploratory statistical analyses used semiparametric Gaussian regression models and progression-free survival (PFS) was assessed using Cox proportional hazard landmark analyses.

Results

As of March 3, 2017, a total of 35 patients with MSS mCRC were treated with CEA-TCB doses ranging from $5 \mathrm{mg}$ to $160 \mathrm{mg} ; 17$ patients were evaluable for PET image analysis. Of the 15 PET-evaluable patients, 8 (53\%) had early (week 4) on-treatment reductions in SUV $_{\max }>15 \%$ with CEATCB plus atezolizumab. Early decreases in SUV ${ }_{\max }$ appeared to correlate with increasing CEA-TCB doses $(P=0.0081)$, with greater and consistent reductions seen at $\geq 80 \mathrm{mg}$. Partial metabolic response was observed in 6 of 15 patients, with all responders treated at $\geq 80 \mathrm{mg}$. Decreases in $\mathrm{SUV}_{\max }$ appeared to be associated with reduction in tumor burden (best change from baseline per RECIST v1.1; $P=0.0061$ ). Depth of reduction in MTV appeared to be associated with longer PFS $(P=0.013)$. In addition, reductions in MTV and TLG appeared to correlate with decreases in soluble CEA (sCEA) at week 6 (MTV, $P=0.013 ;$ TLG, $P=0.034$ ). Data from an expanded patient population treated with CEA-TCB as monotherapy and in combination with atezolizumab will be presented. 


\section{Conclusions}

In patients with MSS $\mathrm{mCRC}$, changes in MTV and SUV max $_{\text {correlated with }}$ longer PFS along with tumor shrinkage, and decreases in 2 FDG parameters, MTV and TLG, correlated with a decline in soluble tumor marker, sCEA. Thus, early on-treatment changes in FDG-PET can serve as a pharmacodynamic biomarker related to treatment efficacy with CEA-TCB.

Trial Registration

NCT02650713

\section{8}

Lactic acid as a mediator of metabolic symbiosis between regulatory $\mathrm{T}$ cells and the tumor microenvironment

McLane Watson', Ryan Whetstone', Rahul Deshpande ${ }^{1}$, Ashely Menk', Nicole Scharping ${ }^{1}$, Brett Morrison ${ }^{2}$, Stacy Gellhaus Wendell ${ }^{1}$

Greg Delgoffe

${ }^{1}$ University of Pittsburgh, Pittsburgh, PA, USA; ${ }^{2}$ Johns Hopkins, Baltimore, MD, USA

Correspondence: Greg Delgoffe (delgoffeg@umpc.edu)

Journal for ImmunoTherapy of Cancer 2017, 5(Suppl 2):08

\section{Background}

The tumor microenvironment (TME) presents a major barrier to effective immunotherapy. Metabolically harsh conditions starve infiltrating effector $T$ cells rendering them ineffective. In contrast, regulatory $T\left(T_{\text {reg }}\right)$ cells, a subset of CD4+ T cells that play a vital role in preventing autoimmunity and maintaining immune homeostasis, thrive in the TME. As a result, the TME becomes more immunosuppressive. It has been shown that $T_{\text {reg }}$ cells have a distinct metabolic profile compared to their effector $T$ cell counterparts. Therefore, we hypothesized that $T_{\text {reg }}$ cells thrive because they are metabolically supported by the TME.

Methods

B16, a mouse model of melanoma, was used. From tumor bearing mice conventional effector $T$ cells and $T_{\text {reg }}$ cells were transcriptionally and metabolically profiled using flow cytometry, proliferation assays, suppression assays, and isotopic flux analysis. A mouse with a $T_{\text {reg }}$ specific deletion of $\mathrm{MCT} 1$, a predominant lactate transporter was generated. A pharmacological inhibitor of MCT1 was used to prevent lactate uptake. Results

Transcriptional and metabolic profiling revealed $\mathrm{T}_{\text {reg }}$ cells from the tumor upregulate a distinct metabolic profile utilizing the glycolytic end-product, lactic acid. Lactic acid has been known to be immunosuppressive, and indeed, it curbed the proliferation of conventional effector T cells in vitro. However, lactic acid had the opposite effect on $\mathrm{T}_{\text {reg }}$ cells, promoting their proliferation. Isotopic flux analysis revealed lactate is utilized by $T_{\text {reg }}$ cells to generate glycolytic intermediates, in part through gluconeogenic pathways. Preventing lactate transport in $\mathrm{T}_{\text {reg }}$ cells through a conditional knockout of MCT1 resulted in mice with normal immune homeostasis, but superior anti-tumor immunity when implanted with melanoma. Similarly, in wild type tumor bearing mice, preventing lactate uptake through a pharmacological inhibitor resulted in decreased intratumoral $T_{\text {reg }}$ proliferation and increased efficacy of checkpoint blockade immunotherapy.

\section{Conclusions}

These data suggest that tumors evade the immune system, in part, by metabolically supporting suppressive $T_{\text {reg }}$ cells with lactic acid. These data also suggest that targeting lactate metabolism may increase the efficacy of checkpoint blockade immunotherapy.

\section{9}

Transcriptional approach to understanding the role of tonic signaling and co-stimulation in CAR T cells

Angela Boroughs ${ }^{1}$, Nemanja D. Marjanovic ${ }^{2}$, Ana P. Castano ${ }^{3}$,

Robert Morris ${ }^{3}$, Livnat Jerby ${ }^{2}$, Felipe Bedoya ${ }^{3}$, Aviv Regev²,

Marcela V. Maus ${ }^{3}$

'Massachusetts General Hospital, Harvard University, Boston, MA, USA;

${ }^{2}$ Broad Institute of Harvard and MIT, Cambridge, MA, USA;

${ }^{3}$ Massachusetts General Hospital, Boston, MA, USA

Correspondence: Angela Boroughs (angelaboroughs@fas.harvard.edu) Journal for Immunotherapy of Cancer 2017, 5(Suppl 2):09

\section{Background}

Chimeric antigen receptors (CARs) targeting CD19 have produced impressive outcomes for the treatment of $B$ cell malignancies. Using RNA sequencing, we determined the transcriptional changes downstream of CARs with different co-stimulation domains pre-and postantigen stimulation.

Methods

T cells were sorted from 3 donors and transduced with one of four CAR constructs. The constructs all contained a CD19-directed scFv fused to one of four different intracellular domains: 4-1BB $, \mathrm{CD} 28 \zeta, \zeta$ and $\Delta \zeta$. Cells were expanded with $C D 3 / C D 28$ beads for 5 days and the rested. On day 12, the T cells were stimulated through their CAR or TCR for 0,4 or 24 hours. T cells were flow-sorted on viable lymphocytes, CAR+, CD3+ and CD4+ or CD8+ and then sequenced using the SmartSeq2 protocol. Reads were aligned and quantified. Differential gene expression calculated using DESeq2. Significant differentially expressed (DE) genes had a FDR cutoff of $<0.05$.

Results

T cells containing CARs have clearly distinct transcriptional signatures both from each other and from untransduced T cells. We identified a gene signature for tonic signaling of all $\zeta$-containing CAR T cells, which was identified in transduced resting $T$ cells prior to antigen stimulation through the CAR. These genes were DE between untransduced or $\Delta \zeta$ vs $4-1 \mathrm{BB} \zeta, \mathrm{CD} 28 \zeta$, $\zeta$ CARs. Furthermore, we identified a set of genes that is DE between $41 B B \zeta$ and CD28 C CAR T cells. Some of these genes such as ENNP2 are DE before antigen stimulation through the CAR, while other genes were significantly DE only after antigen stimulation through the CAR. There was a clear upregulation of HLA Class II genes as well as CIITA, which controls MHCIl upregulation, in activated $41 \mathrm{BB} \zeta$ vs $28 \zeta$ CAR T cells. Across all time points there was an enrichment for human $T_{H} 1$ signature genes in the 41BB compared to the CD28 CD4+ CAR T cells ( $p$-value $<0.0005)$. Cytokines, IL-15 and IL-21 were both significantly upregulated in $41 \mathrm{BB}$ \ vs $\mathrm{CD} 28$ \ CARs by 24 hours after CAR stimulation.

Conclusions

We have identified a transcriptional signature that reflects tonic signaling in CAR T cells bearing the $C D 3 \zeta$ signaling domain, independent of the co-stimulation domain. Differences between $41 \mathrm{BB} \zeta$ and $28 \zeta$ have been previously identified several days following CARantigen stimulation. Here we show many of these differences exist before CAR-mediated activation, indicating a transcriptional effect of tonic signaling. We also defined a transcriptional program in $41 \mathrm{BB} \zeta$ vs $C D 28 \zeta$ CAR T cells that polarizes CD4+ cells to a more $T_{H} 1$ like state pre-and post-signaling.

\section{0}

Gamma secretase inhibition increases recognition of multiple myeloma by BCMA-specific chimeric antigen receptor modified $\mathrm{T}$ cells

Tyler Hill, Margot Pont, Joe Abbott, Jessica Kelliher, Alexander Salter, Steven Liu, Damian Green, Stanley Riddell

Fred Hutchinson Cancer Research Center, Seattle, WA, USA

Correspondence: Margot Pont (mpont@fredhutch.org)

Journal for ImmunoTherapy of Cancer 2017, 5(Suppl 2):010

\section{Background}

B cell maturation antigen (BCMA) is expressed on most cases of multiple myeloma (MM) cases and can be targeted by $T$ cells transduced with an anti-BCMA chimeric antigen receptor (CAR). The extracellular portion of BCMA is cleaved by gamma-secretase ( $\gamma$-secretase), resulting in lower surface expression of BCMA on tumor cells and the presence of soluble BCMA (SBCMA) in the circulation, both of which could hamper BCMA CAR-T cell efficacy and enable escape of BCMAlow MM cells. We investigated whether $\gamma$-secretase inhibitor (GSI) treatment inhibited BCMA shedding, increased surface BCMA levels, and augmented BCMA-specific CAR-T cell antitumor functionality in vitro and in vivo.

Methods

BCMA expression on MM cell lines and primary $\mathrm{CD} 138^{+} \mathrm{MM}$ samples was measured by flow cytometry after treatment with the GSI 
RO4929097. sBCMA levels in patient sera and culture supernatants were measured by ELISA. BCMA-specific CAR-T cells were co-cultured with MM cell lines or primary MM, with or without GSI. Blocking effects of SBCMA on CAR-T cells were measured by titrating recombinant BCMA/Fc into co-cultures. CAR-T cell functionality was measured by cytokine ELISA, CFSE dye dilution, and chromium-release assays. To investigate in vivo CAR-T cell efficacy, MM1.R ${ }^{\text {ffluc }}$-tumor bearing NSG mice were treated with BCMA-specific CAR-T cells alone and in combination with GSI. Tumor burden was monitored by bioluminescence imaging.

Results

BCMA-specific CARs were optimized for functionality by spacer modifications. Patient serum levels of BCMA were found to often exceed $100 \mathrm{ng} / \mathrm{mL}$ and concentrations $>33 \mathrm{ng} / \mathrm{mL}$ inhibited BCMA CAR-T cell function. Treatment with GSI RO4929097 increased BCMA levels on both MM cell lines and primary MM samples in a dose dependent and reversible fashion, and decreased sBCMA shedding. High doses of RO4929097 (>3 mM) inhibited CAR-T cell function, however low doses (0.1-1 mM) were sufficient to upregulate surface BCMA levels on primary MM up to 10-fold, leading to increased recognition by BCMA CAR-T cells. In a preclinical model of myeloma, treatment with RO4929097 increased BCMA on tumor cells in bone marrow and decreased SBCMA in peripheral blood. Treatment of myeloma bearing mice with BCMA CAR-T cells combined with intermittent doses of RO4929097 improved antitumor effects and increased survival as compared to mice that did not receive RO4929097.

\section{Conclusions}

Combining GSI with BCMA-specific CAR-T cells improves tumor recognition and therapeutic benefit in a preclinical model of myeloma. The data provide a rationale for translating this combination to the clinic.

\section{1}

DNA vaccine with PD-1 blockade elicits anti-tumor responses in patients with metastatic, castration-resistant prostate cancer (mCRPC)

Douglas McNeel, Jens Eickhoff, Robert Jeraj, Mary Jane Staab, Jane Straus, Ellen Wargowski, Matthew Scarpelli, Glenn Liu University of Wisconsin, Madison, WI, USA

Correspondence: Douglas McNeel (dm3@medicine.wisc.edu) Journal for ImmunoTherapy of Cancer 2017, 5(Suppl 2):011

\section{Background}

We have previously investigated a DNA vaccine encoding prostatic acid phosphatase (PAP, pTVG-HP) in two trials of patients with PSArecurrent prostate cancer. Favorable changes in PSA doubling time were associated with the development of PAP-specific immunity. A randomized phase 2 trial testing this vaccine is currently underway. In preclinical models, we have found that blockade of regulatory receptors, including PD-1, at the time of T cell activation with DNA vaccination produced anti-tumor responses in vivo. We have also found that patients immunized with this DNA vaccine developed PD-1regulated $\mathrm{T}$ cells. These findings suggested that combined PD-1 blockade with vaccination might elicit superior anti-tumor responses in patients with prostate cancer.

Methods

A clinical trial was designed to evaluate the immunological and clinical efficacy of pTVG-HP when delivered in combination (over 12 weeks) or in sequence (over 24 weeks) with pembrolizumab, in patients with $\mathrm{mCRPC}$. Correlative studies included effects on serial biopsies, different measures of systemic immunity, and exploratory FLT $\mathrm{PET} / \mathrm{CT}$ imaging to evaluate proliferative changes in tumors and vaccine-draining lymph nodes.

Results

26 patients were randomized to treatment and evaluable for response. On-treatment adverse events > grade 2 included 1 episode of fatigue, 1 episode of diarrhea, and 1 episode of autoimmune hepatitis. No grade 4 events were observed. 8/13 subjects (61\%) treated in combination had any decrease in PSA from baseline, with 4/13 (31\%) having a PSA decline $>25 \%$. 1/13 (7\%) treated in sequence had a decrease in PSA from baseline $(p=0.013)$. Several patients treated with the combination experienced decreases in tumor volume by radiographic imaging at 12 weeks. Expansion of peripheral PAP-specific Th1-biased T cells was detected in some patients. Exploratory FLT PET/CT imaging has demonstrated proliferative responses in metastatic lesions and in vaccine-draining lymph nodes. Preliminary evaluation of biopsy specimens has suggested recruitment of activated T cells.

Conclusions

PD-1 pathway inhibitors have demonstrated little clinical activity to date when used as single agents for treating prostate cancer. Our findings demonstrate that combining this blockade with tumor-targeted T cell activation by a DNA vaccine is safe and can augment tumor-specific $T$ cells, detectable within the peripheral blood and by imaging, and result in objective anti-tumor changes. Based on these results, the randomized portion of this trial was closed to accrual, and an expansion arm has been opened to evaluate the safety and clinical efficacy of combination treatment beyond 12 weeks.

Trial Registration

NCT02499835

012

Immune and tumor responses to human IL-10 (AM0010,

Pegilodecakin) alone or in combination with immune checkpoint

blockade

Aung Naing ${ }^{1}$, Deborah J. Wong ${ }^{2}$, Jeffrey R. Infante ${ }^{3}$, Kyriakos Papadopoulos" Raid Aljumaily ${ }^{5}$, W. Michael Korn ${ }^{6}$, Jeffrey G. Schneider ${ }^{7}$, Manish Patel ${ }^{8}$,

Karen A Autio ${ }^{9}$, Gerald S. Falckook ${ }^{10}$, Nashat Y. Gabrail ${ }^{11}$, Bianca Rojo ${ }^{12}$,

Navneet Ratti ${ }^{12}$, Scott McCauley ${ }^{12}$, Annie Hung ${ }^{12}$, Peter Van Vlasselaer ${ }^{12}$,

Gail L. Brown ${ }^{12}$, Edward B. Garon',

Nizar M. Tannir ${ }^{1}$, Martin $\mathrm{Oft}^{12}$

${ }^{1}$ MDACC, Houston, TX, USA; ${ }^{2}$ UCLA, Los Angeles, CA, USA; ${ }^{3}$ SCRI,

Nashville, TN, USA; ${ }^{4}$ START, San Antonio, TX, USA; ${ }^{5}$ OUHSC, OKLAHOMA

CITY, OK, USA; ${ }^{6}$ UCSF, San Francisco, CA, USA; ${ }^{7}$ Winthrop University

Hospital, Mineola, NY, USA; ${ }^{8}$ Florida Cancer Specialists, Sarasota, FL, USA;

${ }^{9}$ MSKCC, New York, NY, USA; ${ }^{10}$ Sarah Cannon at HealthOne, Denver, CO,

USA; ${ }^{11}$ Gabrail Cancer Center, Canton, OH, USA; ${ }^{12}$ ARMO BioSciences,

Redwood City, CA, USA

Correspondence: Martin Oft (martinoft@gmail.com)

Journal for ImmunoTherapy of Cancer 2017, 5(Suppl 2):012

\section{Background}

IL-10 has anti-inflammatory functions and stimulates the cytotoxicity and proliferation of antigen activated CD8+ $T$ cells. $T$ cell receptor mediated activation of CD8+ T cells elevates IL-10 receptors and PD1 , providing the mechanistic rationale for combining AM0010 and anti-PD1 for the treatment of cancer pts. A phase 1 clinical trial investigated the tolerability and anti-tumor activity of AM0010 alone and in combination with anti-PD1 immune checkpoint inhibitors.

We previously reported partial responses in 4 of 16 RCC pts treated with AM0010 monotherapy (Naing et al JCO 2016) and 4 of 8 RCC pts (50\% ORR) and 2 of 5 of NSCLC pts (ORR $40 \%$ ) treated with AM0010 and pembrolizumab. We enrolled phase 1 cohorts of RCC and NSCLC pts on AM0010 + nivolumab as $\geq 2^{\text {nd }}$ line of treatment. Methods

A total of 34 NSCLC pts. were enrolled on AM0010 (10-20mg/kg QD, $\mathrm{SC}$ ) and pembrolizumab ( $2 \mathrm{mg} / \mathrm{kg}$, q3wk IV; $\mathrm{n}=5$ ) or nivolumab (3mg/ $\mathrm{kg}$, q2wk IV; $\mathrm{n}=29$ ). Pts. had a median of 2 prior therapies (PT, range $0-5) .37$ RCC patients were enrolled on AM0010 (10-20mg/kg QD, SC) and pembrolizumab ( $2 \mathrm{mg} / \mathrm{kg}$, q3wk IV; $\mathrm{n}=8)$ or nivolumab $(3 \mathrm{mg} / \mathrm{kg}$, q2wk IV; $n=29$ ), with a median of 1 PT (1-3). Tumor responses were assessed by irRC. Serum cytokines, T cell activation (FACS) and peripheral T cell clonality (TCR sequencing) were analyzed. (Fig. 1).

Results

AM0010 + anti-PD-1 was well tolerated. TrAEs included anemia, thrombocytopenia and fatigue, and were reversible and transient. As of July 30 2017, partial responses (PRs) were observed in 10 of 26 evaluable NSCLC pts (38.5\%), including 3 (of 11 ) with PD-L1+ $<1 \%$ and 4 (of 5) with PD-L1+ >50\% cancers. PRs were observed in 14 of 
34 evaluable RCC pts (41\%). An additional 15 RCC pts had stable disease $(44 \%), 7$ of those had a tumor reduction $>30 \%$. The mPFS and mOS has not been reached, the $\mathrm{mFU}$ is $8.9 \mathrm{~m}$ (range 0.5-26.5). Updated response data including data on delayed responses and the durability of response will be available.

The induction of immune cytokines (IL-18) in the serum, invigoration of CD8+ T cells and the increase of newly expanding $T$ cell clones correlated with objective tumor response to AM0010 monotherapy and AM0010 + anti-PD-1.

\section{Conclusions}

AM0010 in combination with anti-PD-1 is well-tolerated in RCC $\mathrm{pts}$, the recommended phase 2 dose is $10 \mathrm{ug} / \mathrm{kg}$. The robust efficacy data and the observed CD8 T cell activation is promising and encourages the continued study of AM0010 in combination with nivolumab.

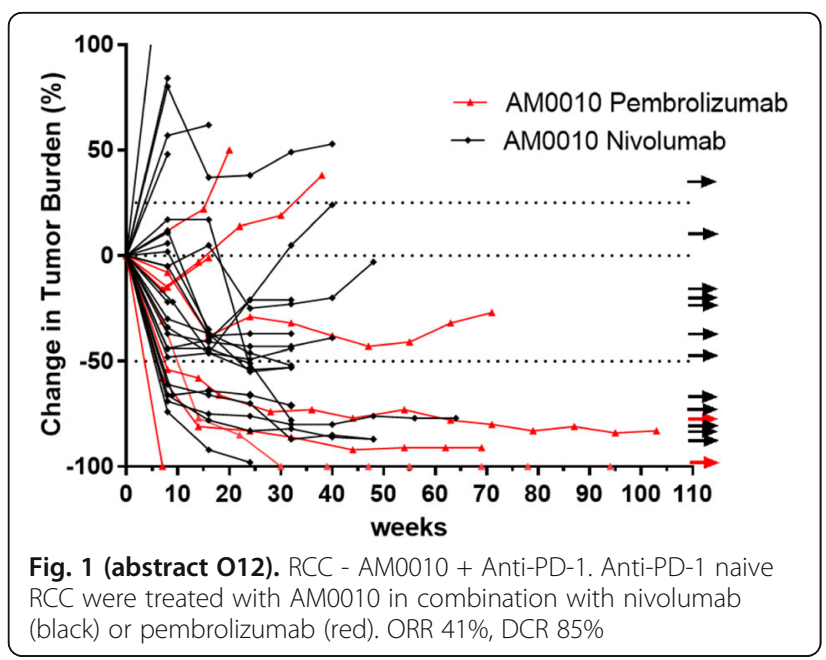

\section{3}

A phase 1 study of TSR-022, an anti-TIM-3 monoclonal antibody, in patients (pts) with advanced solid tumors

Glen J. Weiss', Jason J. Luke' ${ }^{2}$ Gerald Falchook ${ }^{3}$, Zeynep Eroglu ${ }^{4}$ Judy Wang ${ }^{5}$, Erika Hamilton ${ }^{6}$, J. Randolph Hecht ${ }^{7}$, Patricia LoRusso ${ }^{8}$, Joseph Paul Eder ${ }^{8}$, Lorraine Hughes ${ }^{9}$, Jing Wang ${ }^{9}$, Kelli Running ${ }^{9}$,

Kristen McEachern" ${ }^{9}$, Dmitri Bobilev ${ }^{9}$, Antoni Ribas

${ }^{1}$ Western Regional Medical Center Inc., Goodyear, AZ, USA; ${ }^{2}$ University of Chicago Medical Center, Chicago, IL, USA; ${ }^{3}$ Sarah Cannon Research Institute at HealthONE, Denver, CO, USA; ${ }^{4} \mathrm{H}$. Lee Moffitt Cancer Center and Research Institute, Tampa, FL, USA; ${ }^{5}$ Florida Cancer Specialists, Sarasota, FL, USA; ${ }^{6}$ Tennessee Oncology, PLLC, Nashville, TN, USA; 7 University of California Los Angeles - Jonsson Comprehensive Cancer Center, Los Angeles, CA, USA; ${ }^{8}$ Smilow Cancer Hospital at Yale - New Haven, New Haven, CT, USA; ${ }^{9}$ TESARO Inc., Waltham, MA, USA

Correspondence: Antoni Ribas (aribas@mednet.ucla.edu) Journal for ImmunoTherapy of Cancer 2017, 5(Suppl 2):013

\section{Background}

T cell immunoglobulin and mucin-domain containing-3 (TIM-3) is a key immune checkpoint protein that has been implicated in both exhaustion of $\mathrm{T}$ effector cells and immune suppression mediated by regulatory T cells and myeloid cells. Up-regulation of TIM-3 expression on PD-1positive tumor-infiltrating $T$ cells is associated with reduced proliferation and secretion of cytokines important for $\mathrm{T}$ cell mediated antitumor activity in several cancers. Blocking TIM-3 therefore has the potential to restore tumor recognition by $T$ cells and to increase antitumor immunity. TSR-022 is a potent, selective, investigational anti-TIM3 antibody that is in development for the treatment of solid tumors as both a monotherapy and in combination with PD-1 blockade.

\section{Methods}

TSR-022 is being investigated in a multicenter, open-label, first-inhuman phase 1 trial enrolling pts with advanced or metastatic solid tumors who have progressed after, or are intolerant to, available or approved therapies. The primary objective of the current part of this study is to evaluate the safety and tolerability of TSR-022 as a monotherapy and to determine the recommended phase 2 dose of TSR022 as a single agent. Pts received IV infusion of TSR-022 monotherapy every 14 days in six escalating dose levels.

Results

As of July 19, 2017, 31 pts have been treated with monotherapy: 3 pts at $0.03 \mathrm{mg} / \mathrm{kg}, 3$ pts at $0.1 \mathrm{mg} / \mathrm{kg}, 3$ pts at $0.3 \mathrm{mg} / \mathrm{kg}, 9$ pts at $1 \mathrm{mg} / \mathrm{kg}$ (all, 43 days of median follow-up); 7 pts at $3 \mathrm{mg} / \mathrm{kg}$ (28 days of median follow-up), and 6 pts at $10 \mathrm{mg} / \mathrm{kg}$ ( 23 days of median follow up). Adverse events that occurred in $>15 \%$ of patients were fatigue ( 8 pts, $26 \%)$, abdominal pain (7 pts, 23\%), nausea (6 pts, 19\%); elevated ALT, elevated AST, back pain, constipation, and vomiting was each reported in 5 pts (16\%). One dose-limiting toxicity event occurred with TSR-022 monotherapy (immune-related Grade 3 lipase elevation), which did not require treatment modification. No treatment-related serious adverse events were observed. TSR-022 exposure and peripheral receptor occupancy increased in a dose proportional manner from 0.3 to $10 \mathrm{mg} / \mathrm{kg}$. To date, the best response was disease stabilization, observed in patients with various cancer types.

Conclusions

TSR-022 monotherapy was well tolerated across multiple dose levels. Adverse events were manageable and consistent with the safety profiles of other checkpoint inhibitors. Dose-escalation of TSR-022 in combination with an anti-PD-1 antibody is currently ongoing. Trial Registration

ClinicalTrials.gov identifier NCT02817633.

\section{4}

Initial results from phase 1 trial of $\mathrm{M7824}$ (MSB0011359C), a bifunctional fusion protein targeting PD-L1 and TGF- $\beta$, in patients with NSCLC refractory or resistant to prior anti-PD-1/anti-PD-L1 agents

Fabrice Barlesi ${ }^{1}$, Nicolas Isambert ${ }^{2}$, Enriqueta Felip ${ }^{3}$, Byoung Chul Cho ${ }^{4}$, Dae Ho Lee ${ }^{5}$, Julio Peguero ${ }^{6}$, Guy Jerusalem ${ }^{7}$, Nicolas Penel ${ }^{8}$, Esma Saada-Bouzid ${ }^{9}$, Pilar Garrido ${ }^{10}$, Christoph Helwig ${ }^{11}$, Isabelle Dussault ${ }^{12}$, Laureen Ojalvo ${ }^{12}$, James Gulley ${ }^{13}$

${ }^{1}$ Aix-Marseille University, Livon, France; ${ }^{2}$ Centre Georges-François Leclerc, Dijon Cedex, France; ${ }^{3}$ Vall D'Hebron University Hospital, Barcelona, Spain;

${ }^{4}$ Yonsei Cancer Center, Seoul, Republic of Korea; ${ }^{5}$ University of Ulsan College of Medicine, Asan Medical Center, Seoul, Republic of Korea; ${ }^{6}$ Memorial Hermann Texas Medical Center, Houston, TX, USA; ${ }^{7} \mathrm{CHU}$ Liege and Liege University, Liege, Belgium; ${ }^{8}$ Centre Oscar Lambret, Lille, France; ${ }^{9}$ Centre Antoine Lacassagne, Nice, France; ${ }^{10}$ University Hospital Ramón y Cajal, Madrid, Spain; ${ }^{11}$ Merck KGaA, Darmstadt, Germany;

${ }^{12}$ EMD Serono, Billerica, MA, USA; ${ }^{13}$ National Cancer Institute, Bethesda, MD, USA

Correspondence: Fabrice Barlesi (fabrice.barlesi@ap-hm.fr)

Journal for ImmunoTherapy of Cancer 2017, 5(Suppl 2):014

\section{Background}

The transforming growth factor $\beta$ (TGF- $\beta$ ) pathway plays an important role in tumor immune escape and may potentially contribute to the failure of programmed death 1/programmed death ligand 1 (PD-1/PDL1) therapy. M7824 (MSB0011359C) is an innovative first-in-class bifunctional fusion protein composed of a human IgG1 monoclonal antibody against PD-L1 fused with 2 extracellular domains of TGF- $\beta$ receptor II to function as a TGF- $\beta$ "trap." We report initial results on the efficacy and tolerability of M7824 in patients with non-small cell lung cancer (NSCLC) refractory or resistant to prior anti-PD-1/anti-PD-L1 agents. Methods

NCT02517398 is an ongoing, phase 1, open-label trial. In this expansion cohort, patients with advanced NSCLC refractory (progression after treatment initiation) or with acquired resistance (initial disease control with subsequent relapse) to prior treatment with anti-PD-1/ 
anti-PD-L1 therapy received M7824 1200 mg q2w until confirmed progressive disease, unacceptable toxicity, or trial withdrawal. The primary objective is best overall response (BOR) per RECIST v1.1; secondary objectives include safety/tolerability.

Results

To date, 83 heavily pretreated patients $(74.7 \%$ received $\geq 3$ prior therapies) have received M7824 for a median duration of 8 weeks (range: 2-30 weeks) and 23 patients remain on active treatment with a median follow-up of 22.6 weeks (range, 8-35.3 weeks). Preliminary data show initial clinical activity was observed in 19 patients (disease control rate $=22.9 \%$; 2 confirmed partial responses [ongoing at 4.5 and 7.5 months] and 17 patients with stable disease [15 ongoing at 3 months]) based on investigator-assessed BOR. Notably, initial clinical activity was observed both in patients refractory or resistant to prior anti-PD-1/anti-PD-L1 therapy. Fourteen patients (16.9\%) experienced grade $\geq 3$ treatment-related adverse events (AEs), most commonly rash/pruritus $(n=4,4.8 \%)$ and fatigue/asthenia $(n=4,4.8 \%)$. Cutaneous lesions occurred in 3 patients (3.6\%), including keratoacanthoma and squamous cell carcinoma (similar to other TGF- $\beta$-inhibiting agents) and were well managed by surgical excision. One patient discontinued the study due to a treatment-related AE (bullous pemphigoid). One patient died from pneumonia assessed by the investigator as treatment related.

\section{Conclusions}

Based on preliminary data from this still-ongoing trial, treatment with M7824 results in initial clinical activity and a manageable safety profile in heavily pretreated patients with NSCLC refractory or resistant to prior anti-PD-1/anti-PD-L1 agents. Updated data, including results based on biomarkers such as tumor PD-L1 expression and circulating levels of TGF- $\beta 1$ and cytokines, will be presented at the meeting.

Trial Registration

NCT02517398

\section{5}

Neoadjuvant nivolumab versus combination ipilimumab and nivolumab followed by adjuvant nivolumab in patients with resectable stage III and oligometastatic stage IV melanoma: preliminary findings

Sangeetha M. Reddy, Rodabe N. Amaria, Christine N. Spencer, Michael Tetzlaff, Alexandre Reuben, Miles Andrews, Linghua Wang, Scott Woodman, Haifeng Zhu, Jorge Blando, Luis Vence, Shaojun Zhang, Hong Jiang, Vancheswaran Gopalakrishnan, Courtney Hudgens, Khalida Wani, Hussein Tawbi, Adi Diab, Isabella Glitza, Sapna Patel, Wen-Jen Hwu, Michael Wong, Patrick Hwu, Janice Cormier, Anthony Lucci, Richard Royal, Jeffrey E. Lee, Lauren Simpson, Elizabeth M. Burton, Jeffrey E. Gershenwald, Merrick Ross, James Allison, Padmanee Sharma, Michael Davies, Jennifer Wargo

The University of Texas MD Anderson Cancer Center, Houston, TX, USA Correspondence: Jennifer Wargo (jwargo@mdanderson.org) Journal for ImmunoTherapy of Cancer 2017, 5(Suppl 2):015

\section{Background}

Single agent nivolumab (nivo) and combination ipilimumab (ipi) + nivo are efficacious in advanced melanoma, but have not been extensively evaluated in patients with high-risk resectable disease. The optimal neoadjuvant immunotherapy regimen is unknown and development of biomarker specific neoadjuvant trials are critical in understanding mechanisms of response, resistance and informing clinical management. We designed a clinical trial to test these regimens in the neoadjuvant setting coupled with longitudinal sample collection designed to identify strategies to further improve outcomes.

\section{Methods}

Patients with resectable stage III or oligometastatic stage IV melanoma were randomized to nivo $3 \mathrm{mg} / \mathrm{kg}$ IV every 2 weeks for 4 doses or ipi $3 \mathrm{mg} / \mathrm{kg}$ + nivo $1 \mathrm{mg} / \mathrm{kg}$ IV every 3 weeks for 3 doses prior to surgical resection, with both arms receiving adjuvant nivo $3 \mathrm{mg} / \mathrm{kg}$ for 6 months (Fig. 1). Responses were measured at time of surgery as defined by RECIST 1.1 and percent tumor viability in surgical specimen. Blood and tumor samples were collected at baseline, ontreatment, and at surgery.

Results

23 out of a planned 40 patients were enrolled. The response rates (RR) were $33 \%$ for nivo and $67 \%$ for ipi+nivo in evaluable patients $(\mathrm{n}=18$ total, 9 in each arm) (Fig. 2). Pathological complete response (pCR) rates were $22 \%$ for nivo and $56 \%$ for ipi+nivo. Importantly, $22 \%$ of patients in nivo arm experienced disease progression and were ineligible for surgical resection, prompting review by the data safety monitoring board and early trial closure. No grade 3 or higher toxicities were seen with nivo compared to $89 \%$ with ipi+nivo. All patients on ipi+nivo received surgical resection, but $33 \%$ had a surgical delay related to toxicity. RNA-seq analysis of longitudinal tumor samples revealed differential effects of each treatment on the tumor microenvironment as well as between responders ( $R$ ) and nonresponders (NR) within each treatment arm. Immune profiling demonstrated enriched CD8+ T cell infiltrates in on-treatment samples in $R$ versus NR $(p=0.04)$. T cell receptor sequencing showed a lower degree of remodeling from pre- to on-treatment tumor in R vs NR (as measured by Morisita's index, $\mathrm{p}<0.01$ ).

Conclusions

Treatment with neoadjuvant nivo or ipi+nivo has activity in resectable melanoma. Ipi+nivo possessed a higher RR but also higher grade III/IV toxicities whereas nivo led to a higher rate of disease progression and resultant unresectable disease. Ongoing correlative studies will provide insights into the mechanisms of response, toxicity and resistance.

Trial Registration

NCT02519322
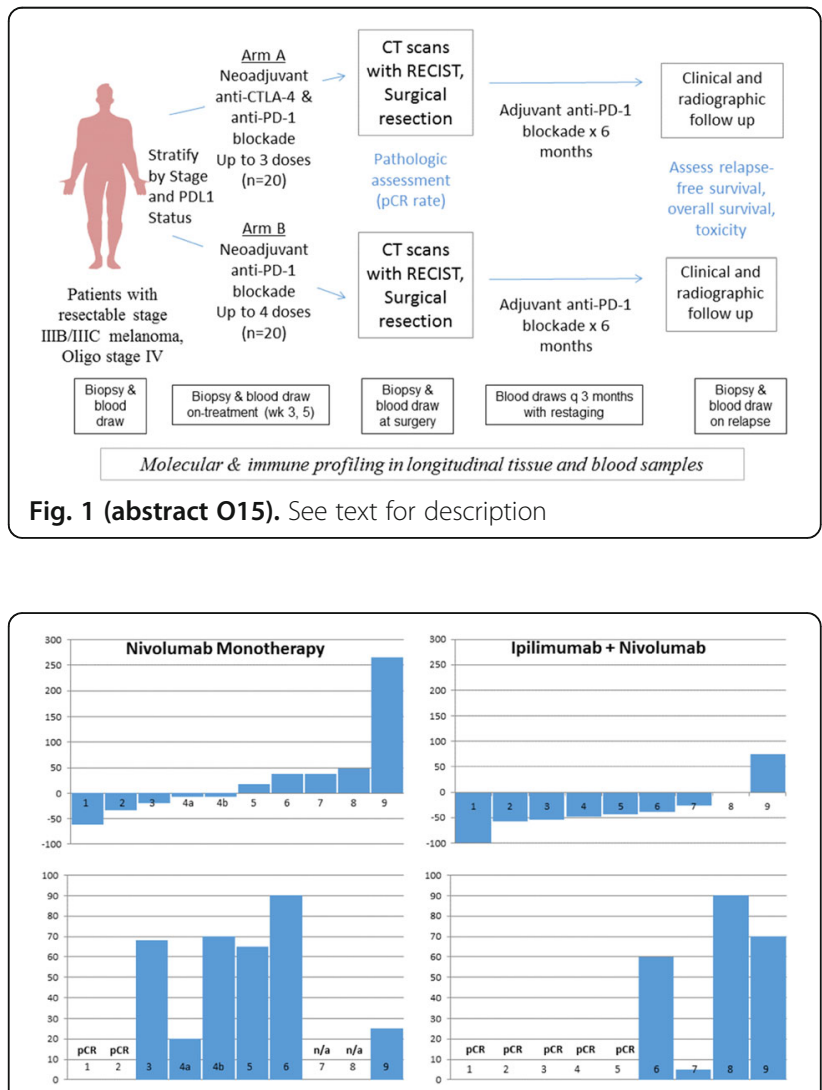

Fig. 2 (abstract 015). See text for description 
016

A phase $1 / 2$ study of CB-839, a first-in-class glutaminase inhibitor, combined with nivolumab in patients with advanced melanoma (MEL), renal cell carcinoma (RCC), or non-small cell lung cancer (NSCLC)

Funda Meric-Bernstam', Michael Gordon², Scott Tykodi ${ }^{3}$, Elaine Lam ${ }^{4}$, Ulka Vaishampayan ${ }^{5}$, Jorge Chaves ${ }^{6}$, Petros Nikolinakos ${ }^{7}$, Alice Fann, Richard Lee ${ }^{9}$, David McDermott ${ }^{10}$, Geoffrey Shapiro ${ }^{11}$, Leena Gandhi ${ }^{12}$ Hussein Tawbi ${ }^{1}$, Shailender Bhatia ${ }^{3}$, Lucas Muigai ${ }^{13}$, Yonchu Jenkins ${ }^{13}$, Sam Whiting ${ }^{13}$, Martin Voss ${ }^{14}$

${ }^{1}$ The University of Texas MD Anderson Cancer Center, Houston, TX, USA; ${ }^{2}$ Pinnacle Oncology Hematology, Scottsdale, AZ, USA; ${ }^{3}$ Seattle Cancer Care Alliance, Seattle, WA, USA; ${ }^{4}$ University of Colorado School of Medicine, Aurora, CO, USA; ${ }^{5}$ Karmanos Cancer Center, Detroit, MI, USA; ${ }^{6}$ Northwest Medical Specialties, Tacoma, WA, USA; ${ }^{7}$ University Cancer \& Blood Center, Athens, GA, USA; ${ }^{8}$ Stanford University Medical Center, Stanford, CA, USA; ${ }^{9}$ Massachusetts General Hospitcal, Boston, MA, USA;

${ }^{10}$ Beth Israel Deaconess Medical Center, Boston, MA, USA; ${ }^{11}$ Medical Oncology, Boston, MA, USA; ${ }^{2}$ NYU Medical Oncology Associates, New York, NY, USA; ${ }^{13}$ Calithera Biosciences, Inc., South San Francisco, CA, USA; ${ }^{14}$ Memorial Sloan Kettering Cancer Center, New York, NY, USA Correspondence: Funda Meric-Bernstam (fmeric@mdanderson.org) Journal for ImmunoTherapy of Cancer 2017, 5(Suppl 2):016

\section{Background}

CB-839 is a first-in-class, oral, highly selective inhibitor of glutaminase that targets tumor glutamine metabolism. Competition between tumor cells and immune cells for nutrients, including glutamine, in the tumor microenvironment can create a metabolic checkpoint that induces local immune suppression. CB-839 inhibits tumor glutamine consumption and increases glutamine availability to support T-cell activity. In pre-clinical models, CB-839 increases intra-tumoral glutamine and enhances antitumor activity of PD-1/PD-L1 inhibitors. This study is evaluating the safety and efficacy of CB-839 in combination with the PD-1 inhibitor, nivolumab, in patients with advanced RCC, MEL, or NSCLC, including cohorts of patients refractory to anti-PD-1/ PD-L1.

\section{Methods}

In dose escalation, patients received CB-839 $600 \mathrm{mg}$ or $800 \mathrm{mg}$ PO BID in combination with standard-dose nivolumab. In expansion, patients are enrolling into one of five cohorts to receive CB-839 $800 \mathrm{mg}$ BID with standard-dose nivolumab: Naïve RCC - anti-PD-1/PD-L1 naïve, previously treated with anti-angiogenic therapy; Rescue RCC on nivolumab with progressive disease (PD) or $>6$ month stable disease without response; Other IO RCC - anti-PD-1/PD-L1 in any previous line of therapy with PD and no history of response; Rescue MEL - on anti-PD-1/PD-L1 with PD; Rescue NSCLC - on anti-PD-1/PDL1 with PD or $>6$ month SD without response. Tumor response assessment is every 8 weeks per RECIST 1.1. Tumor biopsy and blood are collected for biomarker analysis. Bristol-Myers Squibb is providing nivolumab for this study.

Results

The study is ongoing and 50 patients have enrolled to date (42 at $800 \mathrm{mg}$ dose). A maximum tolerated dose of (B- 839 was not reached. One DLT (Gr3 ALT increased) occurred at the $800 \mathrm{mg}$ dose. The combination has been well tolerated; the most common treatment-related $\mathrm{Gr} \geq 3 \mathrm{AE}$ has been ALT increased (4\%). RECIST 1.1 tumor responses by cohort are shown below (Table 1).

There has been one complete response (CR) and two partial responses (PR) out of 7 response-evaluable MEL patients (ORR 43\%). All three responding patients were progressing on anti-PD-1/PD-L1 at study entry and two had progressed on 3 prior immunotherapy regimens. Paired tumor biopsies were available from one MEL responder and analysis showed on-treatment increase in T-effector gene expression including granzyme and perforin.

\section{Conclusions}

Treatment with the CB-839+nivolumab is well-tolerated and has clinical activity in anti-PD-1/PD-L1 refractory patients. Notable responses have occurred in MEL patients progressing on nivolumab at study entry and refractory to multiple prior immunotherapy regimens. Updated safety, biomarker and response data will be presented.

Trial Registration

NCT02771626

Table 1 (abstract 016). See text for description

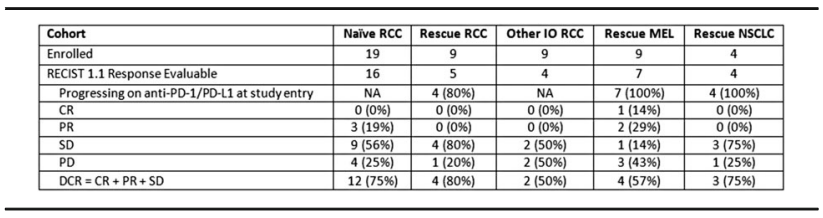

\section{7}

OX40 T-cell costimulatory agonist BMS-986178 alone or in combination with nivolumab in patients with advanced solid tumors: initial phase 1 results

Anthony J. Olszanski ${ }^{1}$, Ignacio Melero ${ }^{2}$, Michael $\mathrm{Ong}^{3}$, Anna Spreafico ${ }^{4}$, Kimberley Heinhuis ${ }^{5}$, Richard Carvajal ${ }^{6}$, Maria Ochoa de Olza ${ }^{7}$, Quincy Chu ${ }^{8}$, Anke Klippel', Yun Shen ${ }^{9}$, Yan Feng ${ }^{9}$, Bryan Barnhart ${ }^{9}$, Alan Korman', Michael Quigley ${ }^{9}$, Paula M. Fracasso ${ }^{9}$, Praveen Aanur', Martin Gutierrez ${ }^{10}$

${ }^{1}$ Fox Chase Cancer Center, Philadelphia, PA, USA; ${ }^{2}$ Clinica Universidad de Navarra, Pamplona, Spain; ${ }^{3}$ The Ottawa Hospital Cancer Centre, Ottawa, ON, Canada; ${ }^{4}$ Princess Margaret Cancer Centre, Toronto, ON, Canada; ${ }^{5}$ The Netherlands Cancer Institute, Amsterdam, Netherlands; ${ }^{6}$ Columbia University Medical Center, New York, NY, USA; ${ }^{7}$ Vall d'Hebron University Hospital, Barcelona, Spain; ${ }^{8}$ Cross Cancer Institute, University of Alberta/ Alberta Health Services, Edmonton, AB, Canada; ${ }^{9}$ Bristol-Myers Squibb, Princeton, NJ, USA; ${ }^{10}$ Hackensack University Medical Center, Hackensack, NJ, USA

Correspondence: Anthony J. Olszanski (anthony.olszanski@fccc.edu) Journal for ImmunoTherapy of Cancer 2017, 5(Suppl 2):017

\section{Background}

With the clinical success of checkpoint blockade, focus has shifted to novel approaches to enhance the benefits of immunotherapy. Activation of the costimulatory tumor necrosis factor receptor family member OX40 may lead to $T$ effector cell activation, inhibition of $\mathrm{T}$ regulatory cell-mediated suppression, and blockade of $\mathrm{T}$ regulatory cell generation. Preclinical data show enhanced antitumor T-cell activity when anti-OX40 is combined with checkpoint inhibitors (eg, anti-PD-1), supporting the potential benefits of this combination to provide deeper, more durable responses than checkpoint blockade alone. BMS-986178 is a fully human IgG1 agonist monoclonal antibody that binds with high affinity to OX40. Here we describe preliminary results of a phase $1 / 2$ a study of BMS-986178 \pm nivolumab (anti-PD-1) in patients with advanced solid tumors (NCT02737475).

Methods

Patients with advanced/metastatic solid tumors with $\geq 1$ prior therapy were treated in this open-label, dose-escalation and dose-expansion study. During escalation, patients received BMS-986178 (20-320 mg) IV Q2W or BMS-986178 (20-320 mg) + nivolumab 240 mg IV Q2W. Safety, immunogenicity, pharmacokinetics, pharmacodynamics, and preliminary antitumor activity were evaluated. Pharmacodynamic analyses included evaluation of OX40, PD-1, Ki67, FOXP3, and CD8 by immunohistochemistry in tumors and immunophenotyping in peripheral blood mononuclear cells. 
Results

As of June 12, 2017, 59 patients were treated with BMS-986178 $(n=20)$ or BMS-986178 + nivolumab $(n=39)$. Approximately one-third of patients in the monotherapy (30\%) and combination (28\%) arms had received prior anti-PD-1/PD-L1-based therapy. Most treatmentrelated AEs were grade 1/2 (Table 1); 1 patient treated with BMS-986178 $160 \mathrm{mg}$ + nivolumab had treatment-related grade 3 pneumonitis that led to discontinuation. The maximum tolerated doses of BMS-986178 \pm nivolumab have not yet been reached. The pharmacokinetics of BMS-986178 \pm nivolumab were linear, and exposure increased proportionally with BMS-986178 doses of 20-320 mg. Preliminary pharmacodynamic activity showed an increase in proliferating T cells and a decrease in FOXP3 cells in tumors with BMS-986178 + nivolumab. Evidence of antitumor activity was observed in several patients treated with the combination, including a patient who had progressed on prior anti-PD-1 therapy; these data will be reported.

Conclusions

BMS-986178, an OX40 agonist, given alone and in combination with nivolumab, was well tolerated; the combination had a safety profile similar to that of nivolumab monotherapy. Treatment with BMS986178 + nivolumab resulted in pharmacodynamic immune changes in tumors and preliminary antitumor activity. Further evaluation of this combination in patients with advanced solid tumors is ongoing Trial Registration

ClinicalTrials.gov, NCT02737475

Table 1 (abstract 017). Treatment-related AEs with BMS-986178 and BMS-986178 + nivolumab

\begin{tabular}{|c|c|c|c|c|}
\hline & \multicolumn{2}{|c|}{$\begin{array}{c}\text { BMS-986178 } \\
n=20\end{array}$} & \multicolumn{2}{|c|}{$\begin{array}{c}\text { BMS-986178 + Nivolumab } \\
n=39\end{array}$} \\
\hline & Any Grade & Grade $3 / 4$ & Any Grade & Grade 3/4 \\
\hline TRAEs, $n(\%)$ & $5(25)$ & $1(5.0)$ & $21(54)$ & $1(2.6)^{\mathrm{a}}$ \\
\hline \multicolumn{5}{|c|}{ TRAEs in $\geq 2$ patients in either treatment arm, $n$} \\
\hline Fatigue & 1 & 1 & 5 & 0 \\
\hline Pyrexia & 1 & 0 & 5 & 0 \\
\hline Arthralgia & 0 & 0 & 4 & 0 \\
\hline Diarrhea & 3 & 0 & 2 & 0 \\
\hline Chills & 0 & 0 & 2 & 0 \\
\hline Hypothyroidism & 0 & 0 & 2 & 0 \\
\hline Nausea & 1 & 0 & 2 & 0 \\
\hline
\end{tabular}

aGrade 3 treatment-related pneumonitis was reported in 1 patient treated with BMS-986178 $160 \mathrm{mg}+$ nivolumab $240 \mathrm{mg}$.

\section{8}

TLR9 agonist harnesses innate immunity to drive tumor-infiltrating T cell expansion in distant lesions in a phase $1 / 2$ study of intratumoral IMO-2125+ipilimumab in anti-PD1 refractory melanoma patients

Cara Haymaker', Marc Uemura', Wen-Jen Hwu', Ravi Murthy ${ }^{1}$

Marihella James', Ankit Bhatta', Salah Eddine Bentebibel', Julie Brevard², James Geib ${ }^{3}$, Kathryn Lipford², Mark Cornfeld ${ }^{3}$, Srinivas Chunduru³,

Cassian Yee ', Scott Woodman', Rodabe Amaria', Sapna Patel', Hussein Tawbi', Isabella Glitza Oliva', Michael Davies', Willem Overwijk', Patrick Hwu', Chantale Bernatchez ${ }^{1}$, Adi Diab

'University of Texas MD Anderson Cancer Center, Houston, TX, USA;

${ }^{2}$ Idera Pharmaceuticals Inc., Cambridge, MA, USA; ${ }^{3}$ Idera Pharmaceuticals, Inc., Exton, PA, USA

Correspondence: Adi Diab (adiab@mdanderson.org)

Journal for ImmunoTherapy of Cancer 2017, 5(Suppl 2):018

\section{Background}

While checkpoint inhibitors (CPI) have transformed melanoma treatment, many patients remain refractory. Pre-clinical models show combination intratumoral IMO-2125 (TLR9 agonist) with anti-CTLA-4 or anti-PD-1 antibody results in improved tumor control compared with either agent alone. We hypothesized that this combination would stimulate local antigen-presenting cells, resulting in activation of anti-tumor $\mathrm{T}$ cells and distant responses.

Methods

Adults with anti-PD-1 refractory, unresectable stage III/IV melanoma were enrolled. IMO-2125, escalating from $4-32 \mathrm{mg}$ is administered under image guidance, intratumorally on weeks 1,2 ,
$3,5,8$, and 11 with standard ipilimumab. Biopsies were obtained in both the injected and distant tumor at baseline, 1 day and 8 weeks (W8) post injection. Immune analyses included phenotypic, activation, and functional characterization of DC subsets and $T$ cells. T-cell repertoire diversity was evaluated by high-throughput CDR3 sequencing and changes in gene expression signatures were assessed by NanoString.

Results

Biopsies were obtained from 17 of the 18 subjects enrolled on the IMO-2125-ipilimumab arm. Of 15 subjects with post-baseline disease evaluations, $9(60 \%)$ showed shrinkage in uninjected tumors with an ORR of $33 \%$ by RECIST 1.1. Fresh tumor biopsies taken $24 \mathrm{~h}$ post IMO2125 injection demonstrated induction of an IFN-a-response gene signature (IRF7) in all patients $(p<0.0001)$, as well as, maturation of the myeloid $\mathrm{DC} 1$ subset $\left(\mathrm{CD}_{1} \mathrm{c}^{+} \mathrm{CD} 303^{-}\right)$by upregulation of $\mathrm{MHC}$ class II (6/12 patients) and upregulation of PD-L1 on malignant cells (10/14 patients). W8 biopsies of the uninjected tumor lesion reveal the induction of $\mathrm{Ki}^{+} 7^{+}$expression by flow cytometry in effector $\mathrm{CD}^{+} \mathrm{T}$ cells in 6 of 7 regressing lesions indicating an abscopal effect of this combination. In 6 patients with both tumor shrinkage and flow cytometry staining of PBMCs, 4 had higher $\mathrm{CD}^{+}{ }^{+}$-cell proliferation in the tumor than in blood on-treatment. CDR3 sequencing shows that the top 50 clones form a larger proportion of the repertoire at W8 suggesting local proliferation in both lesions, as well as, the specific expansion in the distant lesion of clones shared with the injected site in responders.

Conclusions

Combination IMO-2125 and ipilimumab therapy is a promising new treatment for anti-PD-1 refractory melanoma. We show this treatment strategy to result in a local IFN-a-gene signature coupled with mDC1 maturation. Additionally, the hallmark of tumor shrinkage appears to be the presence of $\mathrm{Ki}^{6} 7^{+} \mathrm{CD} 8^{+} \mathrm{T}$ cell effector cells in the uninjected tumor and expansion of clones shared between both lesions (abscopal effect).

Trial Registration

NCT02644967

\section{9}

ENCORE-601: Phase 1b/2 study of entinostat (ENT) in combination with pembrolizumab (PEMBRO) in patients with non-small cell lung cancer (NSCLC)

Leena Gandhi ${ }^{1}$, Melissa L. Johnson ${ }^{2}$, Mateusz Opyrchal ${ }^{3}$, Suresh Ramalingam ${ }^{4}$, Pasi Janne ${ }^{5}$, Abraham Chachoua', Peter Ordentlich ${ }^{6}$, Susan Brouwer ${ }^{6}$, Serap Sankoh ${ }^{6}$, Emmett Schmidt ${ }^{7}$, Michael L. Meyers ${ }^{8}$, Matthew Hellmann ${ }^{9}$

${ }^{1}$ NYU Langone Medical Center, New York, NY, USA; ${ }^{2}$ Sarah Cannon Research Institute, Nashville, TN, USA; ${ }^{3}$ Roswell Park Cancer Institute, Buffalo, NY, USA; ${ }^{4}$ Winship Cancer Institute, Emory University, Atlanta, GA, USA; ${ }^{5}$ Dana Farber Cancer Institute, Boston, MA, USA; ${ }^{6}$ Syndax Pharmaceuticals, Inc., Waltham, MA, USA; ${ }^{7}$ Merck \& Co., Inc., Kenilworth, NJ, USA; ${ }^{8}$ Syndax Pharmaceuticals, Inc., New York, NY, USA; ${ }^{9}$ Memorial Sloan Kettering Cancer Center, New York, NY, USA

Correspondence: Leena Gandhi (leena.gandhi@nyumc.org)

Journal for ImmunoTherapy of Cancer 2017, 5(Suppl 2):019

\section{Background}

Entinostat is an oral, class I selective histone deacetylase (HDAC) inhibitor that has been shown in pre-clinical models to enhance antiPD-1 activity through inhibition of immune suppressor cells in the tumor microenvironment. ENCORE 601 is a Phase $1 \mathrm{~b} / 2$ study evaluating safety and efficacy of ENT plus PEMBRO in NSCLC, melanoma and colorectal cancer patients. We previously reported the results of Phase 1b, which identified ENT 5 mg PO weekly plus PEMBRO 200 mg IV every 3 weeks as the dose to be further explored.

Methods

Efficacy of ENT $5 \mathrm{mg}$ PO weekly plus PEMBRO $200 \mathrm{mg}$ IV q3wks is being assessed in a Simon 2-stage Phase 2 study involving two cohorts of patients with advanced NSCLC: anti-PD-(L)1-naïve (Cohort 1), and progressed on anti-PD-(L)1 (Cohort 2). The primary endpoint is objective response rate. Based on investigator feedback to incorporate Phase $1 \mathrm{~b}$ 
patients dosed at entinostat $5 \mathrm{mg}$ into the Stage 2 go/no go assessment, revised criteria for advancement were $\geq 4$ responses observed out of 17 evaluable patients in Cohort 1 and $\geq 3$ out of 31 in Cohort 2. Tumor biopsies and blood samples for immune correlates were taken pre- and post-treatment.

Results

Enrollment has been completed for Stage 1 of both cohorts. In Cohort 1, 4 of 17 (24\%, 95\% Cl: 7-50) evaluable patients achieved a partial response (PR; 2 confirmed, 2 unconfirmed). In Cohort 2, 3 of 31 (10\%, 95\% Cl: 2-26) evaluable patients achieved a PR (2 confirmed, 1 unconfirmed). As of the data cutoff, the longest duration of response was 24 weeks (and ongoing). Baseline PD-L1 expression for responders was $<1 \%$ ( 1 patient), $1-49 \%$ ( 2 patients) and not available (1 patient) for Cohort 1, and $<1 \%$ ( 2 patients) and not available ( 1 patient) for Cohort 2. 31\% of patients experienced a Grade 3/4 event deemed related to study drug. The most common of these events included hypophosphatemia and neutropenia in Cohort 1, and fatigue, anemia, and pneumonitis in Cohort 2. 13\% of patients discontinued therapy due to an adverse event. Circulating myeloid derived suppressor cells were reduced in both cohorts at Cycle2/Day1 compared to pretreatment. Gene expression analysis of tumor biopsies and association with responses is in progress.

\section{Conclusions}

ENT plus PEMBRO demonstrates anti-tumor activity and acceptable safety in patients with NSCLC who are both naive to and have progressed on prior PD-(L)1 blockade. Based on the responses seen, Cohort 2 has advanced to Stage 2 and is currently enrolling.

\section{0}

Pivot-02: Preliminary safety, efficacy and biomarker results from the Phase $1 / 2$ study of CD-122-biased agonist NKTR-214 plus nivolumab in patients with locally advanced/metastatic solid tumors

Adi Diab ${ }^{1}$, Nizar Tannir ${ }^{1}$, Daniel Cho ${ }^{2}$, Vali Papadimitrakopoulou ${ }^{1}$ Chantale Bernatchez ${ }^{1}$, Cara Haymaker ${ }^{1}$, Salah Eddine Bentebibel ${ }^{1}$ Brendan Curti ${ }^{3}$, Michael Wong ${ }^{1}$, Scott Tykodi ${ }^{4}$, Igor Puzanoff, Ira Smalberg ${ }^{5}$, Ivan Gergel ${ }^{6}$, Mary Tagliaferri ${ }^{6}$, Jonathan Zalevsky ${ }^{6}$, Ute Hoch $^{6}$, Sandra Aung ${ }^{6}$, Michael Imperiale ${ }^{6}$, Wendy Clemens ${ }^{7}$, Harriet Kluger $^{8}$, Michael Hurwitz ${ }^{8}$, Patrick Hwu', Mario Sznol ${ }^{8}$

${ }^{1}$ The University of Texas MD Anderson Cancer Center, Houston, TX, USA; ${ }^{2}$ NYU Medical Oncology Associates, New York, NY, USA; ${ }^{3}$ Providence Cancer Center and Earle A. Chiles Research Institute, Portland, OR, USA;

${ }^{4}$ University of Washington and Fred Hutchinson Cancer Research Center, Seattle, WA, USA; ${ }^{5}$ Roswell Park Cancer Institute, Buffalo, NY, USA;

${ }^{6}$ Nektar Therapeutics, San Francisco, CA, USA; ${ }^{7}$ Bristol-Myers Squibb, Lawrence Township, NJ, USA; ${ }^{8}$ Yale School of Medicine, New Haven, CT, USA

Correspondence: Adi Diab (adiab@mdanderson.org)

Journal for ImmunoTherapy of Cancer 2017, 5(Suppl 2):020

\section{Background}

In patients with advanced solid tumors, NKTR-214, a CD-122-biased agonist, increased newly proliferative CD8+ T cells in the tumor and increased cell surface PD-1 expression, demonstrating a potentially synergistic mechanism with anti-PD-1 therapy. Preliminary safety, efficacy and biomarker results from dose-escalation of the Phase 1/2 study combining NKTR-214 with nivolumab are presented.

Methods

Enrolled patients in dose escalation were checkpoint inhibitor naïve with locally advanced/metastatic melanoma $(\mathrm{mM})$, RCC or NSCLC. NKTR-214 and nivolumab were administered concurrently by IV infusion in the outpatient setting in Q2W or Q3W dosing schedules. Preand on-treatment blood and matched tumor samples were evaluated for changes in immune cell populations, gene expression, and T cell receptor repertoire. Responses were assessed Q8W per RECIST 1.1.
Results

At July 25, 2017, data cutoff, 31 patients were treated across one of 5 dose escalating cohorts of NKTR-214 (@0.003( $n=3)$, @0.006( $n=25)$ or $@ 0.009(\mathrm{n}=3) \mathrm{mg} / \mathrm{kg}$ ) administered with nivolumab given either 240 $\mathrm{mg}$ Q2W or $360 \mathrm{mg}$ Q3W. There were no Grade $\geq 3$ treatment-related adverse events (TRAEs) reported at or below the NKTR-214 $0.006 \mathrm{mg} /$ $\mathrm{kg}$ plus nivolumab cohorts. There were no discontinuations from TRAEs. The most common TRAEs occurring in $\geq 50 \%$ of patients included grade 1-2 fatigue, flu-like symptoms and rash. At the highest dose (NKTR-214 $0.009 \mathrm{mg} / \mathrm{kg}$ + nivolumab), two patients experienced DLTs including hypotension and metabolic acidosis. DLTs were shortlived and manageable with both patients continuing treatment at lower doses. 16 patients with Stage IV mM or Stage IV RCC were evaluable with $\geq 1$ on treatment scan. In $\mathrm{mM}, 1$ complete response was observed plus 4 partial responses ( $n=8)$. In RCC, 4 PRs were observed $(n=8) .5 / 16$ patients had SD, 2 patients had PD. In tumor tissue, there was an up to 450-fold increase in tumor-infiltrating CD8+ T cells from baseline with a limited ( $\leq 2$-fold) increase in T regulatory cells. Infiltrating T cells were newly proliferative with elevated PD-1 expression. In blood, ICOS+ CD8+ T cells increased with treatment representing $13 \%$ of total CD8+ T cells. Updated data will be presented.

Conclusions

Combination of NKTR-214 and nivolumab was well-tolerated and manageable in outpatient setting. Nivolumab-related AEs were not increased by NKTR-214. Immune activation was observed in peripheral blood and tumor microenvironment. Preliminary efficacy with $\mathrm{mM}$ and RCC show encouraging response rates. A recommended Phase 2 dose schedule of Q3W was established (NKTR-214 $0.006 \mathrm{mg} /$ $\mathrm{kg}$ plus nivolumab $360 \mathrm{mg}$ ), and enrollment is underway in expansion cohorts in eight different indications $(n=230)$.

Trial Registration

Trial Registration: NCT02983045

021

Pooled 3-year overall survival data from phase II and phase III trials of nivolumab (NIVO) combined with ipilimumab (IPI) in advanced melanoma

Michael Postow', James Larkin², Jedd D. Wolchok',

Vanna Chiarion-Sileni ${ }^{3}$, F. Stephen Hodi ${ }^{4}$, Piotr Rutkowski ${ }^{5}$,

Jean-Jacques Grob ${ }^{6}$, Kenneth Grossmann ${ }^{7}$, Dirk Schadendorf ${ }^{8}$, Jason Chesney ${ }^{9}$, Michael Smylie ${ }^{10}$, Andrew Hill ${ }^{11}$, Caroline Robert ${ }^{12}$,

Anna C. Pavlick ${ }^{13}$, John Haanen ${ }^{14}$, Grant A. McArthur ${ }^{15}$,

David McDermott ${ }^{16}$, Sheena Demelo ${ }^{17}$, Rafia Bhore ${ }^{17}$, Rene Gonzalez ${ }^{18}$

${ }^{1}$ Memorial Sloan Kettering Cancer Center, New York, NY, USA; ${ }^{2}$ Royal Marsden Hospital, London, UK; ${ }^{3}$ Oncology Institute of Veneto IRCCS, Padova, Italy; ${ }^{4}$ Dana-Farber Cancer Institute, Boston, MA, USA; ${ }^{5}$ Maria Sklodowska-Curie Memorial Cancer Center \& Institute of Oncology,

Warsaw, Poland; ${ }^{6}$ Hospital de la Timone, Marseille, France; ${ }^{7}$ Huntsman Cancer Institute, Salt Lake City, UT, USA; ${ }^{8}$ Department of Dermatology, University of Essen, Essen, Germany; ${ }^{9}$ James Graham Brown Cancer Center, Louisville, KY, USA; ${ }^{10}$ Cross Cancer Institute, Edmonton, $A B$, Canada; ${ }^{11}$ Tasman Oncology Research, Southport Gold Coast, Australia;

${ }^{12}$ Institut Gustave-Roussy, Villejuif, France; ${ }^{13} \mathrm{NYU}$ Medical Oncology Associates, New York, NY, USA; ${ }^{14}$ Netherlands Cancer Institute, Amsterdam, Netherlands; ${ }^{15}$ Peter MacCallum Cancer Centre, Melbourne, Australia; ${ }^{16}$ Beth Israel Deaconess Medical Center, Boston, MA, USA; ${ }^{17}$ Bristol-Myers Squibb, Princeton, NJ, USA; ${ }^{18}$ University of Colorado Cancer Center, Denver, CO, USA

Correspondence: Michael Postow (postowm@mskcc.org)

Journal for ImmunoTherapy of Cancer 2017, 5(Suppl 2):021

\section{Background}

NIVO (anti-PD-1) plus IPI (anti-CTLA-4) improved progression-free survival (PFS) and objective response rate Vs IPI alone in the phase II 
CheckMate 069 and phase III CheckMate 067 trials of treatment-naive patients with advanced melanoma. Recently, a 2-year overall survival (OS) rate of $64 \%$ was reported for patients treated with NIVO+IPI in CheckMate 067 . Here, we report 3-year OS, PFS, and safety results in all randomized patients from CheckMate 069 .

Methods

Patients were randomized 2:1 to NIVO $1 \mathrm{mg} / \mathrm{kg}+\mathrm{IPI} 3 \mathrm{mg} / \mathrm{kg}(\mathrm{n}=95)$ or IPI $3 \mathrm{mg} / \mathrm{kg}$ + placebo $(\mathrm{n}=47)$ Q3W for 4 doses followed by NIVO $3 \mathrm{mg} / \mathrm{kg}$ or placebo Q2W, respectively, until disease progression or unacceptable toxicity.

Results

$55 \%$ of patients in the IPI group crossed over to receive NIVO upon progression. At a median follow-up of 37 months, median OS was not reached (NR) for NIVO+IPI and was 32.9 months for IPI (hazard ratio [HR]: 0.76 ; $P=0.2829$ ), with 3 -year OS rates of $57 \%$ and $46 \%$, respectively. Median PFS was NR for NIVO+IPI and was 3.0 months for IPI (HR: 0.35; P<0.0001). Median duration of response was NR in both groups. Time to subsequent therapy in the NIVO+IPI and IPI groups was NR and 5.4 months, where $63 \%(47 / 75)$ and 19\% (7/37) remain free of subsequent therapy at the 3-year landmark, respectively. The safety profile remained similar to the earlier reports, with grade 3-4 treatment-related adverse events in the NIVO+IPI and IPI groups of $54 \%$ and $24 \% ; 38 \%$ and $9 \%$ discontinued due to any grade adverse events, respectively. Three-year OS rates in patients who discontinued due to toxicity were $68 \%$ for NIVO+IPI and $25 \%$ for IPI; median duration of response was NR in both subgroups.

Conclusions

In summary, NIVO+IPI continues to show a significant improvement in PFS, with consistent safety results, and a favorable 3 -year landmark OS rate. A pooled analysis, which will include updated 3-year OS data from CheckMate 067 , will be presented as well as outcomes in patients off all therapy. Trial Registration

NCT01927419 (CheckMate 069), NCT01844505 (CheckMate 067)

\section{2}

Antibody-mediated neutralization of soluble MIC significantly enhances response to CTLA4 blockade and reduces therapy-induced colitis

Jennifer Wu', Jinyu Zhang ${ }^{2}$

${ }^{1}$ Northwestern University, Chicago, IL, USA; ${ }^{2}$ Medical University of South Carolina, Charleston, SC, USA

Correspondence: Jennifer Wu (jennifer.wu@northwestern.edu) Journal for ImmunoTherapy of Cancer 2017, 5(Suppl 2):O22

\section{Background}

Antibody therapy targeting cytotoxic $\mathrm{T}$ lymphocyte-associated antigen 4 (CTLA4) elicited survival benefits in cancer patients; however, the overall response rate is limited. In addition, anti-CTLA4 antibody therapy induces a high rate of immune related adverse events (IRAEs). The underlying factors that may influence anti-CTLA4 antibody therapy are not well defined. Ligation of the human activation immune receptor natural killer group 2D (NKG2D) has been implicated in optimizing CD8 T cell co-stimulation and pathogenesis of colitis. Herein we investigated the impact of the human NKG2D ligand MIC, which was broadly expressed by human solid tumors, on the therapeutic outcome of anti-CTLA4 blockade.

Methods

We utilized the well-established MIC negative TRAMP and MIC ${ }^{\text {positive }}$ TRAMP/MIC spontaneous pre-clinical tumor models to address our question. We treated cohorts of these animals with anti-CTLA4 antibody or anti-sMIC antibody monotherapy or combination therapy and assessed treatment response and related toxicity. We further corroborate our findings with various indications of $\mathrm{SMIC}^{\text {negative }}$ and SMIC ${ }^{\text {positive }}$ transplantable syngeneic tumor models.
Results

As expected, MIC ${ }^{\text {negative }}$ tumors exhibited nominal response to antiCTLA4 therapy and no response to anti-sMIC therapy. Unexpectedly, animals bearing MIC ${ }^{\text {positive }}$ tumors and with elevated serum SMIC (sMIChi) responded poorly to anti-CTLA4 antibody therapy with significantly shortened survival (Fig. 1). Animals with high levels of serum sMIC had increased lung metastasis and developed colitis in response to anti-CTLA4 antibody therapy (Fig. 2). Co-administration of a sMIC-neutralizing monoclonal antibody B10G5 with the antiCTLA4 antibody alleviated colitis in SMIC $\mathrm{C}^{\mathrm{hi}}$ animals and generated a cooperative anti-tumor therapeutic effect by synergistically augmenting innate and adoptive anti-tumor immune responses. Mechanistically, we show that SMIC downmodulation of CD3z on NK and T cells, which ultimately impairs TCR/CD3 signaling and NK cell receptor signaling and potentially CD4 T cell-mediated tolerance. We further demonstrate that the combination of anti-CTLA4 with anti-sMIC antibody cooperatively primes DC activation, overcomes CD8 T cell tolerance, enhances TCR/CD3 signaling capacity in CD8 T cells, and increases T cell clonality or repertoire complexity in tumor infiltrates.

Conclusions

Our findings imply that a new combination therapy could improve the clinical response to anti-CTLA4 antibody therapy. Our findings also suggest that pre-screening cancer patients for serum SMIC may help in selecting candidates who will elicit a better response to antiCTLA4 antibody therapy.

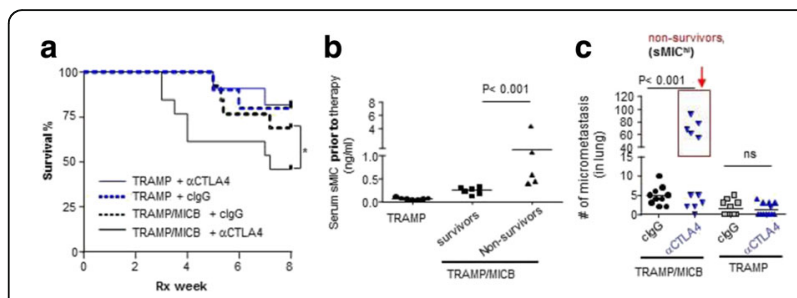

Fig. 1 (abstract 022). High soluble MIC 9sMIC) provokes poor response to anti-CTLA4 therapy

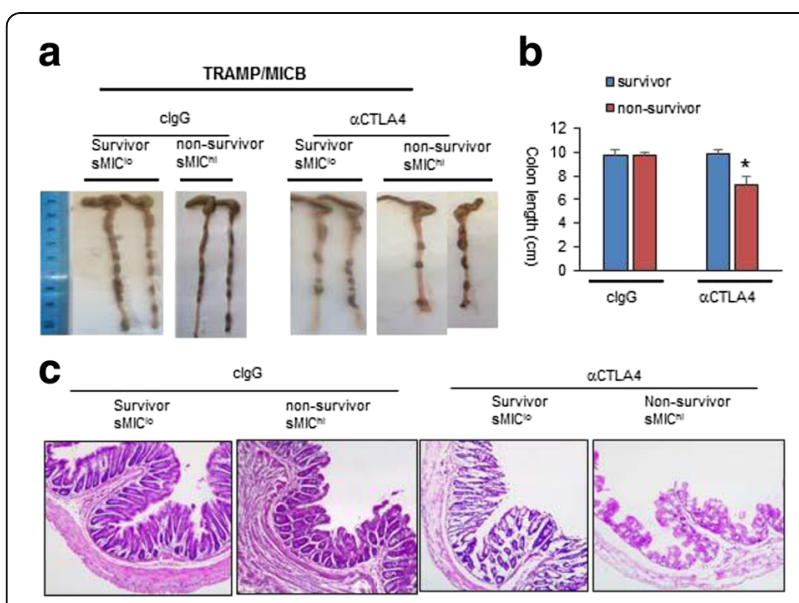

Fig. 2 (abstract 022). High SMIC induces colitis in response to anti-CTLA4 therapy 
023

Multiplex three-dimensional optical mapping of tumor immune microenvironment

Steve Seung-Young Lee, Vytautas P. Bindokas, Stephen J. Kron

University of Chicago, Chicago, IL, USA

Correspondence: Stephen J. Kron (skron@uchicago.edu)

Journal for ImmunoTherapy of Cancer 2017, 5(Suppl 2):023

\section{Background}

The tumor microenvironment is a three-dimensional (3D) system of diverse cellular and non-cellular components whose heterogeneous structure is typically defined by haphazard growth of cancer cells and a disordered microvasculature. Tumor-immune cell interactions occur within this context, providing a challenge for analysis of the infiltrate and anti-tumor immune responses by two-dimensional (2D) methods such as immunohistochemistry (IHC). Toward overcoming the limitations of 2D methods, we introduce Transparent Tissue Tomography (T3) as a tool for quantitative 3D imaging cytometry of the tumor immune microenvironment.

Methods

For T3 imaging cytometry, tumors were sliced into $400 \mu \mathrm{m}$ macrosections to facilitate immunofluorescence staining, optical clearing, and confocal microscopic imaging. Macrosections were stained overnight with panels of four to six directly labeled fluorescent primary antibodies then cleared by D-fructose. The full volume of each macrosection was scanned in each channel using a confocal microscope and tumor images were tomographically reconstructed from the macrosection images. The tumor images were segmented to discriminate cell types, map biomarkers, and perform spatial analysis. Results

As an application of T3 imaging cytometry, we examined the distribution of programmed death-ligand 1 (PD-L1) expression in spontaneous Her2 ${ }^{+}$mammary tumors formed in BALB-NeuT mice. T3 analysis of whole tumors determined PD-L1 expression by tumor cells at the periphery and $\mathrm{CD} 31^{+}$vascular endothelium in the core. For the first time, 3D tomographic projection disclosed vascular PD-L1 expression localized between the endothelium and inner layer of smooth muscle cells. In turn, T3 revealed a strong spatial correlation between $\mathrm{CD} 5^{+}$immune cell distribution and PD-L1 expression. Applying T3 to pharmacokinetic analysis, we observed uneven accumulation of anti-PD-L1 antibody in perfused regions and lack of delivery to hypoxic domains. In addition, 3D vascular mapping showed that the blood vessels wrapped in smooth muscle cell layers restrained anti-PD-L1 antibody extravasation into tumor. Toward translation, T3 was adapted to analyze whole core needle biopsies. Modeling on-treatment biopsy analysis, T3 confirmed a compound effect of radiation and anti-PD-L1 therapy on infiltration of effector cytotoxic lymphocytes such as granzymeB ${ }^{+}$NK cells and T cells.

Conclusions

By assessing multiple tumor parameters simultaneously at cellular resolution, T3 provides a unique window into the heterogeneity of the tumor immune microenvironment. We anticipate that T3 can be applied broadly to facilitate preclinical studies of tumor biology and therapy. In particular, spatial, multiparameter T3 analysis may serve as a tool to improve diagnostic, prognostic and predictive testing of patient biopsies as part of evaluation for immune checkpoint blockade therapy.

\section{4}

First-in-human study with intratumoral administration of a CD40 agonistic antibody: preliminary results with ADC-1013/JNJ-64457107 in advanced solid malignancies

Peter Ellmark', Sandra Irenaeus ${ }^{2}$, Dorte Nielsen ${ }^{3}$, Adnan Deronic ${ }^{1}$ Niina Veitonmäki ${ }^{1}$, Per Norlén ${ }^{1}$, Camilla Wennersten ${ }^{1}$, Jeffrey Yachnin ${ }^{4}$, Gustav Ullenhag ${ }^{2}$

${ }^{1}$ Alligator Bioscience, Lund, Sweden; ${ }^{2}$ Uppsala University, Uppsala, Sweden; ${ }^{3}$ University of Copenhagen, Herlev, Denmark; ${ }^{4}$ Karolinska Institutet and University Hospital, Stockholm, Sweden

Correspondence: Peter Ellmark (pek@alligatorbioscience.com) Journal for ImmunoTherapy of Cancer 2017, 5(Suppl 2):024

\section{Background}

Agonistic antibodies targeting CD40 activate dendritic cells and can expand and activate tumor specific $T$ cells. Preclinical studies have demonstrated that intratumoral administration of CD40 agonists has the potential to improve anti-tumor efficacy and reduce immune related adverse events compared to intravenous administration. Herein, we report the first study of a CD40 agonistic antibody administered intratumorally in cancer patients.

Methods

A Phase I, open label, multicentre study was conducted to evaluate the safety, pharmacokinetics and pharmacodynamics of ADC-1013 in patients with advanced solid tumors who had received established treatments. A 3+3 dose escalation was conducted with a 28-day window (bi-weekly dosing). Twenty-three patients were treated with ADC-1013 intratumorally (dosing from $22.5 \mu \mathrm{g} / \mathrm{kg}$ up to $400 \mu \mathrm{g} / \mathrm{kg}$ ) or intravenously (dosing at $75 \mu \mathrm{g} / \mathrm{kg}$ ). The pharmacodynamic effects observed in the patients were further verified in an hCD40tg mouse model.

Results

Adverse events were primarily fatigue, pyrexia, nausea and vomiting, and were mostly CTCAE Grade 1 or 2 and transient. Two subjects receiving intratumoral bolus injections of $A D C-1013$ into hepatic lesions at a dose of $400 \mu \mathrm{g} / \mathrm{kg}$ experienced Grade 3 abdominal pain assessed as DLTs. Elevations in cytokine levels (MCP-1, TNFa and IL-6) were observed in patients receiving $200 \mu \mathrm{g} / \mathrm{kg}$ and $400 \mu \mathrm{g} / \mathrm{kg}$ of ADC-1013. The systemic ADC-1013 exposure and cytokine release was more pronounced in patients receiving injections in a hepatic lesion than patients receiving injections in a non-hepatic lesion. Treatment with ADC-1013 resulted in a marked decrease in $B$ cell levels in peripheral blood after $24 \mathrm{~h}$, recovering to baseline levels within 8 days. Remaining $B$ cells significantly increased their expression of the cell surface activation marker CD86 $24 \mathrm{~h}$ after first dose, returning to baseline within 8 days.

The pharmacodynamic effects were further studied in hCD40tg mice demonstrating activation of antigen presenting cells such as dendritic cells and subsequent activation of $\mathrm{T}$ cells. Moreover, ADC-1013 treatment in this mouse model acts synergistically with a PD-1 inhibitor.

Conclusions

The results from the first-in-human study of ADC-1013 indicate that intratumoral administration of ADC-1013 into non-hepatic lesions (superficial metastases) is well tolerated at doses up to at least 400 $\mu \mathrm{g} / \mathrm{kg}$, while intratumoral administration of ADC-1013 into hepatic lesions resulted in toxicities associated with cytokine release and Grade 3 adverse events. The pharmacodynamic effects and the preclinical data support further clinical development of ADC-1013 as mono- or combination therapy with e.g. PD-1/PD-L1 targeting antibodies. Janssen is currently developing ADC-1013/JNJ-64457107 for intravenous administration in patients with solid tumors.

\section{5}

Phase I trial of IL-15 superagonist ALT-803 (IL-15N72D:IL-15RaSu/lgG1 Fc complex) in advanced solid tumors: tolerability and correlates of activity

Kim Margolin ${ }^{1}$, Chihiro Morishima², Martin A. Cheever ${ }^{2}$,

Andreanne Lacroix ${ }^{2}$, Steve Fling ${ }^{2}$, Shernan Holtan ${ }^{3}$, Monica Jones ${ }^{4}$, Judy Kaiser ${ }^{2}$, Sylvia Lee ${ }^{5}$, Jeffrey S. Miller ${ }^{3}$, Peter Rhode ${ }^{4}$, Amy Rock $^{4}$ Ann W. Silk ${ }^{6}$, Vamsi Velcheti ${ }^{7}$, Hing C. Wong ${ }^{4}$, Marc S. Ernstoff ${ }^{8}$

${ }^{1}$ City of Hope, Duarte, CA, USA; ${ }^{2}$ Fred Hutchinson Cancer Research Center, Seattle, WA, USA; ${ }^{3}$ University of Minnesota, Minneapolis, MN, USA; ${ }^{4}$ Altor BioScience, Miramar, FL, USA; ${ }^{5}$ University of Washington, Seattle, WA, USA; ${ }^{6}$ Rutgers Cancer Institute of New Jersey, New

Brunswick, NJ, USA; ${ }^{7}$ Cleveland Clinic, Cleveland, OH, USA; ${ }^{8}$ Roswell Park Cancer Center, Buffalo, NY, USA

Correspondence: Kim Margolin (kmargolin@coh.org)

Journal for ImmunoTherapy of Cancer 2017, 5(Suppl 2):025

\section{Background}

IL-15, a common gamma chain ( $\gamma \mathrm{c}$ ) cytokine, stimulates expansion and cytotoxic functions of NK and memory CD8+ T cells. Compared to unmodified IL-15, ALT-803 had superior antitumor activity, low toxicity 
and favorable pharmacokinetics in animal models. These promising data prompted this phase I trial of ALT-803 administered intravenously (i.v.) or subcutaneously (s.c.) to patients with advanced cancers. Methods

ALT-803 was administered at doses of $0.3-20 \mathrm{mcg} / \mathrm{kg}$ weekly i.v. or s.c. for 4 consecutive weeks, every 6 weeks until toxicity or progression. Correlates included pharmacokinetics, immunogenicity, lymphocyte expansion and flow cytometric phenotyping. Clinical endpoints were toxicity and anti tumor activity.

Results

Eleven patients received i.v. ALT-803 (range 0.3 to $6 \mathrm{mcg} / \mathrm{kg}$ ), and 13 patients received s.c. ALT-803 (range 6 to $20 \mathrm{mcg} / \mathrm{kg}$ ) in 9 dose cohorts. Ten had melanoma, 7 renal, 3 head and neck, and 4 lung cancer. The most common dose-related toxicity was injection site reaction with s.C. ALT-803; biopsy showed inflammatory infiltrates. Fatigue, fever and nausea were the next most common adverse events. Maximal increases in white blood cell and lymphocyte counts during cycle 1 were modest across all doses, with means of 1.2-fold (1.2-fold at $20 \mathrm{mcg} / \mathrm{kg}$ s.c.) and 1.5 -fold (1.4-fold at $20 \mathrm{mcg} / \mathrm{kg}$ s.c.) over baseline. NK cell expansion typically peaked on cycle 1 day 15 or 29 , with a mean maximal 2.7-fold increase across all doses, and 3.3-fold for the highest $(20 \mathrm{mcg} / \mathrm{kg})$ s.c. dose cohort. The expansion of circulating CD8+ T cells was modest (mean maximal 1.4-fold increase), but the mean fold increase for the $\%$ positivity of activation marker HLA-DR on CD8+ T cells was 2.9-fold across all dose cohorts and 4.7-fold at $20 \mathrm{mcg} / \mathrm{kg}$ s.c., typically peaking on cycle 1 day 4 . Immunogenicity assays demonstrated no reactivity in patients. Clinical activity was not observed in these heavily-pretreated patients, but combination therapy trials are ongoing. Toxicities were mild, non-dose-limiting and similar to IL-2 and other $\gamma \mathrm{c}$ cytokines. One patient experienced symptomatic diffuse cardiomyopathy that resolved slowly after drug discontinuation and corticosteroid treatment.

\section{Conclusions}

The IL-15 superagonist ALT-803 was well-tolerated with minimal cytokine toxicities in patients with advanced cancer. Substantial increases in NK cells and rapid activation of CD8+ T cells as evidenced by increased HLA-DR expression are promising immunologic effects, providing a strong rationale for combination anticancer immunotherapy with ALT-803.

Trial Registration

NCT01946789

\section{6}

Metabolic adaptations establish immunotherapy resistance in melanoma

Ashvin R. Jaiswal ${ }^{1}$, Shivanand Pudakalakatti ${ }^{2}$, Prasanta Dutta ${ }^{2}$, Liu Arthur ${ }^{2}$, Todd Bartkowiak2, Casey Ager², Cristina Ivan², Michael A Davies²,

James P Allison², Richard Eric Davis ${ }^{2}$, Jennifer Wargo²,

Pratip K Bhattacharya ${ }^{2}$, David Hong ${ }^{2}$, Michael A. Curran ${ }^{2}$

${ }^{1}$ UT MD Anderson Cancer Center, Houston, TX, USA; ${ }^{2}$ The University of

Texas MD Anderson Cancer Center, Houston, TX, USA

Correspondence: Ashvin R. Jaiswal (arjaiswal@mdanderson.org);

Michael A. Curran

Journal for ImmunoTherapy of Cancer 2017, 5(Suppl 2):026

\section{Background}

Despite the success of $T$ cell checkpoint blockade antibodies in treating an array of cancers, the majority of patients still fail to respond to these therapies, or respond transiently and then relapse. The molecular mechanisms which drive lack of response to checkpoint blockade, whether pre-existing or evolved on therapy, remain unclear.

Methods

To address this critical gap in clinical knowledge, we established a mouse model of melanoma designed to elucidate the molecular mechanisms underlying immunotherapy resistance. Through multiple in vivo passages, we selected a B16 melanoma tumor line that evolved complete resistance to combination blockade of CTLA-4, PD1 , and PD-L1, which cures $\sim 80 \%$ of mice of the parental tumor. Using gene expression analysis, proteomics, and immunogenomics, we determined the adaptations engaged by this melanoma to become completely immunotherapy resistant. NMR spectroscopy, Seahorse XF Analysis, flow cytometry, confocal microscopy and western blot analysis provided further insight into the mechanisms driving checkpoint blockade resistance.

Results

Acquisition of immunotherapy resistance by these melanomas was driven by coordinate upregulation of the glycolytic and aldose reductase pathways to create a metabolically hostile microenvironment in which $T$ cell function is profoundly suppressed. When re-introduced into the parental tumor, the genes most closely associated with these metabolic adaptations confer enhanced immunotherapy resistance. We have validated upregulation of these pathways in a unique cohort of melanoma patients who failed dual checkpoint blockade. Additionally, we employed MRI imaging to visualize metabolic changes acquired by resistant tumors in live mice. Clinical application of this technique could provide a much-needed non-invasive tool to predict immunotherapeutic sensitivity of patients.

\section{Conclusions}

Upregulation of glycolytic metabolism and the aldose reductase pathway by melanoma tumor cells cripples T cells in the microenvironment and confers resistance to checkpoint blockade.

\section{7}

Single cell RNA sequencing reveals mechanisms of Merkel Cell Carcinoma escape from intense pressure of $T$ cell immunotherapy Kelly Paulson ${ }^{1}$, Valentin Voillet', Maurizio Perdicchio', Daniel Hunter ${ }^{1}$, William Valente', Felecia Wagener', Samson Koelle², Candice Church ${ }^{3}$, Natalie Vandeven ${ }^{3}$, Hannah Thomas', Aric Colunga ${ }^{3}$, David Koelle ${ }^{3}$, Cassian Yee ${ }^{4}$, Rima Kulikauskas ${ }^{3}$, Robert Pierce ${ }^{1}$, Philip Greenberg', Shailender Bhatia', Jason Bielas ${ }^{1}$, Raphael Gottardo', Paul Nghiem³ Aude Chapuis ${ }^{1}$

${ }^{1}$ Fred Hutchinson Cancer Research Center, Seattle, WA, USA; ${ }^{2}$ Fred Hutchinson Cancer Research Division, Seattle, WA, USA; ${ }^{3}$ University of Washington, Seattle, WA, USA; ${ }^{4}$ MD Anderson, Houston, TX, USA

Correspondence: Aude Chapuis (achapuis@fredhutch.org)

Journal for ImmunoTherapy of Cancer 2017, 5(Suppl 2):027

\section{Background}

$80 \%$ of Merkel cell carcinomas (MCCs) are driven by viral (MCPyV) oncoproteins, rendering them ideal immunotherapy targets. Transfer of MCC-specific T cells alone is insufficient, and half of patients receiving anti-PD1 monotherapy progress. We hypothesized cotransferring MCPyV-specific T cells with checkpoint blockade may enhance efficacy and elucidate interactions between T cells, tumor and the microenvironment. A patient with a dramatic response to $T$ cells plus checkpoint inhibition, whose MCC eventually progressed despite robust $\mathrm{T}$ cell persistence and MCPyV expression, was examined by single-cell RNA sequencing (scRNAseq) to identify the mechanisms of response then immunoevasion.

\section{Methods}

A 59-year-old man with heavily-pretreated, MCPyV-positive MCC ( $>5$ prior therapies including pembrolizumab) received autologous exvivo expanded, HLA B*3502-restricted MCPyV-specific CD8+ T cells $(C D 8+s)$, with sequential addition of pembrolizumab and ipilimumab. A $90 \%$ tumor reduction was sustained for $\sim 1$ year, followed by progression. ScRNAseq was performed on PBMCs and tumor collected before treatment, during response and at progression. Results were compared to flow cytometry and multicolor-IHC.

Results

scRNAseq of PBMCs identified a cluster of intensely-activated CD8+s present exclusively at the time of immunotherapy 
response. Although flow cytometry detected MCPyV-specific $\mathrm{CD} 8+\mathrm{s}$, it was unable to discriminate this proliferative subpopulation. Activated $\mathrm{CD} 8+\mathrm{s}$ in blood corresponded with HLA-DR $+\mathrm{CD} 8+\mathrm{T}$ cell infiltration into the shrinking tumor (including infused cells) and supported a causative role of $\mathrm{CD} 8+\mathrm{s}$ in mediating $M C C$ regression.

scRNAseq of tumor digests was performed to determine the mechanism of acquired immunotherapy resistance. Despite an input of only 10000 cells, the expression profiles of tumor, TIL, macrophages, and fibroblasts could be distinguished. TIL and macrophages expressed transcriptomes that were stable in the pre-immunotherapy and postresistance samples, suggesting these cells were not mediating the acquired resistance. In contrast, tumor cells displayed strikingly different transcriptional profiles before and after development of resistance. Directed analysis revealed tumor-specific loss of only the targeted HLA-B, but not non-targeted HLA-A, at time of progression. This implies intense immunologic and selective pressure from the transferred HLA-B*3502-restricted CD8+s. Unbiased scRNAseq analyses identified significant upregulation of HLA-E, which is an inhibitory signal for NK cells, and helps explain NK avoidance in the context of HLA-loss.

\section{Conclusions}

With small cellular inputs, scRNAseq can provide mechanistic insights beyond traditional $\mathrm{IHC}$ or flow cytometry, here revealing allelespecific HLA downregulation and HLA-E induction by the escaping tumor. Such insights can guide design of improved cellular therapies, in this case suggesting the use of multiple specificities of T cells. Trial Registration

NCT0175845

\section{8}

Functional correlation of increased tumor intrinsic glycolytic activity with resistance to adoptive $\mathrm{T}$ cell therapy

Tina Cascone ${ }^{1}$, Jodi Alicia McKenzie', Rina Mbofung ${ }^{1}$, Simone Punt ${ }^{1}$,

Zhe Wang ${ }^{1}$, Chunyu Xu', Leila Williams' ${ }^{1}$ Zhiqiang Wang',

Christopher Bristow', Alessandro Carugo ${ }^{1}$, Michael Peoples', Lerong $\mathrm{Li}^{1}$

Tatiana Karpinets', Shruti Malu', Caitlin Creasy', Sara Leahey',

Jiong Chen', Chantale Bernatchez' ${ }^{1}$, Y. N. Vashisht Gopal',

Timothy P. Heffernan', Jianhua Hu', Jing Wang', Rodabe N. Amaria',

Levi A. Garraway², Ignacio I. Wistuba', Scott E. Woodman', Jason Roszik', R. Eric Davis', Michael A. Davies', John V. Heymach', Patrick Hwu',

Weiyi Peng

'The University of Texas MD Anderson Cancer Center, Houston, TX, USA;

2Dana-Farber Cancer Institute, Boston, MA, USA

Correspondence: Weiyi Peng (wpeng@mdanderson.org)

Journal for ImmunoTherapy of Cancer 2017, 5(Suppl 2):028

\section{Background}

Adoptive T cell therapy (ACT) has produced impressive responses in a subset of patients with advanced malignancies, particularly those with melanoma, where as many as $50 \%$ of patients may achieve an objective response. However, these responses tend to be transient and only present in a small portion of patients. Thus, there is an urgent need to understand the resistant mechanisms in nonresponders and develop more effective ACT strategies that prevent emergence of resistance or reverse resistance once it occurs. Emerging evidence suggest that tumor intrinsic signaling pathways play a critical role in promoting resistance to $T$ cell-mediated immunotherapy. For example, we recently reported that oncogenic activation of the PI3K pathway resulting from PTEN loss renders melanoma cells refractory to $\mathrm{T}$ cell-mediated therapies and that this could be reverted by targeted inhibition of PI3K signaling. However, work from our laboratory, and others, suggest that melanoma cells may have additional means to circumvent immunotherapy.

Methods

In this study, we employed two independent and unbiased approaches to identify novel molecular determinants of immune resistance. We generated gene expression profiles on PTENdeficient melanoma cell lines from patients to identify alternative immunosuppressive mechanisms and then extended this finding to include non-small cell lung cancer. Then we utilized a new high-throughput shRNA screening platform developed by our group to functionally interrogate immune resistance in melanoma cells.

Results

Results from both analyses implicated tumor-associated glycolysis as a critical pathway that enables tumor cells to evade $\mathrm{T}$ cellmediated antitumor activity. By using samples from melanoma and non-small cell lung cancer patients, we showed that increased expression of glycolysis-related genes is associated with poor $\mathrm{T}$ cell infiltration of tumors. In addition, we found that increased expression of $A L D O A$, which encodes a critical enzyme in the glycolysis pathway, was functionally correlated with reduced sensitivity of tumor cells to T cell-mediated killing. Overexpression of ALDOA impaired $\mathrm{T}$ cell killing of patient-derived melanoma cells, while inhibiting glycolysis restored T cell-mediated apoptosis of tumor cells. More importantly, when we characterized the patterns of gene expression and generated bioenergetic profiles on tumor samples from two non-overlapping ACT-treated patient cohorts, we discovered that tumor glycolytic activity in patients who experienced disease progression following ACT was significantly higher compared to those patients who were responsive to therapy.

Conclusions

Taken together, our results demonstrate that tumor glycolytic metabolism is associated with the efficacy of ACT and identify glycolysis as a candidate target for combinatorial therapeutic intervention.

\section{9}

A dual and opposing role for tumor intrinsic type-II interferon sensing in tumor establishment and acquired resistance Jason Williams, Shuyin Li, Thomas Gajewski, Haochu Huang

University of Chicago, Chicago, IL, USA

Correspondence: Jason Williams (jaybwilliams1@uchicago.edu)

Journal for ImmunoTherapy of Cancer 2017, 5(Suppl 2):O29

\section{Background}

Immunotherapy can augment the host anti-tumor response inducing tumor control and regression. However, in a subset of patients immune selective pressure can drive the outgrowth of resistant tumor cell clones. An understanding of the altered genetic pathways in resistant tumor cell populations is critical to develop therapies to target these tumors.

Methods

To this end, we utilized a whole genome CRISPR library screen to delete genes in B16.SIY cells and selected clones that gained resistance to $T$ cell-mediated killing. One set of mutations was in genes involved in the type-II interferon pathway, namely IFNYR2 and Jak1. To test the role of these genes in subverting the host immune response we generated B16.SIY cells selectively lacking IFNץR2 or Jak1.

Results

In contrast to resistance to T cell-mediated killing in vitro, when implanted into mice, these mutant tumors paradoxically were better controlled in vivo. This phenotype was observed with multiple IFNYR2- and Jak1-deleted B16.SIY lines and using different guideRNAs, and also with a second tumor cell line MC38. Re-introduction of IFNYR2 reverted the phenotype and restored tumor growth. Depletion of host $\mathrm{CD}^{+} \mathrm{T}$ cells eliminated regression of mutant tumors, and increased accumulation of antigen-specific $\mathrm{CD}^{+} \mathrm{T}$ cells was observed in the tumor microenvironment. It seemed likely that IFN- $\gamma$ produced by $\mathrm{CD}^{+}{ }^{+} \mathrm{T}$ cells in the tumor microenvironment might have a dominant negative effect through upregulation of inhibitory factors such as PD-L1 on tumor cells. Indeed, IFNYR2 ${ }^{-/}$and Jak1 ${ }^{-1-}$ tumor cells expressed markedly reduced PD-L1 and IDO in vivo compared to WT tumors. Further, transduction to express PD-L1 restored the ability of IFNYR2 ${ }^{-1-}$ tumors to subvert the host immune response and grow progressively. 


\section{Conclusions}

In summary, we find that the type-ll signaling in tumor cells in some models can be required for the initiation and stabilization of the suppressive tumor microenvironment. Combined with other published data, our results suggest that whether IFN- $\gamma$ on tumor cells is predominantly a positive or negative factor for tumor control may depend on whether inhibitory factors like PD-L1 are dominantly expressed and functional through tumor cells versus host immune cells.

\section{0}

Diversity and composition of the gut microbiome influences responses to anti-PD1-therapy through beneficial changes in innate and adaptive immunity

Vancheswaran Gopalakrishnan', Christine Spencer', Luigi Nezi' ${ }^{1}$,

Alexandre Reuben ${ }^{1}$, Miles Andrews ${ }^{1}$, Tatiana Karpinets ${ }^{1}$, Diego Vicente',

Peter Prieto ${ }^{2}$, Kristi Hoffman', Spencer Wei ${ }^{1}$, Alexandria Cogdill',

Erick Riquelme Sanchez', Florencia McAllister ${ }^{1}$, Michael Wong',

Michael Davies', Alexander Lazar', Jeffrey Gershenwald ${ }^{1}$, Nadim Ajami ${ }^{3}$

Zachary Cooper ${ }^{4}$, Joseph Petrosino ${ }^{3}$, Carrie Daniel-MacDougall',

Michael Tetzlaff', James Allison', Padmanee Sharma', Robert Jenq', Jennifer Wargo

${ }^{1}$ The University of Texas MD Anderson Cancer Center, Houston, TX, USA; ${ }^{2}$ University of Rochester Medical Center, Rochester, NY, USA; ${ }^{3}$ Baylor College of Medicine, Houston, TX, USA; ${ }^{4}$ Medlmmune, Gaithersburg, MD, USA

Correspondence: Jennifer Wargo (jwargo@mdanderson.org)

Journal for ImmunoTherapy of Cancer 2017, 5(Suppl 2):030

\section{Background}

Cancer therapy has benefitted greatly from immune checkpoint inhibitors that target negative regulators of T-cell activation, however responses are variable and not always sustained. Recent evidence in murine models suggests that the gut microbiome can be modulated to enhance responses to immune checkpoint blockade. However, this has not been deeply investigated in patients.

\section{Methods}

We collected buccal and stool samples from a cohort of anti-PD-1 treated metastatic melanoma patients $(n=112)$. Patients were classified as either responders (R) or non-responders (NR) based on RECIST criteria ( $\mathrm{R}=\mathrm{CR}$, $\mathrm{PR}$, or $\mathrm{SD}>6$ months, NR=PD or $\mathrm{SD}<6$ months). $16 \mathrm{~S}$ rRNA and whole-genome shotgun sequencing were performed to characterize the diversity, composition and functional capabilities of the microbiome. Immune profiling (via immunohistochemistry and flow cytometry) was performed in available tumors and serum samples at baseline. Fecal microbiota transplants (FMT) from $R$ versus NR patients were performed in germ-free (GF) mice, tumors were implanted and markers of systemic and anti-tumor immunity queried.

Results

We observed significant differences in the diversity and composition of the gut microbiome in R versus NR to anti-PD- 1 therapy at baseline with no clear differences in the oral microbiome. Specifically, $\mathrm{R}$ had a higher alpha diversity compared to NR (11.2 versus $6.4, p<0.01)$, and an enrichment of the Faecalibacterium genus of the Ruminococcaceae family. In contrast, the Bacteroidales order was found to be enriched in NR. High abundance of Faecalibacterium was also associated with improved progression-free survival $(H R=2.92 .95 \% C . I=1.02-12.52) \mathrm{Im}$ mune profiling demonstrated significantly increased immune infiltrates in baseline tumor samples of $R$ versus NR ( $p=0.04)$, with a positive correlation between CD8+ T-cell density in baseline tumor and blood samples and abundance of beneficial Ruminococcaceae bacteria in the gut microbiome $\left(r^{2}=0.42, p<0.01\right)$. Distinct metabolic signatures were also observed in the gut microbiome with synthetic processes predominating in R and degradative processes predominating in NR. In FMT studies, GF mice transplanted with R-stool demonstrated delayed tumor outgrowth and enhanced responses to anti-PD-L1 therapy $(p<0.01)$. Immune profiling in these mice revealed a higher density of innate and adaptive immune infiltrates in the tumor and gut, and upregulation of PD-L1 in the tumor microenvironment.

\section{Conclusions}

Diversity and composition of the gut microbiome was associated with differential responses to anti-PD-1 therapy, and enhanced systemic and anti-tumor immunity in metastatic melanoma patients. Our results have far-reaching implications and suggest that modifying the gut microbiome could potentially enhance therapeutic responses to immune checkpoint blockade.

\section{1}

Preclinical characterization of a novel STING agonist, MK-1454 Saso Cemerski', Michael Altman ${ }^{1}$, Madhavi Bandi', Wonsuk Chang ${ }^{2}$, Yiping Chen', Long Cui ', Jared Cumming', Lauren Dorosh', Lauren Flateland', Yanhong Ma', Sarah Javaid', Thu Ho ${ }^{3}$, Johnny Kopinja', Jason Laskey ${ }^{1}$, Ellen Minnihan ${ }^{1}$, Laura Price ${ }^{3}$, Robin Mogg ${ }^{3}$, Gregory O'Donnell', Bo-Sheng Pan', Samanthi Perera', Jennifer Piesvaux',

Jeremy Presland', Larissa Rakhilina', Gottfried Schroeder', Michael Slavonic', Steven Smock', Benjamin Wesley Trotter', Daniel Wyss', Qing Zhao ${ }^{4}$, Shuxia Zhao', Svetlana Sadekova ${ }^{5}$, Ying Y Y ${ }^{5}$, Anuradha Khilnani ${ }^{4}$, Archie Tse ${ }^{4}$

${ }^{1}$ Merck Research Laboratories, Boston, MA, USA; ${ }^{2}$ Merck Research Laboratories, Kenilworth, NJ, USA; ${ }^{3}$ Merck Research Laboratories, West Point, PA, USA; ${ }^{4}$ Merck Research Laboratories, Rahway, NJ, USA; ${ }^{5}$ Merck Research Laboratories, Palo Alto, CA, USA

Correspondence: Saso Cemerski (saso.cemerski@merck.com)

Journal for ImmunoTherapy of Cancer 2017, 5(Suppl 2):O31

\section{Background}

Several innate immune danger-sensing pathways have emerged as promising targets for enhancing cancer immunotherapy. In recent years, cyclic dinucleotide agonists of STING (Stimulator of interferon genes) have demonstrated significant anti-tumor efficacy in several mouse syngeneic tumor models and set the stage for STING agonists to be evaluated in human clinical trials.

Methods

Binding of STING agonist MK-1454 to mouse and human STING was evaluated using Biacore. STING activity was monitored in human whole blood and PBMCs, in multiple primary mouse and human cells, cell lines and differentiated myeloid cell subsets using cytokine production and cell activation as readouts. In vivo efficacy studies of intratumorally-dosed MK-1454 were performed in the presence or absence of an anti-PD- 1 antibody in MC38, CT26 and B16F10 syngeneic tumor models. Characterization of MK-1454 activity in primary human tumors was assessed in human histoculture studies. Gene profiling and STING gene signature studies were performed using custom-built Nanostring reagents.

Results

In vitro studies demonstrated potent binding of MK-1454 to human and mouse STING and rapid induction of type-I interferon and proinflammatory cytokine production in human and mouse cell lines and primary cells in a STING-dependent manner. Mechanistic studies were conducted to assess the effect of STING agonism across multiple myeloid cell subsets including monocytic MDSCs, M2 macrophages and TAMs, which have been previously suggested to represent the suppressive myeloid component of the TME. In addition, we describe important effects of STING agonism on mouse and human T cells and highlight some key speciesrelated differences. Critically, we demonstrate that human primary tumors, stimulated ex vivo with MK-1454, respond robustly as indicated in cytokine production and gene profiling studies. Additional studies using in vitro-stimulated human whole blood were used to define a STING gene signature that could represent a valuable biomarker in the clinic. In multiple mouse syngeneic tumor models, MK-1454 induced strong antitumor responses, effecting full tumor eradication upon intratumoral dosing, either as a single agent or in combination with PD-1 blockade, depending on the tumor model and dose level. We also show that MK1454 induced tumor-specific adaptive immune memory as demonstrated by the lack of tumor growth in tumor re-challenge studies. 


\section{Conclusions}

MK-1454, a novel STING agonist, induces potent cytokine responses and immune cell activation in vitro and robust anti-tumor activity in vivo. The preclinical data presented here support the ongoing clinical evaluation of MK-1454 in cancer patients both as monotherapy and in combination with Keytruda.

\section{2}

Phase 1 trial of CA-170, a first-in-class, orally available, small molecule immune checkpoint inhibitor (ICl) dually targeting PD-L1 and VISTA, in patients with advanced solid tumors or lymphomas Yung Jue Bang ${ }^{1}$, John Powderly ${ }^{2}$, Manish Patel ${ }^{3}$, Kyu Pyo Kim ${ }^{4}$.

James Lee ${ }^{5}$, Joshua Brody ${ }^{6}$, Javier Garcia-Corbacho ${ }^{7}$, Sun Young Rha ${ }^{8}$, Funda Meric-Bernstam ${ }^{9}$, Erika Hamilton ${ }^{10}$, Santiago Ponce Aix ${ }^{11}$ Radhakrishnan Ramchandren ${ }^{12}$, Jeffrey Sosman ${ }^{13}$, Myung Ju Ahn ${ }^{14}$, Mario Snozl ${ }^{15}$, Gerald Falchook ${ }^{16}$, Marta Gil Martin ${ }^{17}$, Hongwei Wang ${ }^{18}$ Lisa Adams ${ }^{18}$, Christopher Caldwell ${ }^{18}$, Adam Lazorchak ${ }^{18}$,

Timothy Wyant ${ }^{18}$, Anna Ma ${ }^{18}$, David Tuck ${ }^{18}$, Adil Daud ${ }^{19}$

${ }^{1}$ Seoul National University Hospital, Seoul, Republic of Korea; ${ }^{2}$ Carolina

BioOncology, Huntersville, NC, USA; ${ }^{3}$ Florida Cancer Specialists \& Research Institute, Sarasota, FL, USA: ${ }^{4}$ Asan Medical Center, Seoul, Republic of Korea; ${ }^{5}$ University of Pittsburgh School of Medicine UPMC Cancer Pavilion, Pittsburgh, PA, USA; ${ }^{6}$ Icahn School of Medicine at Mount Sinai, New York, NY, USA; ${ }^{7}$ Hospital Clinic i Provincial, Barcelona, Spain; ${ }^{8}$ Yonsei University Health System - Severance Hospital, Seoul, Republic of Korea; ${ }^{9}$ UT MD Anderson Cancer Center, Houston, TX, USA; ${ }^{10}$ Sarah Cannon Research Institute - TN, Nashville, TN, USA; ${ }^{11}$ Hospital Universitario 12 de Octubre, Madrid, Spain; ${ }^{12}$ Karmanos Cancer Institute, Detroit, MI, USA; ${ }^{13}$ Northwestern University Research, Evanston, IL, USA; ${ }^{14}$ Samsung Medical Center, Seoul, Republic of Korea; ${ }^{15}$ Smilow Cancer Center, New Haven, CT, USA; ${ }^{16}$ Sarah Cannon Research Institute at HealthONE, Denver, CO, USA; ${ }^{17}$ Catalan Institute of Oncology, Bacelona, Spain; ${ }^{18}$ Curis Inc., Lexington, MA, USA; ${ }^{19}$ University of California San Francisco (UCSF), San Francisco, CA, USA

Journal for ImmunoTherapy of Cancer 2017, 5(Suppl 2):032

\section{Background}

Programmed-death 1 (PD-1) and V-domain Ig suppressor of T-cell activation (VISTA) are independent immune checkpoints that negatively regulate T-cell function and are implicated in various malignancies. Pre-clinical studies demonstrated that dual blockade of both checkpoints can be synergistic. CA-170 is a novel oral small molecule ICI that directly targets both PD-L1/L2 and VISTA and demonstrated significant anti-tumor activity in multiple preclinical animal models. Methods

Enrollment initially followed accelerated titration and subsequently switched to $3+3$ design. Cohorts of selected dose levels were expanded with additional patients. The expansion phase allows for enrollment of patients with selected indications known to be sensitive to ICl. Primary objectives: safety, maximum tolerated dose (MTD) and recommended Phase 2 dose. Secondary objectives: pharmacokinetics (PK) and anti-tumor activity. Exploratory endpoints: biomarkers and pharmacodynamic (PD) effects in peripheral blood and tumor tissues.

Results

A total of 34 patients have been treated across 6 dose levels (50 -800 $\mathrm{mg}$ ) with 28 evaluable for safety and 4 still ongoing in cycle 1 . Enrolled tumor types include lung, ovarian, lymphoma, head and neck, renal cell carcinoma, etc.

No DLTs or $\geq$ grade 3 drug-related adverse events (AEs) have been observed thus far. The most common treatment-emergent AEs (all grades) were fatigue $(24 \%)$, gastritis $(20 \%)$, nausea $(20 \%)$, vomiting $(20 \%)$, chills $(16 \%)$, constipation (16\%) and headache (16\%). These were predominantly grade 1 and self-limiting events.

Seven newly enrolled patients are pending restaging. Nineteen patients were evaluable for anti-tumor activity with 12 showing stable disease and 4 on study for $>6$ cycles. An ICl naïve melanoma patient treated at $600 \mathrm{mg}$ had lesion shrinkage by $14 \%$ per RECIST and $23 \%$ per immune-related response criteria by the end of cycle 2 and is currently ongoing on treatment.
CA-170 exhibits approximately dose proportional plasma exposure with $T_{1 / 2} 5-12$ hours. Evidence of peripheral T cell activation was observed with an increased proportion of circulating $\mathrm{CD}^{+}$and $\mathrm{CD} 4^{+}$ T cells expressing activation markers. Additionally, preliminary tumor biopsy data has shown increases in the number of immune cells post-treatment versus pre-treatment.

\section{Conclusions}

The data suggest CA-170 has an acceptable safety profile with preliminary signs of anti-tumor activity and peripheral immune modulation and approximately dose proportional PK profile. MTD has not been reached. These data warrant the continued clinical development of CA-170. Dose escalation is ongoing. Expansion cohorts in selected indications are planned.

Trial Registration

Clinical trial identifier: NCT02812875.

\section{3}

Variance from evidence-based management of immune-related adverse events among healthcare providers: analysis of an online management decision tool

Krista Marcello ${ }^{1}$, Kevin L. Obholz', Timothy A. Ouill', Jeffrey S. Weber ${ }^{2}$

${ }^{1}$ Clinical Care Options, LLC, Reston, VA, USA; ${ }^{2}$ NYU Langone Medical Center, New York, NY, USA

Correspondence: Krista Marcello (kmarcello@clinicaloptions.com) Journal for ImmunoTherapy of Cancer 2017, 5(Suppl 2):O33

\section{Background}

Immune checkpoint inhibitors (ICls) are altering the treatment paradigm in oncology, impacting the care of a rapidly increasing number of patients; but many healthcare providers (HCPs) remain unfamiliar and inexperienced with managing the unique spectrum of immune-related adverse events (irAEs). An online decision support tool was developed to give HCPs easy access to fair-balanced guidance and management algorithms that are patient-specific and extend beyond standard recommendations found in the respective product inserts [1].

Methods

To use the tool, HCPs entered patient symptoms, organ system affected, and the grade or severity of the event along with their planned management strategy. Subsequently, the HCPs using the tool were provided an expert recommendation for management of that specific irAE. Recommendations in the tool comprised algorithms developed from peer-reviewed publications and the personal clinical experience of Jeffrey S. Weber, MD, PhD. Here, we report a comparison of the intended irAE management of HCPs for 2896 cases entered into the tool from 11/9/16 through 7/21/17 with the expert recommendations for those patient cases.

Results

Cases involving $\mathrm{Gl}$ symptoms ( $\mathrm{n}=818 ; 28 \%$ of all cases) were most frequently entered into the tool with renal symptom cases $(n=153$; $5 \%$ of all cases) having the fewest entries (Table 1). The planned irAE management strategy of HCPs differed from the expert recommendations for $49 \%$ of the cases overall with the greatest divergence in neurologic- and endocrine-related cases (56\% and $66 \%$, respectively). The proportion of cases in which the planned management strategies of the HCPs differed from the expert recommendations also varied by symptom grade/severity ranging from $84 \%$ differing for grade 3 adrenal insufficiency to $21 \%$ differing for grade 4 renal toxicity. Overall, the largest proportion of cases with HCP variance from optimal recommended practice occurred with those having intermediate to high symptom severity (grades $2 / 3$ ). Overall, of $648 \mathrm{HCPs}$ who responded to a survey about the impact of the tool on their practice, $93 \%$ indicated that the recommendations provided by the tool either changed or confirmed their management plan.

Conclusions

These data suggest that many HCPs are not optimally managing irAEs associated with $\mathrm{ICl}$ use and that an online tool can provide a resource to improve patient care and safety. A detailed analysis of the tool, including case entries and planned management vs best practice recommendations for each irAE and grade will be presented. 
Reference

1. Clinical Care Options (US). Managing Immune-Related Adverse Events: An Interactive Algorithm Tool [Internet]. Clinical Care Options Oncology (US); 2017 May. Available from: www.clinicaloptions.com/immuneAEtool

Table 1 (abstract 033). See text for description

\begin{tabular}{|l|c|}
\hline Case Queries Entered by HCPs, $\mathbf{n}(\%)$ & $\begin{array}{c}\text { HCP Planned Management Differed } \\
\text { from Tool Recommendation, } \mathbf{n}(\%)\end{array}$ \\
\hline Overall, 2896 (100) & $\mathbf{1 4 1 9 ( 4 9 )}$ \\
\hline Gastrointestinal (colitis, diarrhea), 818 (28) & $364(44)$ \\
\hline Pulmonary (pneumonitis), 608 (21) & $321(53)$ \\
\hline Endocrine, 433 (15) & $285(66)$ \\
\hline Hepatic, 416 (14) & $162(39)$ \\
\hline Dermatologic (rash), 305 (11) & $145(48)$ \\
\hline Neurologic, 163 (6) & $91(56)$ \\
\hline Renal (nephritis) 153 (5) & $51(33)$ \\
\hline
\end{tabular}

\section{4}

Cross-dressed dendritic cells drive anti-tumor immunity

Brendan MacNabb, Douglas Kline', Justin Kline

The University of Chicago, Chicago, IL, USA

Correspondence: Brendan MacNabb (bwm@uchicago.edu)

Journal for ImmunoTherapy of Cancer 2017, 5(Suppl 2):034

\section{Background}

Dendritic cells (DCs) play a central role in the orchestration of antitumor T cell responses. The presentation of tumor-derived peptide antigens on class I major histocompatibility complex molecules (MHC I) by DCs is essential for the activation of antigen-specific $C D 8^{+} \mathrm{T}$ cells. Emerging evidence has revealed that migratory Batf3-lineage DCs play a dominant role in the priming of anti-tumor $\mathrm{CD}^{+} \mathrm{T}$ cell responses against solid tumors. However, the cellular mechanisms of tumor antigen presentation by these DCs have not been formally demonstrated. Batf3-lineage DCs are adept at cross-presenting exogenous antigens on $\mathrm{MHC}$ I molecules, and it has been presumed that this antigen presentation pathway is critical in anti-tumor immunity.

Methods

To determine the contribution of cancer cell-derived MHC I on antigen-specific $\mathrm{CD}^{+} \mathrm{T}$ cell priming, we deleted $\mathrm{H} 2-\mathrm{K}^{\mathrm{b}}$ in the murine acute myeloid leukemia cell line, C1498, using CRISPR/Cas9. Engraftment of $\mathrm{K}^{\mathrm{b}+/+}$ and $\mathrm{K}^{\mathrm{b}-/-} \mathrm{C} 1498$ cells into syngeneic C57BL/6 mice, as well as those lacking TAP-1 (a critical component of the classical cross-presentation pathway), and all $\mathrm{MHC} I\left(\mathrm{~K}^{\mathrm{b}} / \mathrm{D}^{\mathrm{b}--/}\right)$, allowed us to interrogate the importance of different antigen presentation pathways to the anti-tumor $\mathrm{CD}^{+} \mathrm{T}$ cell response.

Results

Consistent with published data, antigen-specific $\mathrm{CD}^{+} \mathrm{T}$ cell priming against localized C1498 tumors was entirely dependent upon Batf3lineage migratory DCs, revealing no role for direct priming by C1498 cells. Surprisingly, the activation of endogenous and adoptivelytransferred tumor-specific $\mathrm{CD}^{+} \mathrm{T}$ cells was absent in tumor-draining lymph nodes of mice harboring localized C1498 $\mathrm{K}^{\mathrm{b}-{ }^{-}-}$tumors, suggesting that antigen cross-presentation by DCs was insufficient to activate anti-tumor $\mathrm{CD}^{+} \mathrm{T}$ cells. Conversely, cancer-derived $\mathrm{MHC}$ । was dispensable for antigen recognition by $\mathrm{CD} 8^{+} \mathrm{T}$ cells in spleens of mice with disseminated leukemia. Further, tumor-derived MHC I was abundantly observed on the surface of DCs isolated from the solid tumor micro-environment, and on migratory DCs in the tumordraining lymph node. However, no cancer-derived MHC I was found on splenic DCs in leukemia-bearing mice, in which antigen-specific $\mathrm{CD}^{+} \mathrm{T}$ cell responses occurred in a TAP-dependent fashion.

\section{Conclusions}

Together, these observations support a model in which the CD8 ${ }^{+} \mathrm{T}$ cell response to an MHC I-expressing, localized cancer is driven by Batf3-lineage DCs cross-dressed with intact tumor-derived peptideMHC I molecules. Furthermore, our data reveal that the processing and presentation of tumor antigens occurs in a fundamentally different manner for solid and hematologic malignancies. These findings could have broad implications both for basic immunology research, and for the development of DC-oriented tumor vaccines.

\section{Poster presentations Biomarkers and Immune Monitoring}

\author{
P1 \\ Characterization of the tumor microenvironment and immune \\ profile in non-small cell lung cancer \\ Andreas Ackermann ${ }^{1}$ Hanny Musa', Johannes Zimmermann², \\ Felix Segerer ${ }^{2}$, Moritz Widmaier ${ }^{2}$, Mindaugas Andrulis ${ }^{3}$ \\ ${ }^{1}$ Boehringer Ingelheim Pharma GmbH \& Co. KG, 88397 Biberach an der \\ Riss, Germany; ${ }^{2}$ Definiens AG, 80636 Munich, Germany; ${ }^{3}$ Klinikum \\ Ludwigshafen, 67063 Ludwigshafen am Rhein, Germany \\ Correspondence: Andreas Ackermann \\ (andreas.ackermann@boehringer-ingelheim.com) \\ Journal for Immunotherapy of Cancer 2017, 5(Suppl 2):P1
}

\section{Background}

Immunotherapies with programmed death receptor-1/programmed death-ligand 1 (PD-1/PD-L1)-blocking antibodies have shown encouraging results in patients with advanced NSCLC, HNSCC, RCC, Melanoma, Hodgkin Lymphoma and other cancer types. Despite a subset of patients exhibiting durable and long lasting benefit, the majority of patients show no signs of efficacy. The identification of the right patient population is often challenging, and although there is an incremental understanding of tumor characteristics and biomarkers bearing limited predictive value, such as PD-L1, there is a clear need for an accelerated development of better predictive biomarkers. This knowledge may permit the guidance of therapyrelevant decisions and potentially increase the clinical benefit of CPIrelated anti-cancer interventions.

Methods

To broaden our understanding of the spatial expression of relevant biomarkers in the tumor microenvironment and gain deeper insights into immune-stimulatory mechanisms, we performed histopathological analysis, image analysis, and gene expression analysis in a complementary approach. In thirty $(n=30)$ NSCLC tissues the expression patterns of preselected biomarkers (CD8 and PD-L1) were conventionally evaluated by a board-certified pathologist and the obtained $\mathrm{H}$-scores were compared with Image Analysis (IA)-derived i-scores. Subsequently, gene expression data for all 30 NSCLC samples were generated in order to complement the histopathological evaluations with mRNA expression patterns.

Results

Computer-based image analysis algorithms for the quantification of CD8 and PD-L1 have been established showing a very high accuracy and concordance compared to conventional histopathological evaluations performed by trained pathologists. The complementary analysis based on image analysis, conventional histopathological evaluation, and gene expression analysis revealed excellent/good agreement. Based on the attained knowledge from the multi-modal approach the image analysis tools were further optimized. Following optimization, preliminary data has demonstrated that the optimized image analysis algorithms and the application of virtual multiplexing approaches permits characterization of samples into four types of tumor microenvironment sensu Teng et al [1]. Subsequently, a further seventy $(n=70)$ NSCLC samples will be interrogated using the optimized image analysis tools and correlated, where applicable, with gene expression analysis.

Conclusions

The insights gained during this complementary analysis will help us to design and implement advanced biomarker investigations in future clinical trials to support the co-development of valuable biomarkers in the field of cancer immunotherapy. The application of advanced image analysis and virtual multiplexing approaches may be suitable vehicles to better characterize the tumor microenvironment, honing precision medicine within the immune-oncology field.

\section{References}

1. Teng, et al. Classifying cancers based on T cell infiltration and PD-L1. Cancer Res. 2015; 75: 2139-45. 
P2

ADXS-PSA immunotherapy increases the magnitude and quality of prostate cancer antigen-specific $T$ cell responses in patients with metastatic castration-resistant prostate cancer

Sandra M. Hayes ${ }^{1}$, Robert G. Petit ${ }^{1}$, Lawrence Fong ${ }^{2}$, Mark Stein ${ }^{3}$, Ronald Tutrone ${ }^{4}$, Anthony Mega ${ }^{5}$, Naomi Haas ${ }^{6}$

${ }^{1}$ Advaxis Immunotherapies, Inc., Princeton, NJ, USA; ${ }^{2}$ UCSF University of California, San Francisco, CA, USA; ${ }^{3}$ The Cancer Institute of New Jersey CINJ Rutgers, Inc., New Brunswick, NJ, USA; ${ }^{4}$ Chesapeake Urology Research Associates, Towson, MD, USA; ${ }^{5}$ Lifespan Oncology Clinical Research, Providence, RI, USA; ${ }^{6}$ University of Pennsylvania Abramson Cancer Center, Philadelphia, PA, USA

Correspondence: Ronald Tutrone (ahmad@advaxis.com)

Journal for ImmunoTherapy of Cancer 2017, 5(Suppl 2):P2

\section{Background}

Active immunotherapies, such as ADXS-PSA, are designed to generate tumor antigen-specific $T$ cell effectors that recognize and kill tumor cells. ADXS-PSA, a highly attenuated Listeria monocytogenes-based immunotherapy that targets prostate-specific antigen (PSA), is currently being evaluated as a treatment for metastatic castration-resistant prostate cancer (mCRPC) in the phase 1/2 KEYNOTE-046 trial as a monotherapy (Part A) and in combination with KEYTRUDA ${ }^{\oplus}$ (Part B). Because tumor antigen-specific $T$ cell responses may be linked to the clinical efficacy of active immunotherapies, we quantified the frequency of functional prostate cancer antigen-specific T cells in the peripheral blood of ADXS-PSA-treated mCRPC patients by ELISpot analysis.

\section{Methods}

ELISpot assays were performed on peripheral blood mononuclear cells (PBMCs) isolated at multiple time points from 9 mCRPC patients who received 3 doses of ADXS-PSA monotherapy in Part A, the ADXS-PSA dose-determining stage, of the KEYNOTE-046 trial (NCT02325557). In addition to peptides from PSA, $\mathrm{CD}^{+}$and $\mathrm{CD} 8^{+} \mathrm{T}$ cell reactivity was assayed to peptides from prostatic acid phosphatase (PAP), prostatespecific membrane antigen (PSMA), prostate stem cell antigen (PSCA), and prostein to determine the extent of antigen cascade/spreading after ADXS-PSA treatment. Secretion of IFNy, TNFa, and the cytolytic granule granzyme $B$ were assayed simultaneously to examine the quality (i.e. degree of multi-functionality) of the T cell responses.

Results

During a 9-week treatment course, which included 3 ADXS-PSA doses, 7/9 patients exhibited increases in the magnitude of the PSA-reactive T cell response, including a patient with undetectable PSA-reactive $T$ cells at baseline. Notably, during the same time course, increases above baseline in the frequency of $T$ cells reactive to one or more of the other prostate cancer antigens was observed in all 9 patients. In addition to increases in the frequency of prostate cancer antigen-specific $T$ cells, all 9 patients exhibited increases in the quality of $T$ cells reactive to at least 1 of the 5 prostate cancer antigens, indicating that ADXS-PSA induces multifunctional T cell responses to a broad range of prostate cancer antigens.

\section{Conclusions}

These results demonstrate that ADXS-PSA monotherapy increases the magnitude and quality of $T$ cell responses specific not only to PSA, its target antigen, but also to other prostate cancer antigens, which is indicative of antigen cascade/spreading. Patient enrollment is ongoing for Part $\mathrm{B}$, in combination with KEYTRUDA ${ }^{\oplus}$.

Trial Registration

ClinicalTrials.gov Identifier NCT02325557

P3

Persistence of 6-thioguanine-resistant T cell clones in a melanoma patient with durable antitumor response following treatment with immune checkpoint blockade

Cindy Zuleger ${ }^{1}$, Michael Newton ${ }^{1}$, Irene Ong ${ }^{1}$, Mark Albertini ${ }^{1,2}$

${ }^{1}$ Carbone Cancer Center at the University of Wisconsin School of Medicine and Public Health, Madison, WI, USA; ${ }^{2}$ William S. Middleton

Memorial Veterans Hospital, Madison, WI, USA

Correspondence: Mark Albertini

Journal for ImmunoTherapy of Cancer 2017, 5(Suppl 2):P3

\section{Background}

Ex vivo selection of T cells resistant to 6-thioguanine can be used to identify $T$ cells with in vivo mutation in the hypoxanthine-guanine phosphoribosyltransferase (HPRT) gene. We hypothesize that in vivo HPRT- mutant T cells (MT) will enrich for in vivo proliferating $\mathrm{T}$ cells and provide a predictive biomarker for melanoma patients treated with immune checkpoint blockade.

Methods

This study examined clonotypic diversity and $T$ cell persistence in peripheral blood mononuclear cells (PBMC) freshly cryopreserved (T0) and in PBMC expanded as mass cultures with (MT) or without (wild-type (WT)) 6-thioguanine selection in a metastatic melanoma patient who achieved a durable antitumor response following treatment with intratumoral a-gal glycolipid injections (IT-AG) followed by Ipilimumab (Ipi). IT-AG (0.1 mg/injection every 4 weeks for 2 doses) was initially administered, and Ipi (3 mg/kg (capped at $125 \mathrm{~kg}$ )/dose every 3 weeks for 4 doses) was then administered 4 weeks later at a time of disease progression. Blood samples were obtained before and 4-weeks after IT-AG as well as 1-, 4-, and 13-months post-Ipi. TCR beta chain (TRB) repertoire of TO, MT and WT were examined via $5^{\prime}$ RACE and Illumina MiSeq sequencing. TRB repertoire of T cells from a tumor sample obtained $\sim 20$ months prior to IT-AG (T-T0) was also evaluated. TRB were collapsed to identical nucleotide sequences to enumerate T cell clones.

Results

Expanded or activated T cell clones, defined by sequences with identical TRB, were detected in T-T0 as well as in TO, WT, and MT at each of the 5 time points. The range of TRB sequences was as follows: T0: 47,602-58,588 (median 54,284); WT: 49,799-58,896 (median 52,277); MT: 35,527-58,217 (median 49,035), and 51,713 in T-T0. The range of distinct TRB amino acid sequences was as follows: T0: 4,682-9,644 (median 5,993); WT: 2,083-4,573 (median 2,702); MT: 84-182 (median 154), and 1850 in T-T0. Clonotypic diversity, measured as the number of unique TRB nucleotide sequences, was substantially reduced in MT compared to either WT or T0 at each time point. Numerous TRB matches were observed between MT and WT and T0, as well as between T-T0 and MT, WT and TO. The patient achieved a durable antitumor response, and several TRB persisted in this patient over a four-year span. Some TRB from this study matched TRB from the literature with defined melanoma specificity.

Conclusions

MT from melanoma patients are enriched for proliferating and/or activated T cells and merit additional study as a biomarker of response to immunotherapy.

\section{P4}

The prognostic and predictive roles of a CD8/PD-L1 signature in cancer

Sonja Althammer ${ }^{1}$, Keith Steele ${ }^{2}$, Moritz Widmaier', Charles Brown ${ }^{2}$ Tze Heng Tan ${ }^{1}$, Lorenz Rognoni ${ }^{1}$, Hélène Kaplon ${ }^{3}$, David Friedrich ${ }^{1}$,

Brandon W Higgs², Günter Schmidt', Koustubh Ranade²,

Marie-Caroline Dieu-Nosjean ${ }^{3}$

'Definiens, Munich, Germany; ${ }^{2}$ Medimmune, Gaithersburg, MD, USA;

${ }^{3}$ INSERM, UMRS 1138, Paris, France

Correspondence: Sonja Althammer (ttan@definiens.com)

Journal for ImmunoTherapy of Cancer 2017, 5(Suppl 2):P4

\section{Background}

Predictive signatures to a-PD1 and a-PD-L1 immunotherapies are active research areas. We previously showed that non-small cell lung cancer (NSCLC) patients with high densities of CD8(+) and PD-L1(+) cells have improved outcome compared to those with high CD8(+) or PD-L1(+) cell densities alone [1]. The applicability of this signature to other indications such as urothelial carcinoma (UC) is uncertain. The potential prognostic contribution of this signature is also unknown.

\section{Methods}

The predictive potential of the CD8/PD-L1 signature in UC, was tested in baseline tumor samples obtained from 43 durvalumabtreated patients in a phase 1/2 clinical trial (NCT01693562). 
To assess the prognostic significance in NSCLC, we analyzed surgically-resected tumors of 134 NSCLC patients that later received chemotherapy (non-IO). Tumors were immunostained for CD8 (SP239) and PD-L1 (SP263) using single stains (UC) or a dual immunohistochemistry (IHC) assay (NSCLC). Densities of CD8(+) and PDL1(+) cells from IHC stained digitized slides were calculated by the Definiens Tissue Phenomics Platform for a total of 183 patient samples.

Results

UC patients with CD8/PD-L1(+) profiles showed improved survival compared to those with CD8/PD-L1(-) profiles $(p<0.06)$, which may help to identify patients with beneficial outcome under a-PD-L1 treatment.

Further, non-IO treated NSCLC patients with CD8/PD-L1(+) profiles did not show improved OS over those with CD8/PD-L1(-) profiles, suggesting that the CD8/PD-L1 signature is predictive of therapeutic response to a-PD-L1 in NSCLC patients (Table 1).

\section{Conclusions}

Our results demonstrate the value of profiling the tumor and its immune-contexture by image analysis for discovery of prognostic and predictive signatures in NSCLC and UC.

Trial Registration

ClinicalTrials.gov Identifier NCT01693562

\section{Reference}

1. Althammer $\mathrm{S}$, et al. Combinatorial CD8+ and PD-L1+ cell densities correlate with response and improved survival in non-small cell lung cancer (NSCLC) patients treated with durvalumab. Immunother Cancer 2016; 4(Suppl 2):91

Table 1 (abstract P4). Clinical Outcomes

\begin{tabular}{|c|c|c|c|c|c|}
\hline Patient subset & Cohort & Treatment & $\mathrm{n}$ & Median OS months $(95 \% \mathrm{Cl})$ & pos \\
\hline $\begin{array}{c}\text { PD-L1/CD8(+) } \\
\text { Prevalence }=50 \%, \\
\text { cutoff }=4.627^{*} 10^{5} \mathrm{cells}^{2} / \mathrm{mm}^{4}\end{array}$ & uc & 10 & 22 & $20(1.9, N R)$ & 0.06 \\
\hline PD-L1/CD8(-) & uc & 10 & 21 & $2.3(1.9,3.3)$ & \\
\hline $\begin{array}{c}\text { PD-L1/CD } 8(+) \\
\text { Prevalence }=36 \% \\
\text { cutoff }=4.858^{*} 10^{5} \text { cells }^{2} / \mathrm{mm}^{4}\end{array}$ & NSCLC & Non-IO & 48 & $51(30,100)$ & 0.48 \\
\hline PD-L1/CD8 (-) & NSCLC & Non-1O & 86 & $52(30,67)$ & \\
\hline
\end{tabular}

P5

Analytical comparison of methods used to assess mismatch repair deficiency

Steven Anderson ${ }^{1}$, Li Cai ${ }^{2}$, Lori Johnson ${ }^{3}$, Bryan McCune ${ }^{2}$

${ }^{1}$ Covance, Durham, NC, USA; ${ }^{2}$ Integrated Oncology, Research Triangle

Park, NC, USA; ${ }^{3}$ Covance, Morrisville, NC, USA

Correspondence: Steven Anderson (sanderson@labcorp.com)

Journal for ImmunoTherapy of Cancer 2017, 5(Suppl 2):P5

\section{Background}

Deficiency in mismatch repair occurs in approximately $15-20 \%$ of sporadic colorectal cancer, and to a lesser degree in a variety of other solid tumors. Mismatch repair deficiency (dMMR) has been associated with disease prognosis and recently has also been linked with potential response to the checkpoint inhibitors pembrolizumab and nivolumab. Defects in the mismatch repair process can be identified by a number of laboratory procedures including immunohistochemical $(\mathrm{IHC})$ methods to assess expression of the mismatch repair proteins (MLH1, MSH2, MSH6 and PMS 2), or by using PCR methods to assess replication fidelity of specific microsatellite sequences in the genome. Tumor samples are indicated as dMMR when one or more the MMR proteins are not expressed, or if the tumor tissue has a high level of microsatellite instability (MSI-H).

\section{Methods}

In this study we compared the performance of the $\mathrm{IHC}$ and PCR methods, to identify colon cancer tumors that demonstrate dMMR. A series of archival formalin-fixed paraffin-embedded samples were used in this comparison study. The IHC staining was performed on the Ventana Benchmark XT system with standard detection chemistry. The specific monoclonal antibody clones were for MLH1 (G168-228, Cell Marque), MSH2 (G219-1129, Cell Marque), MSH6 (44, Ventana) and PMS2 (EPR3947, Cell Marque). For the MSI PCR assay the following microsatellites were evaluated BAT-25, BAT-26, TGF $3 R$ II, D5S346, D17S250 and D2S123. The panel contains both dinucleotide and mononucleotide repeat sequences, and includes the loci recommended by the NCl. PCR fragments were detected by capillary electrophoresis and fragment analysis was used to determined MSI status. If $>2$ loci exhibited evidence of replication defects the sample was considered MSI-H.

Results

In this cohort approximately $40 \%$ of the samples showed absence of tumor staining in either the MLH1/PMS2 or MSH2/MSH6 pathways. All cases demonstrating the MSI-H phenotype also demonstrated lack of expression of one of more MMR proteins. The most common pattern of expression was the loss of MLH1 and/or PMS2, which has been reported as being the most common phenotype in sporadic tumors. For the cases with MMR protein expression, all were considered MSI-stable by PCR.

Conclusions

This comparison study shows an equivalent performance of the two commonly used methods for the detection of mismatch repair deficiency. These data are relevant for selecting the appropriate methodology to assess dMMR when considering the use of checkpoint inhibitors for the treatment of solid tumors.

P6

Characterization of AB928, a dual adenosine A2aR/A2bR antagonist that retains potency under conditions of high albumin and high receptor activation

Devika Ashok, Lisa Seitz, Joanne Tan, Adam Park, Tim Park, Ferdie Soriano, Terry Rosen, Ehesan Sharif, Dillon Miles, Manmohan Leleti, Juan Jaen, Jay Powers, Matthew Walters, Steve Young Arcus Biosciences, Hayward, CA, USA

Correspondence: Devika Ashok (dashok@arcusbio.com)

Journal for ImmunoTherapy of Cancer 2017, 5(Suppl 2):P6

\section{Background}

In many tumors, extracellular adenosine contributes to an immunosuppressed micro-environment (TME) via activation of the $A_{2 a}$ receptor, expressed on lymphocytes, and the $A_{2 b}$ receptor, expressed on myeloid cells. Relative to other tissues like the brain, adenosine concentrations in the TME are much higher. Tumors also contain higher levels ( 3\%) of albumin, to which many drugs bind non-specifically. These factors adversely affect anti-tumor efficacy of drugs previously designed as CNS drugs.

AB928 is a novel, selective, and highly potent small-molecule dual antagonist of $A_{2 a} R$ and $A_{2 b} R$ designed to minimize potency loss associated with non-specific albumin binding. Consequently, AB928 blocks the activation of the $A_{2 a} R$ and $A_{2 b} R$ receptors at high adenosine agonist concentrations, even in the presence of high levels of albumin.

Methods

The activation of $A_{2 a} R$ and $A_{2 b} R\left(G_{s}\right.$-coupled receptors) increases intracellular levels of CAMP and subsequent phosphorylation of the CREB protein, which can be used to determine receptor occupancy/ activation. To assess the potency of AB928 under conditions with high non-specific protein binding and adenosine receptor signaling, $5 \mathrm{mM}$ of synthetic adenosine agonist (NECA) was used to stimulate CREB phosphorylation in human whole blood (WB). Flow cytometry was used to quantify AB928-mediated inhibition of CREB phosphorylation by NECA on $\mathrm{CD}^{+}$and $\mathrm{CD} 4^{+} \mathrm{T}$ cells, NK and B cells. 
Results

AB928 inhibits NECA-induced phosphorylation of CREB with a potency of $88 \mathrm{nM}(n=25)$ and is more potent than other adenosine receptor antagonists currently in clinical development (Table 1). Consistent with $A_{2 b} R$ inhibition, $A B 928$ inhibits CREB phosphorylation in monocytes. In the mouse, inhibition of CREB phosphorylation was evaluated using ex-vivo WB assays, allowing for correlation of compound plasma levels with inhibition of phosphorylated CREB, confirming $90 \%+$ target inhibition between dose intervals.

\section{Conclusions}

In conclusion, $\mathrm{AB} 928$ was designed as a best-in-class dual antagonist of $A_{2 a} R$ and $A_{2 b} R$ with optimal properties for effective shut-down of the effects of adenosine in the TME. AB928 is expected to enter clinical trials in late 2017.

Table 1 (abstract P6). Inhibition by clinical stage adenosine receptor antagonists of NECA (5 $\mu \mathrm{M})$-induced CREB phosphorylation in CD8+ T cells (human WB)

\begin{tabular}{|c|c|}
\hline Compound & $\mathrm{IC}_{50}$ in human WB $(\mathrm{nM})$ \\
\hline AB928 & $88(\mathrm{n}=25)$ \\
\hline CPI-444 & $12,667(\mathrm{n}=2)$ \\
\hline AZD4635 & $2,600(\mathrm{n}=2)$ \\
\hline PBF-509 & $8,030(\mathrm{n}=2)$ \\
\hline
\end{tabular}

P7

Immuno-Oncology companion diagnostics development: a

complex systems approach

Lourdes Barrera (lourdes.barrera@astrazeneca.com)

AstraZeneca, Gaithersburg, DC, USA

Journal for ImmunoTherapy of Cancer 2017, 5(Suppl 2):P7

\section{Background}

Cancer immunotherapy is one of the most important medical advances of our time, and the first approach with the potential to generate long-lasting regressions for all types and stages of cancer. Development of companion diagnostics for emerging immunotherapies is more complicated because they are not dependent on driver mutations in the drug target. Consequently, we need to develop new strategies for the development of immunotherapies.

Methods

One emerging model is to review multiple immune checkpoints and other factors within the tumor that may be contributing to immune exclusion or susceptibility to immune attack. By getting a multifactorial assessment of what is happening within the tumor, investigators should be better able to derive indicators to rationally deliver individualized therapy, either monotherapy or combinatorial immunotherapy. During this review, we will give some examples of how complex systems approach is supporting the development of new biomarkers and potentially companion and complimentary diagnostic tests. Results

We will review some examples of the complex systems approach such as: a cytokine profile determined by data-mining analysis to set into clusters non-small-cell lung cancer patients according to prognosis; a multiscale computational model for spatio-temporal tumor immune response and an IFN-g gene expression signature to correlate with clinical outcomes in PD-L1-treated advanced non-small-cell lung cancer and urothelial carcinoma patients.
Conclusions

- The field of immune-biomarkers aims to characterize this ongoing interaction between the immune system and cancer

- The interplay of these individual elements determines the balance of immune activation versus suppression

- As components and regulators of the immune response, multiple immune-biomarkers, include cell surface proteins, secreted proteins or peptides, and tumor-infiltrating immune cells

- We need to redouble our efforts to be more sophisticated in our use of the powerful technologies at our disposal

- Combining drugs based on increasingly well-understood molecular interactions and attacking complementary cancer hallmarks or distinct cell populations in heterogeneous tumors, is now imperative

- As a result, in the near future, we will develop multiple biomarkers on different samples using different platforms which will result in an integrative lab tests and serial testing which will provide added clinical but, also epidemiological value.

- These next generation of immuno-oncology biomarkers will achieve different questions related with the outcome such as the treatment response, survival and toxicity, must probably at the same time

P8

T cell response profiling in colorectal carcinoma patients reveals an enrichment in responses to specific tumor-associated antigens Wendy Broom ', Kyle Ferber', Johanna Kaufmann', Christine McCoy?, Judy Jacques', Abdulmohammad Pezeshki², Ning Wu', Michael O'Keefe' Crystal Cabral', Yana Ostrovsky', Jason Dobson', Theresa Zhang',

Pamela Carroll1', Khashayarsha Khazaie², Jessica Flechtner ${ }^{1}$

${ }^{1}$ Genocea Biosciences, Cambridge, MA, USA; ${ }^{2}$ Mayo Clinic, Rochester, MN, USA

Correspondence: Wendy Broom (wendy.broom@gmail.com) Journal for ImmunoTherapy of Cancer 2017, 5(Suppl 2):P8

\section{Background}

In colorectal cancer (CRC) the stage of the tumor at the time of diagnosis is considered to be the most important predictor of survival. Several non-invasive diagnostic tests for CRC are in development most focusing on detection of soluble macromolecules or circulating tumor cells. Here, we are exploiting the ATLAS ${ }^{\mathrm{TM}}$ technology, with the ability to identify an individual's $T$ cell recall responses to putative antigens, for profiling tumor-associated antigen (TAA)-specific T cell responses in CRC patients.

Methods

T cell responses to 23 common TAAs previously implicated in CRC were profiled using the ATLAS technology. Libraries composed of full-length open reading frames expressed in $E$. coli alone or together with listerolysin $\mathrm{O}$, thereby targeting antigen presentation to the MHC II or I pathways, respectively, were generated. Peripheral blood mononuclear cells (PBMC) were isolated from $50+$ subjects who were healthy or suffering from different stages of CRC or adenomatous polyps. Peripheral $\mathrm{CD}^{+}$and $\mathrm{CD} 8^{+} \mathrm{T}$ cells were non-specifically expanded using anti-CD3/CD28 beads and $\mathrm{CD}_{14}^{+}$monocytes differentiated into monocyte-derived dendritic cells (MDDCs). E. coli libraries were coincubated with MDDCs for antigen processing and presentation before autologous $T$ cells were added and incubated for $21 \mathrm{~h}$. Recall $\mathrm{T}$ cell responses were measured through detection of interferon $-\gamma$ and TNF- $\alpha$ secretion using a Meso Scale Discovery assay. Normalized cytokine concentrations that differed by $>3$ median absolute deviations from the median concentration secreted in response to non-immunogenic control clones were considered antigen-specific $\mathrm{T}$ cell responses. 
Results

Several TAAs were identified for which specific $T$ cell responses were detected in CRC patients of all stages. The breadth of response varied between individuals, but a strong enrichment of recall responses was observed to a subset of TAAs in CRC patients, to which T cell responses were not detected in healthy individuals. These responses could be attributed to both $\mathrm{CD}^{+}$and $\mathrm{CD}^{+} \mathrm{T}$ cell subsets and appeared to be Th1-polarized based on the predominant secretion of IFN- $\gamma$.

\section{Conclusions}

ATLAS is a highly suitable platform to profile TAA-specific T cell responses in CRC patients despite its classification generally as a tumor type with relatively low immunogenicity. The potential emergence of a specific $T$ cell response profile to a subset of TAAs may open the possibility for the development of a blood-based assay to support early detection and diagnosis of CRC. We are currently investigating the utility of the identified immune signature in detection of premalignant stages and whether it is associated with progression to malignancy.

\section{P9}

\section{Using iPair-TCR ${ }^{\mathrm{TM}}$ and iPair-TCR ${ }^{\mathrm{TM}}$ to track tumor infiltrating} lymphocytes from peripheral blood in a longitudinal breast cancer patient study

Miranda Byrne-Steele, Wenjing Pan, Xiaohong Hou, Brittany Brown, Mary Eisenhower, Alexa Fort, Jian Han

iRepertoire Inc., Huntsville, AL, USA

Correspondence: Miranda Byrne-Steele (msteele@irepertoire.com) Journal for ImmunoTherapy of Cancer 2017, 5(Suppl 2):P9

\section{Background}

Historical tissue samples are typically stored in such a way that make it difficult to access single cell TCR or BCR receptor pairing sequence information, such as samples stored as FFPE slides or tissue stored in Trizol or RNAlater. However, interrogating the bulk TCR or BCR repertoire of these samples is possible by extracting total RNA from such tissue slices or appropriately stored tissue. However, the information regarding the cognate pairing of the alpha and beta chains is lost. We wanted to see if it was possible to identify the most likely paired alpha-beta receptor sequences which likely match the historical tumor infiltrating lymphocyte TCR beta repertoire using circulating PBMCs.

Methods

As part of a longitudinal study, we sequenced the human TCR beta CDR3 fragments from a patient's breast cancer tissue six years ago. The patient's peripheral blood TCR beta repertoire has been examined every 6-9 months since surgical resection by amplifying CD14CD8-CD4+ $T$ cells and various sorted $T$ cell subsets using arm-PCR. Single cells from this patient were recently amplified using iPair$\mathrm{TCR}^{\mathrm{TM}}$ technology directly from the sorted cells. Identified TCR-beta CDR3 regions were used to compare to the longitudinal data sets. Results

Three matching TCR beta sequences to bulk TIL data were identified from 104 sorted single cells. Two of these were private (meaning not shared with any of our database of 75 million CDR3 beta sequences), and one of these contained a paired alpha chain. The third sequence was considered a very public CDR3, meaning that it is present in many of the TCR beta repertoires analyzed within our database.

\section{Conclusions}

Further single cell experiments are being performed to identify more potential pairs matching the TIL data, including additional detailed phenotyping of the circulating lymphocytes. The ability to match paired receptor chains to archived data contained within banked tissue is promising and has important implications for therapeutic development.
P10

Epithelial-mesenchymal transition (EMT) signature was inversely associated with activated CD8 infiltration in non-small cell lung cancer (NSCLC)

Young Kwang Chae', Sangmin Chang ${ }^{1}$, Taeyeong Ko', Jonathan Anker', Sarita Agte ${ }^{2}$, Wade lams', Marcelo Cruz ${ }^{1}$

${ }^{1}$ Northwestern University Feinberg School of Medicine, Chicago, IL, USA;

${ }^{2}$ Robert H. Lurie Comprehensive Cancer Center of Northwestern

University, Chicago, IL, USA

Correspondence: Young Kwang Chae (sangminchang@gmail.com)

Journal for ImmunoTherapy of Cancer 2017, 5(Suppl 2):P10

\section{Background}

EMT is a dynamic process in which epithelial cells acquire traits of mesenchymal cells. It has been known to drive metastasis and drug resistance during progression of cancer, while it is also associated with poor prognosis in cancers such as NSCLC. Though it has been suggested that EMT may contribute to immune escape, a clear relationship is yet to be established [1].

Methods

Gene expression data was obtained from the TCGA database, which contained mRNA-seq expression data of lung adenocarcinoma $(A D C C, n=515)$ and squamous cell carcinoma $(S q C C, n=501)$ patients. The mRNA z-score of 812 immune metagene signatures from previous studies were evaluated using Gene Set Enrichment Analysis (GSEA) [2]. Any immune cell types with a false discovery rate (qvalue) $\leq 10 \%$ were considered as positive infiltrations, and 31 distinct immune cells were analyzed in each tumor sample. The immune landscape of EMT in NSCLC was studied by calculating EMT scores by subtracting the average expression level of 'epithelial' genes $(n=5)$ from 'mesenchymal' genes $(n=24)$. The tumor samples were subsequently classified as EMT-high (EMT scores $\geq$ highest1/3) or EMT-low groups (EMT scores $\leq$ lowest $1 / 3$ ).

Results

There was a significantly lower infiltration of activated CD8 cells in EMT-high groups compared to EMT-low groups in both ADCC and SqCC $(p=<0.001,15.7 \% ; 37.2 \%, p=<0.001,15.5 \%, 40.5 \%$ respectively), as well as decreased infiltration of activated CD4 cells ( $p=0.161$, $25.0 \%$; 31.2\%; $\mathrm{p}=<0.001,26.8 \%$; 48.2\%) (Fig. 1). Effector memory CD4 cells showed significantly lower infiltration in both EMT-high groups of NSCLC ( $p=<0.001,3.5 \% ; 16.8 \%, p=<0.001,3.6 \% ; 16.7 \%)$. EMTscores showed an inverse relationship with infiltrating immune cells, most notably by activated CD8 cells. Increased expression of mesenchymal genes (VIM, ZEB1, ITGB6, etc.) in both ADCC and SqCC resulted in significantly lower infiltration of activated CD4/CD8 cells. However, increased expression of epithelial genes (COL5A1, TJP1) also resulted in significantly lower infiltration of CD8 cells in NSCLC. A significant positive correlation between immunosuppressive cytokine, IL-10 expression and EMT was found in both NSCLCS $(p=<0.001)$. Other cytokines with immunosuppressive functions that showed a significant positive correlation with EMT among others were TGF- $\beta$ and ADCC. EMT-score, or infiltration of CD8 cells among EMT-high/low groups did not show any significant differences in overall survival (OS).

Conclusions

We report for the first time, the association between EMT-score and decreased infiltration of activated CD8 cells in both NSCLCs. This may be related to increased expression of immunosuppressive cytokines such as IL-10 or TGF- $\beta$. Markers of immune exclusion such as EMT scores may be potential biomarkers that predict resistance to immunotherapy.

\section{References}

1. Terry S et al: Mol Oncol 2017; 11(7):824-846

2. Angelova M et al: Genome Biol. 2015; 16:64.doi:10.1186/s13059-015-0620-6. 


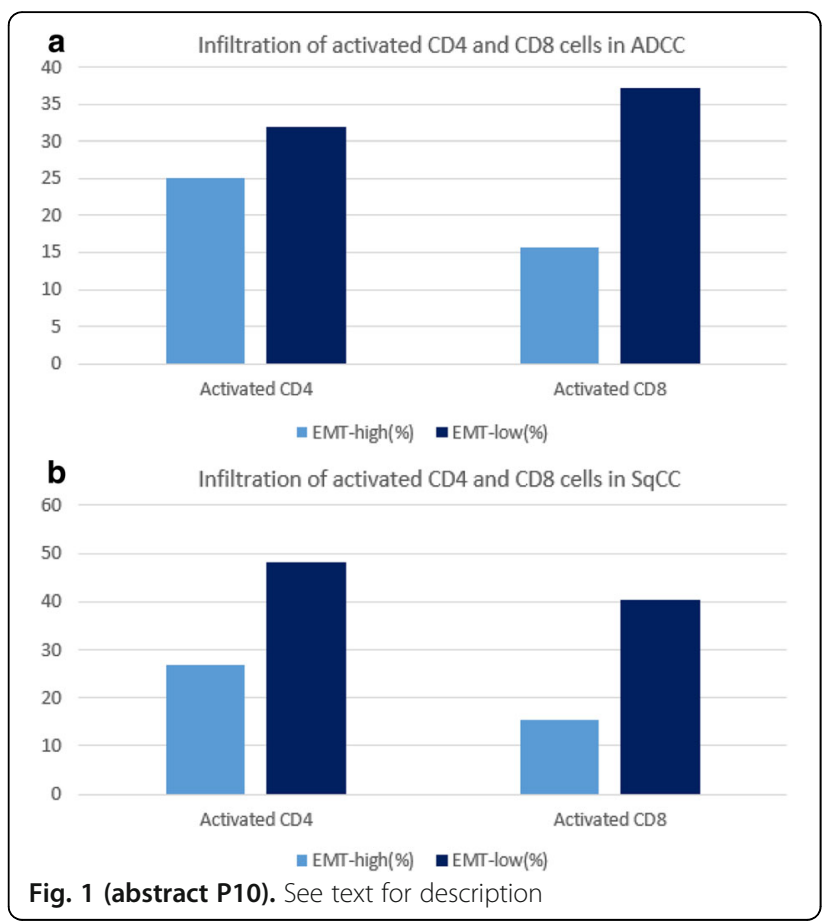

P11

WNT/ $\beta$-catenin pathway is inversely correlated with activated CD8 $\mathrm{T}$ cell infiltration in non-small cell lung cancer (NSCLC)

Young Kwang Chae', Taeyeong Ko', Sangmin Chang ${ }^{1}$, Jonathan Anker', Sarita Agte ${ }^{2}$, Wade lams', Marcelo Cruz'

${ }^{1}$ Northwestern University Feinberg School of Medicine, Chicago, IL, USA;

${ }^{2}$ Robert H. Lurie Comprehensive Cancer Center of Northwestern

University, Chicago, IL, USA

Correspondence: Young Kwang Chae (tyeongko@gmail.com)

Journal for ImmunoTherapy of Cancer 2017, 5(Suppl 2):P11

\section{Background}

It is well known that the WNT/ $\beta$-catenin pathway promotes cell proliferation which is associated with tumorigenesis. While many mechanisms of tumorigenesis have recently been elucidated, one which has particularly garnered attention is the immune escape mechanism [1]. The correlation between WNT/ $\beta$-catenin pathway and immune escape has been investigated in several cancers including melanoma. However the relationship in lung cancer is still largely unknown. Methods

In this study, TCGA data of 515 samples of lung adenocarcinoma (ADCC) and 501 samples of squamous cell lung carcinoma (SqCC) were utilized for analysis of immune landscape and gene expression scores. 17 promotor genes of WNT/ $\beta$-catenin pathway (CTNNB1, DVL1, EP300, LRP6, etc.) and 8 suppressor genes of WNT/ $\beta$-catenin pathway (AMER1, APC, AXIN1, etc.) were utilized for the analysis. The expression score of WNT/ $\beta$-catenin pathway was calculated by subtracting the average of suppressor gene mRNA expression from the average of promotor gene mRNA expression. The tumor samples were then divided into 'low WNT/ $\beta$-catenin expression group'(WNT/ $\beta$-catenin pathway expression scores $\leq$ lowest $1 / 3$ ), and 'high WNT/ $\beta$ catenin expression group'(WNT/ $\beta$-catenin pathway expression scores $\geq$ highest1/3) according to their level of gene expression scores. For immune landscape, Gene Set Enrichment Analysis (GSEA) was utilized to analyze the mRNA z-score of 812 immune metagene signatures from previous studies [2]. Positive infiltration was considered as any immune cell type with a false discovery rate (q-value) equal to or less than $10 \%$ and 31 types of immune cells were analyzed for each tumor sample.
Results

We found that in both ADCC and SqCC, the high WNT/B-catenin score group showed lower infiltration of activated CD8 $T$ cell $(p=0.006,<0.001$ respectively) (Fig. 1). We also found a negative correlation between WNT/ $\beta$-catenin pathway and the expression of immune stimulatory cytokines such as IL-2, IFN-gamma in ADCC $(p=0.003,0.029$ respectively), whereas SqCC showed a negative correlation between WNT/ $\beta$-catenin pathway and the expression of the immune stimulatory cytokines such as IL-2, IFN-gamma and IL$12(p=0.008,0.041,0.018$ respectively). The expression of immune suppressive cytokine such as TGF- $\beta$ showed positive correlation with WNT/ $\beta$-catenin pathway ( $p=0.011$ for $A D C C,<0.001$ for SqCC).

Conclusions

We report for the first time that immune exclusion by WNT/ $\beta$-catenin pathway may also occur in NSCLC. The WNT/ $\beta$-catenin pathway is associated with lower immune stimulatory cytokine secretion, which leads to lower infiltration of activated CD8 T cell. Such findings suggest that WNT/ $\beta$-catenin pathway may serve as a potential biomarker for predicting resistance to immunotherapy.

\section{References}

1. Pai SG, et al. J Hematol Oncol. 2017; 10:101.doi:10.1186/s13045-017-0471-6

2. Angelova M, et al. Genome Biol. 2015; 16:64.doi:10.1186/s13059015-0620-6

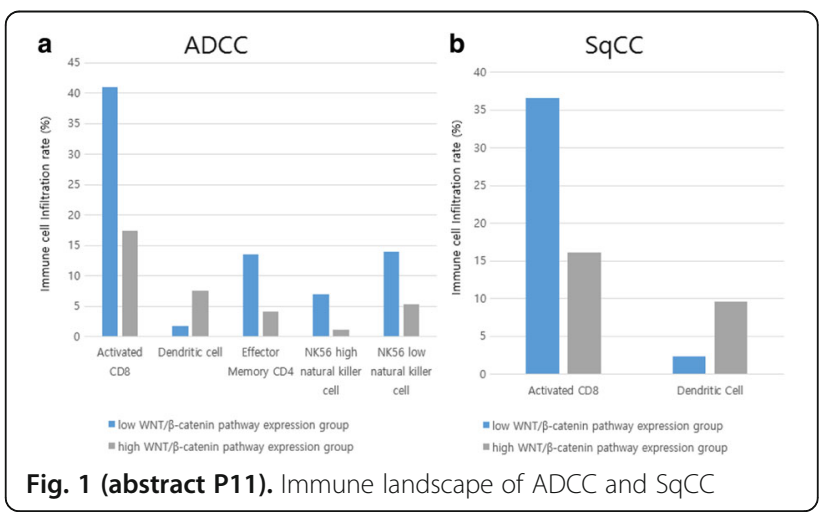

P12

Precision immune monitoring: new 30-parameter flow cytometry uniquely and comprehensively defines immune checkpoint expression and $\mathrm{T}$ cell phenotypes in tumor tissue

Pratip Chattopadhyay', Amber Giles ${ }^{2}$, Leonard Nettey ${ }^{3}$

${ }^{1} \mathrm{NYU}$ Medical School, New York, NY, USA; ${ }^{2} \mathrm{NIH}$, Bethesda, MD, USA; ${ }^{3} \mathrm{VRC}, \mathrm{NIH}$, Bethesda, MD, USA

Correspondence: Pratip Chattopadhyay

(pratip.chattopadhyay@nyumc.org)

Journal for ImmunoTherapy of Cancer 2017, 5(Suppl 2):P12

\section{Background}

The cellular processes that govern $T$ cell activity at the tumorimmune interface involve many different proteins. To better understand these processes, and identify biomarkers of efficacy and toxicity, comprehensive analysis will be needed, through the measurement of as many $T$ cell related proteins as possible. In this abstract, we introduce state-of-the-art, 30-parameter flow cytometry technology for use in immunotherapy research and immune monitoring, and describe the first studies applying this technology to studies of peripheral blood and tumor tissue.

Methods

We developed the first 30-parameter flow cytometry panel focused on the expression of checkpoint molecules. The panel includes CD45, CD3, CD4, and CD8 (to identify major T cell lineages); CD45RO, CCR7, 
CD27, CD28, CD57, CD95, and CD127 (markers of T cell differentiation); PD1, ICOS, CTLA4, LAG3, TIM3, TIGIT, VISTA, GITR, 41BB, and 2B4 (immune checkpoint molecules, involved in T cell exhaustion); CXCR3, CXCR6, and CD103 (trafficking markers); CD25, CD69, and HLA-DR (immune activation markers); and a viability dye (to exclude dead cells from analysis). We also developed a 30-parameter panel that quantifies major cell lineages ( $T, B, N K$, myeloid, dendritic) and their subsets (naive, central memory, effector, plasma, etc.). These panels were applied to peripheral blood and resected tumor tissue from glioblastoma multiformae (GBM) patients, in order to comprehensively define the types of cells present in patient peripheral blood and tumors.

Results

We could clearly identify populations of cells expressing and not expressing each marker. We developed new technical approaches to ensuring robust data analysis, and novel bioinformatics approaches that are easy to understand and accessible to researchers. This will allow broader adoption of this technology. Analysis of GBM tumor tissue revealed about 10 unique subsets of cells, some of which are not found in peripheral blood. Notably, we could easily identify cell types expressing checkpoint molecules that are current therapeutic targets, and define cells expressing combinations of those targets or importantly - none of those targets (Fig. 1). Further analysis allowed comprehensive characterization of these cells, to identify their differentiation status, activation status, and trafficking markers. Conclusions

The technology and approach we have developed will be broadly applicable to phenotyping and monitoring immunotherapy in cancer patients. The technical platform has a number of advantages over other approaches (such as mass cytometry and RNA sequencing), and can be a central technology in precision oncology efforts, which will depend on complete and comprehensive characterization of cells.

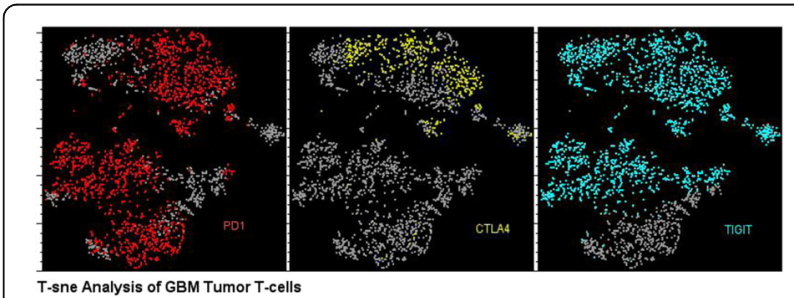

Fig. 1 (abstract P12). T-sne analysis of T cells from GBM tumors

\section{P13}

Pre-existing T cell immunity, mutational burden and stromal TGF- $\beta$ signaling drives clinical responses to atezolizumab in locally advanced or metastatic urothelial carcinoma (mUC) Sanjeev Mariathasan', Shannon J. Turley ${ }^{1}$, Dorothee Nickles ${ }^{1}$ Yulei Wang ${ }^{1}$, Edward E. Kadel III', Hartmut Koeppen', Kobe Yuen', Allessandra Castiglioni', Jillian L. Astarita', Rafael Cubas', Suchit Jhunjhunwala', Romain Banchereau', Yagai Yang', Yinghui Guan' Cecile Chalouni', James Ziai', Yasin Şenbabaoğlu', Steve Lianoglou', Michiel van der Heijden' ${ }^{2}$, Yohann Loriot ${ }^{3}$, Johathan Rosenberg ${ }^{4}$, Lawrence Fong', Ira Mellman', Daniel S. Chen', Marjorie Green' Christina Derleth ${ }^{1}$, Gregg D. Fine', Priti S. Hegde', Richard Bourgon', Thomas Powles ${ }^{6}$

${ }^{1}$ Genentech, Inc., South San Francisco, CA, USA; ${ }^{2}$ Netherlands Cancer Institute, Amsterdam, Netherlands; ${ }^{3}$ Gustave Roussy, Villejuif, France; ${ }^{4}$ Memorial Sloan Kettering Cancer Center, New York, NY, USA; ${ }^{5}$ University of California San Francisco, San Francisco, CA, USA; ${ }^{6}$ Barts Experimental Cancer Medicine Centre, London, UK

Correspondence: Sanjeev Mariathasan

(genevieve.cohen@healthinteractions.com); Shannon J. Turley

Journal for ImmunoTherapy of Cancer 2017, 5(Suppl 2):P13

\section{Background}

Checkpoint inhibitor blockade can result in robust and durable antitumor responses in many cancers. However, only a subset of patients experience long-term clinical remissions. Identifying determinants of response to cancer immunotherapy is critical for extending therapeutic benefit to more patients. Atezolizumab (anti-PD-L1) was approved in the US for the treatment of $\mathrm{mUC}$ based on the single-arm Phase II study IMvigor210 (NCT02108652). Here, we examined the biology underlying primary immune escape and responsiveness to anti-PD-L1 in patients from IMvigor210.

Methods

Cisplatin-ineligible patients previously untreated for mUC or patients who progressed post-platinum therapy received atezolizumab (1200 mg IV q3w). In both cohorts, RECIST v1.1 objective response was a primary endpoint evaluated in all patients and in PD-L1 expression subgroups on tumor-infiltrating immune cells (IC; VENTANA SP142 IHC assay). Exploratory analyzes in evaluable pre-treatment tissues included CD8 IHC, whole-transcriptome RNA sequencing, gene set enrichment analyzes and Lund subtyping. A FoundationOne panel was used to estimate tumor mutation burden (TMB), and whole-exome sequencing data were used for neoantigen prediction. EMT6-grafted BALB/c mice treated with anti-TGF- $\beta$ and/or anti-PD-L1 antibodies were evaluated for tumor growth inhibition and characterization of tumor microenvironment phenotypes.

Results

PD-L1 IC expression and clinical response were positively associated with a CD8+ T-effector expression signature $(P<0.001$ and $P=0.0087$, respectively); patients with complete responses had higher T-effector signatures than did partial or non-responders $(P=0.002)$. Response was also strongly associated with high tumor mutation and neoantigen burden $(P<0.001$ each). Likewise, response was positively associated with the genomically unstable molecular subtype $(P<0.001)$ and pathways involved in proliferation and DNA damage response. Further, nonresponsiveness correlated with expression of a 2-gene TGF- $\beta$ signature $(P<0.001)$, particularly in patients with CD8+ T cells predominantly in the collagen-rich matrix surrounding tumors (immune-excluded tumor phenotype; $P<0.001$ ). Addition of anti-TGF- $\beta$ to anti-PD-L1 in the mouse promoted $T$ cell localization to immune-excluded tumors and reduced tumor growth in vivo.

Conclusions

Pre-existing $T$ cell immunity and TMB are associated with response to atezolizumab in $\mathrm{mUC}$, whereas TGF- $\beta$ signaling in the stroma is a negative indicator of response, especially in immune-excluded tumors, a common phenotype of mUC. Integration of these 3 independent biological features provides a strong basis for understanding clinical outcomes in this setting and suggests that TGF- $\beta$ can interact with the tumor microenvironment to restrain responsiveness to anti-PD-L1 and anti-tumor immunity.

Trial Registration

ClinicalTrials.gov Identifier NCT02108652

\section{P14}

Comprehensive characterization of solid tumor immune profiles

for precision immunotherapy using Immune Report Card

Mary Nesline, Jeffrey Conroy, Sarabjot Pabla, Ji He, Blake Burgher, Vicent

Giamo, Jonathan Andreas, Paul DePietro, Antonios Papanicolau-Sengos,

Mark Gardner, Sean Glenn, Carl Morrison

OmniSeq, Inc., Buffalo, NY, USA

Correspondence: Carl Morrison (Jeffrey.conroy@omniseq.com)

Journal for ImmunoTherapy of Cancer 2017, 5(Suppl 2):P14

\section{Background}

Monoclonal antibodies directed at the inhibitory immune receptors have emerged as successful treatment options for numerous tumor types using both mono and combination modalities. However, responses are not universal and better predictive biomarkers are needed to support precision immunotherapy. We present the findings from patients tested by Immune Report Card (IRC), a validated clinical assay that measures immunotherapeutic targets and immune 
response cycle markers to provide a complete profile for appropriate selection of immunotherapy.

\section{Methods}

300 FFPE cancer samples from patients with diverse histologies were evaluated by IRC using 5 test modes. RNA-seq measures transcript levels of genes related to $T$ cell receptor signaling and tumor infiltrating lymphocytes, DNA-seq is used to estimate mutational burden, MSI-PCR to assess microsatellite instability, Fluorescent in situ hybridization (FISH) to detect PD-L1/2 copy number gain, and FDAapproved Immunohistochemistry to measure PD-L1 protein expression and pattern of expression for PD-L1, CD3, and CD8. Results are interpreted and summarized in an integrated report that comprehensively characterizes the tumor microenvironment to assess likelihood of response.

Results

At least one over expressed marker associated with either an FDA approved checkpoint inhibitor or combination immunotherapy clinical trial was identified for more than $80 \%$ of patients. Among PD-L1 IHC negative patients, the majority overexpressed at least one target with therapies in clinical development. Frequently overexpressed genes with targets in clinical development were identified for antiinflammatory response (TGFB1, IL10), myeloid suppression (CSF1R), checkpoint blockade (PD-L1, LAG3, TIM3), T cell primed (GITR, OX40, CD40, CD137, ICOS), metabolic immune escape (IDO1) and proinflammatory response (IL1B, TNF) phenotypes. Conversely, tumors with low overall immune related expression (i.e. immune deserts) often exhibited high expression of a single marker with a pertinent clinical trial therapeutic opportunity.

\section{Conclusions}

Each of the 5 IRC test modes is necessary for determining the overall likelihood of patient response to FDA approved checkpoint inhibitors. Comprehensive profiling by IRC allows for the identification of patients with over expression of markers with therapeutic targets in clinical development, providing options for patients who are PD-L1 IHC negative. The results also highlight immune phenotypes with therapeutic targets beyond checkpoint blockade, suggesting a complex tumor microenvironment in many tumors. As immune therapy moves from treatment of last resort to first and second line treatment, IRC can provide actionable results for the total tumor immune microenvironment.

\section{P15}

Overexpression of immunotherapeutic targets in the immune desert phenotype

Sarabjot Pabla, Jeffrey Conroy, Sean Glenn, Ji He, Blake Burgher, Vincent

Giamo, Jonathan Andreas, Maochun Qin, Mark Gardner, Antonios

Papanicolau-Sengos, Mary Nesline Carl Morrison

OmniSeq, Inc., Buffalo, NY, USA

Correspondence: Carl Morrison (Jeffrey.conroy@omniseq.com)

Journal for ImmunoTherapy of Cancer 2017, 5(Suppl 2):P15

\section{Background}

Immunotherapy using checkpoint blockade with monoclonal antibodies has gained increasingly high importance in treatment for cancer patients. However, a large proportion of cancer patients do not highly express primary cancer immune biomarkers such as PD-1/PDL1, MSI, and mutational burden, which have been associated with response. Here we present immune-related expression signatures for patients that present with an immune desert phenotype, distinguished by lack of CD8 positive T cells and characteristic T cell receptor signaling expression levels. As part of our clinical immune cell analysis assay, Immune Report Card (IRC), we identified secondary immune biomarkers that are singularly expressed in the otherwise non-inflamed tumor microenvironment and are potential clinical immunotherapy targets.

\section{Methods}

167 formalin-fixed, paraffin-embedded (FFPE) cancer samples of diverse histologies were evaluated by the RNA-seq component of IRC to measure transcript levels of genes related to T cell receptor signaling and tumor infiltrating lymphocytes. Resultant data was QC filtered, normalized and ranked based on an assorted reference population of various tumor types. Gene signatures were determined using these ranked expression values with a rank value $>85$ th percentile considered high. Tumors are also defined as inflamed or noninflamed based upon RNA-seq analysis of CD8, wherein, Tumors in upper 50th percentile of rank for CD8 are considered inflamed, while those in the lower 50th percentile are considered non-inflamed.

Results

The immune desert phenotype represented $28 \%(n=47)$ of the tested samples. 95\% $(n=45)$ tumor were non-inflamed (CD8 Rank < 50). 51\% $(n=24)$ of all samples did not express any gene at a high level. However, $19 \%(n=9)$ of the immune desert phenotype samples have potential for monotherapy targets and another 19\% ( $n=9)$ have underlying biology indication for combination therapy based on the high expression of a singular immune-related gene.

\section{Conclusions}

Immune Report Card allows for the profiling of the tumor immune microenvironment to delineate underlying immune biology of solid tumor samples. With a significant number of non-inflamed tumors lacking high expression of any immune biomarker, IRC results suggests that an underlying biological immune ignorance state exists in the tumor microenvironment of many patients. However, IRC could identify so-called "oasis" targets that could be potentially targeted with mono or combination immune therapy in the immune desert phenotype. With the ever-increasing numbers of FDA-approved therapies and clinical trials, IRC offers a robust tool to identify patients that might benefit from these options.

P16

The immune activated phenotype: secondary immunotherapeutic targets in the primary biomarker negative inflamed tumor

Sean Glenn, Jeffrey Conroy, Sarabjot Pabla, Ji He, Blake Burgher, Vincent Giamo, Jonathan Andreas, Mark Gardner, Antonios Papanicolau-Sengos, Mary Nesline, Carl Morrison

OmniSeq, Inc., Buffalo, NY, USA

Correspondence: Carl Morrison (Jeffrey.conroy@omniseq.com) Journal for ImmunoTherapy of Cancer 2017, 5(Suppl 2):P16

\section{Background}

Immune checkpoint inhibitors are now used to treat many different types of cancer, with some patients demonstrating durable clinical responses. As expected, a significant number of responders express high levels of primary cancer immune biomarkers such as PD-1/PDL1, MSI, or mutational burden. Here we present immune-related expression signatures for patients with an immune activated phenotype that overexpress several pro- and anti-inflammatory genes with or without primary biomarker detection. These immune signatures were identified as part of Immune Report Card (IRC), a comprehensive molecular and immunological assay that uses five testing modes to detect several known markers of the host anticancer immune response.

Methods

167 formalin-fixed, paraffin-embedded (FFPE) cancer samples of diverse histologies were evaluated by IRC to measure transcript levels of genes related to $\mathrm{T}$ cell receptor signaling and tumor infiltrating lymphocytes (RNA-seq) and mutational burden (DNA-seq). Resultant data was QC filtered, normalized and ranked based on an assorted reference population of various tumor types. Gene signatures and mutational burden were determined using these ranked values with a rank value $>85$ th percentile considered high. Tumors are also 
defined as inflamed or non-inflamed based upon RNA-seq analysis of CD8. Tumors in upper 50th percentile of rank for CD8 are considered inflamed, while those in the lower 50th percentile are considered non-inflamed. RNA-seq analysis of CD8 had been previously calibrated against quantitative image analysis using the Aperio platform. Results

The immune activated phenotype represented 20\% $(n=35)$ of the tested samples, of which 32 were inflamed (91\%). $31 \%(n=11)$ of these samples had co-expression of the primary immune biomarker PD-L1 or demonstrated a high mutational burden. However, $66 \%$ $(n=23)$ of the immune activated tumors were primary biomarker negative but instead expressed multiple secondary immune biomarkers that are potential checkpoint inhibitor therapy targets.

\section{Conclusions}

Immune Report Card profiles the tumor immune microenvironment to outline the immune biology of tumor samples. IRC is not only able to identify samples with highly expressed primary immune biomarkers such as PD-L1, MSI, or mutational burden, but using RNAseq can also identify secondary biomarkers in many samples. These secondary biomarkers shed light on the underlying biological immune-state of the tumor microenvironment, potentially identifying additional mono or combination immunotherapies in PD-L1 negative inflamed tumors. With the ever-increasing numbers of FDA-approved therapies and clinical trials, IRC offers a robust tool to identify patients that might benefit from these options.

\section{P17}

The immune-excluded phenotype beyond colorectal cancer

Jeffrey Conroy', Carl Morrison', Sean Glenn', Wiam Bshara²,

Sarabjot Pabla', Blake Burgher', Vincent Giamo ${ }^{1}$, Jonathan Andreas',

Mary Nesline', Mark Gardner ${ }^{1}$, Antonios Papanicolau-Sengos',

Igor Puzanov', Marc Ernstoff ${ }^{2}$

'OmniSeq, Inc., Buffalo, NY, USA; ${ }^{2}$ Roswell Park Cancer Institute, Buffalo, NY, USA

Correspondence: Jeffrey Conroy (Jeffrey.conroy@omniseq.com)

Journal for ImmunoTherapy of Cancer 2017, 5(Suppl 2):P17

\section{Background}

Absence of tumor infiltrating lymphocytes in the tumor proper, referred to as the immune-excluded phenotype, has been directly described in the peer-reviewed literature for colorectal cancer, but indirectly for melanoma and other solid tumors in the field of immunotherapy discussions. As part of our clinical immune cell analysis using a New York State CLEP approved assay, Immune Report Card (IRC), we routinely perform CD8 and CD3 immunohistochemistry (IHC) to determine the infiltrating versus the non-infiltrating (excluded) phenotype for all tumor types.

Methods

For all tumor types tested including carcinoma, sarcoma, and melanoma the immune-excluded phenotype is defined as a restriction of more than $95 \%$ of all CD8+ T cells present in a tumor tissue section to the periphery or interstitial stromal areas and not actively invading nest or groups of neoplastic cells. Tumors are also defined as inflamed or non-inflamed based upon RNA-seq analysis of CD8 from the same tissue section and comparison to a reference population of several hundred prior analyzed samples. Tumors in upper 50th percentile of rank for CD8 gene expression are considered inflamed, while those in the lower 50th percentile are considered noninflamed. RNA-seq analysis of CD8 had been previously calibrated against quantitative image analysis using the Aperio platform. A total of 100 consecutive samples tested, excluding colorectal cancer, were evaluated for the excluded infiltrate phenotype.

Results

The immune-excluded phenotype was identified in all tumor types with the most frequent association seen in lung cancer and the least frequent being melanoma. The immune-excluded phenotype was more common in non-inflamed tumors with a direct correlation to CD8 rank, but was also seen in some moderate to highly inflamed tumors. The most common histological pattern of the immuneexcluded phenotype was restriction of CD8+ T cells to the interstitial stromal areas.

\section{Conclusions}

The immune-excluded phenotype does occur in tumor types other than colorectal cancer. Providing a precisely defined nomenclature and method of testing for the immune-excluded phenotype in inflamed and non-inflamed tumors is important for both clinical and research purposes.

P18

Withdrawn

P19

Genomic determinants of response to pembrolizumab in gastric cancer

Razvan Cristescu, Diane Levitan, Julie Kobie, Andrew Albright, Ping Qiu, Robin Mogg, Jared Lunceford, Xiao Qiao Liu, Xinwei Sher,

Michael Nebozhyn, Gladys Arreaza, Wendy Blumenschein, Mark Ayers,

Terrill McClanahan, Andrey Loboda, David Kaufman, Rita Dalal,

Minori Koshiji

Merck \& Co., Inc., Kenilworth, NJ, USA

Correspondence: Razvan Cristescu (razvan_cristescu@merck.com)

Journal for ImmunoTherapy of Cancer 2017, 5(Suppl 2):P19

\section{Background}

The mutation load of somatic non-synonymous variants (ML) is associated with response to anti CTLA-4 and PD-1/PD-L1 immunotherapies in select tumors, likely because neoepitope formation is not subject to central immune tolerance. An IFNy gene expression profile (GEP) characteristic of a tumor T cell inflamed environment is also related to response to anti PD-1/-L1 therapy. ML and GEP have been reported as predictive in a pan-tumor setting and in HNSCC. This study evaluated relationships between $\mathrm{ML}$ and response, independent predictive value of ML, GEP and PD-L1 IHC in patients with gastric cancer treated with pembrolizumab monotherapy, and the association of response with TCGA molecular subtypes (MSI, EBV, CIN, and GS).

Methods

Whole exome sequencing (WES) and GEP were assessed in FFPE tumor specimens from previously treated patients with gastric cancer from KEYNOTE 059 cohort 1. ML, neoantigen load (NL), EBV status, and clonality were assessed by state-of-the-art WES analytics. GEP score is a weighted sum of normalized expression values of 18 genes. Molecular subtypes were determined using DNA genomic features. Statistical testing of ML and response, ML and GEP or PD-L1 relationship in EBV-negative patients, and association with molecular subtypes were pre-specified.

Results

$85 / 259$ patients (33\%) had WES results, 76 of which had GEP results and 84 of which had PD-L1 results. There were 7 responders (CR or $\mathrm{PR}$ ) and 78 non-responders. Five patients were identified as EBVpositive (EBV+), 3 as MSI-H, 45 as CIN, and 32 as GS. All MSI-H patients and no EBV+ patients were responders. For non-MSI-H responders $(n=4)$, response was not significantly associated with the remaining TCGA subtypes EBV, CIN and GS. In EBV-negative (EBV-) patients, association between $M L$ and objective response (OR) was statistically significant ( $p=7 E-4 ;$ AUROC 0.83 ). ML was not significantly correlated with either GEP or PD-L1. In a joint model, ML was significantly associated with response after adjusting for GEP ( $p=4 E-3)$ or PD-L1 $(p=2 E-3)$ in EBV- patients. NL and clonality-weighted ML were also significantly associated with response in EBV- patients $(\mathrm{p}=2 \mathrm{E}-3$ and $8 \mathrm{E}-4$, respectively). 


\section{Conclusions}

As reported in other indications, ML and GEP or PD-L1 were independently predictive of response to pembrolizumab monotherapy in gastric cancer. This suggests that ML and GEP or PD-L1 may help characterize responses to anti PD-1 therapies and novel treatment regimens in gastric cancer.

Trial Registration

ClinicalTrials.gov Identifier NCT02335411

\section{P20}

Distinct tumor immune profiles in non-small cell lung cancer (NSCLC) revealed by automated quantitative digital image analyzes of immune biomarker densities and spatial relationships Marie Cumberbatch ${ }^{1}$, Elaine Foster ${ }^{2}$, James Clay ${ }^{2}$, Sunita Mistry ${ }^{2}$

Andrew Lawrence, ${ }^{2}$, Christopher Womack ${ }^{2}$, David Fairley ${ }^{2}$

${ }^{1}$ Immune Insight Limited, Macclesfield, UK; ${ }^{2}$ HistologiX Limited,

Nottingham, UK

Correspondence: Marie Cumberbatch

(marie.cumberbatch@immuneinsight.com)

Journal for ImmunoTherapy of Cancer 2017, 5(Suppl 2):P20

\section{Background}

The tumor microenvironment comprises complex interactions between infiltrating immune cells and tumor cells. Understanding these interactions may provide valuable insights into mechanisms of response and resistance, and aid clinical decisions. In the present study, immune cell densities and PD-L1 expression in NSCLC samples were quantified to determine immune status of each sample prior to conducting sophisticated spatial analyzes to establish the proximity and ratio of cytotoxic $\mathrm{T}$ cells and regulatory T cells to tumor expression of PD-L1.

Methods

Serial sections of formalin-fixed paraffin-embedded NSCLC samples $(n=9$; Tissue Solutions, Glasgow, UK) were stained immunohistochemically using well validated assays for CD3, CD8, Foxp3 and PD-L1. Whole slide digital images were generated and viable tumor regions annotated by a clinical pathologist. The immune profile of each sample was established by applying digital image analysis tools available in the Indica Halo ${ }^{\text {TM }}$ platform to determine immune cell densities and PD-L1 expression. Selected images were subsequently registered and spatial analyzes between immune components performed.

Results

Tumor membrane PD-L1 scored by a pathologist ranged from $0 \%$ to $90 \%$ positivity with variable intensity of staining. Digital image analysis of PD-L1 using a membrane algorithm coupled with a classifier tuned to analyze tumor only delivered comparable PD-L1 positivity. Immune cell densities ranged from minimal infiltration up to 2315 , 1512 and 340 cells $/ \mathrm{mm}^{2}$ for CD3, CD8 and Foxp3, respectively. Variable CD8:Foxp3 ratios were observed ranging from 1.3 to 16.5 , and no direct relationship between immune cell infiltration and PD-L1 expression was evident. Spatial analyzes of the proximity of CD8 and Foxp3 to PD-L1 revealed samples $(n=2)$ exhibiting $15-20 \%$ of cytotoxic T cells within $5 \mu \mathrm{m}$ of PD-L1 with few adjacent Foxp3+ cells (CD8:Foxp3 ratio $>10.0)$. In contrast, we identified samples $(n=2)$ where CD8+ T cells were within close proximity $(5 \mu \mathrm{m})$ of the immunosuppressive influences of both PD-L1 and Foxp3+ regulatory T cells (CD8:Foxp3 ratio <2.6).

\section{Conclusions}

We have demonstrated using next generation digital image analysis tools that the potential immunosuppressive influences within close proximity of cytotoxic T cells within a tumor can be mapped. This approach has potential to identify tumors where cytotoxic T cells are in close proximity to PD-L1 and those where cytotoxic T cells may be in the vicinity of additional immunosuppressive influences, thus identifying patients most likely to benefit from different immunotherapies.
P21

Correlation of inflammatory biomarkers and patient-reported outcomes in patients with urothelial carcinoma treated with durvalumab

Arnold Degboe', Srikala Sridhar ${ }^{2}$, Jingsong Zhang ${ }^{3}$, Pralay Mukhopadhyay', Ashok Gupta', Lorin Roskos', Xiang Guo 1 , Terence Friedlander ${ }^{4}$

${ }^{1}$ AstraZeneca, Gaithersburg, MD, USA; ${ }^{2}$ Princess Margaret Cancer Centre, Toronto, ON, Canada; ${ }^{3}$ Moffitt Cancer Center, 12902 USF Magnolia Drive, FL, USA; ${ }^{4}$ UCSF Medical Center, San Francisco, CA, USA

Correspondence: Arnold Degboe (andrea.hammons@PAREXEL.com) Journal for ImmunoTherapy of Cancer 2017, 5(Suppl 2):P21

\section{Background}

A correlation between changes in inflammatory/cachexia biomarkers, tumor shrinkage, and overall survival (OS) has been demonstrated in patients with urothelial carcinoma (UC) receiving durvalumab [1]. The current analysis assessed the relationship between inflammatory/ cachexia biomarkers and patient-reported outcomes (PROs), and differences in PROs between responders versus non-responders in patients with UC treated with durvalumab.

Methods

In a phase $1 / 2$, dose-escalation study of durvalumab, Functional Assessment of Cancer Therapy-Bladder Cancer (FACT-BL) and the European Organisation for Research and Treatment of Cancer Quality of Life Questionnaire (EORTC QLQ-C30) were administered before other procedures at screening, day 1 of doses 1 (d1; baseline assessment), 3 (d29), 4 (d43), 5 (d57), 7 (d85), 9 (d113), and every 8 weeks thereafter. For 126 patients, the association between PRO scores and maximum percent changes in tumor size, albumin level, neutrophil/lymphocyte ratio (NLR), and durvalumab clearance from baseline was assessed by Spearman's correlation analysis.

Results

Pretreatment FACT-BL scores were similar for responders and nonresponders, with patients reporting relatively high health-related quality of life (QoL) at baseline. Compared with non-responders, clinical tumor responders reported statistically significant improvement in FACT-BL total scores (mean change of 17.1 vs -4.13 , $P=0.0005$ ), FACT-BL bladder cancer-specific symptoms (mean change of 5.8 vs $-0.56, P=0.0047$ ), and FACT-BL Trial Outcome Index (mean change of 14.3 vs $-1.9, P=0.0005$ ) at Day 113. Sustained improvement in PROs was observed in $43 \%$ of the responders compared with $12 \%-18 \%$ of non-responders. Fifty percent of patients with a clinical tumor response also reported a clinically meaningful change in UC symptoms at Day 113 using a threshold based on minimum important difference (MID) defined as $1 / 2$ baseline standard deviation, which was sustained over time ( $\geq 2$ consecutive visits with MID improvement vs baseline). Clinical tumor responders also showed higher mean scores and improvement from baseline in EORTC QLQ-C30 functional and global health status/QoL scales.

FACT-BL scores and EORTC QLQ-C30 functioning improvement were correlated significantly with decreased tumor size, increased albumin, decreased NLR, and decreased durvalumab clearance (Table).

\section{Conclusions}

PROs were correlated significantly with inflammatory/cachexia biomarkers and durvalumab clearance in advanced UC; additionally, there was a strong correlation between clinical tumor response and FACT-BL and EORTC QLQ-C30 scores.

Trial Registration

ClinicalTrials.gov Identifier NCT01693562

\section{Reference}

1. Powles T, Jin C, Zheng Y, et al. J Clin Oncol. 2017; 35 (suppl; abstr 3035). 
P22

Up-regulation of a T and NK cell gene signature in peripheral blood is associated with mRNA-based immunotherapy in lung and prostate cancer

Henoch S Hong ${ }^{1}$, Madeleine M Hipp ${ }^{1}$, Fatma Doener', James M Billingsley ${ }^{2}$, Linus Backert ${ }^{3}$, Oliver Kohlbacher ${ }^{3}$, Andreas Schröder ${ }^{1}$ Ute Klinkhardt', Sabine Brutlach ${ }^{1}$, Uwe Peukert', Ulrike Gnad-Vogt ${ }^{1}$, Mariola Fotin-Mleczek', Sven D Koch ${ }^{1}$

${ }^{1}$ CureVac AG, 72076 Tübingen, Germany; ${ }^{2}$ Emory University, Atlanta, GA, USA; ${ }^{3}$ Applied Bioinformatics, University of Tübingen, 72076 Tübingen, Germany

Correspondence: Fatma Doener (fatma.doener@curevac.com)

Journal for ImmunoTherapy of Cancer 2017, 5(Suppl 2):P22

\section{Background}

CV9201 and CV9104 represent novel mRNA-based cancer immunotherapeutics targeting multiple tumor antigens associated with either non-small cell lung cancer (NSCLC) or prostate cancer (PC), respectively. We sought to comprehensively profile transcriptional changes in peripheral blood of cancer patients after repeated mRNA treatment.

Methods

Whole-genome transcriptome profiling was performed in blood samples from 46 patients with localized, intermediate or high risk PC and samples from 22 stage IV NSCLC patients before and after repeated intradermal immunizations with protamine-formulated mRNA. The 46 study participants with PC included a cohort of 16 untreated control subjects. Expression data was analyzed by gene set enrichment analysis and modular approaches using blood transcriptional modules. Phenotypic leukocyte analyzes were performed by flow cytometry.

Results

Peripheral blood samples post mRNA treatment were characterized by an enrichment of various modules consistent with a T and/or NK cell profile in NSCLC as well as PC patients. Notably, this upregulation of effector lymphocyte genes was completely absent in the PC control subjects. We observed an enrichment of a myeloid cell signature at baseline compared to samples derived after mRNA immunotherapy. The T and NK cell gene signature and the myeloid gene signature were inversely correlated and non-overlapping. Furthermore, correlation analyzes between gene expression data with findings from the phenotypic analyzes, suggest changes in the cellular composition of peripheral blood cells after treatment.

\section{Conclusions}

The consistent transcriptional up-regulation of T and NK cell modules in post treatment samples in different cancers and the absence of it in the untreated control cohort suggest an association with intradermal mRNA immunotherapy including its potential immunostimulatory effects.

\section{P23}

Deep molecular and immune-infiltrate stratification of cancer identifies mechanistic subtypes and predicts response to checkpoint inhibition

Janusz Dutkowski, Roy Ronen

Data4Cure, Inc., La Jolla, CA, USA

Correspondence: Janusz Dutkowski (janusz@data4cure.com)

Journal for ImmunoTherapy of Cancer 2017, 5(Suppl 2):P23

\section{Background}

Checkpoint inhibitors are revolutionizing cancer therapy but identifying patients which are likely to achieve clinical benefit remains a major challenge. While relatively simple predictors such as PD-L1 expression or tumor mutation load have shown promise in predicting responders in specific cancer types, they fail to explain or predict response for many patients, suggesting that a more comprehensive understating of biology underlying tumor and immune system interactions and immunotherapy response is needed.

\section{Methods}

We developed an integrated machine-learning system that stratifies cancers using multimodal omics data and associated profiles of immune infiltration and immune pathway activity. This framework holistically determines the underlying pathway-level architecture of genomic and epigenetic alterations in each cancer using a new Model-based Network-informed Stratification approach (MNS, [1] for early version). It further uses a multiscale pathway mapping approach $[2,3]$ to predict subtype-specific immune-infiltrates, activation of key immune pathways, and, ultimately, response to checkpoint inhibition.

Results

The new framework is used to interrogate the tumor-immune system in colorectal and lung adenocarcinomas. Stratification of 533 TCGA lung adenocarcinomas into four subtypes (LUAD_MNS4) allows for reanalysis of pembrolizumab trial data [4] by matching the trial patients to the LUAD_MNS4 to infer immune infiltration and pathway activation signatures for each patient and predict patient response to pembrolizumab (Figure 1). In colorectal cancer, a total of six subtypes (COADREAD_MNS6) are identified along with subtype-specific profiles of pathway mutations and copy-number alterations. Two subtypes are hypermutated and one (COADREAD_MNS6_S4) is associated with high predicted CD8 T cell infiltration. The system also uncovers subtypes S1 and S6 which link WNT/ $\beta$-catenin pathway mutations with immunologically cold tumors, suggesting the possibility of a similar mechanism for immune evasion as recently discovered in specific subtypes of melanoma [5].

Conclusions

Comprehensive systems-level interrogation of tumor genomic architecture and microenvironment may improve our understanding of tumor-immune biology and allow for more accurate patient stratification for immunotherapy.

\section{References}

1. Hofree, Shen, Carter, Gross, Ideker. Network-based stratification of tumor mutations. Nat Methods. 2013;10(11):1108-1115.

2. Dutkowski, Kramer, Surma, Balakrishnan, Cherry, Krogan, Ideker. A gene ontology inferred from molecular networks. Nat Biotechnol. 2013; 31(1):10.1038/nbt.2463.

3. Yu, Kramer, Dutkowski, Sharan R, Ideker T. Translation of Genotype to Phenotype by a Hierarchy of Cell Subsystems. Cell Syst. 2016;2(2):77-88

4. Rizvi et al. Mutational landscape determines sensitivity to PD-1 blockade in non-small cell lung cancer. Science. 2015; 348(6230):124-128.

5. Spranger, Bao, Gajewski. Melanoma-intrinsic $\beta$-catenin signalling prevents anti-tumour immunity. Nature. 2015;523(7559):231-5.

\section{P24}

Systematic literature review of PD-L1 assays, their scoring algorithms and validation metrics

Margarita Udall', Maria Rizzo ${ }^{2}$, Juliet Kenny ${ }^{2}$, Jim Doherty ${ }^{1}$, Eric Faulkner ${ }^{3}$ ${ }^{1}$ Pfizer Inc., New York, NY, USA; ${ }^{2}$ Evidera, London, UK; ${ }^{3}$ Evidera,

Morrisville, NC, USA

Correspondence: Margarita Udall (megancibulas@gmail.com) Journal for ImmunoTherapy of Cancer 2017, 5(Suppl 2):P24

\section{Background}

Programmed cell death protein 1 (PD-1) is a cell-surface receptor expressed on T cells. The interaction between PD-1 and its ligand, programmed death ligand 1 (PD-L1), down-regulates immune responses. Many cancer types express PD-L1 and, via the PD-1/PD-L1 interaction, evade immune destruction. Antibodies targeting this interaction are approved treatments for several cancers with a variety of PD-L1 tests available to measure PD-L1 expression. A systematic literature review was conducted to evaluate these commercially available PD-L1 assays, their scoring algorithms and validation data in bladder/urothelial, lung, gastric, or ovarian cancer. 


\section{Methods}

A search was conducted in Medline and EMBASE and supplemented by abstracts from recent oncology conferences (January 2013 to November 2016). Studies meeting pre-defined criteria (such as cancer types of interest, with validation test data) were extracted and key trends summarized.

Results

26 primary studies were identified from a total of 950 records. All the included studies reported data relating to PD-L1 assays in lung cancer using immunohistochemistry (IHC) testing; one study also reported on bladder/urothelial cancer. Significant heterogeneity was reported among the available tests for PD-L1; for example, cut-offs used by test scoring algorithms to define PD-L1 positivity ranged from $\geq 1 \%$ to $\geq 50 \%$. Studies also differed as to whether they evaluated tumor cells only and/or tumor-infiltrating immune cells. However, all assays developed within clinical-trial settings met recognized acceptance criteria of $\geq 90 \%$ inter-reader concordance. In head-tohead comparisons, there was poor concordance between PD-L1 assays developed outside of clinical trials compared with companion diagnostics developed within clinical trial-settings.

\section{Conclusions}

Published data on PD-L1 testing relates predominantly to IHC assays in lung cancer. Review of this evidence suggests the need to develop standardized testing methods, or, minimally, some common approach for test scoring processes. The current variability of such testing could be a source of confusion among treating physicians and might complicate the uptake of effective treatments.

\section{P25}

A systematic literature review (SLR) of the predictive value of programmed death ligand 1 (PD-L1) tests on clinical outcomes Margarita Udall', Jim Doherty', Amber Martin², Juliet Kenney ${ }^{3}$,

Eric Faulkner ${ }^{4}$, Pratibha Chander ${ }^{1}$, Paul Robbins', SueAnn Dahm ${ }^{1}$

${ }^{1}$ Pfizer Inc., New York, NY, USA; ${ }^{2}$ Evidera, Waltham, MA, USA; ${ }^{3}$ Evidera, London, USA; ${ }^{4}$ Evidera, Morrisville, NC, USA

Correspondence: Margarita Udall (megancibulas@gmail.com)

Journal for ImmunoTherapy of Cancer 2017, 5(Suppl 2):P25

\section{Background}

Promising trial results have led to FDA approval of several drugs that target programmed cell death protein 1 (PD-1) and its ligand (PD-L1). However, the predictive value of PD-L1 expression on clinical outcomes is unclear. Therefore, an SLR was conducted to evaluate evidence on the predictive value of PD-L1 expression in bladder, gastric, lung, and ovarian cancers.

Methods

The SLR included a search of Medline and EMBASE databases from $1 / 1 / 2010$ to $15 / 9 / 2016$ using keywords for PD-L1 paired with terms related to predictive value and relevant abstracts from recent oncology conferences. Studies were selected using pre-defined criteria and then qualitatively summarized.

Results

The SLR identified 29 primary studies. There was limited evidence on bladder $(n=4)$, gastric $(n=1)$, and ovarian $(n=1)$ cancer. Bladder cancer studies showed a trend towards an association between response outcomes and PD-L1 expression, while results were more variable for survival outcomes. Most studies $(n=23)$ were related to lung cancer and were inconsistent. Data for pembrolizumab was most supportive, showing PD-L1 expression is associated with increased probability of response across treatment-naïve, previously-treated, and advanced or metastatic patient subgroups. For the other therapies there was variation across sub-groups. Evidence on the predictive value of combinations of biomarkers was limited to two durvalumab studies, which found improved outcomes when PD-L1 was detected along with additional biomarkers.

\section{Conclusions}

Evidence on the value of PD-L1 as a predictive biomarker is currently heterogeneous and relates mostly to lung cancer. While some studies suggest a correlation between PD-L1 expression and clinical outcomes, the SLR indicated that PD-L1 expression alone may not identify those likely to respond to treatment. Factors such as level of expression, cell phenotype, and variability in testing methods are possible reasons for these differences. Ambiguity in the validity of PD-L1 tests adds complexity regarding its use as a standalone biomarker. Further research is needed to explore the relationship between biomarker expression, cancer phenotypes, and treatment outcomes.

P26

Certain KIR/KIR ligand genotypes influence patient response to immunotherapy in neuroblastoma patient

Paul M. Sondel', Amy K. Erbe ${ }^{1}$, Wei Wang ${ }^{1}$, Lakeesha Carmichael' ${ }^{1}$ Anna Hoefges', KyungMann Kim¹' Wendy B. London², Jacquelyn A. Hank', Mitchell B. Diccianni ${ }^{3}$, Arlene Naranjo ${ }^{4}$, Michael D. Hogarty ${ }^{5}$, Julie R. Park ${ }^{6}$, Alice L. Yu ${ }^{7}$

${ }^{1}$ University of Wisconsin-Madison, Madison, WI, USA; ${ }^{2}$ Boston Children's Hospital/Dana-Farber Cancer Institute, Boston, MA, USA; ${ }^{3}$ University of California San Diego, San Diego, CA, USA: ${ }^{4}$ University of Florida,

Gainesville, FL, USA; ${ }^{5}$ Children's Hospital of Philadelphia, Philadelphia, PA, USA: ${ }^{6}$ Seattle Children's Hospital, Seattle, WA, USA; ${ }^{7}$ University of

California San Diego, San Diego, CA, USA

Correspondence: Paul M. Sondel (aerbe@wisc.edu)

Journal for ImmunoTherapy of Cancer 2017, 5(Suppl 2):P26

\section{Background}

In 2010, a phase III randomized trial (ANBL0032; NCT00026312) conducted by the Children's Oncology Group (COG), found that patients treated with an immunotherapy regimen of dinutuximab, GM-CSF, IL2 and isotretinoin had improved event-free survival (EFS) and overall survival (OS) compared to treatment with isotretinoin alone. Dinutuximab (monoclonal/chimeric anti-GD2 antibody) acts in part via engaging NK cells in antibody-dependent cellular cytotoxicity (ADCC). Killer Immunoglobulin-like Receptors (KIR) are a family of receptors expressed by NK cells that can influence their function. The genotypic profiles of inhibitory KIR/KIR-ligands have previously been shown to influence the response of neuroblastoma patients to immunotherapy. We investigated whether EFS and OS were associated with KIR/KIR-ligand genotypes and this immunotherapy regimen.

Methods

Of the 226 pts randomized in ANBL0032, 174 pts had DNA available to genotype for correlations with outcome (isotretinoin alone: $n=86$; Immunotherapy: $\mathrm{n}=88$; $>5 \mathrm{yr}$ follow-up if no event). KIR gene status was determined via SYBR green melt curve analysis; KIR-ligands were determined by PCR-SSP reactions using the KIR HLA Ligand SSP typing kit (Olerup). Algorithms were used to assess associations of inhibitory KIRs with their respective KIR-ligands and clinical outcome. Log-rank tests and Cox proportional hazards regression models were used to compare EFS/OS by genotype group; adjustment was made for non-proportional hazards as needed using time-dependent covariates.

Results

In this randomized trial of neuroblastoma patients, we found that certain KIR/KIR-ligand genotypes were associated with significantly improved outcome for patients receiving the COG immunotherapy regimen vs. those that received isotretinoin alone; whereas the patients with the complementary KIR/KIR-ligand genotypes had no significant difference in EFS and OS regardless of whether they received immunotherapy vs. isotretinoin alone.

Conclusions

For this study, certain KIR/KIR-ligand genotypes, but not others, are associated with improved outcome for patients treated with immunotherapy compared to isotretinoin alone. As validation of these $\mathrm{KIR/KIR-ligand} \mathrm{findings} \mathrm{has} \mathrm{not} \mathrm{yet} \mathrm{been} \mathrm{performed} \mathrm{in} \mathrm{other} \mathrm{studies}$ comparing patients receiving immunotherapy to those not receiving immunotherapy, further investigation is required. However, if validated, KIR/KIR-ligand genotyping could potentially be used as a predictive biomarker, allowing for administration of this COG im- 
munotherapy regimen to those that might best benefit. Enhancements to anti-GD2 mAb-based therapy, based on preclinical and early clinical data, are being evaluated in efforts to improve its efficacy. Further studies of KIR/KIR-ligand associations with outcome in subsequent trials of immunotherapeutic regimens for children with neuroblastoma will be needed to determine the potential clinical utility of these KIR/KIR-ligand findings.

\section{P27}

Optimized workflow improves the characterization of tumor-infiltrating T cells

Cesar Evaristo, Ramona Siemer, David Agorku, Janina Brauner, Olaf Hardt, Christian Dose, Anne Richter

Miltenyi Biotec GmbH, Bergisch Gladbach, Germany

Correspondence: Cesar Evaristo (cesare@miltenyibiotec.de)

Journal for ImmunoTherapy of Cancer 2017, 5(Suppl 2):P27

\section{Background}

Immunotherapy has proven clinical efficacy and tremendous potential in multiple cancers. Syngeneic mouse tumor models represent the gold standard to analyze effects of immunotherapy, as they possess a fully competent immune repertoire. However, the amount and composition of tumor-infiltrating leukocytes (TILs) is highly variable, complicating the analysis of individual subpopulations. In particular, small subpopulations might escape analysis as they could get lost in the background noise. When working with large cohort sizes, even immunophenotyping of TILs by flow cytometry is time consuming and data processing highly work intensive. Therefore, preenrichment of TILs is highly desirable to increase the sensitivity of analysis and save time and effort during flow cytometry.

Methods

We have established workflows combining tissue dissociation, $\mathrm{T}$ cell isolation and phenotyping. Tumor dissociation was automated using the gentleMACS ${ }^{\mathrm{TM}}$ Octo Dissociator and optimized for epitope preservation. Moreover, isolation of tumor infiltrating $T$ cells was improved by developing new CD4-, CD8- and Pan-T cell specific enrichment reagent for magnetic cell sorting, based on MACS ${ }^{\circledR}$ Technology, direct from dissociated tumor tissue. Finally, we used optimized panels of recombinant REAfinity fluorescently-labelled antibodies to phenotypically characterize tumor infiltrating T cells.

Results

Our workflows were validated in B16.F10, B16-OVA, CT26 and 4T1 mouse tumor models. Optimized tumor dissociation was essential for isolation of specific sub-populations of tumor-specific T cells. Tumorinfiltrating $T$ cells were isolated to purities above $80 \%$ and yields ranging from $60-95 \%$. Time of downstream analysis was reduced up to 50 -fold while enhancing the detection and phenotypic characterization of T cell subpopulations within the tumor.

\section{Conclusions}

Standardized processing of tumor samples and magnetic isolation of tumor infiltrating $T$ cells greatly reduces time and cost of downstream analysis while significantly increasing reproducibility and the quality of data obtained from TIL analysis.

\section{P28}

Profiling of $T$ cell responses to tumor-associated antigens in lung cancer patients treated with checkpoint inhibitors

Wendy Broom, Kyle Ferber, Johanna K. Kaufmann, Judy Jacques, Christine McCoy, Michael O'Keeffe, Crystal Cabral, Yana Ostrovsky, Ning Wu, Jason Dobston, Theresa Zhang, Pamela Carroll, Jessica Flechtner Genocea Biosciences, Inc., Cambridge, MA, USA

Correspondence: Wendy Broom (jessica.flechtner@genocea.com) Journal for ImmunoTherapy of Cancer 2017, 5(Suppl 2):P28

\section{Background}

While the recent approval of checkpoint inhibitor therapies (CPIs) has transformed the therapeutic landscape of advanced lung cancer, the early identification of patients who will benefit from CPIs requires improvement. In addition to neoantigen-specific responses, the success of CPI therapies can in part be attributed to reinvigoration of $T$ cells specific for tumor-associated antigens (TAAs), providing an opportunity to explore using TAA-specific T cell response profiles for patient stratification. Here we utilized ATLAS ${ }^{\mathrm{TM}}$, a high-throughput antigen discovery platform that can profile autologous $\mathrm{CD} 4^{+}$and $\mathrm{CD}^{+} \mathrm{T}$ cell recall responses to putative antigens, to profile TAAspecific T cell responses in NSCLC.

Methods

Based on literature review, 77 common antigens were selected to be cloned as full-length proteins in E. coli, either alone or co-expressed with listerolysin $\mathrm{O}$ to facilitate $\mathrm{MHC}$ II and I presentation, respectively. PBMCs from patients with stage III or IV lung cancer, primarily NSCLC, post-checkpoint inhibitor treatment were analyzed. $\mathrm{CD}^{+}$and $\mathrm{CD} 8^{+} \mathrm{T}$ cells were isolated and non-specifically expanded. Autologous CD14 monocytes were differentiated into monocyte-derived dendritic cells and co-incubated in an ordered array with the bacterial libraries for antigen processing and presentation. T cells were added and IFN- $\gamma$ and TNF-a secretion was quantified in the supernatant after $21 \mathrm{~h}$, using a custom Meso Scale Discovery kit. Significant antigen-specific recall responses were defined as greater than 3 median absolute deviations from the median response to negative control clones.

Results

ATLAS identified broad TAA-specific T cell responses across the cohort of lung cancer patients, with a greater breadth of $\mathrm{CD}^{+}$than $\mathrm{CD}^{+}$response, and little overlap between subsets. In addition to $\mathrm{T}$ cell activation, antigen-specific inhibition of $\mathrm{T}$ cell activity was also observed. T cell response profiles were consistent over at least three weeks in patients for which longitudinal samples were available.

Conclusions

ATLAS is a valuable tool to profile tumor-associated T cell responses in advanced lung cancer non-invasively, providing the opportunity for cohort analysis to identify blood-based signatures that correlate with disease subtype, stage, or responder status to CPI. The consistency of observations over time supports the reproducibility of the platform, validating the utility of ATLAS in anti-tumor response profiling. Future prospective studies will compare T cell response profiles prior to and after CPI therapy to define signatures predictive of clinical response.

\section{P29}

Incorporating premixed staining cocktail for improving the workflow for whole blood immunophenotyping

Iliana Gonzalez, William Redmond, Yoshinobu Koguchi, William Miller,

Tanisha Meeuwsen, Valerie K. Conrad, Ana Howells-Ferreira, Tomasz M. Poplonski

Earle A. Chiles Research Institute, Portland, OR, USA

Correspondence: Iliana Gonzalez (ilianaleti@gmail.com); William

Redmond

Journal for ImmunoTherapy of Cancer 2017, 5(Suppl 2):P29

\section{Background}

Whole blood immunophenotyping is indispensable for monitoring immune responses to cancer immunotherapy. We have successfully introduced an automated liquid handler equipped with a twodimensional barcode reader for the cocktail preparation that enabled us to prepare antibody cocktails for multicolor flow cytometry for more than 800 fresh whole blood patient samples over last two years. Although it is reliable, our current workflow takes up 2-3 hours to prepare cocktails and is not easily scalable to meet an increasing demand. As a solution, we evaluated the compatibility of custommade dried cocktail reagents.

Methods

As brilliant violet dyes are not compatible with the dried reagent format, we have re-established the staining panels for T cells, B cells, NK cells, monocytes and dendritic cells with other fluorochromes. Stained cells are analyzed by CytoFlex (Beckman Coulter).

We will evaluate custom-made dried antibody cocktails based on our revised immunophenotyping panels, focusing on the comparative analysis between liquid and dried cocktails for ease of use and the quality and reproducibility of staining. 
Results

We have successfully reduced from the current 13-color panels to 10-color ones without compromising the ability to detect immune subsets and their activation status by omitting redundant staining. The comparative study between liquid and dried cocktails is underway.

Conclusions

The dried format allows multicolor antibodies to be dried within the analytical tubes, facilitating their ease of use. Unlike liquid cocktails, dried cocktails require no refrigeration, titration, or manipulation before using. Therefore, it not only saves time but also reduces potential errors and obviates the need to manage individual antibodies. With a shelf life of at least 12 months, the reagents also offer potential savings in reagent costs by reducing waste due to expiration or tandem breakdown in standard liquid formulation.

\section{P30}

Mass spectrometry-based test predicts outcome on anti-PD-1 therapy for patients with advanced non-small cell lung cancer, including those with brain metastases

Sarah Goldberg', Lucia Jilaveanu', Harriet Kluger', Veronica Chiang' Amit Mahajan ${ }^{1}$, Bing Xia ${ }^{1}$, Matthew Ribeiro ${ }^{1}$, Heinrich Roder ${ }^{2}$, Joanna Roder $^{2}$, Carlos Oliveira 2 , Julia Grigorieva ${ }^{2}$, Mirte Muller ${ }^{3}$, Anna-Larissa Niemeijer ${ }^{4}$, Robert Schouten ${ }^{3}$, Adrianus de Langen ${ }^{3,4}$, Egbert Smit ${ }^{3}$ ${ }^{1}$ Yale School of Medicine, New Haven, CT, USA; ${ }^{2}$ Biodesix, Steamboat Springs, CO, USA; ${ }^{3}$ Netherlands Cancer Institute, Amsterdam, Netherlands; ${ }^{4} \mathrm{VU}$ University Medical Center, Amsterdam, Netherlands Correspondence: Julia Grigorieva (julia.grigorieva@biodesix.com) Journal for ImmunoTherapy of Cancer 2017, 5(Suppl 2):P30

\section{Background}

Checkpoint inhibition has become a standard of care therapy for advanced non-small cell lung cancer (NSCLC), but only a minority of patients achieves a durable response. PD-L1 expression is often used to identify patients likely to have good outcomes. However, patients with low PD-L1 expression can have long-term treatment benefit and this biomarker is dynamic and requires tumor tissue. A less invasive approach that could provide predictive information regarding treatment outcomes could be useful.

Methods

Pre-treatment blood samples were collected from patients with advanced NSCLC. Cohort A $(n=98)$ received nivolumab, $3 \mathrm{mg} / \mathrm{kg}$ every 2 weeks in a compassionate use program, $59 \%(32 \%)$ in $2^{\text {nd }}\left(3^{\text {rd }}\right)$ line. Patients in cohort B $(n=32)$ had at least one new or progressing brain metastasis and were treated on a clinical trial with pembrolizumab $10 \mathrm{mg} / \mathrm{kg}$ every 2 weeks. Spectra were generated from all samples using a sensitive method of MALDI mass spectrometry and processed to render them comparable. Spectra from cohort A were combined with clinical data using modern machine learning methods to create a test able to identify patients likely to have better or worse overall survival (OS), as assessed by reliable test set evaluation methods. The locked test was applied to cohort B, blinded to all clinical data.

Results

Using mass spectral features associated with acute response and wound healing, we produced a test able to identify patients likely to have better or worse OS (HR=0.41, log-rank $\mathrm{p}=0.005)$ and progression-free survival (PFS) ( $H R=0.56$, log-rank $p=0.022$ ) within cohort A (Figure 1). Test classification was an independent predictor of OS and PFS in multivariate analysis, with 1 year survival of $66 \%$ in the good outcome group and $36 \%$ in the poor outcome group. Thirty-four percent of patients were classified to the good outcome group. In cohort B, 25\% classified to the good outcome group, which had 1 year survival of $88 \%$, compared to $24 \%$ in the poor outcome group (Figure 2). The HR for OS between groups was 0.36 (log-rank $\mathrm{p}=0.062$ ).

\section{Conclusions}

We developed a blood-based test able to identify patients with advanced NSCLC likely to have better or worse survival with anti-PD-1 therapy. It showed promise in a validation cohort of patients with NSCLC with progressing brain metastases. Further validation of the test in additional, larger cohorts is planned and necessary to assess clinical utility.

Trial Registration

Clinical Trials.gov Identifier NCT02085070

\section{P31}

Proteomic biomarker analysis of metastatic melanoma patients treated with anti-PD-1 checkpoint blockade

Paolo Antonio Ascierto ${ }^{1}$, Mariaelena Capone ${ }^{1}$, Antonio Maria Grimaldi ${ }^{1}$

Domenico Mallardo ${ }^{1}$, Ester Simeone ${ }^{1}$, Heinrich Roder ${ }^{2}$, Krista Meyer ${ }^{2}$,

Senait Asmellash ${ }^{3}$, Carlos Oliveira2, Joanna Roder ${ }^{2}$

${ }^{1}$ Istituto Nazionale Tumori IRCCS Fondazione "G. Pascale", Naples, Italy;

${ }^{2}$ Biodesix, Steamboat Springs, CO, USA; ${ }^{3}$ Biodesix, Boulder, CO, USA

Correspondence: Heinrich Roder (julia.grigorieva@biodesix.com)

Journal for ImmunoTherapy of Cancer 2017, 5(Suppl 2):P31

\section{Background}

Biomarkers identifying who will derive benefit from anti-PD-1 therapy from pre-treatment parameters would further clinical understanding of PD-1 blockade and help development of alternative treatments for patients unlikely to benefit. We evaluate the performance of a serum proteomic test, BDX008, in metastatic melanoma patients treated with anti-PD-1 agents and investigate the role of BRAF mutation status. BDX008, a pre-treatment test associated with acute phase reactants, wound healing and complement activation, stratifies patients into two groups, BDX008+ and BDX008-, with better and worse outcomes on immunotherapy. Following development and preliminary validation [1], it has been applied to independent cohorts in melanoma [2] and lung cancer [3].

Methods

Pre-treatment serum samples were available from 71 patients (under IRB); 70 pre-treated with ipilimumab. BRAF mutations were identified in 25 patients; 39 were wild type (WT). BDX008 results were generated by Biodesix blinded to clinical data.

Results

Overall, BDX008+ patients had significantly better OS (HR=0.40 (95\%Cl:0.21-0.76), $\quad \mathrm{p}=0.005)$ and PFS (HR=0.54 (95\%Cl:0.32-0.93), $\mathrm{p}=0.027)$ than BDX008- patients. BDX008 was a significant predictor of disease control $(p=0.002)$ and trended to significance for response $(p=0.056)$. Outcomes were numerically inferior for patients with BRAF mutations (median OS/PFS: 160/85 days for BRAF mutations; 479/181 days for BRAF WT respectively). BDX008+ patients had superior OS $(\mathrm{HR}=0.20$ (95\%Cl:0.07-0.59), $\mathrm{p}=0.004)$ and PFS (HR=0.45 (95\%Cl:0.210.99), $p=0.046$ ) in the BRAF WT subgroup, but not in the BRAF mutation subgroup (OS: $\mathrm{HR}=0.62$ (95\%Cl:0.24-1.59), $\mathrm{p}=0.318$; $\mathrm{PFS}$ : $\mathrm{HR}=0.57$ (95\%Cl:0.24-1.40), $\mathrm{p}=0.223$ ), possibly due to smaller sample size.

Conclusions

BDX008 stratified patients into groups with better and worse PFS and OS. The difference between BDX008+ and BDX008- groups was consistent across BRAF status, but did not reach statistical significance in smaller subgroups. Patients with BRAF mutations may have worse outcomes than those with BRAF WT with anti-PD-1 agents, possibly due to prior targeted therapies, and more previous lines of therapy overall. BDX008 as a possible predictive biomarker warrants further prospective evaluation

\section{References}

1. Grossi F, Rijavec E, Biello F, et al: Evaluation of a Pretreatment Serum test for Nivolumab Benefit in Patients with NWeber J, Martinez A, Roder $\mathrm{H}$, et al: Pre-treatment patient selection for nivolumab benefit based on serum mass spectra. J Immunother Cancer. 2015;3(Suppl 2):P103. 
2. Weber J, Roder H, Asmellash S, et al. A mass spectrometry-based serum test to predict outcome of treatment with nivolumab: Analysis of samples taken during therapy. Cancer Research. 2016;76:4891.3.

3. Grossi F, Rijavec E, Biello F, et al. Evaluation of a Pretreatment Serum test for Nivolumab Benefit in Patients with Non-Small Cell Lung Cancer. J Thorac Oncology. 2017;12:S1322.

\section{P32}

Differential association of myeloid cell and IFN- $\gamma$ associated proteins with clinical response to durvalumab treatment in urothelial bladder cancer

Xiang Guo', Wendy White', Denise Jin², Yanan Zheng ${ }^{2}$, Rajesh Narwal ${ }^{1}$ Yu Gu', Ashok Gupta', Pralay Mukhopadhyay ${ }^{3}$, Brandon Higgs ${ }^{1}$,

Tony W. Ho ${ }^{3}$, Lorin Roskos ${ }^{1}$

${ }^{1}$ Medlmmune, Gaithersburg, MD, USA; ${ }^{2}$ Medlmmune, Mountain View, CA, USA: ${ }^{3}$ AstraZeneca, Gaithersburg, MD, USA

Correspondence: Xiang Guo (andrea.hammons@PAREXEL.com)

Journal for ImmunoTherapy of Cancer 2017, 5(Suppl 2):P32

\section{Background}

Durvalumab, a human monoclonal antibody that binds PD-L1, was recently granted accelerated approval for the treatment of patients with locally advanced or metastatic urothelial carcinoma (UC) who have disease progression during or following platinum-containing chemotherapy. While PD-L1 expression in tumor or infiltrating immune cells has been valuable in the prediction of clinical responses, new biomarkers and prognostic factors should be investigated to support precision medicine approaches.

Methods

In a phase $1 / 2$ trial, durvalumab was administered intravenously at $10 \mathrm{mg} / \mathrm{kg}$ to 182 patients with UC Q2W for up to 12 months. PD-L1 positivity was defined as $\geq 25 \%$ expression in tumor cells or tumorinfiltrating immune cells. Ninety-three serum proteins with inflammatory, growth factor, or metabolic functions were measured by multiplex immunoassay in samples from 158 patients prior to durvalumab treatment.

Results

Univariate analysis identified IL8, CRP, and IL6 to be the most significant prognostic factors among all measured proteins for overall survival (OS) of UC patients receiving durvalumab (log-rank $P<0.00001$ ). Their baseline concentrations were significantly higher in progressive disease (PD) compared with stable disease (SD), complete or partial responders (Kruskal rank sum $P<0.01)$. Fast progressors $(n=36)$ who received only 1-2 doses of durvalumab demonstrated higher baseline levels of the 3 proteins versus progressors who stayed on treatment for $>2$ doses of durvalumab ( $n=60$; Mann-Whitney $P<0.001)$. Similarly, higher macrophage colony-stimulating factor (M-CSF) levels were associated with shorter OS and PD. In contrast, higher IFNy-inducible proteins, CXCL9 and CXCL10, were associated with longer OS, along with TNF-related weak inducer of apoptosis (TWEAK). Using Cox stepwise proportional hazards modeling, we evaluated the impact of protein markers, PD-L1 status, and various clinicopathological factors on OS for 120 patients with complete data for all variables measured. IL8 levels above median showed the highest hazard ratio $(H R=5$, $P<0.001)$, while CRP and IL6 were no longer associated with OS when the effects of other factors were taken into account. CXCL9 and TWEAK levels below median had HRs of $3.4 \quad(P<0.001)$ and 2.9 $(P=0.002)$, respectively, in the multivariate model, which also included low albumin levels $(\mathrm{HR}=4, P<0.001)$, liver metastasis $(\mathrm{HR}=3, P=0.001)$, and low PD-L1 (HR=2, $P=0.03)$.

\section{Conclusions}

Our results demonstrate the association of high serum concentrations of myeloid cell-associated proteins and low concentrations of IFN $\gamma$-inducible proteins with shorter OS in UC patients receiving durvalumab. Myeloid-associated proteins may identify patients with myeloid cell-regulated resistance mechanisms to immune checkpoint blockers, which may be further explored as predictive biomarkers for combination therapy with macrophage-targeting agents.

Trial Registration

ClinicalTrials.gov Identifier NCT01693562

P33

The presence of effector immune cells in human CD200 positive tumor samples supports the CD200 immune checkpoint as a novel therapeutic target

Mark Hamilton ${ }^{1}$, Rui-Ru Ji², Tony Arulanandam', Sharon Barr ${ }^{1}$

${ }^{1}$ Alexion Pharmaceuticals, Inc., New Haven, CT, USA; ${ }^{2}$ Alexion

Pharmaceuticals, Inc., Lexington, MA, USA

Correspondence: Mark Hamilton (mark.hamilton@alexion.com)

Journal for ImmunoTherapy of Cancer 2017, 5(Suppl 2):P33

\section{Background}

CD200 (OX-2) is an immune checkpoint protein expressed by a number of immune cells (e.g. B-cells, T cells, macrophages), and nonimmune cells, (e.g. endothelial cells, neurons). CD200 binds to its receptor (CD200R) expressed on antigen-presenting cells and T cells, and is believed to play an important role in normal immune homeostasis. Overexpression of CD200 by tumor cells implicates CD200 in tumor-mediated immunosuppression and regulation of anti-tumor activity. Samalizumab is a fully humanized therapeutic monoclonal antibody against CD200 and its binding to CD200 disrupts the immunosuppressive CD200-CD200R interaction. Samalizumab is currently under investigation in two Phase 1 trials in patients with acute myelogenous leukemia and solid tumors (NCT02987504 and NCT03013998).

Recent evidence suggests that the presence of immune effector cells within tumors is critical for clinical response to immune checkpoint therapy. Similarly, the expression of the immune modulatory target within the tumor may correlate with response to therapy. The purpose of this study was to identify tumor types which may be most sensitive to samalizumab, based on CD200 expression and the presence of immune effector cell populations within the tumor.

Methods

Publically available tumor gene expression data* was mined to evaluate CD200 expression in multiple tumor types and to develop an immune cell gene signature that may correlate with response to samalizumab. To confirm the gene expression data, we analyzed a series of adult and pediatric tumor sections by immunohistochemistry for expression of CD200 and the infiltration of immune cells.

Results

Gene expression values for CD200 were described as the percentage of samples with expression higher than the median for the set of all tumors. A "samalizumab competent" gene signature was developed from genes which co-correlate with CD200R expression across tumor types, and includes markers of $\mathrm{T}$ cells and macrophages; this signature was used to identify tumor types which harbor CD200Rexpressing immune infiltrates that may mediate sensitivity to samalizumab. CD200 gene expression and gene signature scores were calculated for all tumor samples and expressed as the percentage of tumor samples which express the signature at a higher level than the overall median. Immunohistochemistry and gene expression data were highly concordant.

Conclusions

We explored both CD200 expression and the presence of CD200R+ immune infiltrates in multiple tumor types. These data point to a role for CD200 signaling in certain tumors, and the potential for antitumor activity of samalizumab. 
P34

Pre-existing anti-therapeutic antibodies against Fc-region determinants shared by rituximab and hu14.18K322A are associated with outcome in a phase I trial of hu14.18K322A

Jacquelyn Hank', Jacob Goldberg', Fariba Navid ${ }^{2}$, Amy Erbe', Victor Santana ${ }^{3}$, Jacek Gan', Fenna de Bie', Amal Javaid', Anna Hoefges', Lakeesha Carmichael ${ }^{1}$, Michael Bishop ${ }^{3}$, Michael Meagher ${ }^{4}$,

Stephen Gillies ${ }^{5}$, Janardan Pandey ${ }^{6}$, Paul Sondel ${ }^{1}$

${ }^{1}$ University of WI-Madison, Madison, WI, USA; ${ }^{2}$ Children's Hospital Los Angeles, Los Angeles, CA, USA; ${ }^{3}$ St. Jude Children's Research Hospital, Memphis, TN, USA; ${ }^{3} 3$ St. Jude Children's Research Hospital, Memphis, TN, USA; ${ }^{5}$ Provenance Biopharmaceuticles, Carlisle, MA, USA; ${ }^{6}$ Medical University of South Carolina, Charleston, SC, USA

Correspondence: Jacquelyn Hank (hank@humonc.wisc.edu) Journal for ImmunoTherapy of Cancer 2017, 5(Suppl 2):P34

\section{Background}

Tumor-reactive monoclonal antibody (mAb) therapy has been successful in a growing number of clinical settings, including the use of anti-GD2 mAb as a treatment component for high-risk neuroblastoma. Following treatment with a therapeutic $\mathrm{mAb}$, patients may generate an endogenous antibody response against that mAb. Depending upon the strength and specificity of this antibody against the $\mathrm{mAb}$, this anti-therapeutic antibody response can potentially augment the clearance, or neutralize the molecular functions, of the $\mathrm{mAb}$. For chimeric or humanized therapeutic mAbs, these neutralizing antibodies are often directed against the hypervariable, antigenbinding region (idiotype), of the mAb's Fab components. Patient derived antibody responses directed against non-variable sites on chimeric, humanized, or fully-human mAbs have been less well studied. We describe the detection of Pre-treatment Anti-Therapeutic Antibodies (PATA) in the sera of patients with neuroblastoma who were treated in a Phase I study of the anti-GD2 mAb hu14.18K322A. We sought to serologically characterize these PATA and evaluate for any clinical correlations.

\section{Methods}

Enzyme-linked immunosorbent assays (ELISA) were performed on pre- and post-treatment patient sera to detect the presence of antitherapeutic antibodies. Sera from PATA+ patients were further characterized in ELISA for reactivity against other known antibodies. The association between the PATA data and clinical outcome data were assessed.

Results

Among samples from 38 patients obtained prior to mAb therapy, 9 (24\%) patients demonstrated serological recognition of hu14.18K322A. Unlike the sera from patients who generate post-treatment neutralizing antibody responses, PATA sera did not diminish mAb detection in circulation or inhibit its antigen binding ability in vitro. In 5 of 5 PATA+ patients, IgM is a component of PATA reactivity. Sera from 8 of 9 PATA+ patients demonstrated preferential recognition of the Fc-portions of $14.18 \mathrm{~K} 322 \mathrm{~A}$ and rituximab (both $\operatorname{lgG} 1$ ) as compared with the Fab portions of these mAbs. PATA recognized 14G2a and mouse lgG2a isotype mAbs, but did not recognize mouse lgG1 isotype or the fully human panitumumab (lgG2) mAb. Of the 38 treated patients, only 4 (all PATA+) demonstrated no disease progression for $>2.5$ years without receiving further therapy ( $p=0.002$; Fisher's exact test).

\section{Conclusions}

This study demonstrates an association between the presence of pre-existing antibodies against a tumor-reactive $\mathrm{mAb}$ and a favorable outcome. This association may suggest that PATA are playing a role in augmenting $\mathrm{mAb}$-based anti-tumor effects. Further screening for the presence of non-neutralizing antibodies, analyzes of their clinical correlates, identification of their immunologic targets, and potential anti-tumor mechanism(s) are warranted.

\section{P35}

Withdrawn
P36

Computational tissue analysis-based quantification of tumor-infiltrating leukocytes using morphometrics in immunohistochemistry stained NSCLC samples

Elliott Ergon, Allison Harney, Will Paces, Kristin Wilson, Karen Ryall,

Daniel Rudmann, Brooke Hirsch, Joseph Krueger

Flagship Biosciences, Westminster, CO, USA

Correspondence: Joseph Krueger (aharney@flagshipbio.com)

Journal for ImmunoTherapy of Cancer 2017, 5(Suppl 2):P36

\section{Background}

Quantification of tumor-infiltrating lymphocytes (TILs) is predictive of patient response to immunotherapy regimens. Because of the role of anti-PD-L1 and anti-PD-1 treatments in promoting tumor-specific cytotoxic T cell responses, the identification and quantification of TILs and PD-L1-positive tumor cells by immunohistochemistry (IHC) may provide important information for disease prognosis. Using Computational Tissue Analysis $\left(\mathrm{CTA}^{\mathrm{TM}}\right)$-based morphometric features instead of an IHC-based lymphocyte assay enables a simpler workflow for TIL assessment and can be combined with additional IHC assays, enabling the identification and classification of immune cell subsets within the complex tumor microenvironment.

Methods

Non-small cell lung cancer (NSCLC) samples were stained with IHC assays for CD45, CD3, CD8, and PD-L1. Serial sections were stained with appropriate isotype-negative controls. CTA ${ }^{\mathrm{TM}}$ tools were used to determine the morphometric parameters that identified hematoxylin-stained leukocytes on isotype-stained slides and, once developed, to quantify the total immune cell population in tumor nests and surrounding stroma. The same morphometric ruleset was used to quantify total leukocytes in CD45-, CD3-, CD8-, and PD-L1-stained tissues. The results of this analysis were then compared with the results of the biomarkerspecific assay to evaluate the frequency of biomarker-positive TILs in the context of total infiltrating leukocytes quantified by the $\mathrm{CTA}^{\mathrm{TM}}$ ruleset. Results

Morphometric parameters were established that determined the number of hematoxylin-stained leukocytes correlated with the number of leukocytes identified by CD45 in serial sections. The relative populations of CD3positive and CD8-positive TILs were consistent with available literature findings. The $\mathrm{CTA}^{\mathrm{TM}}$ morphometric ruleset separated PD-L1-positive leukocytes from PD-L1-positive tumor staining in the tumor nests and surrounding stromal tissue.

Conclusions

Methods were developed that use morphometric features to identify the total number of infiltrating immune cells in tumor tissues stained with hematoxylin and eosin or by $\mathrm{IHC}$ biomarker assays. These methods provide an additional dimension of data without requiring additional biomarker staining. In some IHC assays, like anti-PD-L1 assays, both the immune infiltrate and tumor cells may stain positive, and the accuracy and precision of quantifying one population by visual pathology is hindered by the staining of the second population. The CTA ${ }^{\text {TM }}$-based methods can be used to aid pathologists in the interpretation of these types of biomarker assays in which both tumor cells and immune infiltrate may stain positive

\section{P37}

Combining in situ vaccination with checkpoint blockade enhances an endogenous anti-tumor B-cell response resulting in tumor-specific humoral memory

Zachary Morris', Clinton Heinze', Claire Baniel', Peter Carlson',

Jacquelyn Hank', Raghava Sriramaneni', Emily Guy', Jasdeep Kler',

Sara Busche', Stephen Gillies², Hans Loibner ${ }^{3}$, Alan Korman ${ }^{4}$,

Alexander Rakhmilevich', Paul Sondel

${ }^{1}$ Wisconsin Institutes of Medical Research, Madison, WI, USA;

2Provenance Biopharmaceuticals, Carlisle, MA, USA; '3 APEIRON Biologics, Vienna, Austria; ${ }^{4}$ Bristol-Myers Squibb, Sunnyvale, CA, USA

Correspondence: Zachary Morris (cheinze@wisc.edu)

Journal for ImmunoTherapy of Cancer 2017, 5(Suppl 2):P37 


\section{Background}

In a murine model of 5-week established $\left(\sim 200 \mathrm{~mm}^{3}\right)$ melanoma, we previously reported that combined treatment with radiation and intratumor (IT) injection of anti-GD2 hu14.18-IL2 immunocytokine (IC) results in an in situ vaccine effect, rendering most mice disease-free and eliciting tumor-specific T cell-dependent immunologic memory. In the treatment of larger 7-week $\left(500 \mathrm{~mm}^{3}\right)$ melanomas, combining this in situ vaccine with anti-CTLA-4 resulted in greater complete tumor regression $(73 \%)$ and enhanced survival compared to dual combinations of radiation, IT-IC, and anti-CTLA-4. All mice (17/17) rendered disease-free by this triple combination therapy exhibited immunologic memory. Here, we evaluate whether this combination may trigger an endogenous anti-tumor antibody response.

Methods

$\mathrm{GD}^{+} \mathrm{B} 78$ melanoma cells were injected subcutaneously on the flank of C57BL/6 mice. After 5 weeks, B78 tumors were treated with 12Gy, IT injection of hu14.18-IL2 on days 6-10 after radiation, and IP injection of anti-CTLA-4 on days 3, 6, and 9 after radiation. Blood was drawn from mice via facial vein bleeds prior to treatment, at 10 day intervals thereafter to day 50, and from disease-free animals $>90$ days after radiation. Serum from these samples was tested by flow cytometry against the closely related GD2 B16 melanoma (parental to B78) and the unrelated GD2 ${ }^{+}$Panc02-GD2 pancreatic tumor lines for the presence of tumor-specific lgM and IgG antibodies. Functional capacity of tumor-specific serum was tested using ComplementDependent Cytotoxicity (CDC) assays.

Results

A tumor-specific endogenous lgG response was observed against GD2 B16 melanoma in untreated tumor-bearing, untreated mice, and levels of this tumor-specific lgG declined over the first $\sim 20$ days following combined treatment with 12Gy + IT-IC + anti-CTLA-4. Beginning 20-30 days after this treatment, a tumor-specific IgM response against GD2 B16 melanoma was identified in most mice. T and this briefly peaked and then declined by day 50 . A renewed tumor-specific lgG response was observed in serum from mice rendered disease-free with combined in situ vaccine and anti-CTLA-4, and the level of this tumor-specific lgG increased modestly following subcutaneous re-challenge of these mice with B78 cells.

\section{Conclusions}

In this preclinical melanoma model, combined treatment with 12Gy + IT-IC + anti-CTLA-4 augments an endogenous antibody response to the B78 tumor, resulting in a memory humoral response in animals rendered disease-free. This may suggest an opportunity to improve this treatment regimen by using the endogenous anti-tumor B cell response as a biomarker of adaptive anti-tumor immunity.

\section{P38}

A strategy to assess contributions of individual agents in combination immunotherapy trials

Traci Hilton', Christopher Paustian', Yoshinobu Koguchi' ${ }^{2}$, Adi Mehta ${ }^{3}$

Fridtjof Lund-Johansen ${ }^{4}$, Brian Boulmay, Rui Li, Kyle Happel,

Tarsem Moudgil, Sachin Puri, Christopher Dubay, Brenda Fisher,

Rogan Rattray, Mary Campbell

${ }^{1}$ Ubivac, Portland, OR, USA; ${ }^{2}$ Robert W. Franz Cancer Center, Earle A.

Chiles Research Institute, Providence Cancer Center, Portland, OR, USA;

${ }^{3}$ Oslo, Norway; ${ }^{4}$ Oslo University Hospital Rikshospitalet, Oslo, Norway

Correspondence: Traci Hilton (traci.hilton@ubivac.com)

Journal for ImmunoTherapy of Cancer 2017, 5(Suppl 2):P38

\section{Background}

A recent FDA workshop sought discussion on how to demonstrate the contribution of each individual agent in combination immunotherapy trials. Here we outline a strategy of coordinated assessment of $B$ and $T$ cell response kinetics to a complex cancer vaccine as a possible model for such an evaluation. This strategy was applied to assess patients receiving DPV-001 DRibbles ${ }^{\oplus}$, a dendritic cell-targeted microvesicle (proteasome blocked autophagosome) vaccine derived from adenocarcinoma and mixed histology cancer cell lines. It contains multiple TLR agonists and > 200 potential NSCLC antigens, many as prospective altered-peptide ligands or neoantigens. Based on preclinical studies where anti-OX40 significantly $(P<0.05)$ improved survival and apparent cures, a clinical trial of DPV-001 plus anti-OX40 is planned and will use the proposed strategy to monitor the impact of this combination for cancer immunotherapy.

Methods

Patients received induction cyclophosphamide followed by 7 vaccines at 3-week intervals. The first vaccine was given intranodally; subsequent vaccines intradermally. Patients were randomized to receive DRibble alone (A), or with imiquimod (B) or GM-CSF (C). Thirteen pts were enrolled (Arm A: 5; B: 4; C: 4). PBMCs and serum were collected at baseline and at each vaccination to assess changes in antibodies (Ab) (Protoarray, microsphere affinity proteomics (MAP)), cytokines (Quanterix), PBMCs (flow cytometry) and TCR repertoires (Adaptive immunoSEQ).

Results

IgG levels to 5000 proteins was assessed thrice prior to vaccination and at 3-week intervals. For some antigens, IgG responses peaked and then returned to baseline with new $A b$ responses developing or being augmented at each time point. In others, Ab responses were maintained at multiple time points. Ab responses were detected against proteins whose genes were commonly upregulated in NSCLC, in some cases this upregulation was associated with significantly reduced survival (TCGA) Evaluation of CD4 and CD8 T cell clones by TCRSeq identified significantly $(p=0.002)$ increased clonal expansion compared to normal controls $(n=3)$. Similar to Ab responses, T cell clonal expansion exhibited expansion followed by apparent contraction.

Conclusions

This monitoring strategy identified that continued vaccination was associated with induction of new lgG $A b$ responses, inferring that new CD4 T cell responses were developing with repeated vaccination. Expansion and apparent contraction of CD4 and CD8 T cell clones, consistent with basic immunological principles, provides insights into combination immunotherapy strategies that might be used to augment response as well as a method to monitor for that effect.

Trial Registration

ClinicalTrials.gov Identifier NCT01909752

\section{P39}

Use of ex vivo histoculture to identify potential predictive biomarkers for the ICOS agonist antibody, JTX-2011

Heather Hirsch, Jason Reeves, Tong Zi, Amit Desphande, Guang Yang,

Alexander Needham, Jenny Shu, Christopher Harvey, Sriram

Sathyanaryanan, Jennifer Michaelson, Emma Lees, Elizabeth Trehu,

Debbie Law

Jounce Therapeutics, Inc., Cambridge, MA, USA

Correspondence: Debbie Law (hhirsch@jouncetx.com)

Journal for ImmunoTherapy of Cancer 2017, 5(Suppl 2):P39

\section{Background}

ICOS (Inducible T cell CO-Stimulator) is a co-stimulatory molecule expressed primarily on $\mathrm{T}$ lymphocytes. Clinical and preclinical data suggest that ICOS plays an important role in the immune response to cancer. Therefore, we generated JTX-2011, an ICOS agonist antibody currently in clinical development in advanced solid tumors in the ICONIC trial. In preclinical studies, single agent efficacy correlates with the percentage of ICOS-expressing $T$ cells within the tumor. Thus, ICOS expression is being used as a biomarker to enrich for patients in Phase 2 of the ICONIC trial. Building on our biomarkerdriven strategy, we have explored additional potential predictive biomarkers using ex vivo tumor histoculture which allows for in vivo-like analysis of therapies using patient intact tumor tissue. Herein, we report on the results of such analysis, including assessment of the induction of an IFN-gamma gene signature.

Methods

Tumor processing: Fresh human tumor samples were obtained postsurgery through the Cooperative Human Tissue Network. A section of each tumor was cut and fixed for IHC. 300 mM slices of remaining 
tumor were placed in a 6-well plate. Treatments were added into the medium and plates were incubated at $37^{\circ} \mathrm{C}$. Tumor slices were stored in RNAlater after incubation.

RNA extraction and QC: Tumor slices were lysed using Qiagen's TissueLyser processor and FFPE samples were deparaffinized. RNA was extracted from FFPE and fresh tumor samples, quantified using Quibit, and QC'd using AATI's Fragment Analyzer.

Gene expression: Gene expression for interferon signature and other genes of interest were performed using Taqman $\mathrm{QPCR}$ probes or NanoString nCounter using the Human Immunology V2 panel.

IHC: ICOS (Spring SP98) and PD-L1 (CST E1L3N) levels were assessed by IHC.

Results

Treatment of NSCLC and HNSCC histoculture samples with either JTX-2011 or nivolumab, alone and in combination, led to induction of an IFN-gamma gene signature in a subset of the treated samples. This induction correlated with baseline ICOS expression for JTX-2011treated samples, and with PD-L1 expression for nivolumab-treated samples. Additionally, expression of an ICOS RNA gene signature correlated with IFN-gamma induction in JTX-2011-treated samples.

\section{Conclusions}

Ex vivo histoculture is a robust tool that can be used to assess candidate predictive biomarkers, identify novel potential biomarkers, and interrogate post-dose responses to novel therapeutics. As part of the Jounce Translational Platform, we have used it to build on our JTX2011 biomarker-driven strategy as we continue to generate new hypotheses to be tested in the context of the ICONIC trial.

\section{P40}

Deep proteomic and transcriptomic analysis of sorted T cells with a simple, integrated workflow

Kit Fuhrman', Douglas Hinerfeld', Katherine Drake², Gary Geiss'

${ }^{1}$ Nanostring Technologies, Inc., Seattle, WA, USA; ${ }^{2}$ Cytobank, Inc., Santa Clara, CA, USA

Correspondence: Kit Fuhrman (jhorsman@nanostring.com)

Journal for ImmunoTherapy of Cancer 2017, 5(Suppl 2):P40

\section{Background}

In addition to the long-standing efforts to understand and manipulate the immune system in the treatment of autoimmune diseases, the immune system is increasingly becoming a direct target for cancer therapeutics. These cancer immunotherapy efforts require deep biological characterization to better understand and characterize multiple rare cell populations, necessitating next-generation methods to expand or augment current flow cytometry methods. Recent advances in flow and mass cytometry have greatly expanded the number of cell parameters that can be interrogated resulting in an improved understanding of Immune system heterogeneity. These technologies, however, remain limited in the number and types of analytes that can be examined in a single sample.

Methods

We utilized a novel workflow integrating flow cytometry cell sorting with the nCounter ${ }^{\otimes}$ Vantage $3 \mathrm{D}^{\mathrm{TM}}$ RNA:Protein Immune Cell Profiling Assay to deeply characterize multiple flow-sorted immune cell populations. The NanoString nCounter platform enables the highly multiplexed digital analysis of both RNA and protein from a single biological specimen for multiple research applications. This workflow enabled us to interrogate 30 cell surface proteins and 770 immunerelated RNA starting with cells in suspension. We used the unsupervised machine learning algorithms viSNE and SPADE on the Cytobank informatics platform to characterize the expression signatures across all measured proteins and RNA in each of the sorted cell populations.
Results

PBMC were co-stained with both fluorescently-labeled and NanoString DNA barcoded antibodies followed by the isolation of different $\mathrm{T}$ cell populations by standard flow cytometry. Using our novel workflow, we subsequently analyzed 30 proteins and 770 RNA from each sorted population with no additional staining. Demonstrating the value of this workflow in analyzing potentially rare cell populations, cells were titrated to determine the sensitivity of the workflow, producing concordant protein data from 5000 cells down to only 500 cells.

\section{Conclusions}

High-plex RNA data was obtained without the requirement for additional molecular biology methods, such as RNA purification or sequencing library construction, reducing potential for technical biases. Streamlined data analysis and visualization was accomplished by combining Cytobank with $\mathrm{nSolver} \mathrm{r}^{\mathrm{TM}}$ software packages to rapidly analyze this high-plex data. This experiment illustrates '3D Flow' methodology, which is ideally suited for incorporation into cell sorting workflows, simultaneously producing high-plex, multiomic data from multiple rare and potentially clinically-relevant cell populations.

\section{P41}

IgG antibodies correspond with T cell responses to tumor neoantigens

Tyler Hulett', Shawn Jensen', Larry David², Ashok Reddy², Phillip Wilmarth'2, Carmen Ballesteros-Merino', Christopher Dubay', Michael Afentoulis ${ }^{1}$, Bernard Fox

${ }^{1}$ Earle A. Chiles Research Institute, Portland, OR, USA; ${ }^{2}$ Oregon Health \& Science University, Portland, OR, USA

Correspondence: Tyler Hulett (tylerhulett@gmail.com)

Journal for ImmunoTherapy of Cancer 2017, 5(Suppl 2):P41

\section{Background}

Herein we report a survey of humoral and cellular immunity to tumor neoantigens which demonstrates interrelationships between lgG antibody and $\mathrm{T}$ cell responses specific to individual tumor peptides. Although a high background of preexisting autoantibody signals to thousands of normal proteins are frequently observed in IgG biomarker surveys, little work has been done to determine whether such antibodies might represent a broader immunologic history that enhances CD8+ T cell responses or adapts in concert with them. Improved understanding of any antigen-specific relationships between IgG and T cells could lead to improved immune monitoring for cancer patients and a deeper understanding of what features define clinically-relevant tumor antigens.

Methods

We sought to screen for $\lg G$ antibodies to peptides centered at single nucleotide variant and wild-type versions of known mutation sites in our 4T1 vaccine model, and hypothesized that patterns in these antibody profiles would relate to vaccine-induced $T$ cell responses to those same mutation sites. We vaccinated female BALB/c mice with an autophagosome vaccine derived from 4T1 mammary carcinoma cells, then screened serum for IgG binding to a custom array of single nucleotide variant and paired wild-type peptides from those 4T1 cells.

Results

Vaccine-induced IgG signal changes correlated to antigens with stronger-predicted $\mathrm{MHCl}$ binding domains, indicating an overlap between the short peptide antigens recognized by antibody and CD8+ $T$ cells. Interestingly, these IgG signal changes occurred on top of a background landscape of preexisting IgG signals. To determine whether these observations related to T cell immunity, we stimulated $\mathrm{CD} 8+\mathrm{T}$ cells from naïve and vaccinated animals with a diverse selection of the top-predicted $\mathrm{MHCl}$ binding minimal 8-11mer peptides 
matching the mutation sites profiled on the antibody array. Similar to the antibody data, vaccine-induced CD8+ recognition of 4T1 cells was most improved by tumor peptides with stronger-predicted $\mathrm{MHCl}$ affinity. In addition, minimal peptides from Wdr33:H13Y, the mutation site garnering the strongest preexisting and post-vaccination IgG signals, also generated the largest post-vaccination IFNY responses in multiple independent $\mathrm{T}$ cell experiments.

\section{Conclusions}

These results demonstrate that preexisting and post-vaccine serum antibody signals share important features with antitumor $\mathrm{T}$ cell responses. This could have important implications toward understanding why natural surveillance and clinical therapies either succeed or fail in creating immune responses to individual tumor antigens.

\section{P42}

The checkpoint inhibitor TTI-621 (SIRPaFc) stimulates innate and adaptive immune responses in patients with hematologic and solid tumor malignancies

Lisa Johnson', Stephen Ansell ${ }^{2}$, Robert Chen ${ }^{3}$, Ian Flinn ${ }^{4}$, Michael Maris ${ }^{5}$, Owen O'Connor ${ }^{6}$, Alexander Lesokhin ${ }^{7}$, John Thompson ${ }^{8}$, Oleg Akilov ${ }^{9}$, Christiane Querfeld ${ }^{3}$, Matthew Taylor ${ }^{10}$, Meghan Irwin', Tina Catalano', Penka Petrova', Eric Sievers ${ }^{1}$, Robert Uger ${ }^{1}$

${ }^{1}$ Trillium Therapeutics Inc., Mississauga, ON, Canada; ${ }^{2}$ Mayo Clinic, Rochester, MN, USA; ${ }^{3}$ City of Hope, Duarte, CA, USA; ${ }^{4}$ Sarah Cannon Research Institute/Tennessee Oncology, Nashville, TN, USA; ${ }^{5}$ Sarah Cannon Research Institute/Colorado Blood Cancer Institute, Denver, CO, USA; ${ }^{6}$ Columbia University Medical Center, New York Presbyterian Hospital, New York, NY, USA; ${ }^{7}$ Memorial Sloan Kettering Cancer Center, New York, NY, USA; ${ }^{8}$ University of Washington/Seattle Cancer Care Alliance, Seattle, WA, USA; ${ }^{9}$ University of Pittsburgh, Pittsburgh, PA, USA; ${ }^{10}$ Oregon Health and Science University, Portland, OR, Canada Correspondence: Lisa Johnson (lisa@trilliumtherapeutics.com); Robert Uger

Journal for ImmunoTherapy of Cancer 2017, 5(Suppl 2):P42

\section{Background}

CD47 is an immune checkpoint that binds to SIRPa and delivers an anti-phagocytic ("do not eat") signal, suppressing macrophage phagocytosis. Tumor cells frequently over-express CD47 to evade macrophage-mediated destruction. TTI-621 (SIRPaFc) is an immune checkpoint inhibitor that promotes phagocytosis of tumor cells by blocking CD47 and engaging activating FCRs on macrophages. TTI621 may stimulate $T$ cell responses through enhanced antigen presentation and alteration of the tumor microenvironment. Two Phase 1 studies are underway evaluating the safety and tolerability of TTI-621 following intravenous delivery in subjects with hematologic malignancies (Study TTI-621-01; NCT02663518) and intratumoral injection in subjects with solid tumors and mycosis fungoides (Study TTI-62102; NCT02890368). We report ongoing pharmacodynamic assessments from these trials.

Methods

In Study TTI-621-01, subjects with relapsed/refractory hematologic malignancies received weekly IV infusions of TTI-621 at $0.2 \mathrm{mg} / \mathrm{kg}$ as monotherapy or $0.1 \mathrm{mg} / \mathrm{kg}$ in combination with Rituximab. At the investigator's discretion, the protocol allows dose intensification in 0.1 $\mathrm{mg} / \mathrm{kg}$ increments to a maximum of $0.5 \mathrm{mg} / \mathrm{kg}$. Serial blood draws were obtained to assess receptor occupancy, cytokine/chemokine levels, T cell repertoire and immune phenotype. In Study TTI-621-02, TTI-621 was administered intratumorally starting at $1 \mathrm{mg} / \mathrm{injection}$ up to a maximum of $10 \mathrm{mg} /$ injection to subjects with percutaneously accessible solid tumors and mycosis fungoides. Peripheral cytokines/ chemokines, immune phenotype and gene expression were assessed in serial blood draws. Biopsies were collected to assess the impact of TTI-621 on the tumor microenvironment using gene expression, T cell repertoire, and multi-spectral imaging analyzes. Reported data are current as of July 26, 2017.
Results

Subjects receiving IV TTI-621 had increases following infusion in cytokines including MIP- $1 \mathrm{a}$, MIP- $1 \mathrm{~b}$, TNF-a, IL- 6 , and IL-8 with median peak concentrations of $917.7,1644.2,392.2,262.2$, and $400.4 \mathrm{pg} / \mathrm{ml}$ at Week 1 and $168.9,176.4,38.0,17.9 \mathrm{pg} / \mathrm{mL}$ at Week 6 , respectively. NanoString analysis of peripheral blood and tumor tissue pre- and post- intratumoral injection of TTI-621 indicated activation of the innate and complement systems, including modulation of IFN-stimulated genes, such as IFI27. Increases in CD8+ and NK cells were observed in the peripheral blood of subjects. An induction of peripheral T cell clonality was observed in the majority of subjects exhibiting objective responses following IV TTI-621.

Conclusions

Pharmacodynamic analysis demonstrated that TTI-621 stimulates the innate and adaptive immune responses in relapsed/refractory cancer patients. Importantly, changes in the TCR repertoire correlated with objective responses, suggesting that innate checkpoint inhibition with TTI-621 is capable of driving an anti-tumor T cell response.

\section{P43}

Assessment of pharmacodynamic effects of immuno-oncology agents in cynomolgus monkeys using high-content gene expression profiling

Veena Kandaswamy ${ }^{1}$, Amelie Forest ${ }^{1}$, Thompson Doman², Manisha Brahmachary', Krishna Chodavarapu', Jason Manro², John Rhoden², Bing Han², Nate Elliott ${ }^{3}$, Sarah Warren³, Tim Riordan³, Christina Bailey ${ }^{3}$, Ruslan Novosiadly ${ }^{1}$, Michael Kalos ${ }^{1}$, Gerald Hall ${ }^{1}$ ${ }^{1}$ Eli Lilly \& Company, New York, NY, USA; ${ }^{2}$ Eli Lilly \& Company, Indianapolis, IN, USA; ${ }^{3}$ NanoString Technologies, Seattle, WA, USA

Correspondence: Gerald Hall (kandaswamy_veena@lilly.com) Journal for ImmunoTherapy of Cancer 2017, 5(Suppl 2):P43

\section{Background}

Non-human primate studies serve as valuable tools for preclinical safety and pharmacokinetic (PK) evaluation; however, their utility in Pharmacodynamic (PD) assessment of immunomodulatory agents in immuno-oncology is limited. Here, we assessed PD effects of immunomodulatory agents in Cynomolgus monkeys (Macaca fascicularis) using a high-content gene expression platform (nCounter NonHuman Primate (NHP) Immunology Panel, NanoString) that covers 20 immunologically relevant pathways.

Methods

NHP nCounter codeset used in the study, covers 770 genes and spans around 20 immune-related signaling pathways including but not limited to toll-like receptor, interferon signaling, innate and adaptive immune response. Blood was collected from naïve Cynomolgus monkeys, before and at various time points after intravenous administration of experimental immunotherapies. Based on previously generated Quantigene Plex gene expression data from four different studies, nCounter analysis was focused on select time points/samples along with respective controls. Two different sample types, purified RNA from peripheral blood cells and whole blood lysates, were also evaluated.

Results

nSolver software (NanoString) was used for quality control and normalization of the data. The positive control scaling factor was within the recommended range of $0.3-3$, indicating that there were no platform-associated sources of variation. Due to the variability in the negative controls background subtraction was excluded from analysis. Expression levels of housekeeping genes (HKGs) were similar across samples, and all 16 HKGs were included in the analysis. Approximately 300-350 genes were below lower limit of detection under conditions tested and were removed from analysis. The nCounter assay demonstrated comparable performance on purified total RNA and whole blood lysates. Depending on the immunomodulatory agent studied, the number of differentially expressed genes (pre- vs post-dose) ranged from 3 to 76. Principal Component Ana- 
lysis revealed that gene expression levels post-dose segregated from those of pre-dose. Most of the gene expression changes observed were indicative of peripheral innate immune response exemplified by upregulation of genes attributable to monocytes/macrophages and/or granulocytes.

\section{Conclusions}

Taken together, the data indicate that the nCounter gene expression platform was capable of detecting immune-related PD effects of immunomodulatory agents in Cynomolgus monkeys using both RNA and whole blood lysates. High-content molecular platforms such as nCounter, can significantly enhance PD assessment and broaden understanding of immune-related changes in NHP studies which may facilitate informative decision making and PK/PD modeling in clinical trials.

\section{P44}

Patient selection strategies and pharmacodynamic assays for CCR4 antagonists

Abood Okal, William Ho, Brian Wong, Paul Kassner, Gene Cutler

FLX Bio Inc., South San Francisco, CA, USA

Correspondence: Paul Kassner (pkassner@flxbio.com)

Journal for ImmunoTherapy of Cancer 2017, 5(Suppl 2):P44

\section{Background}

Regulatory $T$ cell $\left(\mathrm{T}_{\text {reg }}\right)$-mediated suppression of effector $\mathrm{T}$ cells in the tumor microenvironment (TME) can diminish anti-tumor immune responses. The CCR4 receptor can mediate recruitment and accumulation of $\mathrm{T}_{\text {reg }}$ in the TME making it an ideal target for improving anti-tumor immune responses. We have previously reported on the development of potent and selective CCR4 antagonists. As these move towards the clinic, it is important to have a strategy for selecting patients most likely to respond to this therapy and to measure CCR4 engagement with our inhibitors in these patients once clinical trials begin.

Methods

Data from The Cancer Genome Atlas (TCGA) was mined for expression of relevant genes and signatures. Tumors were classified based on their inferred levels of immune infiltrate, and signatures for $\mathrm{T}_{\text {reg }}$ and $\mathrm{CD}^{+}{ }^{+}$cells. RNAScope ${ }^{\circledast}$ (RNA in situ hybridization) was used on more than 400 tumor biopsy cores to confirm expression levels and localization of key genes. For receptor occupancy, whole blood from healthy donors or cancer patients was incubated with CCR4 antagonist prior to addition of fluorescently-labeled hCCR4 ligand, hCCL22 (A647-hCCL22). At $37^{\circ} \mathrm{C}$ and in the absence of a CCR4 antagonist, A647-hCCL22 stimulates receptor internalization and accumulation of fluorescence signal. Cells were then antibody-stained and interrogated by flow cytometry to measure CCR4-inhibition in $\mathrm{T}_{\text {reg. }}$. Results

Many tumor types show elevated expression of the CCR4 ligands, CCL17 and CCL22, and increases in $\mathrm{T}_{\text {reg }}$ and CD8 signatures, with head and neck (HN), stomach, lung, and breast cancer types showing strong enrichment. Subtype analysis shows further immune signature enrichment in subtypes such as Triple Negative Breast and EBV ${ }^{+}$ stomach cancers. RNAScope analysis supports these observations, with lung and $\mathrm{HN}$, in particular, showing strong clustering of $\mathrm{T}_{\text {reg }}$ and CD8 cells. In the receptor occupancy assay, our CCR4 inhibitors demonstrate dose-dependent reductions in A647-hCCL22-induced internalization. This assay is highly reproducible within donors, on different days, and between operators. This signal stability makes it a robust PD assay for our First-in-Human trials.

Conclusions

Based on RNA expression and in situ hybridization analysis, we hypothesize that patients with a variety of tumors including types of breast, lung and HN cancers, would be good candidates for treatment with CCR4 antagonists. Once these patients are enrolled in clinical trials, robust assays to monitor receptor occupancy and establish pharmacokinetic-pharmacodynamic relationships are ready for deployment.
P45

The inhibitory checkpoint molecule NKG2A is upregulated on tumor infiltrating NK cells and CD8 T cells in human head and neck tumors

Michael Korrer, Young Kim

Vanderbilt University Medical Center, Nashville, TN, USA

Correspondence: Young Kim (michael.j.korrer@vanderbilt.edu)

Journal for ImmunoTherapy of Cancer 2017, 5(Suppl 2):P45

\section{Background}

Immunotherapy has revolutionized cancer therapy by targeting checkpoint molecules found on endogenous immune cells. Presently, the most commonly targeted checkpoint molecules are CTLA-4 and PD-1, which results in response rates of approximately $25 \%$ in head and neck squamous cell carcinomas (HNSCC). To improve treatment, additional immune checkpoint molecules expressed in the tumor microenvironment must be identified. Natural Killer Group 2 A (NKG2A) is an inhibitory receptor found on NK cells and CD8 T cells, the receptor for which is HLA-E, a non-classical MHC molecule often overexpressed in solid tumors. This study aimed to identify if NKG2A is expressed on tumor infiltrating NK cells and CD8 T cells from human HNSCC tumors as it is a potential therapeutic target.

Methods

Fresh human HNSCC tumors were digested with miltenyi human tumor dissacotiation kit and Gentle Macs machine following manufactures instructions. Cells were then stained for surface phenotyping or frozen for functional assays.

Results

We analyzed and compared tumor infiltrating NK cells and CD8 T cells from HNSCC patients with matched PBMC by flow cytometry for the expression of activating and inhibitory receptors. We found a unique population of effector memory PD-1+ NKG2A+ CD8 T cells which was absent from the blood. NKG2A+ PD-1+ CD8 T cells expressed higher levels of CTLA-4 and LAG3 as well as produced lower IFNg than NKG2A- PD-1+ CD8 T cells. Interestingly, NKG2A+ PD-1+ CD8 T cells expressed higher levels of both Perforin and Granzyme $B$, suggesting that these cells are cytotoxically potent. We found a similar upregulation of NKG2A in the NK cell population. NK cells from the primary tumor, but not the blood of HNSCC patients, significantly increased expression of inhibitory KIR2DL4, PD-1 and NKG2A. In addition, we determined that the ligand for NKG2A, HLA$\mathrm{E}$, was abundantly expressed on CD45+ monocytes and T cells, but not on CD45- cells in the tumor.

Conclusions

We believe that this study provides the first characterization of human tumor-infiltrating NKG2A+ PD-1+ CD8 T cells. We have shown that NKG2A+ CD8 T cells express the highest levels of cytotoxic markers, suggesting an enhanced capacity to kill tumor cells, yet also the highest levels of checkpoint molecule expression. As we have also shown that NKG2A is upregulated in tumor infiltrating NK cells, we believe this makes NKG2A an ideal therapeutic target to improve anti-tumor cytotoxic responses. Ongoing studies are underway to determine the effect of inhibiting NKG2A on tumor immune responses.

\section{P46}

The impact of anti-PD-1 treatment on the immune cells and their correlation with the circulating tumor cells in patients with non-small cell lung cancer

Eleni-Kyriaki Vetsika', Despoina Aggouraki ${ }^{1}$, Galaktia Kallergi ${ }^{1}$, Zaharoula Lyristi ${ }^{1}$, Aristeidis Koukos', Despoina Kourougkiaouri ${ }^{1}$, Konstantinos Rounis², Vassilis Georgoulias', Athanasios Kotsakis ${ }^{2}$ ${ }^{1}$ University of Crete, Faculty of Medicine, Heraklion, Greece; ${ }^{2}$ University General Hospital of Heraklion, Crete, Greece, Heraklion, Greece Correspondence: Athanasios Kotsakis (thankotsakis@hotmail.com) Journal for ImmunoTherapy of Cancer 2017, 5(Suppl 2):P46 


\section{Background}

The major immune-check point, programmed cell death-1 (PD-1), plays a pivotal role in tumor immune escape. The interaction of PD-1 with its ligand (PD-L1) results in T cells exhaustion, and the blockade of this interaction can partially restore T cell function. Antibodies targeting PD-1 and PD-L1 have been approved for treatment of advanced Non-Small Cell Lung Cancer (NSCLC). In this pilot study, we aimed to investigate the effect of anti-PD-1 treatment on the frequencies of circulating PD- ${ }^{+}$ and PD-L1 ${ }^{+}$immune cells (ICs), as well as their correlation with circulating tumor cells (CTCs) in NSCLC patients.

Methods

Peripheral blood samples were collected from 24 advanced NSCLC patients before and after 1 and 3 cycles of anti-PD- 1 treatment. Flow cytometry and immunocytochemistry were used to quantify the changes of the frequencies of PD-1- and PD-L1-expressing ICs and CTCs, respectively.

\section{Results}

At baseline, CTCs and PD-L1 ${ }^{+} \mathrm{CTCs}$ were negatively correlated with PD-1 $\mathrm{CD}^{+}\left(\mathrm{p}=0.03\right.$ and $\mathrm{p}<0.0001$, respectively). Moreover, PD- $1^{-} \mathrm{CD} 4^{+} \mathrm{T}$ had a reverse correlation with PD-L $1^{+} \mathrm{CTCs}(\mathrm{p}=0.04)$, whereas PD- $1^{+} \mathrm{CD} 4^{+} \mathrm{T}$ cells were positively correlated with PD-L $1^{+} C T C s(p<0.0001)$. A significant decrease in PD- $1^{+} \mathrm{CD} 4^{+}$and PD- $1^{+} \mathrm{CD} 8^{+} \mathrm{T}$ cells was observed after $3 \mathrm{ad}-$ ministrations of anti-PD- 1 antibody ( $p=0.01$ and $p=0.05$, respectively). In contrast, PD-1 ${ }^{-} \mathrm{CD}^{+}(\mathrm{p}=0.05)$ and $\mathrm{PD}-\mathrm{1}^{-} \mathrm{CD} 8^{+}(\mathrm{p}=0.05)$ levels were increased in response to anti-PD-1 therapy. After one dose of anti-PD-1 therapy, these increased levels of PD- $1^{-} \mathrm{CD} 8^{+}$and PD- $1^{-} \mathrm{CD} 4^{+} \mathrm{T}$ cells were associated with reduced levels of CTCs $(p<0.0001)$. Most importantly, PD-L $1^{+} \mathrm{CTCs}$ were reversely correlated with PD-1 ${ }^{-} \mathrm{CD}^{+}(\mathrm{p}<0.0001)$ and PD- $1{ }^{-} C D 4^{+} T$ cells $(p<0.0001)$ following 3 doses of treatment. Patients, with non-progressive disease after 3 doses of treatment, had reduced percentages of PD- $1^{+} C D 4^{+}(p=0.05)$ and PD- $1^{+} C D 8^{+}$T cells $(p=0.002)$, whereas the percentages of PD- $1^{-} C D 4^{+}(p=0.04)$ and PD-1 $\mathrm{CD}^{+}(p=0.004)$ T cells were increased compared to baseline. Finally, the levels of $\mathrm{CD} 4^{+} \mathrm{PD}-\mathrm{L} 1^{+}$Tregs and PD-L1 ${ }^{+} \mathrm{G}-\mathrm{MDSC}$ (granulocytic-MDSC) were decreased after 1 dose of anti-PD- 1 therapy $(p=0.01$ and $p=0.02$, respectively). No correlation was observed between CTCs and immunosuppressive cells at any time point.

Conclusions

These data indicate that anti-PD-1 therapy seems to exert an effect on circulating immune cells and provides evidence for their possible interaction with CTCs via the PD-1/PD-L1 axis, in NSCLC patients. These findings may pave the way for additional studies in a larger cohort in order to document its impact in NSCLC patients.

\section{P47}

Gene expression analysis of IL-13Ra2 in human adrenocortical carcinoma correlates with poor survival

Abhinav Kumar, lan Bellayr, Raj Puri

Food and Drug Administration, Silver Spring, MD, USA

Correspondence: Raj Puri (abhinav.kumar@fda.hhs.gov)

Journal for ImmunoTherapy of Cancer 2017, 5(Suppl 2):P47

\section{Background}

Adrenocortical carcinoma (ACC) is a rare disease where cancer cells develop in the outer layer of the adrenal gland. Because of unreliable early detection, the five year survival rate of subjects with ACC is only $65 \%$. In a variety of cancers including glioblastoma, ovarian, and pancreatic cancers, expression of the interleukin-13 receptor a2 (IL-13Ra2) has been shown to be a prognostic biomarker and potential target for immunotherapy. Using RNA sequencing data from the Genomic Data Commons (GDC), we analyzed the association between IL-13Ra2 gene expression levels with duration of survival and disease outcome in the ACC patients. Methods

ACC datasets were accessed from the GDC [https://portal.gdc.cancer.gov/legacy-archive] in January 2017. Seventy-nine samples, 48 female and 31 male, were categorized and merged using JMP Genomics. Samples were then evenly distributed into three groups based on low, medium or high levels of IL-13Ra2 expression. Statistical analyzes were performed to compare survival, hormone levels, and tumor reoccurrence with IL-13Ra2 expression.

Results

Subjects with high IL-13Ra2 expressing tumors $(\mathrm{n}=27)$ showed a statistically significant lower survival rate $(56 \%)$ compared to patients with low IL-13Ra2 expressing tumors $(88 \%)(n=26)(p=.0135)$. Subjects with high serum hormone levels (cortisol, estrogen, androgen) exhibited a worse outcome; high IL-13Ra2 expression was associated with a greater incidence of high serum hormone level and poorer survival compared to low IL-13Ra2 expressing subjects. Subjects with new tumor events $(n=35)$ such as reoccurrence or metastasis exhibited a worse survival rate and high IL-13Ra2 expression showed a higher rate of new tumor events when compared to low expression levels $(p=.0042)$. Individuals with new tumor events exhibited a lower rate of survival, whereas individuals with high IL-13Ra2 expression had a low rate of survival regardless of whether a new tumor event occurred or not. Subjects with metastatic tumors $(n=17)$ had a lower survival rate compared to those without metastatic tumors, but no correlation with survival for subjects with medium or high expression levels of IL-13Ra2 was observed.

\section{Conclusions}

IL-13Ra2 expression levels were a major determinant of outcome in ACC subjects; high IL-13Ra2 levels associated with a lower rate of survival, higher incidence rate and worse outcome with excess hormone production, and more new tumor events. Additionally, subjects with higher expression levels of $I L-13 R a 2$ exhibited a low survival rate regardless of new tumor events or metastases. These results indicate that IL-13Ra2 may be a prognostic biomarker in subjects with ACC.

P48

Different impact of immune cell infiltration and HLA class I expression in lymph node vs. cutaneous/subcutaneous metastases as predictive markers in melanoma patients treated with ipilimumab

Tímea Balatoni ${ }^{1}$, Anita Mohos ${ }^{2}$, Eszter Papp ${ }^{1}$, Tímea Sebestyén ${ }^{3}$, Gabriella Liszkay', Judit Oláh', Anita Varga ${ }^{4}$, Zsuzsanna Lengyel ${ }^{5}$, Gabriella Emri'

Soldano Ferrone, Andrea Ladányi

${ }^{1}$ National Institute of Oncology, Budapest, Hungary; ${ }^{2}$ Semmelweis

University, Budapest, Hungary; ${ }^{3}$ St. John's Hospital, Budapest, Hungary;

${ }^{4}$ Albert Szent-Györgyi Medical Center, University of Szeged, Szeged,

Hungary; ${ }^{5}$ University of Pécs, Pécs, Hungary; ${ }^{6}$ Faculty of Medicine,

University of Debrecen, Debrecen, Hungary; ${ }^{7}$ Massachusetts General

Hospital, Harvard Medical School, Boston, MA, USA

Correspondence: Andrea Ladányi (ladanyi@oncol.hu)

Journal for ImmunoTherapy of Cancer 2017, 5(Suppl 2):P48

\section{Background}

Immunomodulatory antibodies targeting immune checkpoints are gaining ground in cancer treatment, with simultaneous efforts to search for clinically usable predictive markers which could help making treatment decisions. The aim of our study was to explore tumorinfiltrating immune cells and HLA class I expression as potential biomarkers of response to ipilimumab and survival in patients with metastatic melanoma.

Methods

In pretreatment surgical tissue samples (52 lymph node and 34 cutaneous or subcutaneous metastases from 30 patients), intratumoral immune cell infiltration was determined using antibodies against a panel of 11 markers (CD4, CD8, CD45RO, CD20, CD134, CD137, FOXP3, PD-1, NKp46, CD16, CD68). Tumor infiltration by each cell type was evaluated with regard to response to treatment and patients' survival. Expression level of HLA class I antigens and its correlation with immune cell densities as well as with response to ipilimumab and disease outcome was also examined.

Results

Immune cell infiltration in lymph node metastases was markedly higher compared to skin or subcutaneous ones in the case of the majority of markers studied (9 of 11). Evaluated separately in the two 
locations, in the group of lymphoid metastases significantly larger amount of $\mathrm{CD}^{+}, \mathrm{CD}^{+}, \mathrm{FOXP3}^{+}, \mathrm{CD} 134^{+}$lymphocytes, $\mathrm{CD} 20^{+} \mathrm{B}$ cells and NKp $46^{+}$NK cells was found in responders compared to nonresponders. The most pronounced differences were seen in the case of FOXP3 and CD8, which also showed association with patients' survival. Infiltration level of $\mathrm{CD}_{45 \mathrm{RO}}{ }^{+}, \mathrm{PD}-1^{+}, \mathrm{CD} 16^{+}$and $\mathrm{CD}^{+} 8^{+}$cells correlated with survival but not with treatment response. On the other hand, analyzing subcutaneous/cutaneous metastases revealed significant associations with clinical response or survival only in the case of $\mathrm{CD}_{16}{ }^{+}$and $\mathrm{CD}^{+} 8^{+}$cells. Furthermore, HLA class I antigen expression level of lymphoid metastases, but not that of skin or subcutaneous ones, showed correlation with $\mathrm{T}$ cell density and with patients' survival.

Conclusions

Our results indicate different predictive impact of immune cell infiltration and HLA class I expression in lymphoid vs. non-lymphoid metastases of melanoma patients treated with ipilimumab. This finding points to the potential importance of analyzing metastases of different locations separately in order to reveal all existing predictive associations, part of which could be obscured in the case of joint evaluation because of high variation in immune cell prevalence.

\section{P49}

Characterization of immune checkpoint marker expression and infiltrating lymphoid and myeloid immune cells in the tumor microenvironment by RNA in situ hybridization

Annelies Laeremans, Na Li, Jeffrey Kim, Xiao-Jun Ma, Emily Park Advanced Cell Diagnostics, Newark, CA, USA

Correspondence: Annelies Laeremans (alaeremans@acdbio.com) Journal for ImmunoTherapy of Cancer 2017, 5(Suppl 2):P49

\section{Background}

Interactions between tumor cells and surrounding immune cells in the tumor microenvironment (TME) play a key role in tumor progression and treatment response with accumulating evidence indicating a crucial role for tumor infiltrating immune cells. Although infiltrating $\mathrm{T}$ cells have been correlated with improved clinical outcome, tumor infiltrated lymphocytes are ineffective in eradicating tumors due to their inhibition by immune checkpoint molecules. Cancer immunotherapy blocking the PD-1/PD-L1 immune checkpoint pathway is an established treatment with long-lasting clinical benefits. However, the majority of patients are resistant or relapse after initial response, showing the need for understanding resistance mechanisms and stratifying patients using predictive biomarkers. Also, immunosuppressive factors in the local TME contribute to tumor evasion by recruiting and modulating regulatory $\mathrm{T}$ cells and myeloid-derived cells.

Methods

In this study, we used RNAscope ${ }^{\circledast}$ ISH to evaluate in situ expression profiles of therapeutic checkpoint targets in the TME of 30 NSCLC and 30 ovarian cancer archived FFPE tissue samples. In addition, immune infiltration of lymphoid and myeloid cells in the TME was investigated.

Results

Specific checkpoint target molecules were visualized in a highly specific and sensitive manner in individual cells within tissue morphological context. Multiple checkpoint molecules, including PD1, PD-L1, TIM3, and CTLA-4, were detected in the same immune environment, especially in highly inflamed tumors. Unexpectedly, we observed tumor cell-intrinsic expression of TIM3, LAG3, PD-L2, and GITR in a subset of samples. Furthermore, PD1 was often co-expressed with other therapeutic checkpoint targets, including LAG3, TIM3, and TIGIT, in the same infiltrating immune cells. Expression patterns of key functional markers FOXP3, IFNg, CXCL10, and CCL22 combined with cell lineage markers CD4, CD8, CD68, and CD163 demonstrated infiltration of lymphoid and myeloid cells across samples. Recruitment of CXCL10-expressing immune cells in local tumor regions was visualized. The presence of regulatory $\mathrm{T}$ cells (FOXP3 $+\mathrm{CD} 4+$ ) was evaluated in relation to CCL22 expression in immunosuppressive local environment. IFNg positive CD8 cells were rarely detected. Conclusions

These findings highlight the utility of RNAscope ${ }^{\circledast}$ ISH in understanding how cancer cells evade the host immune surveillance and ultimately develop resistance against checkpoint blockades. This approach visualizes states of lymphoid and myeloid cells present in the TME by detecting key functional molecules, such as cytokines and chemokines, in addition to immune co-inhibitory and lineage molecules. Considering the highly plastic immune environment with a spectrum of maturation and polarization, characterizing the immunomodulatory phenotype by RNAscope ${ }^{\circledast}$ ISH could offer fundamental insights in contributing factors in immunosuppressive environment.

\section{P50}

Application of the immunoscore as prognostic biomarkers in patients with epithelial ovarian cancer

Shin-Wha Lee', Hee Jung Jung ${ }^{1}$, Young-Jae Lee' ${ }^{1}$, Ha-Young Lee²

Yong-Man Kim', Young-Tak Kim

${ }^{1}$ Ulsan University, ASAN Medical Center, Seoul, Republic of Korea; ${ }^{2}$ ASAN Institute for Life Science, Seoul, Republic of Korea

Correspondence: Shin-Wha Lee (swhlee@amc.seoul.kr)

Journal for ImmunoTherapy of Cancer 2017, 5(Suppl 2):P50

\section{Background}

The analysis of single parameters alone may not provide sufficient insights about complex immune system-tumor interactions. This study is to validate the immune contexture as prognostic biomarkers in high-grade serous ovarian cancer (HGS-OC) and to find new era of immunoscore in HGS-OC.

Methods

We collected FFPE samples from 187 patients with HSOC and produced TMA samples. We accomplished the OPAL multiplex IHC assay for the quantitative analysis of immune markers, including CD4, CD8, CD20, FoxP3, PD-L1, and CK. Multiplex Biomarker Imaging and inForm ${ }^{\circledast}$ Image Analysis Software was used.

Results

FIGO stage III and IV patients were $84.5 \%$ (158/187). The optimal debulking surgery was done in $66.8 \%(125 / 187)$. The 3 -year diseasefree survival and 5 -year overall survival were $35.1 \%$ and $50.0 \%$, respectively. Any single marker was not related to the survival including CD8, FoxP3, and PD-L1. However, high CD8:FoxP3 and CD8:PD-L1 ratios were correlated with the good survival. In cox regression model, the risk factors for HGS-OC survival were FIGO stage (HR $1.784,95 \% \mathrm{Cl}: 1.295-2.457, p<0.001)$ and platinum resistance (HR $4.257,95 \% \mathrm{Cl}: 2.753-6.582, p<0.001)$. Additionally, CD8:PD-L1 ratio was a favorable prognostic factor (HR $0.621,95 \%$ Cl: 0.042-0.917, $p=0.017$ )

\section{Conclusions}

These findings indicate that, although any single immune marker is not related to the survival, CD8:FoxP3 and CD8:PD-L1 ratios provide the positive correlation with the prognosis in HGS-OC. Especially, CD8:PD-L1 ratio is prognostic biomarker which is comparable to clinical biomarkers. The next study for immunoscore is necessary to define immunoscore in ovarian cancer.

P51

FGFR2b expression and baseline immune signature to guide FPA144 development in urothelial cancer

Yeonju Lee, Nerissa Mendoza, Janine Powers, Lee Clark, James

Hnatyszyn, Carmen Ladner, Helen Collins, Kevin P. Baker, Majid Ghoddusi Five Prime Therapeutics, Inc., South San Francisco, CA, USA

Correspondence: Majid Ghoddusi (yeonju.lee@fiveprime.com)

Journal for ImmunoTherapy of Cancer 2017, 5(Suppl 2):P51 


\section{Background}

Five Prime Therapeutics, Inc. has developed an FGFR2b-specific humanized monoclonal antibody, FPA144, to treat cancer patients with overexpression of the FGFR2b receptor. FPA144 blocks ligand binding and has been glycoengineered for enhanced antibodydependent cell-mediated cytotoxicity. Our preclinical models showed FPA144 reprograms the tumor microenvironment by recruiting natural killer cells to the tumor, upregulating PD-L1 expression, and enhancing T cell infiltration [1]. FPA144 is currently in clinical development in gastric and urothelial cancers (UC). FPA144 completed dose escalation in patients with solid tumors and was well tolerated with no dose-limiting toxicity. During dose escalation, a patient with metastatic UC treated with FPA 144 at $3 \mathrm{mg} / \mathrm{kg}$ exhibited a complete response [2]. Currently, UC patients have enrolled in the dose expansion portion of the trial. We characterized baseline immune cells in the tumor microenvironment and their relationship with FGFR2b in UC patients to guide potential development of FPA144 in combination with other therapies.

Methods

We selected a cohort of archival primary UC formalin-fixed paraffinembedded whole tumor sections $(n=34)$ with known FGFR2b expression and performed fluorescent $\mathrm{IHC}$ on these sections using the proprietary FPR2-D mouse monoclonal antibody, which is specific for FGFR2b. We also performed multi-panel multiplex immunofluorescent assays to quantify immune cell compositions in sequential sections of UC samples.

Results

FGFR2b H scores positively correlate with tumor-infiltrating CD163, PD-L1/CD8 and PD-L1/CD68 cells. From baseline immune compositions and FGFR2b expression level, we clustered cases into three distinct groups: 1) Immune desert (low CD8 T cells, tumor-associated macrophages (TAM), and PD-L1 expression in the whole tumor) with low or negative FGFR2b; 2) Immune excluded (high CD8 T cells, TAM, and PD-L1 expression in tumor-associated stroma) with low to moderate FGFR2b; 3) Inflamed (high CD8 T cells, TAM, and PD-L1 expression in the whole tumor) with moderate to high FGFR2b.

\section{Conclusions}

The UC cases with high FGFR2b expression have higher tumorinfiltrating T cells, TAM and PD-L1 expression compared to FGFR2b low or negative tumors. Profiling FGFR2b expression and baseline tumor associated immune cells could help FPA144 development strategy in UC patients. We are currently exploring the utility of combining FPA144 with PD-1/PD-L1 blockade in clinic.

Trial Registration

ClinicalTrial.gov Identifier NCT02318329

\section{Reference}

1. Powers J, et al. FPA144, a therapeutic monoclonal antibody targeting the FGFR2b receptor, promotes antibody dependent cell-mediated cytotoxicity and stimulates sensitivity to PD-1 blockade in the 4T1 syngeneic tumor model in mice. AACR. 2016.2. Ghoddusi M, et al. FGFR2b represents a novel target for treatment of urothelial cancer. EORTC-NCI-AACR Molecular Targets. 2016.

P52

Partially exhausted T lymphocyte directed neoadjuvant immunotherapy in unresectable Stage III melanoma

Lauren Levine, Clinton Wu, Kelly Mahuron, Katy Tsai, Alain Algazi, Michael Rosenblum, Michael Alvarado, Adil Daud

Helen Diller Comprehensive Cancer Center, University of California, San Francisco, San Francisco, CA, USA

Correspondence: Lauren Levine (lauren.levine@ucsf.edu); Adil Daud Journal for ImmunoTherapy of Cancer 2017, 5(Suppl 2):P52

\section{Background}

Programmed death 1 (PD-1) inhibition activates partially exhausted cytotoxic $T$ lymphocytes (peCTL) and induces tumor regression. We have previously demonstrated that the peCTL frequency predicts response to anti-PD-1 monotherapy and combination CTLA-4/PD-1 blockade in metastatic melanoma. However, the utility of this assay in the neoadjuvant setting has not been established. In the current study, 15 patients with unresectable Stage III Melanoma were assessed for response to neoadjuvant anti-PD-1 or anti-PD-1/CTLA-4 inhibition as assigned by peCTL frequency.

Methods

Pretreatment tumor samples from 15 patients with locally advanced melanoma underwent multiparameter flow cytometric analysis. $\mathrm{Pa}-$ tients received neoadjuvant anti-PD-1 monotherapy or combination therapy and best overall response to therapy (BOR) as evaluated by Response Evaluation Criteria in Solid Tumors version 1.1 (RECIST v1.1) and irAE incidence and severity by $\mathrm{NCl}$ Common Terminology Criteria for Adverse Events version 3.0 (CTCAE v3.0) were tabulated by clinicians blinded to immunophenotypic analysis. Patients with low peCTL burden $(<20 \%)$ received anti-PD-1/CTLA-4 inhibitors whereas those with high peCTL (>20\%) were assigned to anti-PD-1 monotherapy. Patients with documented follow up history and evaluable immunophenotype were included in efficacy and safety analysis. Results

Of 82 patients screened, 15 met criteria for further analysis. Patients were $60 \%$ male with mean age of 58 years. Twelve patients had Stage III disease with $L D H<U L N$, and 13 out of 15 patients were BRAF WT. Prior therapy included CTLA-4 monotherapy in 4 of 12 patients, with only 1 patient with prior PD-1 inhibitor exposure or intralesional therapy. Eleven patients received anti-PD-1 monotherapy and 4 received CTLA-4/PD-1 blockade and were evaluable for BOR at time of analysis. Eleven out of 15 patients achieved complete response, with two partial responses, one patient exhibiting stable disease and one patient with progressive disease per RECIST v1.1 criteria. Of the 9 patients that underwent surgery, 6 attained complete pathologic response. Relapse free survival was 26 months and all patients remained alive at time of analysis. Four cases of disease relapse occurred, without correlation with peCTL frequency. Treatment was well tolerated, with grade $1 / 2$ diarrhea, pruritus, arthralgia, and hypothyroidism most frequently reported. Grade 3/4 adverse effects included two isolated cases of gastritis and anaphylaxis.

Conclusions

Our results suggest the efficacy of peCTL directed neoadjuvant treatment in unresectable Stage III melanoma with the large proportion of patients achieving complete treatment response with acceptable toxicity profile. These promising data from this exploratory analysis merit further investigation with a larger cohort validation study.

Trial Registration

UCSF IRB Protocol 138510

P53

The crosstalk between PD-1, CD137 and OX40 in head and neck cancer

Robert Ferris (jil217@pitt.edu)

UPCI, Pittsburgh, PA, USA

Journal for ImmunoTherapy of Cancer 2017, 5(Suppl 2):P53

\section{Background}

Head and neck squamous cell carcinoma (HNSCC) is one of the malignant diseases with highly immunosuppressive tumor microenvironment, which limits the efficacy of cancer therapies. Tumor infiltrating lymphocytes (TIL) express immune checkpoint receptors in the microenvironment, which can be divided into two groups, inhibitory receptors, such as Programmed Death 1 (PD-1), and costimulatory receptors, such as CD137 and OX40. However, the T cell in TIL exhibit dysfunctional status and the inhibitory receptors has more dominant effect than the costimulatory receptor. The aim of this study was to investigate whether there is crosstalk between PD-1, CD137 and OX40 pathways in TIL expressing multiple immune checkpoint receptors. $[1,2,3,4,5]$

Methods

We used PD-L1 (the ligand of PD-1) beads alone or in combination with CD137 or OX40 agonists to treat TIL T cell for 48 hour. Also we used anti-CD3/CD28 beads treated PBL to mimic the TIL environment. 
Results

TIL T cells treated with anti-CD3/CD28/PD-L1 beads expressed less CD137 and OX40, and produced less IFN-g than control-treated TIL T cells. Activated PBL T cells treated with anti-CD3/CD28/PD-L1 beads showed similar results as TIL. CD137 or OX40 stimulation could partially reverse this effect.

Conclusions

The result suggest the potential for intracellular signaling cross-talk. We are investigating the pathways is involved in the PD-1, CD137 and OX40 crosstalk.

\section{References}

1. Pardoll, D. M. The blockade of immune checkpoints in cancer immunotherapy. Nature Reviews Cancer. 2012; 12(4), 252-264.

2. Ferris, R. L. Immunology and immunotherapy of head and neck cancer. Journal of clinical oncology. 2015; 33(29), 3293-3304.

3. Croft, M. Regulation of Pl-3-kinase and Akt signaling in T lymphocytes and other cells by TNFR family molecules. 2013.

4. Chen, S., Lee, L. F., Fisher, T. S., Jessen, B., Elliott, M., Evering, W. \& Wang, H. Combination of 4-1BB agonist and PD-1 antagonist promotes antitumor effector/memory CD8 T cells in a poorly immunogenic tumor model. Cancer immunology research. 2015; 3(2), 149-160.

5. Li, J., Jie, H. B., Lei, Y., Gildener-Leapman, N., Trivedi, S., Green, T. \& Ferris, R. L. PD-1/SHP-2 inhibits Tc1/Th1 phenotypic responses and the activation of T cells in the tumor microenvironment. Cancer research. 2015; 75(3), 508-518.

\section{P54}

Multiplex IHC immuno-oncology panel for standardized profiling

of the immune status based on spatial and functional

characterization of the tumor microenvironment

Svenja Lippok', Florian Leiss ${ }^{1}$, Katrin Schneider ${ }^{1}$, Tobias Wiestler $^{1}$,

René Korn', Dasa Medrikova', Moritz Widmaier', Martin Hager ${ }^{1}$ ',

Ivan Kanchev', Anthony Masci', Gela Sia ${ }^{2}$, Chris Kerfoot ${ }^{2}$,

Lisa Dauffenbach ${ }^{2}$, Ralf Huss ${ }^{1}$

'Definiens AG, 80636 Munich, Germany; ${ }^{2}$ Mosaic Laboratories, Lake Forest, CA, USA

Correspondence: Svenja Lippok (slippok@definiens.com)

Journal for ImmunoTherapy of Cancer 2017, 5(Suppl 2):P54

\section{Background}

Characterization of the immune status of patients will become increasingly important to personalize cancer treatments [1]. As the number of treatment options is expected to dramatically increase in the next years, standardized information that guides treatment decision is highly needed. Therefore, we developed a multiplex ImmunoOncology (IO) panel to profile the immune status of patient tumors into biologically meaningful and clinically actionable categories which support therapy suggestions.

Methods

Three chromogenic IHC assays were developed and optimized for image analysis (PD-L1/CD68/CD3, PD1/FoxP3/CD8, and Granzyme B) using brown (DAB), red and green chromogens for several indications. Definiens' Cognition Network Technology ${ }^{\oplus}$ was used for spatially resolved image analysis of biomarker expression levels combining rulebased object hierarchy detection with machine-learning strategies. Verifications strategies have been developed in preparation for the application in prospective stratification studies. Marker-positive cells were detected and classified as PD-L1+ tumor cells, (exhausted) CD8+ T cells, CD3+ T cells, regulatory $T$ cells, and macrophages. Global and local densities were computed within regions annotated as Tumor Center and Invasive Margin as well as within automatically detected tumor epithelium (TE) and non-epithelial regions (NE).

Results

Based on the measured densities, NSCLC patients were grouped into one of six possible immune status categories and biomarker profiles have been computed. Additionally, heterogeneity between patients within one category is discussed against the backdrop of therapy suggestions and applicability in further indications. The intra-tumoral biomarker heterogeneity is visualized with cell co-occurrence heat maps and regional context maps, providing insights on inhibition status, effector status, and checkpoint blockade (Fig. 1).

Conclusions

Our IO Panel serves as a ready-to-use approach for standardized immune profiling that quantifies the set of seven and subsequently more biomarkers reproducibly on a continuous scale. Definiens' Tissue Phenomics ${ }^{\circledast}$ technology combined with Mosaic's multiplex IHC assays provides next generation spatial and functional resolution of the tumor microenvironment. In the future, we envision applications that facilitate the identification of novel prognostic and predictive signatures, with the goal to support stratification and guide eligibility of patients for a broad range of therapeutics.

Selected maps are shown for two immune status categories belonging to either hot tumors (top) or cold tumors (bottom). A: Light blue: PD-L1 negative T cells, Darker blue: PD-L1 negative T cells (NE), Light Green: Macrophages (TE), Dark Green: Macrophages (NE), Orange: PD-L1 positive T cells, Red: PD-L1 positive tumor cells. B: Red/Light Blue: PD-L1 positive/negative tumor cells, Orange: Macrophages, Green: T cells.

\section{Reference}

1. Yuan, et al. Journal for ImmunoTherapy of Cancer. 2016; 4:3

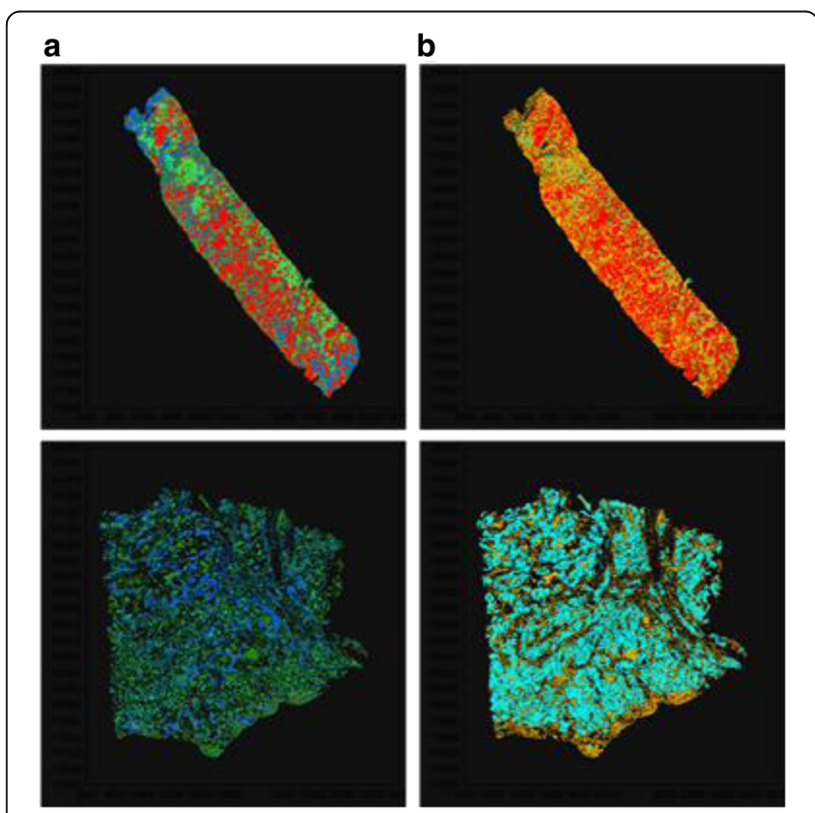

Fig. 1 (abstact P54). Regional context maps displaying potential checkpoint blockade (A) and PD-L1 landscape (B)

P55

Non-fucosylated anti-CTLA-4 antibody enhances vaccine-induced T cell responses in a non-human primate pharmacodynamic vaccine model

John Loffredo, Raja Vuyyuru, Vanessa Spires, Sophie Beyer, Maxine Fox, Jon Ehrmann, Katrina Taylor, John Engelhardt, Alan Korman, Robert

Graziano

Bristol-Myers Squibb, Princeton, NJ, USA

Correspondence: John Loffredo (jtloffredo@uwalumni.com)

Journal for ImmunoTherapy of Cancer 2017, 5(Suppl 2):P55 


\section{Background}

Ipilimumab, which blocks the CTLA-4 pathway, is an effective immunotherapy treatment for melanoma and in combination with nivolumab for other malignancies. To improve the potency of this immune checkpoint inhibitor, the Fc region of ipilimumab was altered to enhance antibody-dependent cellular cytotoxicity (ADCC) activity and thereby the potential for increased T-regulatory cell depletion. Here, we assessed the ability of a non-fucosylated form of ipilimumab (anti-CTLA-4-NF) to modulate $\mathrm{T}$ cell activity in a nonhuman primate pharmacodynamics $(P D)$ vaccine model.

Methods

Mauritian cynomolgus macaques (MCMs) were preselected for the high frequency allele Mafa- $A{ }^{*} 063$ and vaccinated intramuscularly with 2 non-replicating adenovirus serotype 5 (Ad5) viral constructs, encoding simian immunodeficiency virus (SIV) Gag or Nef proteins. Following vaccination, the animals received a single dose of either a vehicle control, ipilimumab, or anti-CTLA-4-NF. Ipilimumab and antiCTLA-4-NF were administered intravenously at either 1 or $10 \mathrm{mg} / \mathrm{kg}$. Peripheral blood was sampled pre-treatment and then longitudinally over 7 weeks. Vaccine-elicited T cell responses and major T cell subsets were measured by flow cytometry, including the use of peptideloaded MHC class I tetramers, and IFN-gamma ELISPOT assays.

Results

A single dose of ipilimumab or anti-CTLA-4-NF resulted in augmentation of antigen-specific $\mathrm{CD}^{+} \mathrm{T}$ cell responses at early (2-3 weeks post-vaccination) and later time points ( $>6$ weeks). The resulting peripheral immune responses included T cells directed against the SIV protein inserts as well as against the Ad5 viral vector itself. Both treatments also enhanced circulating $\mathrm{CD}^{+}$and $\mathrm{CD}^{+}{ }^{+} \mathrm{T}$ cell proliferation as measured by intracellular Ki-67 expression. Anti-CTLA-4-NF demonstrated enhancement of the vaccine-induced $T$ cell responses over that observed in ipilimumab-treated MCMs. This included increased $\mathrm{CD}^{+} \mathrm{CD}^{+}$tetramer ${ }^{+}$frequencies of 2 immunodominant SIV-specific $T$ cell responses by flow cytometry (at 3 weeks postvaccination: Nef RM9 median, $4.16 \%$ with anti-CTLA-4-NF vs $1.95 \%$ with ipilimumab treatment; Gag GW9 median, 3.08\% with anti-CTLA$4-\mathrm{NF}$ vs $1.17 \%$ with ipilimumab treatment).

\section{Conclusions}

Using this non-human primate PD vaccine model, we observed that immune checkpoint blockade with anti-CTLA-4 augments T cell activation and proliferation in circulating blood. These results suggest that, in addition to the use of non-human primates in toxicology models, the evaluation of antigen-specific $T$ cell responses in MCMs provides meaningful preclinical pharmacological assessment of human drug candidates that target immune modulation. The improved $T$ cell activity observed in these studies also lend support for the use of anti-CTLA-4 antibodies for vaccine enhancement, broadening the potential utility of next-generation anti-CTLA-4 antibodies beyond cancer therapy.

\section{P56}

Widespread human $\mathrm{T}$ cell receptor variable gene polymorphism revealed by long amplicon TCRB repertoire sequencing: Implications for the prediction and interpretation of immunotherapy outcome

Tim Looney ${ }^{1}$, Alex Glavin', Sarabjot Pabla ${ }^{2}$, Sean Glenn ${ }^{2}$, Lauren Miller ${ }^{3}$ Denise Topacio-Hall ${ }^{3}$, Elizabeth Linch ${ }^{3}$, Alice Zheng ${ }^{1}$, Jeffrey Conroy², Blake Burgher ${ }^{2}$, Carl Morrison², Geoffrey Lowman', Mark Andersen", Fiona Hyland

${ }^{1}$ Thermo Fisher Scientific, South San Francisco, CA, USA; ${ }^{2}$ OmniSeq, Buffalo, NY, USA; ${ }^{3}$ Thermo Fisher Scientific, Carlsbad, CA, USA

Correspondence: Geoffrey Lowman

(geoffrey.lowman@thermofisher.com)

Journal for ImmunoTherapy of Cancer 2017, 5(Suppl 2):P56

\section{Background}

Human T cell antigen receptors play a critical role in protective adaptive immune responses to infectious disease and cancer, but are also implicated in autoimmune disease and the emergence of severe immune-mediated adverse events during immunotherapy. The antigen specificity of the $T$ cell receptor is determined in part by the sequence of the CDR and Framework regions encoded by the TCRB variable gene. Previous studies of population sequencing data indicate that current antigen receptor allele databases, such as IMGT, fail to capture a significant portion of human variation, though interpretation of this data is challenging. Here we sought to use long amplicon multiplex sequencing of rearranged TCRB receptors to validate putative novel human variable gene alleles previously recovered from 1000 genomes data.

Methods

TCRB rearrangements were amplified from CDNA template from 85 Caucasians undergoing treatment for melanoma using lon AmpliSeq ${ }^{\mathrm{TM}}$-based multiplex Framework 1 and Constant gene primers to produce $\sim 330 \mathrm{bp}$ amplicons. Samples were sequenced in multiplex using the lon $530^{\mathrm{TM}}$ chip to produce $\sim 1.5 \mathrm{M}$ raw reads per sample. Raw data was uploaded to lon Reporter ${ }^{\mathrm{TM}}$ for clonotyping and identification of rearrangements containing variable gene sequences $a b-$ sent from the IMGT database. Putatively novel sequences were compared with those reported in the Lym $1 \mathrm{k}$ database of alleles recovered from 1000 genomes sequence data.

Results

We identified 15 variants of variable gene alleles, absent from the IMGT database, which result in amino acid changes to the CDR or Framework regions of the T cell receptor. Typically, a single individual was found to be heterozygous for a novel variant, though we note two instances of novel alleles that are found in multiple individuals within this cohort. We also find evidence for novel variable gene alleles that are absent from the Lym1k database, potentially due to challenges in inferring receptor alleles from short-read population sequencing studies.

Conclusions

We find evidence for significant human diversity in TCRB variable gene alleles beyond what is currently represented in the IMGT database. TCRB sequencing using multiplex Framework 1 and Constant gene targeting primers is ideally suited for studying the role of T cell antigen receptor diversity in autoimmune disease and the emergence of immune-mediated adverse events during immunotherapy.

\section{P57}

Insights into the tumor microenvironment and therapeutic $\mathrm{T}$ cell manufacture revealed by long amplicon immune repertoire sequencing

Geoffrey Lowman ${ }^{1}$, Tim Looney ${ }^{2}$, Elizabeth Linch ${ }^{1}$, Lauren Miller ${ }^{1}$, Denise Topacio-Hall', Alex Pankov², Alice Zheng ${ }^{2}$, Reidun Hartberg ${ }^{3}$, Hilde

Almåsbak ${ }^{3}$, Tor Espen Stav-Noraas ${ }^{3}$, Anette Kullmann $^{3}$, Fiona Hyland ${ }^{2}$, Mark Andersen

${ }^{1} T$ Thermo Fisher Scientific, Carlsbad, CA, USA; ${ }^{2}$ Thermo Fisher Scientific, South San Francisco, CA, USA; ${ }^{3}$ Thermo Fisher Scientific, Oslo, Norway

Correspondence: Geoffrey Lowman

(geoffrey.lowman@thermofisher.com)

Journal for ImmunoTherapy of Cancer 2017, 5(Suppl 2):P57

\section{Background}

TCRB immune repertoire analysis by next-generation sequencing is emerging as a valuable tool for research studies of the tumor microenvironment and potential immune responses to cancer immunotherapy. Here we describe a multiplex PCR-based TCRB sequencing assay that leverages lon Ampliseq ${ }^{\text {TM }}$ library construction chemistry and the long read capability of the lon $\mathrm{S}^{\mathrm{TM}} 530$ chip to provide coverage of all three CDR domains of the human TCR beta chain. We demonstrate use of the assay to evaluate tumor-infiltrating T cell repertoire features and monitor manufacture of therapeutic T cells.

Methods

To evaluate assay accuracy we sequenced libraries derived from 30 well-studied $T$ cell lymphoma rearrangements, then compared our results with those reported by another commercially available immune repertoire sequencing technology. We next used the assay to profile tumor infiltrating $\mathrm{T}$ cell repertoires for a cohort of 19 
individuals with non-small cell lung cancer. We correlate repertoire features with gene expression profiling data. We then harnessed the long read capability of the assay to profile T cells at various stages of the therapeutic $T$ cell manufacturing process.

Results

Long read TCRB sequencing of 30 reference rearrangements yielded strong linearity in detection of clonal frequencies in reference spikein experiments. We demonstrate the quantitative nature of the assay by studying populations of counted $T$ cells. Profiling of $T$ cell repertoires in non-small cell lung cancer samples revealed a positive correlation between the number of clones detected and CCR7, CXCR5 and CD3 expression. T cell Evenness (i.e. normalized Shannon Entropy) correlated most strongly with myeloid-specific genes and markers for T cell exhaustion and was anti-correlated with IFNG expression. Sequencing of therapeutic $T$ cells during manufacture revealed a steady increase in the evenness of clone sizes during in vitro expansion and allowed for quantification of clonal expansion.

\section{Conclusions}

These results demonstrate: 1) The accuracy and versatility of immune repertoire sequencing using lon AmpliSeq ${ }^{\mathrm{TM}}$ library construction 2) The benefit of combining targeted gene expression and repertoire profiling for studies of the tumor microenvironment 3 ) The utility of repertoire sequencing covering all CDR regions in monitoring the manufacture of therapeutic T cells.

\section{P58}

Public NY-ESO-1 specific TCRs as novel biomarkers for immune monitoring of NY-ESO-1 positive cancer patients

Hailing Lu', Seth Pollack ${ }^{2}$, Neeta Somaiah ${ }^{3}$, Sant Chawla ${ }^{4}$, Patrick Hwu ${ }^{3}$, Marissa Vignali ${ }^{5}$, Julie Rytlewski ${ }^{5}$, Sacha Gnjatic ${ }^{6}$, Jan ter Meulen

${ }^{1}$ Immune Design, Seattle, WA, USA; ${ }^{2}$ Fred Hutchinson Cancer Research Center, Seattle, WA, USA; ${ }^{3}$ MD Anderson Cancer Center, Houston, TX,

USA; ${ }^{4}$ Santa Monica Oncology Center, Santa Monica, CA, USA; ${ }^{5}$ Adaptive Biotechnologies, Seattle, WA, USA; ${ }^{6}$ cahn School of Medicine at Mount

Sinai, New York, NY, USA

Correspondence: Hailing Lu (hailing.lu@immunedesign.com)

Journal for ImmunoTherapy of Cancer 2017, 5(Suppl 2):P58

\section{Background}

T cell clonotypes with shared T cell receptors (public TCRs [pTCR]) are involved in the immune response to chronic viral infections, however, their role in immune responses to cancer is largely unknown. We evaluated the association of NY-ESO-1 specific PTCR sequences and survival in solid tumor patients treated with LV305 or CMB305, which are active immunotherapies based on the dendritic cell targeting lentiviral vector platform $\mathrm{ZVex}^{\oplus}$, expressing the cancer-testis antigen NY-ESO-1.

\section{Methods}

Peripheral blood mononuclear cells (PBMC) were collected before and after patients with NY-ESO-1 positive solid tumors, including soft tissue sarcomas, received therapy with LV305 or CMB305 ( $n=64)$. PBMC were subjected to deep sequencing to study the repertoire of the TCRVB-CDR3 region.

Results

The TCR-VB CDR3 amino acid sequences of three NY-ESO-1 specific pTCR clones obtained through in vitro culture from a LV305 patient with a near complete response were fully conserved in 41/56 (73.2\%) of LV305/CMB305 patients and 54\% of 539 healthy blood donors. Induction of NY-ESO-1 pTCR on LV305 or CMB305 therapy (baseline negative to positive, or doubling of frequency) was observed in $31 \%$ of patients and was associated with a trend towards better overall survival (data as of ASCO 2017). Querying TCR databases from multiple published clinical trials revealed NY-ESO-1 pTCR sequences in blood of patients with melanoma (6/13), renal cancer (1/3), and glioblastoma (6/13), and with a lower incidence in tumor biopsies.

Conclusions

We have identified NY-ESO-1 specific public TCRs in the PBMC of cancer patients undergoing active immunotherapy, as well as in healthy blood donors. In patients, the induction of pTCR appeared to be associated with better survival, whereas their presence in healthy blood donors may indicate frequent low-level baseline T cell immunity against this cancer testis antigen. pTCR should be investigated as a prognostic or predictive biomarker of cancer immunotherapies targeting NY-ESO-1, and possibly other cancer-testis antigens.

\section{P59}

Multi-color flow cytometry comparative analysis with DA-Cell(TM)

centrifugation-less washing

Xavier Le Guezennec (sheila@curiox.com)

Curiox Biosystems, San Carlos, CA, USA

Journal for ImmunoTherapy of Cancer 2017, 5(Suppl 2):P59

\section{Background}

Sample preparation for multi-color flow cytometry analysis requires staining of suspension cells with a mix of fluorescently labelled antibodies. This preparation requires the need to centrifuge and pack cells multiple times in order to wash away the excess of fluorescent antibody unbound to cells. Here, we explore a novel way to stain cell suspension by using wall less DropArray and Laminar flow wash properties of DA-Cell.

Using PBMC samples stained with various multi-color panels of 6,11 and 21 antibodies, standard centrifugation or DA-Cell wash method was performed before acquisition with flow cytometry. We then subjected multi-parametric acquired flow data to unbiased clustering analysis with Cytofkit Bioconductor package.

Clustering Analysis and flow cytometry raw acquisition revealed improved segregation of surface markers intensity and cell population when DA-Cell wash method was used as compared to standard centrifugation method. Human annotation of multiple immune cell population type was also facilitated with DA-Cell wash method as compared to standard centrifugation wash method based on stochastic non-linear embedding (T-SNE) clustering map results. DA-Cell method led in unbiased analysis to identification of CD4 CD57 cells and switch memory B cells absent from standard centrifugation wash method.

The superior separation and identification of cell population with DACell offers a new perspective to study various rare immune cell populations.

Methods

Using PBMC samples stained with various multi-color panels of 6,11 and 21 antibodies, standard centrifugation or DA-Cell wash method was performed before acquisition with flow cytometry. We then subjected multi-parametric acquired flow data to unbiased clustering analysis with Cytofkit Bioconductor package.

Results

Clustering Analysis and flow cytometry raw acquisition revealed improved segregation of surface markers intensity and cell population when DA-Cell wash method was used as compared to standard centrifugation method. Human annotation of multiple immune cell population type was also facilitated with DA-Cell wash method as compared to standard centrifugation wash method based on stochastic non-linear embedding (T-SNE) clustering map results. DA-Cell method led in unbiased analysis to identification of CD4 CD57 cells and switch memory $B$ cells absent from standard centrifugation wash method.

The superior separation and identification of cell population with DA-Cell offers a new perspective to study various rare immune cell populations. 


\section{Conclusions}

The superior separation and identification of cell population with DACell offers a new perspective to study various rare immune cell populations.

Consent to publish

Consent granted.

\section{P60}

Comparative study of flow cytometry analysis between conventional centrifuge method and centrifuge-less DA-Cell ${ }^{\mathrm{TM}}$ method from the perspective of cell retention

Xavier Le Guezennec (sheila@curiox.com)

Curiox Biosystems, San Carlos, CA, USA

Journal for ImmunoTherapy of Cancer 2017, 5(Suppl 2):P60

\section{Background}

The preparation of cell suspension samples for flow cytometry commonly involves staining of cells with a specific fluorescent marker for detection of a cell population or a cell phenotype. For over half a century this standard preparation has required the need to centrifuge and pack cells at $500 \mathrm{~g}$ for multiple times in order to wash away the excess of fluorescent marker unbound to cells. This "worldwide standard" remains however, inherently loses a significant portion of the cell population, particularly when cells are fixed and permeabilized. The biggest challenge imposed by the loss of cells is a wellknown belief and intuition that the loss of cells varies depending on cell types and processing. The biggest challenge imposed by the loss of cells is a well-known belief and intuition that the loss of cells varies depending on cell types and processing.

Methods

Here, we present a new convenient methodology to wash suspension cells based on unique laminar flow properties of DropArray plate technology DA-Cell. This technology offers after multiple wash more than $95 \%$ retention of millions of suspension cells and uses natural gravity without cells packing. The cell retention of $95 \%$ can further increase when incubation time of cells and reagents increases by $10-20 \mathrm{~min}$ as the slight increase of the incubation time helps precipitating cells onto a plate surface by gravity. As an automated method DA-Cell wash is performed conveniently and consistently in 2-4 minutes as compared to a common 20-40 minutes requirement with a centrifugation wash based method.

Results

Application of this new technology on various sample preparation for flow cytometry analysis showed improvements in cell viability, segregation of cell population and detection of rare immune cell population when compared to conventional sample preparation. Various examples of multi-color flow cytometry analysis will be presented to demonstrate the performance of this new technology.

\section{Conclusions}

With minimal hands on time, DA-Cell technology enables researchers to conduct complex multi-parametric flow cytometry cell assays without the cellular stress or long time associated with centrifugation based procedures.

Consent to publish

Consent granted.

\section{P61}

Cellular and genomic disease signature of peripheral blood mononuclear cells in patients with malignant pleural mesothelioma

Zachary R. Mallon', Christophe Poulet ${ }^{1}$, Amanda Enstrom',

Somayeh Honarmand', Aimee L. Murphy', Raffit Hassan²,

Dirk G. Brockstedt ${ }^{1}$, Chan C. Whiting ${ }^{1}$, Nitya Nair ${ }^{1}$

${ }^{1}$ Aduro Biotech, Inc., Berkeley, CA, USA; ${ }^{2}$ National Cancer Institute, Bethesda, MD, USA

Correspondence: Nitya Nair (zmallon@aduro.com)

Journal for ImmunoTherapy of Cancer 2017, 5(Suppl 2):P61

\section{Background}

Recent data on the incidence malignant pleural mesothelioma (MPM) and the continued large-scale use of asbestos throughout the developing world portends an epidemic of asbestos-related disease. MPM is an aggressive and fatal cancer with few treatment options. Recent advances in large scale genomic and high throughput cellular analyzes now provide the tools to more easily attain markers of disease status and potential responsiveness to immunotherapeutics.

Methods

Here we present pre-treatment cellular and genomic biomarker data on a cohort of chemotherapy-naïve MPM patients, and demographically matched healthy donors (HD). MPM patients were enrolled in a Phase $1 \mathrm{~b}$ study utilizing CRS-207, a live, attenuated Listeria monocytogenes strain engineered to express the tumor-associated antigen, mesothelin. Four different multi-color flow cytometry panels were used to provide resolution on major immune cell populations of $T$ cells, $\gamma \delta T$ cells, B cells, dendritic cells, monocytes, and natural killer cells. Together, these panels provided deeper resolution on 39 distinct subpopulations of major immune cell subsets. RNA from these cells was used to perform multiplex gene expression analysis on 770 genes using the Nanostring nCounter PanCancer Pathway Panel.

Results

FACS analysis yielded numerous subpopulations with statistically significant differences between MPM patients and healthy controls. Differences in immune populations were analyzed by median and significant findings included populations of $\mathrm{CD} 4^{+} \mathrm{T}$ cells, $\mathrm{CD} 8^{+} \mathrm{T}$ cells, $B$ cells, classical monocytes, and monocytic myeloid derived suppressor cells. Class comparison and hierarchical clustering of gene expression data revealed genomic markers that were significantly expressed in MPM compared to healthy controls. Immune subset deconvolution of gene expression data provided similar findings as FACS analysis and corroborated this disease signature across experimental platforms.

Conclusions

Understanding a patient's biological disease signature can aide in diagnosis, as well as in making informed choices about therapies amidst the complex and broadening immunotherapeutic landscape. Until recently, existing biomarker data in MPM has been limited to a small number of serological markers and limited immune analysis. Here, we present the first comprehensive report of a MPM disease signature from the cellular and genomic perspectives. Correlation of patient baseline disease signatures with treatment outcome may yield biomarkers predictive of treatment efficacy. Predictive signatures are being investigated in the on-going Phase $1 \mathrm{~b}$ study of CRS207 and chemotherapy, as well as in the Phase 2 study of CRS-207 with pembrolizumab in MPM patients who failed prior treatment.

Trial Registration

ClinicalTrials.gov Identifier NCT01675765; NCT03175172

P62

Circulating T cell subpopulations correlate with immune inflammatory signatures at the tumor site in melanoma and non-squamous non-small cell lung cancer

Nataly Manjarrez-Orduño', Selena Kansal ${ }^{1}$, Laurence Menard',

Sergey Lezhnin ${ }^{2}$, Can Jiang ${ }^{1}$, Paul Kayne ${ }^{1}$, Robin Edwards'

Suzanne Suchard ${ }^{1}$, Paul Fischer ${ }^{1}$, Bijal Kakrecha', Chiang Yu',

Julie Carman', Hongyue Dai ${ }^{2}$, Steven Bernstein', Steven Nadler

${ }^{1}$ Bristol-Myers Squibb, Princeton, NJ, USA; ${ }^{2}$ M2GEN, Tampa, FL, USA

Correspondence: Nataly Manjarrez-Orduño

(nataly.manjarrez@gmail.com)

Journal for ImmunoTherapy of Cancer 2017, 5(Suppl 2):P62

\section{Background}

Checkpoint inhibitors have transformed cancer therapy, although outcomes can be variable. Response to anti-PD1 therapies depends in part on tumor type and characteristics, particularly its mutation burden, as well as pre-existing antitumor immunity. These parameters may constitute the basis for patient stratification strategies for 
several immuno-oncology therapies. This analysis aimed to characterize the relationship between the local immune environment at the tumor site and peripheral immunity in melanoma and nonsquamous non-small cell lung cancer.

\section{Methods}

Melanoma $(n=42)$ and non-squamous non-small cell lung $(n=40)$ FFPE tumor biopsies and peripheral blood mononuclear cells (PBMC) were provided by the Moffitt Cancer Center. All patients were appropriately consented through Total Cancer Care (TCC) protocol. None of these patients had received treatment with checkpoint inhibitors. For local immune responses, we used gene expression data in FFPE tissues to calculate inflammatory signature scores in the tumors [1]. For systemic responses, we performed flow cytometry for T cell subpopulations in PBMC. Control PBMC $(n=27)$ were obtained from the BMS employee volunteer blood donation program.

Results

We observed that there are distinct circulating $T$ cell maturation patterns that distinguish between control samples and those belonging to patients with the studied tumors. We detected particular circulating $\mathrm{T}$ cell profiles in patients with melanoma and non-squamous lung cancer who have an inflammatory milieu in the tumor as determined by inflammatory signature score.

\section{Conclusions}

These findings represent progress in the characterization of peripheral immunity, as it relates to the inflammatory immune status in the tumor. The results also provide potential approaches to measure the immune response which are more easily accessible compared to tumor biopsies.

\section{Reference}

1. Spranger, Bao, Gajewski. Melanoma-intrinsic beta-catenin signaling prevents anti-tumor immunity. Nature. 2015;523: 231-235.

\section{P63}

Measurement of the immune-suppressor nitric oxide within immune cell subsets in patients receiving ipilimumab with a peptide vaccine

Isaac P. Foote', Zhihua Chen'1, Y. Ann Chen'1, Adam W. Mailloux'1, Alisha Agrawal', Braydon J. Schaible', Amod Sarnaik', Jeffrey S. Weber', Anders E. Berglund', James J. Mulé', Joseph Markowitz ${ }^{1}$

${ }^{1}$ Moffitt Cancer Center, Tampa, FL, USA; ${ }^{2}$ NYU, New York, NY, USA

Correspondence: Joseph Markowitz (joseph.markowitz@moffitt.org)

Journal for ImmunoTherapy of Cancer 2017, 5(Suppl 2):P63

\section{Background}

Biomarkers are unavailable to accurately predict clinical response to checkpoint blockade therapy. We employed the SPADE (spanningtree progression analysis of density-normalized events) algorithm to precisely cluster immune cell phenotypes. Because phenotypic identification is laborious (Qiu et al., 2011, Nature Biotechnology, 29(10):887-891), we developed a novel algorithm to address this challenge. We explored whether nitric oxide levels are differentially expressed in distinct immune cell subsets before and after checkpoint blockade therapy, which may serve as a reliable biomarker.

\section{Methods}

Patients with resected stage III/IV melanoma were treated with ipilimumab plus a peptide vaccine. Pre and post treatment peripheral blood mononuclear cells (PBMCs) at week 13 were available for analyzes 44 patients pre-treatment samples, 35 patients had both pre/ post treatment samples; Sarnaik et al., 2011, 17(4):896-906). Two flow cytometry panels (100,000 live cell events; LSR II flow cytometer) were constructed to compare PBMCs collected pre and post ipilimumab/vaccine treatment (Myeloid panel: nitric oxide stain (DAF-FM), HLADR, CD33, CD11b, CD14, CD15, and CD11c; Lymphoid panel: DAF-FM, CD3, CD4, CD8, CD25, CD127, CD56, CD19, and CD11c). Controls included: flow cytometric compensation beads to establish robust compensation matrices, fluorescence minus one controls to set negative and positive gates, isotype controls to control for patient variations, and a live/dead marker. SPADE was utilized to cluster and differentiate cell populations based on marker expression, and then a novel algorithm, MPAT-R (multi-parameter phenotyping analysis tool in R) was developed to quickly determine cell phenotypes in the SPADE tree.

Results

By the MPAT-R algorithm, 58/200 lymphoid and $116 / 200$ myeloid phenotypes were differentially expressed after ipilimumab/vaccine treatment (Wilcoxon signed-rank test, False Discovery Rate (FDR) <0.05). Ten myeloid and 7 lymphoid phenotypes post-treatment trended with relapse-free survival (RFS) at one year $(p<0.05$, FDR $>0.05$, Wilcoxon rank-sum test). Two lymphoid and myeloid populations pre-treatment trended with RFS at one year (Cox regression, Wilcoxon rank-sum test, $\mathrm{p}<0.05$, FDR $>0.05$ ). Two interesting populations associated with increased RFS were: a) Monocytic myeloid cells without NO $\left(\mathrm{HLADR}^{+} \mathrm{CD} 33^{+/ \text {low }} \mathrm{CD} 11 \mathrm{~b}^{+} \mathrm{CD} 14^{+/ \text {low }} \mathrm{DAF}-\mathrm{FM}^{\text {neg }}\right)$ and b) naïve/central memory $T$ cells containing intermediate levels of $\mathrm{NO}\left(\mathrm{CD} 8^{+} \mathrm{CD} 127^{+} \mathrm{CD} 25\right.$

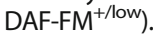

Conclusions

To our knowledge, this is the first application of SPADE to pre/post clinical immunotherapy PBMC samples. The MPAT-R algorithm efficiently detected immune cell phenotypes associated with increased RFS as potential biomarkers for immune checkpoint blockade therapy with ipilimumab. Future studies will focus on the potential immunosuppressive role of NO identified in precise immune cell subsets both in the circulation and in the tumor microenvironment.

P64

Analytical validation of digital spatial profiling - a novel approach for multiplexed characterization of protein distribution and abundance in FFPE tissue sections

Kristi Barker, Heather Metz, Chris Merritt, Lucas Dennis, Philippa Webster, Joseph Beechem

NanoString Technologies, Inc., Seattle, WA, USA

Correspondence: Chris Merritt (cmerritt@nanostring.com)

Journal for ImmunoTherapy of Cancer 2017, 5(Suppl 2):P64

\section{Background}

Characterization of the spatial distribution and abundance of key proteins within tissues enables a better understanding of biological systems in many research areas, including immunology and oncology. However, it has proven difficult to perform such studies in a highly-multiplexed manner on FFPE tissue sections. To address this unmet need, we have developed a novel imaging platform, Digital Spatial Profiling (DSP), designed to simultaneously analyze 10's to 100 's of proteins from discrete regions by detecting oligos conjugated to antibodies that can be released via a UV-cleavable linker. Here we describe the validation of the oligo-conjugated antibodies used with this new approach to highly multiplexed protein analysis. Methods

Various antibodies targeting important immuno-oncology proteins (CD3, CD4, CD8, CD45, PD1, PD-L1, etc.) were tested for specificity and sensitivity. Immunohistochemisty was performed on FFPE human tissues including tonsil and tumor samples, as well as human cell line pellets to evaluate binding specificity in both unconjugated and oligo-conjugated antibodies. The sensitivity and dynamic range of oligo-conjugated antibodies were tested using FFPE cell pellets with target-specific positive and negative cells at different ratios $(0: 100$, 5:95, 20:80, 50:50, 100:0) and specific limits-of-detection (LODs) were determined. An interaction screen was performed to evaluate potential deleterious effects of multiplexing antibodies. Finally, a human tissue microarray (TMA) containing normal and cancer tissues was employed to assess assay robustness.

Results

Immunohistochemical analysis of antibodies integrated into the cocktail displayed indistinguishable staining patterns on control tissues and cell lines for both unconjugated and oligo-conjugated antibodies. Mixed-proportion cell pellet assays revealed strong 
correlations between observed counts above background and positive cell numbers in a region of interest, allowing us to accurately calculate LODs. For example, CD3 displayed a LOD of $4 \%$ when assayed using a cell pellet mixture containing increasing numbers of $\mathrm{CD}^{+}$ CCRF-CEM cells with CD3- HEK293T cells. Antibody interaction studies showed similar count values for antibodies alone or in combination $\left(R^{2}>0.8\right)$. Finally, TMA hierarchical clustering analysis demonstrated expected patterns for immune cells and tumor cells across normal and disease tissue types.

\section{Conclusions}

These results demonstrate that indexing oligo conjugation does not interfere with antibody specificity and that these conjugated antibodies are robust reagents for quantification of protein abundance. Continued work on the DSP platform will expand the library of antibodies accessible for profiling.

\section{P65}

Spatially-resolved, multiplexed digital characterization of protein abundance in FFPE tissue sections: application in preclinical mouse models

Yan Liang, Alison VanSchoiack, JingJing Gong, Chris Merritt ${ }^{1}$

Dwayne Dunaway, Jaemyeong Jung, Isaac Sprague, Sarah Warren, Joseph Beechem

NanoString Technologies Inc., Seattle, WA, USA

Correspondence: Yan Liang (cmerritt@nanostring.com)

Journal for ImmunoTherapy of Cancer 2017, 5(Suppl 2):P65

\section{Background}

Characterization of the abundance, distribution, and colocalization of key immunoregulatory proteins within the tumor microenvironment is necessary for a thorough understanding of tumor immune responses. Historically, immunohistochemistry and immunofluorescence have been used to assess spatial heterogeneity of proteins in tissue slices, but these techniques are of limited utility due to the challenge of measuring multiple targets in parallel. We recently developed a platform to enable spatially-resolved protein detection with the potential to simultaneously quantify up to 800 targets from a single formalin-fixed paraffin-embedded (FFPE) sample slide, termed Digital Spatial Profiling (DSP). To demonstrate preclinical applications of DSP, we have developed an assay to detect and quantify 20 key immuno-oncology (IO) targets in mouse FFPE tissue sections.

Methods

DSP uses DNA oligo tags covalently linked to detection reagents (primary antibodies) via a UV photocleavable linker to identify targets in situ and enable quantitation via the standard $n$ Counter ${ }^{\circledast}$ technology. A slide-mounted FFPE tissue section is incubated with a cocktail of oligo-labeled primary antibodies, and a serial section is stained with low-plex visible/fluorescent probes (e.g. nuclear staining probes, or select antibody pairs such as anti-CD3) to generate an image of the FFPE tissue slice morphology. Regions of interest (ROI) in the tissue/ tumor are then identified and sequentially illuminated with UV light to release the DNA-oligos. Following UV illumination, the photocleaved oligos are released into the aqueous layer above the tissue slice, collected via microcapillary aspiration, and stored in an individual well of a microtiter plate. Oligos are then hybridized to nCounter optical barcodes to permit ex-situ digital counting of as many as 800 different analytes localized within a single ROI, which can be referenced using image capture software.

Results

We demonstrate preclinical applications of this technology by characterization of a panel of immune proteins on mouse FFPE tumor and normal tissue sections. We demonstrate that this approach enables protein detection at single cell resolution, and enables simultaneous multiplexed detection of AKT, B7-H3, Beta-2M,
Beta-Catenin, CD3, CD4, CD8, CD45, CD68, PD-1, PD-L1, GZMB, Ki67, pan-cytokeratin, Stat3 and additional key IO targets.

Conclusions

The ability to measure DNA, RNA, and protein at up-to 800-plex from single slices of FFPE tissue may improve the early evaluation of drug targets with high-resolution spatial information, enable the discovery of key immune biomarkers in mouse tissue (tumors and inflammation et al), and accelerate the preclinical development of immunotherapies.

\section{P66}

Digital spatial profiling platform allows both spatially-resolved, multiplexed measurement of solid tumor and immune-associated protein distribution and abundance using a single FFPE tissue section

Youngmi Kim, Chris Merritt, Giang Ong, Kristi Barker, Jaemyeong Jung, Isaac Sprague, Yan Liang, Sarah Warren, Philippa Webster,

Dwayne Dunaway, Joseph Beechem

NanoString Technologies Inc., Seattle, WA, USA

Correspondence: Youngmi Kim (cmerritt@nanostring.com)

Journal for ImmunoTherapy of Cancer 2017, 5(Suppl 2):P66

\section{Background}

Spatial characterization of immune activations and tumor proteins within tumor tissues enables a better understanding of immunology and oncology. However, it has proven difficult to perform such studies in a highly multiplexed manner using limited samples. To address this unmet need, we have developed an imaging and tissuesampling platform designed to simultaneously analyze hundreds of tumor and immune proteins in a single FFPE tissue section with spatial resolution. Using this approach, we demonstrate that a single FFPE tissue section is enough to obtain multi-dimension data sets, such as solid tumor protein expression, immune activity, and cancer pathways with an emphasis on markers important for cancer immunotherapy.

Methods

We developed a novel optical-barcode based microscope that can spatially resolve up to 800 different proteins or RNAs on a fixed tissue (Digital Spatial Profiling, DSP). DSP probes are not multiplexlimited by spectral resolution. Instead, colors are determined using barcode indexing oligos that are conjugated to antibodies or nucleicacid probes via UV-cleavable linkers. The UV-cleavage is precisely controlled by a programmable digital micromirror device that can illuminate discrete regions ranging from entire tissue microenvironments to single-cell and subcellular regions. Following spatiallydefined UV light exposure, released indexing oligos are siphoned off the tissue surface via a microcapillary tip. For quantification of signal, the UV-cleaved oligos from spatially-resolved regions of interest (ROIs) are hybridized to NanoString barcodes, providing digital counts of the protein or RNA targets in each ROI using standard NanoString nCounter ${ }^{\circledast}$ instruments.

Results

Using this novel approach, we demonstrate multiplexed detection from discrete micro regions within a tumor enables systematic interrogation of tumor protein expression and immune activity inner or outer regions of tumors. In addition, the whole tissue analysis allows us to characterize and link global distribution of tumor protein expression, immune activity and cancer pathways.

Conclusions

The simplicity of the DSP platform allows high-resolution, highmultiplexed, spatially-resolved tumor and immune protein characterization in any laboratory capable of performing immunohistochemistry procedures. DSP platform provides a potential method that can bridge the gap between translational research discovery and clinical applications. 
P67

Standardization of tumor infiltrating lymphocytes isolation for flow cytometric analysis

William Miller, Yoshinobu Koguchi, William Redmond

Earle A. Chiles Research Institute, Portland, OR, USA

Correspondence: William Redmond (wmgaj.miller@gmail.com)

Journal for ImmunoTherapy of Cancer 2017, 5(Suppl 2):P67

\section{Background}

Cancer immunotherapy has become a mainstay of cancer treatment. These agents can target the tumor/lymphocyte interaction in the tumor microenvironment. Therefore, analysis of tumor infiltrating lymphocytes (TIL) needs to be part of comprehensive immunomonitoring in patients receiving immunotherapy. There are two major approaches to isolate TIL, enzymatic digestion and mechanical dissociation. While enzymatic digestion may give better yields, it potentially destroys epitopes. In contrast, mechanical digestion may preserve epitopes, but can suffer from poor yields and reduced viability of TIL. As standardization of TIL isolation for flow cytometry is key to accurate and precise phenotyping of TIL populations, we compared these different approaches for cell yield, epitope preservation and viability.

Methods

Three different isolation techniques were tested: 1) Miltenyi tumor dissociation kit, 2) enzymatic digest, and 3) mechanical isolation. Results

The Milenyi protocol and lab prepared enzymatic digests delivered higher yield of TIL from tumor, but proteases in the enzyme mix cleaved proteins and chemokine receptors on the cell surface of $T$ cells. Mechanical dissociation preserved the integrity of the surface antigens and chemokine receptors, but negatively impacted the ability to breakdown the the tumor matrix and release TIL populations into a homogenous single cell suspension. A combination of mechanical dissociation through sieves and 15 minutes enzymatic digest buffered with HSA at room temperature yielded high recovery of Tregs, retention of surface markers and chemokine receptors and good viability.

Conclusions

The combination of enzymatic digestion and mechanical dissociation provided the best result. Due to small tumor size, frequently $<1 \mathrm{~g}$, and high heterogeneity from tumor to tumor, it is challenging to validate TIL isolation methods for reproducibility and precision. Therefore, we are evaluating a surrogate system using mouse tumors, which allows us to directly compare methods and test proficiency of technologists.

\section{P68}

Immunogenomic evolution and atypical response to atezolizumab in a patient with metastatic triple-negative breast cancer (mTNBC) Luciana Molinero', Yijin Li', Ching-Wei Chang', Sophia Maund', Maureen Berg $^{2}$, Jeanne Harrison ${ }^{2}$, Marcella Fassò ${ }^{1}$, Carol O'Hear ${ }^{1}$, Priti S. Hegde', Leisha A. Emens ${ }^{3}$

${ }^{1}$ Genentech, Inc., South San Francisco, CA, USA; ${ }^{2}$ Johns Hopkins School of Medicine, Baltimore, MD, USA; ${ }^{3}$ Johns Hopkins University School of Medicine, Baltimore, MD, USA

Correspondence: Leisha A. Emens (molinero.luciana@gene.com) Journal for ImmunoTherapy of Cancer 2017, 5(Suppl 2):P68

\section{Background}

mTNBC has poor prognosis and few treatment options. Atezolizumab (anti-PD-L1) demonstrated clinical activity in MTNBC, which has been linked to high levels of tumor-infiltrating lymphocytes (TILs) and PDL1 expression. We describe the immunologic and genomic evolution of TNBC across sequential therapies in a patient with a 31-year history of TNBC and complete response (CR) to atezolizumab.

Methods

A 48-year-old woman enrolled in the Phase la atezolizumab study (PCD4989g) on March 5, 2013. She presented in 1986 with early TNBC and had surgery and radiotherapy (XRT). Two locoregional re- currences were managed with surgery and adjuvant chemotherapy. In 2009, she was diagnosed with MTNBC and was sequentially treated with capecitabine, gemcitabine-carboplatin-iniparib (GCl), XRT and an experimental vaccine. She then received atezolizumab for 1 year with partial response (PR), pseudoprogression and reversion to PR. After 1 year off-treatment, she progressed and reinitiated atezolizumab, achieving a CR. Ten tumor tissues obtained between 2008-2015 were assessed by immunohistochemistry, RNA-seq (Illumina) and DNA-seq (FoundationOne).

Results

Tumor-immune microenvironment (TiME) biomarkers, including CD8, TILs and PD-L1, increased after capecitabine exposure, remaining high after $\mathrm{GCl}$ and XRT and through pseudoprogression on atezolizumab. At relapse post-atezolizumab, expression of TiME biomarkers decreased, particularly PD-L1. Immune-related RNA signatures (T and $B$ cells, cytotoxicity and antigen presentation) confirmed these findings. Angiogenesis signature varied over time and was highest at early time points, lower post-capecitabine, higher before $\mathrm{GCl}$, lowest at pseudoprogression and increased at relapse post-atezolizumab. Proliferation signatures were highest in early tumors, decreasing over time. TNBC subtyping confirmed evolution of TiME from luminal androgen receptor (LAR) to basal-like immune suppressed (BLIS), then to basal-like immune active (BLIA) post-capecitabine and through pseudoprogression, with reversion to BLIS at relapse postatezolizumab. Genomic profiling suggested possible driver mutations in RB1 and TP53. MYC amplification was lost over time, possibly associated with decay of the proliferation signature. The incidence of subclonal somatic mutations peaked post-XRT and were undetectable post-atezolizumab. Likewise, tumor mutational burden was highest post-XRT and lowest at pseudoprogression.

Conclusions

This case report describes the evolution of tumor immunity and molecular subtypes with sequential therapies over time in a patient with TNBC and CR to atezolizumab. Immune, stromal and genomic biomarkers of TiME demonstrated temporal plasticity across multiple therapies, including atezolizumab. These findings suggest that TiME is pliable and may be strategically manipulated to maximize response to immunotherapy.

Trial Registration

ClinicalTrials.gov Identifier NCT01375842

Consent to publish

Written informed consent was obtained from the patient for this publication. A copy of written consent is available upon request.

P69

A pan-cancer diagnostic, prognostic and targetable biomarker pipeline defining the failed immune-response

Anne Monette ${ }^{1,2}$, Antigoni Morou ${ }^{1,2}$, Louise Rousseau ${ }^{2}$, Jean-Baptiste Lattouf', Daniel Kaufmann ${ }^{1}$, Igor Jurisica ${ }^{3}$, Rejean Lapointe ${ }^{1,2}$

${ }^{1}$ University of Montreal, Montreal, QC, Canada; ${ }^{2} \mathrm{CRCHUM}$, Montreal, QC,

Canada; ${ }^{3}$ University of Toronto/7Princess Margaret Cancer Centre and Techna, Toronto, ON, Canada

Correspondence: Anne Monette (anne.monette@mail.mcgill.ca) Journal for ImmunoTherapy of Cancer 2017, 5(Suppl 2):P69

\section{Background}

Tumor infiltrating lymphocytes (TILs) represent positive prognostic and predictive indicators of patient outcomes. Their signature genes are, however, still under tight control by the overwhelming tumor microenvironment. Therefore, despite many of these genes reflecting effector $\mathrm{T}$ cell subsets, their totalities actually belong to a failed immune response (FIR) against cancer. This was best first exemplified by association of renal cell carcinoma (RCC) TILs with poor prognosis, yet was later also validated to be a reversible TIL condition by use of immune-checkpoint modulators. Since circulating $T$ cells can share TIL tumor-reactivity and neoantigen-specificity, we used profiles of circulating RCC CD8 ${ }^{+}$and $\mathrm{CD}_{1} 9^{+}$lymphocytes and their paired TILs and normal tissue infiltrating immune cell (TIIC) counterparts as a model of a FIR signature. Commonalties of this signature were ob- 
served in several other solid cancers and represents a diagnostic, prognostic, and targetable biomarker model pipeline defining novel and feasible immunotherapeutic targets.

Methods

A rapid extraction protocol isolated RNA from $\mathrm{CD}^{+}$effector and CD $19^{+}$antigen presenting cells (APC) isolated from paired TILs, TIICs and PBMCs, and matched normal donor PBMCs. Microarray analysis interrogating the expression of all genes and RNA isoforms, was followed by bioinformatics probing an additional 500 RCC validation cohort. This provided prognostic linkage to biomarker expression, revealing novel antagonistic and agonistic targets, which were refined using clinical datasets from 11,000 additional patients with lung, breast, gastric, and ovarian cancers. Feasible novel immunepromoting targets exclusively expressed by immune cells, and in the context of cancer, included receptors and plasma membraneassociated proteins, or those having pre-existing targeting small molecules. These were further investigated for protein-protein interaction (PPI) and associated pathways, coexpression, and comprehensive literature reviews establishing relevant immune-cell function.

Results

Validation of original hits from the discovery cohort provided strong evidence of which cancers were most related at the TIL level, and PPI revealed that pan-cancer targets were functionally associated in novel pathways $(p=1.85 \mathrm{e}-10)$. Coexpression dynamics reflected key proteins responsible for APC:effector TIL communication, and results also showed that lung and breast cancer TILs and circulating PBMCs were most similar to other cancers, and that a FIR is skewed towards a diminished expression of genes otherwise expressed by the healthy population.

Conclusions

We present a strict, comprehensive pipeline defining a pan-cancer targetable immune-signature having many pre-existing and tested targeting small-molecules, in an effort to develop more feasible and broad reaching diagnostics and precision medicine to counter cancer, foiled by the surge of bystander TIL-signatures.

\section{P70}

Halioseek $^{\mathrm{TM}}$, a dual CD8 and PD-L1 IVD assay to improve NSCLC patients stratification

Florence Monville', Emmanuel Prestat ${ }^{1}$, Laurent Vanhille', Julien Adam² Sergiu Coslet ${ }^{3}$, Caroline Laugé ${ }^{\prime}$, Caroline Davin ${ }^{1}$, Merle Sophie Losch', Luciana Batista ${ }^{1}$, Jacques Fieschi ${ }^{1}$

${ }^{1}$ HalioDx, Marseille, France; ${ }^{2}$ Gustave Roussy Cancer Campus, Villejuif, France; ${ }^{3} \mathrm{MlmAbs}$, Marseille, France

Correspondence: Florence Monville (fabienne.hermitte@haliodx.com) Journal for ImmunoTherapy of Cancer 2017, 5(Suppl 2):P70

\section{Background}

Immune checkpoint inhibitors (ICI) improved non-small cell lung cancer (NSCLC) survival in $20 \%$ of the patients. Their administration is decided based on the expression level of PD-L1 on tumor cells despite limited predictive value of PD-L1 alone. We hypothesized that $\mathrm{ICl}$ action depends on both PD-L1 expression and on the presence of tumor infiltrating lymphocytes (TILs) in the tumor microenvironment. We developed Halioseek ${ }^{\mathrm{TM}}$ according to IVD regulatory processes, a standardized PD-L1/CD8 dual-staining assay. In addition to PD-L1 detection, critical information is provided on TILs through the detection of CD8+ cells on the same tissue section. Halioseek ${ }^{\text {TM }}$ includes a Digital Pathology (DP) analysis module to determine CD8+ cell density and a proximity index between CD8+ and PD-L1+ cells. Here we show the main analytical performance of Halioseek $^{\mathrm{TM}}$ and concordance with two PD-L1 IVD assays.

Methods

Samples are double-stained with anti-PD-L1 (clone HDX3) and antiCD8 (clone HDX1) antibodies, respectively revealed with DAB and fast-red substrates. The assay is fully automated on the Benchmark
XT platform (Ventana). Digital images of stained slides are obtained using a whole slide scanner (Hamamatsu). Percentage of PD-L1 positive tumor cells (PD-L1+ TC) is estimated by a pathologist; DP analysis is performed using Halioseek ${ }^{\mathrm{TM}}$ DP module.

Staining accuracy of PD-L1 by HDX3 antibody was evaluated by comparison with SP263 IVD assay (Ventana) and 22C3 IVD assay (Dako) on 100+ NSCLC including resections and biopsies. Overall, Positive percent and negative percent agreements were assessed at 1\%, 5\%, $10 \%$ and $50 \%$ of PD-L1+ TC corresponding to published clinical cut-offs.

The precision of PD-L1 assay was evaluated in terms of repeatability, inter-primary antibody batches (3 lots), inter-revelation kit batches (3 lots) and inter-instruments (2 stainers) using 332 slides from 11 independent NSCLC samples covering the range of the assay.

Results

- PD-L1 staining accuracy: overall agreements with SP263 were above $90 \%$ and with 22 C3 above $85 \%$.

- PD-L1 precision: none of the tested parameters had a significant impact on results showing the robustness of the assay.

- CD8+ cell density: distribution spanned between 30 and 1200 cells $/ \mathrm{mm}^{2}$; global CV was below $13 \%$.

\section{Conclusions}

Halioseek $^{\mathrm{TM}}$ is a new robust IVD assay leveraging the advantages of DP to combine TILs and PD-L1 quantification within the tumor microenvironment. Halioseek ${ }^{\mathrm{TM}}$ could have a higher predictive performance than existing IVD tests and could fill a major gap in the management of $\mathrm{ICl}$ administration. As next step we intend to investigate predictive performance of the assay on $\mathrm{ICl}$ treated patient's samples.

\section{P71}

Peptide-induced whole blood gene expression analysis for peptide-specific $T$ cell response

Taku Murakami ${ }^{1}$, Keiji Dohi ${ }^{2}$, Kenji Kuwabara², Mieko Ogura',

Masato Mitsuhashi

${ }^{1}$ Hitachi Chemical Co. America, Ltd., Irvine, CA, USA; ${ }^{2}$ Shionogi \& Co., Ltd., Osaka, Japan

Correspondence: Taku Murakami (tmurakami@hitachi-chemical.com) Journal for ImmunoTherapy of Cancer 2017, 5(Suppl 2):P71

\section{Background}

Peptide-based cancer vaccine is a promising approach in cancer immunotherapy. To monitor the efficacy of therapy, a simple tool to monitor specific $\mathrm{T}$ cell response to peptide vaccine is in need. Although MHC multimer and Elispot have been used frequently in various vaccine studies, the former requires specialized reagents and instrument and the latter requires long incubation and imaging instrument.

Methods

Whole blood ex vivo peptide stimulation assay was developed to investigate peptide-specific T cell responses. A $60 \mu \mathrm{L}$ heparin blood was incubated in triplicate with class I peptide for 4 hours at $37^{\circ} \mathrm{C}$ to form a complex among major histocompatibility complex (MHC) peptide - T cell receptor (TCR). Following the incubation, mRNA was isolated and quantified by real-time RT-qPCR. Fold increase of mRNA was calculated against solvent control in triplicate.

Results

We identified that IFNG, CXCL10 and GMCSF mRNA were significantly induced (fold increase $>2, p$ value $<0.05$ ) by CMV peptide in 4,4 and 2 out of the 8 healthy donors, respectively. IFN-gamma Elispot assay confirmed at least 7 of the subjects were positive following 2-week in vitro sensitization. On the other hand, these genes were not induced by HIV peptide, which was corroborated by Elispot with in vitro sensitization. To compare the assay performance with Elispot, 
0 to 300 cells of CMV-peptide specific cytotoxic T-lymphocyte (CTL) were spiked in $1 \times 10^{5}$ cells of PBMC and assayed by both methods. The limit of detection was less than 3 to 10 cells for both assays and a linear correlation between mRNA quantity and Elispot count was obtained (Pearson correlation; IFNG, -0.942 $\left(\mathrm{p}=4.9 \times 10^{-6}\right)$, CXCL10, $-0.690\left(p=1.3 \times 10^{-2}\right)$, and GMCSF -0.888 $\left.\left(p=1.1 \times 10^{-4}\right)\right)$. In a study with 12 samples of whole blood blindly spiked with peptide-specific CTL at 2.5 to $12.5 \times 10^{3}$ cells $/ \mathrm{mL}$, the assay sensitivity and specificity of each mRNA were $17 \%$ and $83 \%$ for IFNG, $100 \%$ and $100 \%$ for CXCL10, and $100 \%$ and $33 \%$ for GMCSF. These data suggest that these markers especially CXCL10 could be a useful marker to monitor peptide-specific T cell response.

\section{Conclusions}

The whole blood mRNA assay developed in this study is useful to measure peptide-specific $T$ cell responses, which could be used to monitor the efficacy of peptide vaccine therapy.

\section{P72}

Analysis of biomarkers from a cohort of advanced melanoma patients previously exposed to immune checkpoint inhibition treated with entinostat (ENT) and pembrolizumab (PEMBRO)

Melissa L. Johnson', Dmitry Gabrilovich², Fang Wang², Rene Gonzalez ${ }^{3}$, Mateusz Opyrchal ${ }^{4}$, David Tamang ${ }^{5}$, Emmett Schmidt ${ }^{6}$,

Michael L. Meyers ${ }^{7}$, Peter Ordentlich ${ }^{5}$, Sanjiv S. Agarwala ${ }^{8}$

'Sarah Cannon Research Institute, Nashville, TN, USA; ${ }^{2}$ The Wistar Institute, Philadelphia, PA, USA; ${ }^{3}$ University of Colorado Comprehensive Cancer Center, Aurora, CO, USA; ${ }^{4}$ Roswell Park Cancer Institute, Buffalo, NY, USA; ${ }^{5}$ Syndax Pharmaceuticals, Inc., Waltham, MA, USA; ${ }^{6}$ Merck \& Co., Inc., Kenilworth, NJ, USA; ${ }^{7}$ Syndax Pharmaceuticals, Inc., New York, NY, USA; ${ }^{8}$ St. Luke's Cancer Center and University Health Network, Easton, PA, USA

Journal for ImmunoTherapy of Cancer 2017, 5(Suppl 2):P72

\section{Background}

Treatment options following immune checkpoint inhibition remain an area of active investigation across multiple cancer indications, including advanced melanoma. Results from the ongoing ENCORE 601 study demonstrated that ENT, a class I selective HDAC inhibitor, combined with PEMBRO elicited a 31\% response rate in a cohort of 13 patients with melanoma progressing on or after a PD-1 blocking antibody. Assessment of blood and tissue-based biomarkers may be useful to understand how patients who respond to ENT plus PEMBRO differ from those who do not.

Methods

ENCORE 601 employs a Simon 2-stage design to assess activity of ENT 5mg PO weekly combined with PEMBRO 200 mg IV every 3 weeks across 4 cohorts: 1) advanced anti-PD-(L)1-naïve NSCLC; 2 ) advanced NSCLC progressed on anti-PD-(L)1;3) advanced melanoma progressed on anti-PD-(L)1; 4) advanced anti-PD-(L)1-naïve microsatellite stable colorectal cancer. Pre-treatment and Cycle 2 Day 15 (C2D15) biomarker analysis included gene expression by Nanostring Pan-Cancer Immune Profiling Panel, PD-L1 expression by immunohistochemistry, and levels of CD8+ T cells and LOX1+CD15+ MDSCs by immunofluorescence staining on paraffin embedded human tissue sections. Phenotypic evaluation of immune cell subsets was conducted in peripheral blood samples collected pre-treatment, C2D1 and C2D15.

Results

All patients enrolled in Stage 1 of the melanoma cohort $(n=13)$ received a prior anti-PD-1 therapy with eight also receiving prior ipilimumab, and two receiving prior BRAF inhibitors. Four patients (31\%) had a partial response (PR; 3 confirmed, 1 unconfirmed), and four additional patients (31\%) had stable disease. Pre-and post-treatment tissue samples were evaluated for PD-L1 expression ( $n=10$ pre; 7 post), gene expression ( $n=7$ pre; 7 post), CD8+ T cells ( $n=9$ pre; 9 post) and MDSCs ( $n=9$ pre; 9 post). In 3 of the 7 patients whose tissue samples were analyzed for gene expression (2 with confirmed PRs, one with progressive disease), non- inflamed tumors at pre-treatment were converted to inflamed. Increases in both T cells and MDSCs were observed only in those tissue samples converted from non-inflamed to inflamed. Overall, regardless of clinical outcome, we noted a decrease in MDSCs $(-35.7 \% ; n=9)$ and an increase in CD8+ T cells $(47.4 \% ; n=9)$ between pre-and post-treatment biopsies.

Conclusions

ENT plus PEMBRO shows promising activity with an overall response rate of $31 \%$ observed in Stage 1. Enrollment to Stage 2 continues. Preliminary biomarker analysis supports our hypothesis that the addition of ENT restores inflammation in the tumor microenvironment necessary for successful re-treatment with an anti-PD-(L)1 blocker.

\section{P73}

First-in-human neoadjuvant study of the immunogenomic impact of the oral IDO inhibitor epacadostat (INCB024360) on the tumor microenvironment of advanced ovarian cancer

Junko Matsuzaki ${ }^{1}$, Amit Lugade', Wiam Bshara', Katherine Collins ${ }^{1}$, Sarah Warren², Patrick Danaher' ${ }^{2}$, Lucas Dennis ${ }^{2}$, Antonios Papanicolau-Sengos' ${ }^{1}$, Leonard D'Amico ${ }^{3}$, Nirasha Ramchurren ${ }^{3}$, Angela Omilian' ${ }^{1}$ Kevin Eng?', Sebastiano Battaglia', Robert Newton ${ }^{4}$, Peggy Scherle ${ }^{4}$, Lance Leopold ${ }^{4}$, Steven Fling ${ }^{3}$, Richard Shine ${ }^{3}$, Martin Cheever ${ }^{3}$, Melissa Geller ${ }^{5}$, Kunle Odunsi ${ }^{1}$

${ }^{1}$ Roswell Park Cancer Institute, Buffalo, NY, USA; ${ }^{2}$ Nanostring, Seattle, WA, USA; ${ }^{3}$ CITN, Seattle, WA, USA; ${ }^{4}$ Incyte Corporation, Wilmington, DE, USA; ${ }^{5}$ University of Minnesota, Minneapolis, MN, USA

Correspondence: Kunle Odunsi (kunle.odunsi@roswellpark.org)

Journal for ImmunoTherapy of Cancer 2017, 5(Suppl 2):P73

\section{Background}

The tryptophan catabolizing enzyme indole-amine 2,3 dioxygenase 1 (IDO1) has been identified as a potent immunosuppressive mechanism that fundamentally alters the ovarian tumor microenvironment (TME) and effector T cell function in ovarian cancer. Epacadostat is a novel oral IDO1 inhibitor that suppresses systemic tryptophan catabolism and is currently being evaluated in ongoing clinical trials. Although the pharmacodynamics (PD) effects of epacadostat in reducing kynurenine concentration in plasma has been demonstrated in pre-clinical and clinical studies, it is currently unknown whether the PD effects will occur at the TME and alter it to become more immunogenic.

Methods

Seventeen patients with newly diagnosed stage III or IV ovarian cancer underwent pre-treatment tumor biopsy and paracentesis. Subjects were scheduled to receive $600 \mathrm{mg}$ epacadostat twice daily for 2 weeks, followed by standard tumor debulking surgery. Peripheral blood and tumor specimens were utilized to assess the phenotype and functional status of multiple immune populations in the TME, define changes in the tumor genomic profile and gene expression by whole-exome sequencing, Nanostring, and RNAseq, TCR clonal evolution, and impact on humoral immune responses to cancer-testis antigens.

Results

Expression profiling identified an upregulated IFNy induced gene signature in a subset of post-treatment tumor specimens, indicative of reactivation of IFNY signaling in the TME. Likewise, in increase in CD8 ${ }^{+} \mathrm{T}$ cells was detected in some, but not all subjects. Of note, IFNy signaling and increases in $\mathrm{CD}^{+}$frequency was not consistently observed in all subjects. Evidence of reduced IDO1 enzyme activity, determined by Kyn:Trp ratio, was observed not only in plasma but also in ascites fluid for a subset of patients ( 5 of 7 evaluable ascites fluid specimens). Although IDO1 activity was blocked, IDO1 expression within the TME was not affected by epacadostat. Mitochondrial spare respiratory capacity of T cells in peripheral blood increased in 8 of 12 patients after treatment. Additional immunogenomic analyzes are underway to identify molecular and metabolic pathways impacted by epacadostat along with determining the clonal evolution of T cells with reactivity to mutational antigens. 


\section{Conclusions}

This is the first demonstration of the effect on tumor tissue of inhibition of IDO1 enzyme activity. IDO1 inhibition by epacadostat resulted in an increase in $\mathrm{CD}^{+} \mathrm{T}$ cell and IFNg gene signatures in the TME. Additional multi-dimensional immunogenomic data from ongoing analyzes will be presented.

Trial Registration

ClinicalTrials.gov Identifier NCT02042430

\section{P74}

Increasing the levels of anti-beta glucan antibodies by administration of intravenous immunoglobulin (IVIG) induces immunopharmacodynamic (IPD) responses of a novel immunotherapeutic Imprime PGG

Nadine Ottoson ${ }^{1}$, Keith Gorden', Blaine Rathmann', Xiaohong Qiu', Ben Harrison', Michele Gargano', Nandita Bose', Gautam Jha², Arkadiusz Dudek $^{3}$

${ }^{1}$ Biothera Pharmaceuticals, Eagan, MN, USA; ${ }^{2}$ The University of Minnesota, Minneapolis, MN, USA; ${ }^{3}$ HealthPartners Institute, St. Paul, MN, USA

Correspondence: Nadine Ottoson (nottoson@biothera.com) Journal for ImmunoTherapy of Cancer 2017, 5(Suppl 2):P74

\section{Background}

There is a critical need for rational combination immunotherapies that have mechanism-driven predictive biomarkers. Imprime PGG (Imprime), is a novel, intravenously (i.v.) administered innate immunomodulator currently in clinical development as a combination therapy with checkpoint inhibitors in biomarker-selected patients. Imprime is a soluble $\beta$-glucan PAMP that requires immune complex formation with serum anti-beta glucan antibodies (ABA) for its functionality. Ex vivo human studies, a healthy volunteer phase I trial and retrospective analyzes of clinical studies have demonstrated that IPD changes and clinical responses mediated by Imprime correlated with serum $A B A$ levels. Ex vivo studies have also shown that innate immune functionality in subjects with lower $A B A$ values can be restored by supplementation with purified ABA or ABA-containing IVIG. Herein we present a case study of a cancer patient with low ABA levels demonstrating enhanced Imprime-induced PD responses post IVIG administration.

Methods

A 54-year-old female with metastatic colorectal adeno-carcinoma was offered immunotherapy with bevacizumab, cetuximab and Imprime as part of a compassionate use program after she could not tolerate first line therapy with FOLFOX and bevacizumab. The patient was dosed in 4 week cycles for 12 cycles. Imprime and cetuximab were administered i.v. weekly. Bevacizumab was administered every 4 weeks from cycle 2 through 8 . Evaluation of serum $A B A$ levels from cycles 1-6 confirmed low values in this patient. To boost ABA levels, IVIG was added to dosing beginning at cycle 6. ABA, complement, and cytokine levels in serum (ELISA and Luminex), Imprime binding and immune cell phenotyping (flow cytometry) were measured prior to and within 30 minutes after Imprime administration.

Results

Compared to the weeks prior to adding IVIG to the dosing regimen, IVIG infusion resulted in increased serum ABA levels at the End of Infusion (EOI), which then dropped to the baseline levels in the subsequent weeks. Concomitant with the ABA increase, serum $\mathrm{C} 5$ a levels peaked at the EOI. Furthermore, serum chemokines such as IL-8 also increased at EOI with the most pronounced change observed during cycles 7 and 8 . Increased ABA levels also correlated with significant Imprime binding on neutrophils and monocytes. Importantly, minimal PD changes were observed with Imprime dosing alone in the cycles prior to IVIG administration. Disease remained stable for 10 months.

\section{Conclusions}

These human data provide the first evidence of rescue of Imprimedriven PD responses in a cancer patient by supplementation of $A B A$, a crucial pre-requisite for the therapeutic activity of Imprime.
P75

iSEND is an algorithmic model specific for advanced non-small cell lung cancer patients treated with PD-1/PD-L1 inhibitors

Wungki Park', Vaia Florou', Sandra Algaze', Diana Saravia',

Deukwoo Kwon ${ }^{1,2}$, Gilberto Lopes ${ }^{1}$

${ }^{1}$ University of Miami Miller School of Medicine, Miami, FL, USA; ${ }^{2}$ Sylvester Comprehensive Cancer Center, Miami, FL, USA

Correspondence: Gilberto Lopes (wungkipark@gmail.com)

Journal for ImmunoTherapy of Cancer 2017, 5(Suppl 2):P75

\section{Background}

We have shown that the iSEND model may be predictive of clinical outcomes for advanced NSCLC (aNSCLC) patients treated with nivolumab but not with chemotherapy or TKI. ${ }^{[1]}$ However, little is known about its potential performance for other advanced solid cancer patients treated with PD-1/PD-L1 inhibitors (PD-1/PD-L1i).

Methods

We evaluated the clinical outcomes of 370 solid cancer patients who received PD-1/PD-L1 $\mathrm{i}$ and compared the performance of iSEND in aNSCLC $(n=203)$ and other advanced solid cancers $(n=167)$. Patients were either treated with nivolumab $(n=237)$, pembrolizumab $(n=109)$, or atezolizumb $(n=24)$. As described in our previous reports, the iSEND model (immunotherapy, Sex, ECOG [Performance status], NLR [Neutrophil-to-Lymphocyte Ratio] \& DNLR [Delta NLR = NLR after treatment - pretreatment NLR]) was developed. We stratified each treatment group by iSEND and compared progression free survivals (PFS) and clinical benefit rates (CBR) at $12+/-2$ weeks in the iSEND Good, Intermediate, and Poor groups.

Results

Median follow-up was 9.1 (95\% Cl: 8.0-10.2). In the aNSCLC, The 3-, 6, 9-, and 12 -months PFS rates were $83 \%, 70 \%, 62 \%$, and $58 \%$ in the iSEND Good group, $71 \%, 40 \%, 29 \%$ and $20 \%$ in Intermediate group, and $60 \%, 18 \%, 18 \%$, and $18 \%$ in Poor group, respectively $(p<0.0001)$. Median PFS was unreached in iSEND Good, 4.2 months; $95 \% \mathrm{Cl}[2.8-$ 5.6] in iSEND Intermediate, and 2.9 months; $95 \% \mathrm{Cl}$ [2.7-3.1] in iSEND Poor groups. (Fig. 1). In contrast in other advanced solid cancers, median PFSs were 5.4, 8.2, and 3.7 months in iSEND Good, Intermediate, and Poor groups, respectively $(p=0.415)$. The area under the curves (AUC) of the iSEND score for CBR at $12+/-2$ weeks for aNSCLC patients treated with PD-1/PD-L1i was 0.720 , (95\% Cl: 0.648-0.793, $p<0.0001)$. The AUCs of iSEND for CBR in other advanced solid cancers was not significant (Fig. 1).

Conclusions

In our single-institution retrospective cohort, the iSEND model showed a predictive potential specifically for advanced NSCLC patients treated with PD-1/PD-L1i but not for other advanced solid cancers treated with PD-1/PD-L1i.

\section{Reference}

1. Saravia D, Laderian B, Park W, Desai A, Vargas F, Elias R, Warsch S,

Mudad R, Ikpeazu C, Ishkanian A, Balfe L, Jahanzeb M. Journal of Thoracic Oncology. 2016; 12(1):S1327.

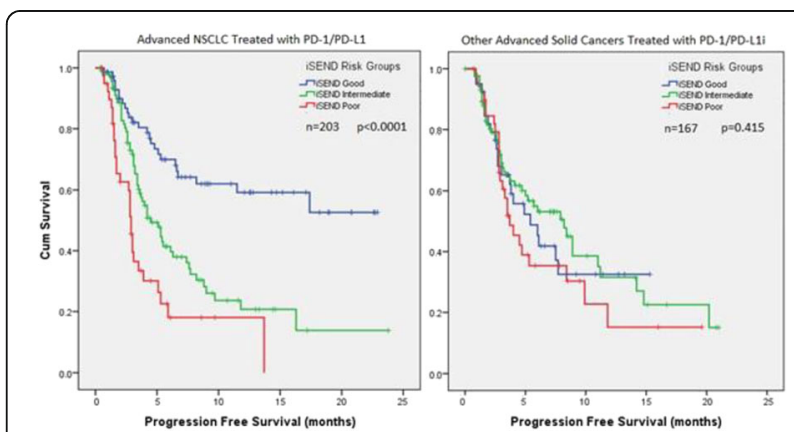

Fig. 1 (abstract P75). Kaplan-Meier curves for PFS in aNSCLC vs. other advanced solid cancers treated with PD-1/PD-L1i 
P76

Usefulness of automated multidimensional flow cytometry analyzes for monitoring the status of T and APC compartments in breast cancer patients under neoadjuvant chemotherapy

David Bernal-Estévez', Carlos Parra-López ${ }^{2}$

${ }^{1}$ Fundación Salud de los Andes, Bogota, Colombia; ${ }^{2}$ Universidad

Nacional de Colombia, Bogota, Colombia

Correspondence: Carlos Parra-López (caparral@unal.edu.co)

Journal for ImmunoTherapy of Cancer 2017, 5(Suppl 2):P76

\section{Background}

Immune monitoring systems based on flow cytometry (FC) are usually analyzed by manual strategies of gating based on twodimensional plots. Recently the forthcoming of new technologies such as CyTOF and advances of multi-parametric FC analyzes demand new approaches to analyze massive immune related data. New algorithms like SPADE, FLOCK, tSNE, and more recently CITRUS, have provided new tools for automated multidimensional FC analysis. These tools have been used mainly for the cellular hierarchy analysis of bone marrow derived cells and in leukemia patients but to a lesser extend for immune-monitoring of patients under antitumor therapy. In this work we explore the usefulness of these automated systems for the immune-monitoring of breast cancer patients under neo-adjuvant chemotherapy.

Methods

We used data from an in vitro system to monitor by FC various immunological readouts in T and APCs in PBMCs from breast cancer patients (BCPs) before, and after three cycles of neo-adjuvant antitumor therapy with Doxorubicin and Cyclophosphamide (A/C). We applied different automated algorithms including SPADE, FLOCK and CITRUS to process FC data on the status of T and APC compartments in these patients.

Results

Using CITRUS, we detected ex vivo alteration in the frequency of Dendritic Cells (DCs) with a plasmacytoid phenotype in BCPs patients before treatment that was recovered to the levels observed in healthy donors after three cycles of $A / C$ chemotherapy. Regarding $T$ cells, we confirmed that before therapy $T$ cells in BCPs exhibited marked unresponsiveness to a polyclonal stimulus evidenced by a deficient TCR internalization and low expression of CD25, CD69, and CD154 that was also recovered in BC patients after chemotherapy.

\section{Conclusions}

Our results let us to argue that automated systems are useful tools for the immune-monitoring by FC of BCPs under chemotherapy and for the analysis of cell populations with a complex immunephenotype not analyzable through manual gating.

\section{P77}

The novel IL-2 cytokine immune agonist NKTR-214 harnesses the adaptive and innate immune system for the treatment of solid cancers

Salah Eddine Bentebibel', Chantale Bernatchez', Cara Haymaker', Michael Hurwitz', Patrick Hwu', Mario Sznol' ${ }^{2}$, Nizar Tannir?', Sandra Aung ${ }^{3}$, Michael Imperiale ${ }^{3}$, Mary Tagliaferri ${ }^{3}$, Christie Fanton ${ }^{3}$, Ernesto lacucci ${ }^{3}$, Jonathan Zalevsky ${ }^{3}$, Ute Hoch ${ }^{3}$, Adi Diab

${ }^{1}$ The University of Texas MD Anderson Cancer Center, Houston, TX, USA; ${ }^{2}$ Yale School of Medicine, New Haven, CT, USA; ${ }^{3}$ Nektar Therapeutics, San Francisco, CA, USA

Correspondence: Salah Eddine Bentebibel

(mvphillips@phillipsgilmore.com)

Journal for ImmunoTherapy of Cancer 2017, 5(Suppl 2):P77

\section{Background}

The novel, CD-122 biased PEGylated IL-2 cytokine immune agonist NKTR-214 harnesses the potent immune stimulatory benefits of the IL-2 pathway to maximize anti-tumor responses, and minimize unwanted biological effects. NKTR-214 has been safely administered in the outpatient setting, with low-grade hypotension as the most clinically significant adverse event. NKTR-214 robustly increases expression of activation and proliferation markers on immune cells in blood and tumor. Evidence of clinical activity has been observed in IO naïve and relapsed/refractory patients. Here we describe the phenotype of proliferating $T$ cells in the blood and present an indepth characterization of tumor-infiltrating immune cells using gene expression analysis and TCR profiling.

Methods

Pre- and on-treatment blood and tumor biopsies from patients receiving 0.003 to $0.009 \mathrm{mg} / \mathrm{kg}$ NKTR-214 monotherapy were analyzed. Blood samples ( $\mathrm{n}=22$ pts) were analyzed with 16-color multiparameter flow-cytometry. Tumor biopsies ( $n=10 \mathrm{pts})$ were analyzed for gene expression using the Nanostring PanCancer immune panel. Differentially expressed genes in pre- and on-treatment samples were identified and mapped to public databases for pathway enrichment analysis. Tumor T cell receptor (TCR) sequencing was conducted at Adaptive Biotechnologies, and analyzed using the $\mathrm{R}$ package LymphoSeq.

Results

In blood samples, we observed increased expression of activation markers (ICOS and OX40) as well as co-inhibitory receptors (PD-1, CTLA-4, TIM-3 and Lag-3) in both CD4 ${ }^{+}$and $\mathrm{CD} 8^{+}$T cells proliferating in response to NKTR-214. Differentially expressed genes $(n=54)$ mapped to several innate and adaptive immune system pathways, including NK cell mediated cytotoxicity, IL-2, IL-12, and TCR signaling pathways. Pairwise comparison of matched baseline and ontreatment tumor biopsies confirmed increased expression of genes associated with $T$ cell signaling (ie. CD3G/D/E, CD247, CD8A and CD8B) and function (ie. PRF1, GNLY, GZM, and IFNG), as well as upregulation of several immune checkpoint and costimulatory genes (ie. PD1, PDL1, LAG3, IDO, and CTLA4). Gene expression changes were observed at all dose levels and across tumor types studied. Tumor TCR sequencing indicates changes in clonality with a trend towards increased frequency of specific clones on-treatment.

Conclusions

A single dose of NKTR-214 modulates both the innate and adaptive immune system. Changes in clonality and increased frequency of specific clones suggest efficient remodeling of the T cell repertoire. Upregulation of checkpoint and costimulatory genes provides insights for potential combination therapies. Based on the favorable safety profile and strong correlative biomarker data, a phase 1/2 trial combining NKTR-214 and nivolumab is currently enrolling.

\section{P78}

Immune, molecular and T cell repertoire landscape of 235 resected non-small cell lung cancers, paired normal lung and peripheral blood mononuclear cells

Alexandre Reuben', Rachel Gittelman², Jiexin Zhang', Runzhe Chen', Kelly Quek', Luis Vence', Irina Fernandez-Cubelo ${ }^{1}$, Carmen Behrens', Jianjun Gao ${ }^{1}$, Erik Yusko ${ }^{3}$, Ryan Emerson ${ }^{3}$, Sharon Benzeno ${ }^{3}$,

Marissa Vignali ${ }^{3}$, Christopher Tipton ${ }^{4}$, Ali Jalali' ${ }^{1}$, Won-Chul Lee', Jun Li ${ }^{1}$, Xifeng $\mathrm{Wu}^{1}$, Yuanqing Ye ${ }^{1}$, Agda Eterovic ${ }^{1}$, Latasha Little ${ }^{1}$, Curtis Gumbs' Vancheswaran Goplakarishnan', Chantale Bernatchez', Cara Haymaker', Marie-Andrée Forget ${ }^{1}$, Lorenzo Federico ${ }^{1}$, Tina Cascone ${ }^{1}$, Harlan Robins ${ }^{5}$, Emily Roarty', Jaime Rodriguez', Edwin Parra', Jennifer Wargo', James Allison', Padmanee Sharma', Jianhua Zhang', Jack Lee', Boris Sepesi', Stephen Swisher', Don Gibbons ${ }^{1}$, John Heymach', Andy Futreal ${ }^{1}$, Ignacio Wistuba ${ }^{6}$, Jianjun Zhang ${ }^{6}$

${ }^{1} \mathrm{MD}$ Anderson Cancer Center, Houston, TX, USA; ${ }^{2}$ Adaptive Biotechnologies, Seattle, WA, USA: ${ }^{3}$ Adaptive Technologies, Seattle, WA, USA; ${ }^{4}$ Emory University School of Medicine, Atlanta, GA, USA; ${ }^{5} \mathrm{MD}$ Anderson Cancer Center, Seattle, WA, USA; ${ }^{6}$ MD Anderson, Houston, TX, USA

Journal for ImmunoTherapy of Cancer 2017, 5(Suppl 2):P78

\section{Background}

Non-small cell lung cancer (NSCLC) is characterized by a high mutational load. Accordingly, it is also among the tumor types that respond to immune checkpoint blockade, presumably mediated by the anti-tumor T cell response. However, the lung is continuously exposed to the outside environment, which may result in a continuous state of inflammation against outside pathogens rather than tumor 
cells. Therefore, further investigation into the $\mathrm{T}$ cell repertoire and $\mathrm{T}$ cell phenotypes across normal lung and tumor is warranted.

\section{Methods}

We performed T cell receptor (TCR) sequencing and whole exome sequencing (WES) on PBMC, normal lung, and tumor from 235 NSCLC patients. Of these patients, 96 were also subjected to WES and TCR sequencing. We further performed Cytometry by Time-of-Flight (CyTOF) on 10 NSCLC tumors and paired normal lung tissues to phenotype immune and T cell subsets.

Results

Comparison of the T cell repertoire showed 9\% (from 4\% to 15\%) of $\mathrm{T}$ cell clones were shared between normal lung and paired tumor. Furthermore, among the top 100 clones identified in the tumor, on average 57 (from 0 to 95 ) were shared with paired normal lung tissue. Interestingly, T cell clonality was higher in the normal lung in $89 \%$ of patients suggesting potential differences in the immune response and immunogenicity. A substantial number of somatic mutations were also identified not only in NSCLC tumors (average 566; from 147 to 2819), but also in all morphologically normal lung tissues (average 156; from 50 to 2481). CyTOF demonstrated striking differences in the immune infiltrate between normal lung and tumor, namely a unique GITR+ T cell subset $(0.96 \%)$ which was entirely restricted to the normal lung. Conversely, increases in regulatory $T$ cell frequency (CD4+FoxP3+) were observed in the tumor $(10.4 \%$ vs $1.7 \%$ in normal lung), further highlighting the differences in T cell phenotype and response across normal lung and tumor.

Conclusions

These results suggest that a substantial proportion of infiltrating $T$ cells in NSCLC tumors may be residential T cells associated with response to environmental factors. However, normal lung and NSCLC tumors carry $\mathrm{T}$ cells of distinct phenotypes including increases in immunosuppressive T cells within the tumor which may further highlight the differences in the anti-tumor immune response.

\section{P79}

T cell inflamed gene expression profile (GEP) analysis of pembrolizumab- and ipilimumab-treated patients with advanced melanoma in the multicenter, randomized, open-label phase 3 KEYNOTE-006 study

Antoni Ribas ${ }^{1}$, Caroline Robert ${ }^{2}$, Jacob Schachter ${ }^{3}$, Georgina Long ${ }^{4}$, Ana Arance ${ }^{5}$, Jean-Jacques Grob ${ }^{6}$, Matteo Carlino ${ }^{7}$, James Larkin ${ }^{8}$ Andrea Webber ${ }^{9}$, Robin Mogg ${ }^{9}$, Mark Ayers ${ }^{9}$, Jared Lunceford ${ }^{9}$, Fang Liu ${ }^{9}$, Michael Nebozhyn ${ }^{9}$, Andrew Albright ${ }^{9}$, Wendy Blumenschein ${ }^{9}$, Clemens Krepler ${ }^{9}$, David Kaufman ${ }^{9}$, Nageatte Ibrahim ${ }^{9}$, Adil Daud ${ }^{10}$ ${ }^{1}$ University of California, Los Angeles, Los Angeles, CA, USA; ${ }^{2}$ Gustave Roussy and Paris-Sud University, Villejuif, France; ${ }^{3}$ Ella Lemelbaum Institute for Melanoma, Sheba Medical Center at Tel Hashomer, Ramat Gan, Israel; ${ }^{4}$ Melanoma Institute Australia, University of Sydney, Mater Hospital and Royal North Shore Hospital, Sydney, Australia; ${ }^{5}$ Hospital Clinic de Barcelona, Barcelona, Spain; ${ }^{6}$ Aix Marseille University, Hôpital de la Timone, Marseille, France; ${ }^{7}$ Westmead and Blacktown Hospitals, Melanoma Institute Australia, and University of Sydney, Blacktown, Australia; ${ }^{8}$ Royal Marsden Hospital, London, UK; ${ }^{9}$ Merck \& Co., Inc., Kenilworth, NJ, USA; ${ }^{10}$ University of California, San Francisco, San Francisco, CA, USA

Correspondence: Antoni Ribas (aribas@mednet.ucla.edu) Journal for ImmunoTherapy of Cancer 2017, 5(Suppl 2):P79

\section{Background}

The independently validated $\mathrm{T}$ cell-inflamed GEP has been consistently associated with response to the anti-programmed death 1 (PD-1) antibody pembrolizumab. We analyzed gene expression in baseline tumor samples from the KEYNOTE-006 study of pembrolizumab versus the anti-cytotoxic T-lymphocyte-associated antigen 4 (CTLA-4) antibody ipilimumab to determine the relative predictive effects of the T cell-inflamed GEP on clinical outcomes in advanced melanoma.

Methods

Patients were randomized to pembrolizumab $10 \mathrm{mg} / \mathrm{kg}$ every 2 or 3 weeks or 4 doses of ipilimumab $3 \mathrm{mg} / \mathrm{kg}$ every 3 weeks. Total RNA from baseline tumor samples was isolated from $4-\mu \mathrm{m}$ formalin-fixed, paraffin-embedded tissue sections and analyzed on the NanoString nCounter gene expression platform. Overall survival (OS) was the prespecified primary end point at final analysis; progression-free survival (PFS) and best objective response (BOR) were secondary end points. For efficacy comparison of pembrolizumab versus ipilimumab across $T$ cell-inflamed GEP levels, the scores were categorized into tertiles (low: GEP < - 0.28 ; middle: $-0.28<$ GEP $<0.07$; high: GEP > 0.07 ), as prespecified in the statistical analysis plan, and Cox proportional hazards modeling was used to compare the treatment effect of the pooled pembrolizumab arms versus ipilimumab within each subgroup. Data cutoff for clinical analyzes was December 3, 2015.

Results

Of 834 enrolled patients, gene expression data were available for 427 (309 pembrolizumab; 118 ipilimumab) with OS/PFS data and for 375 $(269 ; 106)$ with BOR data. Baseline characteristics were similar between the GEP and overall patient populations. Association of the T cell-inflamed GEP with OS was confirmed within each treatment group $(P<0.0001$ pembrolizumab; $P=0.0127$ ipilimumab). Evaluation of the between treatment group effect on OS was significant in all tertile subgroups (hazard ratio $[\mathrm{HR}]=0.61, P=0.0282$ [low]; $\mathrm{HR}=0.60$, $P=0.0224$ [middle]; and $\mathrm{HR}=0.44, P=0.0042$ [high]) but was most pronounced in the high group. Qualitatively similar results were observed for PFS. Although the T cell-inflamed GEP was not associated with BOR among ipilimumab-treated patients (area under the receiver operating characteristic [AUROC] $=0.511 ; P=0.4723$ ), it was associated with BOR among pembrolizumab-treated patients (AUROC $=0.696 ; P<0.0001$ ). Association between the $T$ cell-inflamed GEP and tumor PD-L1 expression by immunohistochemistry and the relationship with clinical outcomes will be presented.

Conclusions

T cell-inflamed phenotype as measured by the GEP shows a differential association with OS for pembrolizumab versus ipilimumab, which may be explained by the ability of the GEP score to predict BOR in the tumor microenvironment with anti-PD-1, but not anti-CTLA-4, therapy.

Trial Registration

ClinicalTrials.gov Identifier NCT01866319

\section{P80}

Testing systems immune monitoring by mass cytometry for clinical correlative research

Caroline Roe, Allison Greenplate, Jonathan Irish

Vanderbilt University, Nashville, TN, USA

Correspondence: Jonathan Irish (caroline.e.roe@vanderbilt.edu) Journal for ImmunoTherapy of Cancer 2017, 5(Suppl 2):P80

\section{Background}

Systems immune monitoring during cancer treatment can track therapy response and reveal biomarkers [1]. In metastatic melanoma, this approach has implicated proliferating $T$ cell subsets as a cellular effector mechanism for checkpoint inhibitors [2, 3]. Aims here were: 1 ) develop a robust cancer immune monitoring panel for multi-center clinical correlative research conducted by a core, and 2) generate pilot data to train and test computational tools employing machine learning algorithms.

Methods

Peripheral blood mononuclear cells (PBMC) were collected with informed consent from melanoma patients with local Institutional Review Board (IRB) approval and in accordance with the Declaration of Helsinki ( $\mathrm{N}=10$ samples from 5 patients). For each patient, PBMC were collected before and approximately 3 weeks post-treatment with anti-PD1 immunotherapy. A commercially available antibody kit, Fluidigm's Complete Human T cell Immuno-Oncology panel kit, was chosen to focus on T cell subsets, memory, activation, and immunooncology proteins. Also tested was the addition of proliferation marker Ki67 and cell identity markers CD19 and CD14. Data were collected using a Helios mass cytometer, normalized using normalization beads, and analyzed with viSNE, Cytobank, and MEM [4] following established methods [5]. 
Results

Individual patient immunophenotypes were consistent pre- and postanti-PD-1 therapy. Addition of markers to the commercial antibody sets was straightforward. CD19 and CD14 proved unnecessary for automatic identification of cell subsets by viSNE and MEM. Ki67 enabled detection of T cell proliferation. Because Ki67 is less abundant in cells, mass cytometry panel design should be used to detect Ki67 in a channel with high sensitivity.

\section{Conclusions}

Use of mass cytometry and commercially available metal conjugated antibodies provided a robust method for systems immune monitoring in cancer therapy compatible with correlative research in larger clinical studies.

\section{References}

1. Greenplate, et al. Systems immune monitoring in cancer therapy. Eur J Cancer. 2016; 61:77-84.

2. Huang, et al. T cell invigoration to tumor burden ratio associated with anti-PD-1 response. Nature. 2017.

3. Spitzer, et al. Systemic immunity is required for effective cancer immunotherapy. Cell. 2017;168(3): 487-502 e15.

4. Diggins, et al. Characterizing cell subsets using marker enrichment modeling. Nat Methods. 2017;14(3):275-278.

5. Diggins, Ferrell, Jr., Irish. Methods for discovery and characterization of cell subsets in high dimensional mass cytometry data. Methods. 2015; 82:55-63.

\section{P81}

The immunomodulatory effects of cancer therapy on IFN-gamma responses in the periphery

Laura Rosa Brunet ${ }^{1}$, James Crooks ${ }^{2}$, Andrew MacDonald ${ }^{2}$, Samuel LaBrie ${ }^{3}$, Dominic Eisinger

${ }^{1}$ Immodulon Therapeutics Ltd, Uxbridge, UK; ${ }^{2}$ Manchester University, Manchester, UK; ${ }^{3}$ Myriad RBM, Austin, TX, USA

Correspondence: Laura Rosa Brunet (Irb@immodulon.com)

Journal for ImmunoTherapy of Cancer 2017, 5(Suppl 2):P81

\section{Background}

The immune system is recognized as an important ally in the fight against cancer. Indeed patients' survival outcome can be predicted by their immune response bias at the tumor site. With the ever expanding landscape of cancer therapy options, including immunotherapeutic agents, it has become critical to determine how patients' immune responses are affected by treatment. In light of the challenges of repeatedly accessing tumor tissue, investigating immune responses in the periphery assumes increased importance.

\section{Methods}

IMAGE-1 is a randomised open-label, phase II, first-line, proof of concept study (NCT01303172), investigating the combination of IMM101, an immunomodulatory treatment comprising of heat-killed whole cell Mycobacterium obuense (NCTC13365), with Gemcitabine for the treatment of advanced pancreatic cancer. Treatment with IMM-101+Gemcitabine increased median survival to 7.0 months in metastatic pancreatic cancer patients compared to 4.4 months following treatment with Gemcitabine alone (1). We measured levels of IFN-gamma, a critical cytokine for anti-tumor responses, in patients' serum samples. To overcome technical difficulties associated with measuring serum IFN-gamma, we used an immunoassay $(\mathrm{LLOQ}=9.5 \mathrm{fg} / \mathrm{ml})$ developed by Myriad RBM for the Quanterix Simoa ${ }^{\mathrm{TM}}$ platform. This approach provides ultra-sensitive measurement of biomarkers, achieving orders-of-magnitude greater sensitivity than conventional immunoassay platforms.

Results

We report that peripheral IFN-gamma levels increased following initiation of treatment (Gemcitabine \pm IMM-101). In the initial 50 serum samples analyzed, there was a statistically significant increase in IFNgamma levels from time 0 (screening/randomization; $0.12 \mathrm{pg} / \mathrm{ml}, 95 \%$ $\mathrm{Cl} 0.084,0.18 \mathrm{pg} / \mathrm{ml})$ to week $13(0.41 \mathrm{pg} / \mathrm{ml}, 95 \% \mathrm{Cl} 0.26,0.66 \mathrm{pg} / \mathrm{ml})$, when patients had been scheduled to receive 3 cycles of Gemcitabine $\left(1000 \mathrm{mg} / \mathrm{m}^{2}\right.$ for 3 consecutive weeks out of 4$) \pm \mathrm{IMM}-101$ (1 $\mathrm{mg}$, intradermally on 6 occasions). Our previous data show that IMM-101 activates primary murine and human dendritic cells, and in murine models instigates IFN-gamma production by a variety of innate and adaptive immune cells. We investigated whether patients that received IMM-101 in addition to Gemcitabine had increased serum IFN-gamma levels. Preliminary data suggest that patients treated with Gemcitabine+IMM-101 $(\mathrm{n}=15)$ had higher IFN-gamma secretion at week $13(0.57 \mathrm{pg} / \mathrm{ml}, 95 \% \mathrm{Cl} 0.33,0.98 \mathrm{pg} / \mathrm{ml})$ than patients $(\mathrm{n}=9)$ treated with Gemcitabine alone $(0.24 \mathrm{pg} / \mathrm{ml}, 95 \% \mathrm{Cl}$ $0.099,0.58 \mathrm{pg} / \mathrm{ml}$ )

Conclusions

We are in the process of confirm these findings in further serum samples from IMAGE-1 patients and addressing the specific cellular sources of IFN-gamma in murine experimental models.

\section{Reference}

1. Dalgleish, et al. Randomised, open-label, phase II study of gemcitabine with and without IMM-101 for advanced pancreatic cancer. Br J Cancer. 2016;115:989-96

\section{P82}

Potential biomarkers in $\mathrm{mRCC}$ patients treated with nivolumab Maria Sayapina', Dmitry Nosov², Nikita Savelov ${ }^{3}$, Nina Lyubimova ${ }^{1}$, Yuriy Timofeev

${ }^{1}$ N.N. Blokhin Russian Oncology Research Center, Moscow, Russian Federation; ${ }^{2}$ The Central Clinical Hospital for Presidential Affairs, Moscow, Russian Federation; ${ }^{3}$ The Moscow City Oncology Hospital No. 62, Istra, Russian Federation

Correspondence: Maria Sayapina (maria.sayapina@mail.ru)

Journal for ImmunoTherapy of Cancer 2017, 5(Suppl 2):P82

\section{Background}

Checkpoint inhibitors produce meaningful survival benefit in mRCC patients (pts). Predictive biomarkers of response to anti-PD1 therapy are still under evaluation in clinical trials. Prospectively we explored biomarkers that might have potential predictive and prognostic value in mRCC pts on nivolumab therapy.

Methods

Patients with $\mathrm{mRCC}$ who had 1 or more prior lines of therapy received nivolumab at $3 \mathrm{mg} / \mathrm{kg}$ q2w ( $\mathrm{n}=23$ ) from 2015 to 2017. Blood samples were collected at baseline and 2 mo after treatment start Serum levels of TGF- $\beta 1$, IL-17A, and soluble PD-1 (sPD-1) were measured by ELISA. Pre-treatment specimens from primary tumors $(n=17)$ were evaluated for PD-L1 and FOXP3 expression on tumorinfiltrating immune cells (IC) using the IHC assay (SP142; EP340).

Results

With a median follow-up of 10 mo, PFS was 4 mo $(95 \% \mathrm{Cl}$ : 1,37$10,04)$ and 1-year PFS was $17 \%$, median overall survival was not reached. The optimum diagnostic cut-off value (mean $+2 \mathrm{SD}$ ) for TGF- $\beta 1$ was $20 \mathrm{ng} / \mathrm{mL}$ (sensitivity 43,75\%, specificity $100 \%$ ), for IL$17 A$ was $0,5 \mathrm{pg} / \mathrm{mL}$ (sensitivity $40 \%$, specificity $88,23 \%$ ), for sPD-1 was $0,45 \mathrm{ng} / \mathrm{mL}$ (sensitivity $40 \%$, specificity $88,8 \%$ ). Higher TGF- $\beta 1$ level at baseline was associated with shorter PFS ( 6 vs 1,5 mo; $p=0,03$ ). Higher baseline IL-17A ( $p=0,0174)$ and $s$ PD- $1(p=0,039)$ levels were significantly associated with higher ORR $(21,7 \%)$. Using the $\geq 0,5 \%$ and $\geq 1 \%$ cut-off for PDL- 1 expression, $11 / 17(64 \%)$ and $8 / 17(47 \%)$ pts were considered PD-L1 positive respectively. When a cut-off of $\geq 0,5 \%$ was used clinical effect (tumor shrinkage $>10 \%$ ) was higher in the PD-L1-positive group than in the PD-L1-negative group $54,5 \%$ vs $33,3 \%)$. Using the $\geq 10 \%$ cut-off for FOXP3, 11/17 pts were considered positive (64\%). Clinical effect was $45 \%$ in the FOXP3-positive group and $50 \%$ in the FOXP3-negative group. Expression of PD-L1 and FOXP3 on IC didn't not have a significant effect on PFS ( $p>0,05)$.

\section{Conclusions}

Our results demonstrated improved survival benefit in patients with lower levels TGF- $\beta 1(<20 \mathrm{ng} / \mathrm{mL})$. Elevated baseline levels of 
IL-17A $(>0,5 \mathrm{pg} / \mathrm{mL})$ and sPD-1 $(>0,45 \mathrm{ng} / \mathrm{mL})$ were significantly associated with ORR. PD-L1 and FOXP3 IC expression have no predictive or prognostic value for pre-treated RCC pts receiving nivolumab therapy.

\section{P83}

Characterizing cancers by prevalence on the PD-L1/CD8 axis

Guenter Schmidt ${ }^{1}$, Keith Steele ${ }^{2}$, Armin Meier', Thomas Herz'

Tze Heng Tan ${ }^{1}$, Brandon Higgs ${ }^{2}$, Sonja Althammer ${ }^{1}$, Ralf Huss

${ }^{1}$ Definiens AG, Munich, Germany; ${ }^{2}$ Medlmmune LLC, Gaithersburg, MD, USA

Correspondence: Guenter Schmidt (gschmidt@definiens.com)

Journal for ImmunoTherapy of Cancer 2017, 5(Suppl 2):P83

\section{Background}

Checkpoint inhibitor-based immunotherapies are successful across various cancers, particularly, type I cancers, characterized by tumoral PD-L1 expression and by a repertoire of tumor-infiltrating lymphocytes, respond well to therapies targeting PD1/PD-L1 [1]. While it has been shown that combinatorial CD8(+) and PD-L1(+) cell densities provide predictive value for durvalumab in NSCLC [2], we present in this study profiling results for multiple indications. The findings are set into the context of tumor mutation burden (TMB) which has been proposed as additional predictor for response to immunotherapies [3].

\section{Methods}

Image analysis (Definiens, Munich, Germany) detected PD-L1(+) tumor and $\mathrm{CD} 8(+)$ immune cells in pathologist annotated tumor center in serial, IHC-stained tissue sections (Ventana SP263 and SP239 resp.) from multiple cancer indications. Automatic image alignment enabled the computation of tile-based (size $=64 \mu \mathrm{m})$ cell coverage statistics: percentage of tiles with at least one PD-L1(+) (CD8(+) resp.) cell, and co-occurrence C-score [4] of tiles containing at least one PD-L1(+) and one CD8(+) cell. The indications were ranked per TMB as published in [3], where the authors used comprehensive genomic profiling using the FoundationOne assay (Cambridge MA, USA).

Results

In Fig. 1, PD-L1(+) and CD8(+) low and high patients were defined by median cut-points (1\% PD-L1(+) tiles, 36\% CD8(+) tiles). The population prevalences in the PD-L1 $(+)^{\text {High }} \mathrm{CD} 8(+)^{\text {High }}$ group correlate with mutational burden (Spearman's $r=0.96, p<0.01$ ), with the notable exception of BLAD (similar prevalence reported in [5]). PANC prevalence dominates the PD-L1 $(+)^{\text {Low }} \mathrm{CD} 8(+)^{\text {High }}$ group $(>2 \sigma)$. The $\mathrm{CD} 8(+)^{\text {Low }}$ groups are heterogenous in prevalences.

Figure 2 shows the mean PD-L1(+) and CD8(+) C-score in all cancers of this study. Mutation-high NSCLC is characterized by high C-scores, whereas mutation-low PANC is characterized by low C-scores, suggesting dysfunctional CD8(+) cells or tumor specific mutations suppressing response to IFN $\gamma$.

\section{Conclusions}

This study presents an automated profiling method for cancers, which may provide clinical decision support for which type of tumor a certain type of immunotherapy works best. While our findings are consistent with population statistics on tumor mutational burden, they highlight the relevance of spatial analysis in tissue for a deepened understanding of the mechanisms of diseases.

\footnotetext{
References

1. Teng, et al. doi:10.1158/0008-5472.CAN-15-0255

2. Althammer, et al. doi:10.1186/s40425-016-0191-4

3. Chalmers, Connelly, et al. doi:10.1186/s13073-017-0424-2

4. Novak, et al. doi:10.1111/j.1365-2486.2011.02482.x

5. Faraj, et al. doi:10.1016/j.urology.2014.10.020
}

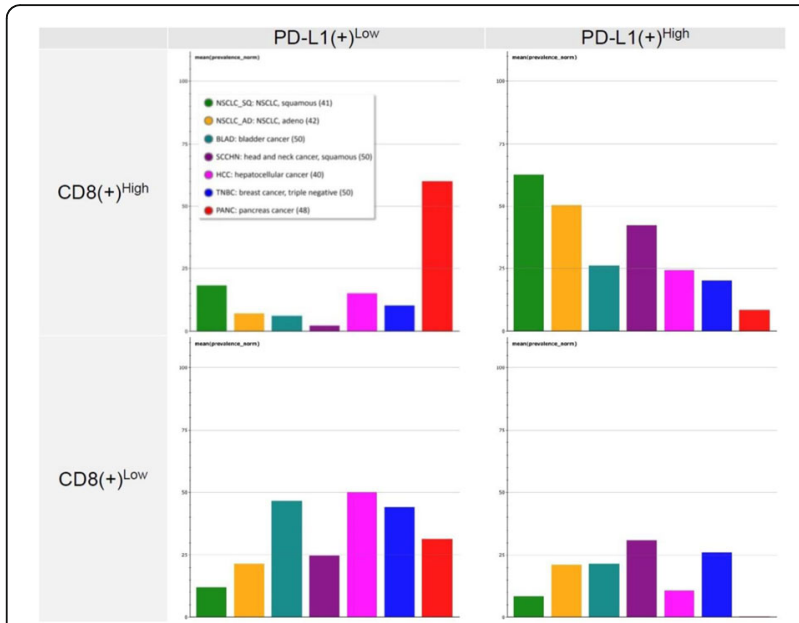

Fig. 1 (abstract P83). Cancer prevalences in the PD-L1(+) and CD8(+) low and high groups. Prevalences of seven cancer indications (see inset, with number of patients), grouped by classification into PD-L1(+) and $\mathrm{CD} 8(+)$ low and high tile percentage tumors. The indications were sorted with respect to decreasing TMB as defined in [3]

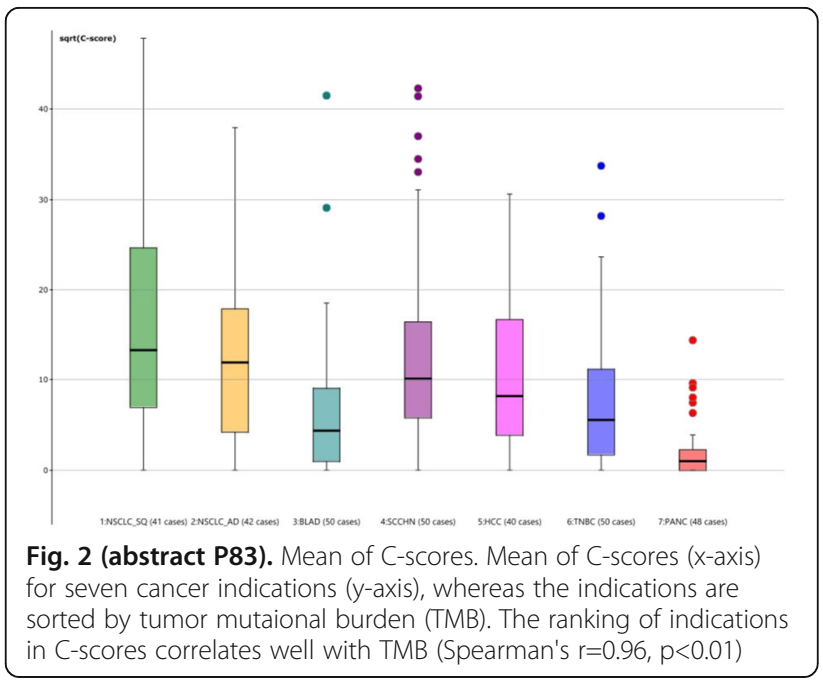

P84

Relevance of the microenvironment and MHC class I mediated immune escape mechanisms of tumors for anti-tumoral immune responses

Barbara Seliger (barbara.seliger@uk-halle.de)

Institute of Medical Immunology, Halle, Germany

Journal for ImmunoTherapy of Cancer 2017, 5(Suppl 2):P84

\section{Background}

Abnormalities of classical and non-classical MHC class I antigens were frequently found in tumor cells of distinct origin resulting in evasion of tumor cells from $T$ cell-mediated immune surveillance. The underlying molecular mechanisms of these alterations are complex and include transcriptional, epigenetic and posttranscriptional control of MHC class $\mathrm{I}$ antigens and components of the antigen processing machinery 
(APM). Furthermore, the escape of tumor cells from immune surveillance is also linked to changes in the cellular composition of the tumor microenvironment and peripheral blood mononuclear cells.

\section{Methods}

Tumor cells were analyzed for the expression of HLA class I APM components by qPCR, Western blot analysis and/or flow cytometry. Immunohistochemistry and multispectral imaging was employed to determine the frequency, localization and topology of suppressor and effector immune cells.

Results

While impaired expression of classical MHC class I and HLA-G is often due to transcriptional downregulation, alterations in the histone acetylation and methylation appear not to be so frequent. Defects in the IFN signal transduction, oxidative status of MHC class I antigens as well as altered expression of immune modulatory microRNAs and RNA-binding proteins affects the expression of MHC class I APM and/ or HLA-G. In addition, the extracellular matrix protein biglycan has been shown to increase MHC class I surface expression, which was associated with enhanced immune cell responses and reduced expression of members of the TGF- $\beta$ pathway. However, not only tumor-, but also therapy-induced effects are responsible for shaping MHC class I antigen expression. Furthermore, high levels of immune suppressive cells in the tumor were associated with a worse prognosis, but in particular the distance of immune cells to each other, and to tumor cells has prognostic value. This could be further extended by combining the immune cell infiltration with MHC class I APM component expression. In addition, downregulation of MHC class I APM component expression has been shown to occur could upon adoptive cell therapy.

Conclusions

Thus, these data suggest that the molecular make up of tumor cells might shape the immune response, which might serve as biomarkers for prediction of immune-based therapies. In conclusion, a better understanding of the immune evasion mechanisms is still required for improving T cell-based, individualized immunotherapies, and appears to be of importance in the development of resistances to these therapies, but opens also new venues for therapeutic intervention.

\section{P85}

HAAH and MMP9 are complementary cancer biomarkers and potentially enhanced predictors of metastasis

Mark Semenuk, Eleanor Ghanbari, Anokhi Cifuentes, Anjali Kalra, Michael Lebowitz, Hossein Ghanbari

Panacea Pharmaceuticals Inc, Gaithersburg, MD, USA

Correspondence: Mark Semenuk (msemenuk@panaceapharma.com) Journal for ImmunoTherapy of Cancer 2017, 5(Suppl 2):P85

\section{Background}

When used together, complementary biomarkers associated with mediators of cancer cell mobility and invasiveness should be useful early disease and patient outcome predictors. The present investigation is an initial quantitative assessment of HAAH (aspartyl (asparaginyl) beta hydroxylase) and MMP9 (matrix metalloproteinase 9) in serum/serum exosomes from cancer patients to evaluate their concerted role in metastasis.

Cancer-specific cell surface HAAH functions by enzymatically modifying a number of motif-restricted protein targets including Notch. It thereby indirectly triggers events leading to metastasis. MMP9 is a well-known predictor of metastasis due to its inherent effect on the process of proteolytically-assisted tumor cell escape, albeit not completely useful as a cancer biomarker on its own. We propose here that up-regulated $\mathrm{HAAH}$ is a prerequisite for metastasis and that in turn MMP9 is an enabler of this process.

Methods

We detect serum and exosomal $\mathrm{HAAH}$ by a simultaneoushomologous ELISA format using an in house manufactured reagent kit comprising pre-coated microplates and pre-formulated reagents.
Serum and exosomal MMP9 was detected with a commercial reagent kit ELISA (Abcam). Exosomes were prepared using a 50\% polyethylene glycol $6000 / 0.5 \mathrm{M} \mathrm{NaCl}$ solution added to serum, centrifugation, and reconstitution. CEA positive cancer and healthy serum samples were commercially obtained (Complex Antibodies) or through off site collaborators.

Results

Similar recovery of HAAH (58.3\%) and MMP9 (54.0\%) from exosomes compared to the source CEA positive cancer serum samples was observed, suggesting exosome co-localization. Further, both biomarkers were quantified in a larger investigation of samples obtained from high risk volunteers that potentially could have cancer. In this ongoing field study of 48 accumulated serum samples, 9 were positive for HAAH. Of these 9 samples 6 were positive for MMP9. Therefore at least three subjects had elevated HAAH that was not paralleled by increased MMP9 levels.

\section{Conclusions}

While HAAH and MMP9 are both expected to be closely associated with metastatic activity of cancer cells, both co-localize in cancer derived exosomes, and both appear to be regulated by the same transcription factor(s), their expression in serum samples are mostly coincident but sometimes may differ. This may explain differences in metastatic potential. These studies are ongoing and focused upon determining whether using both biomarkers could lead to a more accurate prediction of metastatic potential.

\section{P86}

Altered immune cell repertoire and activity after checkpoint blockade immunotherapy with nivolumab in renal cell carcinoma Michael Staehler ${ }^{1}$, Chiara Massaㄹ. Annabel Spek', Daniel Bethmann², Jurgen Bukur ${ }^{2}$, Anja Mueller ${ }^{2}$, Claudia Wickenhauser ${ }^{2}$, Bernard A. Fox ${ }^{3}$, Barbara Seliger ${ }^{2}$

${ }^{1}$ Ludwig Maximilian University, Munich, Germany; ${ }^{2}$ Martin Luther University, Halle, Germany; ${ }^{3}$ Robert W. Franz Cancer Research Center,

Portland, OR, USA

Correspondence: Michael Staehler (mstaehler@me.com)

Journal for ImmunoTherapy of Cancer 2017, 5(Suppl 2):P86

\section{Background}

Programmed cell death protein-1 (PD-1) and its ligand (PD-L1) provide tumor cells with mechanisms to escape from immune surveillance. Antibodies directed against these checkpoint molecules have been developed and successfully employed for the treatment of solid tumors including renal cell carcinoma (RCC). Despite promising clinical benefits, only $20-30 \%$ of tumor patients respond to these therapies. In order to search for predictive or prognostic biomarkers the immune cell repertoire in the peripheral blood of RCC patients was determined prior and during anti-PD-1 therapy with the PD-L1 antibody nivolumab.

Methods

We included 43 patients who had an indication for second line therapy for clear cell RCC with nivolumab. Blood specimen and tumor samples were collected at baseline and three months postNivolumab onset and at a clinical event such as progression or remission.Cell differentiations were performed as well as using multicolor flow cytometry and nanostring technology. In addition, tumor biopsies obtained before treatment were analyzed by multispectral imaging (MSI).

Results

Peripheral blood and tumor lesions of seven RCC patients have been analyzed in detail: 1/7 RCC patients had a stable disease (14\%), 3/7 (43\%) patients a partial or complete remission. One RCC patient had serious adverse effects upon treatment resulting in discontinuation of therapy, while two other RCC patients had a progressive disease and therefore were shifted to chemotherapy. Immunohistochemical evaluation by MSI indicated a higher frequency of Foxp3+ cells in the three progressive RCC patients in the stroma of the tumor, and particularly in the central core, whereas lower levels of CD163+ 
macrophage were present in the core of the tumor. Increased levels of CD4+ Foxp3+ regulatory T cells (Treg) were also present in the peripheral blood of progressive RCC patients. During the therapy, variation in the Treg frequency of peripheral blood did not correlate with clinical outcome of patients, whereas a reduction in HLA-DR+ antigen presenting cells did. Nanostring analyzes highlighted enhanced levels of interleukin (IL)-2 transcript in the peripheral blood of the two progressive RCC patients, in contrast to the diminished IL2 mRNA levels in the three responders.

\section{Conclusions}

These data suggest that the evaluation of a larger number of RCC patients combining these three different techniques might allow identifying a set of reliable markers that predict responsiveness to nivolumab therapy in these patients.

\section{P87}

Results of epigenetic-based quantitative PCR assisted immune cell counting analysis in bavituximab SUNRISE trial subgroup Nikoletta L. Kallinteris', Thomas O. Kleen², Min Tang', Shen Yin', Tobi Guennel ${ }^{3}$, Jennifer Lai', Victor Nowakowski², Steven Olek², Steve King ${ }^{1}$, Joseph S. Shan ${ }^{1}$

${ }^{1}$ Peregrine Pharmaceuticals, Tustin, CA, USA; ${ }^{2}$ Epiontis GmbH, Berlin, Germany; ${ }^{3}$ Precision Medicine, Frederick, MD, USA

Correspondence: Nikoletta L. Kallinteris (lisa_s@msn.com)

Journal for ImmunoTherapy of Cancer 2017, 5(Suppl 2):P87

\section{Background}

SUNRISE (NCT01999673), a global, double-bind, randomized Phase III trial of docetaxel plus bavituximab $(D+B)$ or docetaxel plus placebo $(\mathrm{D}+\mathrm{P})$ in previously treated non-squamous non-small cell lung cancer (NSCLC), demonstrated similar overall survival (OS) in the intent-totreat population $(n=597)$. In the subgroup of 93 patients who received subsequent immune checkpoint inhibitors $(\mathrm{ICl})$, median overall survival (mOS) was not reached $(95 \% \mathrm{Cl}, 15.2-\mathrm{NA})$ in the $\mathrm{D}+\mathrm{B}$ group $(n=46)$ and was 12.6 months $(95 \% \mathrm{Cl}, 10.4-17.8)$ for patients in the $D+P$ group $(n=47)$ (HR for death, $0.46 ; P=0.006)$. Epigeneticbased quantitative real-time PCR assisted cell counting (qPACC) of immune cells in blood was used to potentially identify predictive biomarkers.

Methods

DNA was isolated from peripheral blood mononuclear cells (PBMC) from randomized patients and treated with bisulfite leading to conversion of de-methylated cytosine (epigenetically active) residues into uracil, but left methylated ones unaffected. The de-methylation status of DNA regions, previously identified of being specific to respective immune cell phenotypes sorted by FACS: CD3+, CD4+, CD8 +, TFH, TH17, PD1, Foxp3, naïve CD8, MDSC, CD14+, NK56+, B-cells, GNLY, and CCR6+, was quantitated by qPACC. The number of demethylated gene copies per biomarker was quantitated and translated into \% total of cells in the sample. Each biomarker was classified as high or low based on the median value across all patients and correlated to OS. Hazard ratios (HR) and confidence intervals (Cl) were estimated using a Cox proportional-hazards model.

Results

Pre-treatment (pre-tx) samples were evaluable by qPACC for 62 (32 D $+B, 30 \mathrm{D}+\mathrm{P}$ ) out of the 93 patients who received $\mathrm{ICl}$ as next line therapy after the SUNRISE assigned treatment. High pre-tx ( $\geq$ median) levels correlated with statistically significant $O S$ benefit favoring $D+B$ for the following biomarkers: CD3+ $(H R=0.37, p=0.023), C D 4+$ $(H R=0.32, \quad p=0.012), \quad C D 8+\quad(H R=0.42, \quad p=0.036), \quad P D-1 \quad(H R=0.33$, $\mathrm{p}=0.017)$, GNLY $(\mathrm{HR}=0.25, \mathrm{p}=0.011)$, FoxP3 $(\mathrm{HR}=0.34, \mathrm{p}=0.032)$, naïve CD8+ (HR=0.32, $p=0.034)$, B-cells ( $H R=0.24, p=0.007)$, MDSC (HR=0.33, $\mathrm{p}=0.037) \mathrm{NK} 56+(\mathrm{HR}=0.29, \mathrm{p}=0.026)$. Low $(<)$ pre-tx levels of THF correlated with OS favoring $D+B(H R=0.34, p=0.033)$. No OS difference was observed for pre-tx high or low levels for TH17, CCR6, or CD14+. Conclusions

High pre-tx levels ( $\geq$ median) of circulating immune cells including $T$ cells, B-Cells, MDSCs, NK cells correlated with significant improvement of $\mathrm{OS}$ in patients who received $\mathrm{D}+\mathrm{B}$ then $\mathrm{ICl}$ compared to $\mathrm{D}+\mathrm{P}$ then $\mathrm{ICI}$ in the SUNRISE trial. These results support further investigation of these markers in future bavituximab clinical trials.

\section{P88}

CD96, a new candidate for checkpoint blockade in human

hepatocellular carcinoma

Zhigang Tian, Cheng Sun

Institute of Immunology, The Key Laboratory of Innate Immunity and Chronic Disease (Chinese Academy of Medical Science), School of Life Sciences and Medical Center, University of Science \& Technology of China, Hefei, China

Correspondence: Zhigang Tian (charless@ustc.edu.cn) Journal for ImmunoTherapy of Cancer 2017, 5(Suppl 2):P88

\section{Background}

It was reported that murine CD96 functions as a checkpoint in natural killer (NK) cell functional exhaustion. To date, blocking CD96 has not been demonstrated to be of substantial benefit in patients with cancer. Here, we demonstrated that human CD96 works as a checkpoint in hepatocellular carcinoma (HCC) patients.

Methods

In this study, we analyzed CD96 expression and the related dysfunction of NK cells located in intra- or peritumor regions of liver tissue samples from HCC patients, in addition to analyzing disease outcomes.

Results

Through the use of paired peritumoral tissues (PT) and IT from 236 HCC patients, we found that increased expression of CD96 on NK cells in intratumoral but not peritumoral regions, along with increased expression of its ligand CD155 and a poor prognosis. Then we investigated the expressions of CD226, TIGIT and CD96 (Immunoglobulin-like family members and share the common ligand CD155) in 54 fresh tumor tissues from HCC patients and 20 normal livers from health controls. We observed increased CD96 and decreased TIGIT expressions in NK cells from IT of HCC patients, and an increased number of $\mathrm{CD}^{+} 6^{+}$NK cells in HCC patients is associated with poor clinical characteristics and prognosis.

Human $\mathrm{CD}^{+} 6^{+}$NK cells from health controls exhibited functional exhaustion, showing decreased IFN- $\gamma$ and TNF-a productions, impaired cytolysis in response to in vitro stimulation. CD96 blockade can enhance NK cytotoxicity on CD155 expressing target cells, and play a protective role against tumor through boosting NK cell immune response. Global transcriptomic analysis of sorted $\mathrm{CD}^{+} 6^{+}$and $\mathrm{CD}^{-}$ hepatic NK cells further demonstrated exhausted features of CD96 ${ }^{+}$ NK cells comparing to CD96 NK cells.

Further, we demonstrated that high levels of TGF- $\beta$ in HCC patients induced this increased expression of CD96 in NK cells. Blocking TGF$\beta$ specifically inhibited CD96 expression in NK cells. In addition, we compared other two receptors, CD226 and TIGIT, which share common ligand CD155 with CD96, and found CD96 play a more important role in NK exhaustion.

Conclusions

Overall, these data demonstrate that targeting CD96 is a potential approach for cancer immunotherapy.

\section{P89}

Plasma-derived exosomes carrying CTLA-4, PD-1 and PD-L1 in head and neck squamous cell carcinoma patients treated with immunotherapy is associated with disease outcome

Marie-Nicole Theodoraki ', William E. Gooding ${ }^{2}$, Robert L. Ferris ${ }^{1}$

Theresa L. Whiteside

'University of Pittsburgh, Pittsburgh, PA, USA; ${ }^{2}$ Hillman Cancer Center, Pittsburgh, PA, USA

Correspondence: Marie-Nicole Theodoraki

(marie.nicole.theodorakis@gmail.com)

Journal for Immunotherapy of Cancer 2017, 5(Suppl 2):P89 


\section{Background}

Exosomes, the smallest subset of extracellular vesicles, present in body fluids serve as a cellular communication network. In the tumor microenvironment, exosomes carry messages from the tumor to local and distant cells and are also potential biomarkers of tumor progression. Exosomes express immunomodulatory molecules and shape antitumor immunity.

Methods

The role of exosomes as circulating biomarkers of response to immunotherapy was evaluated by monitoring changes in the protein cargo of exosomes isolated from plasma of patients with head and neck squamous cell carcinoma (HNSCC) treated with a combination of Cetuximab, Ipilimumab and radiotherapy. We retrospectively evaluated changes in the exosome protein cargo at multiple time points during therapy to assess the correlation of these changes with clinical outcome. Patients $(n=18)$ with previously untreated advanced HNSCC enrolled in the phase I clinical trial (NCT01935921) donated plasma specimens at baseline, during (week 5, week 14), after (6 months) and long-term after immunotherapy. Exosomes were isolated from plasma by size exclusion chromatography. The protein content of exosomes in fraction \#4 (30-150nm vesicles) was measured. Immunocapture with biotinylated anti-CD3 mAb was used to separate T cell-derived from non-T cell-derived exosomes. CD3+ exosomes bound to streptavidin-labeled beads were harvested. The uncaptured exosomes, derived from CD3- cells, were bound to beads using biotinylated anti-CD63 mAb. Flow cytometry-based antigen detection on bead-bound exosomes was performed using labeled Abs specific for CTLA-4, PD-1 and PD-L1. Ratios of positive exosomes/total plasma exosomes were assessed for correlation with patients' recurrence status at last follow-up (median of 14 months).

Results

Increases in the protein levels of total exosomes during therapy associated with disease recurrence. Decreases in frequency of CTLA-4+ exosomes in CD3+ and CD3- fractions or of PD- 1 in the CD3+ fraction occurred as early as 5 weeks into therapy. Patients segregated into two groups based on increasing vs. decreasing PD-L1+ exosomes/total exosomes. 4 patients with increasing CTLA-4+ and PD-L1 + exosomes during therapy had recurrence and died of disease. The $5^{\text {th }}$ patient with this "unfavorable" exosome profile remains disease free (NED). 13 patients who had "favorable" profiles (decreased CTLA$4+$ and PDL1+ exosomes) have not recurred.

Conclusions

Changes in the ratio of exosomes carrying checkpoint proteins/total exosomes during immunotherapy associated with oncologic outcome. The checkpoint molecules content of T cell-derived vs non- $T$ cell-derived exosomes defined "favorable" and "unfavorable" immune profiles that associated with the patients' responses to therapy. Plasma exosomes in cancer patients emerge as early markers of response to immune therapies and outcome.

\section{P90}

RNA-based immune gene set signatures demonstrate immune modulation by RXDX-106, a novel TAM (TYRO3, AXL, MER) family small molecule kinase inhibitor

Elizabeth Tindall, Heather Ely, Yumi Yokoyama, Colin Walsh, Erin Lew, Amy Diliberto, Robin Nevarez, Jack Lee, lan Silverman, Joanne Oh Gary Li, Amanda Albert, Jason Christiansen, Robert Shoemaker Ignyta, San Diego, CA, USA

Correspondence: Elizabeth Tindall (etindall@ignyta.com)

Journal for ImmunoTherapy of Cancer 2017, 5(Suppl 2):P90

\section{Background}

The TAM family of receptor tyrosine kinases, including TYRO3, AXL, and $M E R$, is pivotal in regulating the immune system. In the tumor microenvironment, TAMs promote anti-inflammatory/pro-tumorigenic activities. RXDX-106 is a potent and selective TAM inhibitor in preclinical development, shown to have immunomodulatory effects. We herein illustrate that (1) MC38, a syngeneic mouse model of colorectal cancer, is sensitive to RXDX-106, and (2) using RNA sequencing, we demonstrate RXDX-106 treatment results in the recruitment of various immune cell subtypes to the tumor microenvironment. Flow cytometry analysis supports key RNA sequencing findings.

Methods

MC38 tumor-bearing C57BL/6 mice were treated with and without RXDX-106 for 7 days. Tumor tissue was collected for cellular and molecular characterization to measure immune modulation, via flow cytometry and total RNA sequencing respectively. Single sample gene set enrichment analysis (ssGSEA) was applied to RNA sequencing data to calculate normalized enrichment scores (NES) for 15 adaptive and 13 innate immune cell types, based on previously published gene sets. RNA-sequencing significance levels are presented as false discovery rates (FDR) to correct for multiple hypothesis testing. Significance of flow cytometry data are indicated by probability (P) values calculated using the two-tailed T-test.

Results

MC38 tumors. A high proportion (66\%, 33 of the top 50) of individual genes differentially expressed between treated and untreated mice have immunomodulatory roles. Furthermore, sSGSEA suggested a significant enrichment $(F D R<0.01)$ of $T$ cell subtypes, including $T$ follicular helper (Tfh) cells (FDR=0.0005), Type $1 \mathrm{~T}$ helper (Th1) cells (FDR $=0.0005$ ), and central memory $C D 8+T$ cells ( $F D R=0.0008$ ) with RXDX-106 treatment. flow cytometry data revealed a significant increase in overall tumor infiltrating lymphocyte (TIL) recruitment $(P<0.0001)$, as well as a non-significant trend towards increased CD8 $+\mathrm{T}$ cell infiltration in RXDX-106 treated mice. Innate immune cells, such as plasmacytoid dendritic cells ( $\mathrm{pDCs} ; \mathrm{FDR}=0.0005$ ) and macrophages (FDR=0.0005), also had significantly elevated NES values in treated mice. A negative correlation ( $r=-0.7)$ between macrophage NES and M2 secreted VEGFA expression was observed and may suggest infiltrating macrophages are polarized to the pro-inflammatory/ anti-tumorigenic M1 phenotype upon treatment with RXDX-106. Flow cytometry data supported the observed increase in tumor associated macrophages $(P=0.0008)$, and demonstrated $M 1$ polarization by a highly significant $(P<0.0001)$ increase in $M 1 / M 2$ ratio with $R X D X$ 106 treatment.

Conclusions

We have demonstrated the application of an RNA sequencing based approach to detect immunomodulatory effects and provided evidence supporting immunomodulatory activity of RXDX-106 in vivo.

P91

A harmonized evaluation system for expression of HLA class I antigens in formalin-fixed paraffin embedded cancer tissues

Toshihiko Torigoe ${ }^{1}$, Nobuyoshi Hiraoka ${ }^{2}$, Naoya Nakamura ${ }^{3}$,

Eri Atsuyama ${ }^{4}$, Shingo Toji ${ }^{5}$, Yuji Heike ${ }^{4}$

${ }^{1}$ Sapporo Medical University School of Medicine, Sapporo, Japan;

${ }^{2}$ National Cancer Center Hospital, Tokyo, Japan; ${ }^{3}$ Tokai University School of Medicine, Isehara, Japan; ${ }^{4}$ St Luke's International University, Tokyo,

Japan; ${ }^{5}$ Medical Biological Laboratories Co., Ltd., Ina, Japan

Correspondence: Nobuyoshi Hiraoka (torigoe@sapmed.ac.jp); Yuji

Heike

Journal for ImmunoTherapy of Cancer 2017, 5(Suppl 2):P91

\section{Background}

Expression of human leukocyte antigen class I antigens (HLA-I) on tumor cells is crucial for target recognition of cytotoxic T cells (CTLs). Therefore, it should be an important biomarker for CTL-based cancer immunotherapy to evaluate the expression levels of HLA-I on tumor cells. A monoclonal anti-pan HLA-I antibody, EMR8-5 is the first reliable antibody that is suitable for immunohistochemistry (IHC) of formalin-fixed paraffin embedded (FFPE) tissues [1]The aim of this study is to establish the harmonized protocol to evaluate expression levels of HLA-I on cancer cells in FFPE tumor tissues. 


\section{Methods}

First of all, we determined a standardized method of staining, and proposed tentative criteria to evaluate expression levels of HLA-I in tissue sections; $(+++)$ as $\geq 90 \%$ of cancer cells are strongly positive, in which the staining density is equal or stronger than that in lymphocytes or endothelial cells, (++) as $<90 \%$ and $\geq 50 \%$ of cancer cells are strongly positive, $(+)$ as $<50 \%$ and $>10 \%$ of cancer cells are strongly positive or $>10 \%$ of cancer cells are weakly positive, in which the staining density is weaker than that in lymphocytes or endothelial cells, and $(-)$ as $\leq 10 \%$ of cancer cells are positive. Reference IHC photo atlas was made for each type of cancer. After IHC, the microscopic images were converted into digital photo files, and the immunolabeled cells were analyzed on the standardized calibrated monitors (ColorEdge CG2420, EIZO, Japan). Then we examined interobserver reproducibility of the criteria by multi-institutional study using each 20 cases of 7 cancers (colorectal, breast, prostate, pancreatic, ovarian, lung, and kidney cancers), which had been surgically resected in National Cancer Center Hospital, Tokyo.

Results

IHC with anti-pan HLA-I antibody was performed using automated IHC slide staining system. Expression profiles of HLA-I were characteristic in each cancer type. We made reference photo atlas of IHC to harmonize the evaluation for each type of cancer. According to the reference atlas, four certified pathologists scored the expression levels of HLA-I independently. Inter-observer reproducibility for each cancer was within moderate to substantial agreement levels (Fleiss's kappa values were within 0.5 to 0.7 ).

\section{Conclusions}

Expression of HLA class I antigens can be evaluated with an acceptable reproducibility in several cancers using FFPE tissues.

\section{Reference}

1. Pathol. Int. 2012;62;303-8

\section{P92}

Early biomarker correlates of severe neurologic events and cytokine release syndrome in ZUMA-1, a multicenter trial evaluating axicabtagene ciloleucel in refractory aggressive non-hodgkin lymphoma

Frederick Locke', Marika Sherman², John Rossi ${ }^{2}$, Allen Xue ${ }^{2}$, Michelle Mojadidi ${ }^{2}$, Sattva Neelapu ${ }^{3}$, Armin Ghobadi ${ }^{4}$, Lazaros Lekakis ${ }^{5}$, David Miklos ${ }^{6}$, Caron Jacobson? ${ }^{7}$, Olalekan Oluwole ${ }^{8}$, Tanya Siddiqi ${ }^{9}$, Yi Lin ${ }^{10}$, Patrick Reagan ${ }^{11}$, William Go ${ }^{2}$, Jeff Wiezorek ${ }^{2}$, Adrian Bot ${ }^{2}$

${ }^{1}$ Moffitt Cancer Center, Tampa, FL, USA; ${ }^{2}$ Kite Pharma, Santa Monica, CA, USA; ${ }^{3}$ The University of Texas MD Anderson Cancer Center, Houston, TX, USA; ${ }^{4}$ Washington University School of Medicine, Saint Louis, MO, USA; ${ }^{5}$ University of Miami Health System, Sylvester Comprehensive Care Center, Miami, FL, USA; ${ }^{6}$ Stanford University School of Medicine, Stanford, CA, USA; ${ }^{7}$ Dana-Farber Cancer Institute, Boston, MA, USA; ${ }^{8}$ Vanderbilt-Ingram Cancer Center, Nashville, TN, USA; ${ }^{9}$ City of Hope National Medical Center, Duarte, CA, USA; ${ }^{10}$ Mayo Clinic, Rochester, MN, USA; ${ }^{11}$ University of Rochester Medical Center, Rochester, MN, USA

Correspondence: Sattva Neelapu (kate.trueblood@nexusggmed.com) Journal for ImmunoTherapy of Cancer 2017, 5(Suppl 2):P92

\section{Background}

Axi-cel, an autologous anti-CD19 chimeric antigen receptor (CAR) T cell therapy, demonstrated an $82 \%$ objective response rate including $54 \%$ complete remissions in refractory aggressive NHL patients. ${ }^{1} \mathrm{NE}$ and CRS are associated with CAR T cell therapy. ${ }^{1}$ Post-treatment levels of serum analytes, measured after the onset of clinical toxicities, were associated with severe NE and CRS. ${ }^{2}$ Lymphodepleting conditioning-related (day 0 ) and early post-CAR T cell treatment (day 1) biomarkers associated with NE and CRS may provide important mechanistic insight and inform toxicity management.

\section{Methods}

Serum samples were obtained pre and post axi-cel infusion, from 101 refractory aggressive NHL patients in the Phase 2 ZUMA-1 (NCT02348216) trial [1]. A panel of 44 analytes representing major categories of immune function were measured as described before. [2] Post hoc statistical analyzes evaluated associations of analyte levels on day 0 and day 1 , in patients with/without grade $\geq 3 \mathrm{NE} / \mathrm{CRS}$. Univariate Wilcoxon two-sample tests were applied and adjusted for multiplicity, to identify important co-variates. Multivariate machine learning (random forest analysis [3]) was conducted to rank the most influential co-variates.

Results

Grade $\geq 3$ NE were observed in 28/101 (28\%) patients. Those developing grade $\geq 3 \mathrm{NE}$ had increased $\mathrm{IL}-15$ and decreased perforin ( $P=0.0006$ and $P=0.0011$, respectively) at Day 0 , and increased IL-15, MCP-1 and IL-6 ( $P=0.0021,0.0037$ and 0.0085 , respectively) and decreased perforin $(P=0.0025)$ at Day 1 . Multivariate analysis identified identical analytes as the univariate analysis and ranked the importance of analytes relative to each other. Grade $\geq 3$ CRS was observed in 13/101 (13\%) patients. Those developing grade $\geq 3$ CRS had no statistically significant differences in analyte levels at Day 0 , and increased IL-15 at Day 1 (adjusted $P=0.026$ ).

\section{Conclusions}

These analyzes indicate IL-15 in NE/CRS pathogenesis post- antiCD19 CAR T cells treatment, consistent with previous reports $[4,5,6]$. Association between early serum markers (day 0 and 1 ) and grade $\geq 3 \mathrm{NE}$ may support development of clinically useful prevention and treatment algorithms.

Trial Registration

ClinicalTrials.gov Identifier NCT02348216

\section{References}

1. Locke, et al. \#CT019. AACR. 2017.

2. Locke, et al. \#CT020. AACR. 2017

3. Segal and Xiao. WIREs Data Mining Knowl Discov. 2011.

4. Turtle, et al. JClinOncol. 2017.

5. Rossi, et al. \#46. SITC. 2016

6. Kochenderfer et al. JCO. 2017.

P93

Small molecule binders of Ly6K inhibit tumorigenic growth and induce immunity via TGFb/Stat1 and PDL1 pathway

Megha Kumar', Jinya Chen', Idalia Cruz', Purushottam Tiwari', Aykut Uren ${ }^{1}$, Maksymilian Chruszcz ${ }^{2}$, Michael B. Atkins', Louis M. Weiner', Geeta Upadhyay

${ }^{1}$ Lombardi Comprehensive Cancer Center, Georgetown University Medical center, Washington, DC, USA; ${ }^{2}$ Lombardi Comprehensive Cancer Center, Georgetown University Medical center, Columbia, SC, USA

Correspondence: Geeta Upadhyay (gu6@georgetown.edu)

Journal for ImmunoTherapy of Cancer 2017, 5(Suppl 2):P93

\section{Background}

We have previously shown that members of the Ly6 gene family, namely Ly6E, Ly6D, Ly6H and Ly6K are expressed in multiple types of solid human cancers and that the increased expression of these genes is associated with poor outcome [1]. We also have found that Ly6K and Ly6E are required for in vivo tumor growth and anti-tumor immune response [2]. Ly6K is an attractive target due to its cancer cell-specific expression; normal organ expression of Ly6K is limited to testis.

Methods

We used surface plasmon resonance technology to identify two small molecule binders of Ly6K. These molecules showed strong binding to Ly6K (kd range 1-2mM) but not to Ly6E and Ly6D. 
Results

Combined small molecule treatment led to reduced TGFb signaling and reduced PDL1 expression in MDA-MB-231 cells. Homology modeling showed that the two small molecules bind to the $\mathrm{N}$-terminus and C-terminus of the mature Ly6K protein, respectively. Treatment of E0771 tumors in a syngeneic $\mathrm{C} 57 \mathrm{BI} / 6$ model with individual small molecules reduced tumor growth, while combination treatment with the two small molecules completely eliminated tumor growth. The treatment was halted after tumor disappearance. Three weeks later, the cured mice were re-challenged with E0771 cells, with complete, durable protection against tumor growth (Fig. 1).

\section{Conclusions}

These results suggest that Ly6K inhibitors have anti-tumor properties and induce host-protective anti-tumor immunity. We are currently investigating the molecular basis of these findings in vivo. Our data implicate the novel biomarker Ly6K as a therapeutic target and immune modulator in multiple tumor types with high expression of Ly6K.

\section{References}

1. Luo L, McGarvey P, Madhavan S, Kumar R, Gusev Y, Upadhyay G. Lymphocyte antigens 6 complex gene family members drive tumorigenesis. Oncotarget. 2016; 7(10):11165-93, DOI:10.18632. PMID: 26862846

2. AlHossiny, Luo L, Frazier W, Steiner, Gusev, Kallakury, Glasgow, Creswell, Madhavan, Kumar, Upadhyay. Lymphocyte antigens 6 complex K/E-transforming growth factor-beta axis promotes breast cancer tumorigenesis.Cancer Research.Published Online April 11, 2016; doi: 10.1158/0008-5472.CAN-15-2654. PMID: 27197181

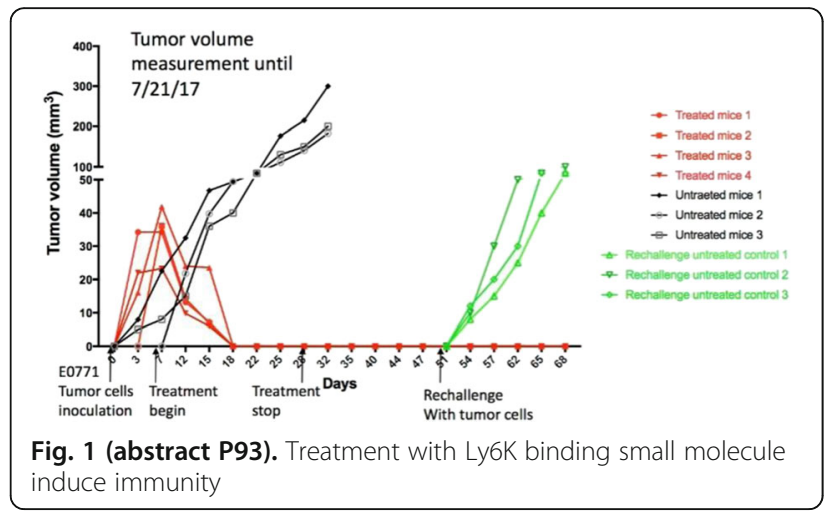

P94

The RareCyte ${ }^{\circledR}$ platform for identifying rare antigen-specific circulating CD4 and CD8 T cells in Merkel cell carcinoma Lance U'Ren', Natalie Vandeven², Erika Hayes ${ }^{3}$, Jennifer Chow ${ }^{1}$, Jackie Stilwell ${ }^{1}$, Paul Nghiem $^{2}$, Eric Kaldjian

${ }^{1}$ RareCyte Inc., Seattle, WA, USA; ${ }^{2}$ University of Washington, Seattle, WA, USA; ${ }^{3}$ Benaroya Research Institute, Seattle, WA, USA

Correspondence: Lance U'Ren (luren@rarecyte.com)

Journal for ImmunoTherapy of Cancer 2017, 5(Suppl 2):P94

\section{Background}

Merkel cell carcinoma (MCC) is an often-lethal skin cancer associated with the Merkel cell polyomavirus (MCPyV) in $80 \%$ of cases. Although checkpoint immunotherapies have proven efficacious for a cohort of MCC patients, there is still a need to identify predictive biomarkers.
Tumor antigen-specific T cells in peripheral circulation can be reflective of their phenotype within the tumor microenvironment making them attractive candidates as cellular biomarkers. However, the frequency of MCC antigen-specific CD4 and CD8 T cells can often be below the detection limit of flow cytometry making them difficult to find. We thus employed the highly sensitive RareCyte platform to detect and phenotype rare antigen-specific CD4 and CD8 T cells.

Methods

MCC patient peripheral blood mononuclear cells (PBMC) was stained and plated onto chamber well slides and imaged on the CyteFinder ${ }^{\circledR}$ 6-channel fluorescent scanning microscope. CD4 and CD8 T cells were identified by MCPyV-specific tetramer staining, and characterized with various exhaustion markers, including PD1, LAG3, TIM3, and CTLA4. Single MCPyV-specific CD4 T cells were picked and placed individually into tubes using an integrated computercontrolled retrieval system (CytePicker ${ }^{\circledast}$ ). RNA was extracted and single cell sequencing was performed on the alpha and beta chains of the $\mathrm{T}$ cell receptor (TCR).

Results

We consistently identified MCPyV -specific CD4 and CD8 T cells below the detection limit of flow cytometry without the need for enrichment or large blood volumes. Furthermore we have high confidence in the cells identified and retrieved due to the morphologic information obtained with imaging that is not possible by flow cytometry. We were able to identify patients that had exhausted MCPyV-specific T cells in circulation and characterize the expression of exhaustion markers. We were also able to sequence the TCR from single rare MCPyV-specific CD4 T cells retrieved using the platform.

Conclusions

Using this approach to identify rare but functionally important MCPyV-specific T cells will enhance our understanding of the immune response against this oncogenic virus and may help guide patient selection for checkpoint therapies.

P95

Development and application of a 6-channel immunofluorescence assay for investigating PDL1 and IFR1 expression on circulating tumor cells

Lance U'Ren, Daniel Campton, Jackie Stilwell, Eric Kaldjian

RareCyte Inc, Seattle, WA, USA

Correspondence: Lance U'Ren (luren@rarecyte.com)

Journal for ImmunoTherapy of Cancer 2017, 5(Suppl 2):P95

\section{Background}

There is a need for non-invasive predictive biomarkers of response to anti-PD1/PDL1 therapies. Assessment of circulating tumor cells (CTCs) is a rational approach to non-invasive sampling of tumors to understand PDL1 phenotype. IFN-gamma signals through the JAK/STAT cascade to induce PDL1 via the Interferon Regulatory Factor-1 (IRF1) transcription factor and is a potent inducer of PDL1 expression in tumor cells. Previous studies comparing PDL1 and IRF1 tissue staining of melanoma showed that IRF1 had a higher predictive value than PDL1 of response to anti-PD1/PDL1 therapy [1]. Using CTC models and the RareCyte platform for CTC identification, we developed a multi-parameter assay that allows simultaneous PDL1 and IRF1 assessment after CTC identification. We applied this assay to clinical samples of breast and Merkel cell carcinoma (MCC).

Methods

Peripheral blood from normal donors or cancer patients was collected into RareCyte blood collection tubes. PDL1(+) and PDL1(-) CTC models were created by culturing A549 overnight with or without $10 \mathrm{ng} / \mathrm{ml}$ INF-gamma. The A549 cells were then spiked into normal donor blood and buffy coats isolated from $7.5 \mathrm{ml}$ of blood by AccuCyte ${ }^{\oplus}$ separation and spread onto slides. Slides were stained with a 6-marker panel that included antibodies to pan-cytokeratin (CK), 
EpCAM, CD45, CD45, PDL1, IRF1, and a nuclear dye on the Leica Bond Rx auto-stainer. Slides were scanned with CyteFinder ${ }^{\circledR}$ and CTCs identified by CK and/or EPCAM positivity and negative CD45 staining. Confirmed CTCs were then assessed for expression of PDL1 and IRF1; cell compartment was also recorded for IRF1 staining.

Results

In unstimulated A549s, PDL1 staining was absent but cytoplasmic staining of IRF1 was often detectable. In contrast, IFN-gamma stimulated A549s demonstrated PDL1 staining and strong nuclear staining for IRF1. In patient samples, PDL1 was identified in a minority of CTCs in both breast cancer and MCC. PDL1(+) CTCs identified had concomitant strong nuclear IRF1 signal. IRF1 was seen in the majority of the PDL1(-) CTCs but staining could be either cytoplasmic and/or nuclear

\section{Conclusions}

In this study we demonstrate the feasibility of combining PDL1 and IRF1 biomarkers with CTC identification for non-invasive assessment. The cellular distribution of IRF1 in combination with PDL1 expression may add predictive value to the assay.

\section{Reference}

1. Smithy, Moore, Pelekanou, Rehman, Gaule, Wong, Neumeister, Sznol, Kluger, Rimm. J Immunother Cancer. 2017; 5:25.

\section{P96}

Tumor mutation burden, microsatellite instability and chromosomal instability analysis using low pass whole genome sequencing of single circulating tumor cell

Angel Rodriguez, Jerry Lee, Ramsay Sutton, Rhett Jiles, Yipeng Wang, Mark Landers, Ryan Dittamore

Epic Sciences, San Diego, CA, USA

Correspondence: Yipeng Wang (yipeng.wang@epicsciences.com) Journal for ImmunoTherapy of Cancer 2017, 5(Suppl 2):P96

\section{Background}

Tumor Mutation Burden (TMB), Microsatellite Instability (MSI) and Chromosomal Instability (CIN) represent the majority of Genomic instability (GI) in metastatic patients. Recent studies show that TMB and MSI are emerging immune checkpoint inhibitor drug sensitivity biomarkers, and CIN is a sensitivity marker for PARP inhibitors. Assessment of $\mathrm{Gl}$ in bulk tumor samples is well explored, but it is limited by sample availability and tumor heterogeneity. Analysis of ctDNA is feasible for TMB and MSI but not CIN, and it also suffers in sensitivity and specificity in patients who harbor subclonal Gl, limiting its clinical utility. Epic Sciences' Circulating Tumor Cell (CTC) platform employs a non-enrichment based approach and could provide insight into subclonal heterogeneity. Here we present downstream single cell Gl assay(s) for the detection of TMB, MSI and CIN from individual CTCs.

Methods

Contrived samples were prepared by spiking prostate cancer cell lines, LNCaP, PC3 and VCaP, into healthy donor blood. Red blood cells were lysed, nucleated cells deposited onto slides, slides immunofluorescently stained (DAPI, CK, CD45, and Androgen Receptor), and identified cancer cells individually isolated from the slides. Each recovered cell was lysed, whole genome amplified (WGA), shotgun library prepared, and low pass whole genome sequenced using Illumina NextSeq 500. Data were analyzed for TMB scores and large scale transitions. MSI was measured using Qiagen Type-It microsatellite PCR kit for four sites. Samples from metastatic castration resistant prostate cancer (mCRPC) patients were included to evaluate clinical feasibility.

Results

TMB scores for LNCaP (average 652) were significantly higher than PC3 (558), VCaP (548), and WBC from healthy donor (540) with $p<0.01$. MSI assay confirmed that LNCaP is the only MSI-H cell line with insertions/deletions found in 3 of 4 microsatellite sites. No MMR deficiencies were found in PC3 and VCaP. LST analysis shows PC3 (average 33) and VCaP (33) have much higher LST scores than LNCaP (11). A wide range of TMB (375-861) and LST scores (0-70) are observed inter- and intra- MCRPC patient samples.

Conclusions

These data demonstrate the feasibility of detecting three types of genomic instabilities at the single cell level using the Epic Sciences CTC Platform. Inter- and intra-patient heterogeneity is observed in the small patient cohort. Additionally, the results confirm that MSI and HRD are likely mutually exclusive driver events, driving tumor selection in MCRPC. Further studies are on-going to investigate the correlation of GI markers with PARPi and I/O checkpoint inhibitor responses.

P97

Simultaneous characterization of rare immune cell subpopulations and PD-L1 expressing CTCs in peripheral blood of cancer patients Adam Jendrisak, Angel Rodriguez, Jiyun Byun, Ryon Graf, Yipeng Wang, Mark Landers, Ryan Dittamore

Epic Sciences, San Diego, CA, USA

Correspondence: Yipeng Wang (yipeng.wang@epicsciences.com) Journal for ImmunoTherapy of Cancer 2017, 5(Suppl 2):P97

\section{Background}

Expression of PD-L1 on tumor and immune markers in tumor tissue are associated with improved response to PD-1 and PD-L1 checkpoint inhibitors. However, each alone has limited predictive utility. Multimodal characterization of both the tumor and host immune system is an unmet medical need for the improved prediction of response to immunotherapy. Metastatic lesions are likely to be undersampled and require a liquid biopsy, given tumor heterogeneity and evolution and temporal changes in the host immune system. We sought to examine expression of PD-L1 on circulating tumor cells (CTCS) as well as characterize rare immune cell populations with a non-invasive liquid biopsy. Examining dynamic biomarker changes in longitudinal samples could enable the development of novel diagnostic tools for response prediction and pharmacodynamics studies related to immunotherapy.

Methods

Blood samples from lung cancer patients were collected and shipped to Epic Sciences. Contrived samples were also developed by spiking cancer cell lines into healthy donor blood. Red blood cells were lysed, and nucleated cells plated onto glass slides. Slides were stained with DAPI as well as multiple immune cocktails and imaged. Targets included pan-CK, CD45, PD-L1, CD4, CD8, Ki-67, LAG-3, and TIM-3. Approximately 3 million nucleated cells per slide were examined through advanced digital pathology pipelines to detect and quantify changes in T cell populations to infer immune activation, exhaustion, suppression, and circulating tumor burden.

Results

Epic Sciences' rare cell detection platform has an analytically validated limit of detection of $1 \mathrm{cell} / \mathrm{mL}$ of blood. Three immuno-panels were developed to profile leukocyte subpopulations and CTCs simultaneously. PD-L1+ leukocytes detected in 30 out of 33 lung cancer patients, and the incidence of PD-L1+ leukocytes ranged from $0 \%$ to $0.138 \%$ (average $0.0213 \%$, median $0.0118 \%$ ). PD-L $1+$ CTCs were observed in the presence of both high and low counts of PD-L1+ leukocyte populations.

Conclusions

The low limit of detection of the Epic Sciences CTC platform coupled with ability to archive patient blood samples allowed for retrospective precise quantification of leukocyte subpopulations and PD-L1 expression on CTCs retrospectively. Development of a liquid biopsy 
based platform that can simultaneously measure immune biomarkers in CTCs as well as on leukocytes will allow for real time assessment and monitoring of response to immune checkpoints inhibitors.

\section{P98}

Immunological profiling of baseline and resected biopsies from locally/regionally advanced/recurrent melanoma treated with neoadjuvant combination ipilimumab $(3 \mathrm{mg} / \mathrm{kg}$ or $10 \mathrm{mg} / \mathrm{kg}$ ) and high dose IFN-a2B

Sarah Warren', Priyanka Vallabhaneni', Jack El-Sawada², Andrew White', Xing Ren', Alessandra Cesano', Joseph Beechem', Ahmad Tarhini ${ }^{1}$ NanoString Technologies, Inc., Seattle, WA, USA; ${ }^{2}$ Hillman Cancer Center, University of Pittsburgh Medical Center, Pittsburgh, PA, USA Correspondence: Sarah Warren (swarren@nanostring.com) Journal for ImmunoTherapy of Cancer 2017, 5(Suppl 2):P98

\section{Background}

Melanomas are the most responsive tumor type to immune checkpoint blockade, but clinical response rates remain suboptimal. Gene expression profiling of the pretreatment tumor biopsies is an attractive approach to developing predictive biomarkers of response because it is able to directly measure the activity of the immune system within the tumor. Furthermore, characterization of the transcriptional changes induced by treatment allows insights into the mechanism of action of the therapy. UPCI 11-063 is a clinical trial to assess the safety of two doses of ipilimumab combined with high dose IFN-a2B in the neoadjuvant setting. Both treatment regimens induce clinical responses, with reduced toxicity observed in the low dose ipilimumab arm. This study is a follow on biomarker analysis to investigate gene expression in pretreatment and post treatment biopsies.

Methods

This was a Phase I trial of 30 patients with locally/regionally advanced/recurrent melanoma who received either high $(10 \mathrm{mg} / \mathrm{kg})$ or low $(3 \mathrm{mg} / \mathrm{kg}$ ) dose ipilimumab plus high dose IFN-a2B. Tumor biopsies collected prior to treatment and at the time of surgery (6-8 weeks after starting neo-adjuvant treatment) were profiled on the NanoString ${ }^{\circledast}$ platform

Results

Transcriptional profiling of tumor biopsies collected prior to treatment or at the time of surgery was performed in order to find patterns of gene expression that correlated with pathological complete response ( $p C R)$, radiologic response (RR) or recurrence-free survival (RFS). In the baseline tumor biopsies, a number of genes were positively associated with $\mathrm{pCR}$, including several members of the WNT signaling pathway. The T cell inflamed Gene Expression Profile (GEP), a signature of a peripherally suppressed immune response in the tumor, was associated with longer RFS. Differential gene expression of paired pre- and post-treatment biopsies identified a number of genes upregulated in response to ipilimumab/IFN treatment, including signatures of multiple immune cell populations, including $T$ cells and macrophages, which were associated with $\mathrm{PCR}$.

\section{Conclusions}

The combination of ipilimumab/IFN as a neoadjuvant for treatment of melanoma has the potential to improve response rates by activating quiescent immune responses via multiple pathways. Expression profiling of pre and post treatment samples suggested a mobilization of immune cells to the tumor, consistent with activation of local immunity. The GEP, which predicts response to pembrolizumab in a variety of solid tumors, may also be useful in the setting of ipilimumab/IFN. Further work in this or similar sample sets may extend these observations.

Trial Registration

ClinicalTrails.gov Identifier NCT01608594
P99

Biomarker analysis from the OpACIN trial (Neo-/adjuvant

ipilimumab + nivolumab (IPI+NIVO) in palpable stage 3 melanoma) Lisette (EA) Rozeman', Lorenzo Franchi', Thomas Kuilman', Oscar Krijgsman²,

Alexander (ACJ) van Akkooi', Pia Kvistborg', Hans van Thienen',

Bauke Stegenga ${ }^{3}$, Dana Cullen 3 , Brian Lamon ${ }^{3}$, John (JBAG) Haanen?',

Alessandra Cesano ${ }^{4}$, Sarah Warren ${ }^{4}$, Annegien Broeks ${ }^{1}$, Ton Schumacher',

Christian Blank ${ }^{1}$

${ }^{1}$ Netherlands Cancer Institute, Amsterdam, Netherlands;

2o.krijgsman@nki.nl, Netherlands, Netherlands; ${ }^{3}$ Bristol-Myers Squibb, Lawrence Township, NJ, USA; ${ }^{4}$ NanoString Technologies, Seattle, WA, USA

Correspondence: Sarah Warren (swarren@nanostring.com) Journal for ImmunoTherapy of Cancer 2017, 5(Suppl 2):P99

\section{Background}

The combination of IPI+NIVO induces high response rates and improved overall survival in late stage melanoma. T cell checkpoint inhibition is of greatest value at the moment of TCR triggering and therefore dependent on the amount of antigen present, indicating that adjuvant immunotherapy will work most efficiently, when initiated prior to surgery.

Methods

Two-arm Phase $1 \mathrm{~b}$ feasibility trial consisting of 20 high risk AJCC stage $3 \mathrm{~B} / \mathrm{C}$ melanoma patients with palpable nodal disease receiving the combination of IPI $3 \mathrm{mg} / \mathrm{kg}$ and NIVO $1 \mathrm{mg} / \mathrm{kg}$, either adjuvant four courses after surgery, or split neo-adjuvant and adjuvant.

Results

In this update 20 patients are evaluable. Neo-adjuvant application of IPI+NIVO was feasible and no surgery-associated adverse events were attributed to (neo-) adjuvant therapy. 18/20 patients had to stop earlier due to grade $3 / 4$ toxicities. Neo-adjuvant IPI+NIVO reduced tumor load in $8 / 10$ patients $(3 \mathrm{pCR}, 4$ near $\mathrm{pCRs}$ [minimal remaining micro metastases], 1 pPR [ $<50 \%$ vital tumor cells], 1 pSD and 1 pPD). So far, none of the responders in the neo-adjuvant arm has relapsed. Relapse was observed for the 2 non-responders within the neoadjuvant arm and for 3 patients within the adjuvant arm.

We will present at the detailed biomarker analysis, comprising DNA and RNA sequencing, NanoString ${ }^{\circledR}$ RNA and protein profiling of PBMC, NanoString digital spatial profiling of tumors, multiplex immunohistochemistry of tumors and TCR sequencing. We found that mutational load did not correlate with response and that IFN signature, inflamed signature and BATF3 signature were mainly positive predictive. Using NanoString spatial profiling, we found that PD-L1, PD$1, \beta 2 \mathrm{M}$ were associated with favorable outcome.

Conclusions

The combination of IPI+NIVO in the (neo-)adjuvant treatment setting for high risk stage 3 melanoma patients is promising and currently tested in an international phase 2 randomized trial comparing different combination schemes (OpACIN-neo trial, NCT02977052) with the aim of preserving efficacy, but reducing toxicity. Biomarkers identifying patients responding upon neo-adjuvant IPI+NIVO and remaining relapse-free for a long time, will help to select the patients that need and don't need to be exposed to IPI+NIVO associated toxicity.

Trial Registration

ClinicalTrials.gov Identifier NCT02437279

\section{P100}

Pretreatment gene expression correlation with clinical response to pembrolizumab or nivolumab in metastatic melanoma

Sarah Warren ${ }^{1}$, Patrick Danaher ${ }^{1}$, Tobias Schatton ${ }^{2}$

${ }^{1}$ NanoString Technologies, Seattle, WA, USA; ${ }^{2}$ Brigham-Women's Hospital, Boston, MA, USA

Correspondence: Sarah Warren (swarren@nanostring.com)

Journal for ImmunoTherapy of Cancer 2017, 5(Suppl 2):P100 


\section{Background}

PD-1 checkpoint blockade with the therapeutic antibodies, pembrolizumab or nivolumab, improves survival in patients with metastatic melanoma. However, not all patients experience clinical benefit. The NanoString $T$ cell Inflamed Gene Expression Profile (GEP) measures genes associated with CD8+ T cells, natural killer cells, IFN signaling, and antigen presentation that are reflective of a pre-existing, PD-1suppressed adaptive immune response within the tumor, and associated with positive treatment outcomes to pembrolizumab in advanced-stage melanoma and other solid tumors. Here, we evaluate the GEP in pre-treatment tumor biospecimens obtained from patients receiving either nivolumab or pembrolizumab. Further, this study examines differential gene expression in PD-1-responsive vs. non-responsive melanomas to identify potential resistance mechanisms, which could be targets for therapeutic intervention.

Methods

Formalin-fixed, paraffin-embedded pre-treatment tumor biopsies from patients receiving either nivolumab or pembrolizumab in the community setting were profiled with NanoString's nCounter gene expression codesets. A total of 110 patients were included from three independent cohorts. Clinical benefit to PD-1 therapy was defined as an immune-related complete or partial response. PD-L1 expression on tumor cells was evaluated by immunohistochemistry in 36 samples from the pembrolizumab-treated cohort using the $22 \mathrm{C} 3$ anti-PD-L1 antibody clone, and staining was scored by two independent pathologists.

Results

The GEP predicted clinical benefit from pembrolizumab $(n=85)$, but was not predictive for nivolumab $(n=25)$, although the small sample size may limit statistical power. We plan to expand the nivolumab cohort prior to presentation of these results. Additionally, the GEP was more predictive of response to pembrolizumab than PD-L1 IHC, as assessed by ROC curves. Furthermore, clinical response to either therapeutic antibody was associated with an enrichment of genes expressed by lymphoid cells, as determined by cell type-specific gene expression. Additional analyzes of the total cohort are ongoing and will be presented.

Conclusions

The TIS predicted clinical response to pembrolizumab, but appears not to predict response to nivolumab. Validation and further studies will be required to determine if this observation holds true in larger cohorts, and if it relates to differences between the two agents.

\section{P101}

Multiplex biomarker detection in FFPE tumor samples

Mike Millar, Louise Welch, Dawn Lyster, Mariana Beltran, Sevi Giakoumelou

Aquila BioMedical, Edinburgh, UK

Journal for ImmunoTherapy of Cancer 2017, 5(Suppl 2):P101

\section{Background}

Immuno-oncology (IO) drug discovery research requires a multi-staged approach: pre-clinical in vitro assays, in vivo studies and histological analysis. We demonstrate the power of incorporating a specialist histological approach in formalin fixed paraffin embedded (FFPE) tissues early in the drug development process can provide high value data to complement functional in vitro assay readouts to inform on cancer immunotherapy clinical trial strategy.

Simultaneously imaging multiple IO markers on a section provides morphological, pathological and spatial information of the juxtaposition of different immune cell types (quantified by image analysis techniques). Multispectral imaging approaches:

1. Single immunofluorescence (IF), immunohistochemistry (IHC) and RNAscope (mRNA)

2. Dual and triple IF, IHC and RNAscope

3. Multiplex IF, IHC and RNAscope
4. Mixed multiplex detection of protein, mRNA and/or proteinmRNA

5. Quantitative image analysis

\section{Methods}

$4 \mu \mathrm{m}$ FFPE sections were stained using validated protocols on automated staining platforms (Leica Bond III/RX). Imaging performed using colorimetric and fluorescent scanners.

Results

Automated single-plex IHC or IF demonstrates protein (Figure 1) and mRNA (Figure 2) expression. Multiple protein targets are visualised using colorimetric (Figure 3) and 4-plex protein IF staining (Figures 47). It is possible to stain 6 or 7 markers on one section (Figures $8 \&$ 9), visualising the cellular location and spatial distribution of markers in relation to each other.

\section{Conclusions}

Specialised histological staining approaches utilised during drug discovery research provide valuable insights into the spatial expression of IO biomarkers. A pragmatic approach to antibody selection and validation powerfully combines with RNAscope to facilitate demonstration of novel and challenging IO biomarkers in situ, and can complement pre-clinical in vitro assay data.

\section{P102}

Mining the periphery for tumor-relevant $B$ and $T$ cells

Katherine L. Williams', Lukas Pfannenstiel ${ }^{2}$, Eldar Giladi ${ }^{1}, Y_{\text {Vvonne Leung }}{ }^{1}$, Xiaobin Tang ${ }^{1}$, Shuwei Jiang ${ }^{1}$, Dongkyoon Kim ${ }^{1}$, Kevin Williamson?', Sean M. Carroll', Ngan Nguyen', Gregg Espiritu Santo', Sini Reponen', Xiaomu Chen', Norman M. Greenberg', Daniel Emerling ${ }^{1}$

Wayne Volkmuth?', Brian Gastman², Guy Cavet

${ }^{1}$ Atreca, Inc., Redwood City, CA, USA; ${ }^{2}$ Cleveland Clinic Lerner Research Institute, Cleveland, OH, USA

Correspondence: Brian Gastman (kwilliams@atreca.com); Guy Cavet Journal for ImmunoTherapy of Cancer 2017, 5(Suppl 2):P102

\section{Background}

Identification of tumor-specific $T$ and B cells is key to understanding the immune response to cancer, but tumor tissue is only available in rare circumstances. Here we identify biomarkers for more accurate identification of tumor-reactive B and T cells from peripheral blood.

Methods

Matched peripheral blood and tumor samples from four donors with either advanced head and neck cancer or melanoma were analyzed by flow cytometry using markers including CD3, CD4, CD8, CD19, CD20, CD28, CD38, HLA-DR, NKG2D and TIGIT. From the periphery, we index sorted $\mathrm{CD}^{+} \mathrm{CD}^{+}{ }^{+} \mathrm{PD} 1^{+} \mathrm{T}$ cells and collected $\mathrm{CD} 19^{+} \mathrm{CD} 20$ $\mathrm{CD}^{+} 8^{+}$plasmablasts (PBs), as these are phenotypes likely to harbor tumor-reactive cells, and generated paired $\alpha / \beta$ and heavy/light chains from single cells. For tumor samples, $\mathrm{CD}^{+} \mathrm{T}^{\mathrm{T}}$ cells, CD19 ${ }^{+} \mathrm{CD} 20^{-} \mathrm{CD} 38^{+} \mathrm{PBs}$ and $\mathrm{CD} 19^{+} \mathrm{CD} 20^{+} \mathrm{B}$ cells were sorted and RNA was extracted for deep sequencing of $\beta$ and heavy chains. Tumor-derived cells were deep sequenced to identify the largest possible numbers of clones present in tumor. T cell lineages were defined as having identical $\beta$-CDR3 nucleotide sequences, while $B$ cell lineages were defined as having identical $V_{H}$ genes, and $80 \%$ amino acid similarity in the H-CDR3.

Results

We generated an average of 390 (314-468) paired $\alpha / \beta$ sequences from peripheral blood $\mathrm{CD}^{+} \mathrm{CD} 8^{+} \mathrm{PD} 1^{+} \mathrm{T}$ cells, and $4,692,4,968,7,346$ and 108,032 clones from deep sequencing of tumor $\mathrm{CD}^{+} \mathrm{CD}^{+} \mathrm{T}$ cells. $63 \%$ of peripheral $\mathrm{CD}^{+} \mathrm{PD} 1^{+} \mathrm{T}$ cell clones overlapped with tumor-resident $\mathrm{CD} 8+$ clones. Among peripheral $\mathrm{CD} 8{ }^{+} \mathrm{PD} 1^{+} \mathrm{T}$ cells, CD28- clones had significantly greater overlap with tumor-resident T cell clones than $\mathrm{CD}_{2} 8^{+}$clones ( $\mathrm{p}=0.005$, Fisher's exact test). There was also a trend toward greater overlap with tumor clones for the $\mathrm{CD} 38^{+}$subset of $\mathrm{CD} 8^{+} \mathrm{PD} 1^{+}$peripheral T cells. Additional analyzes are underway to evaluate other T cell markers. 
In analysis of B cell responses in two donors, $53.2 \%$ and $52.4 \%$ of peripheral plasmablast lineages overlapped with tumor B cell lineages. Additionally, the peripheral PBs that overlapped with the tumor population had a higher number of nucleotide mutations than the peripheral PBs that did not overlap ( 32.49 vs $30.08, p=0.06$, two-sided t-test).

\section{Conclusions}

In this study, we demonstrated significant overlap between peripheral plasmablasts, peripheral $\mathrm{PD} 1^{+} \mathrm{CD} 8^{+} \mathrm{T}$ cells and the tumor lymphocyte populations of advanced cancer patients. We further demonstrated that markers such as CD28 may be used to enrich for tumor-resident clones. Subsequent studies will expand upon these analyzes with additional donors and markers.

\section{References}

1. Van der Loos, Houtkamp, de Boer, Teeling, van der Wal, Becker. Immunohistochemical detection of interferon-gamma: fake or fact? J Histochem Cytochem. 2001;49(6):699-710

2. Toth, Mezey. Simultaneous visualization of multiple antigens with tyramide signal amplification using antibodies from the same species. J Histochem Cytochem. 2007;55(6):545-554.

3. Tsujikawa, Kumar, Borkar, Azimi, Thibault, Chang, Balter, Kawashima, Choe, Sauer, El Rassi, Clayburgh, Kulesz-Martin, Lutz, Zheng, Jaffee, Leyshock, Margolin, Mori, Gray, Flint, Coussens. Quantitative multiplex immunohistochemistry reveals myeloid-inflamed tumor-immune complexity associated with poor prognosis. Cell Rep. 2017;19(1):203-217.

\section{P103}

Tumor infiltrating lymphocyte (TIL) percentage as a prognostic biomarker for overall and relapse free survival in Hu14.18-IL2 treated resectable recurrent stage 3 or $\mathbf{4}$ melanoma patients Richard Yang ${ }^{1}$, Mark Albertini ${ }^{2}$, Jacquelyn Hank ${ }^{2}$, Hans Loibner ${ }^{3}$, Stephen Gillies ${ }^{4}$, Paul Sondel ${ }^{2}$, Erik Ranheim ${ }^{2}$

${ }^{1}$ University of Wisconsin - Madison Hospitals and Clinics, Madison, Wl, USA; ${ }^{2}$ University of Wisconsin - Madison School of Medicine and Public Health, Madison, WI, USA; ${ }^{3}$ Apeiron Biologics, Vienna, Austria;

${ }^{4}$ Provenance Biopharmaceuticals, Carlisle, MA, USA

Correspondence: Richard Yang (rkenny168@gmail.com); Paul Sondel Journal for ImmunoTherapy of Cancer 2017, 5(Suppl 2):P103

\section{Background}

Recurrent stage 3 or 4 melanoma patients (pts) have a poor prognosis. The presence of tumor infiltrating lymphocytes (TILs) have shown prognostic value in pt survival in melanoma, particularly for pts receiving checkpoint blockade immunotherapy. We investigated the prognostic value of TILs identified on H\&E sections of tumors from pts who have already undergone one cycle of hu14.18-IL2 (an immunocytokine fusion protein of anti-disialoganglioside antibody and IL-2) immunotherapy compared to hu14.18-IL2 naïve tumors. Methods

Between 2008-2012, twenty-three stage 3 or 4 recurrent, but resectable, melanoma pts were enrolled in CO-05601 (NCT00590824) and randomized to receive the first of 3 courses of hu14.18-IL2 either before or after surgical resection of all sites of disease. At the time of surgery, two pts within this trial were found to be not completely resectable and were taken off study. Following routine pathologic assessment, representative sections of each tumor were coded and given to our board certified hematopathologist (author EAR) for TIL assessment. Scatter plots were made for exploratory analyzes to interrogate the relationship between treated TILs and survival outcomes with stratification based on whether pts had hu14.18-IL2 treatment. Kaplan Meier survival curves interrogated the relationship between TILs and survival outcomes (relapse free survival [RFS] and overall survival [OS]) in relation to treatment status.

Results

There was a significant correlation between increased percentage of TILs and increased RFS $(p=0.0166 ; n=13)$ and OS $(p=0.0223, n=13)$ in pts whose tumors were obtained after hu14.18-IL2 treatment. These correlations were not seen in pts whose tumors were obtained prior to hu14.18-IL2 (not significant, $\mathrm{n}=8$ ). In addition, when comparing $\mathrm{pt}$ tumors with high TILs (above the median of $5 \%, n=11$ ) and low TILs (below $5 \%, n=10)$, there was an increase in RFS ( $p=0.0446$, median survival: high TILs - not reached, low TILs - 4.65 months) and a trend towards increased OS ( $p=0.0540$, median survival: high TILs - not reached, low TILs - 45 months, Fig. 1a-b). Additionally, hu14.18-IL2 treated pt tumors with high TILs ( $n=6$, median RFS and OS not reached) showed significantly increased RFS $(p=0.0159)$ and OS $(p=0.0208)$ compared to hu14.18-IL2 treated pt tumors with low TILs $(n=5$, median RFS - 4.2 months; OS - 41.3 months (Fig. 1C-D), whereas hu14.18-IL2 naïve tumors did not show this relationship.

\section{Conclusions}

These results suggest that resection specimens and biopsies obtained post-hu14.18-IL2 immunotherapy should be further investigated to monitor for TILs, as they may be associated with a beneficial immunotherapeutic effect.

\section{Trial Registration}

ClinicalTrials.gov Identifier NCT00590824

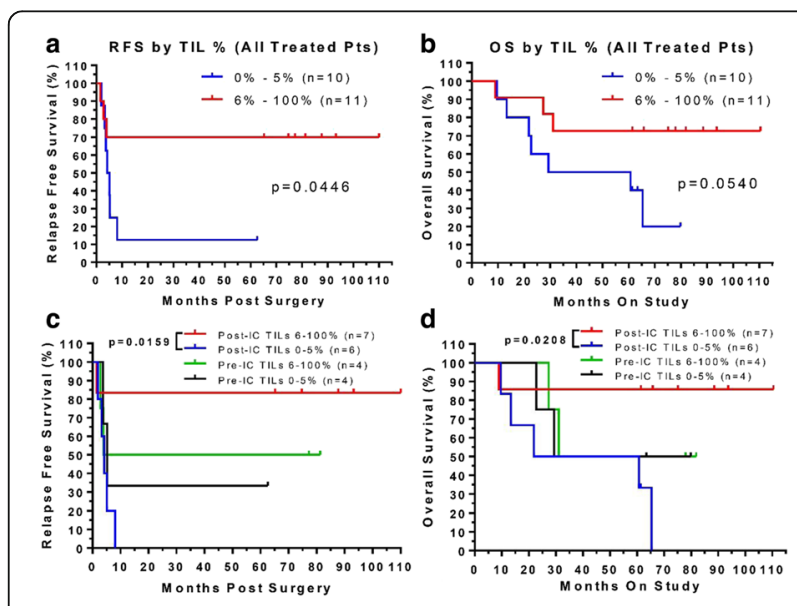

Fig. 1 (abstract P103). TIL Percentage Prognosticates Increased OS and RFS in Hu14.18-IL2 Treated Melanoma Patients

P104

A study of PD-L1 diagnostic assay concordance in urothelial carcinoma

Magdalena Zajac ${ }^{1}$, Marietta Scott ${ }^{1}$, Marianne Ratcliffe ${ }^{2}$, Alan Sharpe ${ }^{1}$, Paul W Scorer ${ }^{1}$, Craig Barker', Hytham Al-Masri ${ }^{3}$, Marlon Rebelatto ${ }^{4}$, Jill Walker ${ }^{1}$

${ }^{1}$ AstraZeneca, Cambridge, UK; ${ }^{2}$ AstraZeneca, Macclesfield, UK; ${ }^{3}$ Hematogenix, Tinley Park, IL, USA; ${ }^{4}$ Medimmune, Gaithersburg, MD, USA

Correspondence: Magdalena Zajac (jubilee.stewart@parexel.com) Journal for ImmunoTherapy of Cancer 2017, 5(Suppl 2):P104

\section{Background}

In urothelial carcinoma (UC), multiple pharmaceutical companies are investigating programmed cell death ligand-1 (PD-L1) expression using different antibody clones, staining protocols, and scoring algorithms. A stronger understanding of the comparative technical performance of these assays will allow appropriate interpretation of 
clinical outcomes for patients with UC treated with different anti-PD1/PD-L1 therapies.

\section{Methods}

325 tumor biopsy samples from patients with UC were assessed using 4 commercially available PD-L1 diagnostic assays: Ventana SP263, Ventana SP142, Dako 28-8 and Dako 22C3. Assays were performed in an CLIA accredited laboratory, per the device protocol and scored for tumor cell (TC) and immune cell (IC) PD-L1 staining. Analytical concordance was calculated pairwise between assays using the Spearman $(\rho)$ rank correlation coefficient. Classification concordance, including agreement between clinically relevant scoring algorithms, was investigated using overall/positive/negative percentage (OPA/PPA/NPA) agreement at multiple cut-offs.

Results

Data indicated good association, with a Spearman correlation coefficient of $\geq 0.8$ for each pairwise comparison of the 4 assays for IC, and $\geq 0.7$ for TC. When applying the SP263 clinical algorithm to 22C3 there is good agreement on positive cases with SP263 (OPA, PPA, NPA $>80 \%$ ) and vice versa.

\section{Conclusions}

Analytically, SP263, 22C3, and 28-8 assays showed similar performance for TC and IC PD-L1 expression. However, while SP142 showed similar analytical performance to the other 3 assays for IC staining, it appeared to be the least sensitive with the fewest samples showing PD-L1 staining on TC. This confirms previous observations from studies on assay performance in NSCLC and HNSCC.

When comparing scoring algorithms, caution should be taken when applying any algorithms utilising TC measures of PD-L1 to SP142 due to differences in sensitivity of the assay. Concordance achieved with SP263 algorithm applied to the 22C3 assay and vice versa builds optimism that those 2 assays could be used interchangeably in informing patient treatment decisions for durvalumab.

\section{P105}

Infino: Bayesian inference to distinguish immune cell expression phenotypes, estimate immune infiltration into tumor microenvironment, and investigate exhausted T cell phenotype

Maxim Zaslavsky', Jacqueline Buros Novik', Eliza Chang ', Alexander Huang ${ }^{2}$, Josephine Giles ${ }^{2}$, Robert Orlowski ${ }^{2}$, E. John Wherry ${ }^{2}$, Jeffrey Hammerbacher ${ }^{1}$

${ }^{1}$ Icahn School of Medicine at Mount Sinai, New York, NY, USA;

${ }^{2}$ Perelman School of Medicine, Philadelphia, PA, USA

Correspondence: Maxim Zaslavsky (maxim@hammerlab.org)

Journal for ImmunoTherapy of Cancer 2017, 5(Suppl 2):P105

\section{Background}

Robust quantification of immune cell infiltration into the tumor microenvironment may shed light on why only a small proportion of patients benefit from checkpoint therapy. Indeed, the immune contexture has recently been associated with prognosis and response to therapy [1], and the exhausted T cell phenotype is likely to be implicated in response to checkpoint blockade [2]. However, traditional measurement of immune cell content around a tumor using cell surface markers is low-throughput, requiring manual intervention by a pathologist. Computational alternatives instead estimate immune cell type abundances by deconvolving the gene expression mixture measured in the tumor microenvironment.

Methods

After constructing synthetic gene expression mixtures with known immune cell abundances, we evaluate the performance of four existing immune cell mixture deconvolution methods [3]. Our analysis discovers that they have high confidence but substantial deconvolution error for certain mixtures. We then propose a new Bayesian method, infino, that learns the relationships between immune cell types to estimate their abundances in RNA-seq data. Modeling these relationships is crucial for our deconvolution ability, enabling investigation of phenotypes of interest.

Results

Using Bayesian generative modeling, infino estimates a probability distribution for the abundance of each immune cell type in an RNAseq mixture. We demonstrate comparable performance to state-ofthe-art infiltrate quantification methods, as well as more rigorous quantification through infino's probabilistic estimates at all levels of the immune cell type hierarchy. Additionally, we extend infino to estimate stromal and tumor cell proportions for a complete accounting of the tumor microenvironment. We present results from a patient cohort, as well as infino's estimation of the exhausted T cell phenotype, to investigate variation in response to checkpoint blockade. Conclusions

By modeling the relationships between immune cell types, our novel Bayesian approach to immune infiltrate quantification produces robust deconvolution of gene expression mixtures like those found in the tumor microenvironment. Our results help probe the differential effectiveness of immunotherapy.

References

1. Galon, Mlecnik, Bindea, Angell, Berger, Lagorce, Lugli, Zlobec, Hartmann, Bifulco, et al.Towards the introduction of the 'Immunoscore' in the classification of malignant tumors. J Pathol. 2014; 232(2):199-209.

2. Sen, Kaminski, Barnitz, Kurachi, Gerdemann, Yates, Tsao, Godec, LaFleur, Brown, et al. The epigenetic landscape of T cell exhaustion. Science. 2016; 354(6316):1165-1169.

3. Hackl, Charoentong, Finotello, Trajanoski. Computational genomics tools for dissecting tumor-immune cell interactions. Nat Rev Genet. 2016; 17(8):441-58

\section{P106}

Bioinformatic analysis of the tumor immune landscape to prioritize combinations with antibody-drug conjugates (ADCs)

Xiaoling Zhang, Song Wu, Brandon W. Higgs, Koustubh Ranade Medlmmune, Gaithersburg, MD, USA

Correspondence: Xiaoling Zhang (zhangx@medimmune.com) Journal for ImmunoTherapy of Cancer 2017, 5(Suppl 2):P106

\section{Background}

Immunotherapy has transformed the treatment of multiple tumors, yet significant unmet need remains. Antibody-drug conjugates (ADCs) have had limited success, with some evidence to support that they can reshape the tumor immune-environment to increase sensitivity to immunotherapy. Thus, combining immunotherapy and ADCs may enhance the benefit of both; however it is unclear how to prioritize such combinations. In this study, 52 ADC targets under clinical investigation were analyzed by bioinformatics approaches to examine the intersection of expression of these ADC targets and the tumor immune landscape to identify targets and pathways that may increase the likelihood of success of ADC through immunotherapy combinations.

\section{Methods}

Thirty-seven ADC targets were analyzed across 16 solid tumor types and 15 ADC targets were analyzed in 2 hematological cancers using transcriptome data in TCGA. Co-expression of ADC targets with inflamed or non-inflamed tumor microenvironment was defined based on a previously reported IFNy mRNA expression signature that showed strong association with ORR and survival in patients treated with durvalumab. Top ranked IFN $\gamma$-associated ADC targets were further analyzed to identify the common molecular signatures shared by these targets. 
Results

Out of 592 combination counts of 37 ADC targets in solid tumors, $163(28 \%)$ were associated with inflamed signature whereas $67(11 \%)$ were associated with non-inflamed signature. Out of 30 combination counts of 15 ADC targets in hematological cancers (AML and DLBCL), $14(47 \%)$ were associated with inflamed signature whereas $5(17 \%)$ were associated with non-inflamed signature. Top ranked ADC targets were associated with inflamed signature in a majority of tumor types (>10 out of 16), including those known to be either highly responsive or less responsive to immune checkpoint inhibitors. In-depth profiling of the top ranked ADC targets identified epithelial-mesenchymal transition (EMT) and tumor invasion/ metastasis to be the most commonly shared signatures among these ADC targets.

\section{Conclusions}

ADC target selection is usually based on differential expression between tumor and normal tissue but the tumor immune landscape is not considered. With the success of immunotherapy, our bioinformatics analysis suggests that selection of ADC targets co-expressed with immune inflamed, EMT and tumor invasion/metastasis signatures may be a strategy to further triage candidate ADC targets to increase the likelihood of success for effective combination of ADC and immunotherapies.

\section{Cancer Vaccines}

\section{P107}

CMV gB/pp65 eVLPs formulated with GM-CSF as a therapeutic vaccine against GBM

Catalina Soare, Tanvir Ahmed, Julie Joseph, Anne-Catherine Fluckiger, Barthelemy Ontsouka, Abebaw Diress, Jasminka Bozic, Marc Kirchmeier, Francisco Diaz-Mitoma, David E. Anderson

VBI Vaccines, Cambridge, MA, USA

Correspondence: David E. Anderson (danderson@vbivaccines.com) Journal for ImmunoTherapy of Cancer 2017, 5(Suppl 2):P107

\section{Background}

Failures of many past therapeutic cancer vaccines can be attributed to several common shortcomings including the inherently poor immunogenicity of the tumor antigens used, the failure to expand CD4

${ }^{+} \mathrm{T}$ helper cell responses that could sustain potent $\mathrm{CD} 8^{+} \mathrm{CTLS}$, and limited breadth of immunity directed against multiple antigens/epitopes that could avoid the rapid immunoselection of tumor escape variants.

Numerous independent laboratories using several techniques (ISH, immunohistochemistry, $\mathrm{T}$ cell recognition of autologous primary tumors) have demonstrated cytomegalovirus (CMV) antigens in over $90 \%$ of GBM tumors. Memory $\mathrm{CD}^{+}$and $\mathrm{CD}^{+}{ }^{+}$cells are most frequently directed against the $\mathrm{gB}$ and $\mathrm{pp} 65$ antigens at frequencies that can exceed $10 \%$. Thus, CMV gB and pp65 represent attractive, highly immunogenic "foreign" antigen components of a vaccine against GBM that can exploit high pre-cursor frequencies of $\mathrm{CD}^{+}$ and $\mathrm{CD} 8^{+} \mathrm{T}$ cells.

Methods

Enveloped virus-like particles (eVLPs) are produced after transfection of HEK 293 cells with a plasmid encoding murine leukemia virus Gag plasmid fused in-frame with CMV pp65 antigen, which gives rise to particles. Co-transfected CMV gB plasmid enables particles budding from the cell surface to incorporate the $\mathrm{gB}$ protein into the lipid bilayer. Results

In ex vivo studies using PBMCs from GBM patients we have demonstrated that $\mathrm{gB} / \mathrm{pp} 65$ eVLPs restimulate both $\mathrm{CD} 4^{+}$and $\mathrm{CD} 8^{+} \mathrm{T}$ cells at frequencies comparable to those observed for healthy $\mathrm{CMV}^{+}$subjects, and that formulation with GM-CSF augments IFN-g and CCL3 secretion. Biodistribution studies have demonstrated that the $\mathrm{gB} /$ pp65 eVLPs remain at the intradermal injection site for at least two weeks, with eVLP particles appearing in draining lymph nodes within a few hours. Mechanistic studies demonstrate that monocyte uptake of eVLP particles induces proinflammatory cytokines, which are amplified by $\mathrm{gB}$ expression on the surface of the gB/pp65 particles. Immunization of $\mathrm{CMV}^{+}$rhesus macaques with a macaque-specific version of the vaccine candidate confirmed the safety of the vaccine and the ability to boost pre-existing IFN-g-secreting T cell responses. Conclusions

An IND for a phase I/lla trial in recurrent GBM patients was filed with FDA in July, with subject enrollment expected in Q4 2017.

\section{P108}

Induction of folate receptor alpha-specific Th17 T cell immunity in ovarian cancer patients

Matthew Block', Kimberly Kalli', Jacob Allred', Courtney Erskine' Allan Dietz', Michael Gustafson', Yi Lin', Keith Knutson², Martin Cannon ${ }^{3}$

${ }^{1}$ Mayo Clinic, Rochester, MN, USA; ${ }^{2}$ Mayo Clinic, Jacksonville, FL, USA;

${ }^{3}$ University of Arkansas for Medical Sciences, Little Rock, AR, USA

Correspondence: Matthew Block (block.matthew@mayo.edu)

Journal for ImmunoTherapy of Cancer 2017, 5(Suppl 2):P108

\section{Background}

The nature of the endogenous immune response to ovarian cancer (OC) is a strong predictor of clinical outcomes; regulatory $\mathrm{T}$ cells (Tregs) predict poor survival, whereas interleukin 17 (IL-17)-producing $\mathrm{T}$ cells (Th17s) are favorably prognostic. We have developed a method by which ex vivo-matured dendritic cells (DCs) can preferentially drive expansion of Th17s [1]. A vaccine utilizing OC-specific Th17-inducing DCs might diminish OC-related Tregs and reduce the rate of OC recurrence.

Methods

Patients with stage IIIC and IV OC were enrolled following completion of initial surgery and chemotherapy. DCs were generated from autologous peripheral blood mononuclear cells (PBMCs) that were cultured according to our Th17-inducing protocol [1] and pulsed with peptides from the OC antigen folate receptor alpha (FRa). Mature antigen-loaded DCs (FRaDCs) were delivered to patients via intradermal injection in a series of five immunizations and up to seven boosters. Patient PBMCs were collected before and after vaccine induction and were assessed via Enzyme-Linked ImmunoSpot (ELISpot) assay for FRa-specific T cells capable of secreting IL-17 or IFNy. ELISpot responses were defined as at least a doubling of FRa peptideor protein-specific cytokine-secreting cells.

Results

Nineteen patients were enrolled and immunized with vaccine, with one patient subsequently excluded due to ineligibility. No grade 3 or higher adverse effects were seen. Vaccination resulted in the development of both Th17 and Th1 immunity to vaccine constituent FRa peptides as well as whole FRa protein in the majority of patients. Antibody responses to FRa were also seen following vaccination in some patients.

\section{Conclusions}

Specific induction of Th17 immunity against self-antigens in cancer patients is feasible and safe. The results of this study pave the way to future trials aimed at determining if vaccine-induced Th17 immunity prevents disease progression and improves survival.

\section{Trial Registration}

ClinicalTrials.gov Identifier NCT02111941

\section{Reference}

1. Cannon, Goyne, Stone, Macdonald, James, Cobos, Chiriva-Internati.Modulation of p38 MAPK signaling enhances dendritic cell activation of human CD4+ Th17 responses to ovarian tumor antigen. Cancer Immunol Immunother. 2013:62:839-849. 


\section{P109}

Anti-NY-ESO-1 immune response and survival benefit after LV305 therapy in patients with advanced sarcoma and other solid tumors Neeta Somaiah ${ }^{1}$, Matthew Block ${ }^{2}$, Joseph W. Kim³ ${ }^{3}$ Geoffrey Shapiro ${ }^{4}$, Khanh Tu Do ${ }^{4}$, Patrick Hwu', Joseph Paul Eder ${ }^{3}$, Robin Lewis Jones ${ }^{5}$, Sacha Gnjatic ${ }^{6}$, Hailing Lu', Jan H. ter Meulen', Michael Chen ${ }^{9}$, Chet Bohac $^{8}$, Frank Hsu ${ }^{8}$, Seth Pollack ${ }^{10}$

${ }^{1} \mathrm{MD}$ Anderson Cancer Center, Houston, TX, USA; ${ }^{2}$ Mayo Clinic, Rochester, MN, USA; ${ }^{3}$ Yale University, New Haven, CT, USA; ${ }^{4}$ Dana Farber Cancer Institute, Brookline, MA, USA; ${ }^{5}$ Royal Marsden NHS, London, UK; ${ }^{6}$ Tisch Cancer Institute, New York, CA, USA; ${ }^{7}$ Immune Design, Seattle, WA, USA; ${ }^{8}$ Immune Design, South San Francisco, CA, USA; ${ }^{9}$ Biostatistics, South San Francisco, CA, USA; ${ }^{10}$ Fred Hutchinson Cancer Research Center, Seattle, WA, USA

Correspondence: Seth Pollack (chet.bohac@immunedesign.com) Journal for ImmunoTherapy of Cancer 2017, 5(Suppl 2):P109

\section{Background}

LV305 is a dendritic cell targeting lentiviral vector encoding NY-ESO1 designed to generate and expand anti-NY-ESO-1 T cells. This firstin-human study showed LV305 is safe, induced anti-NY-ESO-1 CD4/ CD8 T cells, and provided durable disease control with a 1-yr overall survival (OS) of $81 \%$ in sarcoma patients (pts)(N. Somaiah, ASCO 2016). We now present immune response (IR) and long term OS data.

Methods

Previously treated, advanced NY-ESO-1+ solid tumor pts were eligible. Part 1 dose escalation, 4 cohorts received 3 or 4 intradermal injections every 3 weeks of $10^{8}, 10^{9}$ or $10^{10}$ vector genomes $(\mathrm{vg}) /$ dose; $10^{10} \mathrm{vg} /$ dose in part 2 expansion. Anti-NY-ESO-1 antibody (Ab) and T cell IR measured by ELISA, and ELISpot and T cell receptor sequencing, respectively.

Results

As of March 31, 2017, 39 pts (13 synovial sarcoma, 6 myxoid round cell liposarcoma, 4 other soft tissue sarcoma (STS), 1 Ewing's sarcoma, 9 ovarian cancer, 5 melanoma, 1 NSCLC) were enrolled, median (m) duration of observation 17.2 months (mos). For the 24 sarcoma pts (mean age 48.1 yrs, $87.5 \%$ relapsed metastatic, 100\% previously treated), mPFS 4.67 mos and mOS not reached (NR). Exploratory analysis of biomarker data showed: 1) Survival (mos (95\% CI)) and presence of baseline anti-NY-ESO-1 Abs: For all pts combined, mOS was NR $(17.9, N R)$ for NY-ESO-1 $A b+(n=12)$ vs $27.8(10.3,27.8)$ for NYESO-1 Ab- $(n=19)$, HR $0.28 p$ value 0.09 . For STS patients $(n=23), \operatorname{mOS}$ was NR (NR, NR) $(n=8)$ vs $18.8(10.3,27.8)(n=14)$, HR $0.0 p$ value 0.02 , respectively. 2) Survival and LV305 induction of IR: Of 31 pts tested, $52 \%$ pts developed NY-ESO-1 specific T cells; $3 \%$ pts developed antiNY-ESO-1 Abs after LV305 therapy. 3) Survival and IR: For all pts combined, mOS was $27.8(18.8,27.8)$ for $I R+(n=21)$ vs $10.3(4.0, N R)$ for IR- $(n=10)$, HR $0.32 \mathrm{p}$ value 0.06 . Pts who had anti-NY-ESO-1 IR both at baseline and induced had the best survival: mOS was 27.8 (17.9, 27.8) $(n=14)$ vs $8.6(6.0, N R)$ in pts who had no evidence of IR at baseline nor on therapy $(n=4)$, HR $0.17 p$ value 0.018 .

\section{Conclusions}

LV305 is a well-tolerated therapy which induces anti-NY-ESO-1 IR and may improve OS in sarcoma pts. Pts with baseline anti-NY-ESO-1 Abs or induced IR on LV305 therapy appear to have a better survival. Trial Registration ClinicalTrials.gov Identifier: NCT02122861P110

\section{P110}

MEK inhibition augments Talimogene laherparepvec (T-VEC)mediated in vitro melanoma oncolysis and induces in vivo tumor regression

Howard Kaufman, Praveen Bommareddy, Anne Silk, Andrew Zloza, Sachin Jhawar

Rutgers University, New Brunswick, NJ, USA

Correspondence: Howard Kaufman (pkb38@gsbs.rutgers.edu)

Journal for ImmunoTherapy of Cancer 2017, 5(Suppl 2):P110

\section{Background}

Herpes simplex virus, type 1 (HSV-1) encoding GM-CSF (Talimogene laherparepevec; T-VEC) is the first and only FDA approved oncolytic virus for the treatment of melanoma. We recently found that T-VEC induces apoptosis in melanoma cells in vitro, and this effect is enhanced with concurrent MEK inhibition. In this study, we sought to determine how MEK inhibition enhances tumor cell lysis and determine the therapeutic effects of combination treatment in a melanoma xenograft model

Methods

BRAF wild-type and mutated melanoma cell lines were plated in 96-well plates $\left(10^{4}\right.$ cells per well) and treated with T-VEC (MOI 0.001-1.0). Cell viability was assessed by standard MTS assay. After establishing baseline viability results, cells were also treated with MEK inhibitors (Trametinib or PD0325901 at 1-100 nM) and cell viability determined by MTS assay. Viral replication was measured by plaque assay and the infection metric was analyzed using Lumacyte single-cell optical density. Apoptosis was determined by Annexin $V$ flow cytometry and cleaved PARP immunoblotting. For in vivo experiments, NSG mice were challenged with SKMEL-28 $\left(5 \times 10^{6}\right)$ at day 0 and treated with intra-tumoral TVEC $\left(10^{6} \mathrm{PFU}\right)$ biweekly and/or trametinib $(0.1 \mathrm{mg} / \mathrm{kg})$ by oral gavage for 2 weeks. Tumor growth was measured by calipers. Statistical comparisons between treatment groups were determined using the student's $t$ test with $\mathrm{P}<0.05$ being considered statistically significant.

Results

Combination T-VEC and trametinib significantly increased melanoma cell death $(P<0.01)$. A similar effect was seen with a second MEK inhibitor ( $P D$ 0325901) $(P<0.001)$. The combination caused a significant increase in viral replication and increased apoptosis. Combination treatment was associated with morphologic changes in tumor cells and an increased infection metric by Lumacyte. A PCA analysis distinguished virally-infected cells and those treated with both virus and MEK inhibitor from uninfected cells. A significant reduction in tumor volume was also observed in SKMEL-28 NSG xenografts during the combination treatment compared to monotherapy with T-VEC or MEK inhibition (Figs. 1 and 2).

\section{Conclusions}

Combination T-VEC and MEK inhibition enhances tumor cell killing and viral propagation in vitro and induces tumor regression in vivo. Combining MEK inhibitors with T-VEC represents an attractive therapeutic option and further studies are needed to understand the impact of this combination on induction of host anti-tumor immunity.

\section{Reference}

1. Somaiah, Neeta, et al. American Society of Clinical Oncology (ASCO) Annual Meeting. 2016; Abstract \#3093.

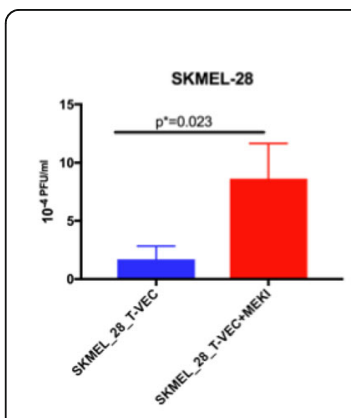

Principal Component Analysis

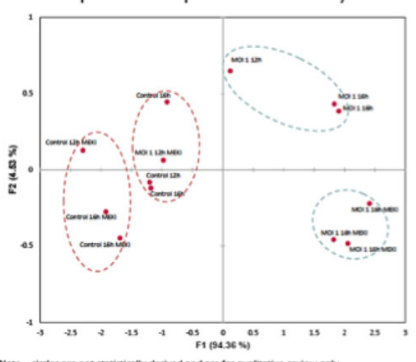

Fig. 1 (abstract P110). MEK Inhibition increases viral titers in T-VEC treated SKMEL28 Melanoma cell line 


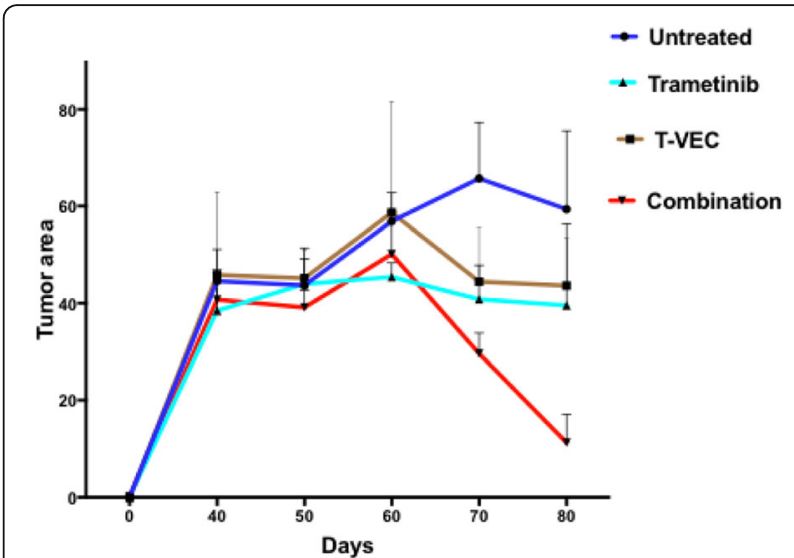

Fig. 2 (abstract P110). Trametinib augments T-VEC mediated tumor regression in Xenograft melanoma model. NSG mice with SKMEL28 xenograft treated with Trametinib and/or T-VEC

\section{P111}

Initial safety and production efficiency of an autologous tumor lysate-loaded yeast cell wall particle vaccine

Tommy Brown 1 , Kaitlin Peace ${ }^{1}$, Myers John', Timothy Vreeland², Diane Hale', Doreen Jackson', Julia Green', Jamie Berry ${ }^{3}$, Clifton Guy', Herbert Garth', Xianzhong Yu ${ }^{4}$, Thomas Wagner ${ }^{5}$, George Peoples ${ }^{6}$ ${ }^{1}$ Brooke Army Medical Center, San Antonio, TX, USA; ${ }^{2}$ University of Texas MD Anderson Cancer Center, Houston, TX, USA; ${ }^{3}$ Womack Army Medical Center, Fort Bragg, NC, USA; ${ }^{4}$ Clemson University, Clemson, SC, USA; ${ }^{5}$ Orbis Health Solutions, Greenville, SC, USA; ${ }^{6}$ Metis Foundation, San Antonio, TX, USA

Correspondence: Tommy Brown (tommy.alan.brown.ii@gmail.com) Journal for ImmunoTherapy of Cancer 2017, 5(Suppl 2):P111

\section{Background}

Yeast cell wall particles (YCWP) have been used to create an autologous tumor lysate, particle-loaded, dendritic cell (TLPLDC) vaccine with encouraging initial clinical results. Direct inoculation of an autologous tumor lysate loaded, particle only (TLPO) vaccine eliminates the need for ex vivo DC loading, greatly reducing production requirements compared to TLPLDC. Our preclinical work showed that YCWP can be loaded with autologous tumor lysate (TL) and capped with silicate (Si) to seal in the TL content and attract a monocytic infiltrate. We have shown that silicate capping enhances TL retention, DC phagocytosis and cytoplasmic delivery of the YCWP contents. Here, we present the initial production efficiency and safety data from an exploratory phase I basket trial of the autologous TLPO vaccine.

Methods

YCWP are created from Saccharomyces cerevisiae by $\mathrm{NaOH} / \mathrm{HCl}$ digestion of all non-cell wall components and washed with isopropanol/ acetone resulting in $\beta$-glycan shells. Autologous TL is created by freeze/thaw cycling of $\geq 1 \mathrm{mg}$ of tumor and loaded into YCWP along with CPG oligonucleotides and a tetanus helper peptide by dissolution and freeze-drying. The TL-loaded YCWP are then capped with $\mathrm{Si}$, aliquoted in single dose vials and cryopreserved. The TL protein content of production batches is determined by measuring the nitrogen content by combustion analysis, while Si cap thickness is calculated based on $\mathrm{Si}$ content by mass spectrometry. The vaccine is administered intradermally at $1.0 \times 10^{8}$ particles monthly $\times 4$ followed by boosters every 3 months until progression.

Results

Nine patients with stage I-IV malignancies(non-small cell lung, pseudomyxoma peritonei, prostate, ovarian, pancreatic, breast, desmoplastic small round cell tumor, esophageal, and renal cell carcinoma) aged 21-85(median 52) have been enrolled and received between 1 to 3 inoculations of TLPO to date. Enrollment is ongoing. Vaccine production has been successful in $100 \%$ of patients. In the production runs of the vaccine, the mean TL protein content was 16.56 $+/-0.56 \%$ of each inoculation by weight. The Si cap thickness was $0.0615+/-0.0009$ microns (approximately 140 molecular layers of Si). In the 17 inoculations thus far, there has been no toxicity >grade 2 . Patients are due for their first set of imaging shortly.

\section{Conclusions}

TLPO vaccine is reliably and efficiently produced from autologous tumor tissue with sufficient loading of TL and sufficient Si capping to maintain TL within the YCWP. The vaccine is well tolerated with minimal toxicity thus far. The favorable production characteristics and safety profile combined with improved production efficiency of the TLPO compared to the TLPLDC vaccine warrant continued development of this vaccine platform.

P112

Telomerase peptide vaccine treatment of patients with locally advanced or metastatic non-small cell lung cancer: Report from a phase I/IIA trial

Tormod Kyrre Guren', Else Marit Inderberg², Paal Fr. Brunsvig', Odd Terje Brustugun ${ }^{3}$, Jon Amund Kyte', Jens Bjørheim ${ }^{4}$, Wenche Rasch ${ }^{4}$,

Claudius H. Reisse', Gustav Gaudernack ${ }^{4}$, Steinar Aamdal ${ }^{1}$

${ }^{1}$ Oslo University Hospital, Oslo, Norway; ${ }^{2}$ University of Oslo, Oslo,

Norway; ${ }^{3}$ Vestre Viken Hospital Trust, Drammen, Norway; ${ }^{4}$ Ultimovacs AS, Oslo, Norway

Correspondence: Paal Fr. Brunsvig (pfb@ous-hf.no)

Journal for ImmunoTherapy of Cancer 2017, 5(Suppl 2):P112

\section{Background}

At the initiation of this study in 2013 second and later lines treatment of patients with stage III or IV NSCLC was chemotherapy or radiotherapy with limited median survival.

UV1 is a therapeutic cancer vaccine consisting of three long synthetic peptides of the enzyme telomerase (hTERT). The UV1 peptides comprise epitopes recognized by $T$ cells from cancer patients experiencing long-term survival following vaccination with first-generation hTERT vaccines. The aim was to investigate the safety, induction of immune response (IR) towards the hTERT peptides and efficacy. Three different UV1-doses were investigated.

Methods

Patients with locally advanced or metastatic NSCLC received UV1 $(100 \mu \mathrm{g}, 300 \mu \mathrm{g}$ or $700 \mu \mathrm{g})$ and $75 \mu \mathrm{g} \mathrm{GM}-\mathrm{CSF}$ as adjuvant [EudraCT No. 2012-001852-20]. All patients had received prior lines of chemotherapy and/or radiation therapy, and had no evidence of disease progression at the time of inclusion. Safety was assessed according to CTCAE v. 4.0 and tumor responses according to RECIST v.1.1. Immune response (IR) against UV1 peptides was monitored by $T$ cell proliferation assays and, if sufficient cell numbers, IFN- $\gamma$ ELISPOT assays. Patients are to be followed up for five years after their first UV1 treatment. Results as of Q2-2017 are reported.

Results

18 patients (10 females), mean age 65.9 yrs. (48-76), with locally advanced or metastatic (stage III or IV) NSCLC were included. Ten patients had adenocarcinoma and eight had squamous cell carcinoma. Six patients were enrolled in each dose group. Patients received between 9 and 18 doses of UV1 each (mean 12.5 doses, total of 224). Treatment was generally well tolerated. Adverse events mainly included injection site reactions and fatigue. No treatment related serious adverse events were reported.

IR was induced against UV1 in 12 of 18 patients (67\%). Fifteen patients were evaluated for tumor response after nine months, 12 patients had SD and three patients had PD. Median overall survival was 23.6 months. After two years, seven patients were alive, two and five in the 300 and $700 \mu \mathrm{g}$ dose group, respectively. 


\section{Conclusions}

UV1 is safe and well tolerated and induce an UV1 specific IR in the majority of the patients (67\%). UV1 is currently under development in different solid tumors as combination therapy.

Trial Registration

EudraCT No. 2012-001852-20

\section{P113}

OncoPeptVAC: A robust TCR binding algorithm to prioritize neoepitope using tumor mutation (DNAseq) and gene expression (RNAseq) data

Papia Chakraborty, Amit Chaudhuri, Vasumathi Kode, Snigdha Majumdar, Priyanka Shah, Malini Manoharan, Ravi Gupta

MedGenome Inc, Foster City, CA, USA

Correspondence: Amit Chaudhuri (papia.c@medgenome.com)

Journal for ImmunoTherapy of Cancer 2017, 5(Suppl 2):P113

\section{Background}

Neoepitopes are tumor-derived immunogenic peptides that arise from intracellular proteolytic processing of somatic mutation in protein coding genes. These peptides bind HLA Class I proteins and are presented on the surface by antigen presenting cells. Productive engagement of HLA Class I-bound peptide with T cell receptor (TCR) activates CD8+ T cells to generate cytotoxic $T$ cells, which mediate lysis of the neoantigen-expressing tumor cells. Neoepitopes can be used as cancer vaccines to prime $\mathrm{CD} 8^{+} \mathrm{T}$ cells against tumor cells. As tumors accumulate hundreds of mutations during cancer development and only a small subset of these are immunogenic, identifying the neoepitopes requires accurate modeling of the steps involved in peptide production, presentation as well as TCR binding.

\section{Methods}

Most current pipelines prioritize neoepitopes based on the expression of mutant proteins and their HLA binding affinity. However, presentation of peptides on the surface is not sufficient to activate T cells. To improve the predictive power of our neoepitope prioritization pipeline and to circumvent selection biases that are inherent with using HLA binding as a proxy for neoepitope prediction, we developed a novel algorithm that prioritizes peptide interactions with the TCR. This algorithm uses features selected by analyzing crystal structures of TCR and HLA-peptide complex present in the Protein Data Bank. A neural network model was built to derive a composite score that includes, besides TCR binding, other features associated with the peptide, such as level of expression of the mutant allele, affinity of HLA binding, affinity of TAP binding and sensitivity to proteasomal processing.

Results

We validated our neural net prediction model on known immunogenic and non-immunogenic peptides and achieved superior accuracy, sensitivity and specificity of prediction compared to using the standard HLA binding affinity of $\leq 500 \mathrm{nM}$. By applying OncoPeptVAC neoepitope prioritization solution to 2.2 million unique somatic mutations, we identified 700 immunogenic peptides derived from recurrent somatic mutations in all cancers. Several of these peptides were validated on a $\mathrm{CD}^{+}{ }^{+} \mathrm{T}$ cell-activation assay.

\section{Conclusions}

Our in silico prioritization platform combined with the cell-based validation method is a powerful tool to identify therapeutic vaccines for personalized cancer immunotherapy applications.

\section{P114}

No toxicity and long-term survival up to 16-years from repeated oncolytic vaccine systemic immunotherapy in advanced melanoma: Teaching us lessons

Brendon Coventry ${ }^{1}$, Carrie Lilly ${ }^{1}$, Tony Michele ${ }^{2}$, Peter Hersey ${ }^{3}$

${ }^{1}$ University of Adelaide, Adelaide, Australia; ${ }^{2}$ Adelaide Oncology \&

Haematology, North Adelaide, South Australia, Australia; ${ }^{3}$ University of Sydney, Sydney, Australia

Correspondence: Brendon Coventry

(brendon.coventry@adelaide.edu.au)

Journal for ImmunoTherapy of Cancer 2017, 5(Suppl 2):P114

\section{Background}

We have previously reported very long-term complete responses with survival of $\geq 5$ years to oncolytic melanoma vaccine therapy for patients with advanced melanoma. The Complete Response (CR) Rate was also higher than expected at $17 \%$ predominantly from therapy with vaccine alone [1]. Importantly, side-effects were negligible with no noticeable toxicity.

Methods

Extended analysis of patient survival was performed to investigate the presence of recurrence, late failure, morbidity, mortality and the longevity of responders.

Standard methods of outcome evaluation (CR, PR, SD, PD and overall survival) including Kaplan-Meier estimates, were performed.

Results

Clinical responses have continued to beyond 10-years with survival periods of now over 15-years in duration for some patients. These responders are being studied in more detail. CR's when obtained were durable in almost all cases.

Conclusions

Clinical responses and long-term survival using vaccine therapy alone is unusual, but continued to beyond 10-years with survival periods of now over 15-years is obtainable and this requires more investigation as a matter of priority. These responders are being studied in more detail. Some $84 \%$ of patients showed some clinically useful response, and no toxicity. These long-term survival [2] and high CR rates results were not from chance alone and were attributable to the vaccine administration \& scheduling most likely creating successful in-vivo immunomodulation.

Trial Registration

Australian Clinical Trials Registry [ACTRN] 12605000425695

\section{References}

1. Responses and survival for advanced metastatic melanoma. J Immunother Cancer. 2014;2: 9-17.

2. Coventry, Baum, Lilly. Long-term survival in advanced melanoma patients using repeated therapies: successive immunomodulation improving the odds? Cancer Manag. Res. 2015;7:93-103.

P115

Inflammatory response after immunotherapy with a yeast-CEA therapeutic cancer vaccine in metastatic medullary thyroid cancer Jaydira Del Rivero', Ann Gramza², Myrna Rausckhorts', Marijo Bilusic ${ }^{1}$, Fatima Karzai ${ }^{1}$, Julius Strauss ${ }^{1}$, Lisa Cordes ${ }^{1}$, William L. Dahut ${ }^{1}$, Jeffrey Schlom', James L. Gulley', Ravi A. Madan

${ }^{1}$ National Cancer Institute/National Institutes of Health, Bethesda, MD,

USA; ${ }^{2}$ Medstar Georgetown Lombardi Comprehensive Cancer Center,

Washington, DC, USA

Correspondence: Jaydira Del Rivero (jaydira.delrivero@nih.gov); Ravi A. Madan

Journal for ImmunoTherapy of Cancer 2017, 5(Suppl 2):P115

\section{Background}

Medullary thyroid cancer (MTC) accounts for approximately $4 \%$ of thyroid carcinomas. Although vandetanib and cabozantinib are approved for metastatic MTC, toxicity limits their use. There are ongoing trials at the $\mathrm{NCl}$ exploring the role of immunotherapy in MTC patients.

Methods

In a series of two clinical trials (including a phase II MTC study; NCT01856920), 30 MTC patients have been treated with a yeastbased vaccine targeting CEA (Bilusic $M$ et al., Immunol Immunother, 2014). The vaccine was scheduled every 2 weeks for 3 months; then monthly for 9 months and quarterly in the second year for patients without radiographic disease progression.

Results

$3 / 30(10 \%)$ of patients developed tumor site-specific inflammatory responses which are not typically seen in MTC. A 39-year-old woman with sporadic metastatic MTC and stable disease on restaging CT after 3 months of vaccine, developed dyspnea one week later. CT scan showed pleural and paricardial effusions at sites of known 
disease. After a work-up yielded no diagnosis, empiric steroids resolved symptoms in 48 hours and a follow-up CT-scan demonstrated resolution of the effusions. This patient from the phase I study was found to have robust antigen-specific $T$ cell responses. Similarly, a 72-year-old woman with sporadic metastatic MTC presented with an enlarging neck mass at a known site of metastatic disease after 21 months of vaccine. Due to difficulty swallowing, she received highdose steroids and empiric antibiotics at an outside hospital with resolution of her symptoms. A follow-up CT reported a decreased in size of the neck mass. Perhaps most interestingly, a 66-year-old woman with MEN2A and metastatic MTC returned to our clinic 17 months after completing the 2-year vaccine course with a rapidly enlarging lymph node at a known site of metastatic disease confirmed by CT. A biopsy of the lymph node was negative for tumor but demonstrated an abundance of CD3+ and CD20+ lymphocytes. The symptoms and lymph node size decreased within 1 week as did the patient's calcitonin in the following weeks.

\section{Conclusions}

We present 3 patients with metastatic MTC who presented with an inflammatory event after treatment with a yeast-CEA therapeutic cancer vaccine. These unexpected inflammatory events may demonstrate the potential for immunotherapy in MTC. A follow-up up phase II is ongoing at the $\mathrm{NCl}$ treating patients (both with previous immunotherapy and immunotherapy-naive) with pembrolizumab (NCT03072160) and analysis of the vaccine study is underway. Informed consent to publish had been obtained from all of the patients.

\section{P116}

Heat-inactivated modified vaccinia virus Ankara (MVA) as vaccine adjuvant

Ning Yang, Stewart Shuman, Taha Merghoub, Jedd Wolchok, Liang Deng

Memorial Sloan Kettering Cancer Center, New York, NY, USA

Correspondence: Liang Deng (dengl@mskcc.org)

Journal for ImmunoTherapy of Cancer 2017, 5(Suppl 2):P116

\section{Background}

Recent discoveries of cancer neoantigens and the potential of the combination of cancer vaccination with immune checkpoint blockade to enhance vaccination effects have generated excitement and renewed interest. Developing novel and effective vaccine adjuvants that can enhance antitumor immune responses is critical for the success of cancer vaccine. We have recently shown that intratumoral injection of Heat-inactivated MVA (Heat-iMVA; by heating MVA at $55^{\circ} \mathrm{C}$ for $1 \mathrm{~h}$ ) generates systemic antitumor effects via a STING-dependent mechanism and requires Batf3-dependent $\mathrm{CD} 103^{+} / \mathrm{CD}_{8} \mathrm{a}^{+}$dendritic cells (DCs). Heat-iMVA infection of conventional DCs (cDCs) induces higher levels of type I IFN and proinflammatory cytokines and chemokines than live MVA. It also induces DC maturation in a STINGdependent manner. The role of Heat-iMVA as a vaccine adjuvant has not been explored previously.

Methods

To test whether Heat-iMVA is an effective vaccine adjuvant, we first tested whether co-administration of chicken ovalbumin (OVA) with Heat-iMVA improves anti-OVA T cell and antibody responses. We then tested whether co-administration of irradiated OVA-expressing B16 cells with Heat-iMVA delays tumor growth in a therapeutic vaccination model. We also tested whether systemic delivery of antiPD-L1 antibody would further improve vaccination efficacy. Mice were intradermally implanted with B16-OVA, they were vaccinated with either irradiated B16-OVA, or B16- OVA + Heat-iMVA three times on the contralateral flank in the presence of absence of anti-PD-L1 antibody. We measured tumor volumes and monitored survival of the mice.

Results

We found that addition of Heat-iMVA increased the percentage of OVA-specific $\mathrm{CD}^{+} \mathrm{T}$ cells and $\mathrm{CD} 4^{+} \mathrm{T}$ cells in the draining lymph nodes and spleens. The induction of OVA-specific $\mathrm{CD} 8^{+} \mathrm{T}$ cells was significantly reduced in Batf3 $3^{-/-}$mice. It also boosted the OVA-specific $\lg \mathrm{G} 2 \mathrm{c}$ and $\lg \mathrm{G} 1$ in the serum. In the therapeutic vaccination model, we found that vaccination with irradiated B16-OVA + Heat-iMVA extended the median survival from 16 days (with irradiated B16-OVA vaccination) to 23 days ( $* *, P<0.01)$. With anti-PD-L1 antibody, vaccination with irradiated B16-OVA + Heat- iMVA extended the median survival from 20 days to 27 days $(* *, P<0.01$ ). In vitro studies demonstrated that Heat-iMVA enhanced antigen cross-presentation by DCs. Conclusions

Our results demonstrate that Heat-iMVA is a safe and potent vaccine adjuvant for peptide and irradiated whole cell vaccinations. Future studies will evaluate the role of Heat-iMVA as a vaccine adjuvant for tumor neoantigens.

P117

DNA-based cancer vaccines designed by SynCon ${ }^{\circledR}$ technology

break tolerance in genetically diverse pre-clinical models

Bradley Garman ${ }^{1}$, Elizabeth K Duperret ${ }^{2}$, Bernadette Ferraro'

Jewell N Walters ${ }^{1}$, Kimberly A Kraynyak', Emma Masteller ${ }^{1}$, Jian Yan',

Amir S Khan', David B Weiner², Laurent M Humeau

${ }^{1}$ Inovio Pharmaceuticals, Inc., Plymouth Meeting, PA, USA; ${ }^{2}$ The Wistar

Institute, Philadelphia, PA, USA

Correspondence: Laurent M Humeau (bradley.garman@inovio.com) Journal for ImmunoTherapy of Cancer 2017, 5(Suppl 2):P117

\section{Background}

Cancer vaccines targeting self-antigens have shown limited efficacy in the clinic due to self-tolerance mechanisms preventing or dampening an immune response against them. To overcome selftolerance, we developed a novel, multi-phase DNA vaccine design strategy (SynCon ${ }^{\oplus}$ technology) that introduces subtle amino acid changes into native cancer antigens. These synthetic immunogens aim to generate cross-reactive $\mathrm{T}$ cells and/or promote epitope spreading. As a proof of concept, we first used SynCon ${ }^{\circledast}$ technology to design a novel DNA immunogen targeting the self-antigen FAP (SynCon ${ }^{\oplus} \mathrm{FAP}$ ) and investigated its ability to break tolerance in outbred CD-1 mice. Compared to commonly used inbred mouse models, CD-1 mice are more relevant for inferring immunogenicity in humans due to their genetic diversity. In addition, we designed a SynCon ${ }^{\circledast}$ immunogen targeting TERT (SynCon ${ }^{\oplus}$ TERT) and tested its ability to break tolerance in non-human primates (NHP), a highly relevant model for immunotherapeutic vaccine development.

Methods

CD-1 mice were immunized with either SynCon ${ }^{\circledR}$ FAP, a mouse native FAP vaccine, or vector control. Three immunizations were administered two weeks apart with electroporation following each immunization. For the NHP study, rhesus macaques were immunized with SynCon ${ }^{\circledR}$ TERT or native rhesus TERT vaccine as a control. Four immunizations were administered four weeks apart with electroporation following each immunization. Vaccine-induced immune responses were evaluated by IFN- $\gamma$ ELISpot using species-specific native peptides.

Results

Compared to native mouse FAP, SynCon ${ }^{\circledR}$ FAP was more immunogenic in CD-1 mice (average 407 ELISpot-forming units (SFUs) per $10^{6}$ splenocytes for SynCon ${ }^{\circledast}$ vs. 160 SFU $/ 10^{6}$ for native), suggesting that SynCon ${ }^{\circledR}$ FAP was capable of breaking tolerance. Importantly, $14 / 15$ mice in the SynCon ${ }^{\circledR}$ FAP group generated an immune response above $100 \mathrm{SFU} / 10^{6}$, compared to only $9 / 15$ mice in the native FAP group. In rhesus macaques, while the native rhesus TERT was not able to induce any detectable immune responses following the fourth immunization, SynCon ${ }^{\circledR}$ TERT elicited an average of 353 SFU/ $10^{6}$, indicating that the SynCon ${ }^{\oplus}$ TERT immunogen was capable of breaking tolerance in NHPs.

Conclusions

Taken together, we demonstrate that SynCon ${ }^{\oplus}$ technology can be used to design DNA immunogens that are capable of breaking tolerance in genetically diverse preclinical models. SynCon ${ }^{\circledR}$ DNA immunogens have the potential to break tolerance, induce immune responses, and provide clinical benefit for patients with diverse HLA haplotypes. Further investigation of SynCon ${ }^{\oplus}$ DNA immunogens in clinical trials is warranted. 


\section{P118}

Gp96-Ig/Costimulator combination vaccine improves $\mathrm{T}$ cell priming, enhances immunity, memory, and tumor elimination Louis Gonzalez, Louise Giffin, Jason Rose, George Fromm,

Suresh De Silva, Taylor Schreiber, Jeff Hutchins

Heat Biologics, Durham, NC, USA

Correspondence: Jeff Hutchins (Igonzalez@heatbio.com)

Journal for ImmunoTherapy of Cancer 2017, 5(Suppl 2):P118

\section{Background}

The recent excitement in the field of immuno-oncology has been driven largely by the clinical success of checkpoint inhibitors. This success is tempered by the fact that monotherapy succeeds in only $10-40 \%$ of patients. It is widely believed that to improve patient outcomes, new approaches that combine treatments with more than one functionality will be required.

Methods

We have developed a next generation cellular vaccine platform - referred to as ComPACT (COMbination Pan-Antigen Cytotoxic Therapy)that incorporates a tumor antigen chaperone (gp96-lg) with T cell costimulation ( $\mathrm{Fc}-\mathrm{OX} 40 \mathrm{~L})$, into a single anti-tumor cell line that secretes them both (Cancer Immunol Res. 2016 Sep 2; 4(9):766-78).

Results

ComPACT primes both antigen-specific CD4+ and CD8+ T cells, and stimulates activation of CD127+KLRG-1- memory precursor cells. Systemic administration of OX40 agonist antibodies led to toxic proliferation of non-specific CD4+ T cells, Tregs and systemic inflammatory cytokine production. Importantly, ComPACT led to high frequencies of IFN $\mathrm{H}$, TNFa+, granzyme-b+ and IL-2+ antigen-specific CD8+ T cells at both priming and boosting, which enhanced rejection of established murine melanoma (B16.F10) and colon cancer (CT26) tumors and increased overall survival.

Conclusions

Here, we have assessed ComPACT in combination with a checkpoint inhibitor and an additional systemic administered T cell costimulator (TL1A) and show that they synergize effectively with antagonist antibody therapies, amplifying antigen-specific $T$ cells, programming a memory response, and eliminating tumors. ComPACT/aPD1 or aPDL1/TL1a combinations may therefore translate into an efficacious approach to treat human cancers.

\section{P119}

Optimization of antigenic composition of a dendritic-cell targeting MIP3a-antigen vaccine and the efficacy of additional IL-10 neutralization therapy in the B16F10 mouse melanoma model James Gordy, Richard Markham Johns Hopkins Bloomberg School of Public Health, Baltimore, MD, USA Correspondence: Richard Markham (jgordy2@jhu.edu) Journal for ImmunoTherapy of Cancer 2017, 5(Suppl 2):P119

\section{Background}

The chemokine MIP3a (CCL20) binds to CCR6 on immature dendritic cells. Vaccines fusing MIP3a to melanoma antigen gp100 have been shown to be moderately effective in therapeutically alleviating melanoma in mouse models. To optimize the therapy, our laboratory is exploring two avenues. First, we added agents to neutralize interleukin 10 (IL-10) at the tumor site (i.t.) or vaccination site to modulate immune responses. Second, we examined the effects of substitution and addition of the highly immunogenic tyrosinase-related protein 2 (TRP2) antigen to the MIP3a-gp100 vaccine construct.

Methods

The current studies utilize the B16F10 syngeneic, transplantable, mouse melanoma model system. The MIP3a-antigen DNA vaccine is administered intramuscularly (i.m.) into the tibialis muscle, followed immediately by i.m. electroporation. Constructs utilized include MIP3a fused to gp100, TRP2, or both. Vaccinations are given therapeutically, beginning at day 3 or 5 post challenge. Tumor sizes, growth, and survival were all assessed. Treatment responses were characterized by flow cytometric analysis of tumor infiltrate. The mechanism of i.t. alL-10 efficacy was explored by RT-PCR and confirmed with a knockout mouse model.

Results

With this therapeutic protocol, we demonstrate for the first time that neutralizing IL-10 at either the vaccination or tumor site enhances the anti-tumor efficacy of a MIP3a-gp100 vaccine, leading to significantly smaller tumors, slower growing tumors, and increases in mouse survival. We discovered that IFNa-4 transcripts in the tumor were significantly upregulated in mice given vaccine and i.t. alL-10 compared to vaccine alone. A mouse model with IFNaR1 knocked out eliminated the protection provided by i.t. alL-10, demonstrating that the additional therapeutic value of i.t. alL-10 is primarily mediated by type-I interferons. Also, we demonstrate that the MIP3agp100-TRP2 dual antigen and the MIP3a-TRP2 vaccine provided better protection compared to the MIP3a-gp100 vaccine but were not significantly different from each other.

\section{Conclusions}

Efficient targeting of antigen to immature dendritic cells with a chemokine fusion vaccine offers a potential alternative approach to the ex vivo dendritic cell antigen loading protocols currently undergoing clinical investigation. Neutralizing IL-10 at both the vaccination site and tumor site have enhanced vaccine efficacy, the latter shown to be due to type-I interferons. Additionally, MIP3a-TRP2 has proven to be a superior vaccine construct than MIP3a-gp100 in our system, and the MIP3a-gp100-TRP2 vaccine did not improve on the protection provided by MIP3a-TRP2. Further potential therapy optimization currently undergoing investigation offers promise for this line of investigation to become a novel melanoma therapy.

P120

Streamlining approaches for the selection of T cell neo-epitopes: from mutanome to therapeutic personalized cancer vaccine

Guilhem Richard', Leonard Moise, ${ }^{1,2}$, Matthew Ardito', Frances Terry', Gad Berdugo ${ }^{3}$, William Martin', Anne De Groot ${ }^{1,2}$

${ }^{1}$ EpiVax Inc., Providence, RI, USA; ${ }^{2}$ University of Rhode Island, Providence, RI, USA; ${ }^{3}$ EpiVax Oncology LLC, Providence, RI, USA

Correspondence: Anne De Groot (jhealey@epivax.com)

Journal for ImmunoTherapy of Cancer 2017, 5(Suppl 2):P120

\section{Background}

Next-generation sequencing has opened the door to precision cancer therapies targeting mutations expressed by tumor cells. However most neo-epitopes selected by traditional $\mathrm{T}$ cell epitope prediction algorithms prove to be non-immunogenic. Poor predictive performance may partially be due to inclusion of mutated epitopes crossconserved with self-epitopes recognized by the $T$ cell receptor of regulatory (Treg), anergic or deleted T cells. Vaccination with selfepitopes can lead to weak effector responses, active immune suppression, and toxicity due to immune-mediated adverse effects.

Methods

We have developed Ancer, an advanced cancer T cell epitope identification and characterization tool that streamlines the selection of Class I and II neo-epitopes. Ancer is leveraging EpiMatrix and JanusMatrix, state-of-the-art tools that have been extensively validated in prospective vaccine studies for infectious diseases [1, 2]. Use of Ancer in the oncology field allows for the prioritization of neo-epitopes exhibiting reduced potential for inducing Tregs, whose activation continues to curtail efficacy of current cancer therapies.

Results

We validated Ancer's predictive accuracy using datasets of HLAbound peptides detected by mass spectrometry, which are independent of training sequence data used in model development. Analysis of sequences from Abelin et al. [3] shows a 96\% agreement between Ancer predictions and peptides eluted from common Class I HLAs, while only $86 \%$ of these sequences are accurately recalled by NetMHC and NetMHCpan. Additional retrospective analyzes of a cancer immunogenicity study [4] demonstrate that Ancer selects immunogenic neo-epitopes with $72 \%$ accuracy, as compared to $21 \%$ accuracy when using public prediction tools. 


\section{Conclusions}

These results demonstrate that Ancer may focus epitope candidate selection on higher value sequences than conventional algorithms. Neo-epitopes with low Treg activation potential may then be used to support the development of safer and more effective vaccines.

\section{References \\ 1. Moise, et al. iVAX: An integrated toolkit for the selection and optimization of antigens and the design of epitope-driven vaccines. Hum. Vaccines Immunother. 2015; 11, 2312-2321. \\ 2. Wada, et al. A humanized mouse model identifies key amino acids for low immunogenicity of H7N9 vaccines. Sci. Rep. 2017; 7, 1283 \\ 3. Abelin, et al. Mass Spectrometry Profiling of HLA-Associated Peptidomes in Mono-allelic Cells Enables More Accurate Epitope Prediction Resource Mass Spectrometry Profiling of HLA-Associated Peptidomes in Mono-allelic Cells Enables More Accurate Epitope Prediction. Immunity 2017; 46, 315-326. \\ 4. Strønen, et al. Targeting of cancer neoantigens with donor-derived T cell receptor repertoires. Science. 2016; 352, 1337-1341.}

\section{P121}

Phase 1 dose escalation trial of CV301

Julius Strauss', Jaydira Del Rivero', Ravi A. Madan', Jennifer Marte', Alanvin Orpia ${ }^{2}$, Eva Wagner ${ }^{3}$, Cesar Pico Navarro ${ }^{4}$, Christopher Heery ${ }^{4}$, Jeffrey Schlom', James Gulley ${ }^{1}$

${ }^{1}$ Center for Cancer Research, National Cancer Institute, Bethesda, MD, USA; ${ }^{2}$ Leidos Biomedical Research, Inc., Bethesda, MD, USA; ${ }^{3}$ Bavarian Nordic, Martinsried, Germany; ${ }^{4}$ Bavarian Nordic, Morrisville, NC, USA Correspondence: James Gulley (chhe@bavarian-nordic.com) Journal for ImmunoTherapy of Cancer 2017, 5(Suppl 2):P121

\section{Background}

CV301 is a poxviral-based vaccine comprising a prime-boost strategy with Modified Vaccinia Ankara (MVA, prime) and fowlpox (FPV, boost), which contain transgenes encoding two tumor associated antigens, CEA and MUC-1, as well as 3 costimulatory molecules (B7.1, ICAM-1 and LFA-3, called TRICOM). Preclinical evidence suggests CV301 activates CEA and MUC-1 specific T cell responses, potentially increasing the clinical benefit of immune checkpoint inhibition. An open label phase $1 / 1 \mathrm{~b} / 2$ trial was initiated to evaluate safety of CV301 alone (phase 1) and in combination with PD-1/L1 inhibition (phase $1 \mathrm{~b}$ ) to be followed by a randomized phase 2 trial evaluating the combination compared with PD-1/L1 inhibition alone. Here, we report the phase 1 safety evaluation of CV301.

\section{Methods}

This open-label, $3+3$ design dose-escalation phase 1 trial evaluated 3 dose levels of the priming dose of CV301 (MVA): 1, 2 or 4 s.c. injections of $4 \times 10^{8} \mathrm{Inf} . \mathrm{U} / 0.5 \mathrm{~mL}$ on days 1 and 29 . FPV booster doses were scheduled for all subjects subcutaneously at a $1 \times 10^{9} \mathrm{Inf} . \mathrm{U} / 0.5$ $\mathrm{mL}$ dose on weeks $9,11,13,15,19,23,27,31,35,39,43,47,51,65$, 78, 91 and 104.

Results

There were no dose limiting toxicities in the phase 1 evaluation of CV301 alone. The minimum required patient number was enrolled in each dose level (DL1: 3, DL2: 3, DL3: 6, total: 12). The most frequent treatment-related AEs were temporary and self-limiting, grade 1/2, and included injection site reactions (erythema, pruritus, pain, induration and/or swelling) and general symptoms (fever/chills, flu-like symptoms, headache, fatigue/weakness, nausea/vomiting, myalgia and arthralgia). Seven of the 12 patients have microsatellite stable colon cancer. Of these seven, two had preliminary evidence of benefit. One patient with stable disease at first restaging has marked reduction $(60 \%$ fall) in tumor markers at 10 weeks. Another patient had unconfirmed partial response at first restaging ( 6 weeks). When 3 month restaging scans indicated progression, a PD-L1 inhibitor was initiated. Subsequently, tumor markers fell by $60 \%$.

\section{Conclusions}

CV301 priming doses (MVA) can be safely administered to at least 4 injection sites concurrently at $4 \times 10^{8} \mathrm{Inf} . \mathrm{U} / 0.5 \mathrm{~mL}$ per site for a total of $1.6 \times 10^{9}$ Inf.U, which is the recommended phase $1 \mathrm{~b}$ and phase 2 dose. Booster doses (FPV) can safely be administered s.c. at $1 \times 10^{9}$
Inf.U/0.5mL. Subsequent analyzes will include immune response data. The trial will continue to enroll phase $1 \mathrm{~b}$ and, subsequently, the randomized phase 2 .

Trial Registration

ClinicalTrials.gov Identifier NCT02840994

\section{P122}

$\mathrm{N}$-terminally extended proline residues influences the HLA class I antigen processing and decreases CTL responses

Ayumi Hongo, Takayuki Kanaseki, Serina Tokita, Vitaly Kochin,

Toshihiko Torigoe

Sapporo Medical University, Sapporo, Japan

Correspondence: Takayuki Kanaseki (arkpon1717@gmail.com);

Toshihiko Torigoe

Journal for ImmunoTherapy of Cancer 2017, 5(Suppl 2):P122

\section{Background}

CD8+ T cells recognize peptide-MHC class I complexes presented on target cell surfaces; however, the precise mechanisms of antigen processing by which $\mathrm{T}$ cell epitopes are generated still remains unclear. Methods

Here we extensively collected $>800$ naturally presented peptide sequences, and statistically analyzed an amino-acid profile of their $\mathrm{N}$ terminal extensions. We combined natural HLA-A24 ligand sequences gained from HLA-ligandome analysis with their corresponding $\mathrm{N}$ extension sequences gained from database search, and analyzed frequency of each amino acid at given positions of $\mathrm{N}$-extensions (Position -15 to -1$)$.

Results

Interestingly, we found that frequency of proline residue (Pro) was significantly reduced between $\mathrm{P}-3$ and $\mathrm{P}-1$, implying that peptide sequences following Pro might not be efficiently presented by HLAA24. To further investigate this hypothesis, we prepared 293T cells expressing a panel of mini-gene constructs that carried $\mathrm{N}$-extensions followed by a model epitope sequence, and ultimately found that presence of Pro at P-3 to P-1 significantly reduced IFN-g production from the CTLs specific to the epitope.

Conclusions

Thus, we consider that presence of Pro could be a new signature in HLA class I antigen processing that attenuates presentation and CTL recognition of the following epitope sequences.

\section{P123}

A novel oncolytic virus expressing multiple immune regulatory factors to change tumor immune microenvironment and to cause lasting complete tumor eradication in animal models

William Jia', JUN Ding ${ }^{2}$, Erica Lee ${ }^{2}$, Dmitry Chouljenko², Guoyu Liu², Yanal Murad'2, Will Liu'², Zahid Delwar', Xuexian Bu'

${ }^{1}$ University of British Columbia, Vancouver, BC, Canada; ${ }^{2}$ Virogin Biotech Ltd, Vancouver, BC, Canada

Journal for ImmunoTherapy of Cancer 2017, 5(Suppl 2):P123

\section{Background}

Oncolytic viruses (OVs) are among the most powerful approaches in cancer immunotherapy -[1]. OVs not only cause cancer cell lysis but more importantly, their infection in tumors induces anti-tumor immune response from the host, resulting in lasting anti-tumor immunity. It has been recognized that anti-tumor immune response requires multiple immune regulatory factors that act synergistically and tumor microenvironment is critical for tumor to grow. Herpes simplex virus type-1 (HSV-1) has been approved by FDA as an oncolytic viral drug to treat melanoma[2]. One advantage of HSV-1 is its large genomic capacity for carrying multiple exogenous genes.

Methods

A HSV-1 oncolytic viral vector (VG161) was constructed to simultaneously express IL12, IL15 with its receptor alpha unit and a PDL-1 blocking peptide. Anti-tumor activity of VG161 was tested in both immune competent mice (CT26 and A20 tumor models) and nude mice for human tumor models (LNCaP and U87). Since CT26 and A20 
are poorly permissive for HSV-1 replication, the mouse tumor models were able to demonstrate the anti-tumor immune response induced by VG161 while oncolytic activity of VG161 was demonstrated in LNCaP and U87 models since the immune system is compromised in those models.

Results

VG161 caused complete tumor eradication in all the models tested and the animals survived tumor-free for many months till sacrificed. VG161 virus induced tumor oncolysis caused complete tumor destruction in both LNCaP and U87 tumors. In the CT26 model, no tumor could be found after re-challenging with the same tumor cells. The anti-tumor immune response by VG161 was significantly stronger than similar viruses that did not express any immune stimulating gene or only express GM-CSF. Furthermore, in an A20 double tumor model, intratumoral injection into the tumor on one side caused tumor regression on both sides. Transcriptom analysis showed significant change in tumor microenvironment. Finally, memory $\mathrm{T}$ cells are evident in the treated animals demonstrated by multiple assays.

Conclusions

VG161 is a novel oncolytic virus that are both strong in stimulating anti-tumor immunity and oncolytic activity. Intratumoral expressing multiple immune regulatory factors by an oncolytic virus may significantly change the tumor immune microenvironment to enhance efficacy of the oncolytic virus.

\section{References}

1. Delwar, Zhang, Rennie, Jia. Nature Reviews: Urology. 2016; 13(6):334-52.

2. Grigg, Blake, Gartrell, Sacher, Taback, Saenger. Semin Oncol. 2016 Dec; 43(6):638-646.

\section{P124}

In vitro mode of action of ilixadencel - a cell-based allogeneic immune primer for intratumoral administration

Grammatiki Fotaki, Chuan Jin, Di Yu, Magnus Essand, Alex Karlsson-Parra Institution of Immunologi, Genetics and Pathology, 75185 Uppsala,

Sweden

Correspondence: Alex Karlsson-Parra (alex.karlsson-

parra@immunicum.com)

Journal for ImmunoTherapy of Cancer 2017, 5(Suppl 2):P124

\section{Background}

Therapeutic cancer vaccines that target personal mutation-derived neoantigens in tumor cells have recently been shown to stimulate a potent immunological anti-tumor response in cancer patients. One obvious way to circumvent major technical difficulties associated with ex vivo production of neoantigen-based vaccines would be to use the patient's own tumor in situ as a direct antigen source by intratumoral administration of a potent immune primer. The immune-priming function would be to promote immunogenic cell death and activation of recruited DCs into T helper 1 polarizing DCs. For this purpose, a cell-based immune primer consisting of cryoperserved proinflammatory allogeneic DCs (international nonproprietary name ilixadencel; previously named INTUVAX), producing high amounts of $\mathrm{T}$ helper 1 associated chemokines and cytokines, has been developed and tested in a phase I/II clinical trial with promising immunological and clinical results [1]. In order to explore the potential mode of action, a batch of GMP-produced ilixadencel was investigated in vitro.

Methods

The effects of soluble factors released by ilixadencel, and cell-to-cell interactions between ilixadencell and co-cultured allogeneic immune cells, on NK cell recruitment and activation, and allogeneic bystander DC maturation and polarization were analyzed in vitro.

Results

Cell migration experiments revealed that allogeneic NK cells migrated towards chemotactic factors produced by ilixadencel. When cocultured with allogeneic NK cells, ilixadencel was found to be superior to IL-2 priming in terms of induced CD69-expression and IFNgamma production by NK cells. The in vitro assay for cytotoxicity further revealed that the tumor-killing ability of NK cells was markedly enhanced after coculture with ilixadencel. Finally, supernatants from ilixadencel, and ilixadencel cocultured with allogeneic PBMCs, induced a phenotypic maturation of bystander DCs producing substantial amounts of IL-12p70 upon subsequent CD40-ligation.

Conclusions

Collectively, the presented in vitro results indicate that intratumorally injected ilixadencel will create a immune-priming environment leading to NK-cell mediated tumor cell death with release of tumorderived antigens, including neoantigens, as well as maturation of bystander endogenous DCs into T helper 1 polarizing mature DCs.

References

1. Laurell, Lönnemark, Brekkan, Magnusson, Tolf, Wallgren, Andersson, Adamson, Kiessling, Karlsson-Parra. Intratumorally injected pro-inflammatory allogeneic dendritic cells as immune enhancers: a first-in-human study in unfavourable risk patients with metastatic renal cell carcinoma. Journal for ImmunoTherapy of cancer. 2017;5:52.

\section{P125}

Immunogenicity of human papillomavirus (HPV) specific DNA vaccine, INO-3112 (HPV16/HPV18 plasmids + IL-12) in HPV+ head and neck squamous cell carcinoma (HNSCCa)

Charu Aggarwal', Roger Cohen', Matthew Morrow², Kimberly Kraynyak², Dawson Knoblock², Joshua Bauml', Gregory Weinstein ${ }^{1}$, Jian Yan², Jean Boyer ${ }^{3}$, Drishty Mangrolia ${ }^{2}$, Sandra Oyola², Susan Duff ${ }^{2}$,

David Weiner ${ }^{4}, \|_{\text {diko }}$ Csiki ${ }^{2}$, Mark Bagarazzi ${ }^{2}$

${ }^{1}$ Perelman School of Medicine at the University of Pennsylvania, Philadelphia, PA, USA; ${ }^{2}$ Inovio Pharmaceuticals, Plymouth Meeting, PA, USA; Inovio Pharmaceuticals, San Diego, CA, USA; ${ }^{3}$ Wistar Institute, Philadelphia, PA, USA

Correspondence: Mark Bagarazzi (dawsondmg@gmail.com) Journal for ImmunoTherapy of Cancer 2017, 5(Suppl 2):P125

\section{Background}

Oropharyngeal HNSCCa is frequently associated with HPV. We hypothesized that immunotherapy with INO-3112 would generate immune responses in patients (pts) with HPV16+ or 18+ HNSCCa.

Methods

This Phase I/lla trial included pts with p16+ locally advanced HNSCCa, ECOG PS 0-1. INO-3112 was delivered IM along with electroporation with the CELLECTRA ${ }^{\oplus}$ device, Q3 weeks $x 4$ doses. Cohort 1 (C1) pts received INO-3112 pre and post-surgery; Cohort 2 (C2) pts received INO-3112 post cisplatin-based definitive chemoradiation. $1^{\circ}$ and $2^{\circ}$ endpoints were safety and immune responses. HPV16/18 specific antibody levels were assessed by ELISA. IFNr ELISpot and flow cytometry were used to quantify antigen specific $T$ cell responses, at each dosing visit and q3 months (mos).

Results

Twenty two (22) pts were treated, completing accrual. C1: $n=6, C 2$ : $\mathrm{n}=16 ; 20$ male, median age 57.5 years $(32-76)$; base of tongue $=10$, tonsil=12; never smoker $=10$. All pts are alive, median follow up is 15.9 mos (1-26). INO-3112 was well-tolerated with no related Grade 3-5 AEs. Peak mean/median antibody responses to HPV16 E7 and HPV18 E7 antigens for 19 pts with evaluable samples were 1:1235/ 1:150 and 1:2853/1:450, respectively. As compared to baseline, 18 pts with evaluable samples showed elevated HPV16/HPV18 specific T cell activity (by IFNr ELISpot). Flow cytometric analysis of PBMC revealed an increased frequency of INO-3112 specific (summed responses to HPV16 and HPV18) CD8+CD38+PD-1+ cells that were positive for Granzyme A or B and perforin from baseline (mean frequency of $1.19 \%$ and $1.097 \%$ for GrzA+Prf+ and GrzB+Prf+ within CD8+CD38+PD-1+, respectively) to post immunotherapy (mean frequency of $7.694 \%$ and 6.698\% GrzA+Prf+ and GrzB+Prf+ within CD8+CD38+PD-1+, respectively) in 7 of 8 patients with evaluable samples. 3 pts have progressed $1 \mathrm{pt}$ received Nivolumab for progressive disease, and remains in CR. Conclusions

These data show that INO-3112 generated HPV-specific peripheral humoral and cellular immune responses in patients with HPV+ HNSCCa. The post therapy increase of INO-3112 specific (summed 
HPV16 and 18 responses) CD38+PD-1+ cells that express lytic granules suggests that INO-3112 promotes CD8+ T cell activation, and that these activated CD8+ T cells may be vulnerable to PD-L1 mediated suppression. Clinical trial information: NCT02163057

Trial Registration

ClinicalTrials.gov Identifier NCT02163057

\section{P126}

Cancer immunotherapy with novel vaccine nanodiscs for efficient elimination of mucosal tumors

Rui Kuai, Yao Xu, Anna Schwendeman, James Moon

University of Michigan, Ann Arbor, MI, USA

Correspondence: Anna Schwendeman (ruikuai@umich.edu); James

Moon

Journal for ImmunoTherapy of Cancer 2017, 5(Suppl 2):P126

\section{Background}

Recently we have reported a novel vaccine nanodisc technology for elicitation of anti-tumor $\mathrm{T}$ cells that penetrate into subcutaneous flank tumors [1]. Here we aimed to apply the nanodisc technology for elimination of mucosal tumors, such as cervical carcinoma and lung tumors. Previous research has suggested that intranasal vaccination can recruit $T$ cells to local mucosal tissues, and may control the growth of lung tumors. However, recruitment of T cells to distal mucosal sites, such as the reproductive tract, is very challenging. In contrast to mucosal vaccination, parenteral vaccination may elicit strong systemic $\mathrm{T}$ cell responses and allow for robust $\mathrm{T}$ cell infiltration into multiple, disseminated mucosal tissues, thus improving the overall therapeutic effect. To test this hypothesis, we established murine models of HPV-induced cancer in reproductive tract and lung metastasis and directly compared the anti-tumor efficacies of subcutaneous vs. intranasal vaccination with nanodiscs.

Methods

The vaccine nanodiscs were prepared by conjugating the HPV antigen peptide and adjuvant $\mathrm{CpG}$ to pre-formed nanodiscs. The resulting vaccine nanodiscs were characterized by dynamic light scattering, gel permeation chromatography, and HPLC. The reproductive tract tumor model was established in C57BL/6 mice by intravaginal administration of luciferase-expressing TC-1 cells - a surrogate for HPV-induced human tumors, such as cervical carcinoma. The lung metastasis model was established by intravenous injection of luciferase-expressing TC-1 tumor cells. Tumor-bearing mice were vaccinated twice, and the growth of TC-1-luc intravaginal and lung tumors was monitored by IVIS. The antigen-specific $T$ cell responses were measured by the tetramer staining assay.

Results

Subcutaneous administration of nanodiscs induced robust expansion of circulating antigen-specific CD8+ T cells with the peak frequency of $\sim 35 \%$, a 12 -fold increase than that achieved by intranasal vaccination $(p<0.0001)$. Furthermore, subcutaneous administration of vaccine nanodiscs eliminated established TC- 1 tumors both in the lungs and reproductive tract by day 20 , whereas intranasal administration only eliminated tumors in the lung but failed to do so for intravaginal tumors.

\section{Conclusions}

Subcutaneous route of nanodisc vaccination is more efficient than intranasal route in terms of elicitation of anti-tumor $\mathrm{T}$ cell responses and elimination of mucosal tumors. These vaccine nanodiscs may offer a promising strategy for treatment of mucosal tumors, including cervical carcinoma and lung metastases.

\section{Reference}

1. Kuai. Nature Materials. 2017;16(4):489-496
P127

Transduction of MAGE-A1, A3, A4, A10 and IL-12 by ZVex ${ }^{\circledR}$, a dendritic cell targeting platform induces robust multi-antigen $T$ cell immune responses without antigenic interference or immunodominance

Tina C. Albershardt, Anshika Bajaj, Jardin Leleux, Tsai-Yu Lin,

Rebecca S. Reeves, Lisa Y. Ngo, Ryan White, Jordan E. Krull,

Reice D. James, Jan ter Meulen, Peter Berglund

Immune Design, Seattle, WA, USA

Correspondence: Jardin Leleux (jardin.leleux@immunedesign.com) Journal for ImmunoTherapy of Cancer 2017, 5(Suppl 2):P127

\section{Background}

Expression of multiple antigens in tandem from viral vaccine vectors often results in immune dominance phenomena, which preclude generation of balanced immune responses to each antigen. To address this issue, we have developed a novel technology that allows for multiple antigens and/or immune-modulators to be expressed in vivo using the integration-deficient, dendritic-cell targeted lentiviral vector platform, ZVex. Utilizing a unique manufacturing process that generates reassortant vectors, we have designed a vector expressing the human cancer testis antigens MAGEA1, MAGEA3, MAGEA4, MAGEA10 and Interleukin 12, in order to generate robust T cell responses specific for all four antigens simultaneously.

Methods

In addition to the backbone plasmids encoding for essential vector components (such as capsid, envelope, Vpx and Rev proteins, polymerase, protease), five identical vector backbones each encoding a different full-length MAGEA or murine IL12 gene were co-transfected into a producer cell line for viral vector production. Because lentiviral vectors package two RNA molecules each, this approach results in the generation of potentially 15 different homozygous or heterozygous vector genotypes. Vector titers were determined by vector and gene-specific RT/PCR and by a cell-based infectivity assay. Recombinant IL-12 was detected by ELISA. Female BALB/c mice were immunized with either reassortant vector products or a ZVex vector expressing single antigens. After two weeks, CD8+ T cell responses were evaluated for multifunctionality via intracellular cytokine staining.

Results

The product was shown to contain nearly equal amounts of MAGE-A1, $\mathrm{A} 3, \mathrm{~A} 4, \mathrm{~A} 10$ (ranging from $2.3-4.9 \times 10^{10}$ genome copies $/ \mathrm{ml}, 1.4-3.9 \times 10^{9}$ infectious units $/ \mathrm{ml}$ ) and to contain IL12 at the expected proportion. A single subcutaneous injection generated specific $T$ cell responses against all four of the encoded MAGEA antigens at similar levels $(0.4 \%$ antigen specific splenic CD8 T cells each). Furthermore, IL-12 was shown to consistently enhance immunogenicity with the response enhancement ranging from 2.9 to 3.8 -fold for each of the four antigens. Conclusions

Expressing multiple MAGE-A proteins and the immune enhancing cytokine IL-12 from a reassortant ZVex vector resulted in robust and balanced antigen specific CD4 and CD8 T cell responses in mice. No evidence of immunodominance or antigenic interference was observed in these experiments. Because MAGE-A1, 3, 4, and 10 antigens are expressed by a large number of solid tumors individually or in combination, this is a potentially broadly applicable, off-the-shelf cancer vaccine.

P128

PD-L1-specific T cells can be activated by interferon-y and have an anti-tumor effect

Mia Thorup Lundsager ${ }^{1,2}$, Mads Hald Andersen ${ }^{1,2}$

${ }^{1}$ Center for Cancer Immune Therapy, Herlev, Denmark; ${ }^{2} \mathrm{O}$ Biotech ApS,

Copenhagen, Denmark

Correspondence: Mia Thorup Lundsager

(Mia.thorup.lundsager@regionh.dk); Mads Hald Andersen

Journal for ImmunoTherapy of Cancer 2017, 5(Suppl 2):P128 


\section{Background}

Checkpoint inhibitory pathways, like the programmed death-1 receptor (PD-1) and its ligand (PD-L1), play key roles in inducing immune tolerance in the tumor microenvironment. PD-L1 is expressed in the tumor microenvironment by cancer cells as well as different immune regulatory cells, like dendritic cells. We have previously shown that the immune system has an anti-cancer mechanism that works via PD-L1-specific effector $T$ cells in vitro. We described spontaneous CD8+ and CD4+ T cell reactivity against PD-L1 in peripheral blood of healthy donors and patients with various cancers. Those results suggested that PD-L1-specific T cells might modulate adaptive immune reactions by reacting to regulatory cells and cancer cells. Here we examined the function of PD-L1-specific T cells in vivo.

\section{Methods}

We hypothesized that PD-L1-specific T cells are present at steady state and are an important factor in regulating the immune system during inflammation. Healthy $\mathrm{C} 57 \mathrm{~B} \mathrm{I} / 6$ mice were injected with IFNy intraperitoneal to simulate inflammation. After 6 days the mice were sacrificed and the spleen was removed for further analysis by Elispot with ex vivo stimulation of a murine PD-L1-derived epitope (mPDL1long). Subcutaneously (s.c.) vaccination with mPD-L1long in Montanide was done in $\mathrm{C} 57 \mathrm{Bl} / 6$ mice to further enhance the response. After a week, the mice were sacrificed and spleen and draining lymph node (dLN) removed and analyzed by Elispot.To test the antitumor effect of the mPD-L1long vaccination, C57BI/6 mice were inoculated with B16F10 cells s.c. and vaccinated two times, and the tumor growth was monitored

Results

Ex vivo stimulation with $\mathrm{mPD}-\mathrm{L}$ 1long resulted in a PD-L1-specific T cell response in the spleen after just two IFNy-injections, indicating a rapidly increase in PD-L1-specific T cells. It was possible to further increase the PD-L1-specific response by one vaccination with $\mathrm{mPD}$ L1long. A strong PD-L1-specific T cell response was found in the spleen and dLN of the MPD-L1long-vaccinated mice compared to the control mice. We also found that $\mathrm{MPDL}$ 1long-vaccination decreased the tumor growth.

\section{Conclusions}

We describe that PD-L1-specific T cells are expanded by IFNyinjections, which suggest that PD-L1-specific T cells are already present and are activated due to a strong activation signal from their cognate targets (i.e. professional antigen-presenting cells) at inflammation sites. PD-L1-specific T cells are easily expanded by vaccination and they have an anti-tumoral effect. Thus, PD-L1-specific T cells are a particularly interesting example of the immune system's ability to influence adaptive immune responses by directly reacting against the immune-suppressive mechanisms employed by cancerous cells.

\section{P129}

Distinct patterns of clonotypic T cell responses in metastatic castration-resistant prostate cancer pts treated with standard sipuleucel-T (sip-T) compared with pts receiving a booster treatment

Li Zhang ${ }^{1}$, Harini Kandadi ${ }^{2}$, Alan Paciorek', Tao He${ }^{3}$, Nadeem Sheikh², Lawrence Fong ${ }^{1}$

${ }^{1}$ University of California San Francisco, San Francisco, CA, USA;

${ }^{2}$ Dendreon Pharmaceuticals Inc., Seattle, WA, USA; ${ }^{3}$ College of Science and Engineering, San Francisco State University, San Francisco, CA, USA Correspondence: Li Zhang (kristin.macintosh@nexusggmed.com) Journal for ImmunoTherapy of Cancer 2017, 5(Suppl 2):P129

\section{Background}

Sip-T is an FDA-approved autologous cellular immunotherapy for asymptomatic or minimally symptomatic mCRPC. Neoadjuvant sip-T induced activated $T$ cell infiltration into prostate tissue [1] and broadened the TCR repertoire within prostate tumor tissue vs nonsip-T-treated patients [2]. To test if sip-T induces long-lasting memory $\mathrm{T}$ cell responses, we compared treatment-induced changes in TCR repertoire between patients who received standard sip-T treatment (STRIDE) vs patients retreated with sip-T as a boost (P10-1).

Methods

In P10-1 ( $\mathrm{N}=8)$, patients were previously treated with sip- $\mathrm{T}$ in an androgen-dependent setting and retreated after a median of 8.6 years [3]. In STRIDE ( $\mathrm{N}=52)$, patients received sip-T with concurrent or sequential enzalutamide [4]. Peripheral blood mononuclear cells (PBMCs) were collected at baseline and during/post-sip-T. Deep sequencing and sequence diversity of the TCR VDJ region were performed using the Immunoseq assay (Adaptive Biotechnologies) and the Shannon diversity index and clonality, respectively. TCR dynamics across time points was evaluated by Morisita's distance, intraclass correlation coefficient, and fold-change analysis [5].

Results

Baseline TCR diversity was similar between the two studies ( $p=0.590$, Shannon frequency; $p=0.700$, clonality; Wilcoxon rank sum test). Significant increases in TCR diversity assessed by clonality were observed from baseline to week $4(p<0.001)$ and week $6(p=0.030)$ in STRIDE, but no significant changes were observed for post-treatment time points in P10-1. Morisita's distance in was significantly higher at week $2(\mathrm{p}=0.040)$ and $4(\mathrm{p}=0.013)$ in STRIDE vs $\mathrm{P} 10-1$, indicating a more consistent TCR repertoire in P10-1 across time points. Percent increases in clones at post-treatment time points were significantly higher in P10-1 vs STRIDE (week 6: $p=0.007$, week 26: $p=0.015$, week 52: $p=0.026$ (Fig. 1). The extent of change within the top 100 most abundant TCR sequences (clonal shuffling) was greater and was initiated earlier in P10-1 vs STRIDE (Fig. 2).

Conclusions

These data indicate that initial sip-T treatment of naïve mCRPC patients programs the TCR repertoire, which is maintained over time consistent with immunologic memory. Retreatment with sip-T expands the number of select $\mathrm{T}$ cell clones persisting up to 1 year after retreatment, which is characteristic of immunological boosting following successful vaccination.

Trial Registration

NCT01338012 (P10-1), NCT01981122 (STRIDE)

\section{References}

1. Fong, et al. Natl Cancer Inst. 2014; 106(11): dju268

2. Sheikh, et al. Cancer Res. 2016; 76: 3711-3718.

3. Beer, et al. Eur J Cancer. 2013; 49 (Suppl. 2): Abstract 2909.

4. Petrylak, et al. Eur J Cancer. 2015; 51: S483.

5. Zhang, et al. BMC Bioinformatics. 2017; 18: 129.

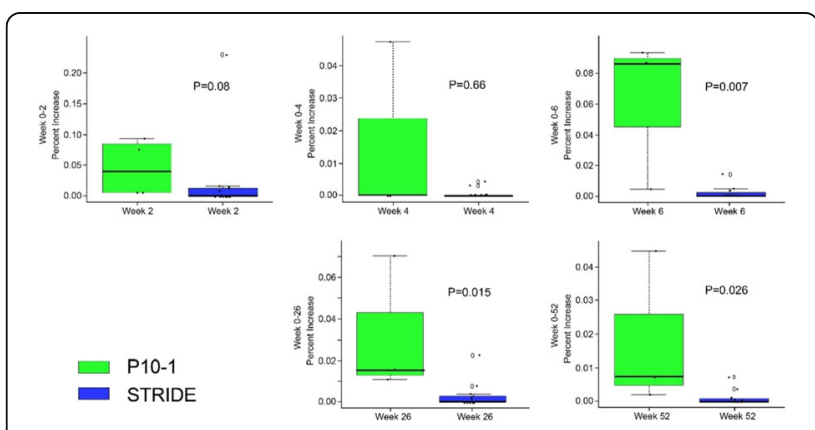

Fig. 1 (abstract P129). Percent increase in clones in MCRPC patients retreated with sip-T (P10-1) compared with patients receiving a late booster treatment (STRIDE) 


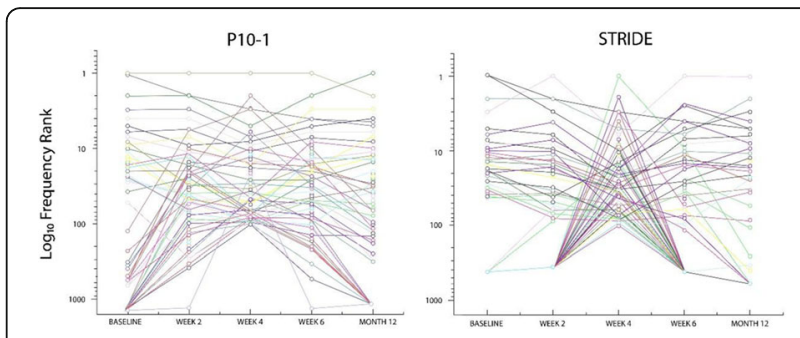

Fig. 2 (abstract P129). Clonal shuffling of top 100 clones at week 4 of sip-T treatment

\section{P130}

In situ vaccination with Flt3L, radiation, and poly-ICLC induces a potent immune response in patients with follicular lymphoma Thomas Marron', Linda Hammerich', Miriam Merad', Adeeb Rahman', Tibor Keler', Thomas Davis², Andres Salazar ${ }^{3}$, Nina Bhardwaj',

Joshua Brody

${ }^{1}$ Icahn School of Medicine at Mount Sinai, New York, NY, USA; ${ }^{2}$ Celldex Inc, Hampton, NJ, USA; ${ }^{3}$ Oncovir, Inc, Washington, DC, USA

Correspondence: Thomas Marron (thomas.marron@mountsinai.org) Journal for ImmunoTherapy of Cancer 2017, 5(Suppl 2):P130

\section{Background}

Lymphomas are the $5^{\text {th }}$ most common cancer in the US; roughly half of these are indolent non-Hodgkin's lymphomas (iNHLs), which outside of allogeneic stem cell transplant remain incurable. A previous trial of in situ vaccination (ISV) in iNHLs combined intratumoral (IT) CpG injection with localized radiotherapy (XRT) with some clinical success. One limitation in this previous trial may have been a paucity of IT dendritic cells (DCs), particularly Batf $3^{+} C D 141^{+}$DCs known to cross-present antigen to T cells, and to express high levels of TLR3. Hence, a novel iteration of ISV was developed which incorporates Flt3-ligand (Flt3L), to increase IT DCs, and poly-ICLC.

Methods

In an ongoing clinical trial patients receive: 1) IT Flt3L for 9 days, 2) XRT two consecutive days, and 3) IT Poly-ICLC on days 10, 14, 17, and weekly thereafter. Primary endpoints are safety/tolerability and overall response. Biopsy tissue and peripheral blood is taken before and during treatment to assess changes in tumor microenvironment by flow cytometry and CyTOF.

In the murine model of ISV in which sub-cutaneous A20 (B cell lymphoma) tumors is injected with Flt3L for 9 consecutive days, irradiated and then treated with poly-ICLC daily for 5 days, systemic anti-PD-1 was administered every 3 days.

Results

Biopsy tissue and peripheral blood from patients confirms that Flt3L induces an influx of CD1c and CD141 DCs into treated tumors, which attain a mature effector (PD-L1 ${ }^{\text {lo }} \mathrm{CD} 80^{\text {hi }}$ ) phenotype following initiation of poly-ICLC; similarly, intratumoral $T$ cells attain a more mature-if not exhausted-phenotype. CyTOF performed on peripheral blood from 2 patients with clinical responses and 4 patients with progressive disease revealed larger populations of more terminally differentiated $\mathrm{T}$ cells with high expression of markers of exhaustion such as PD1, LAG3, and TIGIT (Fig. 1).

Similar findings of an exhausted intratumoral $T$ cell phenotype were seen in the murine ISV model. The addition of anti-PD1 to reverse this exhaustion resulted in significant improvement in survival and tumor regression, with over $75 \%$ of mice clearing tumors (Fig. 2).

\section{Conclusions}

Though small numbers, data from this ISV trial suggest that nonresponders have larger populations of exhausted T cells, also seen in the murine model of this ISV. The addition of PD-1 reverses this exhaustion in mice, and results in significantly improved tumor regression. These findings will inform a novel ISV which will incorporate checkpoint blockade in humans with lymphoma and peripherally accessible solid tumors. Trial Registration

ClinicalTrials.gov Identifier NCT01976585

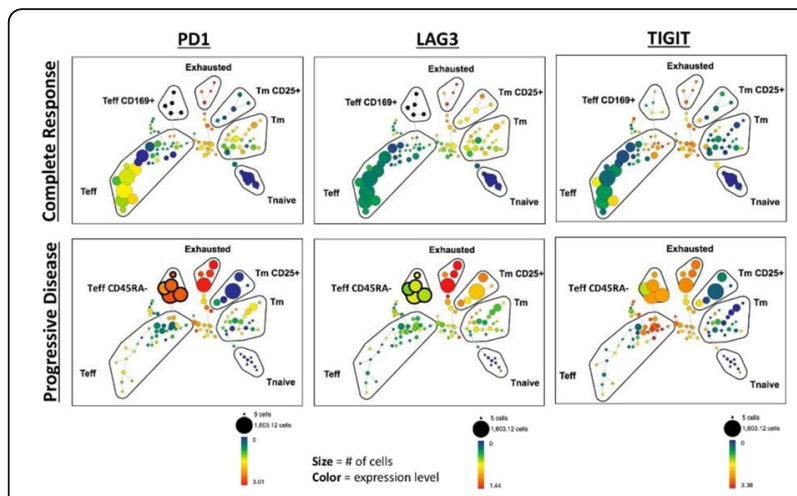

Fig. 1 (abstract P130). CD8 peripheral blood T cells in ISV responders and non-responders

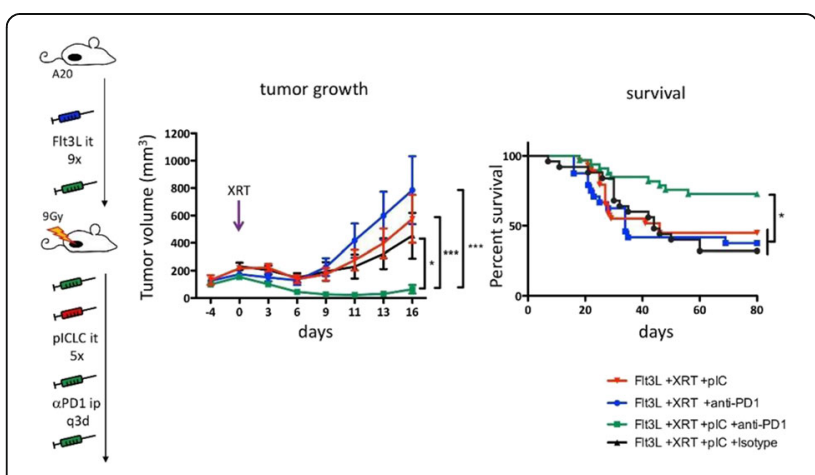

Fig. 2 (abstract P130). Murine ISV + anti-PD1 results

P131

Contrasting roles of cyclooxygenase- 1 and cyclooxygenase- 2 in cell-mediated immunity to Listeria monocytogenes

Courtney McDougal, Erin Theisen, John-Demian Sauer

University of Wisconsin - Madison, Madison, WI, USA

Correspondence: Courtney McDougal (cmcdougal@wisc.edu); JohnDemian Sauer

Journal for ImmunoTherapy of Cancer 2017, 5(Suppl 2):P131

\section{Background}

Listeria monocytogenes is an intracellular bacterium that is currently being developed as a cancer immunotherapeutic platform due to its ability to elicit robust $C D 8^{+} \mathrm{T}$ cell responses. Though the role of cytokines in Listeria-stimulated immunity has been extensively studied, the influence of lipid modulators of inflammation, known as eicosanoids, is less clear. Understanding how eicosanoids impact immunity is critical as current L. monocytogenes-based immunotherapy trials utilize nonsteroidal anti-inflammatory drugs (NSAIDs) as analgesics to alleviate patient discomfort. We hypothesized that eicosanoids, particularly those produced downstream of cyclooxygenases, influence the response to $L$. monocytogenes.

Methods

C57BI/6, microsomal prostaglandin $\mathrm{E}_{2}\left(\mathrm{PGE}_{2}\right)$ synthase-1 -/- (mPGES1), or Cox-1 -/- mice treated with or without indomethacin (broad cyclooxygenase inhibitor), celecoxib (Cox-2 inhibitor), or acetaminophen were immunized with $10^{3}$ DactA/Din/B L. monocytogenes and challenged 30 days later with a lethal dose of wild type L. monocytogenes. Bacterial burdens in the spleens and livers were enumerated at 68-72 hours post infection to assess for protective immunity.

To assess acute $T$ cell responses, mice were immunized with $10^{7}$ DactA/Din/B L. monocytogenes expressing B8R and OVA. B8R specific 
responses were examined at 7 days post immunization for CD8+ $T$ cell populations producing IFNg, TNFa, and IL-2.

Eicosanoid levels were assessed in the spleen from 4 to 48 hours post immunization with $10^{7}$ DactA/Din/B L. monocytogenes. Extracted eicosanoids were analyzed by high performance liquid chromatography and mass spectrometry.

Results

Treatment of mice with the non-specific cyclooxygenase inhibitor indomethacin led to decreased antigen specific $T$ cells and protective immunity, suggesting a role of eicosanoids in the context of L. monocytogenes stimulated immunity. Immunization of $\mathrm{Cox}^{-1 /-}$ deficient mice suggested that cyclooxygenase- 1 activity was detrimental to immunity. In contrast, treatment with celecoxib demonstrated that cyclooxygenase-2 activity was required for immunity. Analysis of eicosanoid levels in the spleen through 48 hours following immunization revealed an acute upregulation of the eicosanoid $\mathrm{PGE}_{2}$ in C57BI/6 mice twelve hours post immunization. Importantly, we found that $\mathrm{PGE}_{2}$ induction is critical for immunity as mice deficient in mPGES-1 showed an impaired cell-mediated immune response. $\mathrm{Fi}$ nally, we demonstrated that the non-NSAID analgesic acetaminophen did not influence the development of cell mediated immunity during $L$. monocytogenes immunization.

\section{Conclusions}

These results indicate that eicosanoids impact the immune response to $L$. monocytogenes. Production of eicosanoids downstream of cyclooxygenase-1 is detrimental to cell-mediated immunity where cyclooxyegenase-2 is critical, particularly through $\mathrm{PGE}_{2}$ production. Understanding how eicosanoids modulate immunity could present novel mechanisms to improve the use of $L$. monocytogenes as an immunotherapeutic.

\section{P132}

Overexpression of androgen receptor (AR) in prostate cancer cells following androgen deprivation increases recognition by ARspecific CD8+ T cells

Melissa Gamat', Brian Olson'1, Joseph Seliski', Thomas Sawicki', Justin Jeffery ${ }^{1}$, Leigh Ellis², Charles Drake ${ }^{3}$, Jamey Weichert ${ }^{1}$, Douglas McNeel ${ }^{1}$

${ }^{1}$ University of Wisconsin, Madison, WI, USA; ${ }^{2}$ Dana Farber Cancer Institute, Boston, MA, USA; ${ }^{3}$ Columbia University, New York, NY, USA

Correspondence: Douglas McNeel (dm3@medicine.wisc.edu) Journal for ImmunoTherapy of Cancer 2017, 5(Suppl 2):P132

\section{Background}

Androgen deprivation therapy (ADT) is the primary treatment for recurrent and metastatic prostate cancer. There has been a lot of interest in combining ADT with targeted immunotherapies, because ADT has demonstrated immunostimulatory properties. In this study, we hypothesized that overexpression of AR as a mechanism of resistance to ADT may result in enhanced AR-specific T cell recognition and cytolysis, suggesting that AR-targeted immunotherapies might specifically best be combined with androgen deprivation.

Methods

We examined the expression of AR in 22RV1 cells cultured in serumreplete or androgen-deprived media and determined whether increased expression of AR would result in increased AR-specific T cell immune recognition. We then examined whether ADT could be combined with a DNA vaccine encoding the AR to increase the antitumor response using two mouse models of prostate cancer (prostate-specific PTEN-deficient mice and MycCaP).

Results

Culturing 22RV1 cells in androgen-deprived medium resulted in increased AR expression compared to 22RV1 cells cultured in complete medium. The overexpression of AR resulted in increased recognition and cytolytic activity of AR-specific CD8+ T cells. When ADT was combined with a DNA vaccine encoding the $A R$, there was improved anti-tumor responses, as demonstrated by smaller tumor volumes and delays in the emergence of castration-resistant tumors using two models of prostate cancer. Furthermore, depletion of CD8+ T cells abrograted this delayed growth of castration resistant tumor growth, demonstrating that the anti-tumor response of the DNA vaccine and ADT is mediated by CD8+ T cells.

Conclusions

Together these data suggest that combining ADT with an ARtargeted vaccine could be investigated for the treatment of prostate cancer to delay or prevent the development of castration resistance due to overexpression of AR.

\section{P133}

A heterologous prime-boost vaccination strategy combining a

Listeria and DNA-based vaccine encoding prostatic acid phosphatase (PAP) elicits a strong antigen-specific, anti-tumor response

Laura Johnson', Dirk Brockstedt ${ }^{2}$, Bill Hanson², Meredith Leong ${ }^{2}$, Peter Lauer $^{2}$, Douglas McNeel ${ }^{1}$

${ }^{1}$ University of Wisconsin, Madison, WI, USA; ${ }^{2}$ Aduro Biotech, Berkeley, CA USA

Correspondence: Douglas McNeel (dm3@medicine.wisc.edu)

Journal for ImmunoTherapy of Cancer 2017, 5(Suppl 2):P133

\section{Background}

Immunotherapies have demonstrated clinical benefit for many types of cancers. Sipuleucel $\mathrm{T}$, an autologous cell-based vaccine targeting the prostate tumor antigen prostatic acid phosphatase (PAP), received FDA approval in 2010 for the treatment metastatic, castration-resistant prostate cancer. Given the cost and logistics of this autologous cell product, off-the-shelf vaccines are highly desirable. We have investigated a DNA vaccine encoding PAP (pTVG-HP) in two clinical trials in patients with biochemically recurrent prostate cancer. In both trials, persistent PAP-specific, Th1-type immunity could be elicited. In the current study, we sought to evaluate whether the immunogenicity and anti-tumor efficacy of this vaccine could be augmented using a prime-boost vaccination strategy with another genetic vaccine approach. Specifically, we investigated a live attenuated double-deleted strain (LADD) of Listeria monocytogenes encoding PAP, given alone or in sequence with the PTVG-HP DNA vaccine, and using a humanized murine tumor model.

Methods

Transgenic mice expressing HLA-A2.01 and HLA-DRB1*0101, but not murine MHC class I or class II (A2/DR), were immunized alone or with heterologous prime-boost strategies using a DNA (pTVG-HP) or listeria-based vaccine (Lm-PAP), each targeting PAP. Splenocytes from immunized mice were evaluated for immune responses to $\mathrm{MHC}$ class I and class II-restricted, PAP-specific epitopes using IFNg ELISPOT and intracellular cytokine staining (ICCS). Anti-tumor response to PAP-expressing syngeneic tumors were also assessed.

Results

A2/DR mice immunized with LM PAP or PTVG-HP alone developed CD4+ and CD8+ PAP-specific immune responses and anti-tumor responses. However, A2/DR mice immunized with Lm-PAP generated primarily an immune response to a PAP-specific MHC class I epitope while mice immunized with the DNA vaccine developed an immune response to PAP-specific, MHC class II epitopes. A2/DR mice primed with pTVG-HP and then boosted with Lm-PAP developed more robust immune responses and anti-tumor responses. The greater antitumor efficacy with this specific sequence was dependent on the development of a CD4+ T cell response and was not due to an adjuvant effect from Listeria. Differences in T cell immunity elicited were also not due to differences in B cell antigen presentation following DNA immunization.

\section{Conclusions}

Prime-boost vaccination with recombinant DNA and Listeria vaccines elicited CD4+ and CD8+ $T$ cell immunity and anti-tumor responses in a relevant murine model. The sequence of immunization, with DNA priming prior to a heterologous immunization approach, was superior, consistent with studies in other systems, and is likely due to the early generation of Th1biased CD4+ T cell immunity. 
P134

Preclinical in vivo model validation for the development of a novel vaccine based strategy targeting IDO1

Souvik Dey ${ }^{1}$, Erika Sutanto-Ward', Arpita Mondal', Katharina Luise Maria Kopp², Mai-Britt Zocca ${ }^{2}$, Ayako Wakatsuki Pedersen², Mads Hald Andersen ${ }^{3}$, Alexander J. Muller ${ }^{1}$

${ }^{1}$ Lankenau Institute for Medical Research, Wynnewood, PA, USA; ${ }^{2} \mathrm{O}$ Biotech ApS, Copenhagen, Denmark; ${ }^{3}$ University of Copenhagen, Copenhagen, Denmark

Correspondence: Alexander J. Muller (muller@limr.org) Journal for ImmunoTherapy of Cancer 2017, 5(Suppl 2):P134

\section{Background}

IDO1 (indoleamine 2, 3-dioxygenase 1) is a tryptophan catabolizing enzyme that fosters a tumor-promoting inflammatory microenvironment. IDO1 acts to subvert T cell immunity at multiple levels, including suppressing effector $T$ cells and inducing/activating Tregs. The therapeutic potential of IDO1 inhibitors, particularly in combination with current 'immune checkpoint' agents, is now being actively explored in multiple clinical trials, including Phase 3 studies. In this context, a particularly intriguing finding has been the observation that humans exhibit MHC class I restricted, cytotoxic T cell reactivity against IDO1-expressing cells. IDO1 reactive $T$ cells are found not only in cancer patients, where pathophysiological elevation of IDO1 has been frequently noted, but even in healthy individuals. This finding implies the existence of a T cell-mediated, counter-regulatory mechanism directed against IDO1. The results from initial clinical studies employing a peptide-based vaccine approach to harness this anti-IDO1 response for the benefit of cancer patients have thus far been encouraging. However, there is a pressing need to establish preclinical models for investigating the underlying basis of the antiIDO1 response in order to inform treatment optimization.

Methods

See Figure Legends.

Results

To develop a surrogate mouse model to mimic the observed MHC Irestricted response to human IDO1 peptides, computer algorithms were used to rank peptides. $\mathrm{H}^{\mathrm{d}}$ restricted peptides were specifically queried to be compatible with the Balb/c strain based, CT26 colon carcinoma model, selected based on high levels of IDO1 expression and responsiveness to IDO1 inhibition reported for these tumors. A subset of these peptides were found to be capable of stimulating IFNg production in a recall response assay. Prophylactic vaccination of mice with each of the peptides produced varying degrees of growth suppression against CT26 tumors, which unexpectedly, did not directly correspond with their effectiveness in the recall response assay. Therapeutic treatment of established CT26 tumors with a combination of peptide vaccine and anti-PD-1 antibody treatment produced a combinatorial anti-tumor response beyond what was achieved with either agent alone. Studies into the underlying nature of the IDO1-directed, anti-tumor response are currently ongoing. Conclusions

As noted initially in humans, our results demonstrate that mice are capable of mounting an effector T cell response against IDO1, confirming that this endogenous protein is somehow excluded from normal tolerance mechanisms. Initial findings that IDO1 peptide derived vaccines can elicit effective anti-tumor responses confirms the utility of mouse tumor models for further exploration and refinement of this novel approach to IDO1 directed cancer therapy.

\section{P135}

PEGylated tumor membrane nano-vesicles for eliciting adaptive immune responses against melanoma

Lukasz Ochyl, James Moon

University of Michigan, Ann Arbor, MI, USA

Correspondence: James Moon (ochyl@umich.edu)

Journal for ImmunoTherapy of Cancer 2017, 5(Suppl 2):P135

\section{Background}

Cancer immunotherapy has progressed greatly over the past decade with the approval of checkpoint blockade therapies and numerous cancer vaccine clinical trials. However, lack of consistent response rates and presence of immune evasion require further improvements. This project utilizes the wide antigen repertoire within tumor cell lysate to elicit cytotoxic T cell lymphocyte (CTL) and humoral responses against tumors in mice. We have prepared membrane vesicles from a model melanoma cell line, B16F10-OVA, and stabilized them by inserting poly (ethylene glycol) (PEG) layer on the surfaces of membrane vesicles. The resulting nano-vesicles effectively drained to secondary lymphoid organs and co-delivered antigens and adjuvants to antigen presenting cells, initiating $\mathrm{T}$ cell and IgG responses against model tumor antigens. Additionally, combination therapy with checkpoint blockade inhibitor, anti-PD-1 antibody, was utilized to enhance the efficacy of the vaccine.

Methods

Tissue culture-grown B16F10-OVA cells were harvested and prepared into cell lysate through freeze-thaw cycles followed by probe-tip sonication. After washing, the remaining fraction was incubated with calcium chloride to allow for fusion of membrane vesicles. The resulting vesicles were washed to remove unincorporated proteins and materials, followed by PEGylation and incorporation of $\mathrm{CpG}$ (a potent TLR9 agonist) by the post-synthesis insertion method. Stabilized nano-vesicles were administered subcutaneously at the tail-base utilizing a prime-boost schedule either in naïve or tumor-bearing C57BL/6 mice.

Results

PEGylated nano-vesicles maintained average hydrodynamic size of approximately $160 \mathrm{~nm}$ with good polydispersity index $(<0.200)$ for over three weeks at $4^{\circ} \mathrm{C}$, whereas cell lysate without PEGylation aggregated within a few days. After subcutaneous administration in C57BL/6 mice, PEGylated membrane vesicles accumulated 2.5 -fold more effectively in the draining lymph nodes, compared with non-PEGylated lysate. Naïve C57BL/6 mice vaccinated with B16F10-OVA vesicles containing $\mathrm{CpG}$ generated modest CTL responses against OVA, while also eliciting IgG antibodies against whole cell lysate. Interestingly, in tumor-bearing mice, vaccination with membrane vesicles with $C p G$ resulted in strong anti-OVA CTL immunity (reaching 5\% of all CD8+ peripheral blood mononuclear cells), which was also enhanced by combination therapy with anti-PD-1 antibody. Vaccinated animals exhibited a prolonged median survival time, compared to untreated animals.

Conclusions

Tumor cell membrane vesicles stabilized by surface PEGylation can be stored and administered as a potent vaccine vehicle. Effective codelivery of antigens and adjuvants to the draining lymph nodes of tumor-bearing animals allowed for enhanced immune responses and prolonged survival in a therapeutic setting.

\section{P136}

Dendritic cell vaccination combined with chemotherapy for advanced or relapsed head and neck cancer patients induces immunological and clinical responses

Shuichi Ota (ogasawara@hokuyu-aoth.org)

Sapporo Hokuyu Hospital, Sapporo, Japan

Journal for ImmunoTherapy of Cancer 2017, 5(Suppl 2):P136

\section{Background}

The prognosis of patients with advanced squamous-cell carcinoma of head and neck is poor with a 5 -year overall survival rate of $20-40 \%$. In the current study, we have evaluated the clinical and immunological responses in patients with advanced or relapsed head and neck cancer who received dendritic cell (DC) vaccination in combination with platinum-based chemotherapy. 


\section{Methods}

Ten patients ( 8 males, 2 females; aged $49-85$ years) were enrolled in the present study. Autologous DCs were generated by culturing adherent mononuclear cells with interleukin-4 and granulocytemacrophage colony stimulating factor. DCs were then loaded with synthetic peptides derived from Wilms' tumor 1 (WT1) and/or MUC1 mucin following maturation by prostaglandin $E_{2}$ and a toll like receptor 4 agonist, OK432. Peptide-loaded mature DCs and OK432 were administered intradermally every 2 weeks for 7 times and repeated biweekly until tumor progression. Induction of vaccine-induced immune responses was evaluated using an ELISpot assay, a HLAtetramer assay and a flow cytometry analysis.

Results

The treatment was well tolerated and none of the patients experienced serious adverse events during the treatment period. Of 10 patients, 5 had stable disease (SD) and 5 had disease progression (PD) after one course of vaccination. Median overall survival was 14.5 months. Survival of patients achieving SD after DC vaccination (responder) was longer than those who did not respond to the treatment (non-responder) (median duration of survival; 17 vs 6 months). ELISpot assay showed the increase in spot-positive cells in both responders and non-responders after one course of vaccination. However, increment in positivity was marked in responders in comparison with non-responders; 24.3 and 3.5 fold in responders and non-responders, respectively. Similarly, HLA-tetramer assay showed the increase in positivity of WT1-specific $\mathrm{CD}^{+} \mathrm{T}$ cells in responders. Percentage of NK and NKT cells were almost same before and after vaccination. On the other hand, percentage of both CD14 ${ }^{+} \mathrm{HLA}-\mathrm{DR}{ }^{-}$and $\mathrm{CD} 15^{+} \mathrm{CD} 11 \mathrm{~b}^{+} \mathrm{CD} 33^{+}$myeloid-derived suppressor cells decreased by $65.1 \%$ and $18.2 \%$, respectively, indicating that DC vaccination may contribute to the reversal of immunosuppression by these cells.

Conclusions

DC vaccine-based immunotherapy combined with conventional chemotherapy was demonstrated to be safe and elicit acquired cellular immune responses correlated with clinical effects. These results suggest that add-on DC vaccination might be a promising novel strategy for the treatment of patients with advanced or relapsed head and neck cancer.

Trial Registration

University hospital Medical Information Network, Japan ID: UMIN000027279

\section{P137}

Immune mechanisms of the response to TG4010, a viral-based vaccine, in patients with advanced non-small cell lung carcinoma Kaidre Bendjama, Eric Quemeneur, Bérangère Bastien, Maud BrandelyTalbot

Transgene S.A., Illkirch-Graffenstaden, France

Correspondence: Eric Quemeneur (quemeneur@transgene.fr)

Journal for ImmunoTherapy of Cancer 2017, 5(Suppl 2):P137

\section{Background}

TG4010 (MVA-MUC1-IL2) is a viral-based vaccine encoding mucin-1 and interleukin-2 which has been developed as an immunotherapy for epithelial cancers displaying abnormal MUC1. TG4010 has shown clinical benefit in patients with advanced NSCLC in combination with chemotherapy in phase II trials, and is currently assessed in combination with nivolumab at UC Davis (NCT 02823990). In this perspective, it has been essential to demonstrate the synergy between TG4010 vaccination and immune checkpoint inhibition; more specifically that improved overall survival (OS) correlates with the onset of a specific and diverse CD8+ repertoire, and to identify patient subpopulations most likely to benefit from TG4010 vaccination.

Methods

Samples were collected from patients of the TIME study. PBMCs were analyzed by using combinatorial MHC multimer staining to detect CD8+ T-cells reactive to MUC1 epitopes and other tumor-associated antigens (TAAs). Monitoring of immune background also included the phenotyping of peripheral blood cells and of circulating cytokines to identify predictive and prognostic biomarkers.

Results

We report that improved OS under TG4010 treatment correlated with development of specific CD8+ T-cell responses against MUC1 epitopes. Interestingly, MUC1 specific response also correlated with the onset of T cell clones against several lung TAAs (11 previously described epitopes for lung cancer were assessed +4 predicted neo-epitopes). These results support the causality of specific $C D 8^{+} T$ cell responses in clinical response to TG4010, and constitute the first clinical report of epitope spreading after vaccination with a viral vector. We could also observe a benefit in subpopulations with unfavorable immune background; OS increased from 9.5 to 14.6 months (HR 0.56 [0.37-0.86]) upon vaccination with TG4010 in patients with high levels of Tregs at baseline, and from 10.3 to 15.2 (HR 0.59 [0.39-0.89]) in patients with low level of innate immunity at baseline (low NK cells).

\section{Conclusions}

Immune monitoring of TG4010-treated patients has confirmed the involvement of MUC1 specific CD8+ T-cell responses, and has demonstrated an original mechanism of epitope spreading. Clinical outcome correlated positively with the diversity of T-cell repertoire, suggesting the major driver function of viral-based vector.

\section{P138}

Tumor-specific CD8 T cell checkpoint expressions in melanoma patients that received adenovirally-engineered DC vaccine +/- IFN-

Patricia M Santos, Lisa H Butterfield, Samuel Du, John M. Kirkwood University of Pittsburgh Hillman Cancer Center, Pittsburgh, PA, USA Correspondence: Lisa H Butterfield (santospm@upmc.edu) Journal for ImmunoTherapy of Cancer 2017, 5(Suppl 2):P138

\section{Background}

Despite durable clinical responses, only $30-40 \%$ of melanoma patients respond to treatment with immune checkpoint blockade. It is critical to identify rational combination approaches that will enhance responses. Combinations of immune checkpoint blockade with cancer vaccines can promote and/or enhance anti-tumor $\mathrm{T}$ cell responses. We developed an adenovirus-based DC vaccine encoding three full length melanoma antigens, Tyrosinase, MART1 and MAGEA6 (AdVTMM2) designed to promote broad, polyclonal CD8+ and CD4+ T cell immunity, and tested it in a Phase I clinical trial to treat late-stage melanoma patients.

\section{Methods}

PBMC from enrolled HLA-A2 ${ }^{+}$patients were banked at baseline, postvaccination (day 43) and at day 101 after either observation or IFN-a administration. Dextramers were used to assess the frequencies of $\mathrm{CD}^{+} \mathrm{T}$ cells specific for peptides encoded by the vaccine (Tyros 369 377, MART- ${ }_{27-35}$, and MAGE-A3 $271-279$ ) before and after vaccination IFN- $\gamma$-producing CD8 T cells specific for tumor antigens were measured by ELISPOT. The expression of CTLA-4, TIM3 and PD- 1 by CD $8^{+}$ $T$ cells were analyzed by multicolor flow cytometry.

Results

There were $17 \mathrm{HLA}^{-A 2^{+}}$out of 35 enrolled patients that received all 3 AdVTMM2 DC vaccines. There was a 2.85 -fold increase in MAGE$\mathrm{A} 3_{271-279}$ and a 1.37 -fold increase in MART-1 ${ }_{27-35}$-specific $T$ cells post-vaccination compared to baseline, and the increased frequency of both tumor-specific CD8 T cells was still evident by day 101. The ELISPOT assay showed a 3.5 -fold increase in MART-1 specific CD8 T cells after vaccination. Patients previously treated with a-CTLA-4 with/without a-PD-1 therapy had more tumor-specific IFN- $y$ producing $T$ cells before and after vaccination compared to patients that received no prior checkpoint blockade. These patients also had lower frequencies of CTLA-4 ${ }^{+} \mathrm{TIM}^{+}$tumor-specific $\mathrm{CD} 8^{+} \mathrm{T}$ cells. While most patients examined expressed PD-1 in $\sim 25 \%$ of tumor-specific CD8 T cells, 2 patients with no prior a-PD-1 treatment had lower levels of PD-1 on tumor-specific $\mathrm{CD}^{+} \mathrm{T}$ cells that correlated with good clinical outcome. IFN-a treatment did not enhance anti-tumor specific CD8 immunity after DC vaccination. 


\section{Conclusions}

Results show that vaccination with AdVTMM2 DC increased the frequency of circulating tumor-specific CD8 T cells. Patients that received checkpoint blockade prior to DC vaccination had a higher number of anti-tumor specific CD8 T cells compared to patients that received DC vaccination alone, and IFN- $a$ did not increase frequencies, suggesting that combining DC vaccination with checkpoint blockade is a rational approach to enhance anti-tumor immunity. Trial Registration

NCT01622933: Multiple Antigen-Engineered DC Vaccine for Melanoma

\section{References}

1. Quoix E, Lena H, Losonczy Get al. TG4010 immunotherapy and first-line chemotherapy for advanced non-small-cell lung cancer (TIME): results from the phase $2 \mathrm{~b}$ part of a randomized, double-blind, placebo-controlled, phase 2b/3 trial. Lancet Oncol. 2016;17: 212-223.

2. Tosch C, et al. Viral-based vaccine TG4010 induces broadening of specific immune response and improves outcome in advanced NSCLC. Submitted.

\section{P139}

A viral vectored vaccine based on shared tumor neoantigens for prevention and treatment of microsatellite instable (MSI) cancers Guido Leoni ${ }^{1}$, Anna Morena D'Alise ${ }^{1}$, Gabriella Cotugno ${ }^{1}$, Federica Mori ${ }^{1}$, Maria Teresa Catanese', Francesca Langone', Imma Fichera' Maria De Lucia ${ }^{2}$, Rosa Vitale ${ }^{1}$, Adriano Leuzzi ${ }^{1}$, Elena Di Matteo ${ }^{1}$, Antonella Folgori', Stefano Colloca', Alfredo Nicosia ${ }^{1,2}$, Armin Lahm', Elisa Scarselli ${ }^{1}$

${ }^{1}$ Nouscom Srl, Rome, Italy; ${ }^{2}$ University of Naples Federico II, Naples, Italy Correspondence: Elisa Scarselli (g.napolitano@nouscom.com) Journal for ImmunoTherapy of Cancer 2017, 5(Suppl 2):P139

\section{Background}

Tumor neoantigens are very appealing targets for cancer vaccines for the low risk of self-tolerance and autoimmunity. Most neoantigens are specific to an individual tumor and therefore can only be utilized for personalized vaccination approaches. There is, however, a group of cancers whose neoantigens are shared among patients: microsatellite instable (MSI) tumors, caused by a defective DNA mismatch repair system. In these tumors, mutations accumulate mainly in microsatellite regions of mononucleotide repeats (MNR) as deletions of 1 nucleotide, and affect a limited number of genes. Here, we describe a method to select shared MSI-associated neoantigens for designing a universal vaccine for prevention and treatment of MSI tumors.

\section{Methods}

Exome and RNA sequencing data were analyzed for 69 colorectal, 85 gastric and 166 endometrial MSI cancers and matched normal samples in the TCGA database. We selected deletions of 1 nucleotide at MNR of coding genes that result in the formation of tumor-specific neoantigens, called frame shift peptides (FSPs). Three key parameters were taken into account by our pipeline to prioritize FSPs for an "offthe-shelf" MSI vaccine: (i) expression of the mutated gene; (ii) sharedness of the mutation among MSI patients; (iii) length of the FSP. An algorithm was then designed to choose the minimal subset of FSPs that ensured optimal coverage of MSI tumors.

Results

Nouscom proprietary pipeline selected 209 FSPs (for a total length of 6021aa) for inclusion in the MSI vaccine. These FSPs were found at expected frequencies in tumor Flash Frozen (FF) biopsies from $6 \mathrm{MSI}$ patients. The $209 \mathrm{FSPs}$ were assembled into 4 artificial genes and cloned into Great Apes adenoviral vectors (GAd) and Modified Vaccine Ankara (MVA). Potent immunogenicity of the vaccine in heterologous prime/boost regimen was demonstrated in mice.

\section{Conclusions}

In targeting a large set of shared neoantigens, the MSI vaccine has a high probability to reach the necessary breadth of immune responses to prevent tumor occurrence in Lynch syndrome carriers affected by a genetic defect of DNA mismatch repair system. Moreover, the vaccine can be used therapeutically in established MSI tumors to potentiate the activity of checkpoint inhibitors.

\section{P140}

NKTR-214 enhances anti-tumor $T$ cell immune responses induced by checkpoint blockade or vaccination

Meenu Sharma', Faisal Fa'ak', Louise Janssen', Hiep Khong',

Zhilan Xiao', Yared Hailemichael', Manisha Singh'1, Christina Vianden',

Adi Diab', Jonathan Zalevsky², Ute Hoch², Willem Overwijk

${ }^{1}$ UT MD Anderson Cancer Center, Houston, TX, USA; ${ }^{2}$ Nektar

Therapeutics, San Francisco, CA, USA

Correspondence: Willem Overwijk (msharma1@mdanderson.org)

Journal for ImmunoTherapy of Cancer 2017, 5(Suppl 2):P140

\section{Background}

High dose IL-2 has been used in treatment of metastatic melanoma and renal cell carcinoma. However, expansion of suppressive Tregs and physiologic toxicities associated with IL-2 treatment, limited its use in anti-cancer therapies. NKTR-214 is an engineered IL-2 cytokine, designed to provide a non-toxic, stable and more efficient alternative to IL-2. NKTR-214 provides sustained activation of the IL-2 pathway through controlled release of active CD122-biased (IL-2RßY) cytokine. In the present study, we assessed the synergy of NKTR-214 with anticancer therapies in treatment of solid murine tumors. We investigated whether NKTR-214 can promote expansion and function of tumor specific CD8+ effector T cells, induced by $T$ cell checkpoint blockade therapy or peptide-based vaccination. We also studied how NKTR-214 impacts the proliferation and apoptosis of effector CD8+ T cells vs. immunosuppressive Tregs.

Methods

To assess synergy between NKTR-214 and checkpoint blockade, CT26 colon carcinoma tumor bearing mice were treated with either NKTR214 or checkpoint blockade or combination of both therapies together. Tumor-specific effector T cell response was also analyzed in blood. To understand the effect of NKTR-214 on antigen-specific CD8 + T cells, we adoptively transferred naïve gp100-specific TCR transgenic PMEL-1 CD8+ T cells into mice bearing established subcutaneous B16 tumors, followed by vaccination (gp100 peptide + anti-CD40 mAb + TLR-7 agonist) alone or in combination with NKTR-214 or IL-2. Mice then received NKTR-214 or IL-2 every 8 days. Tumor growth survival and T cell response in blood was monitored. To identify effect of NKTR-214 on proliferation/apoptosis of effector CD8+ T cells and Tregs, expression of proliferation marker Ki67 and apoptosis marker Annexin $\mathrm{V}$ was also analyzed.

Results

We observed that NKTR-214 demonstrated efficient therapeutic synergy with checkpoint blockade. NKTR-214 also enhanced anti-tumor effect of vaccination and improved survival of mice by potently suppressing tumor growth as compared to vaccination with IL-2. As compared to IL-2, NKTR-214 significantly promoted proliferation of PMEL-1 CD8+ T cells while reduced the proliferation of Tregs in tumor as well as in spleen. Furthermore, Annexin V staining showed that vaccine combined with NKTR-214 supported the survival of PMEL-1 CD8+ T cells while promoting apoptosis of Tregs in tumor. Conclusions

NKTR-214 synergizes with checkpoint blockade and with vaccination and improved the survival, proliferation and tumor infiltration of ef fector CD8+ T cells without promoting the intratumoral accumulation of Tregs. These preclinical results suggested that NKTR-214 can 
exclusively enhance intratumoral CD8+ effector $\mathrm{T}$ cell/Treg ratios to establish anti-tumor immunity.

\section{P141}

A multi-center study of hTERT immunotherapy in adults with solid tumors at high risk of relapse post-standard therapy

Anthony F. Shields ${ }^{1}$, Autumn J. McRee ${ }^{2}$, Jennifer M. Johnson ${ }^{3}$, Weijing Sun ${ }^{4}$, Ashish Chintakuntlawar ${ }^{5}$, Albert J. Sylvester ${ }^{6}$, Naseem Prostak ${ }^{6}$, Kimberly A. Kraynyak ${ }^{6}$, Matthew P. Morrow ${ }^{6}$, Jeffrey M. Skolnik ${ }^{6}$, Robert $H$. Vonderheide

${ }^{1}$ Karmanos Cancer Institute, Detroit, MI, USA; ${ }^{2}$ University of North Carolina, Chapel Hill, NC, USA; ${ }^{3}$ Thomas Jefferson University, Philadelphia, PA, USA; ${ }^{4}$ University of Pittsburgh, Pittsburgh, PA, USA; ${ }^{5}$ Mayo Clinic, Rochester, MN, USA; ${ }^{6}$ Inovio Pharmaceuticals, Plymouth Meeting, PA, USA ${ }^{7}$ Abramson Cancer Center of the University of Pennsylvania, Philadelphia, PA, USA

Correspondence: Jeffrey M. Skolnik (jeffrey.skolnik@inovio.com) Journal for ImmunoTherapy of Cancer 2017, 5(Suppl 2):P141

\section{Background}

Human telomerase (hTERT) consists of a catalytic reverse transcriptase subunit and an internal RNA template that recognize and elongate telomeric DNA ends. hTERT is expressed in $85-90 \%$ human cancer, and can be recognized by cytotoxic $\mathrm{CD} 8^{+} \mathrm{T}$ cells. The administration of optimized full length DNA sequences followed by in vivo electroporation (EP) has generated potent $\mathrm{CD}_{4}^{+}$and $\mathrm{CD} 8^{+} \mathrm{T}$ cell responses against a variety of antigens in preclinical and clinical studies. In this phase 1 dose-escalation study, synthetic optimized DNA plasmids that target hTERT (INO-1400, or mutant hTERT; and INO1401 , or SynCon ${ }^{\circledast}$ TERT) are delivered followed by EP with the CELLECTRA device, to assess the safety, tolerability, and immune effects of immunotherapy with INO-1400 or INO-1401, alone or coadministered with a plasmid encoding for hIL-12 (INO-9012), in adult patients with cancer.

Methods

Following neoadjuvant or adjuvant chemoradiotherapy and/or surgery, and screening and evaluation, patients with one of 9 solid tumors at high risk of relapse but without evidence of residual disease are enrolled to one of 10 arms and receive four doses, four weeks apart, of either INO-1400 or INO-1401 alone or in combination with INO-9012 by intramuscular (IM) injection, followed by EP. Patients are followed for tolerability, immunogenicity and clinical response. Results

As of July 26, 2017, 70 patients have received either INO-1400 or INO-1401 \pm INO-9012. Doses of 2 or $8 \mathrm{mg}$ of INO-1400/01, and of 0.5 or $2 \mathrm{mg}$ INO-9012 have been well-tolerated, with the majority of reported adverse events (AE) being low-grade and related to IM+EP administration. Two related SAEs have been reported: one patient with breast cancer post-breast revision with cellulitis at the prior surgical site; and a second patient with pancreatic cancer with abdominal pain and elevated lipase. One dose-limiting toxicity, rash, has been reported, with no other dose-limiting events to date. Preliminary immunogenicity by ELISpot for antigen-specific interferon-gamma (IFNү) secreting cells suggests that patients can generate an hTERTspecific $\mathrm{CD}^{+} \mathrm{T}$ cell population. The majority of patients continue on study in long-term follow up, with several patients on study for $>1$ year. To date there is no evidence of a dose effect with respect to AEs or immunogenicity, but data assessment is ongoing.

\section{Conclusions}

INO-1400/01 \pm INO-9012 given IM with EP is well-tolerated in adults with solid tumors. Following administration of INO-1400/01, patients can generate hTERT-specific IFN- $\gamma$ secreting cells, suggestive of an ability to break tolerance. Dosing in this study is continuing, and follow-up is ongoing.
Trial Registration NCT02960594

\section{P142}

In situ immunocytokine vaccination, radiation and checkpoint blockade therapy prevents engraftment of brain metastases in a murine melanoma model

Raghava Sriramaneni', Paul A Clark', Clinton Heinze', Emily I Guy',

Jasdeep Kler', Sara Busche', Jonathan A Lubin', Peter M Carlson',

Alexander L Rakhmilevich', Stephen D Gillies², Hans Loibner',

Alan J Korman ${ }^{4}$, Paul M Sondel', John S Kuo ${ }^{1}$, Zachary S Morris ${ }^{1}$

${ }^{1}$ University of Wisconsin, Madison, WI, USA; ${ }^{2}$ UW Madison, Carlisle, MA,

USA; ${ }^{3}$ UW Madison, Vienna, Austria; ${ }^{4}$ UW Madison, Redwood City, CA,

USA

Correspondence: Raghava Sriramaneni (sriramaneni@wisc.edu);

Zachary S Morris

Journal for ImmunoTherapy of Cancer 2017, 5(Suppl 2):P142

\section{Background}

BACKGROUND: Brain metastases develop in $>60 \%$ of advanced melanoma patients, and are now treated with surgery and/or radiation. Recent clinical checkpoint blockade studies show promising results for melanoma brain metastases. We previously showed that combining radiation and anti-CTLA-4 checkpoint blockade with in situ vaccination via intratumoral (IT) injection of hu14.18-IL2 immunocytokine tumor-specific anti-GD2 antibody fused to IL2 improved survival and reduced tumor burden in a syngeneic metastatic lung cancer model. Here we test whether this in situ vaccination strategy of combining local radiation, checkpoint blockade and in situ immunocytokine strategy prevents engraftment of melanoma brain metastases.

Methods

METHODS: GD2- B16 or GD2 ${ }^{+}$B78 (derived from B16 cell line) melanoma xenografts were implanted into flank or stereotactically implanted into brain of C57BL/6 mice. Melanoma flank xenograft were irradiated (12Gy), injected with IT-IC, and/or intraperitoneal antiCTLA-4 given to tumor-bearing mice. Overall survival, immune memory response, and histopathology performed for analysis.

Results

RESULTS: In mice bearing a single B78 flank tumor, $73 \%$ were rendered disease-free by in situ vaccine (12 Gy + IT-IC) + systemic antiCTLA-4 treatment. Testing of these pre-treated mice confirmed immune memory response after greater than 90 days, with all mice rejecting B78 cellular engraftment in contralateral flank, and $60 \%$ rejecting sequential implanted $B 78$ cellular engraftment in brain over 20 days later. Increased CD8+ and CD4+ T cells were observed at implanted brain sites compared to control contralateral striatum. In contrast, all naïve control mice implanted with B78 developed flank or brain melanomas with sparse immune infiltrates. Compared to naïve mice, pre-treated mice showed improved survival after implanting B78 cells into brains (median not reached vs 28 days, $p=0.001$ ). In mice with 5 weeks growth of a B78 flank melanoma xenograft, and a 1 day implanted B16 brain melanoma xenograft, combining in situ IT-IC vaccine + systemic anti-CTLA-4 improved survival compared to anti-CTLA-4 alone (Mean 33 days vs 20 days, $p=0.017$ ). Immunohistopathological assessment of brains from the above treatment cohorts showed a trend toward increased CD4+, CD8+, and CD16+ cells in brain metastases in the in situ vaccinated mice.

Conclusions

CONCLUSIONS: In this preclinical model, in situ vaccination of melanoma flank xenografts with hu14.18-IL2 combined with radiation and anti-CTLA-4 checkpoint blockade enhances the anti-tumor immune response and prevents engraftment of brain melanoma metastases. 


\section{Cellular Metabolism and Antitumor Immunity}

\section{P143}

T cell metabolic insufficiency explains the dysfunctional immune response in renal cell carcinoma

Nicola Annels ${ }^{1}$, Michael Denyer ${ }^{1}$, David Nicol ${ }^{2}$, Muddassar Hussain ${ }^{3}$, Anna Silvanto ${ }^{3}$, Hardev Pandha ${ }^{1}$

${ }^{1}$ University of Surrey, Guildford, UK; ${ }^{2}$ The Royal Marsden Hospital, London, UK; ${ }^{3}$ Frimley Park Hospital, Camberley, UK

Journal for ImmunoTherapy of Cancer 2017, 5(Suppl 2):P143

\section{Background}

Renal cell carcinoma (RCC) is considered an immunogenic tumor with a prominent dysfunctional immune cell infiltrate, unable to control tumor growth. Although tyrosine kinase inhibitors have improved the outlook for some patients, many patients incur disease progression, symptoms and reduced life expectancy. Following the limited success of cytokine therapy, nivolumab checkpoint-inhibitor therapy for second line treatment has reiterated the potential for immune manipulation to significantly impact on this disease. However, the response to nivolumab is limited to $25 \%$ of patients with a median duration of only 12 months. There is an urgent need to improve the potential efficacy of these new targeted immunotherapies. The immune environment created by the tumor recapitulates that present in chronic viral infections in which inappropriate and excessive antigen stimulation leads to intra-tumoral $T$ cell exhaustion. These exhausted $T$ cells exhibit defective proliferative capacities and cytokine production and display an overall phenotype of metabolic insufficiency, characterized by extensive mitochondrial alterations. We set out to comprehensively identify mitochondrial defects in exhausted RCC TILs and correlate these findings with the clinicopathological characteristics of the tumor.

Methods

Tumor cell suspensions were prepared from freshly resected RCC tumors using a combination of mechanical (GentleMACS Dissociator) and enzymatic dissociation. Exhausted T cells were analysed by multicolour FACS on paired PBMC and tumor-cell suspensions using fluorescently conjugated antibodies. The functional status of PBMC and tumor infiltrating lymphocytes (TILs) from RCC patients was evaluated for cytokine production and glucose uptake in response to CD3 cross-linking. The metabolic fitness of sorted stimulated CD3 T cells from dissociated tumors was assessed using a Seahorse XF extracellular flux analyzer and a range of mitochondria-targeted dyes.

Results

Flow cytometric analysis of TILs from late stage RCC specimens expressed a high level of PD-1 which correlated with a depolarized mitochondrial phenotype and abnormal mitochondrial function (defect in oxygen consumption rate with loss of spare respiratory capacity). These exhausted CD8+ TILs displayed a depressed glucose competency and had the lowest cytokine production. This dysfunctional phenotype was specific to TILs from advanced stage cases and not observed in the corresponding blood of RCC patients.

Conclusions

The clinical relevance of mitochondrial alterations within the exhausted RCC TILs will be determined using mitochondria-targeted antioxidant compounds to try to achieve significant functional restoration of the $T$ cells. The ultimate aim is to combine modifying the bioenergetics of the T cells with remodeling of the dysregulated metabolism of the RCC tumor microenvironment to create a more permissive environment for T cell anti-tumor activity.

\section{P144}

Investigating the kynurenine pathway as a central effector mechanism of tumor immune-suppression and its potential as therapeutic target

Luis Felipe Campesato', Sadna Budhu', X. Michelle Zhang ${ }^{2}$, Mark G. Manfredi', Karen McGovern², Jedd D. Wolchok', Taha Merghoub'

${ }^{1}$ Memorial Sloan-Kettering Cancer Center, New York, NY, USA; ${ }^{2}$ Kyn Therapeutics, Cambridge, MA, USA

Correspondence: Jedd D. Wolchok (campesal@mskcc.org); Taha Merghoub

Journal for ImmunoTherapy of Cancer 2017, 5(Suppl 2):P144

\section{Background}

Although immunotherapies have had notable impact on cancer treatment, multiple mechanisms of immune resistance prevent optimal response. The catabolism of Tryptophan into metabolites known as Kynurenines (Kyn) by enzymes like IDO or TDO has been described as a major immunosuppressive player in different tumor types, however, the mechanisms are still poorly understood. Recently it has been shown that Kyn is an agonist of the Aryl hydrocarbon Receptor (AhR), promoting tolerogenic DCs and skewing CD4+T cells towards a regulatory $\mathrm{T}$ cell phenotype. Here we sought to characterize the mechanisms of immunossupression associated with activation of the AhR pathway by Kyn in pre-clinical models of melanoma and to evaluate AhR's potential as therapy target.

Methods

B16 wild-type (B16-WT) and IDO-overexpressing B16 (B16-IDO) melanoma cells were implanted orthotopically in c57bl6 mice. Geneexpression was performed using Fluidigm qRT-PCR system. Macrophages were depleted using clodronate-liposomes $(\mathrm{CL})$ twice a week starting on day 3 after tumor implantation (T.I). Tumor-infiltrating leukocytes were analyzed by Flow Cytometry on days 10, 16 and 23 after T.I. Tumor-bearing mice are under treatment with a specific AhR antagonist (CH-223191) daily at $50 \mathrm{mg} / \mathrm{kg}$ starting at day 7 after T.I. Results

We found that B16-IDO tumors grow at a faster rate than B16-WT in vivo and present reduced expression levels of many Type 1 inflammatory genes, including IFNY, IL1B, TNFa, Granzyme B and CD40. In addition, B16-IDO tumors present higher infiltration of macrophages $\left(\mathrm{CD} 11 \mathrm{~b}^{+} \mathrm{F} 4 / 80^{+} \mathrm{Ly} 6 \mathrm{G}^{-}\right)$, which peaks 16 days after T.I. and persists as tumors progress. B16-IDO TAMs have up-regulation of classic AhRregulated genes (Cyp1a1 and Cyp1b1) and are differentially skewed towards an immunosuppressive M2 phenotype, characterized by higher expression of CD206, STAB1, Fizz1 and lower expression of several M1 markers (IL1, IL6, IL12, NOS2). Also, B16-IDO TAMs have the ability to suppress activation of $\mathrm{CD} 8^{+} \mathrm{T}$ cells in vitro and depletion of TAMs using CL abrogated B16-IDO tumor growth phenotype. Importantly, inoculation of B16-IDOs in $\mathrm{Rag}^{-/-}$and FoxP3-depleted mice abrogated the accumulation of TAMs. Studies using a specific AhR antagonist $(\mathrm{CH}-223191)$ are in progress to evaluate tumor growth and survival in this model.

Conclusions

Here we identified that the activation of the AhR pathway by Kyn in IDO-overexpressing melanomas promotes tumor growth in a macrophage-dependent manner. Importantly, we found that AhRactivated intratumor FoxP3+T cells are associated with the suppressive myeloid phenotype in IDO+ tumors. All together, our findings demonstrate that targeting the Kyn pathway through AhR-inhibition represents a promising approach in cancer patients who are resistant to immune-checkpoint blockade.

\section{P145}

CD38-NAD ${ }^{+}$axis regulates immunotherapeutic anti-tumor $\mathrm{T}$ cell response

Shilpak Chatterjee, Anusara Daenthanasanmak, Paramita Chakraborty, Megan Wyatt, Chrystal Paulos, Xue-Zhong Yu, Shikhar Mehrotra

MUSC, Charleston, SC, USA

Correspondence: Shikhar Mehrotra (chatherj@musc.edu)

Journal for ImmunoTherapy of Cancer 2017, 5(Suppl 2):P145

\section{Background}

Adoptive T cells therapy (ACT) has shown great prominence in immunotherapy of cancer. However, ACT is only able to cure a small proportion of the patients because of the rapid functional exhausting and short-term persistence in tumor bearing host. Therefore, identifying factors governing the maintenance of functional phenotype and survival of anti-tumor $T$ cells is of utmost interest.

Methods

Quantitative PCR (q-PCR), flow cytometry, and metabolomic analysis were used to evaluate the expression of various metabolism and stemness-associated genes as well as protein expression in the T cells. To compare the metabolic commitment in T cells real time 
metabolic flux analyzer (Seahorse Biosciences, USA) and radioactive tracer studies were used. Melanoma epitope gp100 or tyrosinase reactive T cells from either PMEL or TRP-1 mice respectively were used for adoptive T cell transfer $\left(1 \times 10^{6} /\right.$ mouse $)$ in $\mathrm{B} 6$ mice bearing 10 days subcutaneously established B16 melanoma.

Results

Recently, we found that Th1/17 cells which can be differentiated by combining the culture conditions of Th1 and Th17 cells, exhibited durable and superior anti-tumor activity in vivo as compared to Th1 and Th17 alone. Since metabolic fitness affects the persistence and functionality of the adoptively transferred $\mathrm{T}$ cells, we comprehensively characterized the metabolomic profile of Th1, Th17 and Th1/17 cells and identified that increased level of intracellular $N A D^{+}$is a key in regulating the anti-tumor potential of the Th1/17 cells. NAD mediated this effect mainly through providing substrate for the SIRT1 deacetylation activity since pharmacological blockade or genetic ablation of SIRT1 in Th1/17 cells completely abolished its anti-tumor activity. We further provided data in support of this observation by using CD38 deficient T cells which has very high level of NAD due to absence of NAD lyase CD38. We found that CD38 deficient T cells without any in vitro differentiation could mount potent anti-tumor response in mice bearing melanoma.

\section{Conclusions}

These data together suggest that strategies to maintain high level of intracellular NAD in anti-tumor T cells by blocking CD38 will have a potential therapeutic application in improving ACT.

\section{P146}

Co-expression of CD39 and CD103 identifies tumor-reactive CD8

TIL in human solid tumors

Thomas Duhen', Rebekka Duhen', Ryan Montler', Tarsem Mougdil', Bernard A. Fox', Christopher Dubay', Shu-Ching Chang ${ }^{3}$,

Gary Grunkemeier ${ }^{3}$, Jason McDermott ${ }^{4}$, Rom Leidner ${ }^{1}$, Richard B. Bell ${ }^{1}$, Andrew D. Weinberg

${ }^{1}$ AgonOx, Portland, OR, USA: ${ }^{3}$ Medical Data Research Center, Providence Health \& Services, Portland, OR, USA; ${ }^{4}$ Pacific Northwest National Laboratory, Richland, WA, USA

Correspondence: Thomas Duhen (thomas.duhen@agonox.com);

Andrew D. Weinberg

Journal for ImmunoTherapy of Cancer 2017, 5(Suppl 2):P146

\section{Background}

Identifying tumor antigen specific $T$ cells from cancer patients has been a goal of tumor immunologists for several decades.

Methods

none

Results

Here we identified a subset of tumor-infiltrating CD8 T cells (CD8 TIL) characterized by co-expression of CD103 and CD39 in human solid tumors. This cell population was only found in TIL from primary and metastatic tumors, exhibited features of chronic stimulation and displayed characteristics of tissue-resident memory T cells. Double positive CD8 TIL had a distinct TCR repertoire compared to other CD8 TIL subsets, were highly enriched for tumor antigen recognition and efficiently killed autologous tumor cells. Finally, patients with head and neck cancer whose CD8 TIL contained a higher frequency of CD39 ${ }^{+} \mathrm{CD} 103^{+}$cells experienced a greater overall survival.

\section{Conclusions}

This work describes a simple method for detecting tumor-reactive TIL, which should help define mechanisms of current immunotherapies and may lead to the development of future immunotherapies.
P147

Reduced tumor-associated glycosphingolipids in cell membrane lipid rafts and a diminished proliferation rate in mammary breast carcinoma cultures after incubation with glyphosate-based

formulations

Beatrix Kotlan', Gabriella Liszkay', Orsolya Csuka', Jozsef Toth',

Marianna Olah², Miklos Kasler', Andras Szekacs ${ }^{2}$

${ }^{1}$ National Institute of Oncology, Budapest, Hungary; ${ }^{2}$ Agro-Environmental Research Institute, National Agricultural Research and Innovation Centre,

Budapest, Hungary

Correspondence: Beatrix Kotlan (kotlanbea@gmail.com)

Journal for ImmunoTherapy of Cancer 2017, 5(Suppl 2):P147

\section{Background}

The glycosphingolipid-containing cell membrane lipid rafts are important structural and functional cellular components and as so have a primary role in cell integrity and signal transduction. Our question was how glyphosate-based herbicides (intensively used worldwide, leading to environmental and food contamination) their active ingredient glyphosate (revealed as a chemical chelator) and formulants influence the growing potential of mammary carcinomas and other sensitive cell types. Our specific aims were to define the effects of glyphosate and its formulating surfactant on various, intensively growing cell types at cellular and molecular levels.

Methods

Glyphosate-based herbicide formulation Roundup Classic and its polyethoxylated tallowamine detergent POEA were tested in $2 \%$ $50 \mathrm{X}$ diluted and $0.32 \% 300 \mathrm{X}$ diluted forms, respectively, while glyphosate (as isopropylamine salt) (62\%) was $46.3 \mathrm{X}$ diluted; and 5 -step series of dilutions of each stock solution were added into cultures of MCF7, MDA-MD-231 and other sensitive and control (HEK293) cells. Cell proliferation in the cultures was measured with the MTT assay, and microcultures were scanned regularly by inverted light microscopy. Harvested control, and treated cell suspensions were analyzed by immunofluorescence microscopy and high quality RNA was prepared for DNA microarray gene expression analysis.

Results

Reduced proliferation was experienced in the MDA MD-231 and MCF7 cell lines. The effect was different in ER-positive and -negative cells, and a lower tumor-associated glycosphingolipid expression could be detected by indirect immunofluorescence assay and fluorescence microscopy. The expression change of GD3 gangliosides, highly tumor-associated antigens was especially remarkable. First-line gene expression analysis (Agilent GeneSpring GX) and the Ingenuity Pathway Analysis Qiagen revealed different gene expression patterns upon exposure to either Roundup or POEA, or to glyphosate. Differences were also shown in gene expression regulation in the two breast cancer cell lines, indicating the involvement of GD3 synthase gene machinery.

Conclusions

Our data show a new aspect of the glyphosate and Roundup treatments of various cells, emphasizing the cell membrane integrity damaging potential of these xenobiotics via glycosphingolipid-containing lipid rafts. The characteristic transcriptional changes found by gene expression analysis support the diminished GD3 disialylated glycosphingolipide expression found by immunofluorescence microscopy. The study provides important information on the proliferation inhibition and apoptosis promoting effects of adjuvants in glyphosate formulations, attracts attention on epidemiological questions, and provides data to be harnessed in developing novel anti-cancer strategies. 
P148

KHK2455, a long-acting selective IDO-1 inhibitor, in combination with mogamulizumab, an anti-CCR4 monoclonal antibody, in patients with advanced solid tumors: preliminary safety report and pharmacodynamic activity from a first-in-human study Solmaz Sahebjam', Timothy Yap ${ }^{2}$, David Hong ${ }^{2}$, Arpit Rao ${ }^{3}$, Sarah Adams $^{3}$, Sergey Efuni ${ }^{4}$, Dmitri Grebennik ${ }^{4}$, Denis Healy ${ }^{4}$ Eniola Ogunmefun ${ }^{4}$, Yi Liư $^{4}$, Tomonori Tayama ${ }^{4}$, Olivier Rixe ${ }^{3}$ 1 Moffitt Cancer Center and Research Institute and the University of South Florida, Tampa, FL, USA; ${ }^{2}$ The University of Texas MD Anderson Cancer Center, Houston, TX, USA; ${ }^{3}$ University of New Mexico

Comprehensive Cancer Center, Albuquerque, NM, USA; ${ }^{4}$ Kyowa Kirin Pharmaceutical Development Inc., Princeton, NJ, USA

Correspondence: Olivier Rixe (robert.latek@kyowakirin.com)

Journal for ImmunoTherapy of Cancer 2017, 5(Suppl 2):P148

\section{Background}

KHK2455 is an oral, long-acting, once-daily, selective IDO-1 inhibitor. Mogamulizumab is a monoclonal antibody with enhanced antibody dependent cellular cytotoxicity activity against $\mathrm{CCR4}^{+} \mathrm{T}$ cells expressed by Th2 regulatory lymphocytes. Both KHK2455 and mogamulizumab have demonstrated immunologic and antitumor activities during in vitro and in vivo preclinical studies. Clinically, IDO-1 inhibitors have demonstrated synergy in combination with immunotherapy in several tumor types.

Methods

In this first-in-human study, patients with advanced solid tumors will receive escalating doses of oral KHK2455 $(0.3,1,3,10,30$, and 100 $\mathrm{mg}$ once daily) as monotherapy for 4 weeks (Cycle 0 ) followed by combination with intravenous mogamulizumab $1 \mathrm{mg}$ weekly for 4 weeks (Cycle 1) and then biweekly (Cycle 2 and beyond) using a standard $3+3$ design. Cohort expansion will occur at the maximum tolerated dose or the highest tested dose. The primary objective is to determine the safety, tolerability, and dose-limiting toxicities (DLTs) of KHK2455 in combination with mogamulizumab. Secondary objectives include pharmacokinetic, pharmacodynamic, biomarker and immunologic monitoring, and determination of best overall responses.

Results

By May 2017, twelve patients were enrolled in cohorts that received KHK2455 at 0.3 and $1 \mathrm{mg}$ dose level, including patients with head and neck cancer $(n=4)$, ovarian cancer $(n=3)$, osteosarcoma, pancreatic, gastric, gallbladder, and adrenal cancers (each $n=1$ ). Eight patients continue treatment. There were no DLTs. The most frequent adverse events included maculopapular rash, thrush, dysphagia, thrombotic event, and tachycardia, none of which were considered related to KHK2455 although one case of rash (grade 3) was considered related to mogamulizumab.

The effective half-life for KHK2455 exceeded 1.5 and 2.5 days at 0.3 and $1 \mathrm{mg}$ once daily, respectively. Potent dose-dependent inhibition of kynurenine production was demonstrated in plasma samples and ex vivo stimulation assays. Mean change from baseline of kynurenine/tryptophan ratio showed a dose-dependent decrease of $25 \%$ at the $1 \mathrm{mg}$ dose level and no PD effect in serum samples from the 0.3 mg subjects.

Conclusions

Preliminary data from two cohorts ( 0.3 and $1 \mathrm{mg}$ once daily) suggest that KHK2455 in combination with mogamulizumab has a good safety profile, demonstrated a prolonged effective half-life up to 2.5 days, and an ability to suppress kynurenine production in a dosedependent manner. These data support the continuation of KHK2455 dose escalation.

Trial Registration

NCT02867007
P149

In vitro and in vivo characterization of KHK2455, a highly potent and selective indoleamine 2,3-dioxygenase 1 (IDO1) inhibitor with a novel mechanism of action

Motoya Mie', Kana Kunieda', Shoko Koshiba', Takashi Murakami

Shinya Horita', Yuichi Fukuda', Toshihiko Ishii', Ryuichiro Nakai',

Kazuyasu Nakamura ${ }^{3}$

${ }^{1}$ Kyowa Hakko Kirin Co., Ltd., Sunto-gun, Japan; ${ }^{2}$ Saitama Medical

University, Iruma-gun, Japan; ${ }^{3}$ Kyowa Hakko Kirin Co., Ltd., Tokyo, Japan

Correspondence: Kazuyasu Nakamura (robert.latek@kyowakirin.com)

Journal for ImmunoTherapy of Cancer 2017, 5(Suppl 2):P149

Background

IDO1 catalyzes the degradation of tryptophan to kynurenine (kyn) metabolites that can inhibit T cell function and may be one mechanism by which tumors avoid anti-tumor activity. In the clinic, combination of IDO1 inhibitors (IDO1i) with checkpoint inhibitors has been shown to improve response rates in several cancer indications [1][2]. Our IDO1i program identified KHK2455, a highly potent and specific IDO1i with a novel mechanism of action that is currently in a phase I clinical trial.

Methods

Here we describe the pre-clinical characteristics of KHK2455 IDO1 inhibition. In vitro dose-dependent potency and selectivity were analyzed in IDO/TDO expressing 293T cells. In vivo pre-clinical pharmacokinetic (PK) properties, pharmacodynamic (PD) activity and toxicology were examined in mice and in cynomolgus monkeys. Anti-tumor activity of KHK2455 in combination with anti-CTLA-4 antibody (9D9) was demonstrated in B16-F10 derived tumor bearing mice.

Results

KHK2455 had potent inhibition of kyn production in IDO1expressing cells $\left(\mathrm{IC}_{50}=14 \mathrm{nM}\right)$ but not in IDO2 or TDO2-expressing cells $\left(I C_{50}>10,000\right.$ or $\left.1,000 \mathrm{nM}\right)$. Unlike other IDO1i molecules, KHK2455 primarily bound to apo form of IDO1 enzyme and inhibited the enzyme activity in vitro, indicating a unique and possibly more potent mechanism of action.

Mice and cynomolgus monkeys administered KHK2455 demonstrated plasma KHK2455 concentration was increased with increasing dose from $0.1 \mathrm{mg} / \mathrm{kg}$ to $10 \mathrm{mg} / \mathrm{kg}$ (mouse) and from $0.03 \mathrm{mg} / \mathrm{kg}$ to $3 \mathrm{mg} /$ $\mathrm{kg}$ (monkey). Corresponding Kyn/Trp ratios reached a minimum 24 hours after a single oral administration of KHK2455 and the ratio was reduced with dose increment, supporting once daily dosing for clinical study.

KHK2455 demonstrated synergistic tumor growth inhibition with anti-CTLA-4 antibody (9D9) in B16-F10 derived tumor bearing mouse models.

Conclusions

KHK2455 is an IDO1 inhibitor with a novel MOA that potently and selectively inhibits kyn production in preclinical models, and demonstrates synergistic inhibition of tumor growth in a mouse tumor model with anti-CTLA-4 antibody. Together, these data support the clinical evaluation of KHK2455 in a phase I study.

\section{References}

1. Gangadhar, Schneider, Bauer, Wasser, Spira, Patel, Sarkis Balmanoukian, Bauml, Schmidt, Zhao, Jones, Tarhini. Efficacy and safety of epacadostat plus pembrolizumab treatment of NSCLC: Preliminary phase I/II results of ECHO-202/KEYNOTE-037. ASCO 2017.

2. Perez, Riese, Lewis, Saleh, Daud, Berlin, Lee, Mukhopadhyay, Zhou, Serbest, Hamid. Epacadostat plus nivolumab in patients with advanced solid tumors: Preliminary phase I/II results of ECHO-204. ASCO 2017 
P150

Immunomodulatory effects of the senescence-associated secretory phenotype on NK cells in renal cell carcinoma

Andreas Lundqvist, Ann-Kathrin Prinz, Kevin Bohanek, Kristina Witt

Karolinska Institutet, Stockholm, Sweden

Correspondence: Andreas Lundqvist (andreas.lundqvist@ki.se); Kristina Witt Journal for ImmunoTherapy of Cancer 2017, 5(Suppl 2):P150

\section{Background}

Metastatic renal cell carcinoma (mRCC) is highly resistant to chemoand radiotherapy and the 5-year survival rate for mRCC remains low. RCC has long been considered as an immunogenic tumor and infiltration of NK cells rather than T cells is beneficial for patient survival. Thus, augmenting the activity of NK cells in patients with mRCC could lead to prolonged survival. Senescence is a cellular state of cell cycle arrest, but greater metabolic activity. NK cells play an important role in the clearance of senescent tumor cells. Furthermore, senescent tumor cells secrete a variety of factors, i.e. senescenceassociated secretory phenotype (SASP). However, it is unknown if the SASP will alter the activity of NK cells. In this study, we sought to investigate if drug-induced senescence in RCC influence NK cell activity and to further identify rate-limiting factors within the SASP that cause alterations in NK cell activity. With this knowledge, strategies to better augment the activity of NK cells can be developed for patients with $\mathrm{mRCC}$.

Methods

Senescence was induced by treatment with the mdm2-inhibitor Nutlin-3a and detected by senescence-associated $\beta$-gal staining and by ELISA for IL-6 and IL-8. NK cell phenotype, proliferation, IFNgamma production, and lysis were analyzed by flow cytometry, thymidine incorporation, ELISA, and $51 \mathrm{Cr}$-release assay respectively.

Results

RCC cell lines expressing wt p53 are sensitive to mdm2 inhibition and become senescent indicated by higher levels of SA- $\beta$-gal and increased levels of IL- 6 and IL-8 in the supernatants treated compared with untreated RCC cells. Furthermore, changes in the surface expression of MIC A/B and MHC class I was observed in Nutlin-3a compared with untreated cells. Unlike previous studies, no change in the sensitivity between senescent RCC cells to NK cell killing was observed. However, upon exposure of supernatants of senescent RCC, NK cells showed an increased proliferation. In contrast, no changes in NK cell cytotoxicity or degranulation were observed upon co-culture with supernatants of senescent RCC and untreated RCC. Our current focus is to screen the secretome of senescent RCC using to identify components in the SASP that enhance NK cell proliferation.

\section{Conclusions}

Senescent RCC produce factors that enhance the proliferation of NK cells. Secretome analysis to identify what factors within the SASP that are responsible to augment the proliferation of NK cells are currently ongoing. The identification of such factors will lead to increased knowledge to sustain NK cell proliferation and better tailored NK cell-based immunotherapies for patients with RCC.

\section{P151}

Profiling the immunogenic cell death (ICD) mechanisms induced by Nano-Pulse Stimulation (NPS) treatment in mouse B16-F10 melanoma tumors using NanoString technology

Amanda McDaniel', Snjezana Anand ${ }^{2}$, Aman Alzubier ${ }^{1}$, Juliette Berlin ${ }^{1}$, Holly Hartman', Darrin Uecker', Richard Nuccitelli'

'Pulse Biosciences, Hayward, CA, USA; ${ }^{2}$ Pulse Biology, Hayward, CA, USA

Correspondence: Amanda McDaniel

(amcdaniel@pulsebiosciences.com)

Journal for ImmunoTherapy of Cancer 2017, 5(Suppl 2):P151

\section{Background}

Nano-Pulse Stimulation (NPS) is a non-thermal tumor therapy that delivers ultrashort electrical pulses to tumor cells. NPS opens nanopores in the membrane of the ER, allowing the efflux of $\mathrm{Ca}^{2+}$ thus causing ER stress, ROS production and ICD [1]. To date, the primary mechanism of action of most known ICD inducers is ER stress and ROS production leading to intrinsic mitochondrial apoptosis, and the release and translocation of DAMPs [2]. Here we sought to profile the pathways involved in ER stress, apoptotic cell death and the immune response after NPS treatment, using the NanoString PanCancer Immune Panel with an additional 30 spike-in genes designed to investigate cell death pathways.

Methods

C57/B6 albino mice were inoculated intradermally with 1-million B16F10 melanoma cells into the left flank. When tumors reached $\sim 5 \mathrm{~mm}$ in diameter they were treated with NPS (500 pulses, 200 ns in duration applied at $25 \mathrm{kV} / \mathrm{cm}$ at $5 \mathrm{pps}$ ). Tumors were harvested 2-hrs, 4hrs and 24-hrs after treatment and placed into formalin for fixation followed by embedding in paraffin. mRNA was extracted and hybridized to bar-coded probes that correspond to 800 gene transcripts (770 PanCancer Immune Panel; 30 spike-in). Transcripts were read using the NanoString nCounter ${ }^{\circledast}$ and analyzed with nSolver software. Results

NanoString analysis showed that genes involved in ER-stress were upregulated 24-hrs after NPS treatment, including several in key proapoptotic signaling pathways. Studies have demonstrated that calcium released from the ER is taken up by the mitochondria, causing release of cytochrome $\mathrm{C}$ and initiating intrinsic mitochondrial apoptosis [2]. Genes in these pathways were upregulated by 24-hrs, as were those coding for DAMPs, PRRs and the cascade of inflammatory mediators that initiate the maturation of DCs, promoting T cell recognition and priming of the adaptive arm of the immune response.

Conclusions

NanoString profiling revealed that transcripts for specific ER stressrelated factors, intrinsic apoptotic pathways and immune mediators, previously identified as important components of ICD, were upregulated in tumor tissues 24-hrs after NPS treatment. We plan to continue to utilize the NanoString platform in future studies to help us to further understand the cell death and immune mechanisms involved in NPS-treatment.

\section{References}

1. Nuccitelli R, et al. Nano-Pulse Stimulation is a physical modality that can trigger immunogenic tumor cell death. J Immunother Cancer. 2017: 5:32.

2. Gebremeskel, Johnston. Concepts and mechanisms underlying chemotherapy induced immunogenic cell death: impact on clinical studies and considerations for combined therapies. Oncotarget. 2015; 6(39):41600-19.

\section{P152}

41BB costimulation enables PD-1 blockade therapy by inducing $T$ cell mitochondrial function and biogenesis

Ashley Menk, Nicole Scharping, Deanna Dunstane, Greg Delgoffe University of Pittsburgh, Pittsburgh, PA, USA

Correspondence: Greg Delgoffe (avm9@pitt.edu)

Journal for ImmunoTherapy of Cancer 2017, 5(Suppl 2):P152

\section{Background}

Tumors create a suppressive microenvironment that prevents antitumor immunity through a number of mechanisms like recruitment of regulatory $\mathrm{T}$ cells or ligation of co-inhibitory molecules. Targeting these molecules has become a major success in cancer treatment; however, some patients fail to respond to these therapies. It has become clear in recent years that the lack of nutrients in the tumor 
microenvironment may also play an immunosuppressive role and cause resistance to immunotherapy. We have previously shown that $T$ cells infiltrating the tumor have repressed mitochondrial activity and biogenesis that leads to loss of metabolic sufficiency, a state that cannot be rescued by PD-1 blockade therapy alone. The costimulatory molecule $41 \mathrm{BB}$ is present on activated $T$ cells can increase $T$ cell survival and is a major clinical therapeutic target. We hypothesized that $41 \mathrm{BB}$ signaling might provide metabolic support to tumor-infiltrating $T$ cells.

Methods

Non-tumor bearing mice or mice injected with B16 were treated with $200 \mu \mathrm{g}$ anti-PD1 (clone J43, Bio X-Cell) or $50 \mu \mathrm{g}$ anti-41BB (clone 3H3, Bio X-Cell), or respective hamster or rat isotype controls, intraperitoneally every other day for 3 days (in non-tumor bearing experiments) or every other day for the duration of the experiment (in tumor experiments). CD8 T cells were isolated from lymph nodes and tumors and tested for cytokine production by flow cytometry, metabolic function with Seahorse XFe96 Bioanalyzer, and signaling by western blot.

Results

We show that 41BB costimulation of previously activated, murine T cells results in enhanced mitochondrial activity through PGC1a-mediated mitochondrial biogenesis. In naïve $T$ cells, 41BB can substitute for CD28-mediated costimulation, leading to activated T cells with high respiratory capacity and increased mitochondrial activity. Mice treated with agonistic anti-41BB in vivo produced T cells with higher respiratory capacity and oxidative function. In melanoma-bearing mice, 41BB stimulation alone does not induce robust anti-tumor immunity, but leads to increased mitochondrial mass in tumor-infiltrating $T$ cells. Pairing 41BB agonism with PD-1 blockade therapy results in robust anti-tumor immunity and intratumoral $\mathrm{T}$ cell function.

Conclusions

Our results suggest $41 \mathrm{BB}$ functions to bolster $\mathrm{T}$ cell metabolism, highlighting the importance of combining 41BB stimulation with other immunotherapies to support $T$ cells such that they can overcome the metabolic barriers in the tumor microenvironment.

\section{P153}

Nanoparticles to target tumor-draining lymph nodes conjugated with an antagonist for vasoactive intestinal peptide to promote an anti-tumor immune response

Rebecca Pankove1, Sruthi Ravindranathan', Parvin Forghani', David

Francis $^{2}$, Susan Thomas ${ }^{2}$, Edmund Waller ${ }^{1}$

${ }^{1}$ Emory University, Atlanta, GA, USA; ${ }^{2}$ Georgia Institute of Technology, Atlanta, GA, USA

Correspondence: Sruthi Ravindranathan (rpankov@emory.edu); Susan

Thomas; Edmund Waller

Journal for ImmunoTherapy of Cancer 2017, 5(Suppl 2):P153

\section{Background}

Vasoactive intestinal peptide (VIP) is a neuropeptide that regulates co-inhibitory pathways in the immune system. In previous studies, mouse models of bone marrow transplant show the potent immunosuppressive activity of VIP ${ }^{1}$. Further, VIPhyb, an antagonist of VIP, increases the number of effector/memory CD8+ T cells and mature natural killer cells. Additionally, in a mouse leukemia model, VIPhyb downregulates the expression of PD-1 and PD-L1 in activated T cells and dendritic cells ${ }^{1}$. In this study, we hypothesize that in solid tumor immunotherapy, the immunomodulatory effect of VIPhyb can be further augmented by conjugation with nanoparticles (NP) specifically designed to target tumor draining lymph nodes (TDLN) ${ }^{2}$. Since TDLNs are a major tissue involved in draining tumor associated antigens, targeted delivery of VIPhyb to TDLNs could improve the antitumor immune response and hamper overall growth of solid tumors susceptible to immunotherapy.

\section{Methods}

Splenocytes were extracted from WT and VIP-KO mice treated with B16-F0 and B16-F10 melanoma, and analyzed with flow cytometry for Granzyme B (GrZ B) and CD8 expression. VIPhyb was conjugated to a biocompatible, non-toxic $30 \mathrm{~nm} \mathrm{NP}$ through emulsion polymerization and tested for purity and stability. A mixed lymphocyte reaction (MLR) using luciferase-expressing splenic T cells from B6 Luciferase mice were co-cultured with irradiated splenic $T$ cells from FVB mice. VIP, VIPhyb, and VIPhyb-NP at concentrations of $0 \mathrm{ug} / \mathrm{mL}$ to $975 \mathrm{ug} / \mathrm{mL}$ were added to the MLR on 3 consecutive days and their effect on $T$ cell proliferation assessed with bioluminescent imaging.

Results

The addition of VIPhyb and VIPhyb-NP counteracted the inhibitory effects of VIP by increasing T cell proliferation in vitro. VIPhyb had a peak efficacy at a concentration of $300 \mathrm{ug} / \mathrm{mL}$ (Fig. 1) while the effect of VIPhyb-NP was dose-dependent (Fig. 2). Melanoma growth in VIPknockout (KO) mice results in increased amounts of GrZ B and cytotoxic $\mathrm{CD}^{+}$splenocytes (Fig. 3).

Conclusions

Blocking VIP-signaling with VIPhyb, especially when coupled with a TDLN targeting NP, thus represents a potential novel immunotherapeutic approach to bolster an adaptive $\mathrm{T}$ cell response and improve pharmacokinetics for advanced solid tumors in the clinic.

\section{References}

1. Li JM, Darlak KA, Southerland L, Hossain MS, Jaye DL, Josephson CD, Rosenthal H, Waller EK. VIPhyb, an antagonist of vasoactive intestinal peptide receptor, enhances cellular antiviral immunity in murine cytomegalovirus infected mice. PloS one. 2013;8(5)e6: 3381.

2. Thomas SN, Vokali E, Lund AW, Hubbell JA, Swartz MA. Targeting the tumor-draining lymph node with adjuvanted nanoparticles reshapes the anti-tumor immune response. Biomaterials. 2014;35(2):814-24.

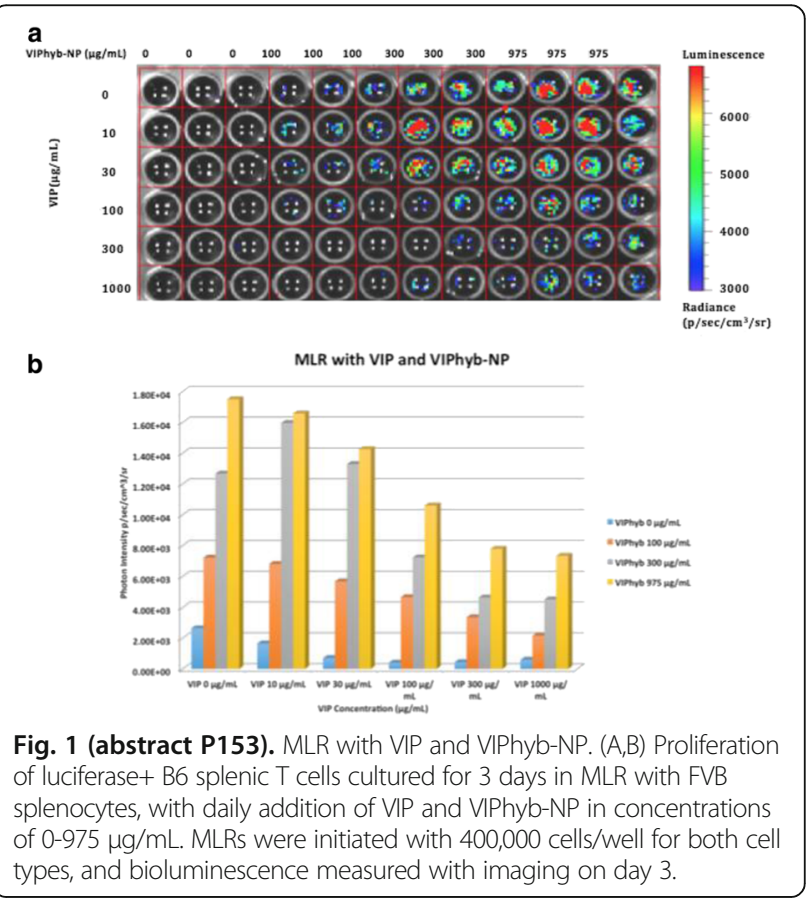



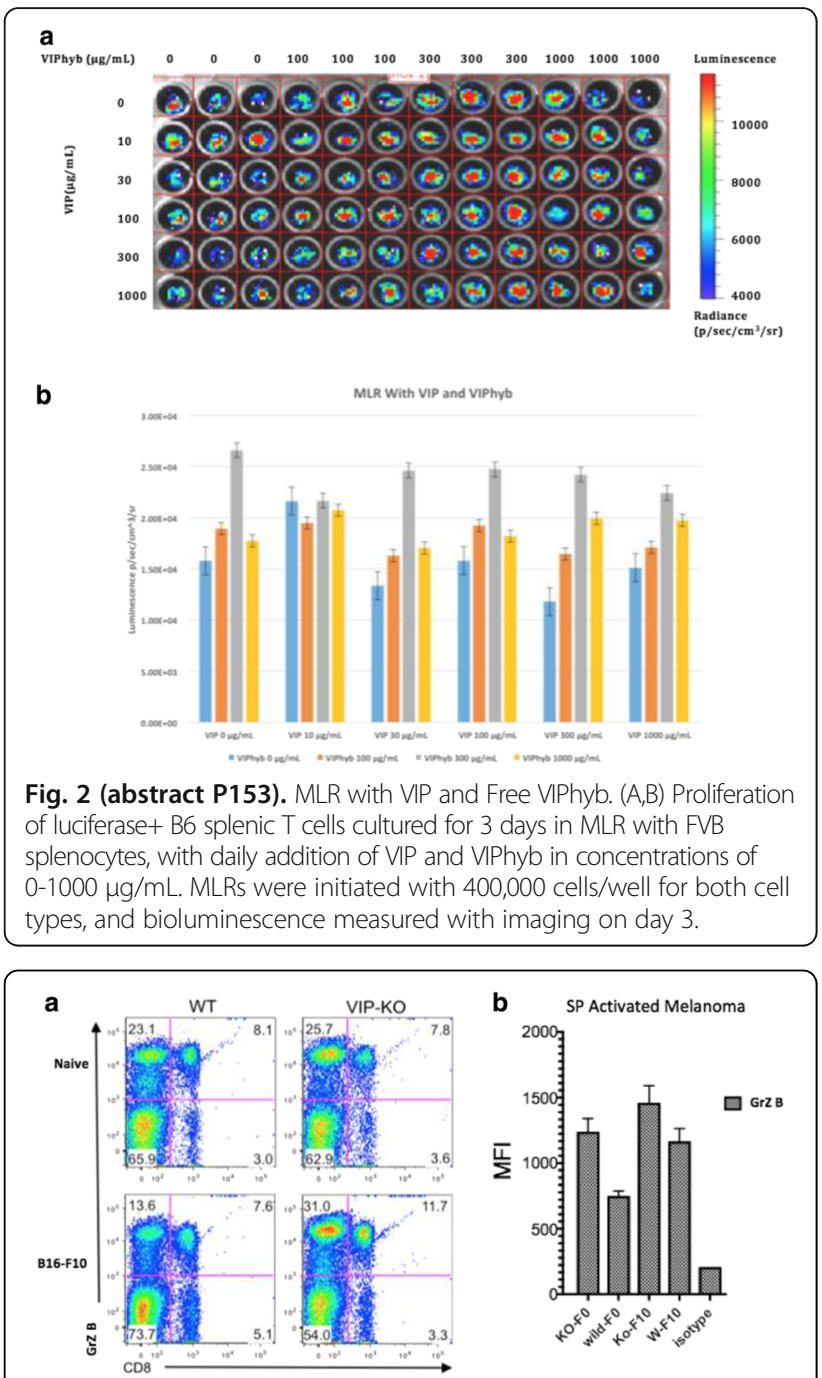

Fig. 3 (abstract P153). Flow Cytometry Analysis of VIP-KO Mice. $(A, B)$ Increased leavels of Granzyme B producing CD8+ T cells in melanoma bearing VIP-KO mice

\section{P154}

Tumor-derived PGD2 and NKp30-B7H6 engagement drives an immunosuppressive ILC2-MDSC axis in acute promyelocytic leukemia Sara Trabanelli', Mathieu Chevalier', Amaia Martinez Usatorre', Alejandra Gomez-Cadena', Bérengère Salomé ${ }^{1}$, Mariangela Lecciso ${ }^{3}$, Valentina Salvestrini ${ }^{3}$, Gabriela Baerlocher ${ }^{4}$, Carmelo Carlo-Stella ${ }^{5}$, Daniela Taurino ${ }^{5}$, Armando Santoro ${ }^{5}$, Orietta Spinelli ${ }^{6}$, Alessandro Rambaldi, Emanuela Giarin ${ }^{7}$, Giuseppe Basso ${ }^{7}$, Cristina Tresoldi ${ }^{8}$, Fabio Ciceri ${ }^{11}$, Luca Mazzarella ${ }^{10}$, Pier Giuseppe Pelicci ${ }^{10}$, Andrew Mc Kenzie ${ }^{9}$, Dominique Vanhecke', Domenico Mavilio ${ }^{12}$, Antonio Curti ${ }^{3}$, George Coukos ${ }^{1}$, Laurent Derré2 ${ }^{2}$ Camilla Jandus ${ }^{1}$ Ludwig Institute for Cancer Research, University of Lausanne, Epalinges, Switzerland; ${ }^{2}$ Lausanne University Hospital (CHUV), Lausanne, Switzerland; ${ }^{3}$ Institute of Hematology Seràgnoli, University of Bologna, Bologna, Italy; ${ }^{4}$ Bern University Hospital, University of Bern, Bern, Switzerland; ${ }^{5}$ Humanitas Cancer Center, Humanitas Clinical and Research Center, Rozzano-Milan, Italy; ${ }^{6}$ Ospedale Papa Giovanni XXIII, Bergamo, Italy; ${ }^{7}$ Clinica di Oncoematologia Pediatrica, University of Padova, Padova, Italy; ${ }^{8}$ Laboratorio Ematologia Molecolare, Biobanca Neoplasie Ematologiche, San Raffaele Hospital, Milano, Italy; ${ }^{9} \mathrm{MRC}$ laboratory of molecular biology, Cambridge, UK; ${ }^{10}$ European Institute of Oncology, Milan, Italy; ${ }^{11}$ San Raffaele Hospital, Milano, Italy; ${ }^{12}$ Humanitas Clinical and Research Center, Rozzano-Milan, Italy Correspondence: Camilla Jandus (berengere.salome@unil.ch) Journal for ImmunoTherapy of Cancer 2017, 5(Suppl 2):P154

\section{Background}

Innate Lymphoid Cells (ILCs) were recently recognized as a distinct family of cells able to play a crucial role in immune processes. They are subdivided into 3 subsets (ILC1, ILC2 and ILC3) that functionally resemble CD4 T helper cells. ILCs are dysregulated in acute myeloid leukemia and are associated with a reduced susceptibility to graftversus-host disease. However, their role in tumor immunosurveillance is still far to be well established. We aim to identify their role in acute promyelocytic leukemia (APL).

Methods

We characterized by multiparametric flow cytometry the ILC compartment present in both the peripheral blood and the bone marrow of 22 APL patients at diagnosis and in remission after all-trans retinoic acid (ATRA) therapy. In parallel, we monitored the presence of myeloid derived suppressor cells (MDSC) and of the different T helper cell subsets. In addition, the serum levels of various cytokines and soluble mediators involved in regulating ILC functions were determined by ELISA or Luminex. We also used 3 APL mouse models, namely FVB/NJ, C57BL/6 and humanized NSG mice injected with APL lines, to address the role of ILC during leukemogenesis and to target in vivo the key players of the newly identified immunosuppressive axis.

Results

We find that, in comparison to the other subtypes of acute myeloid leukemia, APL is specifically characterized by increased ILC2. ILC2 are hyper-activated to produce high amount of IL-13 via the CRTH2PGD2 and NKp30-B7H6 bindings. Through IL-13, ILC2 recruit and promote functional mMDSC both in vitro and in vivo. In line with these findings, APL patients show increased circulating MDSC and elevated concentrations of PGD2 and IL-13. Of note, APL patients that achieve remission show normal frequencies of ILC2 and MDSC as well as normal concentrations of PGD2 and IL-13. The APL mouse models confirm that the enhancement of ILC2 is a consequence of the disease, and directly drives MDSC expansion. By treating the mice with blocking antibodies against NKp30, PGD2 and IL-13, ILC2 and MDSC levels are partially restored, APL growth is delayed and the survival of the mice enhanced.

Conclusions

Our data suggest that APL cells are able to recruit and activate ILC2 through PGD2 secretion and B7H6 expression. ILC2 are in turn able to recruit and activate MDSC, favouring a pro-leukemic environment. The ILC2-MDSC tandem might represent a novel target in tumors with enhanced and hyper-activated ILC2.

\section{P155}

Targeting CSK kinase activity to enhance anti-tumor immunity

Cindy Wang, Carolyn Cao, Lisa Berman-Booty, Jesse Swanson, Rukiye Eraslan, Miguel Sanjuan, Gregory Vite, Lan-Ying Qin, John Hunt, Bryan Fink, Susan Wee

Bristol-Myers Squibb, Princeton, NJ, USA

Correspondence: Cindy Wang (cindy.wang@bms.com) Journal for ImmunoTherapy of Cancer 2017, 5(Suppl 2):P155

\section{Background}

To mount an effective antitumor immune response, a $\mathrm{T}$ cell receptor (TCR) must first recognize a cognate antigen presented by malignant cells. Successful effector T cell activation initiates an intracellular signaling cascade that results in the release of cytotoxins and proinflammatory cytokines that ultimately result in the elimination of diseased antigen-presenting cells. A key regulator of this signaling cascade is the C-terminal Src (CSK) kinase. In lymphocytes, CSK inhibits $T$ cell activation by phosphorylating the SRC kinase family member LCK at tyrosine 505 and thereby terminates the signaling cascade. It is possible that relieving CSK-mediated regulation of TCR signaling could lead to a more robust $\mathrm{T}$ cell response, improving antitumor immunity.

Methods

Both genetic and pharmacologic approaches to inhibit CSK activity were developed. A tamoxifen-inducible transgenic CSK knock-out (KO) mouse was engineered to bypass the embryonic lethality observed with constitutive systemic CSK depletion. Small molecule 
inhibitors of CSK were identified in a high-throughput screen and assessed in vitro for inhibitory activity or in vivo for antitumor efficacy alone or in combination with anti-PD-1.

Results

As expected, loss of CSK enhanced cytokine production and increased the antitumor immune response in the MC38 syngeneic tumor mouse model. Potent and selective CSK small molecule inhibitors, in the presence of TCR stimulation, similarly activated the proximal TCR signaling pathway and increased cytokine production and proliferation. In an in vivo efficacy study, however, an optimized CSK small molecule inhibitor dosed as monotherapy or in combination with anti-PD-1 did not improve the antitumor immune response at the dose levels tested.

\section{Conclusions}

These seemingly discrepant findings in tumor response observed in the tamoxifen-inducible CSK KO mice compared to mice treated with the CSK inhibitor may reflect the need for a more optimized dosing regimen and improved target coverage. In this poster, we will present our findings on the role of CSK inhibition in tumor immunity.

\section{P156}

Immunometabolic requirements for T cell exhaustion

Nicole Scharping, Ashley Menk, Dayana Rivadeneira, Greg Delgoffe

University of Pittsburgh, Pittsburgh, PA, USA

Correspondence: Greg Delgoffe (nes63@pitt.edu)

Journal for ImmunoTherapy of Cancer 2017, 5(Suppl 2):P156

\section{Background}

CD8+ tumor-infiltrating T lymphocytes (TIL) in the tumor microenvironment (TME) are unable to effectively kill targets due to tumor cell and regulatory immune cell-mediated suppression. Poor metabolite availably has also been found to negatively impact TIL, as T cells have high energy demands that are not met in the TME due to competition with surrounding tumor cells. Consequently, tumor growth is allowed to progress as TIL becomes exhausted and dysfunctional. Recently, we found that exhausted TIL also exhibit a profound loss of functional mitochondria, which is due in part to the repression of TIL mitochondrial biogenesis. Enforcing mitochondrial biogenesis in $T$ cells not only lead to increased mitochondrial function, but improved TIL functionality, decreased tumor burden, and increased survival in mouse melanoma. Additional studies suggest that simply causing progressive functional mitochondrial loss is enough to induce $T$ cell dysfunction. Thus, we hypothesize that TIL dysfunction is driven by metabolic insufficiency, and altering the T cells' ability to compete for nutrients will increase TIL functionality.

Methods

T cell metabolic output was measured by Seahorse extracellular flux analysis. The TME was modeled in vitro by co-culturing mouse melanoma tumor cells or $\mathrm{T}$ cell activation beads with transgenic $\mathrm{T}$ cells either in normoxia or hypoxia $\left(1.5 \% \mathrm{O}_{2}\right)$.

Results

To determine the specific drivers of T cell dysfunction, we developed a model system to identify how metabolic insufficiency and chronic activation can induce $T$ cell dysfunction in vitro. Chronically activated $\mathrm{T}$ cells and those cultured in hypoxic conditions were still able to carry out effector function, but chronic activation and hypoxia together induced profound T cell dysfunction, characterized by a PD$1 \mathrm{hi}, \mathrm{Tim}-3+$ phenotype and drastically reduced cytokine production. To specifically understand the contribution of mitochondria to the avoidance of exhaustion, we generated a model in which T cells progressively lose functional mitochondria in vitro. We found that $\mathrm{T}$ cells lacking functional mitochondria could not utilize oxidative phosphorylation, causing decreased cytokine and cytotoxic functionality and increased co-inhibitory marker expression, all hallmarks of TIL. Preliminary data suggests that this dysfunction is not due to energetic insufficiency, but rather to alterations in the epigenetic signature of activated T cells.

\section{Conclusions}

Our data support a model in which chronic activation and metabolic insufficiency show synergistic effects in the promotion of long-term T cell dysfunction. Reversing metabolic insufficiency may be an attractive strategy to improve the efficacy of cancer immunotherapy.

\section{P157}

Mitochondrial Reactive Oxygen Species are a Biomarker of

Metabolic Fitness of T Cells in Tumors

Jessica Thaxton (thaxton@musc.edu)

Medical University of South Carolina, Charleston, SC, USA

Journal for ImmunoTherapy of Cancer 2017, 5(Suppl 2):P157

\section{Background}

Tumor microenvironments drive $\mathrm{T}$ cell metabolic dysfunction resultant in inhibition of anti-tumor capability. Biomarkers that indicate $T$ cell metabolic distress may prove useful to therapeutically target metabolic dysfunction. We hypothesized that accrual of mitochondrial reactive oxygen species (mtROS) may indicate poor metabolic fitness of T cells.

Methods

We used FACS analysis to measure accumulation of mtROS in mouse and human $T$ cells. Further, we FACS sorted mtROS- and mtROS+ T cell subsets to measure spare respiratory capacity, mitochondrial permeability transition pore (MPTP) opening, and dependence on glucose consumption between subsets. In vitro and in vivo mouse and human $\mathrm{T}$ cells were treated with $\mathrm{N}$-acetylcysteine (NAC) as a method to reduce mtROS accumulation and $\mathrm{T}$ cell anti-tumor immunity was measured.

Results

We found that accumulation of mtROS in mouse and human T cells was consistent with $T$ cell aging and cell death. T cells that amassed mtROS had reduced spare respiratory capacity, increased mitochondrial permeability transition pore (MPTP) opening, and shifted toward a dependence on glucose consumption for biofuel. In vitro antioxidant treatment with NAC was able to reduce mtROS accumulation, restore SRC, and inhibit T cell apoptosis. In both mouse models and human tumors we found that $T$ cells accumulate mtROS and that PD-1+ T cell subsets had a particularly robust expression of mtROS. In multiple tumor models treatment with NAC in vivo diminished mtROS accumulation, reduced $\mathrm{T}$ cell death, and remodeled PD1+ T cell populations.

Conclusions

A combination of NAC and anti-PD-1 antibody may greatly augment the effect of either therapy alone. Together our data suggest that mtROS is a biomarker of poor metabolic fitness of T cells in tumors and that anti-oxidant therapies may promote tumor control when combined with checkpoint blockade therapies in the clinical setting.

P158

PBMC-humanized preclinical tumor models serve as a valuable platform to evaluate in vivo activities of novel immune-oncology drug candidates

Lan Zhang ${ }^{1}$, Juan Zhang ${ }^{2}$, Meng Qiao ${ }^{1}$, Xuefei Yan' ${ }^{1}$ Jian Ding ${ }^{1}$,

Fei Chen ${ }^{1}$, Xiaoyu $\mathrm{An}^{3}$, Weibin Tan ${ }^{4}$, Xiaoyan $\mathrm{Fu}^{4}$, WenQing Yang ${ }^{1}$, Qian Shi ${ }^{1}$

${ }^{1}$ CrownBio Science, Inc, Santa Clara, CA, USA; ${ }^{2}$ Cstone Biopharma, Santa Clara, CA, USA; ${ }^{3}$ CrownBio Science Inc, Taicang, China; ${ }^{4}$ Taicang Blood

Center, Taicang, USA

Correspondence: WenQing Yang (wenqing.yang@crownbio.com); Qian Shi Journal for ImmunoTherapy of Cancer 2017, 5(Suppl 2):P158

\section{Background}

Treating cancer by taking advantage of a patient's own immune system has demonstrated considerable success in oncology research. The lack of experimental immunotherapy models is a major obstacle towards better evaluating new immunotherapeutics and testing 
combination strategies. More and more relevant and sophisticated preclinical tumor models are required in the I/O drug discovery industry. Methods

We have established and validated a series of new preclinical in vivo models, PBMC-humanized tumor models, named MiXeno. Human xenogragt tumors were developed and the human immune system was partially established by infusing human PBMCs in NOG/NSG (or similar background) immunocompromised mice.

Results

MiXeno models were established and the inoculation conditions were optimized or sub-optimized. The xenograft tumor establishment and human immune component reconstitution can be achieved either simultaneously or sequentially. These MiXeno models were characterized with regards to reconstitution of T cells, tumor response to anti-PD-1 and anti-CTLA-4CTLA-4 antibodies, and onset of possible graft versus host disease (GVHD) or graft versus tumor response (GVT).

Conclusions

A series of MiXeno tumor models have been established and validated where human immune cells were reconstituted in the mouse system for evaluation of human-origin bispecific antibodies, immune checkpoint inhibitors or immune-modulators. Further studies are needed to expand model collections and to extend their applications in $\mathrm{I} / \mathrm{O}$ space.

\section{P159}

Clinical features and outcomes with talimogene laherparepvec in melanoma patients with comorbidities: a single-center experience Alice Zhou, Daniel Wang, Deborah Wallace, Douglas Johnson, Kristin Ancell

Vanderbilt University Medical Center, Nashville, TN, USA

Correspondence: Alice Zhou (zhoualic@gmail.com); Douglas Johnson Journal for ImmunoTherapy of Cancer 2017, 5(Suppl 2):P159

\section{Background}

Patients with unresectable in-transit or limited cutaneous metastatic melanoma (MM) may benefit from intralesional injections with talimogene laherparepvec (TVEC), a modified oncolytic herpesvirus [1]. However, predictors of response and outcomes in patients with adverse prognostic features (e.g. poor performance status (PS), prior therapy and co-morbidities are not well studied [2].

\section{Methods}

We performed a single-center retrospective analysis of patients with MM treated with TVEC. Age, comorbidities, PS, and prior therapies, as well as treatment details, outcomes, and adverse events were obtained. Durable response rate (DRR) was defined as $>6$ month of progression free survival (PFS).

Results

We identified 27 patients treated with TVEC. Most patients were elderly (median age $74,8>80$ ), $26 \%$ had poor PS (ECOG $>1$ ), and $70 \%$ with $>2$ co-morbidities. Most patients had stage IIIC disease (52\%) and received prior therapies (56\%), including $48 \%$ with immune checkpoint inhibitors (ICI). Overall, there was a DRR of 44\%; median PFS (mPFS) was 5.9 months $(\mathrm{m})$ and median overall survival (mOS) was not reached (median follow-up of $10.4 \mathrm{~m}$ ). Smaller disease burden (largest lesion diameter of $<2 \mathrm{~cm}$ ) was associated with substantially improved DRR (63\% vs. $11 \%$ ), mPFS (not reached vs. $2.1 \mathrm{~m}$ ) and mOS (not reached vs. $10.8 \mathrm{~m}$ ) compared with bulkier tumors. Poor PS was associated with significantly worse mPFS $(1.6 \mathrm{~m}$ vs not reached, $\mathrm{p}=.007$ ) and $\mathrm{mOS}$ ( $4 \mathrm{~m}$ vs not reached, $\mathrm{p}<.0001)$ compared to patients with ECOG 0-1, although occasional durable responses were noted (14\% DRR). There was no association with clinical outcomes for prior ICI (DRR $42 \%$ vs. $46 \%$ ) or age $>75$ years (DRR $43 \%$ vs. $46 \%$ ).

\section{Conclusions}

Overall, TVEC produced responses in patients regardless of age or prior ICl therapy. Poorer survival was associated with poor PS and higher initial disease burden. These findings will need to be validated in larger prospective studies.

\section{References}

1. Ott PA, Hodi FS. Talimogene Laherparepvec for the Treatment of Advanced Melanoma. Clinical Cancer Research. 2016; 22: 3127-3.

2. Andtbacka RHI, Kaufman HL, Collichio F, Amatruda $\mathrm{T}$, Senzer $\mathrm{N}$, Chesney J, Delman KA, Spitler LE, Puzanov I, Agarwala SS, Milhem M, Cranmer L, Curti B, Lewis K, Ross M, Guthrie T, Linette GP, Daniels GA, Harrington, K, Middleton MR, Miller WH, Zager JS, Ye Y, Yao B, Li A, Doleman S, VanderWalde A, Gansert J, Coffin RS. Talimogene Laherparepvec Improves Durable Response Rates in Patients with Advanced Melanoma. Journal of Clinical Oncology. 2015; 33: 2780-88.

\section{Cellular Therapy Approaches}

\section{P160}

Chimeric antigen receptors interact with multiple endogenous T cell proteins and induce tonic signaling through phosphorylation of endogenous CD3Z

Maria C. Ramello, Ismahène Benzaid, Brent M. Kuenzi, Maritza Lienlaf, Wendy M. Kandell, Daniel N. Santiago, Mibel Pabón-Saldaña, Lancia Darville, Bin Fang, Uwe Rix, Sean Yoder, Anders Berglund, John Koomen, Eric Haura, Daniel Abate-Daga

H. Lee Moffitt Cancer Center and Research Institute, Tampa, FL, USA Journal for ImmunoTherapy of Cancer 2017, 5(Suppl 2):P160

\section{Background}

Immunotherapies based on chimeric antigen receptor (CAR)-T cells are expected to become the first line of treatment for multiple malignancies [1]. However, their mechanism of action is not fully understood. In order to gain insight into the molecular events that govern CAR-T cell activity, we conducted a systematic analysis of the CAR "protein interactome" and "signalosome" in human primary CAR-T cells.

Methods

Interactome analysis was based on immunoprecipitation of CARs followed by protein identification using mass spectrometry (LC-MS/ MS). Technical validation of the results was performed using Western blot and flow cytometry. A variation of the SILAC [2] method was used for signalosome analyses, allowing for in-vitro stimulation of human primary CAR-T cells with pancreatic cancer cells. Metabolic labeling was used for post-hoc analysis of the phosphoproteome in the CAR-T cell compartment.

Results

We found that second-generation CARs interacted with, and induced spontaneous phosphorylation of, the endogenous CD3Z. This phenomenon was not exclusive of any specific scFv, or any costimulatory, hinge, or transmembrane modules. However, the intensity and site of phosphorylation were affected by the choice of transmembrane and co-stimulatory domains, respectively. CAR signalosome analysis revealed activation of multiple signaling networks including TCR signaling, Actin Cytoskeleton Signaling, Glycolysis, among others, in concordance with the changes in gene expression patterns observed by microarray analyses. By comparing second- versus third-generation CARs sharing the same antigen-binding domain (anti-PSCA), we observed that the ability of a CAR to engage the endogenous $C D 3 \zeta$ was associated with stronger phosphorylation of downstream secondary messengers, and superior antitumor efficacy in vivo.

Conclusions

We report a detailed analysis of the CAR interactome and signalosome, and we describe the establishment of tonic signaling mediated by CAR-induced phosphorylation of endogenous CD3 molecules. In addition, we present new immunoproteomic tools for the study of the relationship between CAR structure and T cell function. We plan to integrate the knowledge gained in these studies to optimize combination therapies, and to design the next generation of CAR-T cells, integrating customized sensor/effector circuits for superior therapeutic efficacy [3]. 


\section{References}

1. Abate-Daga D, Davila ML. CAR models: next-generation CAR modifications for enhanced T cell function. Mol Ther Oncolytics. 2016; 3:16014.

2. Ong SE, Kratchmarova I, Mann M. Properties of 13C-substituted arginine in stable isotope labeling by amino acids in cell culture (SILAC). J Proteome Res. 2003; 2:173-81.

3. Gordley RM, et al. Engineering dynamical control of cell fate switching using synthetic phospho-regulons. Proc Natl Acad Sci U S A. 2016; 113: 13528-13533.

\section{P161}

Engineering adoptive T cell therapy for efficacy in ovarian cancer

Kristin Anderson", Breanna Bates ${ }^{1}$, Edison Chiu², Nicolas Garcia',

Christopher Morse', Philip Greenberg ${ }^{2}$

${ }^{1}$ Fred Hutchinson Cancer Research Center, Seattle, WA, USA; ${ }^{2}$ University of Washington, Seattle, WA, USA

Correspondence: Philip Greenberg (ande8527@uw.edu)

Journal for ImmunoTherapy of Cancer 2017, 5(Suppl 2):P161

\section{Background}

Over 20,000 women are diagnosed with ovarian cancer annually, and $>50 \%$ will die within 5 years. This rate has changed little in the last 20 years, highlighting the need for innovative therapies. A promising new strategy has the potential to control tumor growth without toxicity to healthy tissues, by employing immune $T$ cells engineered to target proteins uniquely overexpressed in tumors. Recent technological advances have helped identify and validate Wilms' Tumor Antigen 1 (WT1) and mesothelin (MSLN) as valid immunotherapy targets in ovarian cancer, as these proteins contribute to malignant and invasive phenotypes and have limited expression in healthy cells.

Methods

$T$ cells were engineered to express high-affinity $T$ cell receptors (TCRs), using lenti- or retro-viral transduction. The peritoneally disseminated ID8 $8_{\mathrm{VEGF}}$ mouse ovarian cancer model in immunocompetent mice was used for preclinical studies. Transcriptome profiling (ThermoFisher), flow cytometry, intracellular cytokine stimulation assays, mass spectrometry, the Seahorse platform, and immunohistochemical analyses were performed to characterize the human and murine tumor microenvironment (TME), and to measure intra-tumor T cell phenotypes and function.

Results

Using either patient-derived cell lines or mouse ID8 $8_{\text {VEGF }}$ ovarian tumor cells, we found that $\mathrm{T}$ cells engineered to express either a human or mouse WT1- or MSLN- specific high-affinity TCR can kill human and murine ovarian tumor cells respectively in vitro. Moreover, in the ID8 ${ }_{\text {VEGF }}$ in vivo murine model, adoptively transferred TCRengineered $T$ cells preferentially accumulated within established tumors, diminished ovarian tumor growth and prolonged mouse survival. However, our data also revealed that the TME can limit engineered $T$ cell persistence and killing capacity. Immunosuppressive cells, inhibitory ligands that reduce $\mathrm{T}$ cell function, and cell deathinducing ligands are abundant within ID8 $8_{\text {VEGF }}$ tumors. Cellular and molecular analyses of human ovarian cancer specimens showed similar TME-mediated obstacles exist for human T cell therapy. The ovarian cancer TME is a nutrient- and oxygen-deprived milieu, which has protean effects on $T$ cell function. Ongoing studies exploring strategies to disable specific TME elements that facilitate immune evasion by ovarian cancer and are common to human and murine tumors will be discussed. Both direct modulation of cellular and molecular components of the TME and T cell engineering are being pursued to overcome critical obstacles and thereby promote $\mathrm{T}$ cell survival and function.

Conclusions

Strategies that modulate inhibitory pathways have the potential to enhance $\mathrm{T}$ cell function and improve anti-tumor efficacy by reducing limitations posed by the TME. Our studies suggest such efforts will enhance therapy of ovarian cancer.

\section{P162}

Preclinical evaluation of BCMA-specific TAC receptor-engineered T cells for multiple myeloma

Ksenia Bezverbnaya, Craig Aarts, Christopher Helsen, Galina Denisova,

Arya Afsahi, Carole Evelegh, Derek Cummings, Jonathan Bramson

McMaster University, Hamilton, ON, Canada

Correspondence: Jonathan Bramson (ksenia.bezverbnaya@gmail.com)

Journal for ImmunoTherapy of Cancer 2017, 5(Suppl 2):P162

\section{Background}

Multiple myeloma is an incurable hematologic malignancy, with poor prognosis and limited therapeutic options for patients who relapse post-transplant. Recent clinical trials with chimeric antigen receptor (CAR) $T$ cells redirected against B-cell maturation antigen (BCMA) confirmed that myeloma is susceptible to treatment with engineered $T$ cells $[1,2]$. We constructed a novel chimeric receptor, called the $T$ cell antigen coupler (TAC), which was designed to co-opt signalling through the native $T$ cell receptor to provide engineered $T$ cells with a natural way of auto-regulating their activity. Herein, we discuss our results with several BCMA-TAC receptors.

Methods

We generated a series of BCMA-TAC receptors using previouslydescribed and novel BCMA-specific single chain antibody fragments (scFvs) in the antigen-binding domain. Functionality of primary human $T$ cells engineered with BCMA-TACs was evaluated in vitro and in vivo. Anti-tumor efficacy was measured using a xenograft myeloma model based on a luciferase-expressing KMS-11 cell line.

Results

Upon activation with $\mathrm{BCMA}^{+}$myeloma cells in vitro, BCMA-TAC T cells proliferated, secreted cytokines, and killed target cells in an antigenspecific manner. In vivo, treatment of mice bearing disseminated myeloma with $4 \times 10^{6} \mathrm{BCMA}^{-\mathrm{TAC}^{+}} \mathrm{T}$ cells consistently resulted in rapid disease regression, with many mice clearing their myeloma completely, and showing no signs of relapse 6 months after T cell infusion. These results were reproducible across multiple experiments, with $T$ cells derived from different donors performing equally well in male and female myeloma-bearing mice. It was interesting to note that relapses were typically extramedullary, occurring at sites distinct from the primary site of disease, indicating that relapse was not a consequence of failure of the engineered $\mathrm{T}$ cells to clear the primary tumor site.

Conclusions

Our findings support further evaluation of BCMA-TAC T cells in human trials. Understanding the mechanisms of extramedullary relapse should provide strategies to enhance therapeutic efficacy.

\section{References}

1. Berdeja J, Lin Y, Raje N, et al. First-in-human multicenter study of bb2121 anti-BCMA CAR T cell therapy for relapsed/refractory multiple myeloma: Updated results. J Clin Oncol. 2017; 35 supp: abstr 3010.

2. Fan F, Zhao W, Liu J, et al. Durable remissions with BCMA-specific chimeric antigen receptor (CAR)-modified T cells in patients with refractory/relapsed multiple myeloma. J Clin Oncol. 2017; 35suppl: abstr LBA3001.

P163

Enhanced functional profile of CAR-T cells generated in the presence of mTOR kinase inhibitor

Archana Brahmandam, David Swart, Jason D. Fontenot, Lucas J. Thompson Juno Therapeutics, Inc., Seattle, WA, USA

Correspondence: Archana Brahmandam

(archana.brahmandam@junotherapeutics.com); Lucas J. Thompson

Journal for ImmunoTherapy of Cancer 2017, 5(Suppl 2):P163

\section{Background}

Manufacture of CAR-T cells requires activation and expansion of $\mathrm{T}$ cells for clinical dosing. This process results in a portion of the $T$ cell drug product that may, in some cases, be driven to a state of terminal "exhaustion", in which multipotency and $T$ cell replicative 
potential are diminished. Drivers of exhaustion can include signaling through $\mathrm{PI3}$ kinase (PI3K)-mTOR, which drives rapid growth and active effector response. Inhibition of PI3K-mTOR was shown to promote memory $\mathrm{T}$ cell differentiation and improved efficacy in adoptive immunotherapy models [1, 2, 3]. We explored whether inhibition of mTOR kinase (TORK) during CAR-T cell production would promote improved expansion, survival and function in response to cognate antigen.

Methods

We used a CD19-directed CAR-T cell which contains a 41BB-CD3z endodomain. CAR-T cells were generated by lentiviral transduction and subsequent expansion in vitro prior to cryopreservation. During expansion, cells were cultured with or without inhibitors (TORKi and pan-PI3K/mTOR inhibitor). Cryopreserved samples were thawed and assessed for function (target killing, cytokine secretion, and intracellular cytokine expression) and expansion or survival after stimulation through the CAR. Stimulation of CAR-T cells was by co-culture with CD19-expressing cells or anti-idiotypic antibody binding.

Results

When cultured under sustained antigen conditions, inhibitormodified CAR-T cells displayed increased expansion with enhanced survival upon antigen withdrawal. Inhibitor-modified CAR-T cells killed antigen-bearing target cells at levels similar to controls, but secreted higher cytokine levels. The enhanced cytokine functionality of inhibitor-modified CAR-T cells was retained throughout sustained antigen exposure.

Conclusions

The observed enhanced expansion and survival of inhibitor-modified CAR-T cells may lead to a potential for improved in vivo persistence. Additionally, the ability of inhibitor-modified CAR-T cells to retain improved cytokine capacity through sustained antigen exposure indicates that these cells may be resistant to functional exhaustion. Taken together, these data suggest that improved clinical durability of response could be achieved through use of TORK-inhibitors during CAR-T cell manufacturing.

\section{References}

1. Pearce EL, Walsh MC, Cejas PJ, Harms GM, Shen H, Wang LS, Jones RG, Choi Y: Enhancing CD8 T Cell Memory by Modulating Fatty Acid Metabolism. Nature. 2009; 460(7251):103-7.

2. Araki K, Turner AP, Shaffer VO, Gangappa S, Keller SA, Bachmann MF, Larsen CP, Ahmed R: mTOR regulates memory CD8 T cell differentiation. Nature. 2009; 460(7251):108-11.

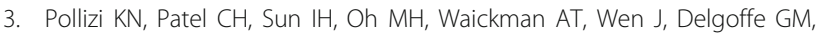
Powell JD: mTORC1 and mTORC2 selectively regulate CD8+ T cell differentiation. J Clin Invest. 2015; 125(5):2090-2108.

\section{P164}

A temporal and spatial analytical system to measure CAR-T cell function

Ana Castano1, Angela Boroughs ${ }^{1,2}$, Marcela Maus ${ }^{1,2}$

${ }^{1}$ Massachusetts General Hospital, Charlestown, MA, USA; ${ }^{2}$ Harvard

Medical School, Charlestown, MA, USA

Correspondence: Ana Castano (ACASTANO@mgh.harvard.edu)

Journal for ImmunoTherapy of Cancer 2017, 5(Suppl 2):P164

\section{Background}

The cell functional assays that currently exist to test the in vitro functional performance of CAR -T cells are indirect read outs. The field could benefit from a continuous live-cell imaging and analysis of the spatial and temporal kinetics of the effector- target interactions in simulated effects of the tumor environment. Ideally, these assays, could be implemented in high throughput to evaluate and isolate perturbations that illuminate the basic biological principles and allow for testing of specific hypotheses.

Methods

We generated and tested primary human CAR T cells as effector cells in co-culture assays with antigen-matched target cell lines. Cells were co-cultured for $48 \mathrm{~h}$ on 96 well plates, and evaluated using the Essen BioScience IncuCyte Live-Cell Imaging System, an automated microscope that resides inside the culture incubator (Fig. 1). We analyzed spatial and kinetic imaging in both phase contrast and fluorescence. Scans were scheduled and images collected and developed to define and test a Processing Definition analysis system. We established the parameters to be measured in all images and defined the metrics of object counts, average area, and mean intensity to be evaluated over time.

Results

The dynamic antigen-specific lysis of target cells could be visualized and quantified during the entire analysis period. The kinetics of the morphologic, proliferative, and surface area occupancy of both T cells and tumor cells were quantified. Our initial significant findings include: rapid clustering of CAR-T cells surrounding cancer target cells, and migration and proliferation of CAR-T cells stimulated by the presence of antigen. In addition, we simulated an inhibitory tumor microenvironment by the addition of human $T$ regulatory cells to the culture, and noted changes in the spatial and temporal function of primary human CAR-T cells. Each one of these aspects was quantified.

\section{Conclusions}

This processing definition analysis system allowed us to measure and quantify biological parameters that allow us to understand the dynamic nature of CAR T cells over time in simulated tumor microenvironments. This system has potential to be tested as a potency assay, which is currently an unmet need in the field of cell-based immunotherapy.

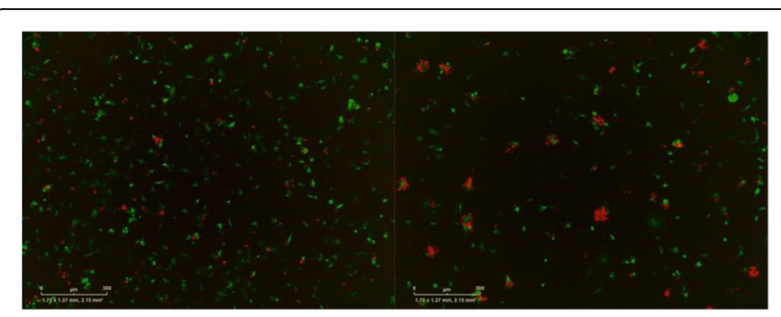

Fig. 1 (abstract P164). U87-GFP cell line and anti-EGFR-CART cells vs unspecific CAR-T. Glioblastoma cells (U87, modified to express Green Fluorescent Protein), incubated with CAR T cells (also expressing red mCherry). Left, photomicrograph immediately after mixing; right, photomicrograph of the same area 24 hours later

\section{P165}

High throughput label-free impedance-based technology for kinetic in vitro functional potency assessment of immune cell-mediated cytolysis and immune checkpoint modulation

Fabio Cerignoli, Biao Xi, Garret Guenther, Lincoln Muir, xBrandon Lamarche, Nikhil Mittal, David Untiet, Yama Abassi ACEA Biosciences, San Diego, CA, USA

Correspondence: Fabio Cerignoli (fcerignoli@aceabio.com) Journal for ImmunoTherapy of Cancer 2017, 5(Suppl 2):P165

\section{Background}

In vitro characterization of reagents efficacy for cancer immunotherapy protocols is necessary before moving to expensive animal models and clinical studies. However, current standard assays like Chromium-51 release, ATP-based luminescence cell monitoring or flow cytometry are difficult to implement in a high throughput environment and are based on end point methodologies that are unable to capture the full dynamic of the immune response. Here we present an impedance-based platform for monitoring cytotoxic activity of immune cells in the context of cancer immunotherapy assays. Methods

The technology detects cell death and proliferation of adherent cells by measuring changes in conductance of microelectrodes embedded in 96 and 384-wells cell culture plates, without the use of labeling or cell modification. Because immune cells are unable to bind the microelectrodes, the technology monitors directly adherent target cells proliferation/survival without signal interference by effector cells. 
Furthermore, the approach can be adapted to suspension target cells, like B cell leukemia, through adhesion mediated by coating antibodies.

Results

We provide a large set of validation including most of the tolls that are currently utilized in the immunotherapy space: CD19 Car T models, EpCAM/CD3 BiTE antibodies and immune checkpoint inhibitor combination therapies. Based on our observation, PD-1 specific antibody can dose-dependently modulate the potency of humanderived PBMC against PC3 prostate cancer cell lines. Using PD-1 checkpoint antibody in combination with antibodies directed against other checkpoint proteins including Tim-3, Lag-3 and in combination with PDL-1 antibody, we can demonstrate either additive effects or no net increase in potency. Data comparison with Annexin V staining/Flow Cytometry shows perfect correlation between the drop in impedance signal and \% of apoptotic cells, as well as correlation between immune checkpoint protein expression in PBMC populations and immune blockade.

\section{Conclusions}

In summary, we have developed a quantitative and reproducible functional assay that can be used to characterize immune cytotoxic response across the temporal scale, an aspect that is otherwise very difficult to assess with more canonical end point assays. Thanks to the availability of 384-wells format and minimal sample handling, the technology is also ideally suited for applications in large drug screening campaigns or therapeutic protocol validation directly on patient samples.

\section{P166}

H3.3K27M mutation-derived novel neoantigen - characterization of the HLA-A2-binding epitope and a specific T cell receptor for development of $\mathrm{T}$ cell-based immunotherapy

Zinal Chheda', Gary Kohanbash', Kaori Okada', Naznin Jahan', John Sidney ${ }^{2}$, Matteo Pecoraro ${ }^{3}$, Diego Carrera' ${ }^{1}$, Shruti Shrivastav ${ }^{1}$, Shuming

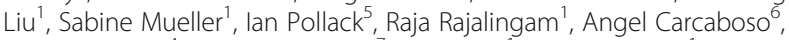
Matthias Mann ${ }^{4}$, Alessandro Sette', Yafei Hou', Hideho Okada'

${ }^{1}$ University of California, San Francisco, San Francisco, CA, USA; ${ }^{2}$ La Jolla Institute, La Jolla, CA, USA; ${ }^{3}$ Max Planck Institute of Biochemistry, Martinsried, Germany; ${ }^{4}$ Max-Planck-Institut für Biochemie, Martinsried, Germany; ${ }^{5}$ University of Pittsburgh School of Medicine, Pittsburgh, PA, USA; ${ }^{6}$ Institut de Recerca Sant Joan de Deu, Barcelona, Spain; ${ }^{7}$ La Jolla Institute For Allergy and Immunology, La Jolla, CA, USA

Correspondence: Hideho Okada (zinal.bt@gmail.com)

Journal for ImmunoTherapy of Cancer 2017, 5(Suppl 2):P166

\section{Background}

The median overall survival for children with diffuse intrinsic pontine glioma (DIPG) is less than a year. The majority of diffuse midline gliomas (DMG), including over $70 \%$ of DIPG, harbor an amino-acid substitution from lysine $(K)$ to methionine $(M)$ at the position 27 of histone 3 variant $\mathrm{H} 3.3$ (the H3.3K27M mutation hereafter) [1]. $\mathrm{H} 3.3 \mathrm{~K} 27 \mathrm{M}$ mutation is associated with shorter survival compared to patients with non-mutated H3.3.

Methods

In vitro stimulation of human $\mathrm{CD}^{+}{ }^{+} \mathrm{T}$ cells with a 10-mer peptide encompassing the $\mathrm{K} 27 \mathrm{M}$ mutation ( $\mathrm{H} 3.3 \mathrm{~K} 27 \mathrm{M}$ peptide, hereafter) led to generation of specific CTL clones and CDNA for T cell receptor (TCR) $\alpha$ - and $\beta$-chains was isolated. Mass spectrometry analysis was conducted to determine whether the H3.3K27M-derived epitope is presented on human leukocyte antigen (HLA)-class I of $\mathrm{HLA}^{-\mathrm{A} 2^{+}}$ $\mathrm{H} 3.3 \mathrm{~K}_{2} 7 \mathrm{M}^{+}$glioma cells. T cells transduced with the TCR were evaluated for cytotoxicity against $\mathrm{HLA}-\mathrm{A2}^{+} \mathrm{H} 3.3 \mathrm{~K}_{2} 7 \mathrm{M}^{+}$glioma cells in vitro and in mice bearing glioma xenografts.

Results

As predicted by the NetMHC3.4 algorithm, a competitive binding inhibition assay demonstrated that the H3.3K27M peptide, but not the corresponding non-mutant peptide, has an excellent binding affinity to HLA-A2. Importantly, mass spectrometric analysis of $\mathrm{H} 3.3 \mathrm{~K} 27 \mathrm{M}^{+}$glioma cells demonstrated that the H3.3K27M peptide is naturally processed and presented by HLA class I.H3.3K27M-specific $\mathrm{CD} 8^{+} \mathrm{T}$ cell responses were detected in patients' peripheral blood mononuclear cells by IFN- $\gamma$

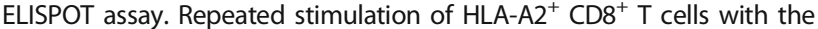
$\mathrm{H} 3.3 \mathrm{~K} 27 \mathrm{M}$ peptide led to establishment of H3.3K27M-reactive $\mathrm{CD}^{+} \mathrm{T}$ cell clones. From one such clone, CDNA for TCR $\alpha$ - and $\beta$-chains were cloned into a retroviral vector. Human $\mathrm{HLA}-\mathrm{A2}^{+} \mathrm{T}$ cells transduced with the TCR efficiently killed HLA-A2 ${ }^{+} \mathrm{H} 3.3 \mathrm{~K}_{2} 7 \mathrm{M}^{+}$glioma cells in an antigen- and HLA-specific manner. Adoptive transfer of TCR-transduced T cells significantly suppressed the progression of orthotopic xenografts in mice. Furthermore, alanine-scanning assays demonstrated that there are no known human proteins that share the set of key amino acid residues required for recognition by the TCR, strongly suggesting that the TCR could be safely used in patients without causing off-target toxicity (Figs. 1, 2, 3, 4, 5).

Conclusions

These data provide us with a strong basis for developing peptidebased vaccines as well as adoptive transfer therapy using autologous T cells transduced with the TCR.

\section{Reference}

1. Schwartzentruber J, Korshunov $\mathrm{A}$, Jabado $\mathrm{N}$ et. al. Driver mutations in histone $\mathrm{H} 3.3$ and chromatin remodelling genes in paediatric glioblastoma. Nature. 2012; 482:226-31.

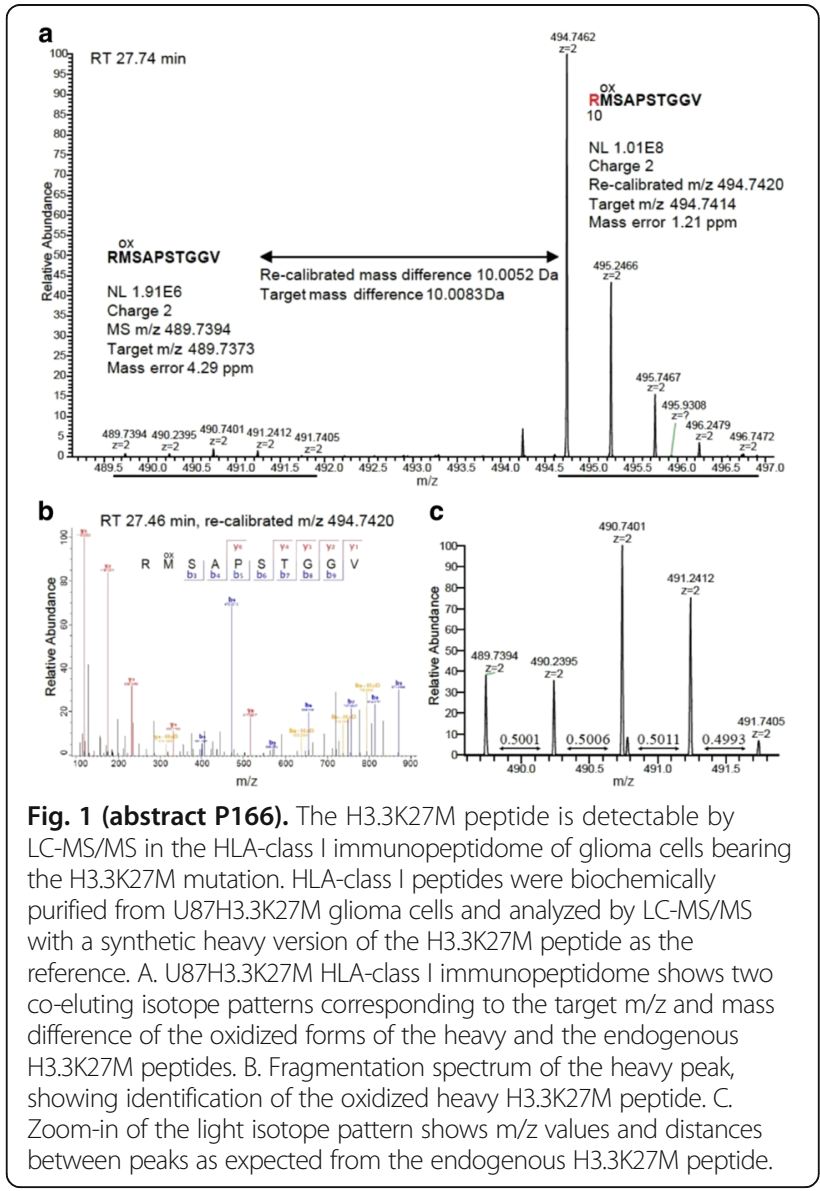



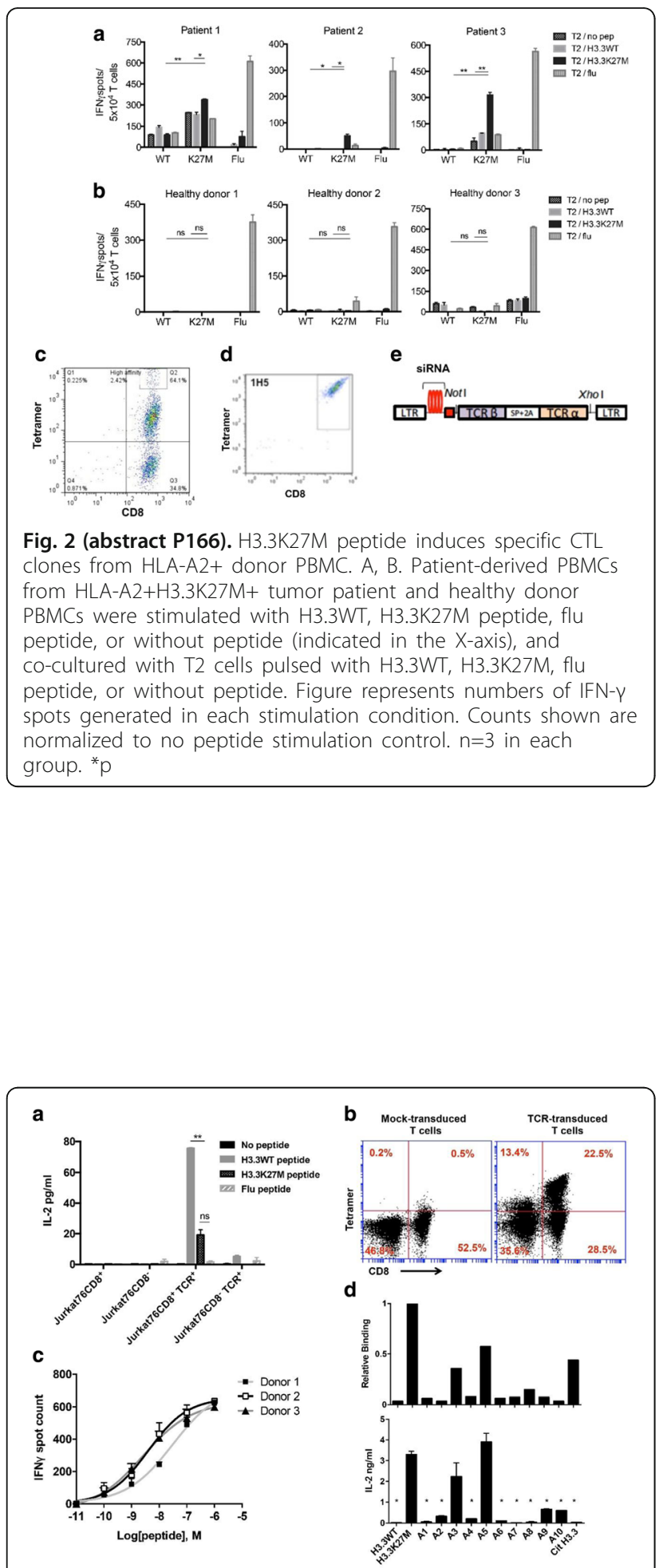

Fig. 3 (abstract P166). Characterization of the functional avidity of TCR in recognition of HLA-A2-peptide complex and alanine scanning for safety of TCR use. A. T2 cells loaded with or without H3.3K27M peptide, H3.3WT peptide or irrelevant HLA-A2 binding flu peptide $(10 \mu \mathrm{g} / \mathrm{ml})$ were co-cultured with either control or TCR-transduced Jurkat76CD8+ cells and Jurkat76CD8- cells in 1:1 ratio and assessed for IL-2 production by ELISA. Data represent three independent experiments with similar results. * $p$

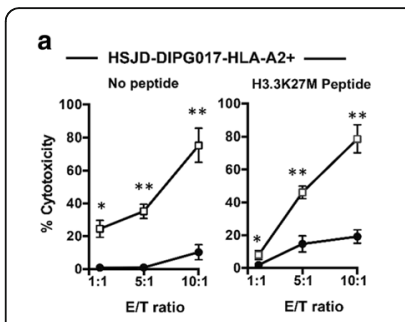

C — HSJD-DIPG019-HLA-A2neg -
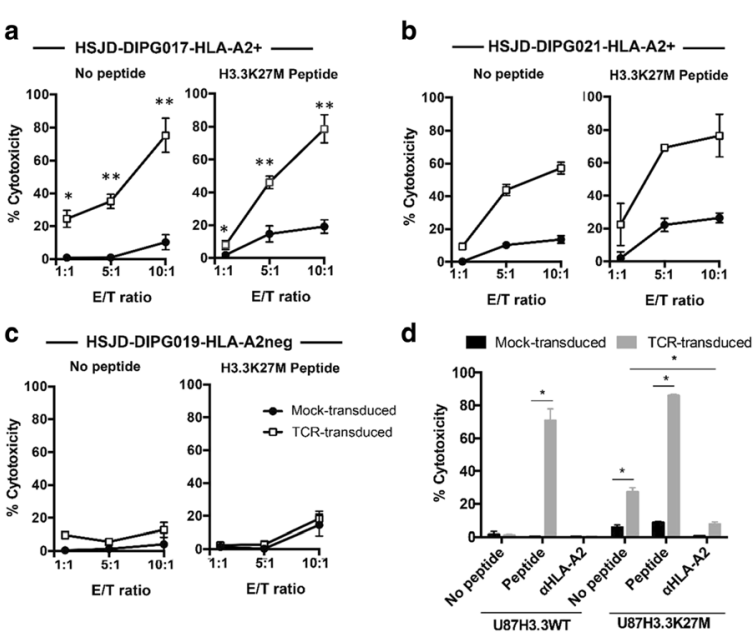

Fig. 4 (abstract P166). TCR-transduced T cells lyse H3.3K27M+HLAA2+ glioma cells in an HLA-A*0201- and H3.3K27M-dependent manner. Cytotoxicity of TCR-transduced T cells was evaluated by lactate dehydrogenase (LDH) cytotoxicity assay. A,B, C. TCR-transduced or mock-transduced T cells were co-cultured with H3.3K27M+HLAA*0201+ HSJD-DIPG-017 cells, HSJD-DIPG-021 cells or control H3.3K27M+HLA-A*0201- HSJD-DIPG-019 cells at E/T ratio of 1, 5, and 10 for 24 hrs. D. TCR-transduced or control T cells were co-cultured with HLA-A*0201+ U87H3.3K27M cells or U87H3.3WT cells at E/T ratio of 5 . Each group was assessed in triplicate. Data represent two independent experiments with similar results. ${ }^{*} p$

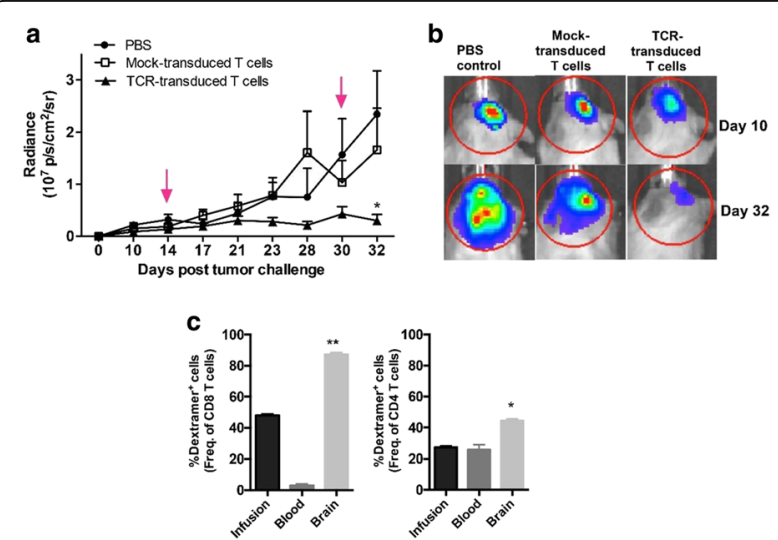

Fig. 5 (abstract P166). Adoptive transfer of TCR-transduced T cells but not mock-transduced T cells results in inhibition of intracranial H3.3K27M+ glioma in NSG mice. NSG mice bearing intracranial U87H3.3K27M luciferase+ gliomas received intravenous infusion with PBS, mock-transduced T cells or TCR-transduced T cells. A. Tumor growth is presented as radiance $(107 \mathrm{p} / \mathrm{s} / \mathrm{cm} 2 / \mathrm{r})$ using $\mathrm{BLI}(\mathrm{n}=8$ per group). Arrows indicate days on which mice received treatment. B. Representative BLI images of mice on Day 10 and on Day 32 post tumor inoculation. The background BLI signals were defined based on the levels seen in non-tumor bearing mice. C. Preferential accumulation of TCR+ T cells in the tumor site. At the time of intravenous infusion, approximately $50 \%$ and $30 \%$ of the infused CD8+ and CD4+ T cells, respectively, were TCR-Dextramer+. On Day 2 following second intravenous infusion, the percentage of Dextramer+ cells among CD8+ $T$ cells and CD4+ T cells were evaluated in the peripheral blood and the brain of mice that received TCR-transduced T cells. Data indicate \% Dextramer+ cells among total live CD8+ or CD4+ T cells ( $n=5$ per group). *p 
P167

Translation of highly functional CMV-induced "adaptive" NK cells to treat cancer

Sarah Cooley ${ }^{1}$, Frank Cichocki ${ }^{1}$, Bob Valemehr ${ }^{2}$, David McKenna ${ }^{1}$,

Jeffrey Miller

${ }^{1}$ University of Minnesota, Minneapolis, MN, USA; ${ }^{2}$ FATE Therapeutics,

San Diego, CA, USA

Correspondence: Sarah Cooley (cool0023@umn.edu)

Journal for ImmunoTherapy of Cancer 2017, 5(Suppl 2):P167

\section{Background}

Cytomegalovirus (CMV)-induced "adaptive" natural killer (NK) cells express more adaptive marker NKG2C and maturation marker CD57, are epigenetically reprogrammed, and have unique functional properties including increased cytokine production after target exposure, increased antibody-dependent cellular cytotoxicity (ADCC), enhanced persistence, and inherent resistance to myeloid derived suppressor cells and regulatory $\mathrm{T}$ cells. We have shown that higher frequencies of adaptive NK cells in patients who reactivate CMV after reduced in tensity hematopoietic cell transplantation have lower rates of relapse. Thus, in partnership with Fate Therapeutics, we have developed FATE-NK100, a first-in-class allogeneic NK cell therapy product enriched for cells with "adaptive" phenotype and function.

Methods

CD3 and CD19 depleted peripheral blood NK cells from CMV seropositive donors are cultured with IL-15 and a small molecule inhibitor of glycogen synthase kinase (GSK) 3.

\section{Results}

Inhibition of GSK3 during ex vivo expansion led to marked increases in the expression of T-BET $(p=0.0005)$, ZEB2 $(p=0.0001)$ and BLIMP-1 (0.0002), which are transcription factors that drive late-stage NK cell maturation. Further, the cultured cells produce significantly more TNF and IFN- $\gamma$ and mediate better ADCC as compared to conventional NK cells. Relative to control cultures with IL-15 alone, FATENK100 cells expressed significantly more KIR $(p=0.014)$, LFA-1 $(p=$ $0.011)$ and 2B4 ( $p=0.009)$ and markedly lower levels of NKG2A ( $p=$ 0.002). A Phase I clinical trial testing FATE-NK100 given after lymphodepleting chemotherapy (Fludarabine $25 \mathrm{mg} / \mathrm{m} 2 \times 5$ days and Cyclophosphamide $60 \mathrm{mg} / \mathrm{kg} \times 2$ days) followed by subcutaneous IL-2 (6 doses of 6 million units every other day) to treat refractory AML has opened to patient accrual. The first patient treated at the lowest dose cohort $(1 \times 10 \wedge 7 \mathrm{TNC} / \mathrm{kg})$ showed enhanced persistence of FATE-NK100 at Day +14 (45\% donor, with an absolute FATE-NK100 cell count of 27 cells/uL of blood). A second Phase 1 clinical trial treating patients with recurrent ovarian cancer via intraperitoneal administration of FATE-NK100 will open in Q3 of 2017.

\section{Conclusions}

FATE-NK100 is a first-in-class, adaptive NK cell therapy product for adoptive cancer immunotherapy. The data demonstrate FATE-NK100 has highly-differentiated properties and superior antitumor activity. Clinical trials of FATE-NK100 in refractory AML, ovarian cancer and advanced solid tumors in combination with monoclonal antibody therapy are being conducted.

Trial Registration

NCT03081780 and NCT03213964

\section{P168}

$C B L B$ gene editing potentiates the effector function of

tumor-targeted engineered-TCR human T cells

Andreia S. Da Costa ${ }^{1}$, Alexandra Croft', Vidya Dhanapal ${ }^{2}$, Michael Dinsmore ${ }^{2}$ Georgia Giannoukos ${ }^{2}$, Kiran Gogi', Gregory Gotta², Fred Harbinski²,

Christopher J. Wilson'², G. Grant Welstead², Jason D. Fontenot ${ }^{1}$

'Juno Therapeutics, Inc, Seattle, WA, USA; ${ }^{1}$ Editas Medicine, Cambridge, MA, USA

Correspondence: Andreia S. Da Costa

(andreia.costa@junotherapeutics.com)

Journal for ImmunoTherapy of Cancer 2017, 5(Suppl 2):P168

\section{Background}

Providing optimal outcomes with engineered T cell therapies in solid tumors will likely require strategies to overcome suppressive and immune-evasive mechanisms often present in solid tumor environments. The E3 ligase, CBLB, functions as a negative regulator of $\mathrm{T}$ cell activation and acts as an intracellular immune checkpoint regulator. In mice, Cblb deletion has been reported to induce autoimmunity and enhanced rejection of spontaneous and implanted tumors [1] due to loss of $\mathrm{CD} 28$ dependent $\mathrm{T}$ cell activation. We investigated the functional consequences of CRISPR/Cas9 mediated CBLB deletion in human engineered $T$ cells in response to antigen in the presence or absence of co-stimulation.

Methods

Human $\mathrm{CD} 4+$ and $\mathrm{CD} 8+\mathrm{T}$ cells were isolated from healthy donor apheresis, activated and, in some cases, transduced to express an engineered TCR (eTCR). Non-transduced or eTCR transduced T cells were transfected with CBLB targeted guide RNA (gRNA) in complex with Cas9 and expanded in the presence of added cytokines prior to analysis. $C B L B$ deletion was confirmed by sequencing and western blot analysis. Responses to TCR stimulation with or without costimulation were analyzed following culture of CBLB edited nontransduced or eTCR transduced T cells, with anti-CD3 (with or without anti-CD28) or HLA-matched T2 cells pulsed with a peptide recognized by the eTCR (with or without CTLA-4-lg), respectively. Cytokine production, targeT cell killing and proliferation were assessed at 24-72 hours post initiation of culture.

Results

CBLB editing in T cells resulted in a $>90 \%$ reduction in CBLB expression as compared to transfection controls. Edited $T$ cells displayed enhanced production of IFNY, IL-2 and TNFa in response to suboptimal TCR stimulation (in the absence of anti-CD28 or in the presence CTLA-4-lg mediated inhibition of co-stimulation). Edited T cells also demonstrated greater proliferative capacity and survival rates in response to antigenic stimulation in the absence of supplemental cytokines. Furthermore, CBLB-edited eTCR transduced T cells were more efficient killers of antigen presenting target tumor cells with an EC50 approximately 10-fold lower than that observed for unedited controls.

\section{Conclusions}

Manipulation of co-stimulatory molecules by tumor cells can, in some contexts, limit the effectiveness of adoptive $T$ cell therapies. Our data show that CBLB editing of eTCR transduced T cells can enhance antigen sensitivity, cytokine production, proliferation and cytotoxicity in the absence of CD28 co-stimulation. These data suggest that CBLB editing may boost $T$ cell function in a suppressive tumor environment.

\section{Reference}

1. Stromnes I M, et al. J Clin Invest. 2010;120:3722-3734.

\section{P169}

Mass-spectrometry-guided target selection and TCR profiling: towards the development of safer TCR-engineered T cell therapies Thorsten Demberg , Mamta Kalra', Leonie Alten², Amir Alpert', Sebastian Bunk ${ }^{2}$, Jens Fritsche ${ }^{2}$, Oliver Schoor ${ }^{2}$, Dominik Maurer ${ }^{2}$, Ali Mohammed ${ }^{1}$, Yannick Bulliard', Harpreet Singh', Carsten Reinhardt', Toni Weinschenk², Steffen Walter

${ }^{1}$ Immatics US Inc., Houston, TX, USA; ${ }^{2}$ Immatics Biotechnologies GmbH,

Tuebingen, Germany; ${ }^{3}$ Immatics Biotechnologies $\mathrm{GmbH}$, Martinsried, Germany

Correspondence: Steffen Walter (demberg@immatics.com)

Journal for ImmunoTherapy of Cancer 2017, 5(Suppl 2):P169

\section{Background}

A major constraint for the broad and safe applicability of Adoptive Cellular Therapy (ACT) is the limited number of known tumor-specific targets, especially for solid tumors. For T cell receptor (TCR)-based 
ACT approaches, presentation of the targeted HLA-peptide complex on normal tissues can lead to on-target/off-tumor toxicity. Independently, TCRs with promiscuous peptide-binding motif are known to cause dramatic off-target adverse events.

\section{Methods}

Here, we present the capabilities of our XPRESIDENT ${ }^{\circledR}$ platform to not only identify novel tumor targets, but also screen TCR candidates against off-target toxicities in absence of relevant in vivo models. The XPRESIDENT platform allows for direct identification of HLA-restricted peptides via ultra-high sensitive mass spectrometry. Peptide sequencing of a wide-ranging collection of healthy tissues and tumor biopsies enabled the discovery of a pipeline of $>80$ targets overrepresented in human tumors. Immatics' high-throughput TCR platform can generate large panels of unique, target-specific TCR sequences from the natural human repertoire. Positional scanning helps identify the peptide-binding motif of lead TCR candidates, and TCRs showing cross-recognition to analogous peptides expressed in healthy tissues (XPRESIDENT) can therefore be negatively-selected at an early preclinical stage.

Results

In addition to expression data, we will present a comprehensive preclinical data package of some of our lead TCR candidates. GMPcompliant manufacture of ACTengine $T$ cells shows robust TCR expression and T cell expansion. ACTengine $T$ cells preserve a stemcell-like memory phenotype, which is known to correlate with longer persistence in vivo. Finally, ACTengine $\mathrm{T}$ cells selectively recognize and kill tumor cell lines presenting the target peptide, while sparing target-devoid primary healthy cells.

\section{Conclusions}

The XPRESIDENT-guided targets currently considered for ACTengine are presented in various solid and liquid tumor types, while not detected on healthy tissues. Immatics' TCR-based ACTengine ${ }^{\oplus}$ programs, in collaboration with MD Anderson Cancer Center, aim to deliver safer, best-in-class immunotherapies.

\section{P170}

Exploiting CD4 T cells for adoptive cell therapy in cancer

Pierre Dillard, Marit Myrhe, Nadia Mensali, Sylvie Pollmann, Gunnar Kvalheim, Gustav Gaudernack, Sebastien Wälchli, Else Marit Inderberg

OUS-Radiumhospital Oslo Cancer Cluster incubator (OCCi), Oslo, Norway Correspondence: Sebastien Wälchli (pierre.dillard@rr-research.no); Else

Marit Inderberg

Journal for ImmunoTherapy of Cancer 2017, 5(Suppl 2):P170

\section{Background}

T cell based immunotherapy represents an attractive strategy for the treatment of cancer. Whereas cellular anti-tumor immune responses have typically been attributed to CD8 T cells, CD4 T cells play a critical role in tumor elimination and the priming and maintenance of CD8 T cell responses. Combining HLA class I- and class II-restricted TCRs for T cell redirection may provide a more potent therapeutic effect in adoptive T cell therapy. Furthermore, HLA class II-restricted TCRs may be of therapeutic value both in haematopoietic malignancies and in melanoma where tumor cells frequently express HLA class II.

Methods

We have isolated CD4+ $T$ cells reactive against tumor antigens from patients who experienced clinical benefit from treatment with cancer vaccines targeting universal tumor antigens and frequent neoantigens.

Results

Strong $T$ cell responses against the vaccines or unrelated cancer antigens suggesting epitope spreading correlated with enhanced survival and tumor regression in late stage cancer patients. These HLA class II restricted $\mathrm{T}$ cell clones recognised target cells loaded with long peptides or protein and for some CD4+ T cell clones we could also show direct tumor recognition. TCRs were expressed in expanded donor T cells by mRNA electroporation or retroviral transduction and found functional in both CD8+ and CD4+ T cells producing TNF- $a$, IFN- $\gamma$ with the capacity of target cell killing. We also show preliminary in vivo data for one of our broadly applicable TCRs recognizing a universal antigen, hTERT, presented on one of the most frequent HLA alleles, HLA-DP4.

Conclusions

Our findings open a way to more broadly applicable cancer treatment that will soon be tested in patients.

P171

Preclinical evaluation of mesothelin-specific $T$ cell receptor (TCR) fusion constructs (TRuC ${ }^{T M} s$ ) utilizing the signaling power of the complete TCR complex: a new opportunity for solid tumor therapy Jian Ding, Ekta Patel, Patrick Tavares, Justin Quinn, Rashmi Choudhary, Bonnie Le, Olga Kiner, Qi Wei, Solly Weiler, Patrick Baeuerle,

Robert Hofmeister

TCR2 Therapeutics, Cambridge, MA, USA

Correspondence: Robert Hofmeister (jian@tcr2.com)

Journal for ImmunoTherapy of Cancer 2017, 5(Suppl 2):P171

\section{Background}

Anti-CD19 chimeric antigen receptor (CAR) T cell therapies for B cell malignancies have demonstrated impressive clinical results. However, clinical trials in solid tumors have been much less promising. We reasoned that $\mathrm{T}$ cell activation triggered by CARs is not adequate to overcome the immunosuppressive microenvironment of solid tumors. We therefore developed the T Cell Receptor Fusion Construct $\left(\mathrm{TRuC}^{\mathrm{TM}}\right)$ platform. It is based on the recombinant fusion of a target binding domain to subunits of the $\mathrm{T}$ cell receptor complex other than CD3Z. Different from CARs, which only use the CD3Z of the six available TCR subunits for signaling, $\mathrm{TRuC}^{\mathrm{TM}}$ variants are integrated into the complete TCR and can thereby trigger a more diverse signaling cascade through all TCR components. In this study, we tested the potency of the TRuC ${ }^{\mathrm{TM}}$-T cells targeting the solid tumor antigen mesothelin (MSLN) in preclinical studies.

Methods

We generated a panel of TRuC ${ }^{\mathrm{TM}}$ variants by recombinantly fusing MSLN-specific single-domain antibodies (sdAbs) of high or low target affinity to TCR $\beta, C D 3 \gamma$, or $C D 3 \varepsilon$ subunits, respectively. The constructs were introduced into primary human T cells via lentiviral transduction. After standard stimulation and expansion, $\mathrm{TRuC}^{\mathrm{TM}}$ surface expression and $\mathrm{T}$ cell activation were analyzed by flow cytometry. Cytokine release and tumor cell lysis were investigated in vitro prior to testing the anti-tumor efficacy and persistence of TRuC ${ }^{\mathrm{TM}}$-T cells in a mesothelioma mouse model.

Results

Of the various $\mathrm{TRuC}^{\mathrm{TM}} \mathrm{s}$ made, the $\varepsilon$-TRuC ${ }^{\mathrm{TM}}$ variant showed the best surface expression irrespective of the MSLN-specific binder used. Upon target cell engagement, phosphorylation of CD3E was observed with the MSLN- $\varepsilon T^{T} C^{\mathrm{TM}}$ version but not with MSLN-specific CAR constructs, indicating that the TRuC ${ }^{\mathrm{TM}}$ was integrated into the TCR complex. TRuC ${ }^{\text {TM}}-\mathrm{T}$ cells showed target-dependent upregulation of activation markers CD69 and CD25. Further, TRuC $^{\text {TM }} \mathrm{T}$ cells demonstrated potent killing of MSLN-positive tumor cells and showed increased levels of degranulation marker CD107a. Of note, despite comparable cell lysis potential in vitro, TRuC ${ }^{\mathrm{TM}}-\mathrm{T}$ cells produced less cytokines than CAR-T cells. When tested for anti-tumor activity in a mesothelioma xenograft model, MSLN-specific TRuC ${ }^{\mathrm{TM}}$ - $\mathrm{T}$ cells were more potent than CAR-T cells. TRuC ${ }^{\mathrm{TM}}$-T cells eradicated primary solid tumors and protected mice from a later re-challenge.

Conclusions

Our results demonstrate that $\mathrm{T}$ cells expressing a $\mathrm{TRuC}^{\mathrm{TM}}$ with a lowaffinity MSLN-specific sdAb can potently kill tumor cells in vitro and appear to be superior in potency over CAR-T cells. In our preclinical 
assessment, MSLN-specific $\varepsilon$-TRuC ${ }^{\mathrm{TM}} \mathrm{T}$ cells showed the highest potential for the treatment of MSLN-positive solid tumors.

\section{P172}

iRGD enhances T cells infiltration and augments response to PD-1 gene knockout immunotherapy in gastric cancer

Baorui Liu, Naiqing Ding

The Comprehensive Cancer Centre of Drum Tower Hospital, Medical

School of Nanjing University \& Clinical Cancer Institute of Nanjing

University, Nanjing, China

Correspondence: Baorui Liu (dingng@126.com)

Journal for ImmunoTherapy of Cancer 2017, 5(Suppl 2):P172

\section{Background}

Poor infiltration of activated lymphocytes into tumors can be a fundamental factor limiting their efficacy and impeding the therapeutic effect of the checkpoint blockade immunotherapy[1,2]. A tumorpenetrating peptide, iRGD, has a well-defined role in delivering drugs into extravascular tumor tissues in both the combination regimen and conjugated pattern[3,4]. Here, we explored for the first time whether this cycled peptide could facilitate the infiltration of lymphocytes into tumor and furtherly overcome resistance to PD1 gene knockout immunotherapy.

\section{Methods}

We used polyethylene glycol-conjugated phospholipid (PEG-lipid) derivatives, a time-efficient and versatile platform, to immobilize iRGD on T cell membrane. The ability of iRGD modified or co-applied lymphocytes infiltration was detected in both the 3D tumor spheroids in vitro and subcutaneous tumor model and peritoneal tumor model of gastric cancer in vivo. Furthermore, the synergistic effect of iRGD modification and PD-1 gene knockout in adoptive T cell transfer immunotherapy was examined in a xenograft model of EBV-associated gastric cancer.

Results

In this study, we showed that T cells could be modified by the synthetic iRGD-PEG-lipid without compromising their vitality, expansion, phenotype and effector function. In vitro, co-administration of iRGD could promote the infiltration of $T$ cells while $\mathrm{iRGD}$ modification made $T$ cells spread more extensively throughout the multicellular spheroids. Near infrared results showed that iRGD modification made a tenfold improvement infiltration of $\mathrm{T}$ cells into tumors without a parallel increase in normal tissues. Most importantly, we demonstrated that $\mathrm{R}$ RGD modified T cells had superior antitumor efficiency owing to sufficiently increased T cells infiltration, and exhibited robust synergistic effect with PD-1 gene knockout immunotherapy.

\section{Conclusions}

Our study indicates that modification of T cell membrane with iRGD might be a potent strategy to increase $T$ cells infiltration, thereby overcome the bottleneck of solid tumor immunotherapy.

\section{References}

1. Tang H, Wang $Y$, Chlewicki LK, et al. Facilitating $T$ Cell Infiltration in Tumor Microenvironment Overcomes Resistance to PD-L1 Blockade. Cancer Cell. 2016;29:285-296.

2. Caruana I, Savoldo B, Hoyos V,et al. Heparanase promotes tumor infiltration and antitumor activity of CAR-redirected T lymphocytes. Nat Med. 2015;21:524-529.

3. Sugahara KN, Teesalu T, Karmali PP, et al. Tissue-penetrating delivery of compounds and nanoparticles into tumors. Cancer Cell. 2009;16:510-520.

4. Sugahara KN, Teesalu T, Karmali PP, et al. Coadministration of a tumorpenetrating peptide enhances the efficacy of cancer drugs. Science. 2010:328:1031-1035
P173

Mass cytometry based immunophenotyping of tumor-reactive $T$ cells in human cancer reveals subpopulations with coordinate inhibitory molecule expression

Monika Aleksandra Eiva, Jessica Ann Chacon, Tori Yamamoto, Thomas Garrabrant, Gabor Kari, Andrew Best, Qunrui Ye, Daniel J. Powell University of Pennsylvania, Philadelphia, PA, USA

Correspondence: Daniel J. Powell (monsta@mail.med.upenn.edu)

Journal for ImmunoTherapy of Cancer 2017, 5(Suppl 2):P173

\section{Background}

The presence of tumor infiltrating lymphocytes (TILs) is a positive prognostic factor in various cancers. TIL function can be suppressed by the expression of immune checkpoint molecules (ICMs) and antibody-based blockade of these molecules can re-invigorate suppressed TIL function to evoke tumor regression. However, multiple checkpoint blocking antibodies exist, and approaches to rationally designing complex combination immunotherapies remain elusive. Identifying tumor-reactive TILs in cancer and understanding the complex immunobiology of these cells may drive the rational development of effective combination immunotherapies. CD137 (TNFRSF9, 4$1 \mathrm{BB}$ ) is a biomarker for tumor-reactive TILs in human cancer [1]. CD137+ TILs recognize autologous tumor antigens, produce proinflammatory cytokines, and suppress tumor outgrowth in xenograft models; CD137- TILs do not. CD137+ TILs express ICMs, however, which and how many specific ICMs are coordinately expressed by tumor-reactive TILs is unknown. Accordingly, such knowledge may reveal new opportunities for rationally designed combination therapies.

Methods

Cytometry by time-of-flight (CyTOF) was performed on enzymatically-digested human ovarian cancers to immunophenotype TILs using a 34 analyte panel. CyTOF data were analyzed using viSNE and PhenoGraph algorithms to identify TIL subpopulations. Tumors were treated with anti-PD-1 and/or CD137 agonist antibodies, and TIL cytotoxic molecule expression was analyzed by flow cytometry.

Results

CD137+ TILs preferentially co-express multiple ICMs that may inhibit their anti-tumor abilities (e.g. PD-1, CTLA-4, Tim-3, 2B4, etc.) compared to CD137- TIL. Tumor-reactive TILs selectively sub-cluster into phenotypic subsets revealing coordinate ICM expression with some tumor-reactive TIL subsets expressing more ICMs than others. Agonizing CD137 on TILs significantly increased their cytotoxic phenotype, with elevated IL-2, TNFa, and IFN $\gamma$, while blocking PD-1 on TILs significantly increased the expression of cytotoxic molecules in the CD137+ TIL subsets, but not CD137- TIL. Preliminary results also showed increased patient tumor lysis in response to CD137 agonism. Conclusions

Tumor-reactive TILs co-express multiple ICMs, sub-cluster into defined subsets revealing ICM patterning, and exhibit an enhanced cytotoxic profile in response to PD-1 blockade or CD137 agonizing. The biology underlying this sub-clustering phenomenon remains under investigation and other immunomodulatory antibodies are under assessment using this bio-assay system. Overall, our system provides the potential to predict immunomodulatory combinations that may enhance tumor-reactive T cells' cytotoxic activity and may serve as a guidepost for the rationale design of combination immune checkpoint inhibition trials.

\section{Reference}

1. Ye Q, et al. CD137 accurately identifies and enriches for naturally occurring tumor-reactive T cells in tumor. Clin.Cancer Res. 2014;20:44-55. 
P174

Removal of double stranded RNA using a novel method with RNaselll produces robust mRNA chimeric antigen receptor $\mathrm{T}$ cell therapy

Jessica Foster', Namrata Choudhari', Jessica Perazzelli', Angela Waanders', Julie Storm', Ted Hofmann', Payal Jain', Phillip Storm', Stephan Grupp', Katalin Kariko ${ }^{2}$, Adam Resnick', David Barrett ${ }^{1}$

'The Children's Hospital of Philadelphia, Philadelphia, PA, USA; ${ }^{2}$ BioNTech RNA Pharmaceuticals, Mainz, Germany

Correspondence: Jessica Foster (fosterjb@email.chop.edu) Journal for ImmunoTherapy of Cancer 2017, 5(Suppl 2):P174

\section{Background}

Chimeric Antigen Receptor (CAR) T cells made with mRNA offer a transient and safe alternative to viral CARs, mitigating the concern for persistent unwanted side effects from constitutively active $T$ cells. Previous studies have shown that mRNA CARs are transiently effective, but lack CAR persistence and have struggled to show success across tumor types. We hypothesized the efficacy of mRNA CARs could be improved by utilizing recent advancements in RNA technology including the use of modified uracil and a novel purification method with RNaselll to prevent double stranded RNA (dsRNA) that induces toxicity.

Methods

Using the established CD19 CAR model in B cell acute lymphoblastic leukemia, we created CD19-directed CAR T cells using unmodified, unpurified mRNA and compared them to CAR T cells created using modified uracil and/or purification to remove any aberrant dsRNA. A simple, novel procedure using RNaselll enzyme was used for purification: in vitro transcribed mRNA was incubated with RNaselll for thirty minutes then isolated via phenol-chloroform extraction. CAR T cells were evaluated for cytotoxicity, CAR expression, negative checkpoint regulator expression, and genomic profiling using Nanostring RNA sequencing.

Results

Both modified and purified mRNA CAR T cells showed a two-fold increase in expression of the CAR on their surface initially $(p<0.0001)$, as well as a two-fold improvement in cytotoxic killing of leukemia cells in vitro that persisted for up to five days $(p<0.0001)$. Both the modified and purified mRNA CAR T cells also showed reduced expression of negative checkpoint regulators PD1 and LAG3 compared to original RNA CAR T cells $(p<0.0001)$. However, in vivo studies using a patient-derived xenograft model with a single dose of CAR T cells revealed purified RNA CAR T cells offered the most robust 2-log enhanced suppression of leukemic burden $(p<0.02)$. In addition, genomic profiling revealed a differential pattern of activation among the mRNA CAR T cell constructs, indicating that using different mRNA products can alter the complex and often detrimental activation that occurs with mRNA electroporation.

Conclusions

RNaselll is a novel purification technique that has not yet been reported in the literature for RNA manufacturing. DsRNA is intensely immunogenic; removal allows for increased protein translation, and here provides improved CAR T cell cytotoxicity, in particular in vivo. Our results provide a time efficient purification method that can be easily incorporated into RNA production for use in clinical trials, and poise RNA CARs for increased efficacy as new CAR targets emerge and are being tested.

\section{P175}

A rapid and streamlined method for the culture of Tumor Infiltrating Lymphocytes (TIL) from melanoma tumor fragments Nermin Gerges, Shari Pilon-Thomas, Amod Sarnaik, John Mullinax, Cheryl Cox, Sandra Gonzalez-Rivera, Ludivina Lay, Linda Kelley Moffitt Cancer Center, Tampa, FL, USA

Correspondence: Nermin Gerges (ngerges123@icloud.com); Linda Kelley Journal for ImmunoTherapy of Cancer 2017, 5(Suppl 2):P175

\section{Background}

The Moffitt Cancer Center TIL program has manufactured 45 TIL products for the treatment of melanoma patients. Currently, the prerapid expansion (pre-REP) manufacturing process takes 24-38 days to generate a sufficient cell dose for subsequent clinical dose production, during which time the patient is at risk of disease progression and treatment ineligibility. Here we present a simplified, rapid method for the culture of TIL from melanoma tumor fragments using gas-permeable 24-well culture plates.

Methods

Melanoma tumor fragments $\left(1-3 \mathrm{~mm}^{3}\right)$ were cultured in polystyrene or gas-permeable (G-REX, Wilson-Wolf) 24-well culture plates. Each fragment was cultured in a separate well in complete media supplemented with $\mathrm{IL}-2(6000 \mathrm{IU} / \mathrm{ml})$ and agonistic anti-41BB antibody $(10 \mu \mathrm{g} / \mathrm{ml})$. TILs cultured in polystyrene plates were re-fed and split upon confluence into secondary 24-well polystyrene plates according to standard protocol and harvested on day (D) 24/25 of culture. The optimal media replacement schedule for TIL culture in G-REX wells was investigated and determined to be 3X/week. TILs cultured in G-REX plates were fed 3X/week, kept in their original wells throughout the culture period and harvested on D17/18 or D24/25. Cell count, viability, immunophenotype, and tumor reactivity were assessed.

Results

Sufficient TIL yield for rapid expansion was achieved using a single G-REX well per fragment $(4.4 \mathrm{e} 7 \pm 4.3 \mathrm{e} 7, \mathrm{D} 17 / 18)$ a full seven days prior to a comparable yield from multiple polystyrene wells $(5.1 \mathrm{e} 7 \pm 5.3 \mathrm{e} 7$, D24/25) $(p=0.32)$. Prolonged culture in the G-REX well did not significantly increase the number of TIL per fragment $(4.6 \mathrm{e} 7 \pm 4.4 \mathrm{e}$, D24/25) $(p=0.45)$. TIL grown in G-REX wells showed higher viability $(91 \pm 3 \%)$ on D17/18 compared to polystyrene on D24/25 $(79 \pm 5 \%)$ $(p<0.000001)$. The viability of TIL in G-REX wells decreased on D24/25 $(78 \pm 8 \%)$ suggesting peak expansion occurred at the earlier time point. TIL in G-REX wells showed higher percentages of CD8+ T cells $(88 \pm 10 \%, \mathrm{D} 17 / 18 ; 94 \pm 8 \%, \mathrm{D} 24 / 25)$ than TILs from polystyrene $(76 \pm 14 \%, D 24 / 25 \quad(p<0.001)$ and lower percentages of NK cells

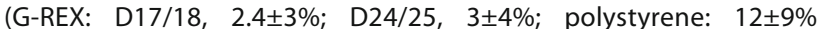
$(p<0.001)$. Tumor-specific activity was similar, as measured by IFN- $\gamma$ secretion between the two culture conditions.

Conclusions

G-REX culture plates allowed us to obtain sufficient pre-REP TIL (60e6 viable cells) for clinical dose manufacture in one week less than conventional polystyrene culture plates. Reducing the manufacturing period by one week potentially decreases patient ineligibility due to disease progression during TIL manufacturing. TIL culture in G-REX 24-well plates from tumor fragments is a simple and rapid method that may facilitate adoption of TIL therapy to other clinical sites.

P176

AdoptCell ${ }^{\oplus}$-NK: Ex vivo generation of highly purified and activated natural killer cells from human peripheral blood in accordance with GMP/GCTP for clinical studies

Yui Harada, Yoshikazu Yonemitsu

Kyushu University, Fukuoka, Japan

Correspondence: Yui Harada (rkfraile@med.kyushu-u.ac.jp)

Journal for ImmunoTherapy of Cancer 2017, 5(Suppl 2):P176

\section{Background}

Cancer immunotherapy has been established as a new therapeutic category since the recent success of immune checkpoint inhibitors and a type of adoptive immunotherapy, namely chimeric antigen receptor-modified T cells (CAR-T). Although CAR-T demonstrated impressive clinical results, serious adverse effects (cytokine storm and on-target off-tumor toxicity) and undefined efficacy on solid tumors are important issues to be solved.

As an alternative adoptive immunotherapy approach, we recently developed a new, simple method to generate highly active and 
expanded human NK cells from PBMCs. NK cells play a crucial role during the innate immune responses against various diseases, including infectious diseases and malignancies. Therefore, adoptive immunotherapy using NK cell is emerging as promising treatments for intractable malignancies; however, there has been still developing because of difficulties in culture, shortage of overall effector numbers, contamination of considerable numbers of T cells, and their limited anticancer potencies. We here established the simple feeder-free method to generate purified $(>90 \%)$ and highly activated NK cells from PBMCs in accordance with GMP/GCTP for clinical studies.

Methods

Under approval of the institutional ethical committee, PBMCs were collected from healthy volunteers by using CliniMACS Prodigy ${ }^{\oplus}$ (automatic/closed system). $\mathrm{CD}^{+}$and $\mathrm{CD} 4^{+}$cells were depleted by CliniMACS beads, and the cells were cultured at a concentration of 5 $\times 10^{5}$ cells $/ \mathrm{ml}$ with high concentration of hIL-2 and $5 \%$ human $A B$ serum for 14 days. Then, we confirmed the expression of surface markers, CD107a mobilization and cell-mediated cytotoxicity against various tumor cells and normal cells with or without monoclonal antibody drugs in vitro and antitumor effects against $\mathrm{K} 562$ in vivo.

Results

Among the several parameters, we found that simply 1 ) only CD3/ CD34-depletion, 2) high dose IL-2, and 3) use of specific culture medium were sufficient to obtain the highly purified, expanded ( 200-fold) and activated CD3 ${ }^{-} / \mathrm{CD}^{2} 6^{+}$NK cells from PBMCs. Almost all activated NK cells expressed lymphocyte-activated marker CD69, and showed dramatically high expression of NK activation receptors (i.e. NKG2D, NKp30, NKp46, etc.), interferon-g, perforin and granzyme B. Importantly, only 2 hours' reaction at effector/target ratio=1:1 was sufficient to kill almost all K562 and Raji cells, and antitumor activity was also representative on tumor bearing mice in vivo. Cytolysis was specific for various tumor cells, but not for normal cells, irrespective of MHC class I expression.

\section{Conclusions}

We now started GMP/GCTP production of this new NK cells and firstin-man clinical trials in use of "haploidentical AdoptCell ${ }^{\oplus}-\mathrm{NK}$ " will be initiated on 2Q 2018.

\section{P177}

Engineering artificial lymph nodes for immunotherapy

John Hickey', Hai-Quan Mao², Jonathan Schneck'

'Johns Hopkins School of Medicine, Baltimore, MD, USA; ${ }^{2}$ Johns Hopkins University, Baltimore, MD, USA

Correspondence: Jonathan Schneck (jhickey8@jhmi.edu) Journal for ImmunoTherapy of Cancer 2017, 5(Suppl 2):P177

\section{Background}

One of the challenges of adoptive immunotherapy is generating enough highly functional CD8+ T cells. Our lab has developed artificial antigen-presenting cells (aAPCs) to bypass immunosuppressed antigenpresenting cells to generate more functional CD8+ T cells. We hypothesized that an additional important variable to control T cell stimulation is the microenvironment-traditionally stimulation occurs just in a plastic culture dish. Inspired by the composition and structure of the lymph node, we engineer stimulatory microenvironments to improve numbers of highly functional antigen-specific T cells.

\section{Methods}

aAPCs were prepared by attaching dimeric antigen-loaded pMHC and anti-CD28 to nanoparticles. These were used to activate B6 CD8 $+T$ cells on either tissue culture plates (TCPs) or on prepared hyaluronic acid hydrogels (Hyd). To remove the need for aAPCs we also conjugated the stimulatory signals directly to the hyaluronic acid hydrogels, which we term an artificial lymph node (aLN). To further mimic the reticular fiber network of the lymph node, polymeric (polycaprolactone) electrospun nanofibers were added to aLNs to form a composite material. On day 7 cells were counted and stained with cognate and non-cognate dimer PMHC. Functionality was measured by staining cytokines produced after 7 days of culture through intracellular staining of INF-g, TNFa, IL-2, and CD107a+ T cells.
Results

aLNs generated more than triple the total antigen-specific CD8+ T cells than those stimulated by aAPCs on TCP (Fig. 1A). Even stimulating with the same aAPCs but just on a hydrogel surface demonstrated nearly double the antigen-specific $T$ cell number. These conditions were also more functional than traditional stimulation methods (Fig. 1B). Using composite aLNs with polymeric nanofibers induced greater numbers of high functional $T$ cells compared to hydrogel aLNs (Fig. 1C).

\section{Conclusions}

We engineered environments which produce high number of functional antigen-specific T cells. Interestingly, having an extracellular matrix hydrogel was a critical factor. We used this data to further design our material to mimic the lymph node by incorporating polymeric nanofibers for similar structure, stiffness, and porosity. Beyond demonstrating the importance of the microenvironment, these data have implications for use of these cells and materials for in vivo immunotherapies.

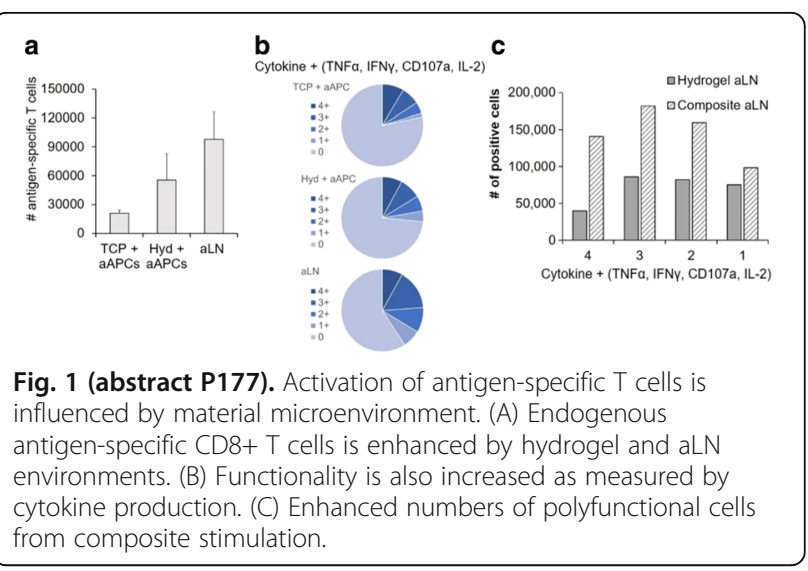

P178

Combined killing of cancer cells and cross presentation of tumor antigen by $\mathrm{V} \gamma 9 \mathrm{~V} \delta 2 \mathrm{~T}$ cells

Gitte Holmen Olofsson', Manja Idorn', Elfriede Nössner ${ }^{2}$, Ramona

Schenker ${ }^{2}$, Reno Debets ${ }^{3}$, Özcan Met ${ }^{1}$, Per thor Straten

${ }^{1}$ Center for Cancer immunotherapy (CCIT), Herlev, Denmark; ${ }^{2}$ Institute for Molecular Immunology, München, Germany; ${ }^{3}$ Erasmus MC Cancer

Institute, Rotterham, Netherlands

Correspondence: Gitte Holmen Olofsson

(gitte.holmen.olofsson@regionh.dk)

Journal for ImmunoTherapy of Cancer 2017, 5(Suppl 2):P178

\section{Background}

The human $V_{Y} 9 \mathrm{~V} \delta 2 \mathrm{~T}$ cells are a unique $\mathrm{T}$ cell type, and recent studies of the biology of $\mathrm{V} \gamma 9 \mathrm{~V} \delta 2 \mathrm{~T}$ cells emphasize the potential exploitation of these cells in immunotherapy of cancer. $V y 9 V \delta 2 T$ cells exhibit dual functionality in that they are both antigen presenting cells (APC) and cytotoxic towards cancer cells.

Methods

In vitro assays that were included in this study were; ELISPOT, chromium cytotox assay, xCELLigence, flow cytometry and zoledronic acid expansion protocol for $\mathrm{V} \gamma 9 \mathrm{~V} \delta 2 \mathrm{~T}$ cells.

Results

We show that Vy9V82 T cells can kill cancer cell lines from various cancer types such as leukemia, melanoma, prostate-, and breast cancer, with a significantly increased killing upon treatment of the cancer cells with Zoledronic acid. In addition, we show that $\mathrm{V} \gamma 9 \mathrm{~V} \delta 2 \mathrm{~T}$ cells take up tumor antigens gp100 and MART-1 (long peptide and recombinant protein, respectively), and process these antigens for presentation of class I restricted peptides in the context of the HLA-A02.01 
molecule, to be recognized by peptide specific cytotoxic CD8 T cells. Moreover, we show that specific inhibition of the proteasome by lactacystin impair recognition by peptide specific CD8 T cells, strongly suggesting proteasome involvement in presentation of the relevant class I restricted peptides.

\section{Conclusions}

The dual functions; killing and antigen presentation combined with the ease of expanding Vy9Vס2 T cells in vitro from peripheral blood lymphocytes to billions of cells, makes $\mathrm{V} \gamma 9 \mathrm{~V} \delta 2 \mathrm{~T}$ cells attractive vehicles for adoptive cell therapy (ACT) in cancer therapy. Thus, Vy9V $22 \mathrm{~T}$ cells are broadly tumor specific killers that concurrently could induce or support tumor specific $\alpha \beta-T$ cell responses.

\section{P179}

NKG2D ligand-inducing immune therapy plus NKG2D+CD8+ T cell therapy overcomes chemoresistance in tumors

Jiemiao Hu, Izhar Batth, Chantale Bernatchez, Xueqing Xia, Patrick Hwu, Richard Gorlick, Shulin Li

The University of Texas MD Anderson Cancer Center, Houston, TX, USA

Correspondence: Shulin Li (jhu1@mdanderson.org)

Journal for ImmunoTherapy of Cancer 2017, 5(Suppl 2):P179

\section{Background}

Though chemotherapy is still the first-line therapy for most cancers, tumors ultimately develop resistance to chemotherapy, allowing tumor relapse and metastasis, the primary cause of cancer patient death. Immune therapy has become a viable alternative for treating chemo-resistant tumors. Notably, CD20/19-CAR-T cell therapy has achieved great success in eliminating leukemia, and immune checkpoint blockers have extended overall survival in melanoma. For most solid tumors, however, neither approach has been effective; the poor efficacy is attributed to tumor heterogeneity, an inert tumor microenvironment, and the difficulty in penetration.

Methods

During the past years, our group has identified a simple combination of an immune stimulatory signal (interleukin-12) and chemotherapy (doxorubicin) that persistently induces NKG2D ligand on tumor cells in vivo across tumor types [1] (Fig. 1) and developed a novel CD28 stimulation based approach for fast induction of NKG2D receptor on CD8+ T cells [2] (Fig. 2).

Results

We show here that adoptive transfer of NKG2D+CD8+ T cells to NKG2D ligand-induced tumors overcame tumor resistance to chemotherapy in both murine and humanized tumor models (Fig. 3).

\section{Conclusions}

The mechanism underlying this tumor-specific and persistent NKG2D ligand-induction therapy has several components: (1) it boosts NKG2D+CD8+ T cell accumulation in tumors by inducing $T$ cell attracting chemokines; and (2) it transforms the tumor microenvironment to enable the accumulated T cells to effectively kill tumor cells by engaging with the induced NKG2D ligand. Furthermore, it is independent of the known transcription mechanism but is dependent on epigenetic modifiers Kat2a/2b. Notably, the stress-induced NKG2D transcription lasts only for 1-2 days, but this Kat2a/2b-dependent induction lasts for at least 8 days and is CD8+ T cell-dependent. Depletion of CD8+ T cells impairs this NKG2D ligand induction in tumors (Fig. 4), impeding the associated tumor eradication (Fig. 5). Details of the molecular and immune mechanism will be discussed.

\section{References}

1. Hu J, Bernatchez C, Zhang L, Xia X, Kleinerman ES, Hung MC, Hwu P, Li S Induction of NKG2D Ligands on Solid Tumors Requires Tumor-Specific CD8+ T Cells and Histone Acetyltransferases. Cancer Immunol Res. 2017.
2. Hu J, Batth IS, Xia X, Li S. Regulation of NKG2D+CD8+ T cell-mediated antitumor immune surveillance: Identification of a novel CD28 activationmediated, STAT3 phosphorylation-dependent mechanism. Oncoimmunology. 2016; 5 : e1252012.

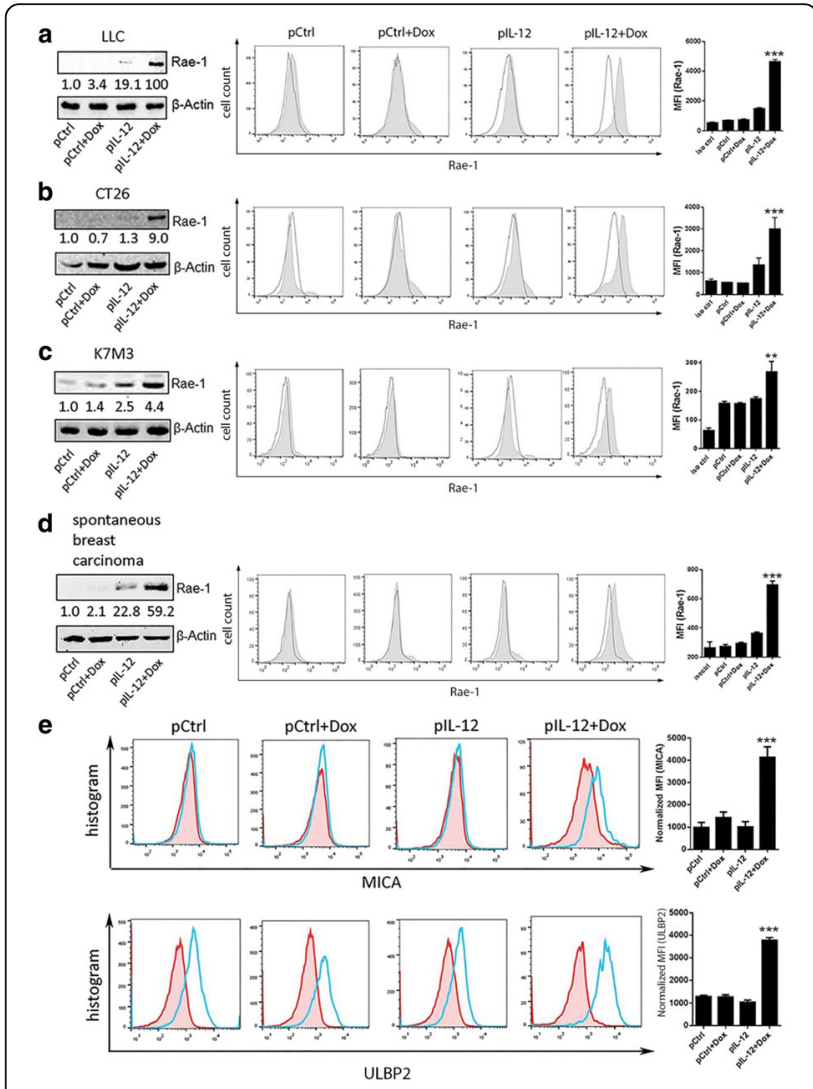

Fig. 1 (abstract P179). Rae-1 is induced by IL12 plus doxorubicin in four tumor models

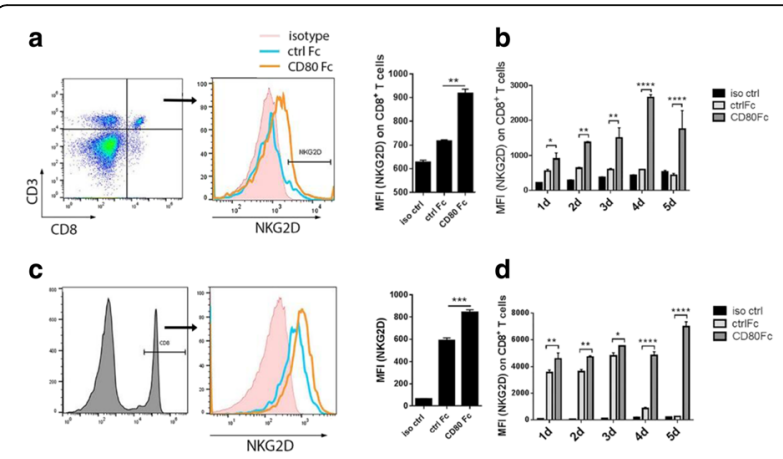

Fig. 2 (abstract P179). CD80 binding-mediated CD28 activation induces sustained expression of the NKG2D receptor on CD8+ T cells 

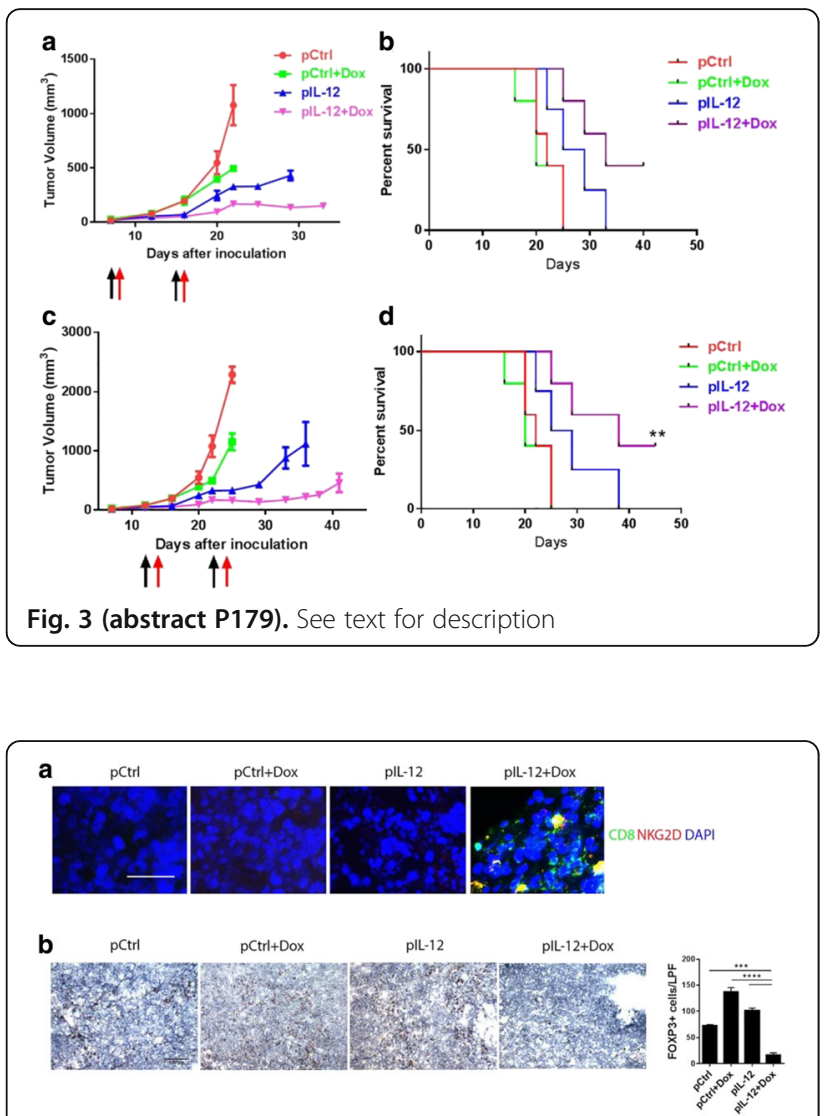

Fig. 4 (abstract P179). IL-12 plus doxorubicin enhanced the accumulation of NKG2D+CD8+ T cells, and reduced the infiltration of Treg cells in tumors

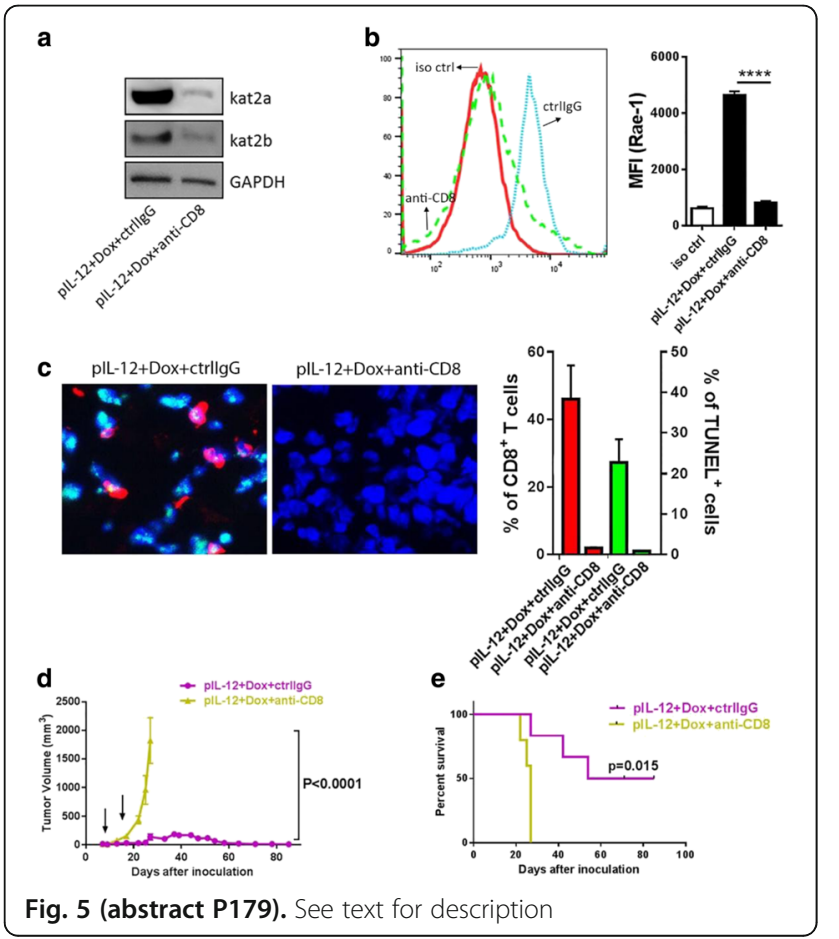

P180

Chemokine receptor engineering of T cells with CXCR2 improves homing towards subcutaneous human melanomas in xenograft mouse model

Manja Idorn', Signe Koggersbøl Skadborg', Lauge Kellerman²,

Hólmfrídur Rósa Halldórsdóttir?', Gitte Holmen Olofsson', Özcan Met ${ }^{1}$

Per thor Straten

${ }^{1}$ Center for Cancer Immune Therapy, Herlev Gentofte Hospital, Herlev, Denmark; ${ }^{2}$ Herlev Hospital, Herlev, Denmark

Correspondence: Manja Idorn (denaxier@hotmail.com)

Journal for ImmunoTherapy of Cancer 2017, 5(Suppl 2):P180

\section{Background}

Adoptive cell therapy (ACT) using in vitro expanded tumor infiltrating T lymphocytes (TILs) from biopsy material represents a highly promising treatment of disseminated cancer. A crucial prerequisite for successful ACT is sufficient recruitment of transferred lymphocytes to the tumor site; however, despite transfer of billions of lymphocytes, $T$ cell infiltration into the tumor post ACT is limited.

Methods

To identify potential chemokine/chemokine receptor axes in melanoma, chemokine expression and secretion of 12 selected chemokines were analysed by PCR and multiplex chemokine assays performed on CDNA and supernatants of 20 human melanoma cell lines. Expression of cognate chemokine receptors, CCR2, CCR4, CCR5, CXCR2, CXCR3, CXCR4 and CXCR6, was assessed by flow cytometry of tumor infiltrating lymphocytes from 10 patients with metastatic melanoma. For expression of selected chemokine receptors, human primary $T$ cells (from PBMC of healthy donors) were genetically engineered using lentiviral transduction. In vitro signalling and chemotaxis via the receptors were assessed by $\mathrm{Ca}^{2+}$ influx assay, and transwell migration assay towards both ligand and melanoma cell line supernatant. In vivo homing of $T$ cells genetically engineered with chemokine receptor, CXCR2, was evaluated in a xenograft NOG mouse model. Mice with subcutaneous human melanomas were treated by ACT of MAGE-A3 specific T cells transduced with either CXCR2 or MOCK.Tumor infiltration of T cells was quantified by flow cytometry and immunohistochemistry.

Results

We found that a majority of malignant melanoma (MM) cell lines expressed chemokines CXCL8/IL-8, CXCL12/SDF-1 and CCL2. Successful engineering of TILs and peripheral blood T cells significantly increased receptor expression of the corresponding chemokine receptors CXCR2, CXCR4 and CCR2. All three chemokine receptors are functional in vitro and show ligand specific transwell migration of engineered T cells as well as increased migration towards MM conditioned medium. In vivo homing was assessed in a xenograft NOG mouse model. Mice with subcutaneous human melamoma were treated with ACT of MAGE-A3 specific T cells transduced with either CXCR2 or GFP. Transducing T cells with CXCR2 increased tumor infiltration. In comparison mock transfected T cells appeared to be allocated to other organ-compartments.

Conclusions

CXCR2, CXCR4 and CCR2 engineered T cells are functional in vitro, and transduction with CXCR2 improve in vivo homing of T cells to tumor site, setting the stage for mixing and matching chemokinereceptor expression to tumor microenvironments. Longitudinal studies assessing cell trafficking and tumor control using in vivo imaging are currently ongoing, and results will be presented at the meeting.

P181

Production of site-specific allogeneic CD19 CAR-T cells by CRISPR-Cas9 for B-cell malignancies

Zinkal Padalia, Ashley Porras, Dakai Mu, Min Jin Lee, Ying Zhang,

Elaine Huang, Ashley Maillet, Jeffrey Li, Matthias John, Lawrence Klein, Sven Ante Lundberg, Seshi Police, Jonathan Terrett, Demetrios Kalaitzidis CRISPR Therapeutics, Cambridge, MA, USA

Correspondence: Demetrios Kalaitzidis (d.kalaitzidis@crisprtx.com) Journal for ImmunoTherapy of Cancer 2017, 5(Suppl 2):P181 


\section{Background}

We have applied CRISPR/Cas9 technologies to develop anti CD19 Allogeneic chimeric antigen receptor T cells (CAR-T) with reduced GVHD potential and reduced rejection potential for the treatment of CD19 positive malignancies. The efficiency of the CRISPR/Cas9 system enables rapid production of homogeneous CAR-T product from prescreened healthy donors and thus can potentially be developed as an "off-the-shelf" therapy for efficient delivery to patients. Autologous CAR-T therapeutics targeting CD19 have shown impressive responses in B-cell malignancies but currently require significant individualized manufacturing efforts and can suffer from manufacturing failures. In addition, these autologous CAR-Ts are produced using retrovirus or lentivirus, for which the variable nature of integration can lead to a heterogeneous product. Allogeneic or "off-the-shelf" CAR-T products with site-specific CAR integration generated with gene editing technologies may address some of these significant challenges seen for Autologous products.

Methods

We have utilized the CRISPR-Cas9 technology in primary human T cells to produce allogeneic CAR-T cells by multiplexed genome editing. We have developed a robust system for site-specific integration of CAR and concurrent multiplexed gene editing in single T cells by utilizing homology-directed repair (HDR) with Cas9 ribonucleoprotein (RNP) and an AAV6-delivered donor template.

Results

With CRISPR/Cas9 editing technology we have achieved high frequency knockout of the constant region of the TCRa gene (TRAC) with $\sim 98 \%$ reduction of TCR surface expression in human primary $T$ cells from healthy donors, which aims to significantly impair graftversus-host disease (GVHD). High frequency knockout of the $\beta$-2microglobulin (B2M) gene could also be obtained, which aims to increase persistence in patients, potentially leading to increased potency overall. TRAC/B2M double knockout frequencies have been obtained in $\sim 80 \%$ of T cells without any subsequent antibody-based purification or enrichment. Human T cells expressing a CD19-specific CAR from within a disrupted TRAC locus, produced by homologydirected repair using an AAV6-delivered donor template, along with knockout of the B2M gene have been consistently produced at a high efficiency. This site-specific integration of the CAR protects against the potential outgrowth of $\mathrm{CD}^{+} \mathrm{CAR}^{+}$cells, further reducing the risk of GVHD, while also reducing the risk of insertional mutagenesis associated with retroviral or lentiviral delivery mechanisms. These engineered Allogeneic CAR-T cells show CD19-dependent T cell cytokine secretion and potent CD19-specific cancer cell lysis.

Conclusions

We are able to use genome editing with the CRISPR-Cas9 system to efficiently create an Allogeneic or "off-the-shelf" CAR-T cell product that demonstrates potent and specific anticancer effects for patients with CD19-expressing human cancers.

\section{P182}

Development of chemically defined medium for ex vivo expansion of natural killer cells

Jessie Kinjo, Vanda S. Lopes, Jessie H.-T. Ni

Irvine Scientific, Santa Ana, CA, USA

Correspondence: Jessie Kinjo (jkinjo@irvinesci.com)

Journal for ImmunoTherapy of Cancer 2017, 5(Suppl 2):P182

\section{Background}

NK cells belong to the innate immune system and are a type of cytotoxic lymphocyte. They comprise between $5-20 \%$ of the human peripheral blood lymphocytes and are characterized by expression of CD56 and absence of $\mathrm{CD} 3\left(\mathrm{CD}^{\circ} 6^{+} \mathrm{CD} 3^{-}\right)$. NK cells recognize and attack abnormal cells, such as cancer or viral infected cells without preactivation. This ability to target and destroy cancer cells makes NK cells valuable for immunotherapy applications. However, this requires large doses of NK cells to be infused into the patient. Furthermore, consistent ex vivo expansion of NK cells retaining their functional properties remains a challenge despite extensive efforts in the field. In this study, we present a chemically-defined medium capable of supporting the ex vivo expansion of NK cells that retain their cytotoxic functionality.

Methods

Peripheral Blood Mononuclear Cells (PBMCs) or NK92 cells (immortalized NK cell line) were stimulated with $100 \mathrm{U} / \mathrm{L}$ IL-2 in the presence or absence of mitomycin C treated K562 feeder cells. Cells were expanded for 14 days, with media changes at days 3, 7, and 10. During the course of media development, we applied our Rational Media Design $^{T M}$ approach to eliminate serum and identify non-animal derived components that could improve NK cell expansion and performance. The performance of media was evaluated by the total nucleated cell count, $\mathrm{CD}_{56}{ }^{+} \mathrm{CD} 3^{-}$percentage, and cytotoxicity against K562 cells.

Results

By using a Rational Media Design ${ }^{\mathrm{TM}}$ approach we were able to formulate a chemically-defined medium that was able to support expansion of NK cells derived from PBMCs and NK92 cells, in the presence or absence of feeder cells. When NK92 cells and PBMCs from multiple donors were compared, our chemically-defined medium delivered a similar or higher specific fold increase of $\mathrm{CD}_{56} 6^{+} \mathrm{CD}^{-}$cells and comparable cytotoxicity profiles over two other commercially available serum-free media.

\section{Conclusions}

The medium we developed is a product that combines translational quality, boasts high performance, and production under cGMP conditions. These features make it ideal for use in clinical studies for applications of both PBMC derived NK or NK92 cells.

\section{P183}

Stem cell differentiation towards tumor-recognizing CD4 T cells provides robust anti-tumor effects in combination with CD8-TCR-T cell therapy

Richard Koya, Junko Matsuzaki, Takemasa Tsuji, Satoko Matsueda, Thinle Chodon, Kunle Odunsi

Roswell Park Cancer Institute, Buffalo, NY, USA

Correspondence: Thinle Chodon (richard.koya@roswellpark.org); Kunle

Odunsi

Journal for ImmunoTherapy of Cancer 2017, 5(Suppl 2):P183

\section{Background}

T cell receptor (TCR) engineering of patient's mature T cells has shown to be a promising approach, but unfortunately, the antitumor effect is largely short-lived. We have recently discovered a novel and distinct subset of human CD4+ Th1 cells and cloned their TCRs which directly recognize NY-ESO-1 tumor antigen naturally presented by MHC II on cancer cells. Our central hypothesis is that CD4TCR-engineered human hematopoietic stem/progenitor cells (hHSC) will lead to durable in vivo supply of fully active Tumor Recognizing (TR)CD4 cells with anti-tumor activity, and provide sustained help to co-injected CD8TCR-transduced effector T cells (which will serve to immediately debulk the tumor), leading to long-lasting tumor rejection.

\section{Methods}

We generated lentiviral vectors for cell transduction with two unique NY-ESO-1 (TR)CD4TCRs (MHC-II restricted HLA-DR1 and DP4), and a novel CD8 (MHC-I HLA-A2.1)TCR for NY-ESO-1. We created new transgenic mouse models based on a highly immunodeficient NSG background with expression of human MHC-II-DP4 and DR1 in order to study hHSC differentiation/function in vivo and to test anti-human cancer activity with our adoptive cell transfer (ACT) platform.

Results

Transduction efficiency of hHSC was high with transgene expression levels of $40-51 \%$ (0.5-1 vector copies/cell); and $65-92 \%$ tetramer+ for mature T cells. We confirmed specific functional activity of all TCRs by co-incubating transduced $\mathrm{T}$ cells with various tumor targets (SKMEL-37, MZ19, or aAPC:K562/DR1/DP4/A2.1 +/- cognate peptides) by ELISA and intracellular staining for IFNy. We injected mice with hHSC transduced with (TR)CD4-TCR and after 2-3 months we confirmed generation of engineered TCR-expressing human T cells by FACS through blood sampling. We then tested anti-tumor efficacy 
in vivo with s.q. injection of human melanoma (MZ19) or human ovarian carcinoma (A2780/A2/NY) cells in mice injected with (TR)CD4-TCR-transduced or non-transduced-control hHSC. We injected a low dose $(5 \times 10 \wedge 5$ cells) of NY-ESO-1 HLA-A2.1 CD8TCRtransduced hPBMC and followed tumor growth. Remarkable tumor control was obtained $(p<0.05)$ in the mouse group that received (TR)CD4-TCR-transduced $\mathrm{hHSC}$ (tumor size $=28 \mathrm{~mm}^{\wedge} 2$; $\mathrm{SE}+/-11$ ) versus mice that received untransduced $\mathrm{hHSC}\left(152.7 \mathrm{~mm}^{\wedge} 2 ; \mathrm{SE}+/-24\right)$ or control untreated (no hHSC, no CD8 T cell ACT) $\left(196 \mathrm{~mm}^{\wedge} 2\right.$; SE+/-40) assessed at day 32 after tumor (A2780/A2/NY) injection.

\section{Conclusions}

Here we demonstrate for the first time that a combined ACT approach of (TR)CD4-TCR-transduced hHSC with CD8-TCR-transduced PBMC leads to a synergistic and efficient in vivo control of tumor burden. These results provide basis to pursue a Phase I/lla clinical trial based on our novel ACT platform to benefit patients with advanced solid tumors.

\section{P184}

Genetic engineering of human NK cells to express CXCR2 improves migration to renal cell carcinoma

Veronika Kremer', Maarten Ligtenberg ${ }^{2}$, Rosa Zendehdel',

Christina Seitz', Annet Duivenvoorden', Erik Wennerberg ${ }^{3}$, Eugenia Colón ${ }^{4}$,

Ann-Helén Scherman Plogell ${ }^{4}$, Andreas Lundqvist

${ }^{1}$ Karolinska Institutet, Stockholm, Sweden; ${ }^{2}$ The Netherlands Cancer Institute, Amsterdam, Netherlands; ${ }^{3}$ Weill Cornell Medicine, New York,

NY, USA; ${ }^{4}$ Stockholm South General Hospital, Stockholm, Sweden

Correspondence: Veronika Kremer (veronika.kremer@ki.se)

Journal for ImmunoTherapy of Cancer 2017, 5(Suppl 2):P184

\section{Background}

Adoptive natural killer (NK) cell transfer is being increasingly recognized as a therapeutic approach to treat cancer patients. However, clinical responses have so far been limited to patients with hematological malignancies. A potential rate-limiting factor in patients with solid tumors is defective homing of the infused NK cells to the tumor site. Chemokines regulate the migration of leukocytes via corresponding chemokine receptors. Various solid tumors, including renal cell carcinoma (RCC), secrete ligands for the chemokine receptor CXCR2. We hypothesize that infusion of NK cells expressing high levels of CXCR2 will result in increased influx of the transferred NK cells into tumors, and improved clinical outcome in patients with cancer.

\section{Methods}

Blood and tumor biopsies from primary RCC patients $(n=14)$ were assessed by flow cytometry and chemokine analysis. Primary NK cells were retrovirally transduced with human CXCR2; transgene expression ranged from 23 to $93 \%$. CXCR2 receptor functionality was determined by Calcium flux and NK cell migration was evaluated in transwell assays.

Results

We detected 10- to 186-fold higher concentrations of CXCR2 ligands in tumors compared with plasma of RCC patients. In addition, CXCL5 levels correlated with the intratumoral infiltration of CXCR2-positive NK cells ( $n=9, p=0.039$ ). However, frequencies of CXCR2-positive NK cells were lower in the tumors compared with peripheral blood $(p=0.0003)$. Moreover, healthy donor NK cells rapidly lost their CXCR2 expression upon in vitro culture and expansion. Genetic modification of human primary NK cells to re-express CXCR2 improved their ability to specifically migrate along a chemokine gradient of recombinant CXCR2 ligands $3.4-$ fold ( $p=0.0083$ ) or RCC tumor supernatants 2- to 2.5 -fold $(p<0.05)$ compared with control cells. The enhanced trafficking resulted in increased killing of target cells $(p=0.0039)$. In addition, while their functionality, including cytotoxicity, degranulation and cytokine production, remained unchanged compared with control cells, CXCR2-transduced NK cells obtained increased adhesion properties and formed $53 \pm 21 \%$ more conjugates with target cells $(\mathrm{p}=0.0128)$.

\section{Conclusions}

To increase the success of NK cell-based therapies of solid tumors, it is of great importance to promote their homing to the tumor site. In this study, we show that stable engineering of human primary NK cells to express a chemokine receptor thereby enhancing their migration is a promising strategy to improve the efficacy of adoptive cellular immunotherapies.

\section{P185}

A novel chimeric $T$ cell receptor that operates through the endogenous TCR diverges in biology from conventional CAR T cells

Vivian Lau, Ksenia Bezverbnaya, Alina Lelic, Anna Dvorkin-Gheva, Joanne Hammill, Arya Afsahi, Galina Denisova, Christopher Helsen, Jonathan Bramson

McMaster University, Hamilton, ON, Canada

Correspondence: Jonathan Bramson (lauwc@mcmaster.ca) Journal for ImmunoTherapy of Cancer 2017, 5(Suppl 2):P185

\section{Background}

T lymphocytes engineered with chimeric antigen receptors (CARs) have generated significant enthusiasm based on remarkable clinical successes in the treatment of B cell leukemias. Conventional CARs seek to recapitulate TCR and costimulatory signals through integration of signaling elements into a single receptor. The robust antitumor activity of CARs is associated with over exuberant T cell activation and cytokine production, often resulting in debilitating toxicities. Our lab has generated a novel chimeric receptor termed T cell antigen coupler (TAC), which is designed to co-opt the native $\mathrm{T}$ cell receptor for cellular activation and retain the MHC-independence of CARs. Comparatively, TAC T cells display enhanced anti-tumor activity and diminished cytokine production in vivo relative to CAR T cells.

Methods

Activated human PBMCs were transduced with lentivirus encoding the chimeric receptors. For RNAseq, T cells were flow sorted based on expression of the chimeric receptor and either CD4 or CD8. Total RNA from three biological replicates was sequenced via Illumina HiSeq platform. Flow cytometry was used to analyze expression of chimeric receptors, memory markers, checkpoint receptors, cytokine production, and T cell proliferation. For proliferation assays, T cells were labeled with CFSE and stimulated with either antigen-coated beads or antigen-expressing cell lines.

Results

Unsupervised hierarchical clustering from RNAseq determined that, regardless of donor, non-stimulated TAC and control T cells had very similar transcriptomes, whereas CAR T cells had a distinct transcriptional profile. Transcripts associated with effector T cells were differentially expressed in CAR T cells relative to TAC and control T cells, suggestive of elevated basal signaling in the CAR T cells. CARs produced cytokines and displayed elevated expression of checkpoint receptors in the absence of antigen exposure, indicative of autoactivation. Consequently, TAC T cells retained a less differentiated phenotype than CAR T cells when analyzed for memory-associated markers such as CD45RA and CCR7. To better understand antigenic requirements for activation, $T$ cells were stimulated with antigen on beads or antigen on cells. While CAR T cells reacted to antigen regardless of the context, TAC T cells responded weakly to protein on beads, demonstrating that TAC T cells have enhanced ability to distinguish antigen context.

Conclusions

These studies confirm that TAC receptors and CARs operate through distinct biology. Understanding the differences between the two chimeric receptors will aid in the selection of diseases that is most suitable for treatment with the individual receptors. 


\section{P186}

Molecular nanomachines for in situ reprogramming of innate immunity

Sandro Matosevic (sandro@purdue.edu)

Purdue University, West Lafayette, IN, USA

Journal for ImmunoTherapy of Cancer 2017, 5(Suppl 2):P186

\section{Background}

Current approaches to adoptive immunotherapy with reprogrammed natural killer (NK) cells present significant obstacles $\bigotimes$ they are cumbersome and require manipulation, expansion and cryopreservation. Personalized immunotherapies with NK cells have also proven difficult because in vivo, they are inhibited by the tumor microenvironment's chronic immune suppressive signals, and are resistant to the uptake of exogenous genetic material. [1] We discuss the development of precision molecular tools for the generation of biodegradable, genome-modifying nanomachines that reprogram, in situ, the function of NK cells, without the need for ex vivo expansion, and can be tracked with precision.

Methods

We synthesized novel nanostructures based on cationic oligomers and quaternary-ternary lipids with multivalent headgroups and disulfide linkers, and polymers based on poly( $\beta$-amino esters). Incorporation of NKG2D-DAP10-CD3 C CARs imparts tumor-targeting ability. Persistent CAR expression is achieved by flanking the gene expression cassette with piggyBac inverted terminal repeats. Continued stimulation is achieved by co-delivering IL-15 and IL-18 genes. The nanomachines also carry transcription activator-like effector nucleases (TALENs) to genomically impart cells with resistance to TGF- $\beta$ inhibition via a dominant-negative TGF- $\beta$ type II receptor, DNT $\beta$ RII.

Results

The structure-function relationships between nanoparticle composition and intracellular trafficking pathways have uncovered structural features driving intracellular motion. On the lipid construct side, we investigated whether lipid headgroups with cationic amine combinations favor Brownian diffusion-driven nucleic acid trafficking, while ester-linked quaternary amines favor cytoskeletal delivery. Improved genetic trafficking was obtained with multivalent cationic lipid-based carriers, while the membrane surface charge was critical in directing motion. Incorporation of a hydroxyalkyl chain capable of hydrogen bonding to neighboring headgroups increases hydration. Serumresistant nanoparticles with positively-charged poly( $\beta$-amino ester) and derivatives are also being studied as biodegradable vehicles, and to generate dual-construct nanostructures. "Coats" of serum-resistant amphiphiles are added to avoid off-target degradation. Ex vivo and in vivo studies are uncovering the functional extent of TALENscarrying nanomachines.

\section{Conclusions}

Taking cues from the efficiency with which viruses infect healthy cells, we are developing nanomachines that self-assemble and disassemble when needed and have the capacity to selectively reprogram NK cells. In doing so, we are challenge the traditional notions of nanoparticle-mediated NK cell engineering and molecular imaging to change the clinical paradigm for personalized in situ immunotherapies. [2]

\section{References}

1. Vitale M, Cantoni C, Pietra G, Mingari MC, Moretta L. Effect of tumor cells and tumor microenvironment on NK-cell function. Eur J Immunol. 2014;44(6):1582-92

2. Shang L, Nienhaus K, Nienhaus GU. Engineered nanoparticles interacting with cells: size matters. J Nanobiotechnology. 2014;12:5

\section{P187}

Efficient non-small lung cancer targeted therapy with TCR-T cell transfer engineered for TGF $\beta$ blockade

Satoko Matsueda, Adekunle Odunsi, Richard Koya

Roswell Park Cancer Institute, Buffalo, NY, USA

Correspondence: Richard Koya (satokomatsueda@gmail.com)

Journal for ImmunoTherapy of Cancer 2017, 5(Suppl 2):P187

\section{Background}

Adoptive T cell therapy (ACT) has showed promising clinical responses for patients with advanced cancer, though more work needs to be accomplished to enhance the effector functions of T cells, as well as counter the immunosuppressive tumor microenvironment (TME). The cancer testis antigen NY-ESO1 is expressed in many cancer types, including Non-Small Lung Cancer (NSLC). NY-ESO1 targeted T Cell Receptors (TCR) have been successfully used to engineer T cells for ACT. We hypothesize that incorporating a dominant-negative TGF $\beta$ receptor II (dnTGFßRII) into the NY-ESO-1 TCR construct to transduce T cells will render these cells insensitive to the immunosuppressive effects of TGF $\beta$, and augment their effector function in the TME.

Methods

We constructed retroviral vectors with an MHC I HLA-A2.1 NY-ESO1 TCR with and without a second transgene, dnTGF $\beta R$ II interspaced with a P2A self-cleaving element. NSG mice were inoculated with HLA-A2.1+, NY-ESO+ lung cancer cells subcutaneously. PBMCs from healthy volunteers were activated with OKT3 antibody plus IL-2 for two days. The activated PBMCs were then transduced with NY-ESO1 TCR with/without dnTGF $\beta$ RII. The transduced cells were analyzed by flow cytometry to confirm NY-ESO1 TCR expression before injection. Equal numbers of TCR+ cells were injected 17 days after tumor inoculation. Mice were then followed for tumor growth. The spleens and tumors were collected for further analysis, including Immunophenotype and functional assays at the endpoint.

Results

We have consistently obtained more than $70 \%$ retroviral vector transduction efficiency as assessed by surface expression via clonotypic V $\beta$ staining. The transduced NY-ESO1 TCR with dnTGF $\beta R$ Il cells were able to resist the progression of inoculated tumor for 49 days $(p<0.01)$. We found that adoptively transferred NY-ESO1 TCR with dnTGF $\beta R$ II cells were effectively infiltrated into tumor microenvironment in higher numbers than in control and eliminated lung cancer cells. Tumor harvest analysis demonstrated that regulatory T cells were significantly lower in NY-ESO1 TCR with dnTGF $\beta$ Rll cells transferred group and characteristic memory phenotypes were observed in these mice. We also obtained data that indicate that epigenetic modulation of NSLC lines can efficiently upregulate NYESO1 and therefore augment the effect our ACT platform.

\section{Conclusions}

Our results strongly support that T cells engineered with NY-ESO1 TCR with dnTGF $\beta R$ II more efficiently target NSLC tumors in vivo, and this provides a rationale to implement our platform towards clinical translation for this difficult to treat cancer.

\section{P188}

A universal killer $\mathrm{T}$ cell for adoptive cell therapy of cancer Nadia Mensali ${ }^{1}$, Pierre Dillard ${ }^{1}$, June H Myklebust ${ }^{1}$, Gjertrud Skorstad ${ }^{1}$ Marit Renée Myhre', Anne Fåne ${ }^{1}$, Else Marit Inderberg', Sébastien Wälchli', Gustav Gaudernack², Gunnar Kvalheim', Michael Hebeisen ${ }^{3}$ ${ }^{1}$ Oslo University Hospital OUS-Radiumhospital, Oslo, Norway; ${ }^{2}$ Oslo University Hospital, Oslo, Norway; ${ }^{3}$ Lausanne University Hospital, Lausanne, Switzerland

Correspondence: Nadia Mensali (nadia.mensali@rr-research.no) Journal for ImmunoTherapy of Cancer 2017, 5(Suppl 2):P188

\section{Background}

T cell-mediated immunotherapy of cancer has achieved remarkable results in hard-to-beat cancers. The main challenge of adoptive $T$ cell transfer (ACT) is its labour intensive and costly production and logistics as well as its dependency on the quality of the patient's T cells. Methods

To overcome these hurdles we have designed a universal cell line for TCR expression by modifying the FDA-approved NK cell line, NK-92. Advantages of using this cell line is that it is easy to expand and can readily be genetically engineered. However, tumor cell recognition and killing by NK-92 is not antigen specific. This can be controlled by introducing an antigen receptor, such as a chimeric antigen receptor (CAR) or, as in the current work, a TCR. We herein present evidence that NK-92 can be modified to become a T cell-like lymphocyte which we named UK-92 cell (Universal Killer). 
Results

UK-92 expressing a therapeutic TCR showed conserved binding capacity to the cognate PMHC. Phosphoflow cytometry results indicated that the introduced TCR was able to mediate intracellular signaling upon either crosslinking or cognate PMHC binding. Our data showed that both early and late TCR signalling players were activated in a TCR-specific manner (anti-CD3/anti-CD28 stimulation) and further in a pMHC specific manner. In vitro functional assays using TCRs isolated from both CD8 and CD4 T cells demonstrated that UK-92-TCR could be stimulated in a pMHC-specific manner and, importantly, could kill tumor cells specifically. We have now shown in vitro that UK-92 cells are as specific and potent as redirected T cells to kill target cells. Finally, encouraging in vivo data showed that mice receiving UK92 cells expressing a therapeutic TCR experienced reduction in tumor load and enhanced survival compared with control mice.

Conclusions

If confirmed, the use of UK92 as a universal cell line might pave the way to truly off-the-shelf therapeutic effector cells for cancer immunotherapy and leading to drastic reduction of cell production time, logistic and cost.

\section{P189}

Cytotoxic dendritic cells have efficient anti-tumor capacity of in a murine T cell lymphoma model

Jürgen Müller, Thomas Waldmann, Sigrid Dubois

$\mathrm{NIH}$, Bethesda, MD, USA

Correspondence: Jürgen Müller (muellerj@mail.nih.gov)

Journal for ImmunoTherapy of Cancer 2017, 5(Suppl 2):P189

\section{Background}

Murine bone marrow-derived dendritic cells are known to possess cytolytic activity that can be directed against various tumor targets. We tested whether this activity can be used to treat tumors in several murine models.

\section{Methods}

We used bone marrow-derived dendritic cells as effectors and the solid tumors B16, MC38, CT26 as well as the novel T cell lymphoma line SJ3 as target cells in vitro. For in vivo studies, mice carrying B16, MC38 or SJ3 were treated with combinations of dendritic cells that had been amplified from bone marrow in culture, GM-CSF and the TLR ligand R848.

Results

Co-cultures with tumor cells showed a strong cytolytic capacity of dendritic cells directed against B16, MC38, CT26 or SJ3 when activated with interferon-gamma and/or TLR ligands. MHC mismatches between DCs and tumor cells did not appear to change the outcome.

We attempted to translate these findings into in vivo settings of murine tumor models. Survivals of mice that carried intravenously injected B16 or MC38 were increased by treatments with GM-CSF and the TLR7/8 ligand R848 but an additional therapy with bone marrow-derived dendritic cells did not change the outcome. In contrast, the survivals of mice that carried the SJ3 T lymphoma line were significantly prolonged in response to DC cell injections.

\section{Conclusions}

Our study adds to our understanding of dendritic cell physiology. It points to the possibility to target their cytotoxic capability in the treatment of T cell lymphomas regardless of MHC compatibility.

\section{P190}

Patient specific automated dendritic cell generation in a closed system

Andrew Kozbial', Lekhana Bhandary ${ }^{1}$, Christopher Eickhoff ${ }^{2}$,

Bradley Collier', Daniel Hoft', Shashi Murthy ${ }^{1}$

${ }^{1}$ Northeastern University, Boston, MA, USA; ${ }^{2}$ Saint Louis University, St

Louis, MO, USA

Correspondence: Shashi Murthy (s.murthy@northeastern.edu) Journal for ImmunoTherapy of Cancer 2017, 5(Suppl 2):P190

\section{Background}

Dendritic cells (DCs) are an attractive vehicle for therapeutic manipulation and are used in antigen-pulsed autologous DC therapies and
DC-stimulated autologous T cell therapies [1, 2]. Given the low abundance of DCs in blood, they are typically generated ex-vivo from monocytes or stem cell precursors using a time-consuming, laborintensive method that is subject to user-variability. We have developed an automated closed system, MicroDEN (Fig. 1), for generating DCs that overcomes these challenges. Similar to the standard plate culture used for dendritic cell generation, MicroDEN uses plastic adherence to enrich monocytes from a mixed PBMC population and, in addition, uses medium perfusion to supply fresh cytokines throughout the duration of monocyte differentiation to DCs.

Methods

Peripheral blood mononuclear cells were seeded into the culture chamber of MicroDEN at a density of $690,00 \mathrm{cells} / \mathrm{cm}^{2}$. Monocytes were enriched by plastic adherence and cultured for 7 days in the presence of $500 \mathrm{U} / \mathrm{mL} \mathrm{IL}-4$ and $800 \mathrm{U} / \mathrm{mL}$ GM-CSF in RPMI medium containing $10 \%$ FBS, which was perfused continuously throughout differentiation. Simultaneous cultures were set up in 6-well plates as a control. Cells were harvested at day 7 .

Results

Flow cytometry data indicated that the iDCs from MicroDEN and 6well plates were phenotypically comparable with regard to expression of DC-SIGN (CD209), CD80, CD83, CD86 and CD14 (Fig. 2). Allogeneic $T$ cell proliferation assays and an antigen-specific assay demonstrated that iDCs generated via MicroDEN are functionally competent and can successfully generate a T cell response (Fig. 3). A higher number of viable iDCs were harvested from MicroDEN ( 2 million iDCs) compared to 6-well plates ( $\sim 0.3$ million iDCs). Moreover, the iDC yield relative to input PBMCs was higher for MicroDEN at $8.4 \%$ compared to $4.8 \%$ for 6 -well plates, indicating that MicroDEN can generate iDCs at a higher efficiency than well plates.

Conclusions

DCs generated by MicroDEN are functionally equivalent to DCs generated by the standard well plate culture. The larger surface area of MicroDEN cartridge allows generation of a higher number of dendritic cells in single run when compared to the standard 6-well plate, thereby reducing time and labor involved in harvesting cells from multiple plates along with variability.

\section{References}

1. Bracci L, Capone I, Moschella F, Proietti E and Belardelli F. Exploiting dendritic cells in the development of cancer vaccines. Expert Rev Vaccines. 2013; 12(10):1195-1210

2. Steinman RM and Banchereau J. Taking dendritic cells into medicine. Nature. 2007: 449(7161):419-426.

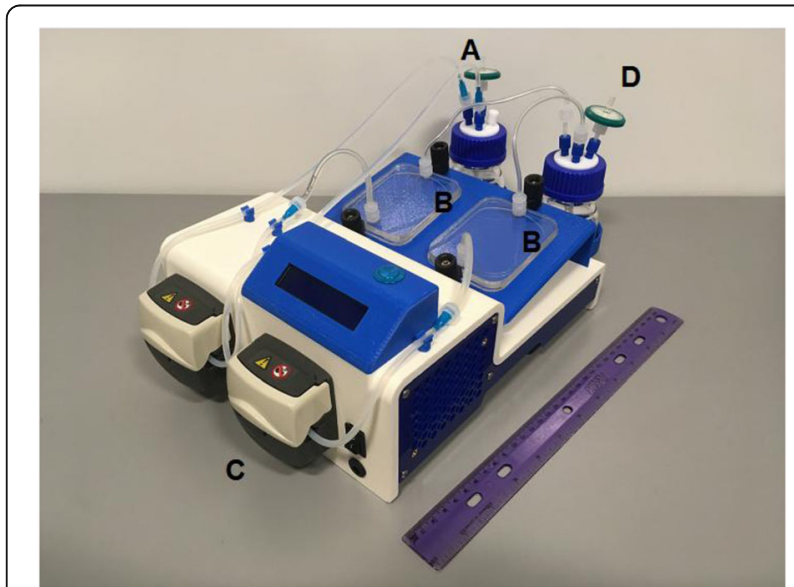

Fig. 1 (abstract P190). MicroDEN device. The inlet reservoir (A) containing medium is connected to the culture chamber (B) via a pump (C) to ensure continuous medium perfusion. The effluent medium is collected in outlet reservoir (D). 


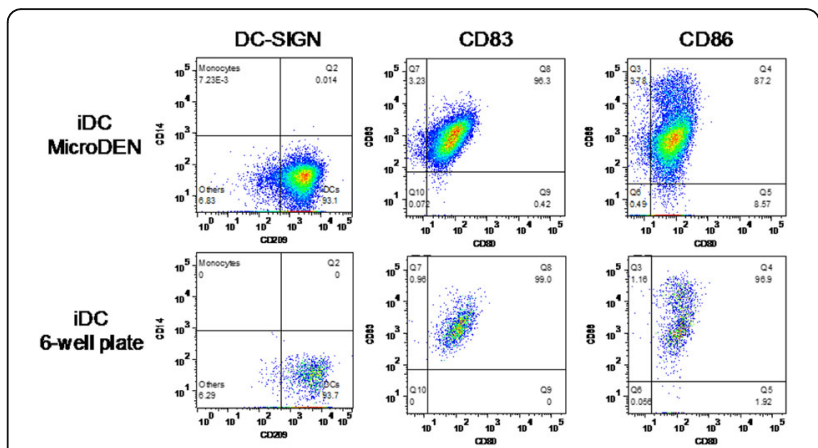

Fig. 2 (abstract P190). Immature dendritic cells generated by MicroDEN (top row) have comparable surface marker profiles to those generated by standard 6-well plate culture (bottom row)

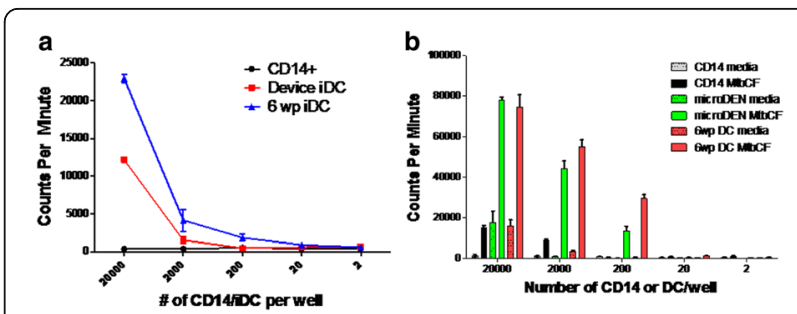

Fig. 3 (abstract P190). T cell response to immature dendritic cells generated in MicroDEN is equivalent to DCs generated in a 6-well plate as measured by (A) Allogeneic T cell proliferation assay and (B) Antigen-specific assay

\section{P191}

Limiting antigen escape in multiple myeloma by dual antigen-targeting of BCMA and TACl using the natural ligand APRIL Maria Ormhoj', Felipe Bedoya ${ }^{2}$, Irene Scarfò ${ }^{2}$, Benjamin Kleinstiver ${ }^{2}$,

J. Keith Joung ${ }^{2}$, Torben Barington ${ }^{3}$, Marcela Maus ${ }^{2}$

${ }^{1}$ University of Southern Denmark and Massachusetts General Hospital Cancer Center, Harvard Medical School, Brookline, MA, USA;

${ }^{2}$ Massachusetts General Hospital Cancer Center, Harvard Medical School, Boston, MA, USA; ${ }^{3}$ Odense University Hospital, University of Southern Denmark, Odense, Denmark

Correspondence: Maria Ormhoj (mormhoj@mgh.harvard.edu); Marcela Maus

Journal for ImmunoTherapy of Cancer 2017, 5(Suppl 2):P191

\section{Background}

Recent clinical trials of CAR T cells directed against B cell maturation antigen (BCMA) have lead to responses including complete remission in patients with multiple myeloma. However, treatment failure due to antigen-loss of BCMA has already been described. The transmembrane activator and calcium modulator and cyclophilin ligand interactor (TACl) is thought to have a redundant role to BCMA in maintaining cell survival, and is highly expressed on multiple myeloma cells. In this study, we utilized the natural ligand for BCMA and $\mathrm{TACl}$, a proliferation inducing ligand (APRIL), as a CAR binding moiety. With this approach we aim to prevent disease relapse due to antigen-escape by dual targeting of multiple surface antigens.

\section{Methods}

We generated CAR constructs with scFv-based anti-BCMA, and APRILbased CARs bearing different hinge and transmembrane domains (CD8 or 4-1BB), all fused to $4-1 B B$ and $C D 3$ zeta. Human primary $T$ cells were lentivirally transduced with anti-BCMA-CAR or APRILbased CARs. Cytotoxicity, proliferation and cytokine production was evaluated in vitro against a panel of cell lines with varying expression levels of $B C M A$ and $T A C l$ and in vivo in a xenograft model of multiple myeloma.

Results

Activation in response to $B C M A+$ or TACl+ target cells, were seen for APRIL-based CARs. Anti-BCMA-CAR was only activated in response to $\mathrm{BCMA}+$ target cells. Both BCMA and APRIL-CD8 hinge/transmembrane CARs displayed antigen-specific cytotoxicity. Interestingly, we found lower levels in cytokine production for APRIL-based CARs compared to anti-BCMA-CAR. This observation is likely to reflect the difference in binding affinity between using APRIL or an ScFv as CAR binding moiety. Altering the hinge/transmembrane domain to 4-1BB in the APRIL-CAR lead to a reduction in cytotoxicity. Ongoing studies, using a xenograft model have shown complete tumor remission in some mice treated with anti-BCMA-CAR or APRIL-CD8 hinge/transmembrane CAR.

Conclusions

In this study, we designed a CAR, based on the natural ligand APRIL, able to recognize both $B C M A$ and $T A C l$ in order to limit potential antigen-escape in multiple myeloma. We found that inclusion of the CD8 hinge and transmembrane region was essential for APRIL CAR function; this region could not be replaced with 4-1BB, despite the fact that both APRIL and 4-1BB are members of the TNF receptor superfamily and bear greater similarity to each other than to CD8. Despite the cytotoxic efficacy of the APRIL CAR against tumor cells, lower levels of effector cytokine production was seen. This is an important finding, since CAR T cell therapy can lead to cytokine release syndrome.

\section{P192}

Oncolytic viruses effectively celiver chimeric antigen receptor targets to triple negative breast cancers

Anthony Park', Saul Priceman' Stephen Forman', Yuman Fong², Nanhai

Chen $^{2}$, Dileshni Tilakawardane ${ }^{1}$, Brook Jeang ${ }^{1}$, John Murad', Michael O'Leary $^{2}$

${ }^{1}$ City Of Hope Beckman Research Institute, Duarte, CA, USA; ${ }^{2}$ City Of Hope, Duarte, CA, USA

Correspondence: Saul Priceman (apark@coh.org); Stephen Forman;

Yuman Fong

Journal for ImmunoTherapy of Cancer 2017, 5(Suppl 2):P192

\section{Background}

Chimeric antigen receptor (CAR) T cell therapy is a promising immunotherapy that has shown impressive clinical responses against CD19+ B-cell hematological malignancies [1]. While significant efforts are underway to translate CAR T cell therapy to solid cancers, the restricted antigen expression of targets such as CD19 is largely absent beyond B-cell malignancies [2]. It is widely appreciated that some solid tumors, like triple-negative breast cancer (TNBC), lung cancer, and liver cancer lack amenable tumor antigens to be effectively and safely targeted by CAR T cells [3]. Thus, novel approaches - particularly ones that are capable of selectively targeting intractable solid tumors - are desperately needed to improve clinical outcomes. Oncolytic viruses (OVs) are a novel and attractive form of immunotherapy for treating tumors since it can target tumor cells selectively - even in the absence of tumor specific antigens.

Methods

We have developed a chimeric OV expressing truncated CD19 (CD19t) to create a targetable antigen on human TNBC cells. PBMCs were isolated from healthy donors, which were lentivirally transduced to express CD19-CAR. Flow cytometric analysis was used to determine killing and activation of CAR T cells. In addition, supernatants of co-culture assays were collected to determine cytokine levels. As a positive control,TNBC cells were lentivirally transduced to express CD19t. NSG mice were used for in vivo studies with subcutaneous tumors, which were infected with OV followed by treatment with CAR T cells.

Results

OV effectively delivered CD19t in triple negative breast cancer cells in a time and multiplicity-of-infection dependent manner. OV infected tumor cells expressing CD19t were bonafide targets of 
CD19-CAR T cells. OV delivery of CD19t in triple negative breast cancer cells induced functional activation and antigen specific tumor targeting by CD19-CAR T cells.

\section{Conclusions}

Our studies have harnessed the exquisite capability of OV to selectively deliver expression of genes in tumors, in our case CD19t, such that TNBC now become de-novo targets for CD19-CAR T cells. By using this combinatorial strategy, we have broadened the utility of CD19-CAR T cells to otherwise target-less tumors, which we anticipate can be applied to a wide array of solid cancers as an effective immunotherapy approach.

\section{References}

1. Grupp SA, et al. CD19-targeted chimeric antigen receptor T cell therapy for acute lymphoblastic leukemia. Blood. 2015.

2. Priceman SJ, et al. Smart CARs engineered for cancer immunotherapy. Curr Opin Oncol. 2015.

3. Palma G, et al. TNBC: looking for the missing link between biology and treatments. Oncotarget. 2015.

\section{P193}

TRuC ${ }^{\mathrm{TM}}-\mathrm{T}$ cells: a novel class of engineered $\mathrm{T}$ cells that power $\mathrm{T}$ cells through the entire T cell receptor complex without MHC restriction

Ekta Patel', Jian Ding', Nicolaus Thorausch², Bonnie Lee', Rashmi Choudhary', Olga Kiner', Janani Krishnamurthy', Justin Quinn', Patrick Tavares', Qi Wei', Solly Weiler', Wolfgang Schamel', Patrick Baeuerle', Robert Hofmeister ${ }^{1}$ ${ }^{1}$ TCR2 Therapeutics, Cambridge, MA, USA; ${ }^{2}$ University of Freiburg, Freiburg, Germany

Correspondence: Robert Hofmeister (ekta@tcr2.com)

Journal for ImmunoTherapy of Cancer 2017, 5(Suppl 2):P193

\section{Background}

T cells expressing chimeric antigen receptors (CARs) have demonstrated remarkable clinical benefit in certain hematological malignancies but so far have struggled to show efficacy in patients with solid tumors. This could be because CARs bypass the complex TCR signaling by utilizing only the $\mathrm{CD} 3 \zeta$ chain in combination with a costimulatory domain and therefore fail to initiate a complete TCR signaling cascade that is necessary to overcome the immunosuppressive tumor microenvironment. Here, we present the preclinical evaluation of a novel T cell engineering platform, which is based on T Cell Receptor Fusion Constructs (TRuCs) that are integrated into the TCR and target CD19+ tumors in a MHC non-restricted fashion. Methods

$\mathrm{TRuC}^{\mathrm{TM}}$ variants were constructed by recombinant fusion of an antiCD19 scFv (FMC-63) to various TCR subunits via a flexible linker sequence. Likewise, CD19-specific CD28 and 41BB $\zeta$ CARs were generated for side-by-side comparison. After stimulation and lentiviral transduction, human primary T cells were analyzed for $\mathrm{TRuC}^{\mathrm{TM}}$ or CAR surface expression. TRuC ${ }^{\mathrm{TM}}$ TCR complex integration was assessed by immunoprecipitation and Western blot analysis. In vitro functional assays were conducted using both a luciferase based cytotoxicity assay and an impedance-based cytotoxicity assay, and cytokine release was measured by Luminex. To evaluate the constructs in vivo, NODSCID-IL2Rg null mice were treated with T cells four days after subcutaneous injection of the Raji tumor cell line.

Results

Of the five CD19-TRuC $C^{\mathrm{TM}}$ variants tested, the $\varepsilon-\mathrm{TRuC}^{\mathrm{TM}}$ variant consistently demonstrated highest surface expression and $\mathrm{T}$ cell activation. Biochemical and signaling pathway analysis revealed that $\mathrm{TRuC}^{\mathrm{TM}}$ variants incorporated into TCR complex and utilized the natural T cell receptor signaling machinery. In vitro anti-tumor activity of the CD3E TRuC was comparable to that of the $\mathrm{CD} 28 \zeta$ and $41 \mathrm{BB} \zeta$ CARs. However, $\varepsilon$-TRuC ${ }^{\mathrm{TM}} T$ cells released significantly less cytokines compared to CAR T cells. Intriguingly, TRuC ${ }^{\mathrm{TM}} \mathrm{T}$ cells were more potent than $\mathrm{CD} 28 \zeta$ or $41 \mathrm{BB} \zeta$ in clearing solid tumors in a subcutaneous Raji mouse model.

Conclusions

We demonstrate that $\mathrm{TRuC}^{\mathrm{TM}}$ variants can effectively reprogram $\mathrm{T}$ cells to recognize tumor surface antigens in a non-MHC-restricted fashion. TRuC $\mathrm{CM}^{\mathrm{T}} \mathrm{S}$ are distinct from CARs in their ability to activate $T$ cells through the entire TCR without and do not require additional costimulatory domains. While TRuC ${ }^{\mathrm{TM}} \mathrm{T}$ cells were equally potent as CAR T cells in eliminating tumor cells in vitro, they induced less cytokine release by $T$ cells. We believe that these features make the $\mathrm{TRuC}^{\mathrm{TM}}$ platform a superior approach to treat cancer with engineered T cells.

\section{P194}

Studies of key quality attributes for TIL product, LN-144

Krit Ritthipichai, Ian Frank, Marcus Machin, Michelle Simpson-Abelson, Michelle Blaskovich, Amanda Stramer, Michael Lotze

lovance Biotherapeutics, Inc., Tampa, FL, USA

Correspondence: Michael Lotze (krit.ritthipichai@iovance.com)

Journal for ImmunoTherapy of Cancer 2017, 5(Suppl 2):P194

\section{Background}

Adoptive T cell therapy with autologous tumor infiltrating lymphocytes (TIL) has demonstrated clinical efficacy in studies conducted by other institutions in patients with metastatic melanoma and cervical carcinoma. Most clinical study reports include exploratory analyses of the infused TIL products intended to identify quality attributes that could relate to product efficacy or safety. We describe progress in the development of a quality control platform for use in the commercial manufacturing of TIL products based on process development studies conducted at lovance and our experience with the production of investigational TIL products.

Methods

The process of generating an autologous TIL product includes a preRapid Expansion Protocol (pre-REP), in which tumor fragments of 1-3 $\mathrm{mm}^{3}$ size are placed in media containing IL-2. During the pre-REP, TIL emigrate out of the tumor fragments and expand in response to IL-2. To further stimulate TIL growth, TIL are expanded using a secondary culture period termed the Rapid Expansion Protocol (REP) that includes irradiated PBMC feeders, IL-2 and anti-CD3. Pharmaceutical products are required to meet analytical criteria that uniquely and appropriately reflect key features of identity, purity, and potency of the product. The identity and purity of cell populations present in TIL products are extensively characterized through flow cytometry analyses of cell surface markers. Flow cytometry is also used to screen for the potential presence of residual melanoma tumor cells in REP TIL products through quantitative assessment of Melanoma-associated Chondroitin Sulfate Proteoglycan (MCSP) expression. Functional analyses relevant to product potency include the measurement of IFN- $\gamma$ secretion in the presence of beads coated with monoclonal antibodies (CD3, CD28, and CD137).

Results

TIL products manufactured by lovance are composed of greater than $97 \% \mathrm{CD} 5^{+} \mathrm{CD}^{+}$cells. Non-T cell population including B cells and NK cells represent fewer than $3 \%$ of cells within the final TIL product. Residual melanoma cells in TIL products were below the limit of detection $\left(<5\right.$ cells $\left./ 10^{6}\right)$ TIL using a specialized assay developed for this assessment. IFN- $\gamma$ secretion by the TIL product following anti-CD3/ CD28/CD137 re-stimulation was consistently $>200 \mathrm{pg} / 10^{5}$ TIL.

Conclusions

Commercial manufacturing of TIL products will require a robust analytical platform to ensure consistent delivery of products meeting the critical quality attributes of cellular therapeutics. Our ongoing research in the development of analytical methodology to fully 
characterize product identity, purity, and potency guides the establishment of formal release criteria to be implemented in commercial production of TIL products at lovance.

\section{P195}

Anti-CD37 chimeric antigen receptor T cells: a new potential therapeutic option for B-cell malignancies

Irene Scarfo ${ }^{1}$, Maria Ormhøj] ${ }^{2}$, Matthew Frigault ${ }^{2}$, Marcela Maus ${ }^{2}$

${ }^{1}$ Massachusetts General Hospital, Charlestown, MA, USA; ${ }^{2} \mathrm{MGH}$, Charlestown, MA, USA

Correspondence: Irene Scarfo (iscarfo@mgh.harvard.edu); Marcela Maus Journal for ImmunoTherapy of Cancer 2017, 5(Suppl 2):P195

\section{Background}

CD37 is a tetraspanin expressed on mature B cells but absent on early progenitors or terminally differentiated plasma cells. CD37 is highly expressed on malignant $B$ cells in non-Hodgkin lymphomas $(\mathrm{NHL})$, including mantle cell lymphoma $(\mathrm{MCL})$, diffuse large B-cell lymphoma (DLBCL), follicular lymphoma (FL), Burkitt lymphoma and B-cell chronic lymphocytic leukemia (CLL); thus, CD37 represents a promising target for B-cell malignancies, particularly for variants that escape existing therapies targeting the common B cell antigens CD19 and CD20

Methods

We designed the first anti-CD37 CAR (CAR-37) for the treatment of Bcell malignancies. Specifically, we designed a second-generation CAR, encoded by a lentiviral vector and bearing a 4-1BB costimulatory domain. We tested two different orientations of a humanized murine antibody-derived single-chain variable fragment $\left(\mathrm{V}_{\mathrm{L}}-\mathrm{V}_{\mathrm{H}}\right.$ or $\left.\mathrm{V}_{\mathrm{H}}-\mathrm{V}_{\mathrm{L}}\right)$ and have generated a pre-clinical data panel to select the most stable and efficacious format

Results

In vitro cytotoxic activity of CART-37 cells was evaluated by coculturing CART-37 cells with CD37-expressing human tumor cell lines (RAJI, OSU-CLL and JEKO-1) at different effector to target ratios. CD37-directed CAR T cells demonstrated antigen-specific activation, proliferation, cytokine production, and cytotoxic activity in vitro in multiple models of B cell malignancy. Next, we assessed the antilymphoma efficacy in vivo in a mantle cell lymphoma model. CAR-37 treatment eliminated the tumor cells within 2 weeks, and mice maintained durable remissions. We were able to detect CAR T cells in the blood of mice after 7 days of injection. Ongoing studies are evaluating the long term persistence of CAR T cells in mice

Conclusions

Taken together these results show that $T$ cells expressing anti-CD37 CAR have substantial activity in vitro and in vivo against $B$ cell malignancies. These findings indicated that CD37-CAR T cells are a novel potential therapeutic agent for the treatment of patients with CD37 expressing tumors.

\section{P196}

FGFR4 specific chimeric antigen receptor (CAR) T cell therapy against rhabdomyosarcoma

Nityashree Shivaprasad', Marielle Yohe ${ }^{1}$, Ying Xiong ${ }^{2}$, Darong $\mathrm{Wu}^{2}$, Peter Azorsa', Adam Cheuck', Joon-Yong Chung', Zhongyu Zhu', Dimiter Dimitrov ${ }^{3}$, Stephen Hewitt ${ }^{1}$, Boro Dropulic ${ }^{2}$, Dina Schneider ${ }^{2}$, Rimas Orentas ${ }^{2}$, Javed Khan ${ }^{1}$

${ }^{1}$ National Institute of Health, Bethesda, MD, USA; ${ }^{2}$ Lentigen Technology Inc., a Miltenyi Biotech company, Gaithersburg, MD, USA; ${ }^{3}$ National Institute of Health, Frederick, MD, USA

Correspondence: Nityashree Shivaprasad (nitya.shivaprasad@nih.gov) Journal for ImmunoTherapy of Cancer 2017, 5(Suppl 2):P196

\section{Background}

Rhabdomyosarcoma (RMS) is the most common soft tissue sarcoma in childhood and adolescence with an annual incidence of 4.5 cases per 1 million children. Patients with high-risk metastatic disease have dismal prognosis and require newer therapeutic approaches. Whole genome and exome sequencing have shown that RMS has a low mutational burden with few directly actionable somatic targets. The fibroblast growth factor receptor 4 (FGFR4) cell surface protein is an attractive therapeutic target as the FGFR4 gene is overexpressed in RMS and FGFR4 protein has been shown to be critical for cell survival, proliferation, and metastasis. In addition, activating mutations in the kinase domain of FGFR4 leads to aggressive tumor growth and RMS metastasis. Here, we hypothesize that FGFR4 provides a specific target for immune-based therapy of RMS. In particular, we are developing $T$ cells genetically modified to express chimeric antigen receptor (CAR) targeting FGFR4.

Methods

To verify specific expression of FGFR4 protein we performed both immunohistochemistry (IHC) and electrochemillumescence (ECL) ELISA assays. To construct CAR-T cells targeting FGFR4, we first screened a single-chain variable fragment (scFv) cDNA library, identified ten specific human anti-FGFR4 scFv binders and cloned into prokaryotic expression vector containing the human lgG1 Fc region. In addition, we have scFv derived from mouse monoclonal antibodies (mAbs) generated from hybridoma technology that is also being tested in CAR format.

Results

We confirmed a significantly increased staining for FGFR4 protein on RMS primary tumors, compared to normal tissues by IHC. FGFR4 expression measured using quantitative $E C L$ assay shows an average of 10 fold higher expression in RMS cell lines compared to normal tissue.

ScFv-Fc binders and the mouse mAbs developed against FGFR4, were first assayed for binding to cell surface FGFR4 on RMS cell lines. Anti-FGFR4 binders also showed positive binding on the cell surface of RMS cell lines. Finally, these anti-FGFR4 scFv FGFR4 CAR constructs were further assessed in human T cells. Four out of ten FGFR4 CAR constructs tested showed cell-mediated cytotoxicity against RMS cell lines. Two lead FGFR CAR, BT53 (murine) and M410 (human), are highly potent in inducing gamma interferon, TNF alpha and cytotoxicity when the FGFR4-CART are co-cultured with RMS cells. Preliminary in-vivo testing of M410 has shown to be effective in eliminating RMS cells in xenograft models.

Conclusions

We demonstrated that FGFR4 is a novel target for immune-based therapy. FGFR4 CAR-T cell therapy offers a potential novel therapeutic intervention for high-risk, refractory and relapsed RMS patients.

P197

Engineered antigen presenting $\mathrm{T}$ cells for the treatment of solid tumor cancers

LeeAnn Talarico, Idelfonso Vicente-Suarez, Katarina Blagovic,

Eritza Chong-Ng, Lauren Jones, Lucas Pomerance, Howard Bernstein

SQZ Biotechnologies, Watertown, MA, USA

Correspondence: LeeAnn Talarico (leeann.talarico@sqzbiotech.com)

Journal for ImmunoTherapy of Cancer 2017, 5(Suppl 2):P197

\section{Background}

CD8+ cytotoxic T lymphocytes (CTL) stimulated by tumor-associated antigens have the potential to target and clear solid tumors, however, current methods for inducing endogenous $T$ cell responses or ex vivo engineering of $\mathrm{T}$ cell receptors have faced challenges. Antigen presenting cells (APCs) play a key role in inducing endogenous activation of CTLs. In this work, we describe the implementation of our CellSqueeze ${ }^{\circledast}$ platform to engineer more effective APCs for use in oncology indications. By enabling efficient cytosolic delivery of target antigens, this platform has demonstrated the ability to induce more effective MHC-I presentation of target antigens and stimulation of CTLs in vivo. This presentation focuses on the use of autologous T cells as APCs in murine disease models and in vitro human systems with the potential to translate to clinical indications.

Methods

MHC-I presentation of antigen was measured with an in vitro model utilizing human primary T cells SQZed with synthetic long peptides (SLPs) encoding human cancer antigens for the given HLA type. 
Treated cells were co-cultured with HLA matched human CTLS enriched in vitro for reactivity to the given cancer antigens, and cytokine secretion was measured by ELISA.

In vivo efficacy was measured with a murine tumor cell line transformed through expression of antigens of interest. T-APCs were SQZed with SLPs encoding the target antigen and administered. Mice were monitored for up to 60 days following tumor inoculation to establish tumor volume and survival.

Results

Our results show that human T-APCS SQZed with SLPs encoding tumor antigens can elicit antigen specific IFN gamma secretion $>20-$ fold above controls in vitro.

Administration of T-APCs SQZed with SLPs to mice elicits a robust expansion of antigen-specific CTLs such that $2.26 \%$ CD8+CD44+ T cells in the blood are tetramer positive following a prime and boost. Additionally, T-APCs confer protection against tumor challenge with $90 \%$ of T-APC treated mice surviving to Day 57 vs. $0 \%$ in untreated controls, $\mathrm{P}$ value $<0.0001$

\section{Conclusions}

This work highlights the cell therapy potential of engineered T cells as antigen presenting cells. The SQZed T-APCs demonstrate robust CTL responses in vivo capable of prophylactically preventing tumor growth and therapeutically treating existing tumors.

\section{P198}

Bispecific antibody armed activated T cells can target drug resistant pancreatic cancer cells and cancer stem like cells effectively

Archana Thakur', Elyse N. Tomaszewski ${ }^{2}$, Lawrence G. Lum

${ }^{1}$ University of Virginia, Charlottesville, VA, USA; ${ }^{2}$ Karmanos Cancer

Institute, Detroit, MI, USA

Correspondence: Archana Thakur (at2fx@virginia.edu)

Journal for ImmunoTherapy of Cancer 2017, 5(Suppl 2):P198

\section{Background}

Pancreatic cancer has the worst survival rate of all cancers. The multidrug resistance (both intrinsic and acquired) is thought to be a major reason for chemotherapeutic ineffectiveness of pancreatic cancer. The multidrug resistance (MDR) phenotype is mostly contributed by the members of the ATP-binding cassette (ABC) transporter superfamily and have been shown to be key mediators of drug efflux and drug resistance in many tumor types. Another player to the refractory pancreatic cancer is the presence of $\mathrm{CD}_{4} 4^{+} / \mathrm{CD} 24^{+} / \mathrm{EpCAM}^{+}$cancer stem like cells (CSCs) that may contribute to the high recurrence rate after clinical remission. Improved and novel therapeutic strategies are needed for pancreatic cancer. This study was designed to investigate whether bispecific antibody armed activated $\mathrm{T}$ cells (BATs) can target drug resistant pancreatic cancer cell lines.

Methods

We used two chemotherapeutic drugs, gemcitabine and cisplatin for generating drug-resistant pancreatic cancer cell lines. MiaPaCa-2 and L3.6, were exposed step-wise to increasing concentrations of gemcitabine and cisplatin ranging from 0.05 to $1.0 \mu \mathrm{M}$. When the cells were adapted to a dose of gemcitabine or cisplatin, the concentration was increased gradually. After 3-4 months of selection, MiaPaCa2 and L3.6 cells were able to survive at $1.0 \mu \mathrm{M}$ of gemcitabine and $0.5 \mu \mathrm{M}$ of cisplatin for both lines.

Results

Flow cytometry data showed increased proportion of $C D 44^{+} / C D 24$ ${ }^{+} / \mathrm{EpCAM}^{+}$cancer stem like cells as well as increased number of $A B C$ transporter $A B C G 2$ positive cells in drug resistant cell lines compared to the parental cell lines. Bispecific antibody (OKT3 $x$ anti-EGFR [EGFRBi] or OKT3 $x$ anti-HER2 [HER2Bi]) armed activated T cells from 6-7 normal donors were as effective in targeting gemcitabine or cisplatin resistant MiaPaCa-2 (Median cytotoxicity was 18\% compared to $13 \%$ of parental cells) and L3.6 (Median cytotoxicity was $44 \%$ compared to $30 \%$ of parental cells) cell lines as parental non drug treated cell lines, produced Th $\mathrm{h}_{1}$ cytokines, IFN-g and TNF-a, and chemokines, MIP-1b and RANTES.

Conclusions

These data suggest that BATs mediated killing of chemoresistant tumor cells and release of $\mathrm{Th}_{1}$ cytokines may modulate the tumor microenvironment to enhance anti-tumor immune responses. Trial Registration

NA

P199

ID01-mediated tryptophan depletion potently inhibits CAR-T

functionality as part of a CAR-T driven adaptive immune resistance response in the tumor microenvironment

Evan P. Thomas, Heidi K. Jessup, Jim Qin, Alex Baturevych, Christina Swanson, Sarah Swenson, Ken Prentice, Hyam Levitsky, Michael Ports

Juno Therapeutics, Seattle, WA, USA

Correspondence: Michael Ports (evan.thomas@junotherapeutics.com) Journal for ImmunoTherapy of Cancer 2017, 5(Suppl 2):P199

\section{Background}

Chimeric antigen receptor T cells (CAR-T) are engineered antigenspecific $T$ cells that are poised to kill tumor cells and release inflammatory cytokines, including interferon-gamma (IFN $\gamma$ ), into the tumor microenvironment (TME). Unlike endogenous intratumoral $T$ cells, CAR-T generally are highly functional upon infusion and may produce a robust pro-inflammatory milieu in the TME. In a mechanism termed adaptive immune resistance [1], tumor cells can upregulate immunosuppressive pathways in response to inflammatory cytokines. One such pathway, indoleamine 2,3-dioxygenase (IDO1), can limit effector $\mathrm{T}$ cell activity through the catabolism of tryptophan, resulting in local amino acid deficiency and accumulation of tryptophan catabolites [2]. Here we evaluated the effect of IDO1 induction in response to multiple CAR-T products.

Methods

Anti-CD19 and anti-ROR1 CAR-T, containing 4-1BB co-stimulatory endo-domains, were generated using healthy donor peripheral blood samples, and exemplary full-scale manufacturing processes. IDO1mediated effects on CAR-T proliferation and cytolytic activity in vitro were evaluated in the presence of IDO1-inducible, target antigenexpressing cell lines and the IDO1-specific inhibitor, epacadostat. In vivo studies utilized a xenograft mouse model with subcutaneous tumors expressing CD19, and capable of upregulating IDO1. Anti-CD19 CAR-T were administered to animals in combination with epacadostat or vehicle.

Results

Antigen-stimulated CAR-T induced IDO1 expression in tumor cell lines, likely through secretion of IFNY, resulting in tryptophan depletion and accumulation of kynurenine. Activation of the IDO1 pathway substantially inhibited CAR-T proliferative capacity, which appeared to be restored in the presence of epacadostat. Studies with tryptophan-free medium indicated that the observed inhibition of CAR-T cells was primarily driven by tryptophan depletion. Mouse models demonstrated that CAR-T may derive a benefit in vivo when administered concomitantly with epacadostat.

Conclusions

It was observed that CAR-T could induce quiescent immunosuppressive mechanisms in the TME through secretion of IFNY. Upon upregulation of IDO1, tryptophan was depleted and the integrated stress response appeared to be activated in CAR-T, limiting proliferation. The IDO1-specific inhibitor epacadostat blocked IDO1 activity and restored CAR-T function. Combination of epacadostat with current CAR-T therapies may be useful across multiple indications, including hematologic and solid tumors. Such a combination may counteract a suppressive mechanism of tryptophan starvation limiting CAR-T performance in the TME, and ultimately, therapeutic efficacy. 


\section{References}

1. Pardoll DM. The blockade of immune checkpoints in cancer immunotherapy. Nat. Rev. Cancer. 2012;12(4):252-264.

2. Munn D, et al. GCN2 kinase in T cells mediates proliferative arrest and anergy induction in response to indoleamine 2,3-dioxygenase. Immunity. 2005;22(5):633-642

\section{P200}

The use of ex-vivo stimulation to generate a neoantigen specific $\mathrm{T}$ cell product for adoptive T cell therapy

Marit M. van Buuren', Joost H. van den Berg², Divya Lenkala', Jessica Kohler $^{1}$, Renate de Boer ${ }^{2}$, Ton N.M. Schumacher ${ }^{2}$, Edward F. Fritsch', John B.A.G. Haanen², Richard B. Gaynor' ${ }^{\text {, Matthew J. Goldstein }}{ }^{1}$

${ }^{1}$ Neon Therapeutics, Cambridge, MA, USA; ${ }^{2}$ Netherlands Cancer Institute, Amsterdam, Netherlands

Correspondence: Marit M. van Buuren

(mvanbuuren@neontherapeutics.com)

Journal for ImmunoTherapy of Cancer 2017, 5(Suppl 2):P200

\section{Background}

A growing body of evidence supports the role of neoantigens as important targets for anti-tumor immune responses. Adoptive $\mathrm{T}$ cell therapy is a powerful modality to treat patients with solid cancers and has demonstrated success in the treatment of melanoma. Current approaches largely focus on products generated from the non-specific expansion of tumor infiltrating lymphocytes (TILs) or the genetic modification of $\mathrm{T}$ cells with $\mathrm{T}$ cell receptors (TCRs) that recognize tumor associated antigens such as NY-ESO1. Here, we describe an approach to generate neoantigen specific $\mathrm{T}$ cell therapies that are specific to and personalized for each individual patient. We have developed an ex vivo method to generate a neoantigen specific $\mathrm{T}$ cell product for use as an autologous adoptive $\mathrm{T}$ cell therapy. The goal of this method is two-fold: (i) to broaden the tumor-specific $T$ cell repertoire by inducing neoantigen specific $T$ cells from the naïve $\mathrm{T}$ cell compartment and (ii) to expand pre-existing, neoantigenspecific memory $\mathrm{T}$ cell responses.

Methods

The model antigens chosen for these studies mimic the type of neoantigens present within a patient's tumor. Naïve T cell responses were induced and studied using neoantigens previously identified from the literature and memory $T$ cells were expanded and studied using viral epitopes against Cytomegalovirus (CMV), Epstein-Barr Virus (EBV), and Influenza. For T cell stimulations, autologous dendritic cells (DCs) were generated from healthy donor apheresis products, loaded with model antigens, and used to stimulate autologous $\mathrm{T}$ cells. Induced $\mathrm{T}$ cells were harvested and analyzed at different timepoints throughout the stimulation protocol to assess the following: antigen-specificity, functionality, phenotype, and fold expansion. Results

We demonstrate here the successful induction of both naïve and memory $\mathrm{T}$ cell responses from healthy donor material. These $\mathrm{T}$ cell responses are:

- Preferentially responsive to mutant epitopes and not their wild-type counterparts

- Have multiple functions (cytokine production and degranulation)

- Show a mostly central memory phenotype.

Can be completed in a timeframe that is therapeutically appropriate for patients with advanced or metastatic cancer.

\section{Conclusions}

Finally, supporting data will be presented demonstrating successful induction of personalized neoantigen specific T cell responses in patient donor material.
P201

Targeting heterogeneous glioblastoma using chlorotoxin-redirected CAR-T cells

Dongrui Wang, Sarah Wright, Wen-Chung Chang, Renate Starr, Xin Yang,

Brenda Aguilar, Alfonso Brito, Aniee Sarkissian, Behnam Badie,

Stephen J. Forman, Michael E. Barish, Christine E. Brown

City of Hope, Duarte, CA, USA

Correspondence: Stephen J. Forman (dowang@coh.org);

Michael E. Barish; Christine E. Brown

Journal for ImmunoTherapy of Cancer 2017, 5(Suppl 2):P201

\section{Background}

Chimeric antigen receptor (CAR)-modified T cells have shown indications of clinical promise against B cell malignancies yet inconsistent efficacy on solid tumors. One of the challenges of CAR therapy for treating solid tumors is the heterogeneity of tumor cells, which allows for antigen escape and tumor recurrence. Glioblastoma (GBM) is one of the most lethal cancers, displaying considerable heterogeneity across patients as well as among intratumoral subpopulations. Therefore, future progress when applying CAR therapy to GBM requires targeting the majority of tumor cells, which would potentially limit opportunities for antigen escape. Chlorotoxin (CLTX) is a peptide component of scorpion venom which, given its demonstrated capability to bind with a wide-range of brain tumors, has been used for identifying tumor cells and for drug delivery. These results open the potential to exploit CLTX for directing T cells against GBM.

Methods

First, we used a fluorescence-conjugated CLTX to verify its specific binding to patient-derived GBM cells and to mouse GBM cells over normal cells from various human tissues. Then $T$ cells were engineered with a CAR utilizing CLTX as the tumor-binding domain. The CLTX-CAR T cells were tested for antitumor efficacy against GBM neurospheres derived from different patients through in vitro co-culture and in vivo orthotopic GBM models. To evaluate the safety of CLTXCAR T cells, we tested their cytotoxicity against normal cells and evaluated potential pathological alterations of CAR treated mice.

Results

We showed that CLTX binds to a panel of primary GBM cells and low-passage GBM spheres with negligible binding to normal cells derived from neural and other tissues. T cells expressing CLTX-directed CAR were activated when exposed to GBM cells as suggested by $T$ cell degranulation, production of cytokines, and formation of immunological synapses. Moreover, these CAR-T cells were able to eliminate GBM cells with varying TCGA subtypes and antigen expression patterns during in vitro co-culture, as well as to eradicate established orthotopic GBM tumors in vivo. In contrast, CLTX-CAR T cells showed minor cytotoxicity against normal human cells. Consistently, despite the CLTX binding to mouse GBM cells, CLTX-CAR T cells caused no pathological alterations of normal mouse tissues, indicating that these cells have few or no off-target effects.

Conclusions

Here, we report the development of a new class of peptide-based CAR exploiting the GBM-binding potential of CLTX. Our study supports the potential utility of CLTX-CAR T cells to effectively target GBM while overcoming heterogeneity and limiting antigen escape.

P202

New therapeutic approach for central nervous system lymphoma by CD19CAR T cells

Xiuli Wang, Ryan Urak, Christian Huynh, Miriam Walter, Laura Lim,

Brenda Aguilar, Alfonso Brito, Aniee Sarkissian, Christine Brown,

Lihua Budde, Leslie Popplewell, Stephen Forman

City of Hope National Medical Center, Duarte, CA, USA

Correspondence: Xiuli Wang (xiuwang@coh.org)

Journal for ImmunoTherapy of Cancer 2017, 5(Suppl 2):P202 


\section{Background}

Central nervous system lymphoma (CNSL) can start in the brain, spinal cord, eye, and/or meninges or result from metastasis of systemic disease. About $90 \%$ are B cell lymphomas. Compared with systemic lymphoma, CNSL carries a worse prognosis. Only a few chemotherapeutic drugs can cross the blood-brain barrier, limiting treatment. T cells genetically engineered with chimeric antigen receptors (CARs) targeting CD19 have shown tremendous potential in the treatment of systemic lymphoma. Although CD19CAR T cell trafficking in cerebrospinal fluid (CSF) is frequently reported, most (if not all) protocols exclude patients with active CNS involvement. Here we investigate the feasibility and efficacy of CD19CAR T cells to treat CNSL.

\section{Methods}

Human B cell lymphoma Daudi cells were injected intracranially into NSG mice and allowed to engraft for 5 days. T cells genetically modified with CD19CAR lentivirus were administered via three delivery routes: intracranial local infusion (ic, $1 \times 10 \wedge 6$ cells); intracerebroventricular (icv, $1 \times 10 \wedge 6$ cells); and intravenous injection (iv, $3 \times 10 \wedge 6$ cells). Results

We observed in separate experiments that both a single i.c infusion and a single i.c.v delivery of CD19CAR T cells were able to completely eradicate CNS lymphoma in all mice by day 14 post CAR T cell infusion; and that a single dose of i.v infusion induced significant antiCNSL activity with a slightly delayed response as compared to i.c and i.c.v treatment and all mice achieved complete remission 21 days post $T$ cell infusion. CAR T cells were detected in peripheral blood obtained from retro-orbital bleeding, not only in the i.v treated mice, but also in i.c.v treated mice 28 days after CAR T cell infusion, suggesting that i.c.v not only controls CNSL but may also play a role in immune surveillance for systemic tumors. To confirm this, we established an NSG CNS B cell lymphoma model by also inoculating subcutaneous tumors on the animal's flank, 3 weeks prior to i.c tumor injection into the same mouse. CD19CAR T cells were delivered via i.c.v 5 days after i.c. tumor injection. CAR T cell injection resulted in complete remission of both the brain tumor and the flank tumor 14 days after CAR T cell administration. All mice survived more than 300 days. We also found that i.c.v. delivered CD19CAR T cells were resistant to tumor re-challenge.

Conclusions

In conclusion, intracerebroventricular delivery of CD19CAR T cells is a promising and feasible therapeutic approach for both primary central nervous system lymphoma and systemic lymphoma with concurrent CNS involvement.

\section{P203}

A cryopreserved TIL product, LN-144, generated with an abbreviated method suitable for high throughput commercial manufacturing exhibits favorable quality attributes for adoptive cell transfer

Seth Wardell1', Maria Fardis'², Michael T. Lotze', Ian Frank', Amanda Stramer ${ }^{1}$, Jyothi Sethuraman', James Bender ${ }^{2}$, Michelle Abelson?

Lavakumar Karyumpudi ${ }^{1}$

${ }^{1}$ IOVANCE Biotherapeutics Inc, Tampa, FL, USA: ${ }^{2}$ IOVANCE

Biotherapeutics Inc., San Carlos, CA, USA

Correspondence: Maria Fardis (seth.wardell@IOVANCE.com); Michael T.

Lotze

Journal for ImmunoTherapy of Cancer 2017, 5(Suppl 2):P203

\section{Background}

Existing methods for generating clinical TIL products involve open operator interventions followed by extended incubation periods to generate a therapeutic product. IOVANCE Generation 1 process takes approximately 6 weeks and yields a fresh product. To bring TIL therapy to all patients that may benefit from its potential, IOVANCE has developed an abbreviated 22-day culture method, Generation 2, suitable for centralized manufacturing with a cryopreserved drug product capable of shipment to distant clinical sites [1]. Generation 2 represents a flexible, robust, closed, and semi-automated cell production process that is amenable to high throughput manufacturing on a commercial scale. Drug products generated by this method have comparable quality attributes to those generated by IOVANCE Generation 1.

Methods

Qualified methods to assess proliferation, phenotype, and function were applied to in-process and final drug products generated by both IOVANCE TIL expansion methods to determine fit within the internal target product profile. TIL expansion was assessed by triplicate automated count. Immunophenotyping was performed to determine identity, purity, as well as relative levels of activation, memory, and exhaustion of the cell product. Cellular function was evaluated as the ability of the cell product to secrete IFN- $\gamma$ in response to CD3, CD28, and $4-1 \mathrm{BB}$ receptor engagement.

Results

TIL cultured with the abbreviated Generation 2 method achieved doses comparable to IOVANCE Generation 1 (Generation 2: mean $4.29 \times 10^{10}$ nucleated cells $n=12$, Generation 1: mean $5.84 \times 10^{10}$, $\mathrm{n}=28$ ). Thawed Generation 2 drug products were similar to Generation 1 in terms of T cell purity, with similar ratios of CD4 to CD8 cells and memory subsets. Generation 2 drug products displayed an increased ability to produce INF- $\gamma$ upon reactivation relative to Generation $1(p=<0.0001[M F 1])$

\section{Conclusions}

The IOVANCE Generation 2 process produces a potentially potent TIL product with comparable quality attributes to Generation 1. Generation 2 products exhibit high levels of co-stimulatory molecules, low levels of exhaustion markers, and retain the ability to secrete cytokine upon reactivation. The abbreviated 22-day expansion platform allows for the rapid generation of clinical scale doses for patients in urgent need of therapy. The cryopreserved drug product introduces critical logistical efficiencies allowing flexibility in distribution. The IOVANCE expansion method overcomes traditional barriers to the wider application of TIL therapy.

References

1. Jin J, Sabatino M, Somerville R, Wilson JR, Dudley ME, Stroncek DF, Rosenberg SA. Simplified method of the growth of human tumor infiltrating lymphocytes in gas-permeable flasks to numbers needed for patient treatment. J Immunother. 2012;35(3):283-92.

P204

Reenergizing the tumor infiltrating $T$ cells by intratumoral delivery of BCG to treat bladder cancer and prevent recurrence

Gang Xin ${ }^{1}$, Gopit Shah², William See ${ }^{2}$, Weiguo Cui ${ }^{1}$

${ }^{1}$ Blood Research Institute, Blood Center of Wisconsin, Milwaukee, WI,

USA; ${ }^{2}$ Medical College of Wisconsin, Milwaukee, WI, USA

Correspondence: Weiguo Cui (Gang.Xin@BCW.edu)

Journal for ImmunoTherapy of Cancer 2017, 5(Suppl 2):P204

\section{Background}

Bladder cancer remains one of the most common malignancies, which is difficult to treat with high rates of recurrence. Given the recent astonishing clinical results in treating hematological malignancies, the adoptive cell transfer (ACT) of tumor-reactive T cells holds great promise to address this urgent need [1]. However, the efficacy of such approach is curtailed when treating solid tumors, such as bladder cancer. The primary hurdles which must be overcome for immunotherapy to be effective against bladder cancer include 1) clonal types and quantity of tumor-specific T cells adoptively transferred into tumor-bearing hosts are too low to mount adequate responses, 2) a large portion of adoptively transferred anti-tumor $T$ cells fail to migrate to the tumor and 3) the immunosuppressive microenvironment within the tumor induces a rapid loss of $\mathrm{T}$ cell effector function.

Methods

To overcome these challenges, we designed an innovative approach that capitalized the synergistic effect between pathogen-based immunotherapy and ACT for treating bladder cancer (Fig. 1). The tumor-reactive CD8 T cells were genetically engineered with a secondary bacterial TCR to generate dual-specific CD8 T cells, that can 
recognize both a tumor associated antigen (TAA) and a bacterial antigen (BA). In this study, the bladder tumor bearing mice were treated with ACT intravenously using a small number of these dual-specific CD8 T cells and accompanied by intratumoral injection (I.T.) of a low dose of BCG.

Results

The BCG infection alone sufficiently reduced the number of Treg and MDSC in the tumor microenvironment. In addition, the dual-specific CD8 T cells expanded robustly and migrated to the tumor bed in response to the infection. At the same time, these tumor-reactive CD8 $T$ cells recognized the tumor antigen and executed killer functions, which resulted site-specific tumor regression and protection against recurrence.

\section{Conclusions}

Our approach not just breaks the tumor mediated immunosuppression, but also provokes a strong anti-tumor immune reaction that lead to primary tumor eradication and long-term protection. Overall, this proof-of-principle study demonstrated the feasibility of this strategy, and open new avenues to treat bladder cancer.

\section{Reference}

1. Xin G, Schauder D, Jing W, Jiang A, Joshi N, Johnson B, Cui W. Pathogen boosted adoptive cell transfer immunotherapy to treat solid tumors. Proceedings of the National Academy of Sciences. 2017;114, no. 4: 740-745.

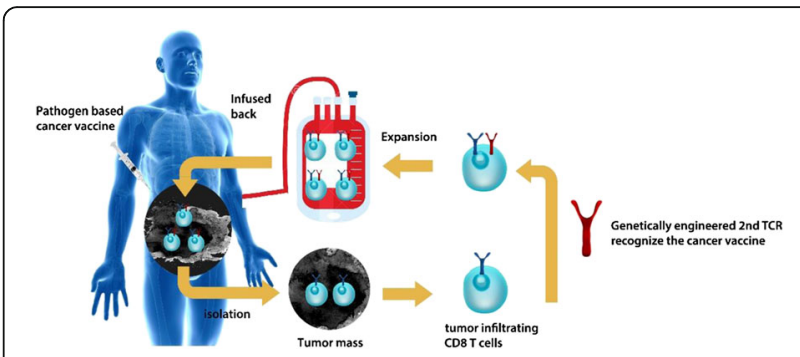

Fig. 1 (abstract P204). The conceptual model of ReACT in treating bladder cancer

\section{P205}

Chimeric antigen receptor (CAR) T cells incorporating linker and/or transmembrane domains derived from TNFRSF19 demonstrate superior anti-tumor activity

Ying Xiong, Darong Wu, Boro Dropulic, Rimas Orentas, Dina Schneider Lentigen Technology Inc., Gaithersburg, MD, USA

Correspondence: Dina Schneider (ying.xiong@lentigen.com)

Journal for ImmunoTherapy of Cancer 2017, 5(Suppl 2):P205

\section{Background}

Chimeric antigen receptor $T$ cells redirected to the $B$ cell antigen CD19 (CAR19) are an emerging treatment for a range of B cell malignancies. The functional relationship between the amino acid sequence of the CAR linker, the trans-membrane domain, and CAR T function is, however, poorly understood. We replaced the extracellular linker or trans-membrane domains from previously reported CD8 constructs with novel sequences derived from the tumor necrosis factor superfamily member TNFRSF19, with an aim to enhance the anti-tumor activity of CAR19.

Methods

Human T cells were transduced with CAR-encoding lentiviral vectors featuring linker and trans-membrane domains of various lengths derived from CD8, TNFRSF16, or TNFRSF19. CARs expression and activity was assessed by flow cytometry, CTL activity and cytokine production. Results

CART comprised of both linker (long or short) and trans-membrane domains of TNFRSF19 were the most potent in overnight in vitro killing and cytokine release assays (IFNg, TNFa and IL-2); however, the expression of CAR molecules on the surface of transduced T cells, as measured by flow cytometry, was low or undetected. When CART and Raji tumors were co-incubated at low E:T ratio (1:1) for up to 9 days, all constructs demonstrated a surprising cyclic pattern of CAR surface up-regulation. Constructs comprised of TNFRSF19 linker domains had very low CAR expression on day 1, strong up-regulation on days 4-7, and down-regulation by day 9, as tumors were being eliminated. By contrast, the expression of CD8 linker - containing CART cells remained prominent through day 9 , regardless of tumor elimination. The expression of exhaustion markers Tim-3, Lag-3 and PD-1 on the co-incubated T cells was similar between CARs with CD8 or TNFRSF19-derived linkers. CAR constructs with both linker and trans-membrane domains derived from TNFRSF19 cleared the tumors faster than CAR19 variants containing the CD8 linker and/or transmembrane domain.

Conclusions

Here we demonstrate that direct surface detection of CAR expression may not best represent the most active CAR species, unless coincubated with tumor target cell lines. Furthermore, TNFRFS19 derived-domains appear to be superior to CD8 linker and transmembrane domain in in vitro anti-leukemia cell assays.

P206

Tumor-penetrating recombinant protein anti-EGFR-iRGD enhance the extravasation and tumor penetration of lymphocytes in gastric cancer

Anqing Zhu' ${ }^{1}$, Huizi Sha ${ }^{2}$, Baorui Liu²

${ }^{1}$ Clinical College of Traditional Chinese and Western Medicine, Nanjing University of Chinese Medicine, Nanjing, China; ${ }^{2}$ The Comprehensive

Cancer Centre of Drum Tower Hospital, Nanjing, China

Correspondence: Baorui Liu (493668608@qq.com)

Journal for ImmunoTherapy of Cancer 2017, 5(Suppl 2):P206

\section{Background}

Efficient trafficking of T cells to the tumor site is a critical step for success of cancer immunotherapy. Cancer patients with tumors which are highly infiltrated with lymphocytes have shown enhanced survival rates. Although the gene transfer technology such as CAR-T is effective in redirecting $T$ cell activity against tumors, the lymphocytes infiltrating in solid tumor tissues is still limited by the poor tumor penetration of adoptive cells due to the complicated tumor microenvironment such as immunosuppression and aberrant vasculature. The tumor-penetrating peptide iRGD contains both a RGD domain which can target the integrin expressing on activated $T$ cells and a CendR motif increasing the vascular and tissue permeability. We have previously constructed a protein of bispecific targets and high permeability named anti-EGFR-iRGD,which could improve tumor penetration of antitumor drugs into extravascular tumor tissue in a tumor-specific and neuropilin-1-dependent manner.

Methods

Recombinant protein anti-EGFR-iRGD consisting of an anti-EGFR VHH fused to iRGD were expressed in E. coli BL21 and purified by nickelnitrilotriacetic acid affinity chromatography. We use gastric cancer cell lines and tumor-bearing mice to examine the synergy antitumor effect of adoptive immunotherapy combine with anti-EGFR-iRGD. In addition, the ability of recombinant protein anti-EGFR-iRGD to improve lymphocytes infiltration into tumors were analyzed in xenograft mouse model.

Results

In this study, no influence on cell viability, proliferation, phenotype and fuction was observed when lymphocytes co-cultured with antiEGFR-iRGD in vitro. Furthermore, the recombinant protein anti-EGFRiRGD exhibited synergy antitumor activity with adoptive cell therapy (ACT) in tumor cell lines and mice. In adoptive transfer studies, we found increased tumor extravasation and tumor penetration of our lymphocytes by the systematic co-administration with anti-EGFRiRGD for the first time.

\section{Conclusions}

Our results provide new insights for effectively target a higher frequency of lymphocytes to the tumor microenvironment, this will be an important clinical translation to improve immunotherapy outcomes using co-administration ACT of and anti-EGFR-iRGD. 
Reference

1. A. Jemal, F. Bray, M.M. Center, J. Ferlay, E. Ward, D. Forman, Global cancer statistics, CA: a cancer journal for clinicians. 2011; 61:69-90.

\section{Clinical Trials (Completed)}

\section{P207}

Phase II study of anti-CD3 $x$ anti-HER2/neu (HERBi) armed activated T cells (ATC) after neoadjuvant chemotherapy in patients with HER2/neu (0-2+)-negative stage II-III breast cancer

Naresh Bumma', Andrew Kin', Judith Abrams', Abhinav Deol', Amy Weise', Archana Thakur ${ }^{2}$, Lawrence Lum²

${ }^{1}$ Wayne State University/Karmanos Cancer Institute, Detroit, MI, USA; ${ }^{2}$ University of Virginia Cancer Center, Charlottesville, VA, USA

Correspondence: Naresh Bumma (bumman@karmanos.org) Journal for ImmunoTherapy of Cancer 2017, 5(Suppl 2):P207

\section{Background}

Patients with ER/PR negative, Her2/neu negative triple negative breast cancer (TNBC) have dismal outcomes as compared to other subtypes. Based on previous studies, pathological complete remission ( $\mathrm{pCR}$ ) after neoadjuvant chemotherapy is a good surrogate of long-term survival. Many studies have attempted to improve the efficacy of chemotherapy and increase the pCR rate. Currently, anti-Her2 targeting antibodies and chemotherapy are the only approved therapeutic options for primary systemic treatment. We conducted a phase II trial to evaluate safety and efficacy of infusions of anti-CD3 $x$ anti-Her2/neu bispecific antibody (HER2Bi)-armed activated $T$ cells (ATC) after neoadjuvant chemotherapy, surgery, and/or radiation for women with stage II-III operable TNBC who had residual disease at the time of surgical resection

Methods

After informed consent was obtained, patients received standard neoadjuvant chemotherapy followed by surgery. Eligible patients underwent pheresis after completion of standard therapy. T cells collected during pheresis were activated with anti-CD3 and expanded in $100 \mathrm{IU} / \mathrm{ml}$ of IL-2 to generate ATC. After culture, ATC were harvested, armed with HER2Bi, washed, and cryopreserved in 8 aliquots ( 10-15 billion Her2Bi-armed ATC/aliquot) for bi-weekly infusions for 4 weeks in combination with low dose IL-2 and GM-CSF (Fig. 1). Patients then received anthracycline and/or taxane based standard regimens. Following chemotherapy, surgery, and/or radiation, patients were given 10-15 billion Her2Bi-armed ATC twice per week for 4 weeks beginning starting no sooner than 3 weeks ( \pm 1 week) after completion of standard breast cancer treatment.

Research reported in this abstract was supported by $\mathrm{NIH}$ under award number R01 CA 140314.

Results

Eight patients were enrolled on the protocol. Median age was 44.5yrs (range, 38.5-52). Four patients (50\%) were African American (AA). Two had T2N0, 3 T2N1, 2 T3N1 and 1 T3N2. Everyone received 8 infusions except 1 patient withdrew after 1 infusion due to a grade 3 toxicity experience.

Most common infusion associated adverse events were chills/rigors $(87 \%)$, fatigue $(83 \%)$, nausea $(75 \%)$, fever $(75 \%)$, anemia $(62 \%)$, vomiting $(50 \%)$, hyperglycemia $(50 \%)$, neutropenia $(50 \%)$, rash $(38 \%)$. No dose limiting toxicity was identified in our study. After median follow up of 4.2 years, 3 of 8 patients had disease progression with median time to progression in these pts being 2.1 years.

\section{Conclusions}

We conclude that Her2Bi-armed ATC infusions in these patients were safe and it may improve progression free survival in this high risk group. This immunotherapeutic modality warrants further investigation.

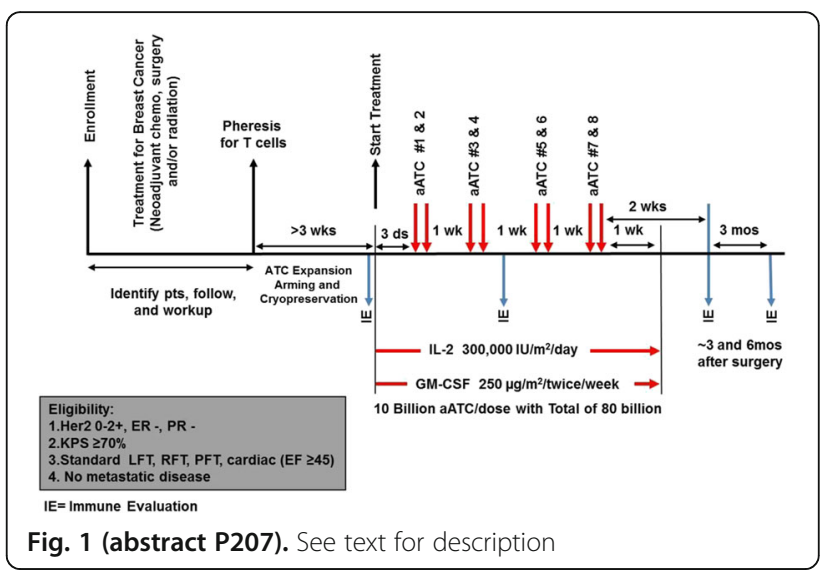

P208

Safety, efficacy and biology of the gp100 TCR-based bispecific T cell redirector, IMCgp100 in advanced uveal melanoma in two Phase 1 trials

Richard Carvajal', Takami Sato ${ }^{2}$, Alexander N. Shoushtari ${ }^{3}$, Joseph Sacco ${ }^{4}$, Paul Nathan ${ }^{5}$, Marlana Orloff ${ }^{2}$, Pippa Corrie ${ }^{6}$, Neil Steven ${ }^{7}$, Jeff Evans ${ }^{8}$, Jeffrey Infante ${ }^{9}$, Mario Sznol ${ }^{10}$, Clive Mulatero ${ }^{11}$, Omid Hamid ${ }^{12}$, Leonel Hernandez-Aya ${ }^{13}$, Nicola Little ${ }^{14}$, Cheryl McAlpine ${ }^{15}$, David Krige ${ }^{15}$, Namir J. Hassan ${ }^{16}$, Sanjay Patel ${ }^{16}$, Ann-Marie Hulstine ${ }^{17}$, Christina M. Coughlin ${ }^{17}$, Mark R. Middleton ${ }^{18}$

${ }^{1}$ Columbia University Medical Center, New York, NY, USA; ${ }^{2}$ Thomas Jefferson University, Philadelphia, PA, USA; ${ }^{3}$ Memorial Sloan Kettering Cancer Center, New York, NY, USA; ${ }^{4}$ Clatterbridge Cancer Center, Liverpool, UK; ${ }^{5}$ Mount Vernon Cancer Center, Middlesex, UK;

${ }^{6}$ Addenbroke's Hospital, Cambridge, UK; ${ }^{7}$ University of Birmingham, Birmingham, UK; ${ }^{8}$ Beatson Cancer Centre, Glasgow, UK; ${ }^{9}$ Sarah Cannon Cancer Research Institute, Nashville, TN, USA; ${ }^{10}$ Yale University Cancer Center, New Haven, CT, USA; ${ }^{11}$ Spire Leeds Hospital, West Yorkshire, UK; ${ }^{12}$ The Angeles Clinic, Santa Monica, CA, USA; ${ }^{13}$ Siteman Cancer Center, St. Louis, MO, USA; ${ }^{14}$ Department of Biostatistics, Alderley, UK;

${ }^{15}$ Biomarkers, Milton, UK; ${ }^{16}$ Immunocore, Ltd., Milton, UK; ${ }^{17}$ Immunocore, Ltd., Conshohocken, PA, USA; ${ }^{18}$ University of Oxford, Oxford, UK

Correspondence: Richard Carvajal (richard.carvajal417@gmail.com) Journal for ImmunoTherapy of Cancer 2017, 5(Suppl 2):P208

\section{Background}

Uveal melanoma (UM) is characterized by low PD-L1 expression, low mutational burden and limited efficacy with checkpoint inhibition. IMCgp100 is a bispecific T cell redirector with an affinity-enhanced TCR recognizing gp100 and an anti-CD3 scFV.

Methods

Two phase 1 trials evaluated safety, pharmacokinetics, pharmacodynamics and efficacy for IMCgp100 administered IV weekly in HLAA2 patients (pts): a first in human (FIH) study enrolling pts with melanoma including a cohort with advanced UM ( $n=16$, NCT01211262) and a second study of an intra-patient dose escalation (IE) regimen for pts with advanced UM ( $n=19$, NCT02570308). Endpoints included overall response rate (ORR) by RECISTv1.1, progression free survival (PFS), and overall survival (OS). The IE Phase 1 allowed treatment beyond progression assessed by modified irRC if specific clinical criteria were met.

Results

The safety profile of IMCgp100 was consistent between trials with the most frequent adverse events ( $A E$, any grade) including rash 
(90\%), pruritus (90\%), and edema (63\%). The most frequent grade $3 /$ $4 \mathrm{AE}$ was hypotension (16\%, IE study, $9 \% \mathrm{FIH}$ study). The recommended phase 2 dose (RP2D) in the FIH trial was $50 \mathrm{mcg}$ (DLT of hypotension). The IE schedule was designed to mitigate toxicity, with reduced doses of $20 \mathrm{mcg}$ and $30 \mathrm{mcg}$ administered at weeks 1 and 2 , respectively, and dose escalation at Day 15 . The RP2D was $68 \mathrm{mcg}$ (DLT of transaminase elevation). The ORR in the FIH and IE trials was $20 \%$ (3/15 evaluable) and $11 \%$ (2/19 evaluable), respectively. Five pts (26\%) in the IE trial achieved minor responses (10-29\% SLD reduction), including pts treated with prior checkpoint inhibition and with an elevated LDH. Median PFS in the FIH and IE trials (RECISTv1.1) was 3.7 months and 5.6 months, respectively. The 1 year PFS rate by irRC in the IE study was $62 \%$. The 1-year OS rate in the FIH and IE trials is $73 \%(95 \% \mathrm{Cl}[52,99])$ and $79.5 \%(95 \% \mathrm{Cl}[55,93])$, respectively. Median OS has not been reached with a minimum of 16 and 8 months follow-up in the FIH and IE trials, respectively. Within 3 doses of IMCgp100 (day 16), immunofluorescence studies reveal an influx of PD-1+/CD8+ T cells in the tumor bed with PD-L1 expression. Peripheral cytokines indicate activation of immune responses within 24 hours of the first dose.

\section{Conclusions}

These studies demonstrate preliminary immune biology, safety and promising efficacy in advanced UM. The pivotal trial with IE dosing is open in advanced UM (NCT03070392).

\section{P209}

Randomized trial of autologous dendritic cell vaccines versus autologous tumor cell vaccines in metastatic melanoma Robert Dillman', Edward McClay², Thomas Amatruda ${ }^{3}$, Andrew Cornforth $^{4}$, Carol Depriest ${ }^{5}$

${ }^{1}$ Hoag Cancer Institute, Newport Beach, CA, USA; ${ }^{2}$ Institute for Melanoma Research \& Education, Encinitas, CA, USA; ${ }^{3}$ Minnesota Oncology, Fridley, MN, USA; ${ }^{4}$ TCR2 Therapeutics, Cambridge, MA, USA; ${ }^{5}$ Hoag Hospital, Franklin, TN, USA

Correspondence: Robert Dillman (robert.dillman55@gmail.com) Journal for ImmunoTherapy of Cancer 2017, 5(Suppl 2):P209

\section{Background}

Metastatic melanoma cells contain high numbers of nonsynonymous mutations, making this cancer a prime target for immunotherapies including patient-specific vaccines that utilize autologous tumor associated antigens (TAA). In a 74-patient trial metastatic melanoma patients treated with autologous tumor cell vaccines (TCV) consisting of autologous irradiated tumor cells (ITC) had a median overall survival (OS) of 20.5 months and 5-year OS of $28 \%$. [1] In a subsequent 54-patient trial similar patients treated with a dendritic cell vaccine (DCV) consisting of autologous dendritic cells loaded with TAA from autologous ITC had a median OS greater than 5 years and a 5year OS of 50\%. [2] An open-label randomized phase 2 trial was initiated to compare TCV and DCV.

Methods

Short-term autologous tumor cell lines were established from resected metastatic lesions and served as TAA sources. Vaccines were injected with 500 micrograms of granulocyte-macrophage colony stimulating factor (GM-CSF) weekly for 3 weeks, then at weeks 8, $12,16,20$, and 24. Key objectives were OS, adverse events (AE), and delayed type hypersensitivity (DTH) reactions to injections of intradermal tumor cells.

Results

Forty-two patients were enrolled and all treated as randomized. An interim analysis showed superiority of DCV, but median follow up was less than 2 years and minimum follow up was only 6 months. [3] As of the final analysis, all patients have been followed for 5 years or until death with no patients lost to follow up. DCV was associated with longer OS: median 43.4 versus 20.5 months ( $95 \% \mathrm{Cl}, 18.6$ to $>60$ versus 9.3 to 32.3 months). Cox regression analysis identified tumor burden at the time of randomization and treatment arm as significant independent variables. Cox proportional hazards model revealed a $70 \%$ reduction in the risk of death in the DCV arm (hazard ratio $=0.304$, $\mathrm{p}=0.0053,95 \% \mathrm{Cl}, 0.131$ to 0.702 ). There was no association between efficacy and DTH reactions to tumor cell skin tests. The most common treatment-related $\mathrm{AE}$ were mild to moderate local injection site reactions and flu-like symptoms; grade 2 treatmentrelated $\mathrm{AE}$ were more frequent in the TCV arm.

Conclusions

As a therapeutic vaccine, DCV appears to be superior to TCV for autologous TAA presentation. Autologous DCV is associated with minimal toxicity and extended long-term survival benefit in patients with metastatic melanoma.

Trial Registration

ClinicalTrials.gov (NCT00436930)

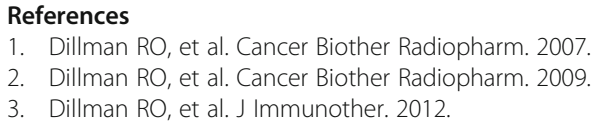

\section{P210}

Pilot trial of sipuleucel-T, with or without pTVG-HP DNA vaccine, in patients with metastatic, castration-resistant prostate cancer (mCRPC)

Ellen Wargowski, Laura Johnson, Glenn Liu, Jens Eickhoff, Mary Jane Staab, Douglas McNeel

University of Wisconsin, Madison, WI, USA

Correspondence: Douglas McNeel (dm3@medicine.wisc.edu)

Journal for ImmunoTherapy of Cancer 2017, 5(Suppl 2):P210

\section{Background}

Sipuleucel-T is an anti-tumor vaccine approved by FDA for the treatment of advanced metastatic prostate cancer. Sipuleucel-T targets the prostate-specific antigen prostatic acid phosphatase (PAP). We have investigated PAP as a target antigen using a DNA vaccine, pTVG-HP, in patients with earlier stages of prostate cancer, and have demonstrated that this vaccine can elicit Th1-biased PAP-specific immunity. The current trial was designed to evaluate these two vaccines together, in a prime-boost fashion, to determine whether booster immunizations with pTVG-HP could augment PAP-specific effector and memory T cells following treatment with sipuleucel-T.

Methods

18 patients with asymptomatic, metastatic, castration-resistant prostate cancer were treated in a single-institution trial from 2013 to 2016 in which they were randomized to receive sipuleucel-T alone, as per standard of care, or sipuleucel-T followed by pTVG-HP administered as an intradermal injection with $200 \mu \mathrm{g} \mathrm{GM-CSF}$ as adjuvant every 2 weeks for 4 immunization, and then quarterly at months 6 and 9. Serial blood draws were obtained for immune analyses up to one year. CT scans and bone scans were performed at 3-month intervals.

Results

10/18 patients completed treatment per protocol, with 8 experiencing disease progression requiring discontinuation. No unanticipated adverse events were observed, and there were no treatment-associated adverse events greater than grade 2. Th1biased PAP-specific $T$ cell responses (defined as significant antigen-specific IFNg or granzyme B ELISPOT responses that were at least 3-fold over baseline, with a frequency of at least 1:100,000 peripheral blood cells, and detectable at least twice post-treatment) were detected in 11/18 individuals, and not statistically different between study arms. Antibody responses to PAP were significantly higher in patients receiving PTVG-HP booster immunizations. Median time to progression was less than 6 months and not statistically different between study arms; median overall survival was 31 months.

\section{Conclusions}

Prime-boost vaccination with sipuleucel-T and a DNA vaccine encoding the same antigenic target is feasible, and without obvious toxicity. Th1 immunity to PAP was elicited with sipuleucel-T and was not significantly augmented with booster immunization. Antibody 
immunity to PAP was augmented with pTVG-HP DNA immunization, a finding that has not been observed in subjects treated with pTVGHP alone.

Trial Registration

NCT01706458

\section{P211}

Phase 2 trial exploring allogeneic vaccine therapy in late-stage, second-line non-small cell lung cancer

Christopher Meldrum', Eckhard Podack², Jonathan Serody ${ }^{3}$, Varada

Bidargaddi $^{3}$

${ }^{1}$ Pique Therapeutics, Inc., Durham, NC, USA; ${ }^{2}$ Pique Therapeutics, Inc., Coconut Grove, FL, USA; ${ }^{3}$ University of North Carolina at Chapel Hill, Chapel Hill, NC, USA

Correspondence: Christopher Meldrum

(cmeldrum@piquetherapeutics.com)

Journal for ImmunoTherapy of Cancer 2017, 5(Suppl 2):P211

\section{Background}

Lung carcinoma is the leading cause of cancer in the United States, and non-small cell lung cancer (NSCLC) is the most common form [1]. Long-term outcome for patients with late-stage NSCLC receiving front-line chemotherapy is poor with few patients alive more than 30 months after therapy. Recent approaches using checkpoint inhibitor therapy (CPI) have shown promise, although tumors with specific genetic mutations may not respond to CPI. [2] Vaccine treatment has the potential of inducing immune responses in patients with lung cancer who poorly respond to $\mathrm{CPI}$. Previous Phase 1 results in treating second-line, late-stage NSCLC with an allogeneic whole-cell vaccine transfected with B7.1 and HLA (PT 107) were promising [3]. Methods

We randomized in a 2:1 format 94 patients with advanced (stage IIIB/ IV) NSCLC to receive intradermal injection of an irradiated whole cell allogeneic lung cancer vaccine that had been engineered to express HLA-A1 and B7.1 (PT 107 arm) or placebo (control arm). Patients in the drug-treated arm received up to 9 intradermal vaccinations of PT 107 every 2 weeks.

Results

The baseline characteristics of the patients enrolled are shown below (Table 1).

Median overall survival for the PT 107 arm was 14.9 months versus 6.7 months for the control arm, with a hazard ratio of 0.71 (one-sided $\mathrm{p}=0.188$ ). Median progression-free survival was 2.8 months (PT 107 arm) versus 2.6 months (control arm), with a hazard ratio of 0.65 (one-sided $\mathrm{p}=0.074$ ). Median time to progression was 4.0 months (PT $107 \mathrm{arm}$ ) versus 2.5 months (control arm), with a statistically significant hazard ratio of 0.45 (one-sided $p=0.016$ ). Safety results were consistent with previous clinical experience, with no drug-related serious adverse events reported (Table 2).

\section{Conclusions}

While not powered to detect highly significant differences in outcome, this study demonstrated that treatment with PT 107 was associated with an improvement in overall survival and a significant improvement in time to progression compared to placebo therapy. These data provide strong rationale for a larger phase 3 clinical trial, as well as approaches to combine PT 107 with standard of care CPI.

\section{Trial Registration}

CTRI/2010/091/001348

\section{References}

1. American Cancer Society. Cancer Facts \& Figures. 2017

2. Lee CK, et al. Checkpoint Inhibitors in Metastatic EGFR-Mutated Non-Small Cell Lung Cancer - A Meta-Analysis. J Thor Oncol. 2017;12: 403-407.

3. Raez LE, et al. Allogeneic Vaccination With a B7.1 HLA-A Gene-Modified Adenocarcinoma Cell Line in Patients With Advanced Non-Small-Cell Lung Cancer. J Clin Oncol. 2004; 22: 2800-2807.
Table 1 (abstract P211). Summary of Patient Baseline Characteristics

\begin{tabular}{|c|c|c|c|c|c|}
\hline & & $\begin{array}{lll}\text { PT } 107 \\
\end{array}$ & Control & 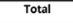 & $\begin{array}{l}\text { p-value } \\
\end{array}$ \\
\hline \multirow[t]{4}{*}{ ECOG } & & & & & 0.3787 \\
\hline & ECOG Performance Status 0 & $27(43 \%)$ & $10(32 \%)$ & $37(39 \%)$ & \\
\hline & ECOG Performance Status 1 & $27(43 \%)$ & $18(58 \%)$ & 45 (48\%) & \\
\hline & \begin{tabular}{|l|l|}
$E C O G$ Performance Status 2 \\
\end{tabular} & $9(14 \%)$ & $3(10 \%)$ & $12(13 \%)$ & \\
\hline \multirow[t]{6}{*}{ Type } & & & & & 0.8196 \\
\hline & Bronchioloalveolar carcinoma & $2(3 \%)$ & 0 & $2(2 \%)$ & \\
\hline & Lung adenocarcinoma & $26(41 \%)$ & $15(48 \%)$ & $41(44 \%)$ & \\
\hline & $\begin{array}{l}\text { Lung adenocarcinoma with bronchial } \\
\text { features }\end{array}$ & $2(3 \%)$ & $1(3 \%)$ & $3(3 \%)$ & \\
\hline & Non-small cell lung cancer, NOS & $18(29 \%)$ & $7(23 \%)$ & $25(27 \%)$ & \\
\hline & Squamous cell lung carcinoma & $15(248)$ & $8(26 \%)$ & 23 (248) & \\
\hline \multirow[t]{3}{*}{ Stage } & & & & & 0.6849 \\
\hline & Stage IIII & $23(37 \%)$ & $10(32 \%)$ & $33(35 \%)$ & \\
\hline & Stage IV & $40(63 \%)$ & $21(68 \%)$ & $61(65 \%)$ & \\
\hline \multirow[t]{4}{*}{ Smoking Habit } & & & & & 0.3678 \\
\hline & Never & $33(52 \%)$ & $15(48 \%)$ & $48(51 \%)$ & \\
\hline & Current & $3(5 \%)$ & $4(13 \%)$ & $7(7 \%)$ & \\
\hline & Former & $27(43 \%)$ & $12(39 \%)$ & $39(42 \%)$ & \\
\hline \multirow[t]{3}{*}{$\begin{array}{l}\text { Gender } \\
\end{array}$} & & & & & 0.7652 \\
\hline & Male & $47(75 \%)$ & $24(77 \%)$ & $71(76 \%)$ & \\
\hline & $\begin{array}{c}\text { Female } \\
\end{array}$ & $16(25 \%)$ & $7(23 \%)$ & $23(24 \%)$ & \\
\hline \multirow[t]{4}{*}{ Age } & & & & & 0.6569 \\
\hline & $n$ & 63 & 31 & 94 & \\
\hline & Mean (SD) & $55.2(12.9)$ & $54.1(8.6)$ & $54.8(11.6)$ & \\
\hline & Median & 58.0 & 55.0 & 57.0 & \\
\hline
\end{tabular}

Table 2 (abstract P211). Summary of Adverse Events

\begin{tabular}{|c|c|c|c|c|c|}
\hline Adverse Events (AE) & & & & & \\
\hline & Subjects with at least $1 \mathrm{AE}$ & $33(52 \%)$ & $12(39 \%)$ & $45(48 \%)$ & \\
\hline \multicolumn{6}{|l|}{$\begin{array}{l}\text { AE Relationship to Study } \\
\text { Drug }\end{array}$} \\
\hline & Definite & 0 & 0 & 0 & \\
\hline & Probable & $4(6 \%)$ & 0 & $4(4 \%)$ & \\
\hline & Possible & $2(3 \%)$ & $1(3 \%)$ & $3(3 \%)$ & \\
\hline & Unlikely & $1(2 \%)$ & $2(7 \%)$ & $3(3 \%)$ & \\
\hline & Unrelated & $26(41 \%)$ & $9(29 \%)$ & $35(37 \%)$ & \\
\hline \multicolumn{6}{|l|}{$\begin{array}{l}\text { Serious Adverse Events } \\
\text { (SAE) }\end{array}$} \\
\hline & Subjects with at least $1 \mathrm{SAE}$ & $15(24 \% 6)$ & $5(16 \%)$ & $20(21 \%)$ & \\
\hline \multicolumn{6}{|l|}{$\begin{array}{l}\text { SAE Relationship to } \\
\text { Study Drug }\end{array}$} \\
\hline & Definite & 0 & 0 & 0 & \\
\hline & Probable & 0 & 0 & 0 & \\
\hline & Possible & 0 & $1(3 \%)$ & $1(1 \%)$ & \\
\hline & Unlikely & 0 & 0 & 0 & \\
\hline & Unrelated & $15(24 \%)$ & $4(13 \%)$ & $19(20 \%)$ & \\
\hline \multicolumn{6}{|l|}{ Grade 3+ Toxicity } \\
\hline & Subjects with Grade 3+ Toxicity & $25(40 \%)$ & $10(32 \%)$ & $35(37 \%)$ & \\
\hline \multirow{6}{*}{$\begin{array}{l}\text { Grade 3+ Toxicity } \\
\text { Reationshipio to Study } \\
\text { Drug }\end{array}$} & & & & & \\
\hline & Definite & 0 & 0 & 0 & \\
\hline & Probable & $2(3 \%)$ & 0 & $2(2 \%)$ & \\
\hline & $\begin{array}{llll}\text { Possible } \\
\end{array}$ & $1(2 \%)$ & 1 (3\%) & $2(2 \%)$ & \\
\hline & Unlikely & $1(2 \%)$ & $1(3 \%)$ & $2(2 \%)$ & \\
\hline & Unrelated & $21(33 \%)$ & $8(26 \%)$ & $29(31 \%)$ & \\
\hline
\end{tabular}

P212

Tumor immune infiltration in patients with early stage breast cancer after preoperative treatment with IRX-2

David B. Page1, Joanna Pucilowska', Nikki Moxon', Staci Mellinger',

Alison Conlin', James Imatani ${ }^{1}$, Shaghayegh Aliabadi-Wahle', Valerie

Conrad $^{1}$, Maritza Martel', Kelly Perlewitz', Neil L. Berinstein², James E. Egan $^{2}$, William L. Redmond', Walter J. Urba

'EACRI, Portland, OR, USA; ${ }^{1}$ IRX Therapeutics, NY, NY, USA

Correspondence: David B. Page (David.Page2@providence.org)

Journal for ImmunoTherapy of Cancer 2017, 5(Suppl 2):P212

\section{Background}

The IRX-2 biologic is an injectable cancer immunotherapy containing multiple cytokines derived from stimulated lymphocytes. In preclinical models, it activates $T$ cells, natural killer cells and differentiates immature dendritic cells into mature antigen-presenting cells. In a previous phase II trial, neoadjuvant IRX-2 increased tumor-infiltrating lymphocytes (TILs) and shrank tumors in resectable head/neck squamous carcinoma (SCC). ${ }^{1}$ Since stromal TILs (sTILs) are associated with improved survival and neoadjuvant chemotherapy response in early stage breast cancer $(\mathrm{ESBC})$, we conducted a phase $\mathrm{lb}$ trial to evaluate the feasibility 
of preoperative IRX-2, and its effect on TIL recruitment and immune priming within breast tumors, regional lymphatics, and blood.

Methods

Patients with early stage (I-III) breast cancer indicated for standard-ofcare surgical lumpectomy/mastectomy were enrolled in this phase $\mathrm{Ib}$ trial. Twenty-one days prior to surgery, all patients received a single low dose of cyclophosphamide $\left(300 \mathrm{mg} / \mathrm{m}^{2}\right)$ to facilitate T-regulatory (Treg) cell depletion, followed by 10 days of subcutaneous peri-areolar injections of IRX-2 into the affected breast $(1 \mathrm{~mL} \times 2$ at tumor axis and at $90^{\circ}$ ). The primary endpoint was feasibility. The secondary endpoint was blinded assessment of sTILs by the 2015 San Antonio working group criteria $^{2}$ (measured as \% of total area); exploratory endpoints included comprehensive immune monitoring of TILs and blood.

Results

As of August 2017, 12 patients are enrolled and evaluable. Each enrollee received all planned injections with no treatment-related surgical delays, complications, or grade III/IV toxicities. Treatment was associated with increases in sTILs in 6/12 subjects, corresponding with a mean $52 \%$ relative increase (range: $-25 \%$ to $+166 \%$ ) and $6 \%$ absolute increase in sTILs (range: $-5 \%$ to $+23 \%, p=0.02$ paired t-test). Increases in T cell activation markers (ICOS, HLADR, and CD38) and decreases in peripheral T-reg quantity, proliferation (Ki67), and function (GzmB) were observed in blood during and 1 month following IRX-2.

\section{Conclusions}

Peri-lymphatic IRX-2 was well tolerated with preliminary evidence of TIL recruitment, peripheral lymphocyte activation, and T-reg depletion. Further assessment by Nanostring analysis, TCR sequencing, and multispectral immunohistochemistry is underway. Study enrollment is ongoing, including patients for a pre-neoadjuvant-chemotherapy triple-negative ESBC cohort. Trial Registration

NCT02950259

\section{References}

1. Whiteside $T L$, Butterfield $L H$, Naylor PH, et al. A short course of neoadjuvant IRX-2 induces changes in peripheral blood lymphocyte subsets of patients with head and neck squamous cell carcinoma. Cancer Immunol Immunother. 2012; 61:783-788.

2. Salgado R, Denkert C, Demaria $S$, et al. The evaluation of tumorinfiltrating lymphocytes (TILs) in breast cancer: recommendations by an International TILs Working Group 2014. Ann Oncol. 2015;26:259-271.

\section{P213}

Efficacy and tolerability of tremelimumab in locally advanced or metastatic urothelial carcinoma

Padmanee Sharma', Joo Hyuk Sohn², Sang Joon Shin ${ }^{2}$, Do-Youn $\mathrm{Oh}^{3}$

Bhumsuk Keam ${ }^{3}$, Hyo Jin Lee', Jean-Luc Canon', Ewa Kalinka-Warzocha', Marlies Langenberg ${ }^{7}$, James Allison ${ }^{8}$, Yong Ben ${ }^{9}$, Ritesh Kataria ${ }^{9}$, Salvatore Ferro ${ }^{9}$, Kobby Asubonteng ${ }^{9}$, LuC Dirix ${ }^{10}$

${ }^{1} \mathrm{MD}$ Anderson Cancer Center, Houston, TX, USA; ${ }^{2}$ Yonsei University Severance Hospital, Seoul, Republic of Korea; ${ }^{3}$ Seoul National University Hospitial, Seoul, Republic of Korea; ${ }^{4}$ Chungnam National University Hospital, Daejeon, Republic of Korea; ${ }^{5}$ Grand Hopital de Charleroi, Charleroi, Belgium; ${ }^{6}$ Polish Mother's Memorial Hospital-Research Institute, Lodz, Poland; ${ }^{7}$ University Medical Center Utrecht, Utrecht, Netherlands; ${ }^{8}$ University of Texas MD Anderson Cancer Center, Houston, TX, USA; ${ }^{9}$ AstraZeneca, Gaithersburg, MD, USA; ${ }^{10}$ St-Augustinus Ziekenhuis, Wilrijk, Belgium

Correspondence: Padmanee Sharma (andrea.hammons@PAREXEL.com) Journal for ImmunoTherapy of Cancer 2017, 5(Suppl 2):P213

\section{Background}

The efficacy of anti-CTLA-4 antibody monotherapy in metastatic urothelial carcinoma (UC) is not yet known. Tremelimumab was evaluated in patients with advanced solid tumors in a phase 2 multicenter, open label study. We report a planned analysis of safety and efficacy in a cohort of patients with locally advanced/metastatic UC.

Methods

Eligible patients were adults with histologically or cytologically confirmed UC who had progressed on, were ineligible for, or refused prior chemotherapy. Patients received tremelimumab $750 \mathrm{mg}$ intravenously every 4 weeks (Q4W) for 7 doses, followed by $750 \mathrm{mg}$ Q12W for 2 doses, for up to a total of 12 months or until disease progression, initiation of another anticancer therapy, or unacceptable toxicity. The primary endpoints were safety (evaluated by Common Terminology Criteria for Adverse Events v4.0) and confirmed objective response rate (ORR).

Results

As of April 5, 2017, 32 patients from 8 sites in 5 countries had received treatment and were eligible for efficacy analysis. All had stage IV disease and the majority had received prior platinum-based treatment. Median follow-up for overall survival (OS) was 4.5 months (range, 0.2 -12.0 ). Objective response was observed in 6 patients (ORR 18.8\%; 95\% $\mathrm{Cl}, 7.2 \%-36.4 \%)$, including 2 with a complete response. Responses occurred as early as 8 weeks after initiation of treatment (median time to response, 3.3 months) and were durable (median duration of response not reached). For the available PD-L1 dataset, response was observed in both PD-L1 high and PD-L1 low/negative patients (PD-L1 expression assessed using the Ventana SP263 assay; PD-L1 high = staining on $\geq 25 \%$ of tumor or immune cells). Median progression-free survival and OS were 3.7 months $(95 \% \mathrm{Cl}, 2.1-9.0)$ and 9.6 months $(95 \% \mathrm{Cl}, 7.2$, not estimable), respectively. Treatment-related adverse events (AEs) occurred in $53.1 \%$ of patients; grade $\geq 3$ treatment-related AEs and treatment-related serious AEs occurred in $18.8 \%$ of patients each. Four patients (12.5\%) discontinued treatment due to an AE. There were no treatment-related deaths. Biomarker analysis will be presented.

Conclusions

Tremelimumab monotherapy showed favorable clinical activity and an encouraging and manageable safety profile in patients with locally advanced/metastatic UC.

Trial Registration

NCT02527434

P214

T cells transfer and boost anti-breast cancer immunity after stem cell transplant

Lawrence G Lum¹, Archana Thakur', Sri Vidya Kondadasula², Joseph P

Uberti', Voravit Ratanatharathorn ${ }^{2}$

${ }^{1}$ University of Virginia, Charlottesville, VA, USA; ${ }^{2}$ Karmanos Cancer

Institute, Detroit, MI, USA

Correspondence: Archana Thakur (at2fx@virginia.edu)

Journal for ImmunoTherapy of Cancer 2017, 5(Suppl 2):P214

\section{Background}

We investigated whether cellular and humoral anti-breast cancer immunity induced by infusions of OKT3 x HER2 bispecific antibody armed activated T cells (BATs) can be transferred after high dose chemotherapy (HDC) and autologous stem cell transplant (SCT) by immune activated T cells (ATC) obtained after BATs infusion.

Methods

Eight metastatic breast cancer (MBC) patients received 8 infusions of BATs, low dose IL-2, and GM-CSF. Seven to 14 days after the last BATs infusion, the patients were leukapheresed for immune T cells. Immune T cells were expanded and cryopreserved for 8-15 booster infusions after SCT.

Results

Six of $8 \mathrm{MBC}$ patients were evaluable. There were no dose-limiting toxicities or delays in engraftment. One patient had sepsis after SCT and was treated successfully. Four of 6 patients exhibited increases in antibreast cancer (BrCa) cytotoxicity, NK activity, and IFN- $y$ Elispots after infusions of BATs and these responses were seen 2 weeks after SCT and persisted up to 2 years post-SCT. We verified the specificity of immune responses by multiple tests that provide evidence of $T$ cell and serum antibody reactivity to multiple 9-mer peptides (three HER-2, two pooled EGFR and three CEA peptides) in post IT and post SCT samples. Analysis of $V \beta$ repertoire and the quantitation of peptide-specific $C D 8^{+} T$ cells demonstrated that there were $T$ cell clones that could bind to the HLApeptide complex in higher frequencies in post IT and of memory $\mathrm{T}$ cell clones in ATC boost post SCT. Likewise, there were epitope specific antibody induction after infusions of HER2 BATs and transfer of memory B cells in ATC boost after SCT. A significant correlation $(r=1.0$; $\mathrm{p}<0.002$ ) between immune ATC cytotoxicity directed at BrCa cells and TTP strongly suggests that more robust vaccinations with a $\mathrm{Th}_{1}$ shift in cytokine profiles can lead to clinical benefit. 


\section{Conclusions}

Multiple HER2 BATs infusions may result in immunogenic epitope/antigen spreading and development of the broad and durable T- and Bcell memory responses after multiple infusions of HER2 BATs and "transfer of immunity" by immune ATC after SCT that parallels with the clinical responses.This study shows that adoptive transfer of immune $\mathrm{T}$ cells after SCT accelerates reconstitution of cellular, humoral anti-BrCa immunity and may delay TTP.

Trial Registration

NCT00027807

NCT00020722

\section{P215}

Randomized phase $\mathrm{I} / \mathrm{Il}$ pilot study using all-trans retinoic acid to target myeloid-derived suppressor cells in melanoma patients undergoing Ipilimumab therapy

Richard P. Tobin, Kimberly R. Jordan, William A. Robinson, Dana Davis, Virginia F. Borges, Rene Gonzalez, Karl D. Lewis, Martin D. McCarter University of Colorado Anschutz Medical Campus, Aurora, CO, USA Correspondence: Martin D. McCarter (richard.tobin@ucdenver.edu) Journal for ImmunoTherapy of Cancer 2017, 5(Suppl 2):P215

\section{Background}

Immune checkpoint inhibitors have improved overall survival rates for many cancers, yet the majority of patients do not respond to treatment and succumb to disease progression [1]. One tumorrelated mechanism limiting the efficacy of immunotherapies is the recruitment and expansion of myeloid-derived suppressor cells (MDSCs), a heterogeneous population of immature immunosuppressive myeloid cells, associated with poor outcomes in melanoma patients $[2,3]$. Therefore, enhancing the efficacy of current immunotherapies by targeting MDSCs throughout treatment is an attractive strategy to improve response rates and effectiveness.

\section{Methods}

Following an IRB approved protocol, a total of ten patients were recruited for this randomized controlled clinical trial. Advanced melanoma patients were randomized into two arms, standard of care Ipilimumab (Arm A), or Ipilimumab plus ATRA (Arm B). MDSC gene expression was measured by qRT-PCR. Flow cytometry was used to quantify circulating MDSC populations and T cell activation.

Results

Here we show that in vitro treatment with all-trans retinoic acid (ATRA) decreases the expression of immunosuppressive genes (PDL1, IL10, TGFB, NADPH oxidase 1, and indoleamine 2,3-dioxygenase) in human MDSCs. Furthermore, the addition of ATRA to standard of care Ipilimumab therapy is safe and significantly decreases the frequency of circulating MDSCs compared to Ipilimumab treatment alone in advanced-stage melanoma patients $(p=0.017)$ (Figure 1). Additionally, throughout therapy, the frequency of circulating MDSCs inversely correlated with the frequency of activated CD8+T cells $(p=0.021)$.

\section{Conclusions}

These results illustrate the importance of MDSCs in immunotherapy resistance and provide evidence that targeting MDSCs in cancer patients may augment future immunotherapeutic approaches. The strategy of targeting MDSCs in combination with current immunotherapies appears to be a viable and safe therapeutic approach. Trial Registration

This trial is registered at clinicaltrials.gov as NCT02403778.

\section{References}

1. Hodi FS, O'Day SJ, McDermott DF, Weber RW, et al. Improved survival with ipilimumab in patients with metastatic melanoma. N Engl J Med 2010;363:711-23

2. Sade-Feldman $M$, Kanterman J, Klieger $Y$, et al. Clinical significance of circulating CD33+CD11b+HLA-DR- myeloid cells in Stage-IV melanoma patients treated with ipilimumab. Clin Cancer Res 2016.

3. Jordan KR, Amaria RN, Ramirez O, et al. Myeloid-derived suppressor cells are associated with disease progression and decreased overall survival in advancedstage melanoma patients. Cancer Immunol Immunother 2013;62:1711-22.
P216

Long-term survival in patients with advanced melanoma, renal cell carcinoma, or non-small cell lung cancer treated with nivolumab Suzanne L. Topalian', F. Stephen Hodi², Julie R. Brahmer ${ }^{3}$, Scott N. Gettinger ${ }^{4}$, David C. Smith ${ }^{5}$, David F. McDermott ${ }^{6}$, John D. Powderly ${ }^{7}$ Jeffrey A. Sosman ${ }^{8}$, Michael B. Atkins ${ }^{9}$, David R. Spigel ${ }^{10}$, Scott J. Antonia $^{11}$, Alexander Drilon ${ }^{12}$, Rafia Bhore ${ }^{13}$, Fareeda Hosein ${ }^{13}$, Mario Sznol ${ }^{4}$

'Johns Hopkins Bloomberg Kimmel Institute for Cancer Immunotherapy, Baltimore, MD, USA; ${ }^{2}$ Dana-Farber Cancer Institute, Boston, MA, USA; ${ }^{3}$ Sidney Kimmel Comprehensive Cancer Center, Johns Hopkins University, Baltimore, MD, USA; ${ }^{4}$ Yale Cancer Center, New Haven, CT, USA; ${ }^{5}$ University of Michigan Comprehensive Cancer Center, Ann Arbor, MI, USA; ${ }^{6}$ Beth Israel Deaconess Medical Center, Dana-Farber/ Harvard Cancer Center, Boston, MA, USA; ${ }^{7}$ Carolina Bio-Oncology Institute, Huntersville, NC, USA; ${ }^{8}$ Northwestern University Medical Center, Chicago, IL, USA; ${ }^{9}$ Georgetown University Medical Center - Lombardi Comprehensive Cancer Center, Washington, DC, USA; ${ }^{10}$ Lung Cancer Research Program, Sarah Cannon Research Institute, Nashville, TN, USA;

${ }^{11}$ Moffitt Cancer Center, Tampa, FL, USA; ${ }^{12}$ Memorial Sloan Kettering Cancer Hospital, New York, NY, USA; ${ }^{13}$ Bristol-Myers Squibb, Princeton, NJ, USA

Correspondence: Suzanne L. Topalian (steve.kavakellis@parexel.com) Journal for ImmunoTherapy of Cancer 2017, 5(Suppl 2):P216

\section{Background}

Nivolumab, a fully human lgG4 antibody that inhibits programmed death receptor-1 (PD-1), is FDA-approved for treating advanced melanoma (MEL), renal cell carcinoma (RCC), non-small cell lung cancer (NSCLC), and several other malignancies. Here we report the longest available overall survival (OS) outcomes for any PD-1/PD ligand-1 (PD-L1) inhibitor using data from patients with treatment-refractory MEL, RCC or NSCLC who received nivolumab in the phase 1 CA209-003 study.

Methods

Eligible adult patients had progressive cancers after 1-5 previous systemic therapies, with no prior immunotherapy (e.g., anti-PD-1, anti-PDL1, anti-cytotoxic T-lymphocyte antigen-4 [CTLA-4] antibodies). Patients enrolled in 2008-2012 received nivolumab (0.1-10.0 mg/kg) every 2 weeks in 8-week cycles for up to 96 weeks, unless they developed progressive disease, a complete response, unacceptable toxicity, or withdrew consent. Tumor response was assessed by RECIST v1.0 after each treatment cycle. Patients with stable disease or tumor regression, who experienced off-therapy disease progression within 1-year of stopping nivolumab, could resume nivolumab for $\leq 96$ weeks.

Results

270 patients with advanced MEL $(\mathrm{N}=107), \mathrm{RCC}(\mathrm{N}=34)$, or NSCLC $(\mathrm{N}=129$; squamous, $\mathrm{n}=54$; non-squamous, $\mathrm{n}=74$; unknown histology, $\mathrm{n}=1$ ) received nivolumab. Most patients were heavily pretreated: 194 (71.9\%) had received $\geq 2$ prior regimens, and $112(41.5 \%) \geq 3$ prior regimens. Minimum follow-up for OS was 58.3 (MEL), 63.9 (RCC), 58.3 (squamous NSCLC), and 60.8 (non-squamous NSCLC) months. OS outcomes are shown in the table (Table 1). Long-term safety in these cohorts has been reported previously [1-3]. Long-term (5-year) survivors, patients with durable response, and retreated patients will be characterized by clinical and demographic factors, treatments received, and depth of tumor response.

Conclusions

Nivolumab therapy demonstrates durable survival in some heavily pretreated patients with advanced MEL, RCC, or NSCLC. Characterizing long-term survivors and the potential benefits of retreatment with nivolumab will inform future treatment strategies.

Trial Registration

ClinicalTrials.gov: NCT00730639

\section{References}

1. Topalian SL, Sznol M, McDermott DF, et al. Survival, durable tumor remission, and long-term safety in patients with advanced melanoma receiving nivolumab. J Clin Oncol. 2014;32:1020-30.

2. McDermott DF, Drake CG, Sznol M, et al. Survival, durable response, and long-term safety in patients with previously treated advanced renal cell carcinoma receiving nivolumab. J Clin Oncol. 2015;33:2013-20. 
3. Gettinger SN, Horn L, Gandhi L, et al. Overall survival and long-term safety of nivolumab (anti-programmed death 1 antibody, BMS-936558, ONO-4538) in patients with previously treated advanced non-small-cell lung cancer. J Clin Oncol. 2015;33:2004-12.

Table 1 (abstract P216). See text for description

\begin{tabular}{|l|c|c|c|c|}
\hline & $\begin{array}{c}\text { MEL } \\
(\mathbf{n}=107)\end{array}$ & $\begin{array}{c}\text { RCC } \\
(\mathbf{n}=\mathbf{3 4})\end{array}$ & $\begin{array}{c}\text { Squamous } \\
\text { NSCLC } \\
(\mathbf{n}=54)\end{array}$ & $\begin{array}{c}\text { Non-squamous } \\
\text { NSCLC } \\
(\mathbf{n}=\mathbf{7 4})\end{array}$ \\
\hline $\begin{array}{l}\text { Median OS, months } \\
\text { (95\% Cl) }\end{array}$ & $\begin{array}{c}20.3 \\
(12.5-37.9)\end{array}$ & $\begin{array}{c}22.4 \\
(12.5-48.6)\end{array}$ & $\begin{array}{c}9.2 \\
(7.3-12.5)\end{array}$ & $\begin{array}{c}10.1 \\
(5.8-13.7)\end{array}$ \\
$\begin{array}{l}\text { 3-year OS rate, \% } \\
\text { (95\% Cl) }\end{array}$ & $\begin{array}{c}42.3 \\
(32.7-51.6)\end{array}$ & $\begin{array}{c}40.1 \\
(23.6-56.0)\end{array}$ & $\begin{array}{c}20.3 \\
(10.5-32.4)\end{array}$ & $\begin{array}{c}17.0 \\
(9.0-27.2)\end{array}$ \\
$\begin{array}{l}\text { 4-year OS rate, \% } \\
\text { (95\% Cl) }\end{array}$ & $\begin{array}{l}35.3 \\
(26.2-44.5)\end{array}$ & $\begin{array}{c}37.0 \\
(21.1-53.0)\end{array}$ & $\begin{array}{c}16.3 \\
(7.6-27.8)\end{array}$ & $\begin{array}{c}15.3 \\
(7.7-25.3)\end{array}$ \\
\hline 5-year OS rate, \% & 34.2 & 27.7 & 16.3 & 15.3 \\
(95\% Cl) & $(25.2-43.4)$ & $(13.9-43.5)$ & $(7.6-27.8)$ & $(7.7-25.3)$ \\
\hline
\end{tabular}

P217

Clinical outcomes for the phase 2, single-arm, multicenter trial of JCAR015 in adult B-ALL (ROCKET Study)

Daniel J. DeAngelo ${ }^{1}$, Armin Ghobadi ${ }^{1}$, Jae H. Park ${ }^{3}$, Shira N. Dinner ${ }^{4}$ Gabriel N. Mannis, Matthew A. Lunning ${ }^{6}$, Samer K. Khaled ${ }^{7}$, Amir T. Fathi ${ }^{8}$, Ivana Gojo ${ }^{9}$, Eunice S. Wang ${ }^{10}$, Michael R. Bishop ${ }^{11}$, Heather Hughes $^{12}$, Samuel Blackman ${ }^{12}$, James Whitmore ${ }^{12}$, Nikolaus S. Trede ${ }^{12}$, Mark Gilbert ${ }^{12}$, William G. Wierda ${ }^{13}$

${ }^{1}$ Dana-Farber Cancer institute, Boston, MA, USA; ${ }^{3}$ Memorial SloanKettering Cancer Center, New York, NY, USA; ${ }^{4}$ Northwestern Medicine, Feinberg School of Medicine, Chicago, IL, USA; 5 UCSF Helen Diller Family Comprehensive Cancer Center, San Fransisco, CA, USA; ${ }^{6}$ Unversity of Nebraska Medical Center, Omaha, NE, USA; ${ }^{7}$ City of Hope, Duarte, CA, USA; ${ }^{8}$ Massachusetts General Hospital, Boston, MA, USA; ${ }^{9}$ Johns Hopkins Medicine, Baltimore, MD, USA; ${ }^{10}$ Roswell Park Cancer Institute, Buffalo, NY, USA; ${ }^{11}$ The University of Chicago Medicine, Chicago, IL, USA; ${ }^{12}$ Juno Therapeutics, Inc., Seattle, WA, USA; ${ }^{13}$ The University of Texas MD

Anderson Cancer Center, Houston, TX, USA

Correspondence: Daniel J. DeAngelo

(jessica.radtke@junotherapeutics.com)

Journal for ImmunoTherapy of Cancer 2017, 5(Suppl 2):P217

\section{Background}

Promising results have been reported in phase 1 studies of CD19-specific chimeric antigen receptor (CAR)-based cellular therapy for relapsed/refractory (R/R) aggressive B-cell malignancies. Based on a phase 1, single-center trial of a CD19-specific, CD28CD3z CAR T cell product candidate (19-28z) demonstrating high overall response rate (ORR) with manageable toxicity in adult BALL patients (NCT01044069), a phase 2, single-arm, multicenter trial evaluated a similar CD19-CAR T cell product candidate (JCAR015) [ROCKET; NCT02535364]. ROCKET was stopped due to unexpected fatal neurotoxicity (NTX), involving cerebral edema. Herein we report clinical outcomes from this trial.

Methods

ROCKET evaluated the safety and efficacy of two JCAR015 infusions $\left(10^{6}\right.$ and $3 \times 10^{6}$ cells $/ \mathrm{kg}$ separated by $>14$ days), in ECOG 0-2 adult patients with morphologically-R/R CD19 positive B-ALL. Primary endpoint was ORR defined as CR/CRi $>28$ days post-last dose. Patients with R/R disease were eligible, including prior-allo-transplant (HSCT), extramedullary disease and prior CNS leukemia. Bridging chemotherapy was permitted during manufacturing. Prior to first infusion, patients were restaged for morphologic disease and received lymphodepleting chemotherapy (LD) with either fludarabine (Flu) $\left(25 \mathrm{mg} / \mathrm{m}^{2} \times 3 \mathrm{~d}\right)$ and cyclophosphamide (Cy) $(30-60 \mathrm{mg} / \mathrm{kg} \times 1 \mathrm{~d})$ or single-dose Cy-only $\left(1-3 \mathrm{gm} / \mathrm{m}^{2}\right)$.

Results

ROCKET enrolled 82 patients, 57 underwent apheresis; 38 toxicityevaluable patients received $>1$ infusion. Median age was 39 (19-69 years) with a male preponderance (74\%), median prior lines of therapy was 2 $(1,7), 37 \%$ had previously undergone HSCT and $50 \%$ had received prior blinatumomab (Table 1). Primary efficacy analysis included 32 patients with morphologic disease ( $>5 \%$ blasts) prior to LD with Flu/Cy (8/32) or Cy-only (24/32). ORR per investigator assessment was 14/27 (52\%, 95\%Cl: $32-72 \%$ ) among response-evaluable patients. Median overall survival for all patients was 7.3 months (95\% Cl: $5.2,12.7)$ and 12.7 months (7.3, NR) among responders with median follow-up of 12.6 and 11.9 months, respectively. Median relapse-free survival was 4.4 months (2.1, $\mathrm{NR}$ ) and 9/14 (64\%) responders were alive at last follow-up.

Severe (Gr3-5) NTX and Gr3-4 CRS occurred in 20/38 (52\%) and 8/38 (21\%) patients, respectively. Notably, vascular leak in five cases of cerebral edema was restricted to the CNS, except for one patient with incidental radiographic findings compatible with interstitial pulmonary edema.

\section{Conclusions}

JCAR015 demonstrated anti-leukemia activity in adult B-ALL that was negatively impacted by fatal NTX. Efficacy compared favorably to standard therapy in this patient population with high unmet need [1]. NR: not reached

\section{References}

1. Gökbuget: Blood Cancer Journal.2016;6: e473.

Table 1 (abstract P217). Demographics or ROCKET patients

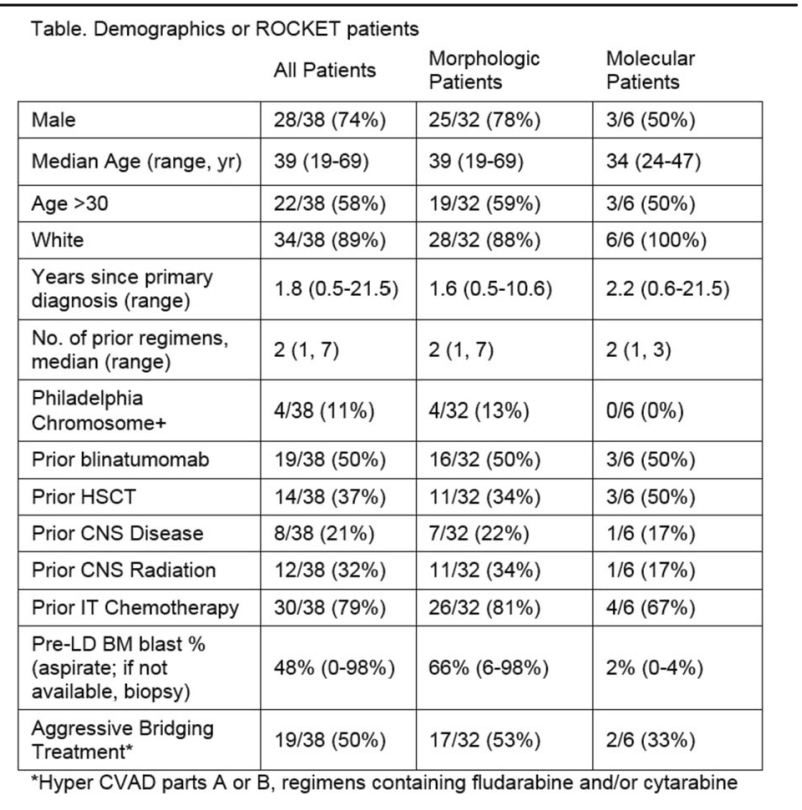

\section{Publisher's Note}

Springer Nature remains neutral with regard to jurisdictional claims in published maps and institutional affiliations. 